\title{
Doctor thesis
}

Investigation of high-precision $\Lambda$ hypernuclear spectroscopy via the $\left(\mathrm{e}, \mathrm{e}^{\prime} \mathrm{K}^{+}\right)$reaction

$$
\left(\mathrm{e}, \mathrm{e}^{\prime} \mathrm{K}^{+}\right) \text {反応を用いた }
$$

高分解能 $\Lambda$ ハイパ一核分光の研究

Department of Physics, School of Science, Tohoku University

Daisuke Kawama 




\begin{abstract}
The study of $\Lambda$ hypernuclear structure is very interesting in point of the understanding of the interaction between $\Lambda$ and nucleon ( $\Lambda N$ interaction) and its "strange" structure itself due to the containment of a $\Lambda$ hyperon which has a strangeness as a new degree of freedom. In the several way to study the $\Lambda$ hypernuclei, the $\left(\mathrm{e}, \mathrm{e}^{\prime} \mathrm{K}^{+}\right)$reaction spectroscopy is a powerful tool for the precise investigation of $\Lambda$ hypernuclear structure.

The purpose of the preset thesis is the establishment of the experimental design with the efficient data analysis method for the $\left(\mathrm{e}, \mathrm{e}^{\prime} \mathrm{K}^{+}\right)$hypernuclear spectroscopic experiment in the wide mass region (from $\mathrm{A}=7$ to $\mathrm{A}=52$ ). It is very challenging to perform the $\left(\mathrm{e}, \mathrm{e}^{\prime} \mathrm{K}^{+}\right)$spectroscopic experiment with such a heavy target, because of the huge electron background due to the bremsstrahlung process. In the experiment, it is required to obtain the necessary hypernuclear yield, suppressing the background event ratio. We achieved these requirements by newly constructing the high resolution electron spectrometer (HES) and splitter magnet (SPL) dedicated to the $\left(\mathrm{e}, \mathrm{e}^{\prime} \mathrm{K}^{+}\right)$spectroscopic experiment.

The HES consists of two quadrupole magnets and a dipole magnets (Q-Q-D) with a momentum resolution of $d p / p=3 \times 10^{-4}$ at $p=0.84 \mathrm{GeV} / c$. It was used being vertically tilted by 6.5 degree so as to optimize signal to noise ratio and hypernuclear yield.

The SPL is a dipole magnet. The experimental target was placed at the entrance of this magnet. The role of the SPL is to separate four kind of particles; scattered kaons, photons created by the bremsstrahlung, the post beam and scattered electrons. In addition, since the SPL is a part of the kaon and electron spectrometers. We designed the magnet shape carefully considering these points.

The experiment was performed with $2.344 \mathrm{GeV} / c$ electron beam from CEBAF at Jefferson Lab. The experimental setup consists of the HES, SPL and HKS (high momentum resolution kaon spectrometer). The HKS is also a Q-Q-D type spectrometer with the momentum resolution of $d p / p=2 \times 10^{-4}$ at $p=1.2 \mathrm{GeV} / c$.

In the data analysis, the particle momentum calibration was the most important procedure. At the initial point, the particle momentum was obtained from the calculated magnetic field map of the spectrometer whose accuracy is an order of $10^{-2}$. The initial momentum was calibrated by two step, the the magnetic field map improvement and the calibration with known masses of $\Lambda / \Sigma^{0}$ which were observed by the $\mathrm{CH}_{2}$ target data. As a result of the calibration, the momentum resolutions of HKS and HES were estimated as $4 \times 10^{-4}$ and $6 \times 10^{-4}$, respectively. Though these values are the double of the designed value, it was achieved to obtain the $\Lambda / \Sigma^{0}$ peaks with the same order of the designed energy from the original calculated magnetic field.

The cross section was calculated with the several estimated factors. The averaged $\mathrm{p}\left(\gamma^{*}, \mathrm{~K}^{+}\right) \Lambda$ cross section in the HKS acceptance, $\left(0.90<\cos \left(\theta_{K^{+}}^{C M}\right)<1.0\right)$ was calculated as $227 \pm 12 \pm 26$ [nb/sr], which is consistent within the error bar with the other experiment results of $\mathrm{p}\left(\gamma, K^{+}\right) \Lambda$. The obtained yield of the peak was almost same as the designed value with the considered detector efficiencies. The observed hypernuclear spectrum of ${ }_{\Lambda}^{12} \mathrm{~B}$ was also consistent with the other experimental results.

These analysis result represents that the experimental setup including the newly constructed HES and SPL worked and the calibration procedure of this unique experimental setup is basically established.
\end{abstract}





\section{Contents}

1 Introduction $\quad 1$

1.1 The role of hypernuclear physics in the modern physics . . . . . . . . . . 1

1.1.1 Hyperon and hypernucleus . . . . . . . . . . . . . . 1

1.1.2 Significance of hypernuclear spectroscopy . . . . . . . . . . . . . . 2

1.2 Historical background of the hypernuclear physics . . . . . . . . . . . . 4

1.2.1 Emulsion experiments via $\left(\mathrm{K}^{-}, \pi^{-}\right)$reaction . . . . . . . . . . . . 4

1.2.2 Counter experiments via the $\left(\mathrm{K}^{-}, \pi^{-}\right)$reaction . . . . . . . . . . 5

1.2.3 Counter experiments via the $\left(\pi^{+}, \mathrm{K}^{+}\right)$reaction . . . . . . . . . . . 6

1.2.4 Counter experiments via the $\left(\mathrm{e}, \mathrm{e}^{\prime} \mathrm{K}^{+}\right)$reaction . . . . . . . . . . 6

1.2.5 $\gamma$-ray and decay pion spectroscopy . . . . . . . . . . . 7

1.3 Hypernuclear production mechanisms . . . . . . . . . . . . . . . . 7

1.3.1 Comparison of each reaction . . . . . . . . . . . 8

1.3.2 Characteristics of the $\left(\mathrm{e}, \mathrm{e}^{\prime} \mathrm{K}^{+}\right)$reaction for hypernuclear spectroscopy 9

1.4 The purpose of the present study . . . . . . . . . . . . . . . . . 10

1.4.1 Physics motivations . . . . . . . . . . . . . . . . . 10

1.4.2 The goal of the present thesis . . . . . . . . . . . . 14

$2 \Lambda$ hypernuclear spectroscopy via the $\left(\mathrm{e}, \mathrm{e}^{\prime} \mathrm{K}^{+}\right)$reaction $\quad 17$

2.1 The elementary process of $\left(\mathrm{e}, \mathrm{e}^{\prime} \mathrm{K}^{+}\right)$reaction $\ldots \ldots \ldots . . \ldots 17$

2.2 Background sources of the $\left(\mathrm{e}, \mathrm{e}^{\prime} \mathrm{K}^{+}\right)$reaction . . . . . . . . . . . . . . 19

2.2.1 Background in scattered electron side . . . . . . . . . . . . . . 19

2.2.2 Background in kaon side . . . . . . . . . . . . . . . . 23

2.3 Previous $\left(\mathrm{e}, \mathrm{e}^{\prime} \mathrm{K}^{+}\right) \Lambda$ hypernuclear spectroscopic experiments at JLab Hall-

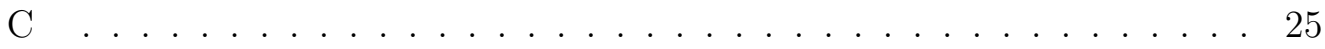

2.3.1 The first generation experiment (E89-009) . . . . . . . . . . 25

2.3.2 The second generation experiment (E01-011) . . . . . . . . . 28

2.4 The design concepts of the third generation experiment (E05-115) . . . . 31

2.4.1 Hypernuclear yield . . . . . . . . . . . . . . . . . . 33

2.4 Resolution ..................... 36

3 Experimental setup overview and trigger system 38

3.1 CEBAF electron accelerator facility . . . . . . . . . . . . . . . . . 38

3.1 .1 CEBAF overview . . . . . . . . . . . . . . . . . . . . . . . . . . . . . . . .

3.1 .2 Beamline apparatuses . . . . . . . . . . . . . . . 40

3.1 .3 Hall-C beamline . . . . . . . . . . . . . . . . . . . . . . . . . 42

3.1.4 Beam Raster . . . . . . . . . . . . . . . . . . . . . . . . . . 44 
3.2 Experimental condition and entire setup . . . . . . . . . . . . . 46

3.2 .1 Kinematics . . . . . . . . . . . . . . . . . . . . . . . 48

3.2.2 Particles to be detected . . . . . . . . . . . . . . . . 48

3.2.3 Tilt Method . . . . . . . . . . . . . . . . . . . . . 48

3.2.4 Expected singles rate and background suppression . . . . . . . . . . 50

3.2.5 Apparatus requirement from analysis . . . . . . . . . . . . . . 52

3.3 Experimental target and target system . . . . . . . . . . . . . . 53

3.3.1 Experimental target . . . . . . . . . . . . . . 53

3.3.2 Target system overview . . . . . . . . . . . . . . . . 55

3.3.3 Target ladder . . . . . . . . . . . . . . . . . . . . . 57

3.3 .4 Water-cell target . . . . . . . . . . . . . . . 59

3.3.5 BeO target and Target viewer . . . . . . . . . . . . . . . . 60

3.4 The SPL magnet . . . . . . . . . . . . . . . . . . . . . . . . 61

3.5 HKS magnets . . . . . . . . . . . . . . . . . . . . . . . . . . 64

3.5.1 Magents specification and alignment . . . . . . . . . . 65

3.5.2 Magnetic field . . . . . . . . . . . . . . . . . . . . . 65

3.5.3 Sieve slit and collimator . . . . . . . . . . . . . . . . 65

3.5.4 HKS magnets support . . . . . . . . . . . . . . . . . 70

3.6 HKS detectors . . . . . . . . . . . . . . . . . . . . . . . . 71

3.6.1 Drift chambers $(\mathrm{KDC} 1, \mathrm{KDC} 2) \ldots \ldots \ldots \ldots 71$

3.6.2 TOF counters . . . . . . . . . . . . . . . 72

3.6.3 Aerogel Cherencov counters . . . . . . . . . . . . . . . 77

3.6.4 Water Cherencov counters . . . . . . . . . . . . . . . . . 77

3.6.5 Backing coil for Cherencov counters . . . . . . . . . . . . . . . . 79

3.6.6 HKS background events and singles rate of HKS detectors . . . . . 83

3.7 HES magnets . . . . . . . . . . . . . . . . . . . . . . 86

3.7.1 Magnet specification . . . . . . . . . . . . . . 86

3.7.2 Magnetic field of HES . . . . . . . . . . . . . . . . . . 86

3.7.3 Sieve slit and collimator . . . . . . . . . . . . . . . 88

3.7.4 HES movable base . . . . . . . . . . . . . . . . . . . . . . 92

3.8 HES detectors . . . . . . . . . . . . . . . . . . . . . . . . . . 92

3.8.1 Drift Chambers . . . . . . . . . . . . . . . . . . 92

3.8.2 TOF counters . . . . . . . . . . . . . . . . . . . 96

3.8.3 Singles rate of HES detectors . . . . . . . . . . . . . . . . . 98

3.9 Trigger . . . . . . . . . . . . . . . . . . . . . . . . . . . . . . . . . . . 99

3.9.1 Trigger overview . . . . . . . . . . . . . . . . . . . . . 99

3.9.2 Tohoku Universal Logic module (TUL-8040) . . . . . . . . . . . . . 100

3.9.3 HKS single trigger . . . . . . . . . . . . . . . . . . . . . 101

3.9 .4 HES single trigger . . . . . . . . . . . . . . . . . 103

3.9.5 Coincidence trigger . . . . . . . . . . . . . . . . . . . . 103

3.9.6 Typical trigger rate and Trigger Supervisor . . . . . . . . . . . 103

3.10 Data acquisition system _ . . . . . . . . . . . . . . 105

3.10 .1 CODA . . . . . . . . . . . . . . . . . 105

3.10 .2 EPICS . . . . . . . . . . . . . . . . . 106

3.11 Data summary . . . . . . . . . . . . . . . . 106 
4 Design of the HES $\quad 107$

4.1 Experimental design of E05-115 experiment . . . . . . . . . . . . 107

4.1.1 Kinematics and optics . . . . . . . . . . . . . . . . . 107

4.1.2 The estimation of momentum resolution by first order . . . . . . . . 108

4.2 SPL Magnet . . . . . . . . . . . . . . . . . . . . . . 110

4.2.1 The role and design of SPL magnet . . . . . . . . . . . . . 111

4.2.2 SPL magnetic field calculation . . . . . . . . . . . . . . 113

4.3 HES Magnets . . . . . . . . . . . . . . . . . . . . 116

4.3.1 HES optics design . . . . . . . . . . . . . . . . . . . . 117

4.3.2 HES magnetic field . . . . . . . . . . . . . . . . . . . . 117

4.3 .3 Kinematics matching with HKS . . . . . . . . . . . . . . 119

4.4 HES tilt method . . . . . . . . . . . . . . . . . . . . . . 119

4.4.1 Fundamental of "tilt method" . . . . . . . . . . . . . . . . 119

4.4 .2 Tilt angle optimization . . . . . . . . . . . . . . . . . . . 122

4.5 Resolution and yield estimation . . . . . . . . . . . . . . . . . 123

4.5.1 Estimation of HES momentum resolution . . . . . . . . . . . . . . 123

4.5.2 Estimation of mass spectrum resolution . . . . . . . . . . . . . . . . 125

4.5.3 Hypernuclear yield estimation . . . . . . . . . . . . . 126

5 Data analysis 128

5.1 Analysis overview . . . . . . . . . . . . . . . . . . . . . . . . . . 128

5.2 Analysis on the HKS side . . . . . . . . . . . . . . . . . . . . 129

5.2.1 Particle tracking . . . . . . . . . . . . . . . . . . . . . . . . . 129

5.2.2 Kaon particle identification . . . . . . . . . . . . . . . . 132

5.3 Analysis on the HES side . . . . . . . . . . . . . . . . . . . . . . . . . 138

5.4 Coincidence time analysis of HKS and HES . . . . . . . . . . . . . . . . 142

5.4 .1 Coincidence time spectrum . . . . . . . . . . . . . . . . . . . . 142

5.4.2 Mixed event analysis for background estimation . . . . . . . . . . 144

5.5 Missing mass calibration . . . . . . . . . . . . . . . 145

5.5.1 Momentum reconstruction and calibration overview . . . . . . . . . 145

5.5.2 Target energy loss correction . . . . . . . . . . . . . . . . . 147

5.5.3 Raster correction . . . . . . . . . . . . . . . . . . . . . . . . . 148

5.5.4 Magnetic field improvement with the Sieve Slit data . . . . . . . . . 151

5.5.5 Direct calibration of the reconstruction function . . . . . . . . . 153

5.5 .6 Systematic errors of mass scale . . . . . . . . . . . . 155

5.6 Cross section . . . . . . . . . . . . . . . . . . . . . . . . . . 159

5.6 .1 Acceptance . . . . . . . . . . . . . . . . . . 162

5.6.2 The number of incoming electron and virtual photon . . . . . . . 165

5.6.3 Efficiencies and correction factors . . . . . . . . . . . . . . 167

5.6.4 Systematic errors for cross section . . . . . . . . . . . . . . 173

6 Experimental results and discussion $\quad 174$

6.1 Analysis of the $\mathrm{CH}_{2}$ target data . . . . . . . . . . . . . . . 174

6.1.1 Fitting function for mass spectrum . . . . . . . . . . . . . . . . 174

6.1.2 $\mathrm{CH}_{2}$ target missing mass spectrum . . . . . . . . . . . . . . . 174

6.1 .3 Evaluation of the resolution . . . . . . . . . . . . 175 
6.1.4 Cross section of the $\mathrm{p}\left(\gamma^{*}, \mathrm{~K}^{+}\right) \Lambda / \Sigma^{0}$ reaction . . . . . . . . . 178

6.2 Experimental results and discussion for ${ }_{\Lambda}^{12} \mathrm{~B} \ldots \ldots . \ldots . \ldots . \ldots 181$

6.2 .1 Mass spectra . . . . . . . . . . . . . . . . . . . . . 181

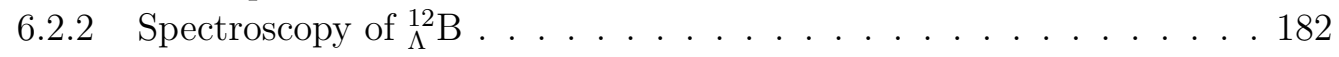

7 Conclusion and outlook $\quad 192$

$\begin{array}{ll}\text { A Mass references used in spectra calculation } & 198\end{array}$

B Experimental results and discussion for ${ }_{\Lambda}^{7} \mathrm{He}$ and ${ }_{\Lambda}^{10} \mathrm{Be} \quad 199$

B.1 Mass spectra . . . . . . . . . . . . . . . . . . . . . . . . . 199

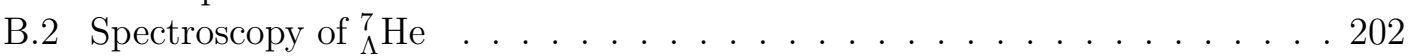

B.3 Spectroscopy of ${ }_{\Lambda}^{10} \mathrm{Be} \ldots \ldots \ldots \ldots$. . . . . . . . . . . . . . . . . . . . . . .

B.3.1 Comparison with mirror symmetric ${ }_{\Lambda}^{10} \mathrm{~B}$ data and theoretical calcu-

lation . . . . . . . . . . . . . . . . 207

C Parameters for the mass scale calibration $\quad 212$

C.1 Kinematic parameters . . . . . . . . . . . . . . . . . . . . . 212

C.2 Offset of the missing mass . . . . . . . . . . . . . . . . . . . . 212 


\section{List of Figures}

1.1 Flowchart of $\Lambda$ hypernuclear study . . . . . . . . . . . . . . . . 3

$1.2 \Lambda$ hypernuclear chart . . . . . . . . . . . . . . . . 5

1.3 Hyperon recoil momentum as a function of projectile momentum . . . . . 6

1.4 Schematic of the hypernuclear production via $\left(\mathrm{K}^{-}, \pi^{-}\right)(\mathrm{left})$ and $\left(\pi^{+}, \mathrm{K}^{+}\right)$ (right) reaction . . . . . . . . . . . . . . . . 8

1.5 Schematic of the hypernuclear production via the $\left(\mathrm{e}, \mathrm{e}^{\prime} \mathrm{K}^{+}\right)$reaction $\ldots . \quad 8$

1.6 Experimental results of ${ }_{\Lambda}^{4} \mathrm{H}$ and ${ }_{\Lambda}^{4} \mathrm{He} \ldots \ldots \ldots \ldots \ldots \ldots$

1.7 Calculated level structures of ${ }_{\Lambda}^{7} \mathrm{He},{ }_{\Lambda}^{7} \mathrm{Li}^{*}$ and ${ }_{\Lambda}^{7} \mathrm{Be} \ldots \ldots \ldots \ldots \ldots . . \ldots \ldots$

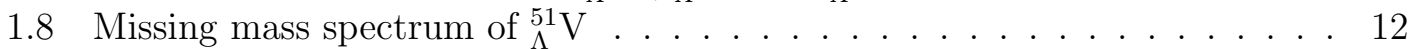

1.9 Calculated cross section of ${ }_{\Lambda}^{52} \mathrm{~V} \ldots \ldots \ldots \ldots \ldots \ldots$

$1.10 A$ dependence of $\Lambda$ single particle energy . . . . . . . . . . . . . . 15

2.1 Kinematics of the $\left(\mathrm{e}, \mathrm{e}^{\prime} \mathrm{K}^{+}\right)$reaction . . . . . . . . . . . . . . . 18

2.2 Scattered electron angular distribution via the $\left(\mathrm{e}, \mathrm{e}^{\prime} \mathrm{K}^{+}\right)$reaction . . . . . . 20

2.3 Scattered kaon angular distribution associated with the photo production of ${ }_{\Lambda}^{12} \mathrm{~B} 12$ ground state doublet . . . . . . . . . . . . . . . . . . . 20

2.4 Total cross section of the $\mathrm{p}\left(\gamma, \mathrm{K}^{+}\right) \Lambda$ reaction . . . . . . . . . . . . 21

2.5 Angular distribution of electron associated with bremsstrahlung process . . 22

2.6 Kinematics of $\mathrm{M} \phi$ ller scattering in the center of mass frame . . . . . . . . . 23

2.7 Angular distribution of electron associated with $\mathrm{M} \phi$ ller scattering . . . . . 24

2.8 Angular distribution of positron background . . . . . . . . . . . . . . . 24

2.9 Experimental setup of JLab E89-009 . . . . . . . . . . . . . . . . 26

$2.10{ }_{\Lambda}^{12} \mathrm{~B}$ spectrum measured in JLab E89-009 experiment . . . . . . . . . . . . 27

2.11 Experimental setup of JLab E01-011 . . . . . . . . . . . . . . 28

2.12 Angular distribution of the virtual photon / bremsstrahlung associated electron . . . . . . . . . . . . . . . . . 30

2.13 Concept of the tilt method . . . . . . . . . . . . . . . 31

$2.14{ }_{\Lambda}^{12} \mathrm{~B}$ spectrum measured in JLab E01-011 experiment . . . . . . . . . . . 32

2.15 The design concept of E05-115 experiment . . . . . . . . . . . . . . 34

2.16 Comparison angular distribution of bremsstrahlung electron with different

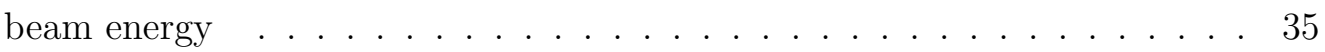

2.17 Comparison of angular acceptance between ESPS and HES . . . . . . 35

2.18 Schematic of the scattered electron and post beam track in the SPL . . . . 37

3.1 Schematic view of CEBAF electron accelerator . . . . . . . . . . . . . . 39

3.2 HallC beamline . . . . . . . . . . . . . . . . . . . . . 43

3.3 Picture of SPL vacuum chamber . . . . . . . . . . . . . . . . 45 
3.4 Schematic of beam trajectory around SPL . . . . . . . . . . . . . 45

3.5 Picture of raster pattern on the $\mathrm{CH}_{2}$ target . . . . . . . . . . . . . 46

3.6 Schematic view of the entire setup . . . . . . . . . . . . . . . 47

3.7 Picture of entire setup . . . . . . . . . . . . . . . . . 47

$3.8 \beta$ vs momentum correlations for pion, kaon and proton . . . . . . . . . 50

3.9 Picture of tilted HES . . . . . . . . . . . . . . . . . . . . 51

3.10 The effect of beam energy shift on the $P_{K}$ vs $P_{e^{\prime}}$ correlation $\ldots \ldots$. . . 53

3.11 Concept of SS tune . . . . . . . . . . . . . . . . . 54

3.12 Schematic of whole target system . . . . . . . . . . . . . . 56

3.13 Target system in the SPL magnet . . . . . . . . . . . . . . . . 56

3.14 Schematic of target ladder . . . . . . . . . . . . . . . . . . . . . 57

3.15 Picture of target plate attached in target ladder . . . . . . . . . . . . 58

3.16 Schematic view of the water-cell target . . . . . . . . . . . . . 61

3.17 Schematic view of Target Viewer . . . . . . . . . . . . . . . 62

$3.18 \mathrm{BeO}$ target monitoring during experiment . . . . . . . . . . . . . . 62

3.19 Schematic view of SPL magnet . . . . . . . . . . . . . . . 63

3.20 Picture of SPL magnet . . . . . . . . . . . . . . . . . . . . 64

3.21 Schematic view of HKS magnets . . . . . . . . . . . . . . . . . . . . . . . . . . . . . 64

3.22 Picture of HKS magnets . . . . . . . . . . . . . . . . . . . 65

3.23 Alignment of the HKS to the SPL magnet . . . . . . . . . . . . . 67

3.24 Excitation curve of KD magnet . . . . . . . . . . . . . . . 67

3.25 Picture of HKS Sieve Slit . . . . . . . . . . . . . . . . . . . . 68

3.26 CAD drawing of HKS Sieve Slit and collimator . . . . . . . . . . . . 69

3.27 HKS Sieve Slit pattern at reference plane . . . . . . . . . . . . . . . . 69

3.28 HKS magnets and common movable base . . . . . . . . . . . . . . . 70

3.29 HKS detector setup . . . . . . . . . . . . . . . . . . . . . . . 71

3.30 Picture of HKS detector setup . . . . . . . . . . . . . . . . . . 72

3.31 Schematic view of KDC . . . . . . . . . . . . . . . . 73

3.32 The definition of HKS coordinate system with KDC configuration . . . . . 74

3.33 Schematic view of KTOF-1X counters . . . . . . . . . . . . . . 75

3.34 Schematic view of KTOF-1Y counters . . . . . . . . . . . . . . 75

3.35 Schematic view of KTOF-2X counters . . . . . . . . . . . . . . 76

3.36 Schematic view of $\mathrm{AC}$ counters . . . . . . . . . . . . . . . . 77

3.37 Schematic view of $\mathrm{WC}$ counters . . . . . . . . . . . . . . . . 79

3.38 Momentum dependence of $\beta$ in HKS acceptance . . . . . . . . . . . . . . 80

3.39 Reflectivity comparison between acrylic and teflon . . . . . . . . . . . . . 81

3.40 Transmittance comparison of WC window materials candidates . . . . . . . 81

3.41 Typical photocathode spectral response . . . . . . . . . . . . . . . . . 82

3.42 Result of WC cosmic ray test . . . . . . . . . . . . . . . . . . . . . . . . . . . . . . . . . . 83

3.43 Basic concept of backing coil . . . . . . . . . . . . . . . . . . . . 84

3.44 Pictures of backing coil winded around AC and WC PMT . . . . . . . . 84

3.45 Monte Carlo simulation result of HKS background events . . . . . . . . . 85

3.46 Schematic view of HES spectrometer system . . . . . . . . . . . . . . 86

3.47 Picture of HES magnets . . . . . . . . . . . . . . . . . . . 87

3.48 Picture of HES Sieve Slit . . . . . . . . . . . . . . . . . . . . . . . . 88

3.49 CAD drawing of HES Sieve Slit and collimator plate . . . . . . . . . . . 89 
3.50 CAD drawing of HES Sieve Slit . . . . . . . . . . . . . . . . . . . 89

3.51 HES collimator position during experiment . . . . . . . . . . . . . . 90

3.52 HES Sieve Slit pattern at reference plane . . . . . . . . . . . . . . . 91

3.53 CAD drawing of HES movable base . . . . . . . . . . . . . . . . . . . . 92

3.54 HES detector setup . . . . . . . . . . . . . . . . . . . . 93

3.55 Picture of HES detectors . . . . . . . . . . . . . . . . . . . . . 93

3.56 Mechanical drawing of EDC1 . . . . . . . . . . . . . . . . . . 94

3.57 Picture of EDC1 . . . . . . . . . . . . . . . . . . . . . . . . . . 95

3.58 Coordinate system and wire configuration of EDC1 . . . . . . . . . 96

3.59 Schematic view of EHODO . . . . . . . . . . . . . . . . 97

3.60 Picture of EHODO . . . . . . . . . . . . . . . . . . . . . . 97

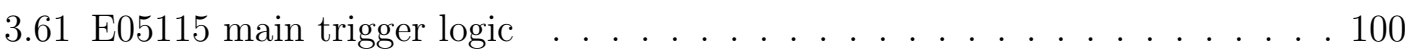

3.62 Picture of TUL-8040 f . . . . . . . . . . . . . . . 100

3.63 Schematics of a HKS grouping . . . . . . . . . . . . . . . . . 102

3.64 HKS trigger logic . . . . . . . . . . . . . . . . . . . . . . . 102

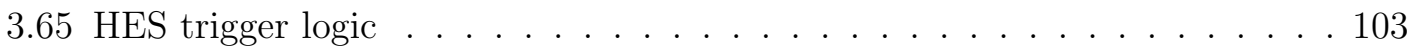

3.66 Logic around TS . . . . . . . . . . . . . . . . . . . . 104

3.67 CODA flow chart . . . . . . . . . . . . . . . . . . . . . . . . . . . . . . . . .

4.1 Kinematics of the $\left(\mathrm{e}, \mathrm{e}^{\prime} \mathrm{K}^{+}\right)$hypernuclear production reaction . . . . . . . . 108

4.2 Definition of HKS reference plane . . . . . . . . . . . . . . . . . . . . 109

4.3 Definition of HES reference plane . . . . . . . . . . . . . . . . . . . . 109

4.4 Vacuum chamber and pole shape of SPL magnet . . . . . . . . . . . . . . . . . . . . . 11

4.5 Particle coming to the $e^{\prime}$ exit of the SPL . . . . . . . . . . . . . . . . . 112

4.6 SPL 3D model in TOSCA calculation . . . . . . . . . . . . . . . . . 113

4.7 BH curve used in SPL model . . . . . . . . . . . . . . . . . . . . . . . . 114

4.8 Excitation curve of SPL magnet (measured and TOSCA) . . . . . . . . . 115

4.9 Magnetization of the SPL pole piece . . . . . . . . . . . . . . . 116

4.10 Typical tracks of the HES . . . . . . . . . . . . . . . . . . . 117

4.11 ED 3D model in TOSCA calculation . . . . . . . . . . . . . . . 118

4.12 EQ1 and EQ2 3D model in TOSCA calculation . . . . . . . . . . . 118

4.13 BH curve used in HES model . . . . . . . . . . . . . . . . . . . . . . . . . 119

4.14 Excitation curve of EQ1 magnet (measured and TOSCA) . . . . . . . . . 120

4.15 Excitation curve of EQ2 magnet (measured and TOSCA) . . . . . . . . . 120

4.16 Excitation curve of ED magnet (measured and TOSCA) . . . . . . . . . 121

4.17 Correlation between HKS momentum and HES momentum . . . . . . . . . 121

4.18 Definition of the tilt angle and spectrometer offset . . . . . . . . . . . . . 122

4.19 Figure of merit changing tilt angle value . . . . . . . . . . . . . 123

4.20 Estimation of HES momentum resolution . . . . . . . . . . . . . . . . . 124

4.21 Estimated Missing mass resolution in the Geant4 simulation . . . . . . . 125

5.1 E05115 analysis flow chart . . . . . . . . . . . . . . . . . 128

5.2 The relation drift time between drift length . . . . . . . . . . . . . 130

5.3 Typical plane resolution of KDC . . . . . . . . . . . . . . . . . . . . 131

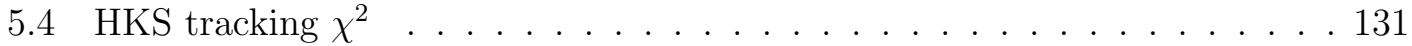

5.5 Typical $\beta$ distribution . . . . . . . . . . . . . . . . . . 132 
5.6 Typical $m^{2}$ distribution . . . . . . . . . . . . . . . . . . . . . . . . . . . 133

5.7 A typical NPE distribution of an layer of AC and scatter plot with $m^{2}$ distribution . . . . . . . . . . . . . . . . . . . 134

5.8 Typical fitting result of kaon with remained pion background . . . . . . . . 134

5.9 Typical AC NPE offline cut efficiency . . . . . . . . . . . . . . . . . . . . 135

5.10 A typical NPE distribution of an layer of WC and scatter plot with $m^{2}$ distribution . . . . . . . . . . . . . . . 136

5.11 A normalized NPE distribution of an layer of the WC and a scatter plot with $m^{2}$ distribution . . . . . . . . . . . . . . . . . . . . . . . 136

5.12 Typical WC NPE offline cut efficiency . . . . . . . . . . . . . . . 137

5.13 Typical fitting result of kaon with remained proton background . . . . . 138

5.14 Kaon PID on the $m^{2}$ distribution . . . . . . . . . . . . . . . . . . 139

5.15 Kaon cut using $m^{2}$ distribution . . . . . . . . . . . . . . . . . . . . . 140

5.16 The relation drift time between drift length . . . . . . . . . . . . . 141

5.17 Residual of the EDC1 and the EDC2 . . . . . . . . . . . . . . . . 141

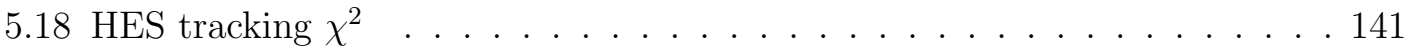

5.19 Typical coincidence time spectrum . . . . . . . . . . . . . . . . 143

5.20 The correlation between coincidence time spectrum and $m^{2}$. . . . . . 143

5.21 Basic idea of the mixed event analysis . . . . . . . . . . . . . . . 144

5.22 Result of the mixed event analysis . . . . . . . . . . . . . . . . . . 144

5.23 Simulated energy loss spectrum in $\mathrm{CH}_{2}$ target . . . . . . . . . . . . 148

5.24 Correlation between missing mass $(\Lambda)$ and $x$ at target . . . . . . . . . 150

5.25 Raster correction effect on $\Lambda$ and $\Sigma^{0}$ peak . . . . . . . . . . . . . . . 150

5.26 Position of the SPL and KQ1 magnet . . . . . . . . . . . . . . . . . 152

5.27 The modified HKS SS pattern . . . . . . . . . . . . . . . . . 153

5.28 The modified HES SS pattern . . . . . . . . . . . . . . . . . . . 153

5.29 The acceptance division for the $\chi^{2}$ calculation . . . . . . . . . . . 155

5.30 Procedure of momentum tuning . . . . . . . . . . . . . . 155

5.31 Flowchart of simulation data analysis . . . . . . . . . . . . . . 157

5.32 The result of the calibration with simulated ${ }_{\Lambda}^{12} \mathrm{~B} \ldots \ldots \ldots \ldots$

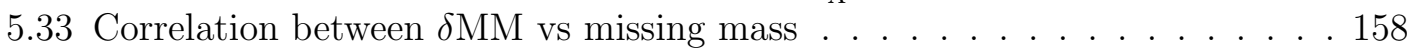

5.34 HKS solid angle . . . . . . . . . . . . . . . . . . . . . . . 162

5.35 HES solid angle . . . . . . . . . . . . . . . . . . . 163

5.36 Correlation between $\theta_{K^{+}}$and $\phi_{K^{+}} \ldots \ldots \ldots \ldots$. . . . . . . . . . . . . . . . . . . . .

5.37 Correlation between $x_{K^{+}}^{\prime}$ and $y_{K^{+}}^{\prime} \ldots \ldots \ldots \ldots$. . . . . . . . . . . . . . . . .

5.38 Correlation between $\theta_{e^{\prime}}$ and $\phi_{e^{\prime}} \ldots \ldots$. . . . . . . . . . . . . . 164

5.39 Correlation between $x_{e^{\prime}}^{\prime}$ and $y_{e^{\prime}}^{\prime} \ldots \ldots \ldots \ldots$. . . . . . . . . . . . . . . . . . . . . . . . . . . . . . . .

5.40 The selected region for the cross section calculation . . . . . . . . . . . 165

5.41 The virtual photon flux,$\Gamma(\omega) \ldots \ldots \ldots$. . . . . . . . . . . . . . . . . . .

5.42 ETOF1 hit pattern . . . . . . . . . . . . . . . . . . . . 168

5.43 The momentum dependence of kaon decay factor . . . . . . . . . . . 172

5.44 Computer live time factor for several run conditions . . . . . . . . . . . . 172

6.1 Comparison of the fitting function with simulated ${ }_{\Lambda}^{12} \mathrm{~B}$ missing mass $\ldots . .175$

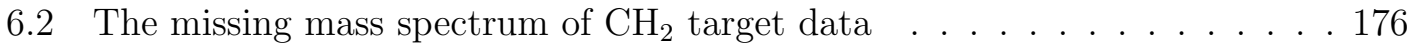

6.3 The relation between $\delta\left(p_{K^{+}}\right)$and $\delta\left(p_{e^{\prime}}\right) \ldots \ldots \ldots \ldots \ldots$ 
6.4 The missing mass spectrum for $\Lambda$ and $\Sigma^{0}$ with the vertical scale of differential cross section . . . . . . . . . . . . . . . . . . 179

6.5 Important kinematic parameters for $\Lambda$ and $\Sigma^{0} \ldots \ldots$. . . . . . . . 180

6.6 The missing mass spectrum for ${ }_{\Lambda}^{12} \mathrm{~B}$ with counts on the vertical scale . . . . 181

6.7 The missing mass spectrum for ${ }_{\Lambda}^{12} \mathrm{~B}$ with the vertical scale of differential cross section . . . . . . . . . . . . . . . . . . 183

6.8 The fitted missing mass spectrum of ${ }_{\Lambda}^{12} \mathrm{~B}$ (Vertical axis : differential cross section) . . . . . . . . . . . . . . . . . . 184

6.9 The missing mass spectrum of ${ }_{\Lambda}^{12} \mathrm{~B}$ obtained in JLab E89-009 experiment . 185

6.10 The missing mass spectrum of ${ }_{\Lambda}^{12} \mathrm{~B}$ obtained in JLab E94-107 experiment . 186

6.11 The missing mass spectrum of ${ }_{\Lambda}^{12} \mathrm{~B}$ obtained in JLab E01-011 experiment . 187

6.12 The missing mass spectrum of ${ }_{\Lambda}^{12} \mathrm{C}$ obtained by $\left(\pi^{+}, \mathrm{K}^{+}\right)$reaction . . . . 189

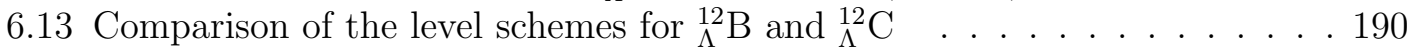

B.1 The missing mass spectrum for ${ }_{\Lambda}^{7} \mathrm{He}$ with counts on the vertical scale . . . 200

B.2 The missing mass spectrum for ${ }_{\Lambda}^{10} \mathrm{Be}$ with counts on the vertical scale . . . 201

B.3 The missing mass spectrum for ${ }_{\Lambda}^{7} \mathrm{He}$ with the vertical scale of differential cross section . . . . . . . . . . . . . . . . . . . 203

B.4 The fitted missing mass spectrum for ${ }_{\Lambda}^{7} \mathrm{He}$ (Vertical axis : differential cross section) . . . . . . . . . . . . . . . . . 204

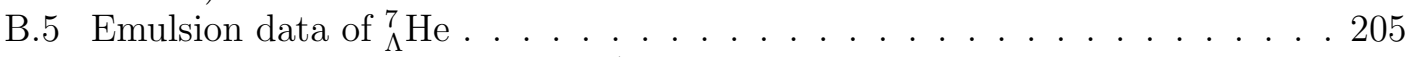

B.6 The missing mass spectrum for ${ }_{\Lambda}^{7} \mathrm{He}$ obtained in JLab E01-011 experiment 206

B.7 The missing mass spectrum for ${ }_{\Lambda}^{10} \mathrm{Be}$ with the vertical scale of differential cross section . . . . . . . . . . . . . . . . . . . . 208

B.8 The fitted missing mass spectrum of ${ }_{\Lambda}^{10} \mathrm{Be}$ (Vertical axis : differential cross section) . . . . . . . . . . . . . . . . . . 209

B.9 The missing mass spectrum of ${ }_{\Lambda}^{10} \mathrm{~B}$ obtained by $\left(\pi^{+}, \mathrm{K}^{+}\right)$reaction . . . . 210

B.10 Level scheme of ${ }_{\Lambda}^{10} \mathrm{Be},{ }_{\Lambda}^{10} \mathrm{~B}$ and their core nuclei . . . . . . . . . . . . . . 211

C.1 Background effect on the mass scale calibration _ . . . . . . . . . . 213 


\section{List of Tables}

1.1.1 Properties of light quarks . . . . . . . . . . . . . . . . . . . 2

1.1.2 List of hyperons $(\mathrm{S}=-1) \ldots \ldots \ldots \ldots \ldots \ldots$

1.3.1 Summary of reactions to produce hypernuclei $\ldots \ldots \ldots \ldots$

2.3.1 Specification of SOS . . . . . . . . . . . . . . 26

2.3.2 Specification of HKS and ESPS . . . . . . . . . . . . . . . . . 29

2.3.3 Comparison of the singles rate, hypernuclear yield of scattered electron side and luminosity . . . . . . . . . . . . . . . . . . . . . . . 29

2.4.1 Experimental target used in E89-009, E01-011 and E05-115 . . . . . . 33

3.1.1 The main parameter of CEBAF . . . . . . . . . . . . . . . . . 39

3.1.2 Beamline apparatus in E05-115 experiment . . . . . . . . . . . . . . . . 42

3.1.3 The beam raster applied during the experiment . . . . . . . . . . . . . 46

3.2.1 E05-115 experimental condition and spectrometer specification . . . . . . . 49

3.2 .2 Estimated singles rates for each target . . . . . . . . . . . . . 51

3.3.1 The target list . . . . . . . . . . . . . . . . . . 5 55

3.3.2 The melting point and expected max. temp. of the target . . . . . . 58

3.3 .3 The target plate list . . . . . . . . . . . . . . . . . . . 59

3.4.1 SPL magnet parameters . . . . . . . . . . . . . . . . . 63

$3.5 .1 \mathrm{KQ1}$ and KQ2 parameters . . . . . . . . . . . . . . . . . . . . 66

$3.5 .2 \mathrm{KD}$ parameters . . . . . . . . . . . . . . . . . 66

3.5.3 HKS magnetic field summary . . . . . . . . . . . . . . . . . . 66

3.5 .4 HKS SS parameters . . . . . . . . . . . . . . . . . . . . . 69

$3.6 .1 \mathrm{KDC}$ parameters . . . . . . . . . . . . . . . . . . . . . 73

$3.6 .2 \mathrm{KTOF}$ parameters . . . . . . . . . . . . . . . 76

$3.6 .3 \mathrm{AC}$ parameters . . . . . . . . . . . . . . . . . . . 78

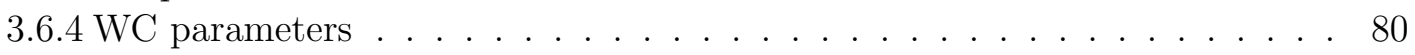

3.6.5 List of the backing coil parameters . . . . . . . . . . . . . . . . . . . . . . . . . . . 83

3.6.6 Summary of HKS detector singles rate . . . . . . . . . . . . . . . . . . . . . 85

3.7 .1 EQ1 and EQ2 parameters . . . . . . . . . . . . . . . 87

3.7 .2 ED parameters . . . . . . . . . . . . . . . . . 87

3.7.3 List of HES collimator position for each run conditions . . . . . . . . . . . 90

3.7.4 HES SS parameters . . . . . . . . . . . . . . . . . . . . . . . . . . . . . . . . . . . . . . . . . . . . . .

3.8.1 EDC1 parameters . . . . . . . . . . . . . . . . . . . . . . . . . . . . . . . . . . . . . . . . . . . . 95

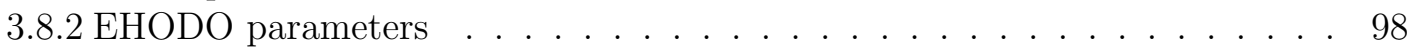

3.8.3 Summary of HES detector singles rate . . . . . . . . . . . . . . . . . . 98

3.9.1 Properties of TUL . . . . . . . . . . . . . . . . . . . . 101 
3.9 .2 Typical trigger rate for some conditions . . . . . . . . . . . . . . . . . 104

3.11.Run Summary . . . . . . . . . . . . . . . . . . . 106

4.1.1 E05-115 optics and kinematic parameters . . . . . . . . . . . . . 107

4.2.1 Momentum and exit angle of SPL magnet . . . . . . . . . . . . . . . 112

4.2.2 Material and mesh size in TOSCA 3D model . . . . . . . . . . . . . . . . . 114

4.3.1 HES magnetic field summary . . . . . . . . . . . . . . . . . . 116

4.3.2 Material and mesh size in HES TOSCA 3D model . . . . . . . . . . . . . 118

4.5.1 List of HES materials and their properties used in the simulation . . . . . 124

4.5.2 The list of the estimated yields . . . . . . . . . . . . . 127

5.2 .1 Calculated HKS FP resolution . . . . . . . . . . . . . . . . . . . 130

5.2 .2 Kaon cut efficiencies by AC NPE cut . . . . . . . . . . . . . . . . . . 135

5.2 .3 Kaon cut efficiencies by WC NPE cut . . . . . . . . . . . . . . . . . 137

5.2.4 Kaon cut efficiencies by $m^{2}$ cut . . . . . . . . . . . . . . . . . . . . 139

5.3.1 Calculated HES FP resolution . . . . . . . . . . . . . . . . . . . . . . . . . 142

5.4 .1 Kaon cut efficiencies by coin-time cut . . . . . . . . . . . . . . . . . 142

5.5.1 The number of terms of the $F 2 T$ function . . . . . . . . . . . . . . . 147

5.5.2 Energy loss at several targets . . . . . . . . . . . . . . . . . 149

5.5 .3 Obtained angular resolution from the SS data . . . . . . . . . . . . 153

5.5.4 The leakage effect for each peak . . . . . . . . . . . . . . . 157

5.6.1 List of the experimental efficiencies and factors . . . . . . . . . . . . 160

$5.6 .2 N_{\gamma^{*}}$ values used for the calculation of cross section . . . . . . . . . . . 167

5.6.3 HES trigger efficiency for several run conditions . . . . . . . . . . . . 167

5.6.4 HES tracking efficiency for several run conditions . . . . . . . . . . . . . . 169

5.6.5 HKS trigger efficiency for several run conditions . . . . . . . . . . . . . 169

$5.6 .6 \mathrm{HKS}$ tracking efficiency for several run conditions . . . . . . . . . . . 169

5.6.7 Properties of the HKS materials for kaon absorption factor estimation . . . 171

5.6.8 List of the kaon absorption factors . . . . . . . . . . . . . . . 173

5.6 .9 List of $\mathrm{K}^{+}$decay channels . . . . . . . . . . . . . . . . . . . . 173

5.6.10The systematic errors derived from experimental conditions . . . . . . . . 173

6.1.1 Fitting results of $\Lambda / \Sigma^{0}$ peaks ． . . . . . . . . . . . . . . . . 175

6.1.2 Considerable factors for the missing mass resolution . . . . . . . . . . . 177

6.1.3 Obtained differential cross sections and total cross sections of the $\mathrm{p}\left(\gamma^{*}, \mathrm{~K}^{+}\right) \Lambda / \Sigma^{0}$

reactions . . . . . . . . . . . . . . . . . . . . 179

6.1.4 Obtained differential cross sections and total cross sections of the $\mathrm{p}\left(\gamma^{*}, \mathrm{~K}^{+}\right) \Lambda$

reactions . . . . . . . . . . . . . . . . . . . 180

6.2.1 Obtained binding energies, excitation energies and width of ${ }_{\Lambda}^{12} \mathrm{~B} \ldots \ldots 2$

6.2.2 Obtained differential cross sections and total cross sections of ${ }_{\Lambda}^{12} \mathrm{~B} \ldots \ldots 3$

6.2.3 Comparison of kinematic conditions between hypernuclear experiments at

JLab . . . . . . . . . . . . . . . . . . . . . . 186

6.2.4 Comparison of excitation energies and width of ${ }_{\Lambda}^{12} \mathrm{~B} \ldots \ldots . . \ldots 188$

6.2.5 Comparison of excitation energies and width of ${ }_{\Lambda}^{12} \mathrm{~B} \ldots \ldots . . \ldots 188$

6.2.6 Obtained differential cross sections and total cross sections of ${ }_{\Lambda}^{12} \mathrm{C} \ldots \ldots 0$

6.2.7 Comparison of the differential cross section with the present results and theoretical calculation . . . . . . . . . . . . . . . . . . . 191 
A.0.1Particle mass references f . . . . . . . . . . . . . . . . . 198

B.2.1Obtained binding energies, excitation energies and width of ${ }_{\Lambda}^{7} \mathrm{He}$. . . . . . 202

B.2.2Obtained differential cross sections and total cross sections of ${ }_{\Lambda}^{7} \mathrm{He}$. . . . . 202

B.2.3Comparison of excitation energies and width of ${ }_{\Lambda}^{7} \mathrm{He} \ldots . . .20 . .206$

B.3.1Obtained binding energies, excitation energies and width of ${ }_{\Lambda}^{10} \mathrm{Be}$. . . . . 207

B.3.2Obtained differential cross sections and total cross sections of ${ }_{\Lambda}^{10} \mathrm{Be}$. . . . 207

B.3.3Obtained excitation energies and relative yields of ${ }_{\Lambda}^{10} \mathrm{~B} \ldots \ldots . . . . .210$

C.1.1The number of events with and without applying the $\chi^{2}$ cut . . . . . . 212 


\section{Chapter 1}

\section{Introduction}

A hypernucleus is a many-body system which consists of nucleons and hyperons. The spectroscopic study of hypernuclei is one of the most effective tools to understand the strong interaction in low energy regions and then it has been developed for the past several decades. First, this chapter introduces the significance of hypernuclear physics and its roles to play in the modern physics in section 1.1. After that, the historical background of hypernuclear physics is explained in section 1.2 comparing the reactions to produce hypernuclear in section 1.3. Finally, in section 1.4, the goal of the present thesis are shown.

\subsection{The role of hypernuclear physics in the modern physics}

\subsubsection{Hyperon and hypernucleus}

Understanding of the strong interaction is one of the most important issues remained in the modern physics. Basically, the strong interaction is mediated by gluons in quark level and QCD can describe the mechanism. Though QCD is a powerful tool for the description of high energy regions where perturbative QCD is effective, it is difficult to calculate QCD in low energy regions where momentum transfer is less than order of 1 $\mathrm{GeV}$. In such region, lattice QCD is the most promising tool for the description of the strong interaction from the first principle. Though it is developing with recent remarkable growth of computer technology, still impractical in low energy region.

In the low energy regions, some models of meson exchange between hadrons is popular for the practical calculation at this moment. In particular, the interaction between nucleons has been studied well phenomenologically with thousands of scattering experimental data. However, it is very difficult to expand the nucleon interaction to the general hadron interaction because of the lack of scattering experimental data, even in the case of hyperon $(Y)$-nucleon $(N)$ interaction which is the first step of the expansion of the nucleon interaction.

Table 1.1.1 shows the properties of quarks. A nucleon consists of up and down quarks and a hyperon is a hadron which includes strange quarks. Though the mass of an up quark is almost the same as that of a down quark, the mass of a strange quark is heavier than 
these quarks. This is the reason why we cannot simply expand the nucleon interaction to the $Y-N$ interaction. Furthermore, since it is very difficult to handle high quality hyperon beam because of its short lifetime, experimental data for the study of the $Y-N$ interaction is sparse. Table 1.1.1 shows the list of hyperons which consist of up, down and strange quarks. For example, the lightest hyperon is $\Lambda$ particle and its lifetime is the order of $10^{-10} \mathrm{sec}$ corresponding to $c \tau=7.89 \mathrm{~cm}$.

Table 1.1.1: Properties of light quarks. The masses in the table are current-quark masses.

\begin{tabular}{ccc}
\hline \hline Name & Mass $\left[\mathrm{MeV} / c^{2}\right]$ & Electric Charge \\
\hline down & $m_{d} \sim 1.5$ to 3.3 & $-1 / 3$ \\
up & $m_{u} \sim 3.5$ to 6.0 & $2 / 3$ \\
strange & $m_{s} \sim 104$ & $-1 / 3$ \\
\hline \hline
\end{tabular}

Table 1.1.2: List of hyperons $(\mathrm{S}=-1)$.

\begin{tabular}{ccccc}
\hline \hline Name & Mass $\left[\mathrm{MeV} / c^{2}\right]$ & Quark & $I\left(J^{P}\right)$ & Lifetime $[\mathrm{sec}]$ \\
\hline$\Lambda$ & 1115.68 & uds & $0\left(\frac{1}{2}^{+}\right)$ & $2.63 \times 10^{-10}$ \\
$\Sigma^{-}$ & 1197.45 & dds & $1\left(^{+}{ }^{+}\right)$ & $1.48 \times 10^{-10}$ \\
$\Sigma^{0}$ & 1192.64 & uds & $1\left(\frac{1}{2}^{+}\right)$ & $7.4 \times 10^{-20}$ \\
$\Sigma^{+}$ & 1189.37 & uus & $1\left(\frac{1}{2}^{+}\right)$ & $0.80 \times 10^{-10}$ \\
\hline \hline
\end{tabular}

In addition to the interest in hadronic interaction, it is also important motivation to study a hypernucleus as a many-body system of hadrons. Ordinary nuclei, that is a many-body system of nucleons, has been studied through some models such as cluster models and shell models. In the case of hypernuclei, it is being discussed whether these kinds of models including s-quarks can be applied, and it is valid for each hypernucleus or not.

\subsubsection{Significance of hypernuclear spectroscopy}

A hypernucleus is a bound state of hyperons in nucleons. Since a scattering experiment using a hyperon beam is hard to perform, study of hypernuclei plays an important role to investigate the property of the $Y-N$ interaction. In particular, $\Lambda$ hypernuclei has been ever studying because $\Lambda$ is the lightest hyperon and its production cross section is higher than other hyperons.

Figure 1.1 shows the flowchart of the $\Lambda$ hypernuclear study. Firstly, the theoretical calculation of hypernuclear configuration is necessary to interpret the obtained hypernuclear spectroscopic data. In other words, the model calculation has a role of "bridge" to derive some the $\Lambda$-N interaction information from the spectroscopic data of hypernuclei. In addition, unique characters of hypernuclei are discovered from the comparison between the model calculation and experimental data. 


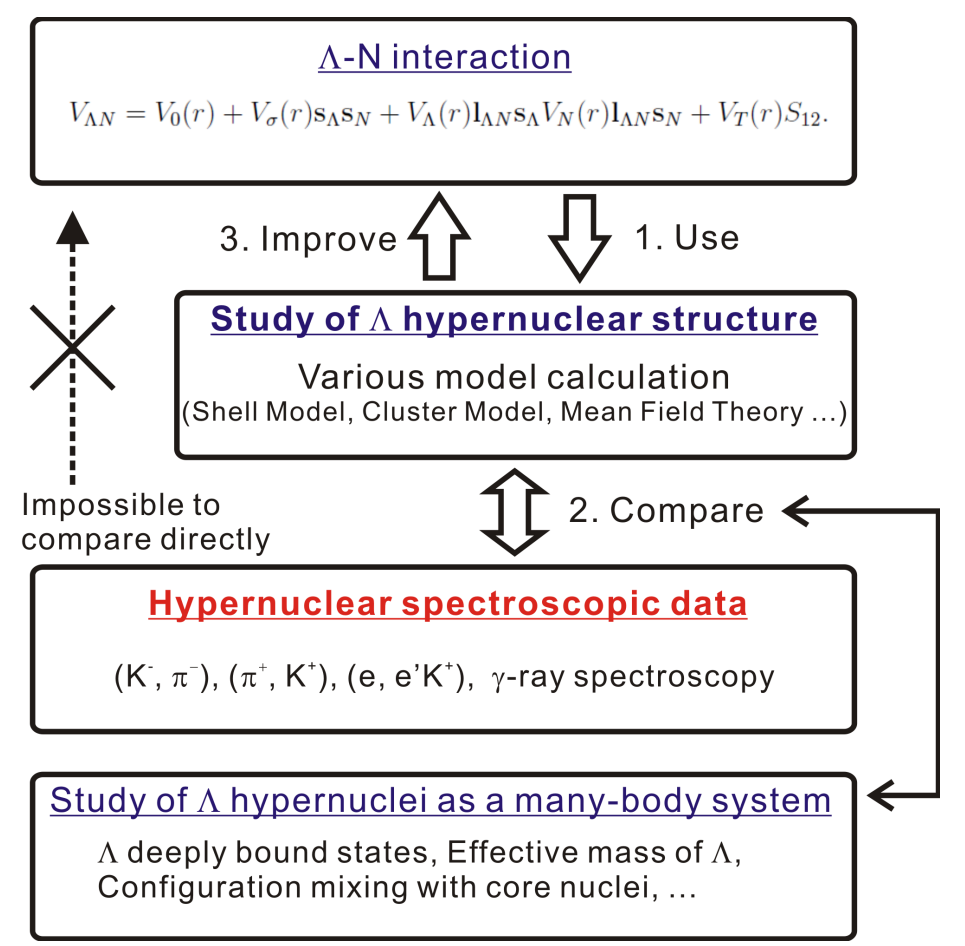

Figure 1.1: Flowchart of $\Lambda$ hypernuclear study.

The description of the $\Lambda$-N interaction is as follows. Assuming that a $\Lambda$ hypernuclear wave function can be decomposed into a core nucleus and a $\Lambda$ hyperon, the hypernuclear Hamiltonian is written as below [1]:

$$
H_{H Y}=H_{N}+H_{\Lambda}+\sum_{i} V_{\Lambda N_{i}}
$$

$H_{\mathrm{N}}$ is the Hamiltonian for a core nucleus ([2], [3]) and $H_{\Lambda}$ is a $\Lambda$ single particle Hamiltonian. Remained $V_{\Lambda N_{i}}$ represents a potential for a effective $\Lambda$ - $N$ interaction. This effective $\Lambda-N$ interaction is often constructed via a G-matrix calculation, starting from a two-body interactions in free space. One-boson-exchange models such as Nimegen [4], [5] and Jülich interaction [6], [7] are often used to describe the effective $\Lambda-N$ interaction.

For the case of $p$-shell hypernucleus, the effective $\Lambda-N$ interaction can be represented in the following form [8] [9]:

$$
V_{\Lambda N}=V_{0}(r)+V_{\sigma}(r) \mathbf{s}_{\Lambda} \mathbf{s}_{N}+V_{\Lambda}(r) \mathbf{l}_{\Lambda N} \mathbf{s}_{\Lambda} V_{N}(r) \mathbf{l}_{\Lambda N} \mathbf{s}_{N}+V_{T}(r) S_{12} .
$$

Here $\mathbf{s}$ and $\mathbf{l}$ denote the spin and orbital angular momentum and

$$
S_{12}=3\left(\sigma_{\Lambda} \hat{\mathbf{r}}\right)\left(\sigma_{N} \hat{\mathbf{r}}\right)-\sigma_{\Lambda} \sigma_{N}
$$

is the tenser force term $\left(r=\left(\mathbf{r}_{\Lambda} \times \mathbf{r}_{N}\right) /\left|\mathbf{r}_{\Lambda} \times \mathbf{r}_{N}\right|\right)$, respectively. Formation of the lowlying excited states of $p$-shell $\Lambda$ hypernuclei can be described with the radial integrals of the five terms in equation 1.2. The integrated value, which is defined as $V_{0}, V_{\sigma}, V_{\Lambda}, V_{N}$ 
and $V_{T}$, respectively can be determined by the fit to the data of $p$-shell $\Lambda$ hypernuclear spectroscopic data. In particular, $\gamma$-ray spectroscopy data are significant for this study because of its high energy resolution (about a few $\mathrm{keV}$ ).

Another unique aspect of hypernuclei is that a hypernucleus has contains "impurity", that is nothing other than hyperons. A hyperon is free from Pauli blocking in an ordinary nuclei because it contains at least one strange quark. Therefore a hyperon can penetrate into the nuclear interior and form deeply bound hypernuclear states which cannot be measured in the ordinary nuclear structure. In the case of ordinary nuclei, deeply bound states can be investigated via the nucleon knockout reactions such as $\left(e, e^{\prime} p\right)$ and $(p, 2 p)$, but the nuclear bound states get broader for the deeper bound states. For instance, the width of $1 d$-shell proton states of ${ }^{208} \mathrm{~Pb}$ reach as $5 \mathrm{MeV}$, which is too wide to do a precise spectroscopic study.

\subsection{Historical background of the hypernuclear physics}

The first hypernucleus was identified through the decay in a nuclear emulsion in 1953 [10]. After that, the hypernuclear experiments have been developed through following four stages.

1. Emulsion experiments via the $\left(\mathrm{K}^{-}, \pi^{-}\right)$reaction at $\mathrm{CERN}^{1}$ and $\mathrm{BNL}^{2}$ in 1960 's.

2. Counter experiments via the $\left(\mathrm{K}^{-}, \pi^{-}\right)$reaction at CERN and BNL from early-1970's to 1980 's.

3. Counter experiments via the $\left(\pi^{+}, \mathrm{K}^{+}\right)$reaction at BNL and KEK from mid-1980's to 1990's.

4. Counter experiments via the $\left(\mathrm{e}, \mathrm{e}^{\prime} \mathrm{K}^{+}\right)$reaction at JLab from 2000's.

Figure 1.2 shows the hypernuclear chart obtained by the above experiments. The history of hypernuclear experiment is explained in this section along above four stages and referring the hypernuclear chart. The detail of each reaction is explained section 1.3.

\subsubsection{Emulsion experiments via $\left(\mathrm{K}^{-}, \pi^{-}\right)$reaction}

The study of hypernucleus with a $\mathrm{K}^{-}$beam started with emulsion techniques at CERN and BNL. In this experiment, only light hypernuclei $(\mathrm{A} \leq 16)$ were observed through their weak decay process. Furthermore, experimental data is limited to the binding energies of ground state and the excited states could not be investigated except for a few case. However, it is important to obtain the binding energies of the ground states of hypernuclei with a small systematic error of the emulsion measurement. In particular, the binding energies of ${ }_{\Lambda}^{7} \mathrm{He},{ }_{\Lambda}^{10} \mathrm{Be}$ and ${ }_{\Lambda}^{12} \mathrm{~B}$ are important for the present thesis. The detail of these data will be discussed in chapter 6 .

\footnotetext{
${ }^{1}$ European Organization for Nuclear Research, Geneve, Switzerland

${ }^{2}$ Brookhaven National Laboratory, NY, US
} 


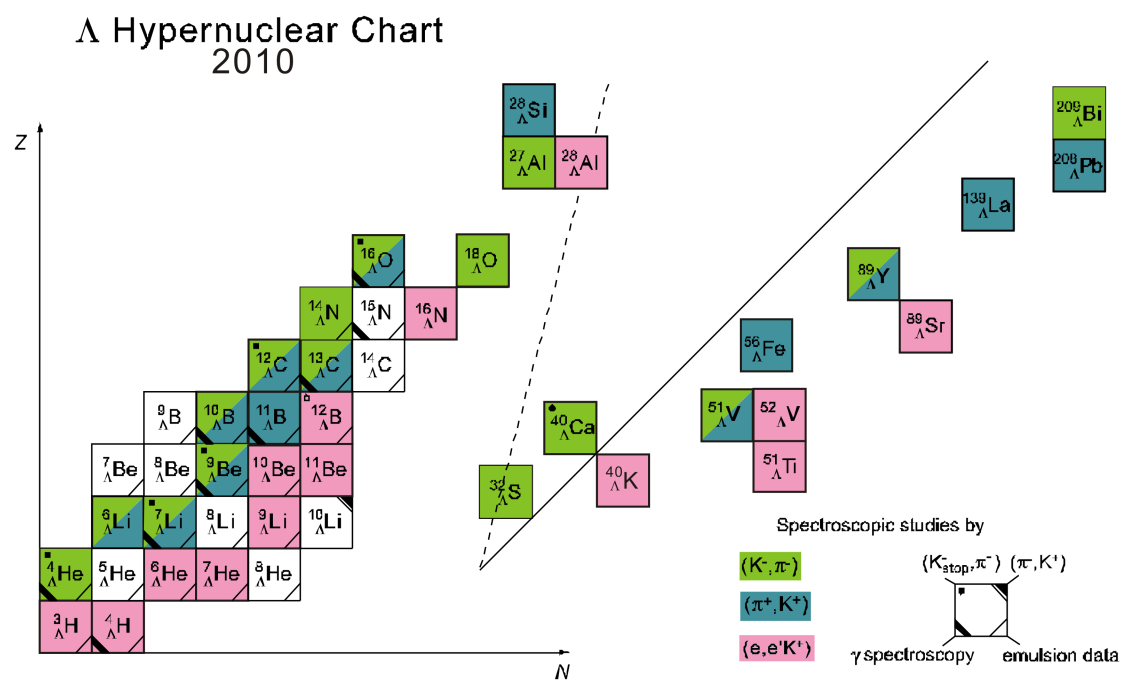

Figure 1.2: $\Lambda$ hypernuclear chart. For the $\left(\mathrm{e}, \mathrm{e}^{\prime} \mathrm{K}^{+}\right)$reaction spectroscopy, it contains the hypernuclear "candidates" that is possible to measure by the $\left(\mathrm{e}, \mathrm{e}^{\prime} \mathrm{K}^{+}\right)$reaction.

\subsubsection{Counter experiments via the $\left(\mathrm{K}^{-}, \pi^{-}\right)$reaction}

In the early 1970's, counter experiments started at CERN and later in BNL using a K ${ }^{-}$ beam. This method made it possible to carry out the spectroscopic experiments including excited states of hypernuclei.

At the beginning of this stage, $\left(\mathrm{K}_{\text {stop }}^{-}, \pi^{-}\right)$reaction was used because this method does not need to measure kaon. The $\gamma$-ray transitions from the excited states [11] and the first spectroscopic experiment with a magnetic spectrometer via ${ }^{12} \mathrm{C}\left(\mathrm{K}_{\text {stop }}^{-}, \pi^{-}\right){ }_{\Lambda}^{12} \mathrm{C}$ reaction $[12]$ is worthy of special mention.

This method taken over by the noble in-flight $\left(\mathrm{K}^{-}, \pi^{-}\right)$reaction in almost recoilless condition for various $\Lambda$ hypernuclei up to mass number $A=209$ [13, 14]. In particular, $p$-shell hypernuclei were studied well and it was found that the spin-orbit splitting of $\Lambda$ orbits are quite small [15].

Studies of hypernuclei via the $\left(\mathrm{K}^{-}, \pi^{-}\right)$reaction are powerful tool particulary for $p$ shell hypernuclei. Nevertheless, sometimes the statistics were not enough because of low intensity kaon beam and a limited energy resolution of the spectra in these era. Additionally, the $\left(\mathrm{K}^{-}, \pi^{-}\right)$reaction favors the excitation of substitutional states because the momentum transfer of the reaction was small in the available kaon beam energy at that time. Therefore the $\left(\pi^{+}, \mathrm{K}^{+}\right)$reaction become to be used for the study of various hypernuclei, as mentioned in the next subsection in 1980's. However, if an enough intensity of kaon beam is available, this problem will be overcome and $\mathrm{J}_{-} \mathrm{PARC}^{3}$ start running right now aiming to the next decade. A high-intensity kaon beam can be used there, then the $\left(\mathrm{K}^{-}, \pi^{-}\right)$reaction will be the one of the important tools to investigate hypernuclei.

\footnotetext{
${ }^{3}$ Japan Proton Accelerator Research Complex, Tokai, JAPAN
} 


\subsubsection{Counter experiments via the $\left(\pi^{+}, \mathrm{K}^{+}\right)$reaction}

From mid-1980's counter experiments via the $\left(\pi^{+}, \mathrm{K}^{+}\right)$reaction began at the AGS in BNL $[16,17]$ and later $12 \mathrm{GeV}^{\mathrm{PS}}$ in $\operatorname{KEK}^{4}[18,19,20,21,22]$. As mentioned in the next section, the $\left(\pi^{+}, \mathrm{K}^{+}\right)$reaction is a powerful tool to measure variable states of hypernuclei because of its large momentum transfer (figure 1.3). In particular, the superconducting kaon spectrometer (SKS) placed at K6 beamline in KEK $12 \mathrm{GeV}$ PS plays a significant role for the investigation of various hypernuclear spectroscopy with high resolution of 1.45-2.0 $\mathrm{MeV}$ (FWHM) and high statistics [19, 20, 21, 22]. With this fine resolution, various peak structures were measured in the wide range of the hypernuclear spectra including deeply bound states such as $p_{\Lambda}$ states. Particularly, the observation of fine peak structure for light hypernuclei such as ${ }_{\Lambda}^{7} \mathrm{Li},{ }_{\Lambda}^{9} \mathrm{Be},{ }_{\Lambda}^{10} \mathrm{~B},{ }_{\Lambda}^{12} \mathrm{C},{ }_{\Lambda}^{13} \mathrm{C}$ and ${ }_{\Lambda}^{16} \mathrm{O}$ provided important information. For heavier hypernuclei, ${ }_{\Lambda}^{28} \mathrm{Si},{ }_{\Lambda}^{51} \mathrm{~V},{ }_{\Lambda}^{89} \mathrm{Y},{ }_{\Lambda}^{139} \mathrm{La}$ and ${ }_{\Lambda}^{208} \mathrm{~Pb}$ were measured and it was found that the single particle behavior of $\Lambda$ in hypernuclei (see section 1.4).
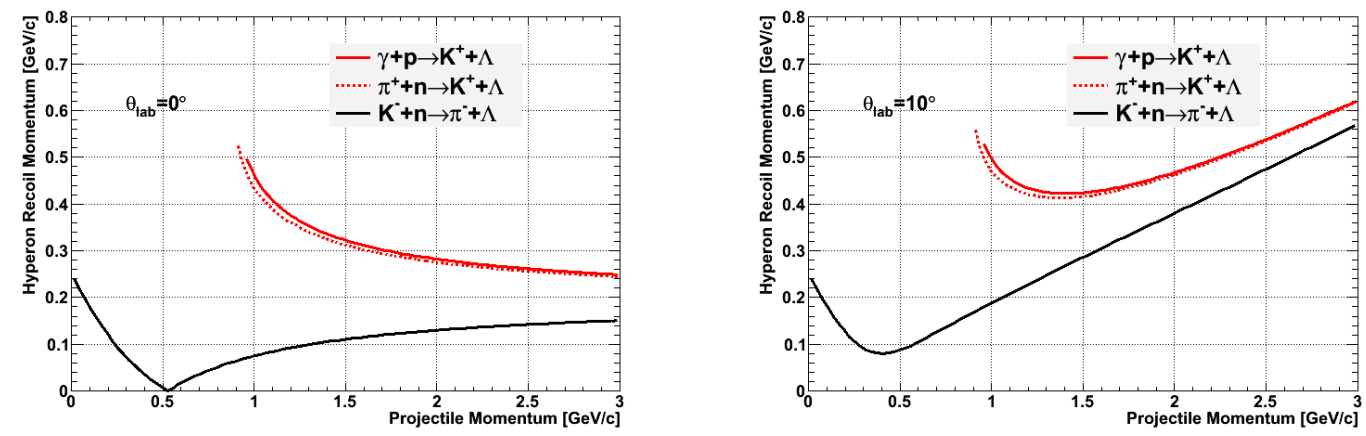

Figure 1.3: Hyperon recoil momentum as a function of projectile momentum.

\subsubsection{Counter experiments via the $\left(e, e^{\prime} K^{+}\right)$reaction}

In 2000, the first hypernuclear spectroscopic experiment (E89-009) via the (e, $\left.\mathrm{e}^{\prime} \mathrm{K}^{+}\right)$reaction was performed in $\mathrm{JLab}^{5}$ Hall-C with ${ }^{12} \mathrm{C}$ target. This method can achieve an energy resolution of several hundred $\mathrm{keV}$ because of the high quality electron beam of JLab. In addition, it is possible to create neutron-rich hypernuclei with this reaction because the $\left(\mathrm{e}, \mathrm{e}^{\prime} \mathrm{K}^{+}\right)$reaction converts neutron into $\Lambda$. This experiment proved that this noble method with the high quality electron beam is possible.

After that, we newly designed and constructed high resolution kaon spectrometer (HKS) dedicated to the hypernuclear spectroscopic experiment. Furthermore, we also introduced new experimental configuration to avoid huge background in scattered electron side so called "tilt method". The second experiment (E01-011) in 2005 implemented these new techniques and successfully took experimental data for ${ }^{7} \mathrm{Li},{ }^{12} \mathrm{C}$ and ${ }^{28} \mathrm{Si}$ targets proving the techniques worked well.

For the third experiment (E05-115), we designed and constructed a new high resolution electron spectrometer (HES) for the spectroscopy of medium hypernuclei up to mass

\footnotetext{
${ }^{4}$ High Energy Accelerator Research Organization, Tsukuba, JAPAN

${ }^{5}$ Thomas Jefferson National Accelerator Facility, Newport News, VA, US
} 
number $A=52$. The experiment was carried out successfully in 2009, aiming to take spectroscopic data for ${ }^{7} \mathrm{Li},{ }^{9} \mathrm{Be},{ }^{10} \mathrm{~B},{ }^{12} \mathrm{C}$ and ${ }^{52} \mathrm{Cr}$ targets.

This is the overview of the $\left(\mathrm{e}, \mathrm{e}^{\prime} \mathrm{K}^{+}\right)$reaction. The detail of this reaction and history of the experiments carried out in JLab Hall-C will be explained in chapter 2 .

\subsection{5 $\gamma$-ray and decay pion spectroscopy}

It should be mentioned that the $\gamma$-ray hypernuclear spectroscopy technique using Ge detector has been developed from the end of the 1990's. This is a technique to measure the level scheme of hypernuclei observing the $\gamma$-ray emitted by the hypernuclear level transition. The hypernuclei were mainly created by the $\left(\mathrm{K}^{-}, \pi^{-}\right)$and $\left(\pi^{+}, \mathrm{K}^{+}\right)$reactions. Therefore, this method can determine the energy spacing of hypernuclear states with the excellent energy resolution of Ge detector of a few $\mathrm{keV}$ (FWHM). The provided energy spacing data is important for the understanding of the $\Lambda \mathrm{N}$ interaction and unique characteristics of hypernuclei [23].

Furthermore, a new method of decay pion spectroscopy is now under developing. The principle of this method is to measure the emitted pion from the weak decay of hypernucleus such as ${ }_{\Lambda}^{4} \mathrm{H} \rightarrow{ }^{4} \mathrm{He}+\pi^{-}$. This experiment has been performed in the 1980's using stopped $\left(\mathrm{K}^{-}, \pi^{-}\right)$reaction at $\mathrm{KEK}[24]$ and the peak of ${ }_{\Lambda}^{4} \mathrm{H}$ was measured at that time. In future, the decay pion spectroscopy via the $\left(\mathrm{e}, \mathrm{e}^{\prime} \mathrm{K}^{+}\right)$reaction at JLab and MAINZ is now under planning. By using a thin target, the energy resolution of the experiment is estimated to be about $100 \mathrm{keV}$.

\subsection{Hypernuclear production mechanisms}

The history of hypernuclear spectroscopy was explained in the last section and three hypernuclear production reactions were mainly used for the spectroscopic study; the $\left(\mathrm{K}^{-}, \pi^{-}\right),\left(\pi^{+}, \mathrm{K}^{+}\right)$and $\left(\mathrm{e}, \mathrm{e}^{\prime} \mathrm{K}^{+}\right)$reactions. These reactions are compared each other and summarized in table 1.3. In this section, the mechanism of the three reactions will be explained and compared each other.

Table 1.3.1: Summary of reactions to produce hypernuclei. Incident energy is a typical value used in the past experiment.

\begin{tabular}{ccccc}
\hline \hline Reaction & Reaction type & $\begin{array}{c}\text { Cross Section } \\
{[\mu \mathrm{b} / \mathrm{sr}]}\end{array}$ & $\begin{array}{c}\text { Incident energy } \\
{[\mathrm{GeV} / \mathrm{c}]}\end{array}$ & $\begin{array}{c}\text { Recoil momentum } \\
{[\mathrm{MeV} / \mathrm{c}]}\end{array}$ \\
\hline in-flight $\left(\mathrm{K}^{-}, \pi^{-}\right)$ & $\mathrm{n}$ to $\Lambda$ & $10^{3}$ & 0.8 & $q<100$ \\
stopped $\left(\mathrm{K}^{-}, \pi^{-}\right)$ & $\mathrm{n}$ to $\Lambda$ & $10^{2}$ & 0 & $\mathrm{q}=250$ \\
$\left(\pi^{+}, \mathrm{K}^{+}\right)$ & $\mathrm{n}$ to $\Lambda$ & 10 & 1.0 & $q>300$ \\
$\left(\mathrm{e}, \mathrm{e}^{\prime} \mathrm{K}^{+}\right)$ & $\mathrm{p}$ to $\Lambda$ & $10^{-1}$ & 2.0 & $q>300$ \\
\hline \hline
\end{tabular}




\subsubsection{Comparison of each reaction}

Figure 1.4 shows the hypernuclear production mechanism via the $\left(\mathrm{K}^{-}, \pi^{-}\right)$reaction and the $\left(\pi^{+}, \mathrm{K}^{+}\right)$reaction. Both reactions use a meson beam and a neutron in the target nuclei for $\Lambda$ creation, but the $\left(\mathrm{K}^{-}, \pi^{-}\right)$reaction is strangeness exchange reaction and the $\left(\pi^{+}, \mathrm{K}^{+}\right)$reaction create $s-\bar{s}$ pair associatively. Therefore, according to the OZI rule, the cross section of $\left(\pi^{+}, \mathrm{K}^{+}\right)$reaction is suppressed compared with that of $\left(\mathrm{K}^{-}, \pi^{-}\right)$reaction. The typical value of different cross sections is the order of $10^{3} \mu \mathrm{b} / \mathrm{sr}$ in in-flight $\left(\mathrm{K}^{-}, \pi^{-}\right)$ reaction and the order of $10 \mu \mathrm{b} / \mathrm{sr}$ in the $\left(\pi^{+}, \mathrm{K}^{+}\right)$reaction. Thus, the $\left(\pi^{+}, \mathrm{K}^{+}\right)$reaction experiment compensate the hypernuclear yield by a higher intensity beam compared to the kaon beam used in $\left(\mathrm{K}^{-}, \pi^{-}\right)$reaction experiment. Therefore detectors used in the $\left(\pi^{+}, \mathrm{K}^{+}\right)$reaction need to overcome the high rate. This is the reason why the $\left(\pi^{+}, \mathrm{K}^{+}\right)$ reaction experiment was difficult and performed later than $\left(\mathrm{K}^{-}, \pi^{-}\right)$reaction experiment.

Figure 1.5 is a schematic of hypernuclear production mechanism via the $\left(\mathrm{e}, \mathrm{e}^{\prime} \mathrm{K}^{+}\right)$ reaction. This reaction also create $s-\bar{s}$ pair associatively via the electromagnetic reaction and the cross section of the reaction is the order of $0.1 \mu \mathrm{b} / \mathrm{sr}$, two orders of magnitude less than that of $\left(\pi^{+}, \mathrm{K}^{+}\right)$reaction. Therefore, we need a high intensity electron beam which makes experiment much more difficult than the hadronic reaction experiments. The high intensity beam itself can be easily obtained, but the singles rate of the detectors get to $\mathrm{MHz}$ order. This high rate make the experiment extremely difficult.
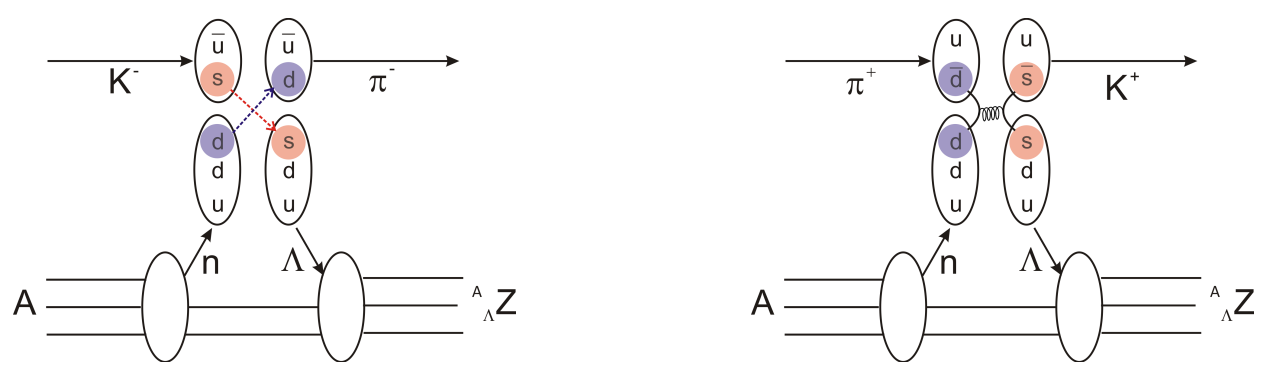

Figure 1.4: Schematic of the hypernuclear production via $\left(\mathrm{K}^{-}, \pi^{-}\right)$(left) and $\left(\pi^{+}, \mathrm{K}^{+}\right)$ (right) reaction.

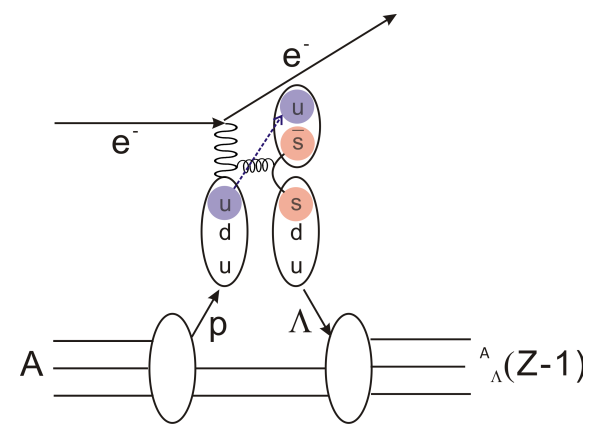

Figure 1.5: Schematic of the hypernuclear production via the $\left(\mathrm{e}, \mathrm{e}^{\prime} \mathrm{K}^{+}\right)$reaction.

Figure 1.3 shows the hyperon recoil momentum as a function of a projectile momentum for the three reactions. In order to create hypernucleus, the hyperon recoil momentum 
is important because hyperon has to stay bound in target nuclei and the probability of the station, so called "sticking probability", depends on it. If the momentum is too large compared to the Fermi momentum (about $270 \mathrm{MeV} / c$ ), hyperon will leave from target nuclei and the sticking probability will be small. Therefore the recoil momentum has to be smaller than the Fermi momentum. However, if the recoil momentum is too small the hyperon keep same orbit as the original nucleon.

In the case of the $\left(\mathrm{K}^{-}, \pi^{-}\right)$reaction which is endothermal reaction and the recoil momentum is low. In particular, the recoil momentum of the in-flight $\left(\mathrm{K}^{-}, \pi^{-}\right)$reaction is less than $100 \mathrm{MeV} / c$ at projectile momentum $0.8 \mathrm{GeV} / c$, then the kaon react with a nucleon in outer shell. This is why the in-flight $\left(\mathrm{K}^{-}, \pi^{-}\right)$reaction is difficult to measure a deeply bound state of $\Lambda$ hypernucleus and favor to excite substitutional state at zero degree. In the case of the stopped $\left(\mathrm{K}^{-}, \pi^{-}\right)$reaction, the recoil momentum is $250 \mathrm{MeV} / c$ and various states of hypernuclei can be excited.

On the other hand, the recoil momentum of the $\left(\pi^{+}, \mathrm{K}^{+}\right)$and the $\left(\mathrm{e}, \mathrm{e}^{\prime} \mathrm{K}^{+}\right)$reaction is about $300 \mathrm{MeV} / c$, which is much larger than that of the in-flight $\left(\mathrm{K}^{-}, \pi^{-}\right)$reaction. Then, it becomes possible to excite deeply bound states of $\Lambda$ hypernuclei, although the sticking probability becomes smaller in this region.

\subsubsection{Characteristics of the $\left(e, \mathrm{e}^{\prime} \mathrm{K}^{+}\right)$reaction for hypernuclear spectroscopy}

The advantages of the $\left(\mathrm{e}, \mathrm{e}^{\prime} \mathrm{K}^{+}\right)$reaction for hypernuclear spectroscopy can be summarized as follows.

1. To convert a proton into a $\Lambda$. Since the $\left(\mathrm{K}^{-}, \pi^{-}\right)$and $\left(\pi^{+}, \mathrm{K}^{+}\right)$reaction convert a neutron into a $\Lambda$, the $\left(\mathrm{e}^{\prime} \mathrm{e}^{\prime} \mathrm{K}^{+}\right)$reaction can create neutron-rich hypernuclei compared to these created by $\left(\mathrm{K}^{-}, \pi^{-}\right)$or $\left(\pi^{+}, \mathrm{K}^{+}\right)$reaction, particularly in light hypernuclei. The study of neutron-rich hypernucleus play an important role for the study of $Y-N$ interaction, such as the study of $\Lambda \mathrm{N}-\Sigma \mathrm{N}$ coupling. The hypernuclei created by each reaction can be seen in figure 1.2.

2. Large recoil momentum of hyperon. The significance of momentum transfer is explained above.

3. Sizable spin flip/non-flip amplitude at zero degree direction because photon has spin $=1$. Therefore, both spin-flip and spin-non-flip hypernuclear states are excited with significant cross sections.

4. Primary electron beam can be used. In particular, the high quality beam of JLab bring technically important feature because of following reason.

- High energy resolution beam is available. In the case of JLab, the beam energy resolution $\Delta E / E$ is less than $10^{-4}$ at $\mathrm{E}=2.344 \mathrm{GeV}$, i.e. $\Delta E \leq 200 \mathrm{keV}$. Therefore, the total energy resolution can reach to a few hundred $\mathrm{keV}$, which is hard to achieve in the case of the meson beam experiment such as the $\left(\mathrm{K}^{-}, \pi^{-}\right)$ or $\left(\pi^{+}, \mathrm{K}^{+}\right)$experiment. 
- Thin target can be used with keeping practical hypernuclear yield because of the high intensity beam of JLab. There are two merits to use thin target. One is the reduction of energy struggling and multiple scattering in target and result in the improvement of total energy resolution. Another merit is that high purity isotope targets can be used easily compared to the $\left(\mathrm{K}^{-}, \pi^{-}\right)$or $\left(\pi^{+}, \mathrm{K}^{+}\right)$experiment and various hypernuclei can be measured.

- Determination of absolute binding energy due to the usage of proton target. The elementary process, $\mathrm{p}\left(\mathrm{e}, \mathrm{e}^{\prime} \mathrm{K}^{+}\right) \Lambda / \Sigma^{0}$ can be measured with a proton target and the mass spectrum can be calibrated using well-known $\Lambda$ and $\Sigma^{0}$ mass.

As listed above, the $\left(\mathrm{e}, \mathrm{e}^{\prime} \mathrm{K}^{+}\right)$spectroscopic experiment is a powerful tool to investigate hypernuclei precisely. Meanwhile, the experiment becomes challenging because of the huge background generated by the electron beam. The background events are generated by the bremsstrahlung and $\mathrm{M} \phi$ ller scattering process in the experimental target and the rate at the very forward angles were the order of $200 \mathrm{MHz}$ in the first generation experiment during physics production run $\left(22 \mathrm{mg} / \mathrm{cm}^{2} \mathrm{CH}_{2}\right.$ target, $0.67 \mu \mathrm{A}$ beam). This point is the most important for the experimental design, especially with the consideration of hypernuclear yield and the signal to noise ratio. The detail about the background and the experimental design will be discussed in chapter 2 .

\subsection{The purpose of the present study}

The present study is aiming to the investigation of the high-precision $\Lambda$ hypernuclear spectroscopic experiment via the $\left(\mathrm{e}, \mathrm{e}^{\prime} \mathrm{K}^{+}\right)$reaction in the wide mass region from $A=7$ to $A=52$. As mention above, the experimental data was taken as the third generation experiment in JLab Hall-C, with the setup dedicated to this experiment. In this section, the physics motivation to carry out this challenging experiment and the goal of the present thesis will be described.

\subsubsection{Physics motivations}

The E05-115 experiment is aiming to carry out a precise hypernuclear spectroscopic experiment. Experimental target are listed in table 2.4, the purpose of each targets can be explained as following:

Spectroscopy of light $\Lambda$ hypernuclei $\left({ }_{\Lambda}^{7} \mathrm{He}\right.$ and $\left.{ }_{\Lambda}^{10} \mathrm{Be}\right) \quad$ Recently, theoretical studies of $p$-shell hypernuclei have been progressed. In particular, calculation of the binding energies of $p$-shell hypernuclei are being performed based on the several models and interactions.

One of the important results of such calculation is the cluster model calculation performed by Hiyama et al. $([25,26]$. Her calculation has been done for a $T=1$ multiplet with mass number $A=7,{ }_{\Lambda}^{7} \mathrm{He},{ }_{\Lambda}^{7} \mathrm{Li}^{*}$ and ${ }_{\Lambda}^{7} \mathrm{Be}$, within the framework of an $\alpha+\Lambda+N+N$ fourbody cluster model. Interactions between each cluster which has large influences on the calculation were determined so as to reproduce reasonably the experimentally observed low-energy properties of the $\alpha N, \alpha \Lambda, \alpha N N$, and $\alpha \Lambda N$ subsystems. 
In addition to these interactions, the two-body $\Lambda N$ interaction was also adjusted so as to reproduce the $0^{+}-1^{+}$splitting of ${ }_{\Lambda}^{4} \mathrm{H}$ which were measured by emulsion experiment ([27]) and the $\gamma$-ray spectroscopic experiment using NaI ([28], [29]). The experimental results are summarized in figure 1.6 as the level structures of ${ }_{\Lambda}^{4} \mathrm{H}$ and ${ }_{\Lambda}^{4} \mathrm{He}$. According to Hiyama et al. [30], the coulomb force between a proton and a $\Sigma$ (appeared in the $\Lambda \mathrm{N}-\Sigma \mathrm{N}$ coupling force) does not explain this difference (in this study, the binding energies of ${ }_{\Lambda}^{4} \mathrm{H}$ and ${ }_{\Lambda}^{4} \mathrm{He}$ were calculated as $-2.28[\mathrm{MeV}]$ and $-2.33[\mathrm{MeV}]$, respectively). The difference between the binding energies of ${ }_{\Lambda}^{4} \mathrm{H}$ and ${ }_{\Lambda}^{4} \mathrm{He}$ seems to be attributable to not only the electromagnetic interaction, but also the charge symmetry breaking (CSB) components in the $\Lambda N$ interaction. The CSB effect is introduced phenomenologically in this calculation and the results on the $A=7$ isotriplet level structures are summarized in figure 1.7.

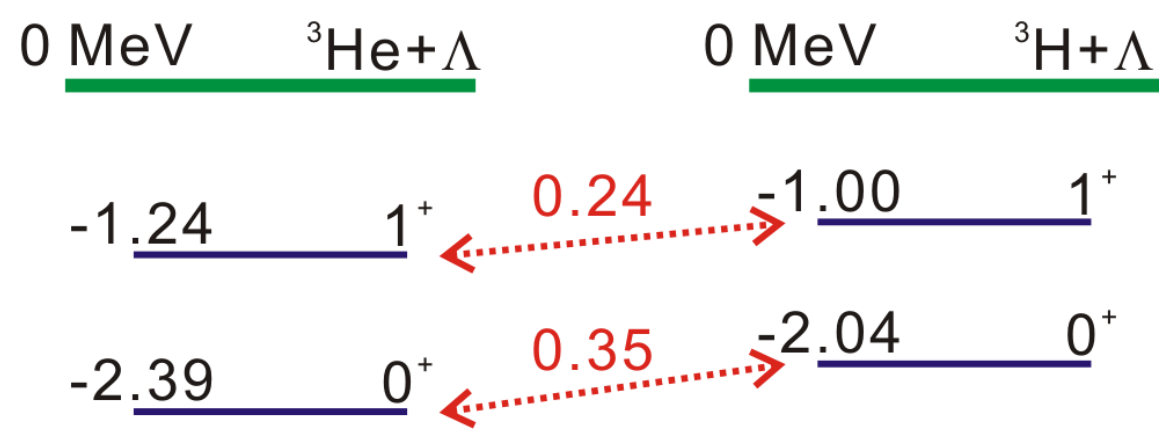

Figure 1.6: Experimental results of ${ }_{\Lambda}^{4} \mathrm{H}$ and ${ }_{\Lambda}^{4} \mathrm{He}$.

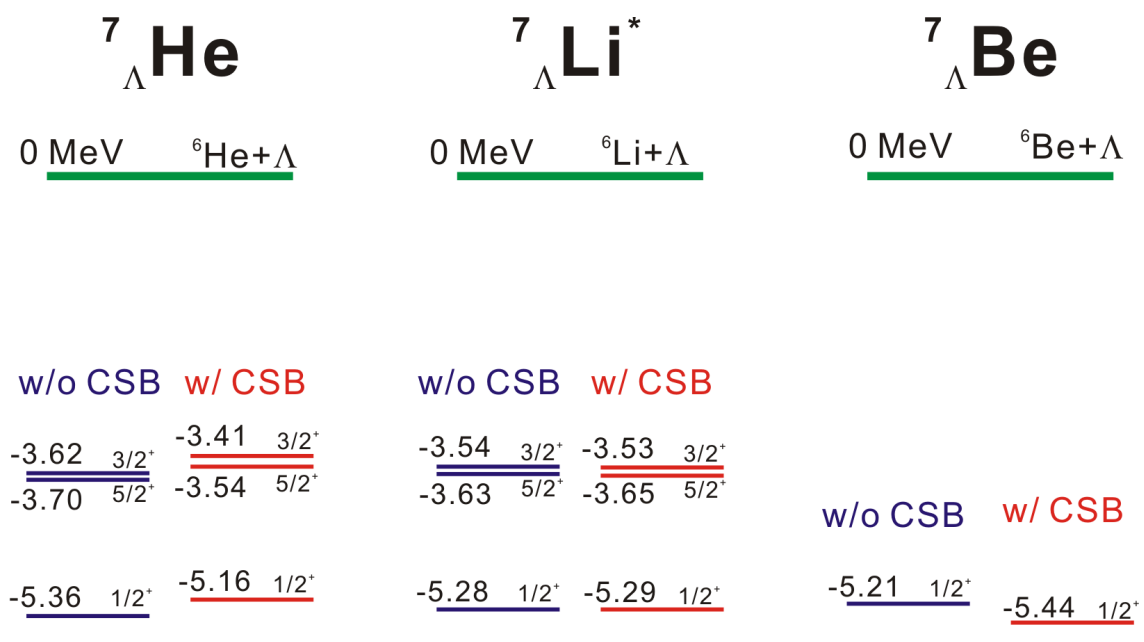

Figure 1.7: Calculated level structures of ${ }_{\Lambda}^{7} \mathrm{He},{ }_{\Lambda}^{7} \mathrm{Li}^{*}$ and ${ }_{\Lambda}^{7} \mathrm{Be}([26])$.

According to the calculation results shown in 1.7, the effect of CSB appears to be about $200 \mathrm{keV}$ in as the binding energy of ${ }_{\Lambda}^{7} \mathrm{He}$. Because we can use a proton target (see section 1.3) in the $\left(\mathrm{e}, \mathrm{e}^{\prime} \mathrm{K}^{+}\right)$hypernuclear spectroscopic experiment, the binding energies of hypernuclei can be determined in the accuracy of $100 \mathrm{keV}$. 
Moreover, the calculation of the $T=1 / 2$ multiplet with mass number $A=10{ }_{\Lambda}^{10} \mathrm{Be}$, $\left.{ }_{\Lambda}^{10} \mathrm{~B}\right)$ also show interesting results about the CSB. The ${ }_{\Lambda}^{10} \mathrm{Be}$ spectrum can be studied simultaneously in our experiment, and the ${ }_{\Lambda}^{10} \mathrm{~B}$, which is the mirror hypernucleus of ${ }_{\Lambda}^{10} \mathrm{Be}$, was studied by the $\left(\pi^{+}, \mathrm{K}^{+}\right)$reaction[20].

These hypernuclei need to be created by the $\mathrm{p}$ to $\Lambda$ reaction, which is satisfied by the $\left(\mathrm{e}, \mathrm{e}^{\prime} \mathrm{K}^{+}\right)$reaction. In addition to this point, the precise measurement is possible by the reaction. Therefore, the $\left(\mathrm{e}, \mathrm{e}^{\prime} \mathrm{K}^{+}\right)$is a powerful and unique tool for the study of CSB effect in $\Lambda N$ interaction.

Spectroscopy of medium heavy $\Lambda$ hypernuclei $\left({ }_{\Lambda}^{52} \mathrm{~V}\right)$ One reason why we performed ${ }_{\Lambda}^{52} \mathrm{~V}$ spectroscopy is the study of fine structures for the medium-heavy $\Lambda$ hypernuclei. As described later, there are no hypernuclear data in this mass region via the $\left(\mathrm{e}, \mathrm{e}^{\prime} \mathrm{K}^{+}\right)$reaction spectroscopy because of the experimental difficulties. However, several experimental data via the $\left(\pi^{+}, \mathrm{K}^{+}\right)$reaction [22] is existing and there are some discussions about the fine structures of such hypernuclei. For example, figure 1.8 is a missing mass spectrum of ${ }_{\Lambda}^{51} \mathrm{~V}$. One can find major structures corresponding $\Lambda$ single particle states $\left(s_{\Lambda}, p_{\Lambda}\right.$ and $\left.d_{\Lambda}\right)$ and other structures. While the measure structures have the configurations of $0 f_{7 / 2}^{-1}$ neutron-hole and a $\Lambda$ hyperons in each orbit, there are other states which are interpreted as having configurations of $0 d_{3 / 2}^{-1}, 1 s_{1 / 2}^{-1}$ and other deeper neutron holes.

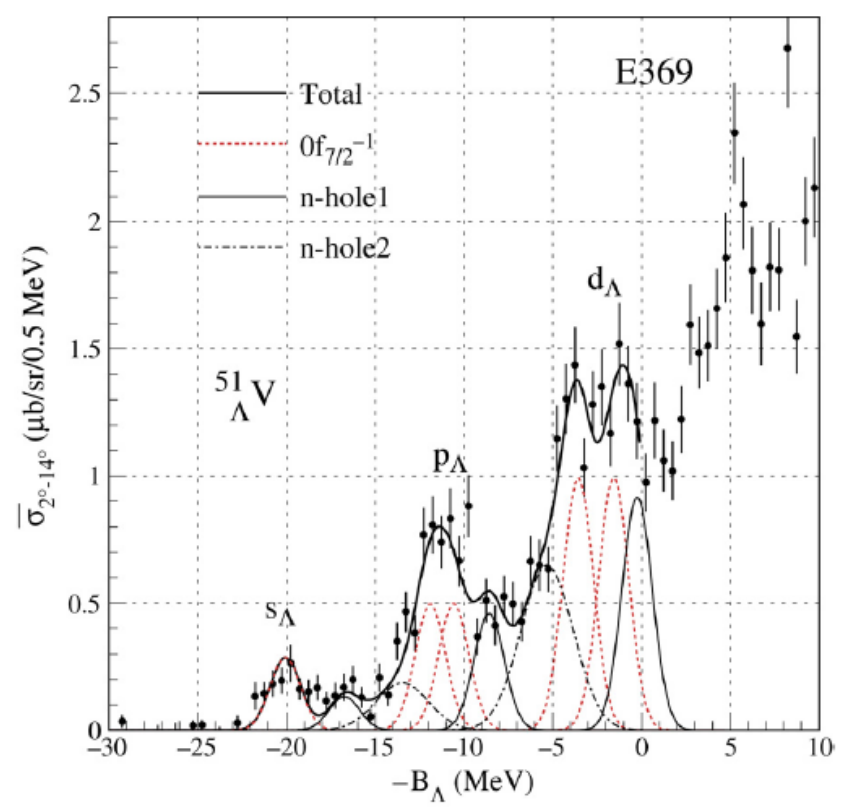

Figure 1.8: Missing mass spectrum of ${ }_{\Lambda}^{51} \mathrm{~V}$ via the $\left(\pi^{+}, \mathrm{K}^{+}\right)$reaction [22].

The ${ }_{\Lambda}^{52} \mathrm{~V}$ likely to have similar structure with that of ${ }_{\Lambda}^{51} \mathrm{~V}$, and the calculated excitation function for ${ }_{\Lambda}^{52} \mathrm{~V}$ is shown in figure 1.9. The ${ }^{52} \mathrm{Cr}$ target nucleus has four protons in the uppermost $0 f_{7 / 2}^{-1}$ shell and the neutron is $j j$-closed. Therefore it is easy to understand that the major peak series are based on the conversion of the 0f7/2 protons into the $\Lambda$ particle 
sitting in the $s, p, d$, and $f$ orbits. In addition, there would be several states originating from the core nuclear configuration such as deeper proton hole orbits of $\left(0 d_{3 / 2}^{-1}\right)_{p}$ and $\left(0 d_{5 / 2}^{-1}\right)_{p}$. It is very interesting to investigate such complicated structure with the energy resolution of sub-MeV, because the study of such structure will provide us with the $\Lambda$ single particle potential and deformation of the nucleus by putting $\Lambda$ hyperon. In addition, from the study of splitting of the major peak, it is possible to study the effect of twobody $\Lambda$-N spin-orbit interaction. The $\Lambda$ hyperon has large angular momentum states in this situation and the effect of $\Lambda$-N spin-orbit interaction might be a few $100 \mathrm{keV}$ level, which might be measured with the energy resolution of the $\left(\mathrm{e}, \mathrm{e}^{\prime} \mathrm{K}^{+}\right)$spectroscopy. The $\left(\mathrm{e}, \mathrm{e}^{\prime} \mathrm{K}^{+}\right)$reaction can provide much more precise spectrum (sub-MeV energy resolution) and it becomes possible to do precise investigation about the structures of medium-heavy $\Lambda$ hypernuclei.

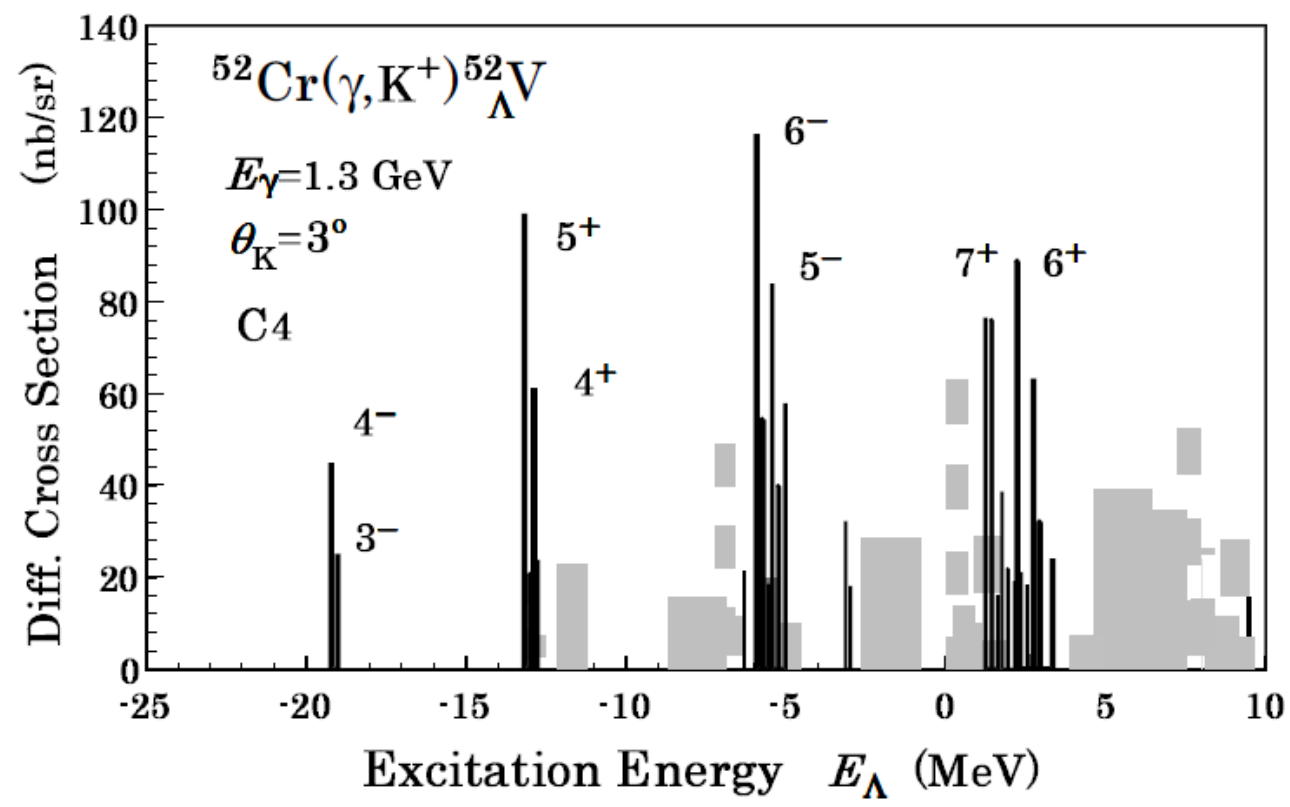

Figure 1.9: Calculated cross section of ${ }_{\Lambda}^{52} \mathrm{~V}$ in DWIA using C4 model [31]. The artificial ls-splitting is assumed as $0.17(2 \mathrm{l}+1)$ in $\mathrm{MeV}$. The gray regions are originated from deeper proton hole orbits.

Moreover, it is also important to study the $\Lambda$ single particle potential by systematic studies of the $\Lambda$ hypernuclei in wide mass region. In general, a $\Lambda$ hypernuclear potential can be represented using the Woods-Saxon form as following:

$$
\begin{aligned}
U_{\Lambda} & =V_{0}^{\Lambda} f(r)+V_{L S}^{\Lambda}\left(\frac{\hbar}{m_{\pi} c}\right)^{2} \frac{1}{r} \frac{d f(r)}{d r} \mathbf{l} \mathbf{s} \\
f(r) & =[1+\exp ((r-R) / a)]^{-1} .
\end{aligned}
$$

In the above equation, $V_{0}^{\Lambda}$ and $V_{L S}^{\Lambda}$ represents central and spin-orbit potentials, respectively. $V_{\Lambda}$ was obtained as $-31 \mathrm{MeV}$ with the experimental data of $\left(\pi^{+}, \mathrm{K}^{+}\right)$reaction[23], 
which is about $2 / 3$ of the nuclear potential for nucleon. This value is consistent with the depth determined by emulsion data. An example of the calculation results are shown in figure 1.10 with several data obtained by emulsion experiments $[27,32],\left(\pi^{+}, \mathrm{K}^{+}\right)$reaction spectroscopic experiments $[23,16,20,22]$ and the $\left(\mathrm{e}, \mathrm{e}^{\prime} \mathrm{K}^{+}\right)$spectroscopic experiments [33]. The dashed line in the figure represents a calculated binding energy by Woods-Saxon potentials with a depth of $28 \mathrm{MeV}$ and a radius parameter $r_{0}=1.128+0.439 A^{-2 / 3}[34]$.

The above is one example to describe the $\Lambda$ single particle potential with the WoodsSaxon form, but actually there are a lot of discussions. For example, the density dependence of the potential is discussed by Millener et al.[34], and they studied the shape of the Woods-Saxon-based potential. It is also interesting to study the $\Lambda N N$ three-body force and $\Lambda$ effective mass in the potential by Skyrme-Hartree-Fock approach and the study of $\Lambda$ single particle potential up to $A=208$ is studied [35], [36].

Treatment by mean-field theories is also effective to describe the properties of a $\Lambda$ in nuclear matter or deep inside nuclei, that is, heavy hypernculei. In such region, the distinguishability of a $\Lambda$ hyperon as a baryon in a nucleus is raised as an interesting questions. It is said that the mass dependence of the $\Lambda$ single particle energy could be changed whether a $\Lambda$ hyperon can keep its identity as a baryon or not. In other words, this can be said the difference between the quark picture or the baryon picture. There are theoretical study by quark-meson coupling model [37], the density dependent relativistic hadron field theory $[38,39]$. The precise experimental results can provide the understanding of $\Lambda$ behavior deep inside nuclei through these studies.

Such studies reveal the behavior of $\Lambda$ hyperon in nuclei as an "impurity", which can be a probe of deep inside nuclei. This study can be extended to the study of $\Lambda$ hyperon in nuclear matter $(A \rightarrow \infty$ limit). Such study is very important input for the theory about neutron stars, because the neutron stars have very high density which can be described with the existence of hyperon inside the stars. The reliable experimental inputs of $\Lambda$ binding energies play an important role to reduce the ambiguity of the theoretical parameters.

So far, experimental input of $\Lambda$ hypernuclear binding energy was obtained by the spectroscopy via $\left(\pi^{+}, \mathrm{K}^{+}\right)$reaction whose mass resolution is about $2 \mathrm{MeV}$. However, the $\left(\mathrm{e}, \mathrm{e}^{\prime} \mathrm{K}^{+}\right)$reaction spectroscopy can determine reliable absolute binding energy with the mass resolution of sub-MeV and an accuracy of $100 \mathrm{keV}$ level. Therefore, the systematic hypernuclear spectroscopy via the $\left(\mathrm{e}, \mathrm{e}^{\prime} \mathrm{K}^{+}\right)$reaction is a valuable data for this kind of study and further understanding of the $\Lambda$ hypernuclei.

\subsubsection{The goal of the present thesis}

The present thesis is aiming to the way to establish the experimental setup of the $\left(\mathrm{e}, \mathrm{e}^{\prime} \mathrm{K}^{+}\right)$ reaction spectroscopy and its evaluation through the obtained experimental data. In order to achieve the purpose, firstly the detail character of the $\left(\mathrm{e}, \mathrm{e}^{\prime} \mathrm{K}^{+}\right)$reaction will be described in chapter 2 . The understanding of the reaction is essential to design the experimental setup. The design principle of the experiment will be explained with some merits and difficulties of the $\left(\mathrm{e}, \mathrm{e}^{\prime} \mathrm{K}^{+}\right)$spectroscopy in this section.

In chapter 3 , the constructed experimental setup and detector specifications will be described, together with the actual experimental conditions and the trigger setup. The most remarkable point of the present setup is the newly constructed HES and the Splitter 


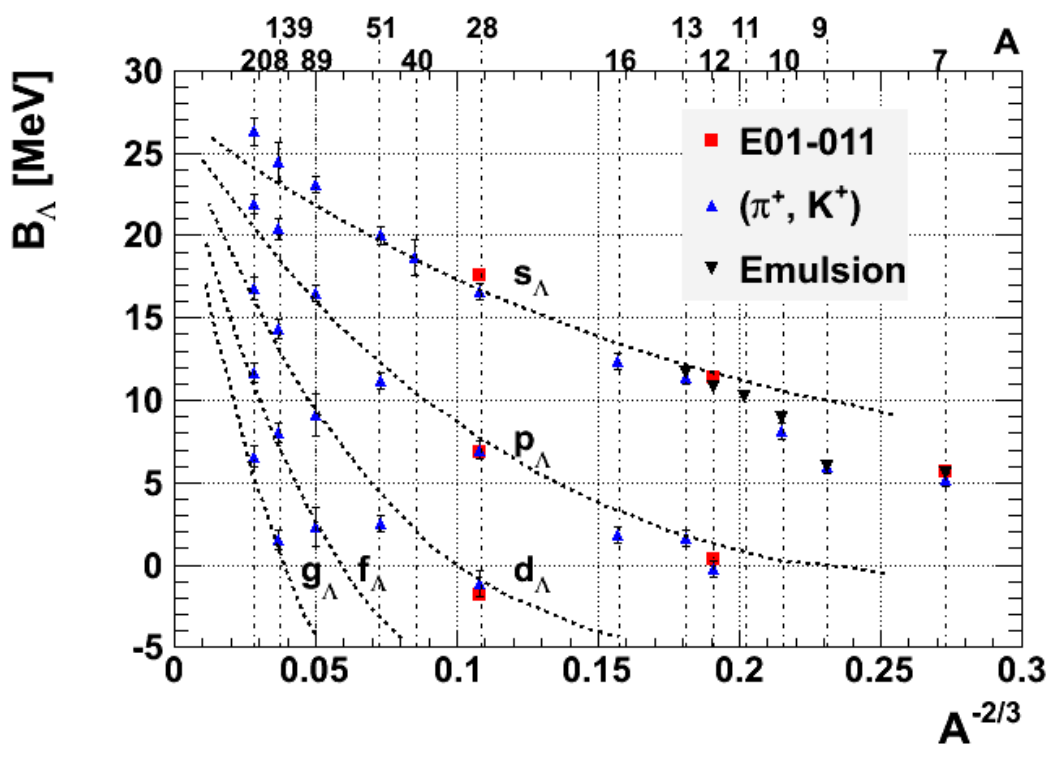

Figure 1.10: $A$ dependence of $\Lambda$ single particle energy measured by emulsion experiments $[27,32],\left(\pi^{+}, \mathrm{K}^{+}\right)$reaction spectroscopic experiments $[23,16,20,22]$ and the $\left(\mathrm{e}, \mathrm{e}^{\prime} \mathrm{K}^{+}\right)$ spectroscopic experiments [33]. The dashed line represents a calculated binding energy by Woods-Saxon potentials with a depth of $28 \mathrm{MeV}$ and a radius parameter $r_{0}=1.128+$ $0.439 A^{-2 / 3}[34]$. 
magnet (SPL). They were designed carefully with consideration of the $\left(\mathrm{e}, \mathrm{e}^{\prime} \mathrm{K}^{+}\right)$reaction attributes, and the design detail of the spectrometer will be explained in chapter 4 .

In order to obtain hypernuclear mass spectra, an unique analysis procedure to tune momentum reconstruction functions was used. This procedure will be described in chapter 5. At the final chapter, the experimental setup and the analysis procedure will be evaluated through the mass spectra of $\Lambda$ and $\Sigma^{0}$ via the elementary process $\mathrm{p}\left(\mathrm{e}, \mathrm{e}^{\prime} \mathrm{K}^{+}\right) \Lambda / \Sigma^{0}$. In addition, the obtained spectrum of ${ }_{\Lambda}^{12} \mathrm{~B}$ will be compared with the other experimental results for the discussion of the consistency and absolute mass scale. 


\section{Chapter 2}

\section{$\Lambda$ hypernuclear spectroscopy via the $\left(\mathrm{e}, \mathrm{e}^{\prime} \mathrm{K}^{+}\right)$reaction}

In order to determine the experimental parameters, it is very important to discuss the feature of the $\left(\mathrm{e}, \mathrm{e}^{\prime} \mathrm{K}^{+}\right)$reaction.

Thus, firstly the elementary process of $\left(\mathrm{e}, \mathrm{e}^{\prime} \mathrm{K}^{+}\right)$reaction will be explained in section 2.1. One of the experimental difficulties for the $\left(\mathrm{e}, \mathrm{e}^{\prime} \mathrm{K}^{+}\right)$spectroscopic experiment is the huge background coming from the electromagnetic process. This factor has to be considered for the experimental design, and the background event process will be explained in section 2.2. After the explanation of the basic feature of the $\left(\mathrm{e}, \mathrm{e}^{\prime} \mathrm{K}^{+}\right)$reaction, the purposes and results of previous two $\left(\mathrm{e}, \mathrm{e}^{\prime} \mathrm{K}^{+}\right)$hypernuclear experiments carried out in JLab Hall-C will be described in section 2.3. At the final section of this chapter, the experimental design concepts of the present experiment will be explained based of the feature of the $\left(\mathrm{e}, \mathrm{e}^{\prime} \mathrm{K}^{+}\right)$ reaction.

\subsection{The elementary process of $\left(e, e^{\prime} K^{+}\right)$reaction}

The elementary process of hyperon electroproduction is described as follows:

$$
\mathrm{e}+\mathrm{p} \rightarrow \mathrm{e}^{\prime}+\Lambda+\mathrm{K}^{+} \text {. }
$$

Figure2.1 represents the $\left(\mathrm{e}, \mathrm{e}^{\prime} \mathrm{K}^{+}\right)$reaction kinematics and the definition of four-momenta of each particles in the center of mass $(C o M)$ frame. This reaction has two reaction planes, one is a electron scattering plane and another is hadronic reaction plane. In the electron scattering plane, initial and final electron four-momenta are defined as follows:

$$
\begin{aligned}
P_{e} & =\left(E_{e}, \vec{p}_{e}\right), \\
P_{e^{\prime}} & =\left(E_{e^{\prime}}, \vec{p}_{e^{\prime}}\right) .
\end{aligned}
$$

An electron reacts with a proton electromagnetically via virtual photon $\left(\gamma^{*}\right)$, and its four momentum is defined as

$$
Q \equiv(\omega, \vec{q})
$$

The relation of momenta in electron scattering plane can be described in next equations:

$$
\begin{aligned}
\omega & =E_{e}-E_{e^{\prime}}, \\
\vec{q} & =\vec{p}_{e}-\vec{p}_{e^{\prime}} .
\end{aligned}
$$


In the hadronic reaction plane, four momenta of kaon, proton and hyperon are defined as follows:

$$
\begin{aligned}
P_{K^{+}} & =\left(E_{K^{+}}, \vec{p}_{K}^{+}\right), \\
P_{N} & =\left(E_{N}, \vec{p}_{N}\right), \\
P_{Y} & =\left(E_{Y}, \vec{p}_{Y}\right) .
\end{aligned}
$$

An azimuthal angle $\phi_{K^{+}}$is defined as the the angle between the scattering plane and reaction plane.

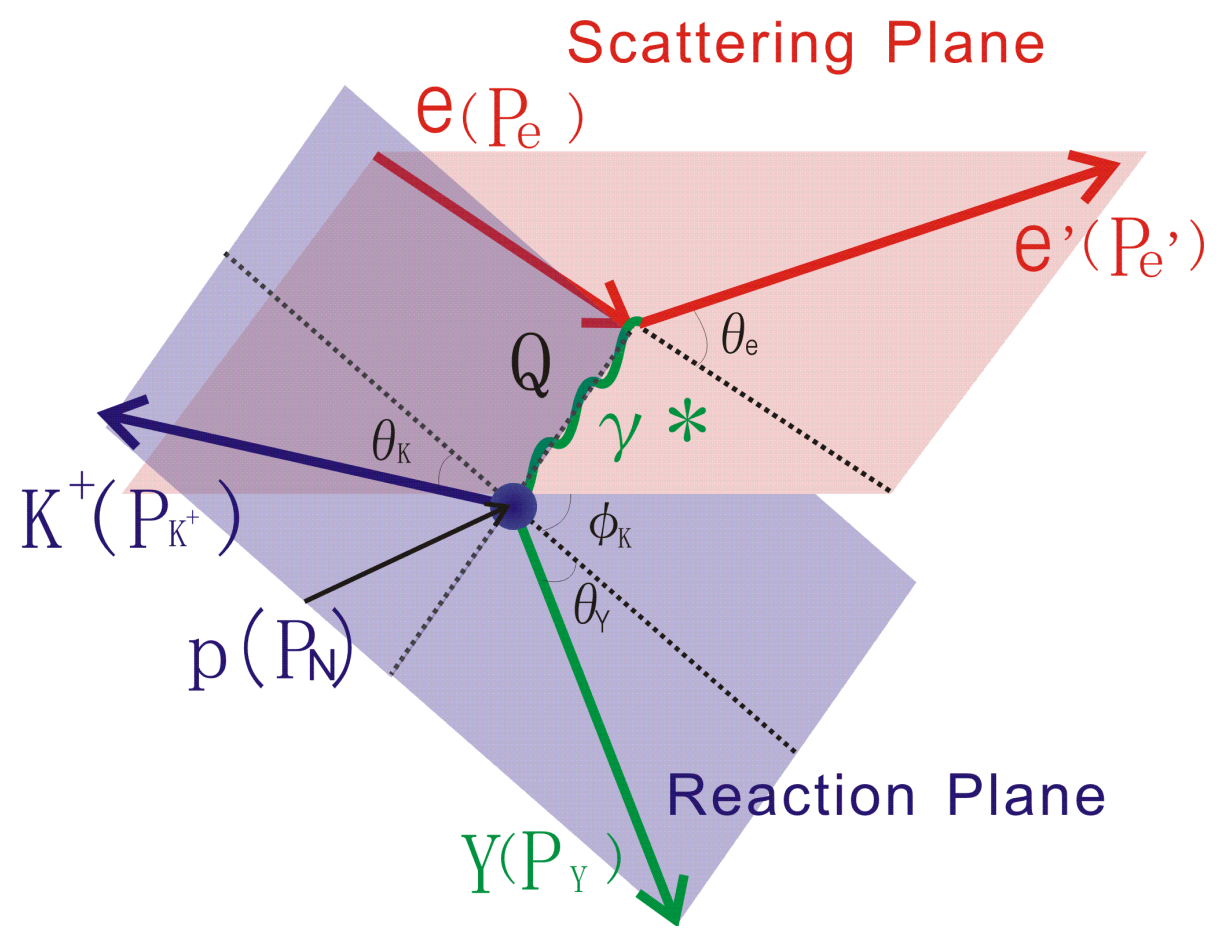

Figure 2.1: Kinematics of the $\left(\mathrm{e}, \mathrm{e}^{\prime} \mathrm{K}^{+}\right)$reaction.

The cross section of this reaction is described as

$$
\frac{d^{3} \sigma}{d E_{e^{\prime}} d \Omega_{e^{\prime}} d \Omega_{K^{+}}}=\frac{\alpha^{2}}{Q^{4}} \frac{E_{e^{\prime}}}{E_{e}} L_{\mu \nu} W^{\mu \nu}
$$

$L_{\mu \nu}$ and $W^{\mu \nu}$ are a lepton tensor and a hadron tensor, respectively. These tensors can be calculated as follows, using Feynman's rule and Trace theorem [40, 41];

$$
\begin{aligned}
L^{\mu \nu}= & 2\left(P_{e^{\prime}}^{\mu} P_{e}^{\nu}+P_{e^{\prime}}^{\nu} P_{e}^{\mu}\right)-\left(P_{e^{\prime}} \cdot P_{e}-m_{e}^{2}\right) g^{\mu \nu} \\
W^{\mu \nu}= & \frac{1}{(2 \pi)^{3}} \frac{M_{N} M_{\Lambda}}{E_{N} E_{\Lambda}} \int d p_{K^{+}} \frac{p_{K^{+}}^{2}}{2 E_{K^{+}}} d \mathbf{p}_{\Lambda} \delta\left(p_{\Lambda}+p_{K^{+}}-p_{N}-q\right) \\
& \times \frac{1}{2} \sum_{\text {spin }} J^{\mu}\left(p_{K^{+}} p_{\Lambda}, p_{N} q\right) J^{\nu}\left(p_{K^{+}} p_{\Lambda}, p_{N} q\right),
\end{aligned}
$$


where $J^{\mu}\left(p_{K^{+}} p_{\Lambda}, p_{N} q\right)$ is the hadron current matrix, which is expressed in [40]. Calculation result of 2.10 using equation 2.11 and 2.12 with the kinematics shown in figure 2.1 is as following:

$$
\begin{aligned}
\frac{d^{3} \sigma}{d E_{e^{\prime}} d \Omega_{e^{\prime}} d \Omega_{K^{+}}}= & \frac{\alpha^{2}}{Q^{4}} \frac{E_{e^{\prime}}}{E_{e}} \frac{1}{1-\epsilon}\left[\left(W^{x x}+W^{y y}\right)+\epsilon\left(W^{x x}-W^{y y}\right)+2 \epsilon \frac{Q^{2}}{\omega^{2}} W^{z x}\right. \\
& \left.-\sqrt{2 \epsilon(1+\epsilon)} \sqrt{\frac{Q^{2}}{\omega^{2}}}\left(W^{z x}+W^{x z}\right)\right]
\end{aligned}
$$

where $\epsilon$ is defines as:

$$
\epsilon \equiv\left(1-2 \frac{Q^{2}-\omega^{2}}{Q^{2}} \tan ^{2}\left(\frac{\theta_{e}}{2}\right)\right)^{-1}
$$

Calculating 2.14 the triple-differential cross section of this reaction is described as below:

$$
\frac{d^{3} \sigma}{d E_{e^{\prime}} d \Omega_{e^{\prime}} d \Omega_{K}}=\Gamma\left[\frac{d \sigma_{T}}{d \Omega_{K}}+\epsilon \frac{d \sigma_{L}}{d \Omega_{K}}+\epsilon \frac{d \sigma_{P}}{d \Omega_{K}} \cos 2 \phi_{K}+\sqrt{2 \epsilon(1+\epsilon)} \frac{d \sigma_{I}}{d \Omega_{K}} \cos \phi_{K}\right] .
$$

In 2.16 ,

$$
\Gamma \equiv \frac{\alpha E_{\gamma}}{2 \pi^{2} Q^{2}} \frac{E_{e^{\prime}}}{E_{e}} \frac{1}{1-\epsilon} .
$$

is a virtual photon flux with virtual photon energy in Lab frame $E_{\gamma} \equiv \omega+\frac{Q^{2}}{2 M_{N}} . \sigma_{T}, \sigma_{L}$, $\sigma_{P}$ and $\sigma_{I}$ are called transverse, longitudinal, polarization and interference cross section, respectively. The angular distribution of virtual photon flux is shown in figure 2.2. As shown in this figure, electrons associated with the virtual photons have a peak at zero degree. Therefore it is essential to detect electrons emitted as forward as possible to increase the hypernuclear yield.

Figure 2.3 shows the angular distribution of the kaons associated with photo production of ${ }_{\Lambda}^{12} \mathrm{~B}$ ground state doublet with a DWIA calculation in Lab frame [42]. Since the cross section is large around zero degree, it is also essential to put kaon acceptance as forward as possible, as well as the case of the scattered electrons.

Figure 2.4 is an experimental result of kaon photoproduction cross section [43], plotted with the total energy. It can be seen that the cross section in the region of $1.1 \leq E_{\gamma} \leq 1.6$ $[\mathrm{GeV}]$ is higher than that of another region. On the other hand, according to figure 1.3, the recoil momentum of $\Lambda$ hyperon become lower for higher $E_{\gamma}$ at $\theta_{K}=0$ [deg]. Since the sticking probability is higher for the lower $\Lambda$ recoil momentum and we detect kaon emitted at very forward angle, higher $E_{\gamma}$ is preferable, as far as considering only sticking probability. We conclude to select the central $E_{\gamma}$ as $1.5[\mathrm{GeV}]$.

\subsection{Background sources of the $\left(\mathrm{e}, \mathrm{e}^{\prime} \mathrm{K}^{+}\right)$reaction}

\subsubsection{Background in scattered electron side}

The background events in the scattered electron side are electrons associated with the Bremsstrahlung process $\left(e_{B}^{\prime}\right)$ and $\mathrm{M} \phi$ ller scattering process $\left(e_{M}^{\prime}\right)$. The Bremsstrahlung 


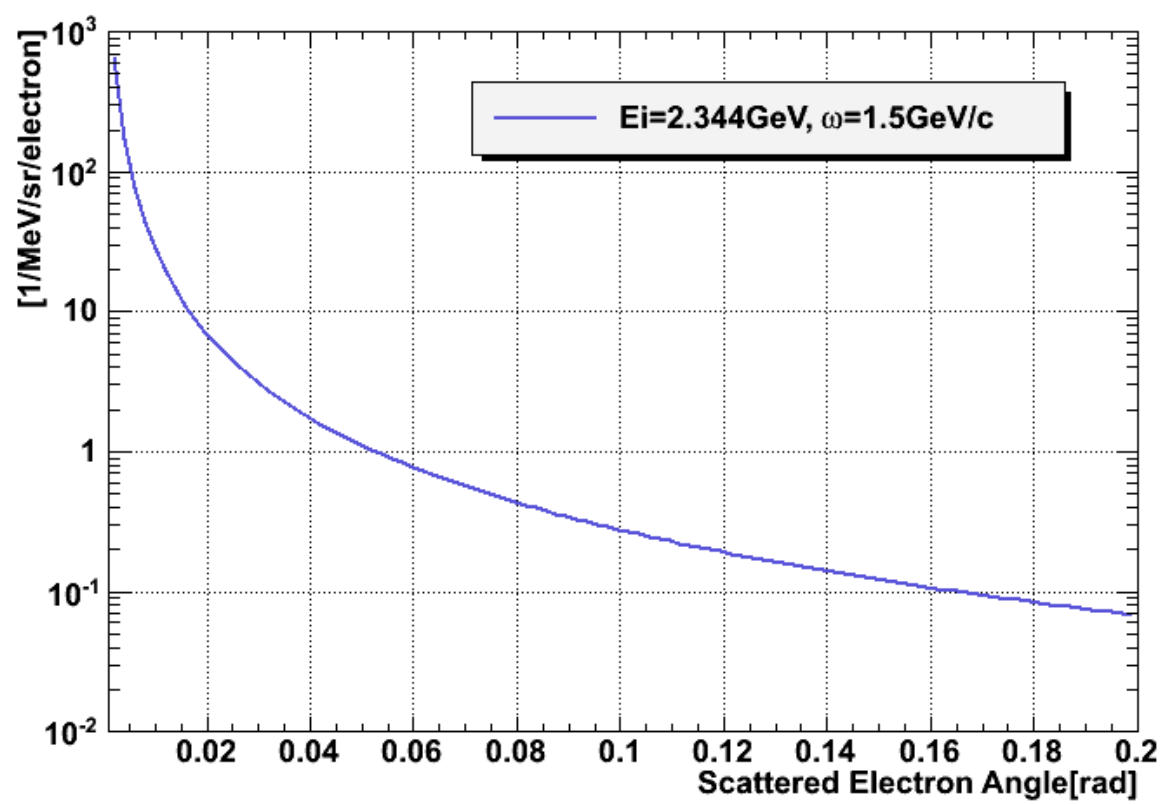

Figure 2.2: Scattered electron angular distribution via the $\left(\mathrm{e}, \mathrm{e}^{\prime} \mathrm{K}^{+}\right)$reaction.

$\left(\mathrm{e}, \mathrm{e}^{\prime} \mathrm{K}^{+}\right)$kaon angular distribution, harm. osci, DWIA

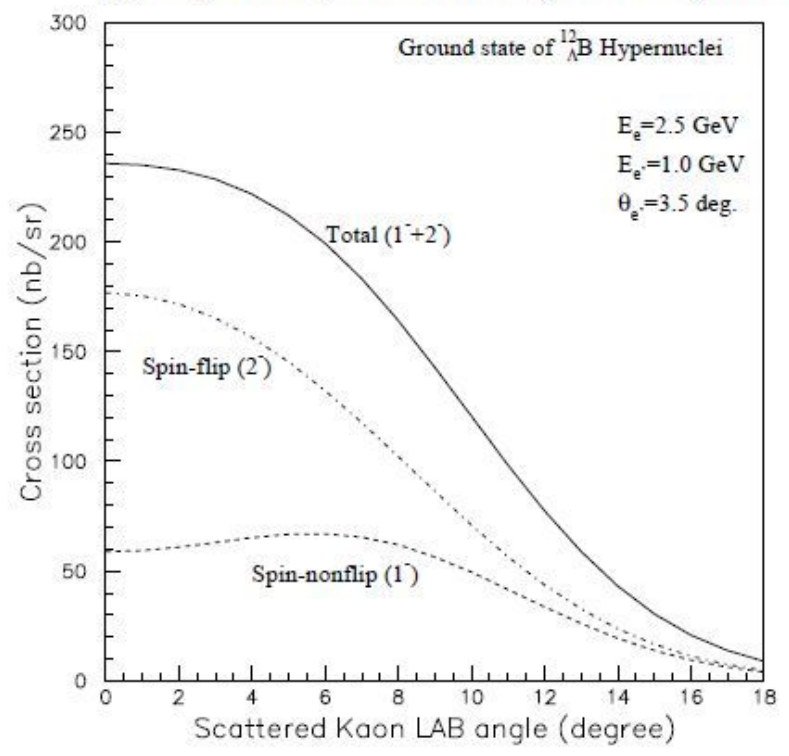

Figure 2.3: Scattered kaon angular distribution associated with the photo production of ${ }_{\Lambda}^{12} \mathrm{~B} 12$ ground state doublet calculated with DWIA method assuming $E_{i}=2.5[\mathrm{GeV}]$, $E_{e^{\prime}}=1.0[\mathrm{GeV}]$ and $\theta_{e^{\prime}}=3.5[\mathrm{deg}]$. 


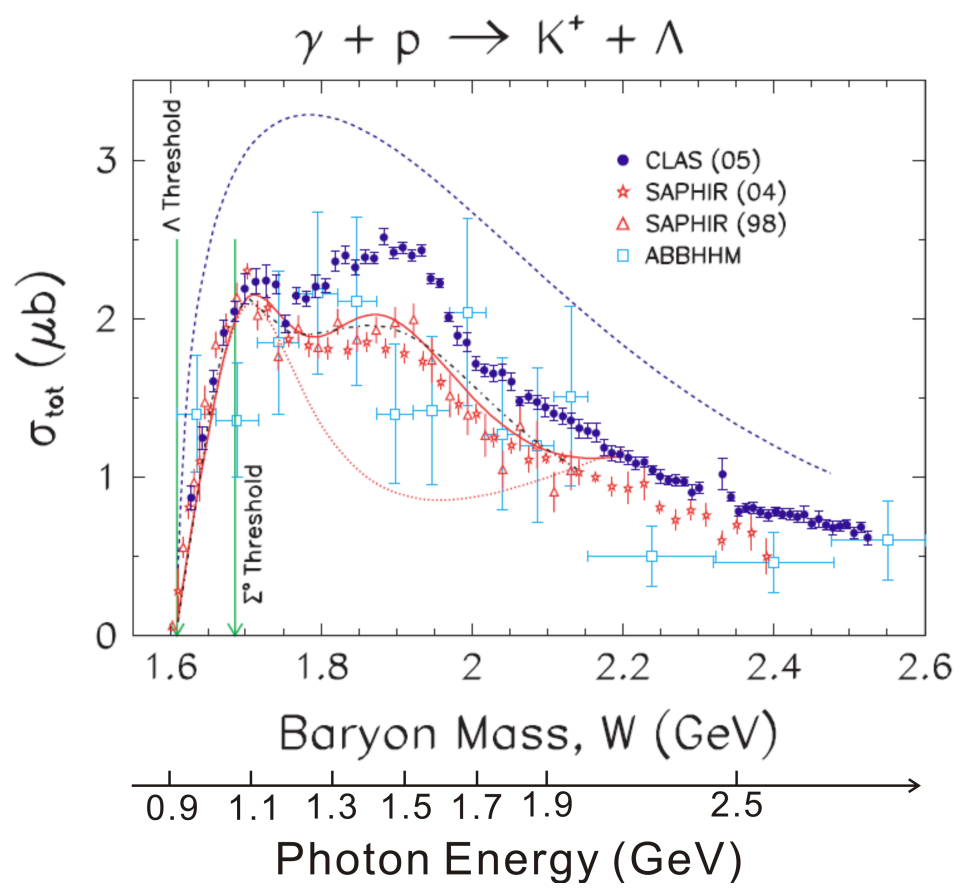

Figure 2.4: Total cross section of the $\mathrm{p}\left(\gamma, \mathrm{K}^{+}\right) \Lambda$ reaction [43].

process is caused by the electric field of the target nuclei and the electrons associated with the Bremsstrahlung photon can be the background event. The $\mathrm{M} \phi$ ller scattering process is caused by the reaction with the beam and electrons in the target atom. It is essential to know the feature of these backgrounds such as angular and momentum dependences, for the design and carrying out of the $\left(\mathrm{e}, \mathrm{e}^{\prime} \mathrm{K}^{+}\right)$experiment.

\section{Bremsstrahlung}

The cross section of Bremsstrahlung in a nucleus is discussed by Tsai et al. [44]. The cross section is roughly proportional to the $Z^{2}$ ( $Z$ is atomic number of the target), which is essential for the experimental design. Because this means the background events for scattered electron side also increase in proportion to $Z^{2}$. The cross section can be expressed as the following equation:

$$
\frac{d^{2} \sigma_{b}}{d \Omega_{\omega} d \omega}=\frac{2 \alpha^{3} E^{2}}{\pi \omega m_{e}^{4}}\left[b_{1}\left(Z^{2}+Z\right)+b_{2}\left(X-2 Z^{2} f\left((\alpha Z)^{2}\right)\right)\right],
$$

where

$$
\begin{aligned}
b_{1} & =\frac{2 y-2}{(1+l)^{2}}+\frac{12 l(1-y)}{(1+l)^{4}}, \\
b_{2} & =\frac{2-2 y+y^{2}}{(1+l)^{2}}-\frac{4 l(1-y)}{(1+l)^{4}},
\end{aligned}
$$




$$
\begin{aligned}
y & =\frac{\omega}{E}, \quad l=\left(\frac{\theta_{\omega} E}{m_{e}}\right)^{2} . \\
X & =X^{e l}+X^{\text {inel }} \\
& =Z^{2}\left[\log \frac{a^{2} m_{e}^{2}(1+l)^{2}}{a^{2} t_{\text {min }}^{\prime}+1}-1\right]+Z\left[\log \frac{a^{\prime 2} m_{e}^{2}(1+l)^{2}}{a^{\prime 2} t_{\text {min }}^{\prime}+1}-1\right], \\
a & =\frac{184.15}{(2.718)^{1 / 2} Z^{1 / 3} m_{e}}, \\
a^{\prime} & =\frac{1194}{(2.718)^{1 / 2} Z^{2 / 3} m_{e}}, \\
t_{\text {min }} & =\left[\frac{\omega m^{2}(1+l)^{2}}{2 E(E-\omega)}\right]^{2}, \\
f(x) & =1.202 z-1.0369 z^{2}+1.008 \frac{z^{2}}{1+z} .
\end{aligned}
$$

In above equations, $X^{e l}$ and $X^{\text {inel }}$ are corresponding to simple elastic and atomic form factor. $t_{\min }$ is the minimum momentum transfer to the atom which is used for the calculation of the $X . f(x)$ is Coulomb correction to the one-photon exchange approximation.

Figure 2.5 is the angular distribution of $e_{B}^{\prime}$. The distribution has very sharp peak at zero degree This is very important characteristic for the background suppression.

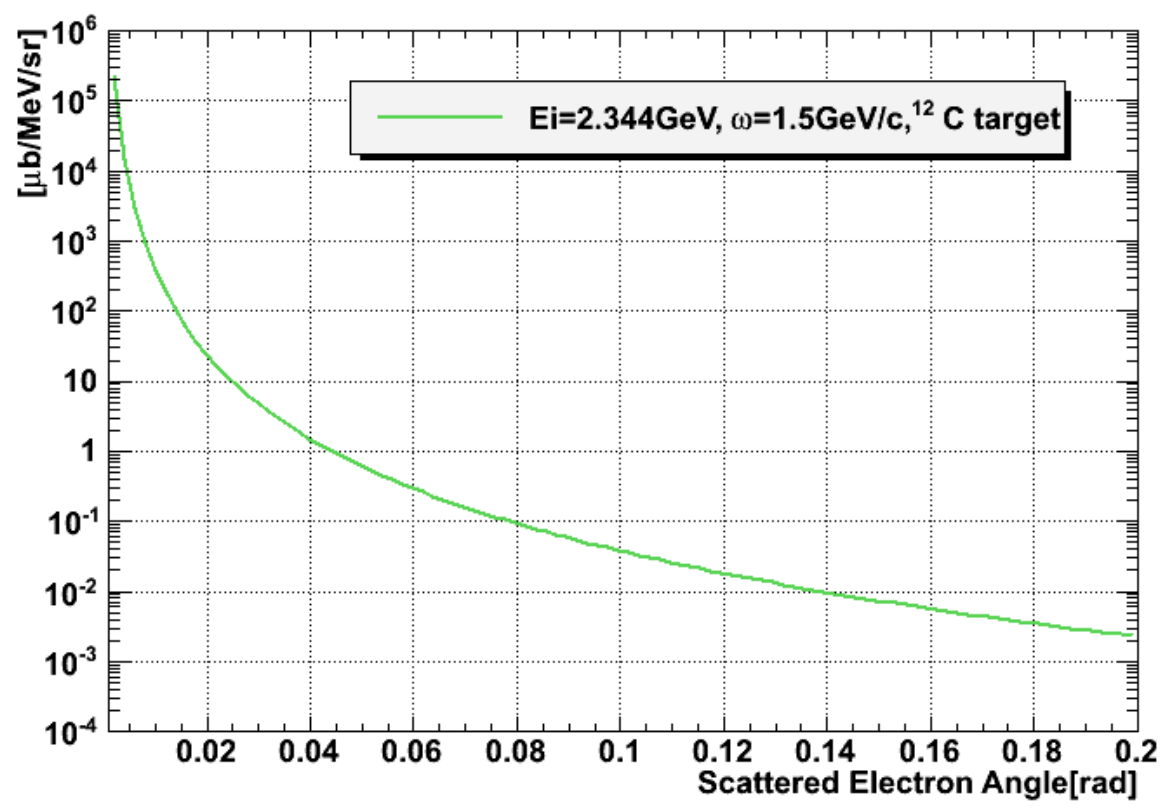

Figure 2.5: Angular distribution of electron associated with bremsstrahlung process. 


\section{M $\phi$ ller Scattering}

$\mathrm{M} \phi$ ller scattering is an electron-electron scattering process (figure 2.6). The cross section of the reaction in $C o M$ frame can be written as equation 2.19 [45]. In this equation, $E_{C M}$ is energy of incident electron and $\theta_{C M}$ is the angle between incident electron and scattered electron (see figure 2.6) in $C o M$ frame.

$$
\left(\frac{d \sigma_{m}}{d \Omega}\right)_{C M}=\frac{\alpha^{2}\left(2 E_{C M}^{2}-m_{e}^{2}\right)^{2}}{4 E_{C M}^{2}\left(E_{C M}^{2}-m_{e}^{2}\right)^{2}}\left[\frac{4}{\sin ^{4} \theta_{C M}}-\frac{3}{\sin ^{2} \theta_{C M}}+\frac{\left(E_{C M}^{2}-m_{e}^{2}\right)^{2}}{\left(2 E_{C M}^{2}-m_{e}^{2}\right)^{2}}\left(1+\frac{4}{\sin ^{2} \theta_{C M}}\right)\right]
$$

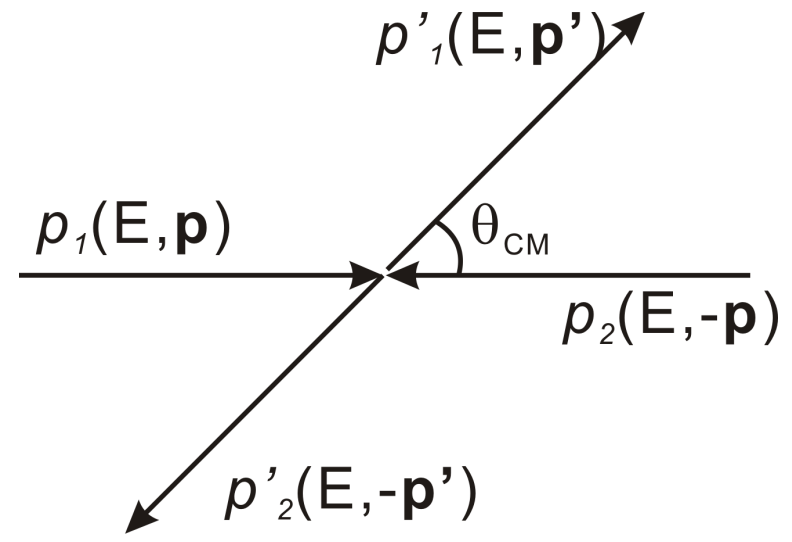

Figure 2.6: Kinematics of $\mathrm{M} \phi$ ller scattering in the center of mass frame.

\subsubsection{Background in kaon side}

Dominant background particles emitted to the kaon side are pions, protons and positrons. These background can be basically rejected by Cherencov counters in trigger level, the rejection method will be explained in chapter 3. However, since the counting rate of the positron background is very high (order of $100 \mathrm{MHz}$ ), it is required to suppress the counting rate itself.

The source of positron background is $\mathrm{e}^{-} / \mathrm{e}^{+}$pair creation from bremsstrahlung photon. Therefore, the positron background is emitted at the very forward angles because of Lorentz boost. Figure 2.8 shows a simulation result of the angular and momentum distribution of the positron background in Lab frame. One can see the positron background can be suppressed by avoiding the zero degree direction of the kaon angular acceptance. From these results, kaon angular acceptance should be determined so as to avoid zero degree in the case of the $\left(\mathrm{e}, \mathrm{e}^{\prime} \mathrm{K}^{+}\right)$hypernuclear spectroscopy. 


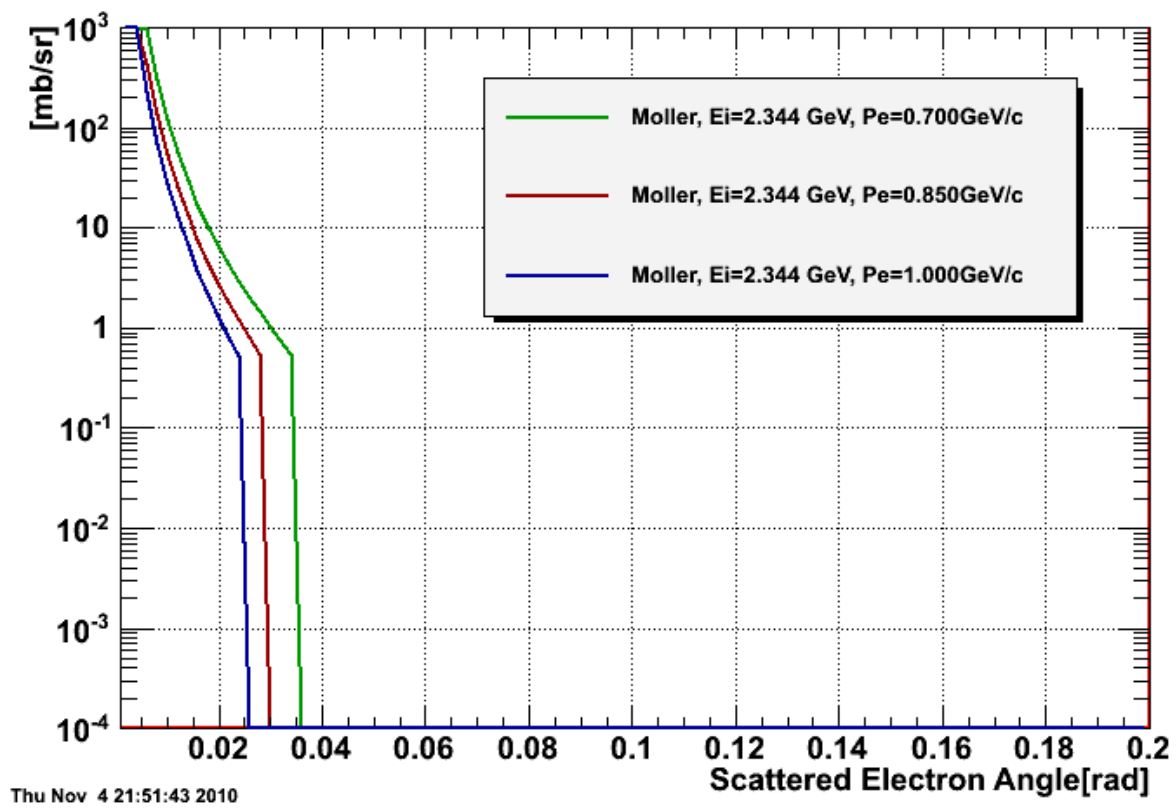

Figure 2.7: Angular distribution of electron associated with $\mathrm{M} \phi$ ller scattering.

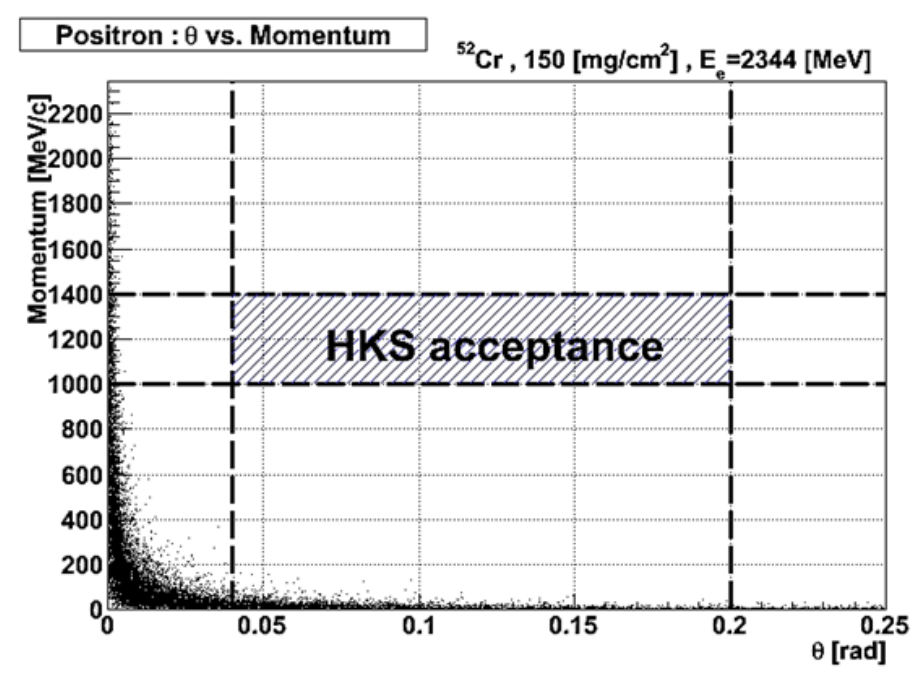

Figure 2.8: Angular distribution of positron background. Hatched region is the acceptance of HKS which was used as the kaon spectrometer in the JLab second generation experiment (E01-011) and the third generation experiment (E05-115). 


\subsection{Previous $\left(\mathrm{e}, \mathrm{e}^{\prime} \mathrm{K}^{+}\right) \Lambda$ hypernuclear spectroscopic ex- periments at JLab Hall-C}

In the last section, the feature of the $\left(\mathrm{e}, \mathrm{e}^{\prime} \mathrm{K}^{+}\right)$elementary process and dominant background source of the $\left(\mathrm{e}, \mathrm{e}^{\prime} \mathrm{K}^{+}\right)$reaction were explained. The feature can be summarized as follows.

1. Angular distributions of both kaons and electrons have a peak at forward angle.

2. Background particles associated with elecromagnetic process come in both side. These particles emitted at extremely forward angles.

Therefore, in order to do an experiment with high yield, it is important to put both spectrometers in the forward direction with suppressing the background particles. However, basically a $\left(\mathrm{e}, \mathrm{e}^{\prime} \mathrm{K}^{+}\right)$spectroscopic experiment needs two spectrometers, and it is impossible to put both spectrometer in zero degree because of physical interference. A solution to solve this problem is putting a dipole magnet around the target in order to deflect the positive and negative charged particles to each spectrometer. This dipole magnet is called as "splitter magnet".

We carried out hypernuclear spectroscopic experiments in JLab Hall-C for two times before the present experiment, and these experiments were based on this method. We developed the experiments by introducing new experimental apparatus in order to expand the mass region of the produced hypernuclei and improve the mass resolution of the hypernuclei. The physics goals and developments of the experimental technique will be explained in this section.

\subsubsection{The first generation experiment (E89-009)}

We carried out the first $\left(\mathrm{e}, \mathrm{e}^{\prime} \mathrm{K}^{+}\right) \Lambda$ hypernuclear spectroscopic experiment in 2000 at JLab Hall-C. This experiment aimed to study the feasibility of the $\left(\mathrm{e}, \mathrm{e}^{\prime} \mathrm{K}^{+}\right)$hypernuclear spectroscopic experiment with ${ }^{12} \mathrm{C}$ target. In the experiment, two existing spectrometer, the short orbit spectrometer (SOS) and the Enge Split Pole Specrometer (ESPS) were used with the splitter magnet. The SOS was used as a kaon spectrometer and the ESPS was used as a scattered electron spectrometer, as shown in figure 2.9. The SPL was used to deflect the positive and negative charged particles to the SOS and ESPS, respectively. The obtained ${ }_{\Lambda}^{12} \mathrm{~B}$ spectrum is shown in figure 2.10 [46], [47].

There were mainly two problems in this experiment as follows:

1. Zero-degree direction is included in the acceptance for each side. As already mentioned in the last section, there are many background events in the zero-degree direction associated with bremsstrahlung for each side. During E89-009, singles rate is about $200 \mathrm{MHz}$ in ESPS and $100 \mathrm{kHz}$ in SOS. Almost all particles coming to SOS were the positrons.

2. The SOS was not dedicated to $\Lambda$ hypernuclear spectroscopy, especially for its momentum resolution and solid angle. The conclusive result of missing mass resolution is $900 \mathrm{keV}$, which was limited by the momentum resolution of SOS. 


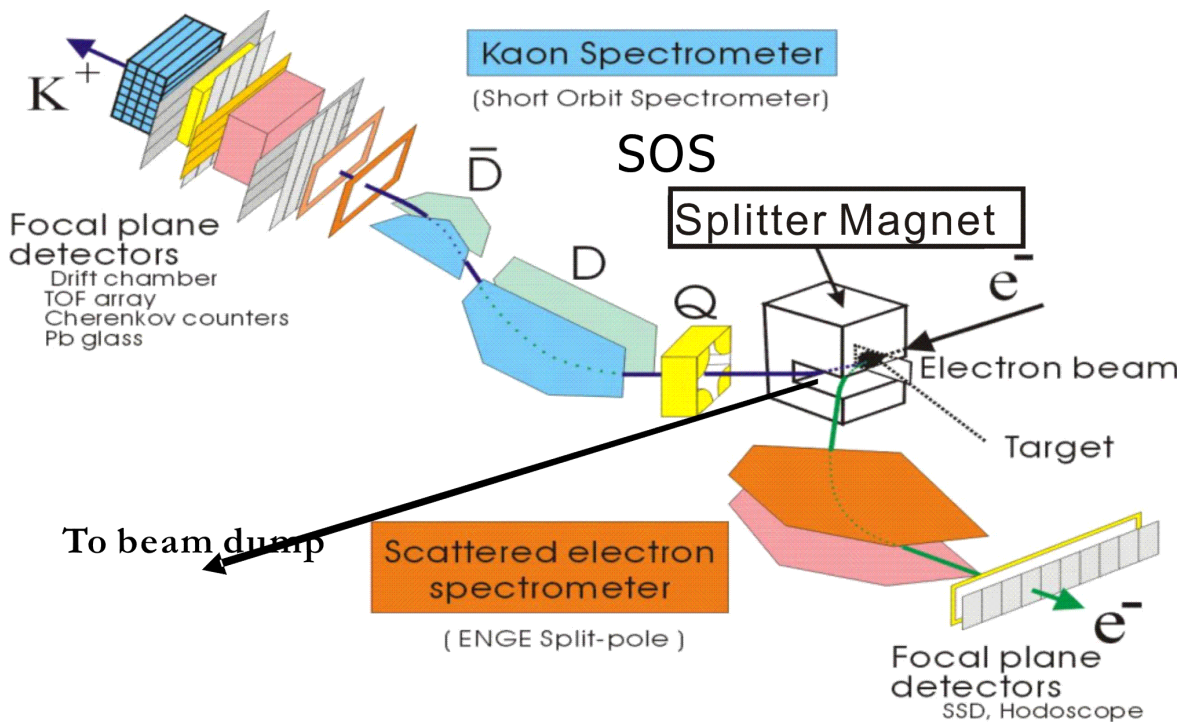

Figure 2.9: Experimental setup of JLab E89-009.

\begin{tabular}{lc}
\hline \hline SOS & QDD, vertical bend \\
Configuration & $1.2 \mathrm{GeV} / c$ \\
Central Momentum & $\pm 20 \%$ \\
Momentum acceptance & $5 \times 10^{-4}(\mathrm{FWHM})$ \\
Momentum resolution & $7.5 \mathrm{msr}$ \\
Solid angle & $10 \mathrm{~m}$ \\
Angular acceptance & $\pm 60 \mathrm{mrad}$ (scattering plane) \\
& $\pm 40 \mathrm{mrad}$ (out of plane) \\
Flight path length & \\
ESPS & $0.283 \mathrm{GeV} / \mathrm{c}$ \\
Central Momentum & $\pm 20 \%$ \\
Momentum acceptance & $5 \times 10^{-4}$ (FWHM) \\
Momentum resolution & $5 \mathrm{msr}$ \\
Solid angle & $\pm 25 \mathrm{mrad}$ (scattering plane) \\
Angular acceptance & $\pm 20 \mathrm{mrad}$ (out of plane) \\
\hline
\end{tabular}

Table 2.3.1: Specification of SOS and ESPS. 


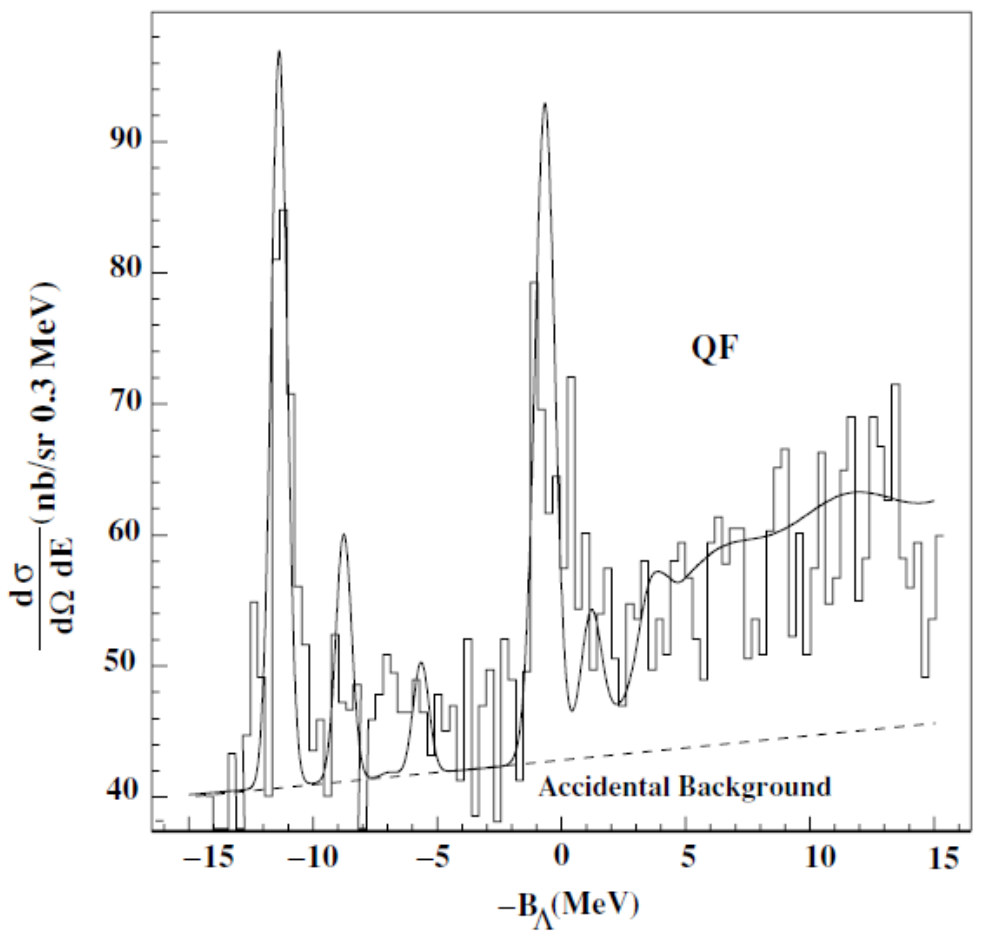

Figure 2.10: ${ }_{\Lambda}^{12} \mathrm{~B}$ spectrum measured in JLab E89-009 experiment. 
However, it is remarkably important that the first sub-MeV hypernuclear spectroscopy was achieved and principle of precise hypernuclear spectroscopy via the $\left(\mathrm{e}, \mathrm{e}^{\prime} \mathrm{K}^{+}\right)$reaction was proved.

\subsubsection{The second generation experiment (E01-011)}

Following the first generation experiment, we started the second generation $\left(\mathrm{e}, \mathrm{e}^{\prime} \mathrm{K}^{+}\right) \Lambda$ hypernuclear spectroscopic experiment in 2005, at JLab Hall-C with experimental target of ${ }^{7} \mathrm{Li},{ }^{12} \mathrm{C}$ and ${ }^{28} \mathrm{Si}$. We improved the following two points in order to overcome the problems found in the E89-009.

1. The "tilt method" was applied. In this method, the scattered electron spectrometer was tilted in order to avoid the huge background distributed around zero degree. The detail principle of the method will be explained later.

Table 2.3.2 is the comparison of scattered electron rate between the E89-009 experiment and the E01-011 experiment. Although the luminosity of E01-011 is almost 200 times higher than that of E89-009, the singles rate of the scatted electron side become $1 / 200$. We figured out that the tilt method worked effectively to reduce the electron singles rate.

2. The new high resolution kaon spectrometer (HKS), which is dedicated to the $\Lambda$ hypernuclear spectroscopic experiment, was designed and constructed. The detail of the HKS will be explained in chapter 3 .

The setup of the experiment is shown in figure 2.11. The splitter magnet and ESPS is used again, but the tilt method was applied. This setup was designed to achieve high resolution (about $500 \mathrm{keV}$ in missing mass resolution) and the high yield for $\Lambda$ hypernuclei.

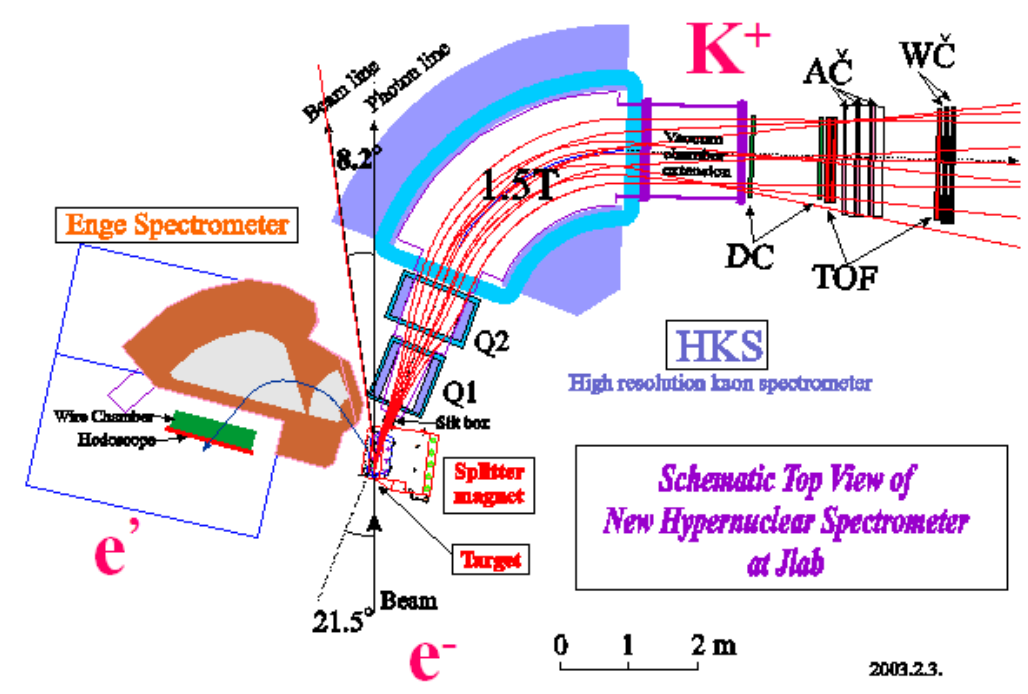

Figure 2.11: Experimental setup of JLab E01-011. 


\begin{tabular}{lc}
\hline \hline HKS & \\
Configuration & QDD, horizontal bend \\
Central Momentum & $1.2 \mathrm{GeV} / c$ \\
Momentum acceptance & $\pm 12.5 \%$ \\
Momentum resolution & $2 \times 10^{-4}(\mathrm{FWHM})$ \\
Solid angle & $15 \mathrm{msr}$ \\
Angular acceptance & \pm 7 degree $(\mathrm{scattering}$ plane $)$ \\
Flight path length & $10 \mathrm{~m}$ \\
ESPS & \\
Central Momentum & $0.276 \mathrm{GeV} / \mathrm{c}$ \\
Momentum acceptance & $\pm 30 \%$ \\
Momentum resolution & $4 \times 10^{-4}(\mathrm{FWHM})$ \\
Solid angle & $7 \mathrm{msr}$ \\
Angular acceptance & $\pm 7.75 \mathrm{mrad}(\mathrm{scattering}$ plane $)$ \\
\hline \hline
\end{tabular}

Table 2.3.2: Specification of HKS and ESPS.

Table 2.3.3: Comparison of the singles rate, hypernuclear yield of scattered electron side and luminosity.

\begin{tabular}{ccccc}
\hline \hline & $\begin{array}{c}\text { Beam } \\
\text { intensity } \\
{[\mu \mathrm{A}]}\end{array}$ & $\begin{array}{c}\text { Target } \\
\text { thickness } \\
{\left[\mathrm{mg} / \mathrm{cm}^{2}\right]}\end{array}$ & $\begin{array}{c}\text { Singles rate } \\
\text { of } e^{\prime} \text { side } \\
{[\mathrm{MHz}]}\end{array}$ & $\begin{array}{c}\text { Hypernuclear }\left({ }_{\Lambda}^{12} \mathrm{~B} \text { gs }\right) \\
\text { yield } \\
{[1 / \text { hour }]}\end{array}$ \\
\hline $\mathrm{E} 89-009$ & 0.67 & 22 & 200 & 0.4 \\
$\mathrm{E} 01-011$ & 30 & 100 & 1 & 5 \\
\hline \hline
\end{tabular}




\section{Tilt method}

The huge background particles going into the electron side are associated with the bremsstrahlung and $\mathrm{M} \phi$ ller scattering processes. Figure 2.12 shows the angular distribution of these background with the "signal" electrons associated to the virtual photon $\left(e_{V}^{\prime}\right)$ which produce $\Lambda / \Sigma^{0}$ or hypernuclei. One can see the signal to background (S/B) ratio is lower in the forward region and higher in the backward region. The "tilt method" utilize this characteristic for the background suppression, i.e, select the slightly backward region as an acceptance by tilting the spectrometer (see figure 2.13).

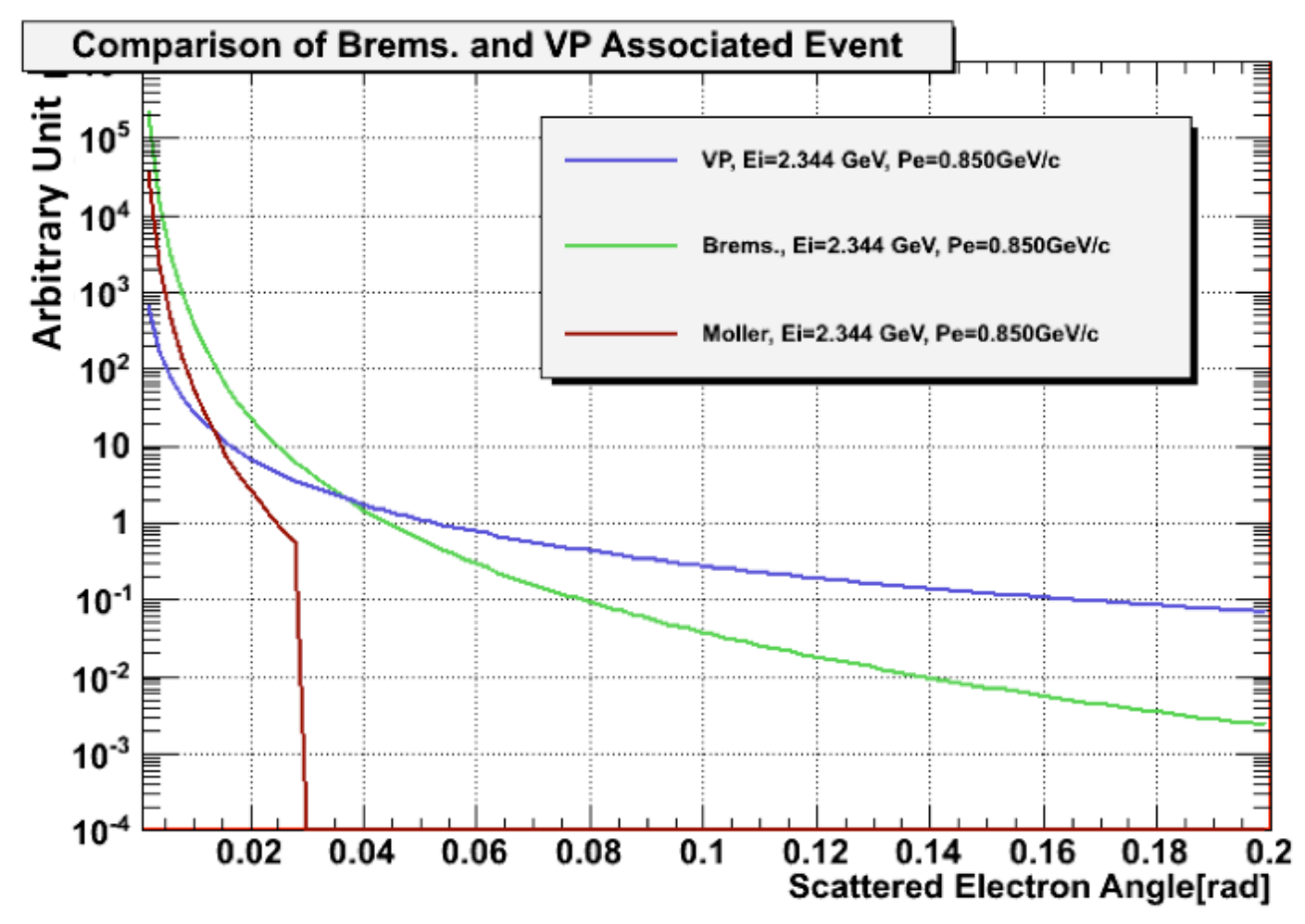

Figure 2.12: Angular distribution of the virtual photon / bremsstrahlung associated electron.

In this experiment, we tilted the ESPS vertically by 7.75 degree. The SPL has horizontal dispersion plane, and the angular acceptance of ESPS is also enlarged to the horizontal direction. Therefore, if we try to reduce the background by "horizontal tilt", the yield of the electrons become lower compared to the case of "vertical tilt". This is the reason why we applied the vertical tilt.

The tilt angle of 7.75 degree was the optimized value from the result of the simulation. The $\mathrm{S} / \mathrm{B}$ ratio bocome to be small in the backward region, but the hypernuclear yield is also decreased in such regions. Then, the optimization of the tilt angle is necessary so as to improve the S/B ratio with obtaining the hypernuclear yield as largest as possible. 

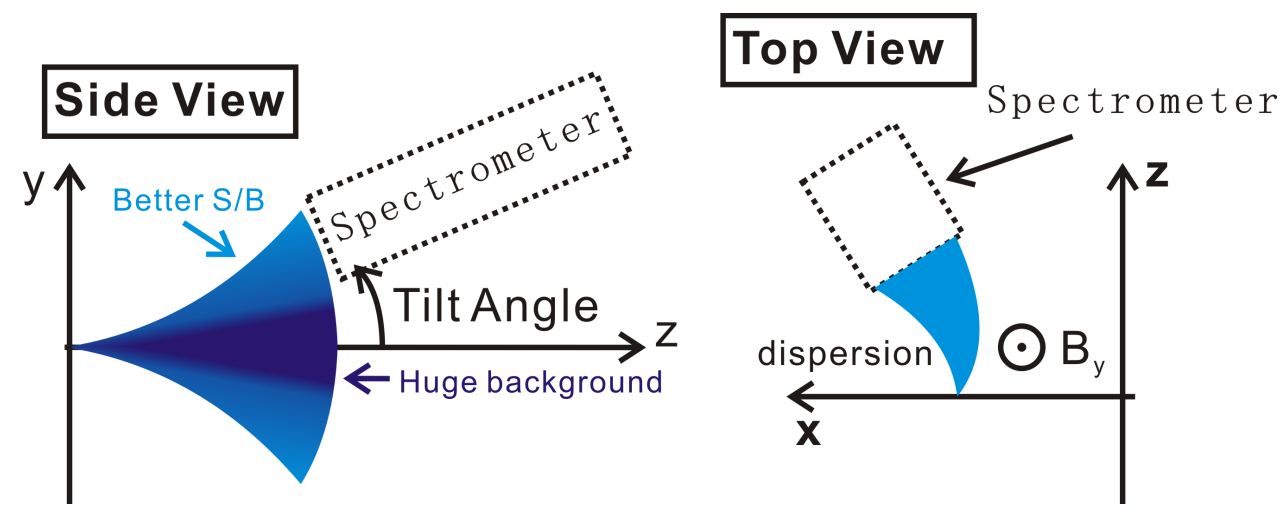

Figure 2.13: Concept of the tilt method. Backward acceptance is selected by titling the spectrometer vertically in order to improve the S/B ratio. The particles are horizontally dispersed by the magnetic field of the SPL.

\section{Result of E01-011 experiment}

Figure 2.14 shows the result of ${ }_{\Lambda}^{12} \mathrm{~B}$ spectrum measured in E01-011 experiment with the energy resolution of $470 \mathrm{keV}$ (FWHM). The spectra of ${ }_{\Lambda}^{7} \mathrm{He}$ and ${ }_{\Lambda}^{28} \mathrm{Al}$ were also measured with this high energy resolution [33]. These results indicate that two new experimental configuration, HKS and tilt method, worked fine. Then, it is essential to use these configurations for further $\left(\mathrm{e}, \mathrm{e}^{\prime} \mathrm{K}^{+}\right)$hypernuclear spectroscopic experiments.

\subsection{The design concepts of the third generation ex- periment (E05-115)}

In the E01-011 experiment, we successfully achieved hypernuclear spectroscopy up to $A=28$. Thanks to the application of the "tilt method", the counting rate became much smaller increasing the hypernuclear yield (see table 2.3.2), compared to the E89009 experiment.

In the third generation experiment (E05-115), the spectroscopy of medium heavy hypernuclei (up to $\mathrm{A}=52$, see table 2.4 as a summary of the targets used in the three experiments) is one of the important physics motivation, as mentioned in chapter 1 . In order to take such a heavy target data, two options could be considered. One is the data taking with very low intensity beam or very backward acceptance. Though this option can take "clean" experimental data with low background condition, the hypernuclear yield become also smaller. Another option is the data taking with high intensity beam and forward acceptance. In this option, the hypernuclear yield become higher, but the exquisite optimization of the beam current and acceptance is necessary because of the huge background. In addition, the improvement of the experimental configuration was essential in either case for effective data taking.

Consequently, in the present E05-115 experiment, we newly developed the high resolution electron spectrometer (HES) and the splitter magnet (SPL) with the assumption of 


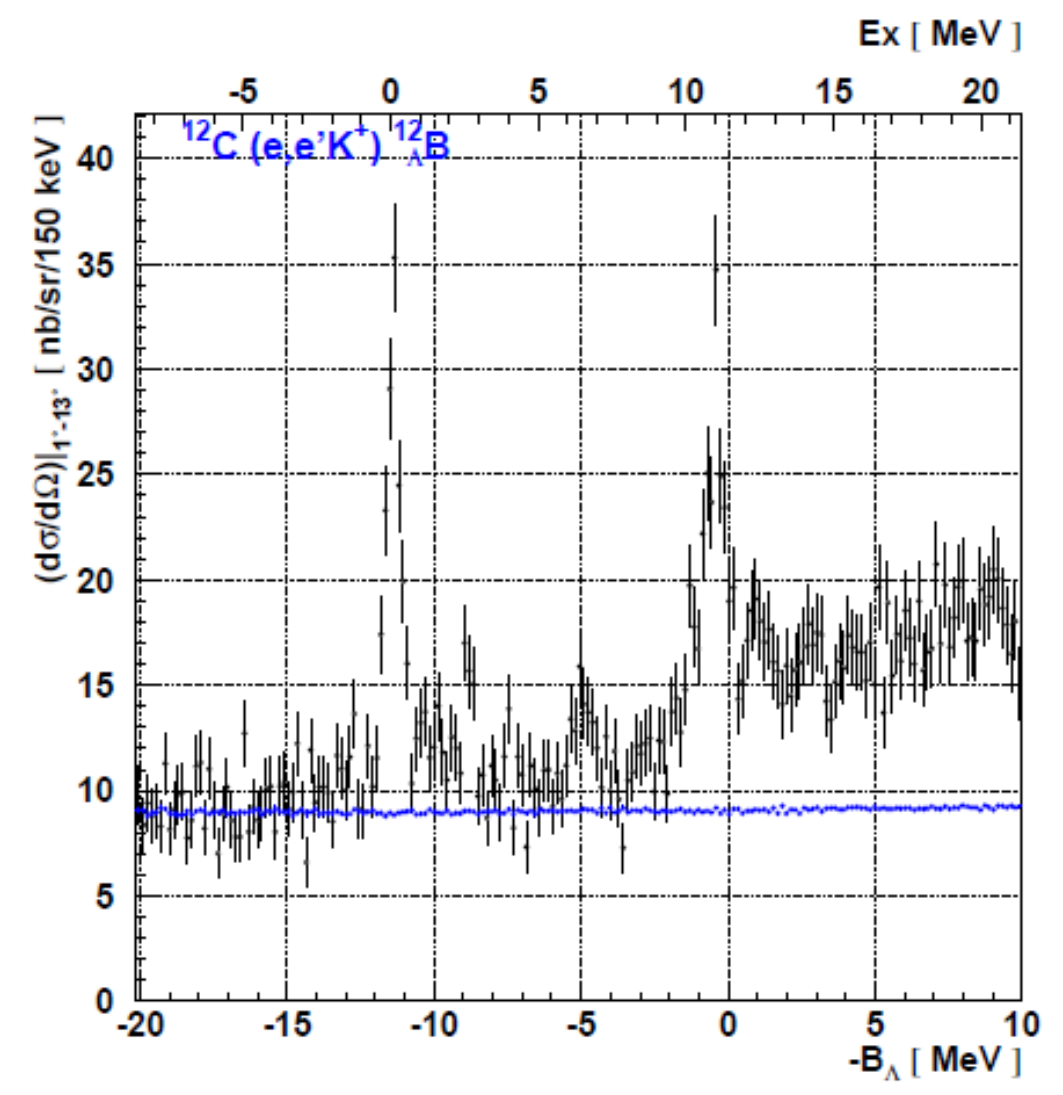

Figure 2.14: ${ }_{\Lambda}^{12} \mathrm{~B}$ spectrum measured in JLab E01-011 experiment [33]. 
using of the "tilt method". This configuration is dedicated to the $\left(\mathrm{e}, \mathrm{e}^{\prime} \mathrm{K}^{+}\right)$hypernuclear spectroscopic experiment with the very forward acceptance and high intensity beam. This configuration made it possible to take with five times larger hypernuclear yield, keeping the counting rate even in the case of ${ }^{52} \mathrm{Cr}$ target. The design concepts of this configuration will be explained in this section.

Table 2.4.1: Experimental target used in E89-009, E01-011 and E05-115.

\begin{tabular}{cc}
\hline \hline $\mathrm{E} 89-009$ & ${ }^{n a t} \mathrm{C}$ \\
$\mathrm{E} 01-011$ & ${ }^{n a t} \mathrm{Li},{ }^{n a t} \mathrm{C},{ }^{28} \mathrm{Si}$ \\
$\mathrm{E} 05-115$ & ${ }^{7} \mathrm{Li},{ }^{9} \mathrm{Be},{ }^{10} \mathrm{~B},{ }^{n a t} \mathrm{C},{ }^{52} \mathrm{Cr}$ \\
\hline
\end{tabular}

\subsubsection{Hypernuclear yield}

One of the important concepts for experimental setup design is obtainment of large hypernuclear yield. In the E01-011 experiment, the counting rate of the ${ }_{\Lambda}^{12} \mathrm{~B}$ ground state was about 5 per hour (see 2.3.2) and this was almost ten times larger than that of E89-009 experiment. However, it was required to increase this number as 30 per hour or more in the present experiment. Otherwise the data taking with ${ }^{52} \mathrm{Cr}$ target is very hard, because it is needed to use lower intensity beam in such a target than that of ${ }^{12} \mathrm{C}$ target case. In order to satisfy this requirement, we applied following points.

1. Higher energy beam $(2.344 \mathrm{GeV})$,

2. New electron spectrometer,

3. Tilt method and its optimization,

4. New splitter magnet.

These concepts are shown diagrammatically in figure 2.15. Each point will be explained in the following parts.

\section{Higher energy beam}

The reason we chose higher energy beam is that the electron background is emitted to more forward angles by the usage of higher energy beam. Figure 2.16 shows angular distribution of $e_{B}^{\prime}$ in two different energy, $1.851 \mathrm{GeV}$ (E01-011 value) and $2.344 \mathrm{GeV}$ (E05-115 value). It is clear that the $e_{B}^{\prime}$ is emitted to more forward angles in the higher energy case. In contrast, the angular distribution of the $e_{V}^{\prime}$ is not changed by the beam energy. Therefore, it is possible to select larger regions in the case of higher energy.

The reason to choose the value of $2.344 \mathrm{GeV}$ is that the energy beam can be provided by the CEBAF accelerator (see section 3.1) as a two pass beam. It is possible to use much higher energy beam, but the momentum of electrons become higher in that case, assuming the $E_{\gamma}=1.5 \mathrm{GeV}$. In such a case, the momentum resolution of the electron become worse compared to the lower beam energy case, because the momentum resolution 


\section{HES (new)}

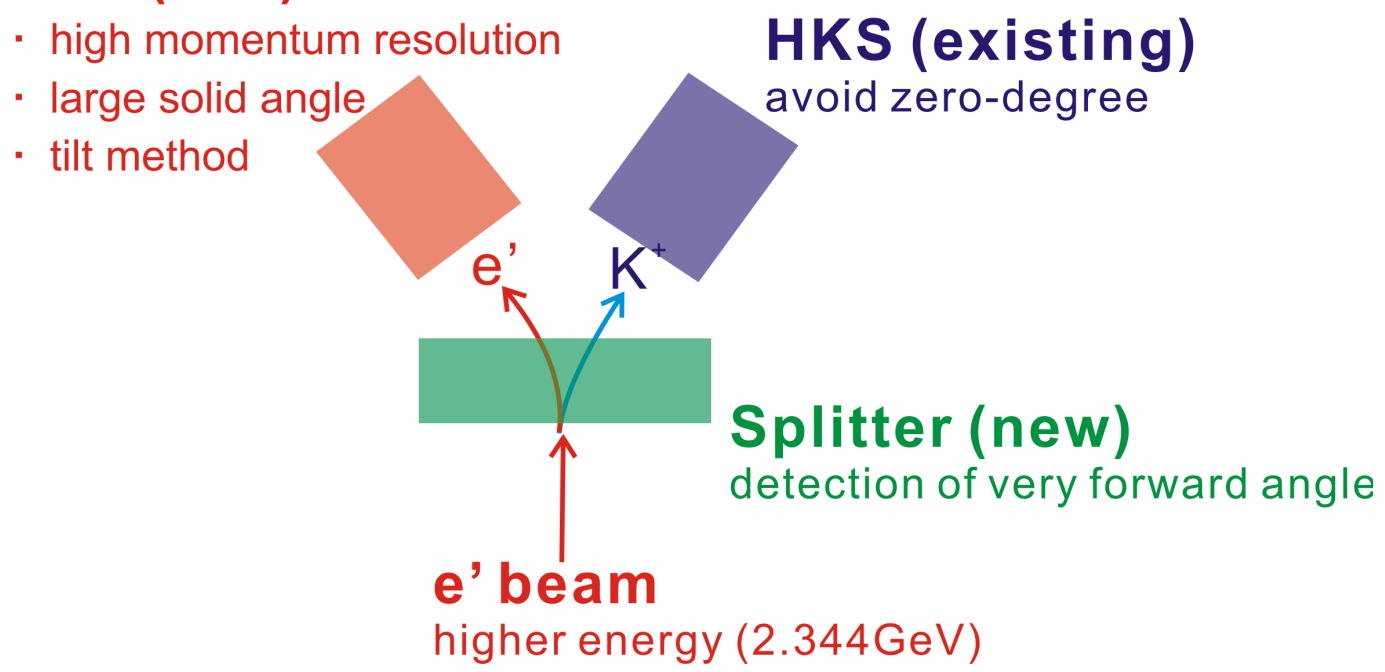

Figure 2.15: The design concept of E05-115 experiment.

of the spectrometer $d p / p$ is almost fixed as an order of $10^{-4}$ and the $d p$ is proportional to the momentum $p$. In the present case, we have existing HKS spectrometer and its momentum resolution is $d p / p=2 \times 10^{-4}$ at $p=1.2 \mathrm{GeV} / c$. It is possible to balance the momentum resolution of the scattered electron with this value if we choose the beam energy of $2.344 \mathrm{GeV}$.

\section{New electron spectrometer}

It is true that much higher beam energy concentrate the background much more forward, but it is required to handle higher momentum scattered electrons. In the present case, $E_{\gamma}$ is kept about $1.5 \mathrm{GeV}$, therefore the central scattered electron momentum $E_{e^{\prime}}$ is calculated as

$$
E_{e^{\prime}}=E_{e}-E_{\gamma}=2.344-1.5=0.844[\mathrm{GeV}]
$$

assuming 2.344 GeV beam. The ESPS which was used in the previous E01-011 experiment cannot handle this momentum beam. Therefore it is required to design new spectrometer for the scattered electrons to be able to handle the electrons of $0.844 \mathrm{GeV} / c$. This new spectrometer is the HES.

For the design of the HES, its momentum and angular acceptance are required to be matched with the existing HKS. Since the momentum acceptance of the HKS is $1.2 \pm 0.15$ $\mathrm{GeV} / c$, the momentum bite of the HES is required to be also about $\pm 0.15 \mathrm{GeV} / c$. The angular acceptance of the HES was based on that of ESPS. However, because of the usage of higher energy beam and changing of the background distribution, the acceptance can be expanded from that of ESPS. Figure 2.17 shows the $x^{\prime}$ vs $y^{\prime}$ distribution of the $e^{\prime}$ side for the ESPS (E01-011 experiment) and the HES. It can be seen that the acceptance for $y^{\prime}$ is expanded in the case of HES. This expansion highly contributes to the increase of hypernuclear yield. 


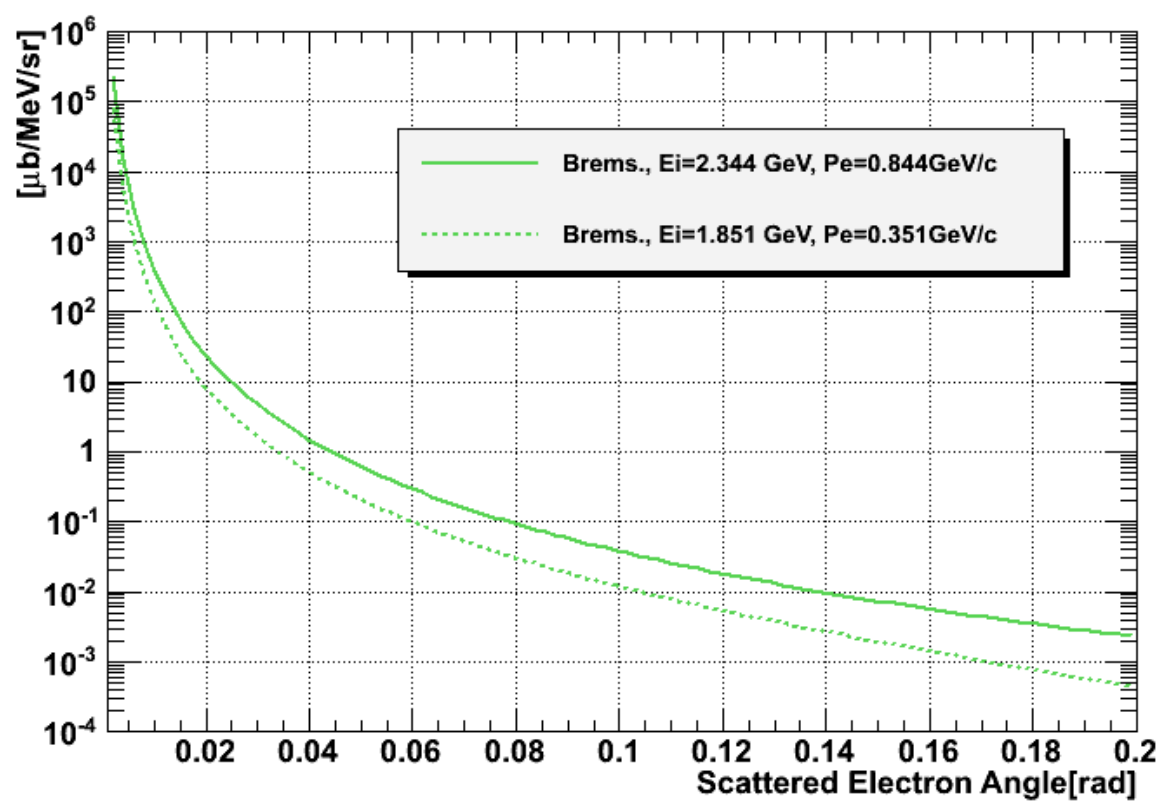

Figure 2.16: Comparison angular distribution of bremsstrahlung electron with different beam energy.
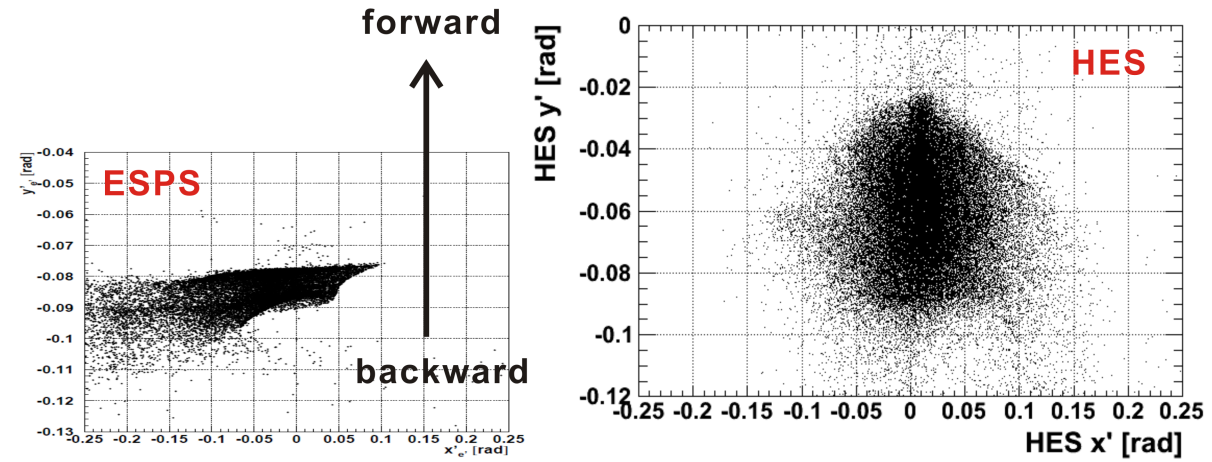

Figure 2.17: Comparison of angular acceptance between ESPS (left) and HES (right), plotting the $x^{\prime}$ vs $y^{\prime}$ distribution at HES. Note that $y^{\prime}$ direction is upside-down. 


\section{Tilt method and its optimization}

E05-115 is aiming to carry out the hypernuclear spectroscopy in the wide mass range up to ${ }_{\Lambda}^{52} \mathrm{~V}$. As already mentioned in section 2.2, the counting rate of background event in scattered electron side is associated with bremsstrahlung which is roughly proportional to the $Z^{2}$ of target. Therefore, the treatment of the background is much more severe than the case of E01-011. As a result of the tilt angle optimization, the HES was used with the tilt angle of 6.5 degree. The detail of the optimization will be explained in section 4.4.

\section{New splitter magnet}

As mentioned in 2.3, splitter magnet (SPL) is an essential equipment for the $\left(\mathrm{e}, \mathrm{e}^{\prime} \mathrm{K}^{+}\right)$ hypernuclear spectroscopic experiment.

The previous splitter magnet was used with the beam energy of about $1.8 \mathrm{GeV}$. The beam energy in the present experiment is higher by $0.5 \mathrm{GeV}$ and the previous splitter magnet cannot handle this beam. Therefore, in the present experiment, we newly designed and constructed the SPL magnet which can be used with the HES and higher beam energy.

The requirement for the new SPL was as follows:

1. Kinematic and optical matching with the HES and HKS,

2. Separation of kaons, scattered electrons, photons and post beam.

Since these requirements have to be satisfied by a dipole magnet, the magnet shape became very strange (see section 3.4 and 4.2 ).

The magnetic field strength can be determined so as to separate the scattered electrons and the post beam. The relation between the magnetic rigidity $(B r)$ and the momentum $(p)$ of a particle can be written as

$$
B r=\frac{10^{9}}{c} p=3.34 p
$$

where $B$ is the magnetic field strength in Tesla, $r$ is the radius of the track in meter and $p$ is in $\mathrm{GeV} / c$. The momentum difference between the scattered electron $(2.34 \mathrm{GeV} / c)$ and the post beam $(0.84 \mathrm{GeV} / c)$ is $1.5 \mathrm{GeV} / c$. Therefore, if we required the difference of these track radius as 3 meter, the necessary magnetic field can be calculated as

$$
B=1.67 \text { [Tesla] }
$$

From this consideration, we determined the SPL maximum magnetic field as 1.7 Tesla. The idea of this calculation is diagrammatically represented in figure 2.18 . These are the basic concepts of the SPL magnet and the detail of design based on the requirements will be described in section 4.2, together with that of the HES.

\subsubsection{Resolution}

HES is required be a high momentum resolution spectrometer with an order of $d p / p \sim$ $10^{-4}$. The main contribution to the resolution of hypernuclear mass spectrum is

- Resolution of the beam momentum, 


\section{Post Beam}

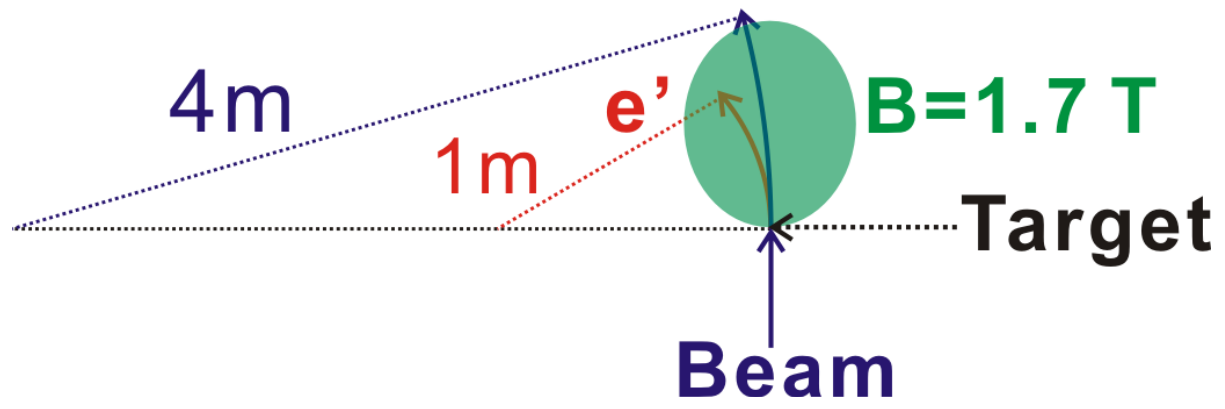

Figure 2.18: Schematic of the scattered electron and post beam track in the SPL. Both tracks are separated by the same magnetic field and they are separated by $3 \mathrm{~m}$ in track radius. The actual track is shown in figure 4.4 .

- Resolution of the kaon momentum,

- Resolution of the scattered electron momentum,

- Particle energy struggling in the experimental target.

The angular resolution of each particle can be neglected in the case of hypernuclear mass spectrum, because the recoil effect for the hypernuclear production is very small.

In the above components, the resolution of beam energy and kaon momentum is already fixed as $\Delta p_{e} / p_{e}<1 \times 10^{-4}$ at $p_{e}=2.344 \mathrm{GeV} / c$ and $\Delta p_{K} / p_{K}=2 \times 10^{-4}$ at $p_{K}=1.2 \mathrm{GeV} / c$, respectively. Namely, the momentum resolutions of these two particles are balanced as $\Delta p=2.4 \times 10^{-4} \mathrm{GeV} / c$. Therefore, the momentum resolution of HES is required be this level. In that case, the required resolution is better than

$$
\frac{\Delta p_{e^{\prime}}}{p_{e^{\prime}}}=2.4 \times 10^{-4} / 0.844 \sim 2.8 \times 10^{-4}
$$

These are the concepts of the third generation experiment. The detail estimation of the HES resolution will be done in section 4.5. As a result of building the experimental setup based on these concepts, we successfully took experimental data of hypernuclei up to $\mathrm{A}=52$. 


\section{Chapter 3}

\section{Experimental setup overview and trigger system}

A hypernuclear spectroscopic experiment via the (e, $\left.\mathrm{e}^{\prime} \mathrm{K}^{+}\right)$reaction (JLab E05-115 experiment) was carried out in JLab Hall C from August 2009 to Octber 2009 using the electron beam of CEBAF, continuous linear electron accelerator. The goal of this experiment is precise determination of the binding energies of various states for $\Lambda$ hypernuclear up to $\mathrm{A}=52$. In order to achieve this purpose, the quality of the electron beam and spectrometers are absolutely essential.

In this chapter, firstly the specification of CEBAF electron accelerator will be explained in section 3.1. Section 3.2-3.8 describe whole experimental setup and each apparatus of the experiment. In particular, the detail of magnets and detectors of HKS (high resolution kaon spectrometer) and HES (high resolution electron spectrometer) will be described. We newly designed HES with the same concept as HKS for this experiment, then the detail design of HES will be explained later in chapter 4, although the basic specifications will be described in this chapter. Finally, the trigger system of the present experiment will be explained in section 3.9 and section 3.11 summarize the experimental data condition.

\subsection{CEBAF electron accelerator facility}

\subsubsection{CEBAF overview}

Continuous Electron Beam Accelerator Facility (CEBAF) at JLab can provide high quality and high luminosity beam which is essential tool for precise measurement of nucleus or nucleon via small cross section reactions.

CEBAF has two electron linear accelerators which can deliver almost $100 \%$ continuous wave $(\mathrm{CW})$ to three experimental halls, Hall A, B and C, as shown in figure 3.1.

The main parameter of CEBAF accelerator is summarized in table 3.1.1. An electron beam is generated at the injector with $1497 \mathrm{MHz}$ short bursts, then divided into three 1497 $\mathrm{MHz} / 3=499 \mathrm{MHz}$ bursts by RF chopper and accelerated by both south and north linac up to $6.0 \mathrm{GeV}$ maximum. After the splitting the beam from the accelerator beam switch yard, finally it is transported to the beamline of each experimental hole with 2 ns bunch structure. The typical value of beam energy spread and emittance are $d E / E<1.0 \times 10^{-4}$ 


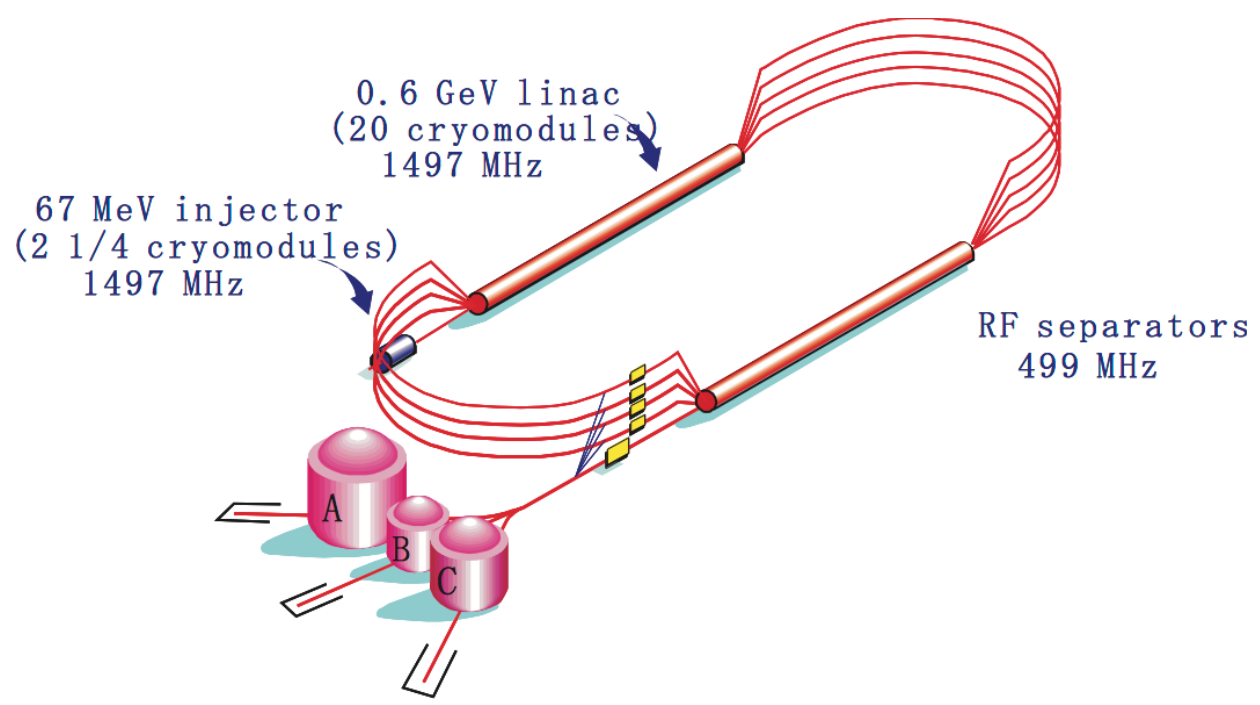

Figure 3.1: Schematic view of CEBAF electron accelerator

Table 3.1.1: The main parameter of CEBAF.

\begin{tabular}{cc}
\hline \hline Maximum Beam Energy & $6 \mathrm{GeV}$ \\
Maximum Beam Intensity & $200 \mu \mathrm{A} / \mathrm{Hall}$ \\
Beam Emittance & $\sim 2 \times 10^{-9} \mathrm{~m} \mathrm{rad}$ \\
Beam Energy Spread & $\leq 1 \times 10^{-4}$ (FWHM) \\
Beam Bunch Interval & $2 \mathrm{~ns}(499 \mathrm{MHz})$ \\
\hline \hline
\end{tabular}


and $\sim 2 \times 10^{-9} \mathrm{~m} \cdot \mathrm{rad}$, respectively. These values correspond to the absolute beam energy spread of $d E<230 \mathrm{keV}$, the beam spot size of $\phi=100 \mu \mathrm{m}$ and the beam angular spread of order of $10^{-2} \mathrm{mrad}$.

CEBAF is unique for a high resolution hypernuclear spectroscopic experiment via the $\left(\mathrm{e}, \mathrm{e}^{\prime} \mathrm{K}^{+}\right)$reaction at the point of 2009 by following reason:

1. Beam energy is larger than $\Lambda$ threshold $(1.6 \mathrm{GeV})$.

2. High intensity beam which can make up for the small cross section of hypernuclei via the $\left(\mathrm{e}, \mathrm{e}^{\prime} \mathrm{K}^{+}\right)$reaction can be used (typically several $10 \mu \mathrm{A}$ ).

3. The energy resolution of beam can achieve $\leq 1.0 \times 10^{-4}$ (FWHM). This value is almost balanced with the energy resolutions of our spectrometer (about $200 \mathrm{keV}$ $($ FWHM $)$ ). We can estimate that our designed energy resolution of whole experimental setup is about $500 \mathrm{keV}$ (FWHM).

4. Small beam spot size (about $\sigma=100 \mu \mathrm{m}$ ) is also important for the high resolution spectroscopic experiment. We assumed that the optics of our spectrometer is so-called "piont-to-point" focus which assumes the beam spot size at target is zero. However, the actual beam spot has its size and if the size become bigger, the momentum resolution of the spectrometers become worse. The value of $100 \mu \mathrm{m}$ is almost same as the position resolution of the tracking detector, therefore this spot size does not affect to the momentum resolution.

5. Duty factor is almost $100 \%$. Since the $\left(\mathrm{e}, \mathrm{e}^{\prime} \mathrm{K}^{+}\right)$hypernuclear experiment is the coincidence experiment of scattered electron and kaon, high duty factor is important for effective data taking.

Recently, MAMI-C at Mainz university in Germany is upgraded and becomes to be another facility which can to carry out the $\left(\mathrm{e}, \mathrm{e}^{\prime} \mathrm{K}^{+}\right)$experiment. KaoS collaboration is studying and preparing for the future experiment [48].

\subsubsection{Beamline apparatuses}

\section{Beam Position Monitor (BPM)}

BPM measures beam position in the beamline and can be used for the beam tuning and beam energy correction. The beam tuning was done at the beginning of the experiment and the detail will be described in the later part of this section. The energy correction is done after the experiment. Since the BPM information was read by ADC event by event, we can correct the beam momentum due to the difference of the beam orbit.

In this experiment, cavity BPM was used. Cavity BPM has two antennas for horizontal direction and vertical direction, respectively. These antennas can measure beam position without affecting beam.

\section{Superharp}

The superharp is an a beam wire scanner which provides absolute beam position readout using a shaft encoder [49]. The advantage of superharp is high precision measurements 
of beam profile and position $(\Delta x \sim 10 \mu \mathrm{m})$ which corresponds to beam energy accuracy of $10^{-3}$. The beam profile measurements can be obtained over a wide beam current range of $1-200 \mu \mathrm{A}$.

The superharps are installed in the arc section of Hall-C beamline and used to calibrate beam position at the beginning of the experiment, because the superharps measure the beam position destructively,. The information was read by EPICS (Experimental Physics and Industrial Control System, a slow readout control system). The detail of EPICS will be described in section 3.10.

\section{Beam Current Monnitor (BCM) and Unser monitor}

The Beam Current Monitor (BCM) consists of an Unser monitor and two RF cavities. In addition to the Unser monitor, a cavity monitor and a Faraday Cup at the injector section of the main accelerator are also used to provide an absolute reference during calibration runs.

Unser monitor is a Parametric Current Transformer which consists of magnetic modulator for lower frequency channel and active current transformer for higher frequency channel. The magnetic modulator provides parametric amplification in the low frequency channel, up to a transition frequency of about $500 \mathrm{~Hz}[50]$.

The continuous beam monitoring has been performed by two RF cavities and Unser was used to calibrate BCM, because the Unser measures the beam current destructively and require dedicated beam time. The calibration has been done with the special runs during beam time and we obtained the BCM systematic error of $\pm 0.2 \mu \mathrm{A}$.

The BCM information was read by EPICS.

\section{Syncrotron Light Interferometry (SLI)}

$\mathrm{SLI}[51]$ is a device to measure the beam energy spread which is measured by the beam width at a dispersive bend located in the transport line to the experimental hall. The advantages of this device is its high spatial resolution with non-invasive measurement by the measurement of interferometry of the synchrotron light produced in the dispersive bend.

Our requirement of upper limit on the RMS momentum spread is $\delta p / p \leq 3 \times 10^{-5}$, which is corresponding to an upper bound on the beam width of $120 \mu \mathrm{m}$. Since the spatial resolution of SLI is $10 \mu \mathrm{m}$ over the beam current range from $20 \mu \mathrm{A}$ to $120 \mu \mathrm{A}$, we can measure the beam width with enough resolution.

The information of SLI was also read by EPICS.

\section{Fast feedback system (FFB)}

FFB is a system used for beam energy and position stabilization[52], which is installed in the arc section of the Hall-C beamline. The mechanism of the system is that the beam position information obtained from BPM can be feed back to the magnet beam current actively.

The system can suppress beam motion by a factor greater than ten, thus achieving the stabilization of relative beam energy fluctuations and beam position to be better than $10^{-5}$ and $20 \mu \mathrm{m}$ on the target, respectively. 


\subsubsection{Hall-C beamline}

Figure 3.2 shows a schematic view of Hall-C beamline. The arc section and alcove section were existing part of the Hall-C beamline used as usual in the experiment. The electron beam from the linac of the CEBAF is delivered to the experimental hall and some beam parameters such as the momentum and intensity can be determined by the apparatuses installed on it.

The other part is the beamline in the experimental hall. We use the Splitter magnet (SPL) which basically deflects positive charged particles and negative charged particles. However, the SPL also needs to separate electrons going to HES and post-beam, in other words, the SPL is a part of beamline and the beam is strongly affected by the SPL magnetic field. Since it was impossible for existing beamline to absorb the influence of SPL and the experimental target position is different from the standard position of Hall$\mathrm{C}$, the beamline inside the hall was newly designed and constructed for our experiment by the collaboration of JLab accelerator group and our experimental group. Table 3.1.2 shows the list of apparatuses inside the hall. Some BPM, BCM and superharps were installed backward and forward of the magnets and used for the beam tuning.

\begin{tabular}{ccccc}
\hline \hline Position & BPM & BCM & Superharp & Distance from target $[\mathrm{cm}]$ \\
\hline \hline A & IPM3C20A & & & \\
\hline B & IPM3H00 & & & 2148 \\
\hline C & IPM3H00A & IBC3H00 & IHA3H00 & \\
& IPM3H00B & & IHA3H00A & \\
& IPM3H00C & & & \\
\hline D & IPM3H00D & IBC3H00E & IHA3H01A & \\
& IPM3H00EH & & IHA3H01B & \\
& IPM3H00EV & & & \\
& IPM3H00F & & & \\
E & & IBC3H01 & \\
\hline \hline
\end{tabular}

Table 3.1.2: Beamline apparatus in E05-115 experiment.

\section{Pre-chicane}

As shown in figure 3.2, the beam entrance of Hall-C is in alignment with Hall-C beam dump. However, as mentioned above, the beam is bent by the SPL magnetic field. Therefore, in order to transfer the beam to the Hall-C beam dump, the bending power of the SPL must be cancelled by putting the other magnetic fields.

We have two options for the cancellation, putting the bending power before or after the experimental target. During the E01-011 experiment, the cancellation was done by the dipole magnet placed after the target (Post-chicane). This method does not need the fine beam tune before target and actually worked. However, it was difficult to handle postbeam because beam halo was generated by multiple scattering at the target. Furthermore, huge background events generated by the beam halo of the post-chicane beamline suffered HKS detectors. These background events also increased radiative level of the beamline. 


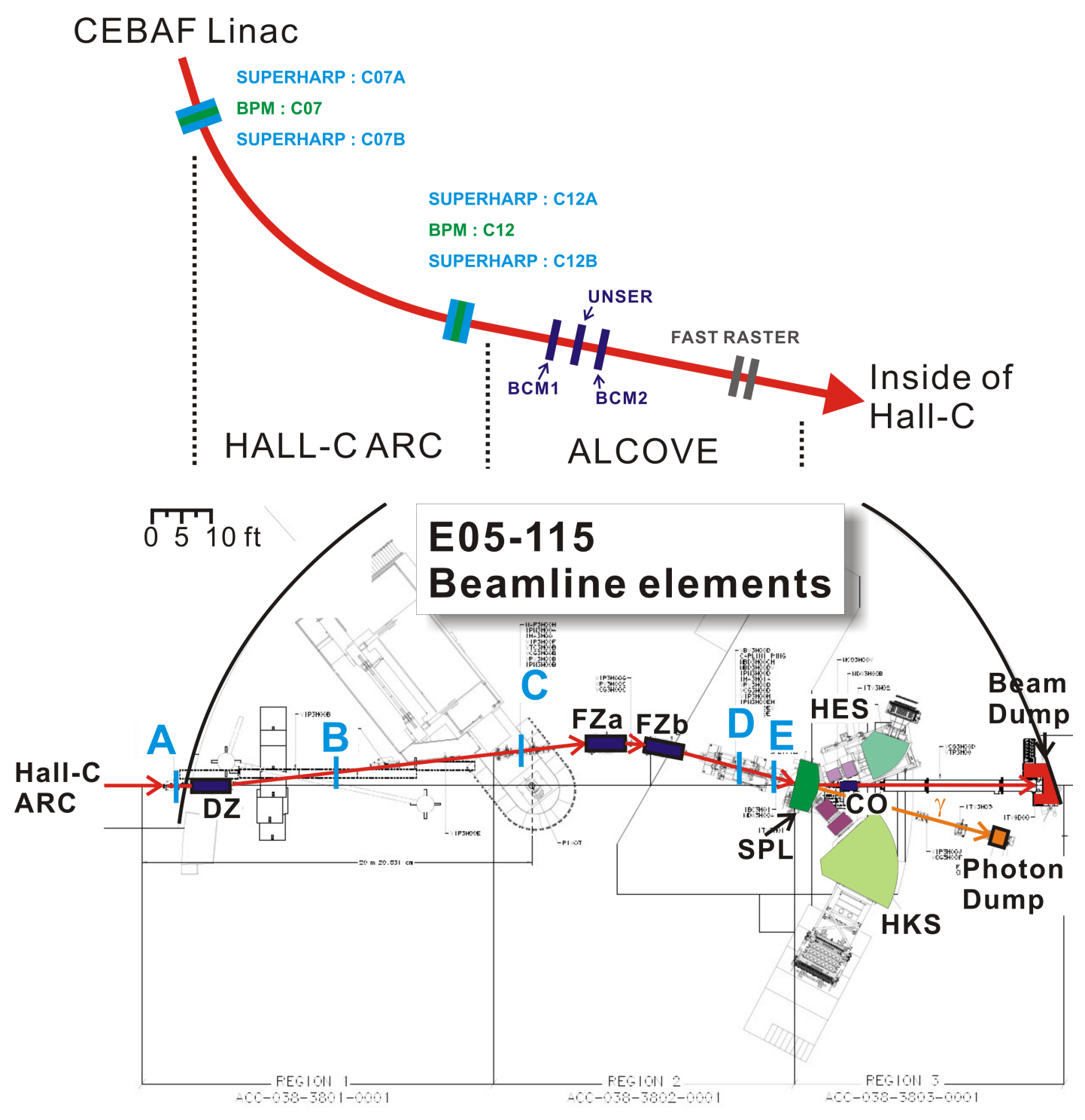

Figure 3.2: Schematics of Hall-C beamline. Upper figure shows the schematic view of Hall-C arc and alcove. Lower figure shows inside of the hall. 
On the other had, "Pre-chicane" is a method to bend the beam before the target as shown in figure 3.2. This method can resolve the problems described above. In the Prechicane method of E05-115 experiment, the beam coming from Hall-C arc is firstly bent to the HES side by DZ magnet placed at the entrance of Hall-C. After that, FZ magnets bend the beam to the other side and finally the beam hits the experimental target placed in the SPL magnet. After the target, the post-beam is bent by SPL magnetic field and transferred to the beam dump. The photons generated in the target by bremsstrahlung goes to the photon dump. Although the beam transfer handling become much more difficult in this method, we applied this method in order to avoid the problems, in particular for the reduction of the radiative level of the beamline.

\section{Beamline tuning}

The beamline magnet parameters were tuned at the beginning of the beam time using pulsed beam in order to avoid high radiative damage on the stuffs around the beamline. Firstly, the tuning before target has been done looking at beam position at target, which was checked by the target viewer and $\mathrm{BeO}$ target (see section 3.3). Basically the parameters were based on the optics calculation of the beamline magnets.

Next, the tuning has been done for the magnets after the target toward beam dump. The largest component in these magnets is the SPL and the post-beam is strongly affected. The beam "position" at target had been already adjusted, but the incoming "angle" which has a big influence on the trajectory of post-beam was not adjusted at that time.

Figure 3.3 shows the picture of SPL vacuum chamber. The SPL vacuum chamber has four exits: HKS exit for kaon, photon dump exit for photon, beam dump exit for post beam and HES exit for scattered electron. In particular, post beam and photon must be delivered to their own exit in order to avoid high radiation around beamline. We checked these tracks using YAG viewer placed just before the photon dump for photon and a beam monitor placed just before the beam dump for post beam. Figure 3.4 is a schematic of the tracks around the SPL. The directions of these tracks are directly affected by the incoming angle of the beam, which is bent from the fringe field of SPL which has a lot of uncertainty. We carefully adjusted the incoming angle of the beam so as to guide each particles to the each exit looking at the monitors, the detectors of HES and HKS and beam position at target.

\subsubsection{Beam Raster}

The spot size of electron beam from CEBAF is less than $100 \mu \mathrm{m}$. This feature is essential for our experiment which requires high resolution of sub-MeV level because the optics of the spectrometers assume so called "point to point focus".

However, in the case of some experimental targets, this beam spot size is so small that the temperature of some targets reach to its melting point. In order to avoid the target melting, we effectively enlarged the beam spot size by moving the beam hit position at target by $25 \mathrm{kHz}$. We called this beam moving "beam raster". The beam raster is realized by the Fast Raster system [53] installed in the Hall-C beam line.

The beam raster is applied in $\mathrm{CH}_{2}$ target run and ${ }^{7} \mathrm{Li}$ target run, as shown in table 3.1.4. For the analysis of these target runs, we assume the point beam spot, similarly 


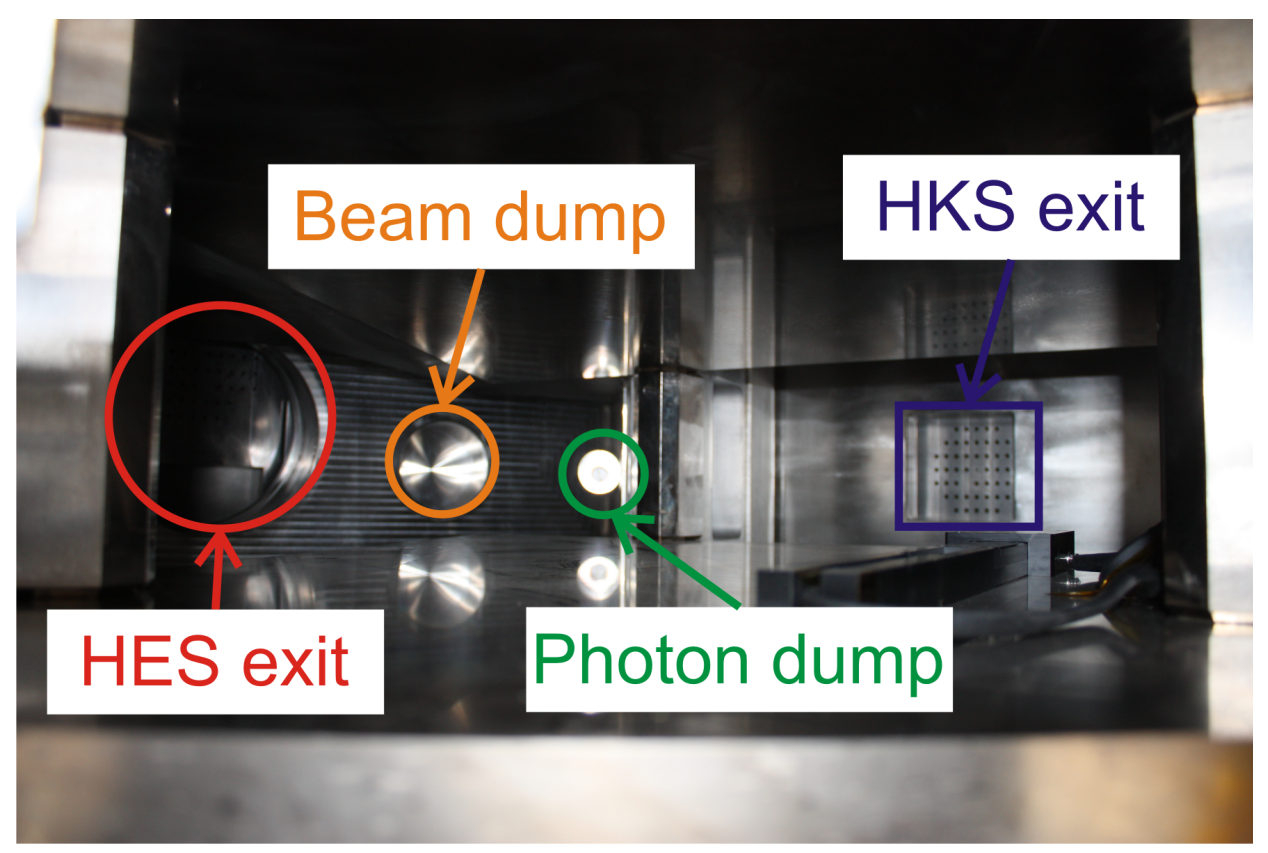

Figure 3.3: Picture of SPL vacuum chamber taken from the target point. There are four exits on the vacuum chamber: HKS exit (kaon), photon dump exit (photon), beam dum exit (post beam) and HES exit (scattered electron). We must tune the beam for each particle to be delivered to the corresponding exit.

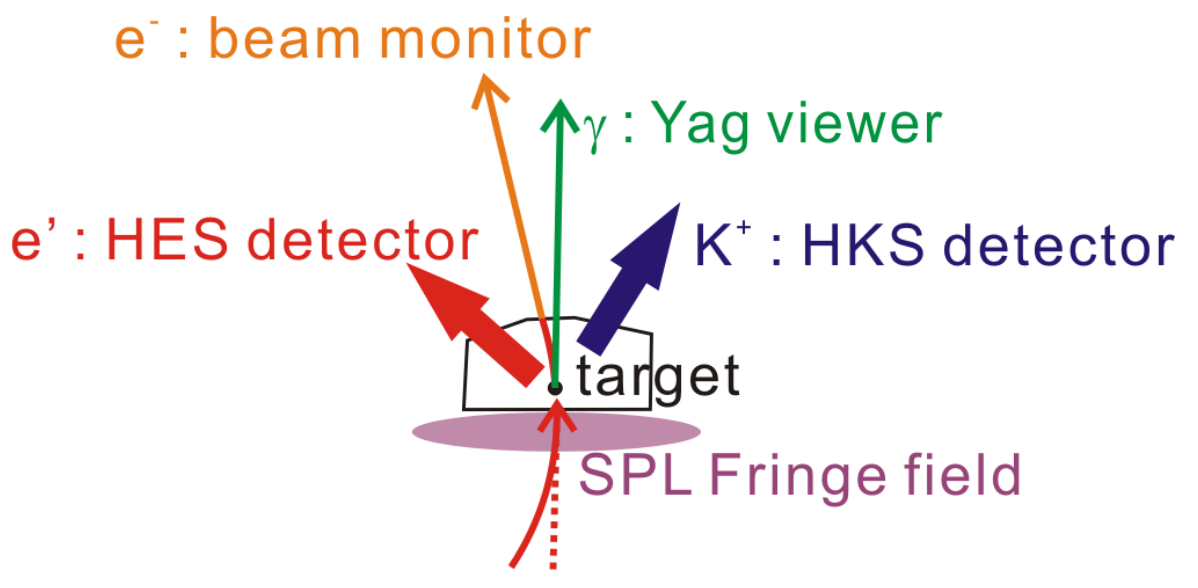

Figure 3.4: Schematic of beam trajectory around SPL. The incoming angle of the beam to the target must be tuned so as to guide each particles to the each exit considering the fringe field effect of the SPL magnet. 
to the other targets. This assumption affect on the reconstructed momentum and mass scale. Therefore we need to apply a correction to cancel the effect of the beam raster (so-called raster correction). The detail of the raster correction analysis will be explain in section 5.5.1.

Table 3.1.3: The beam raster size applied during the experiment. The raster sizes are shown as (horizontal size) $\times$ (vertical size).

\begin{tabular}{ccc}
\hline \hline $\begin{array}{c}\text { Target } \\
\text { Target }\end{array}$ & $\begin{array}{c}\text { Nominal beam } \\
\text { current }[\mu \mathrm{A}]\end{array}$ & $\begin{array}{c}\text { Raster size } \\
{[\mathrm{mm}(\mathrm{h}) \times \mathrm{mm}(\mathrm{v})]}\end{array}$ \\
\hline $\mathrm{CH}_{2}$ & 2 & $3 \times 1$ \\
${ }^{7} \mathrm{Li}$ & 35 & $1 \times 1$ \\
\hline \hline
\end{tabular}

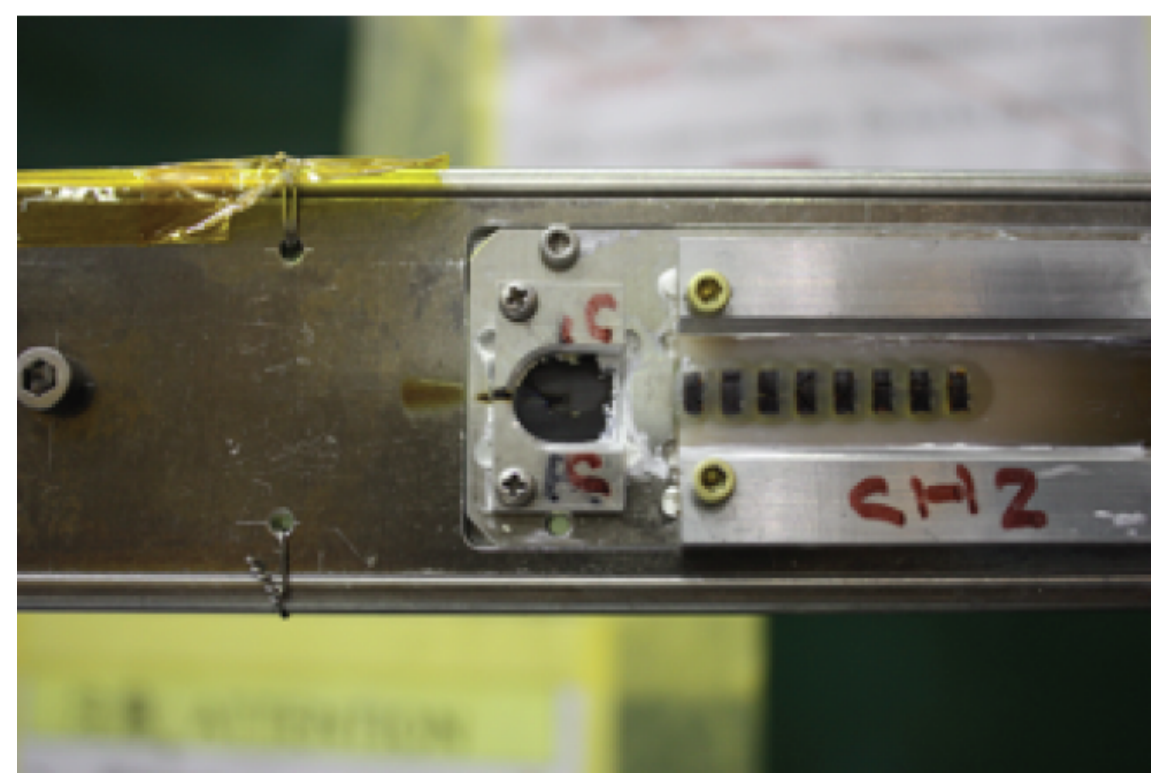

Figure 3.5: Picture of raster pattern on the $\mathrm{CH}_{2}$ target.

\subsection{Experimental condition and entire setup}

Figure 3.6 shows the entire setup of E05-115 experiment and figure 3.7 is a picture of the setup. The entire setup is mainly consists of two spectrometers, HKS and HES, which detect kaons and scattered electrons, respectively. There are also a characteristic magnet, SPL, which deflect kaons, photons, post beam and scattered electron with a dipole magnetic field. The experimental target was placed in the SPL magnet. This setup is our answer to the effective and precise measurement of hypernuclei and the basic idea will be explained in this section. 


\section{E05-115 whole setup}

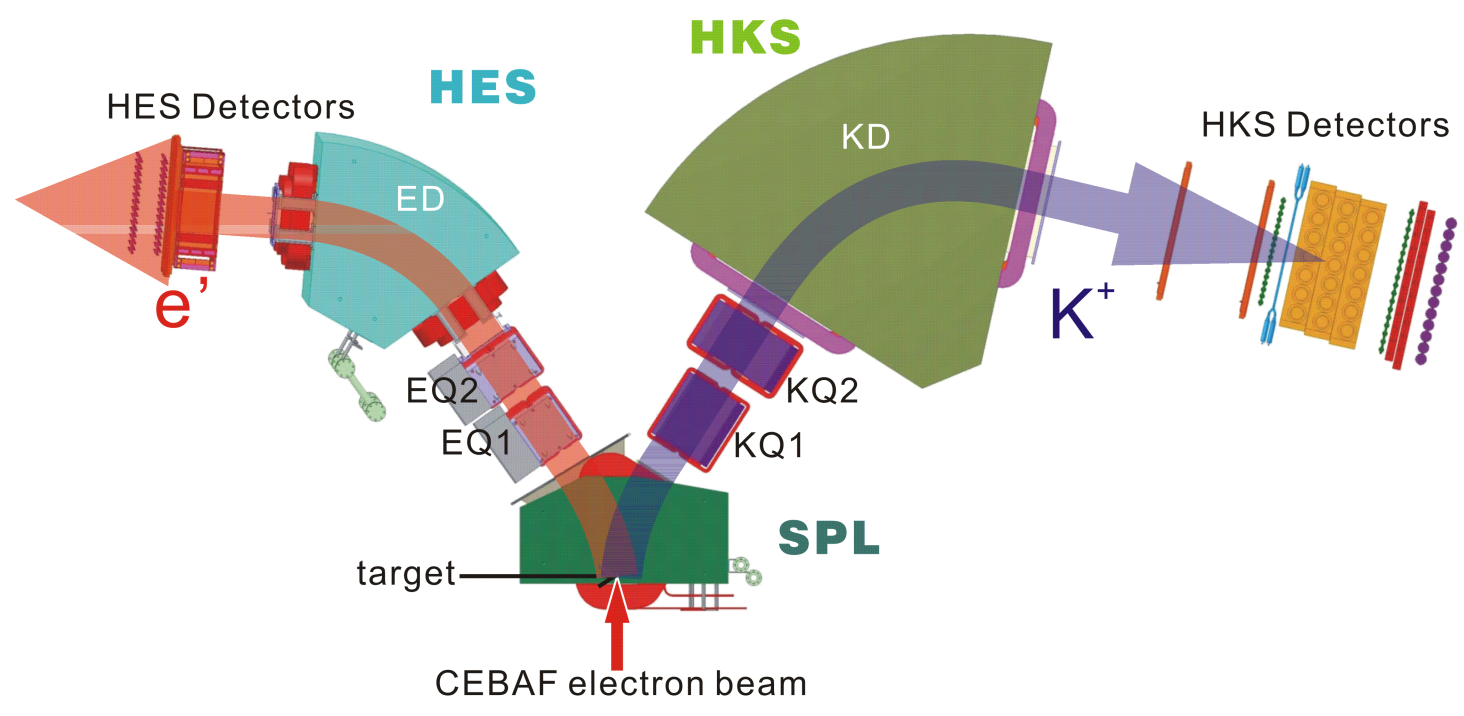

Figure 3.6: Schematic view of the entire setup.

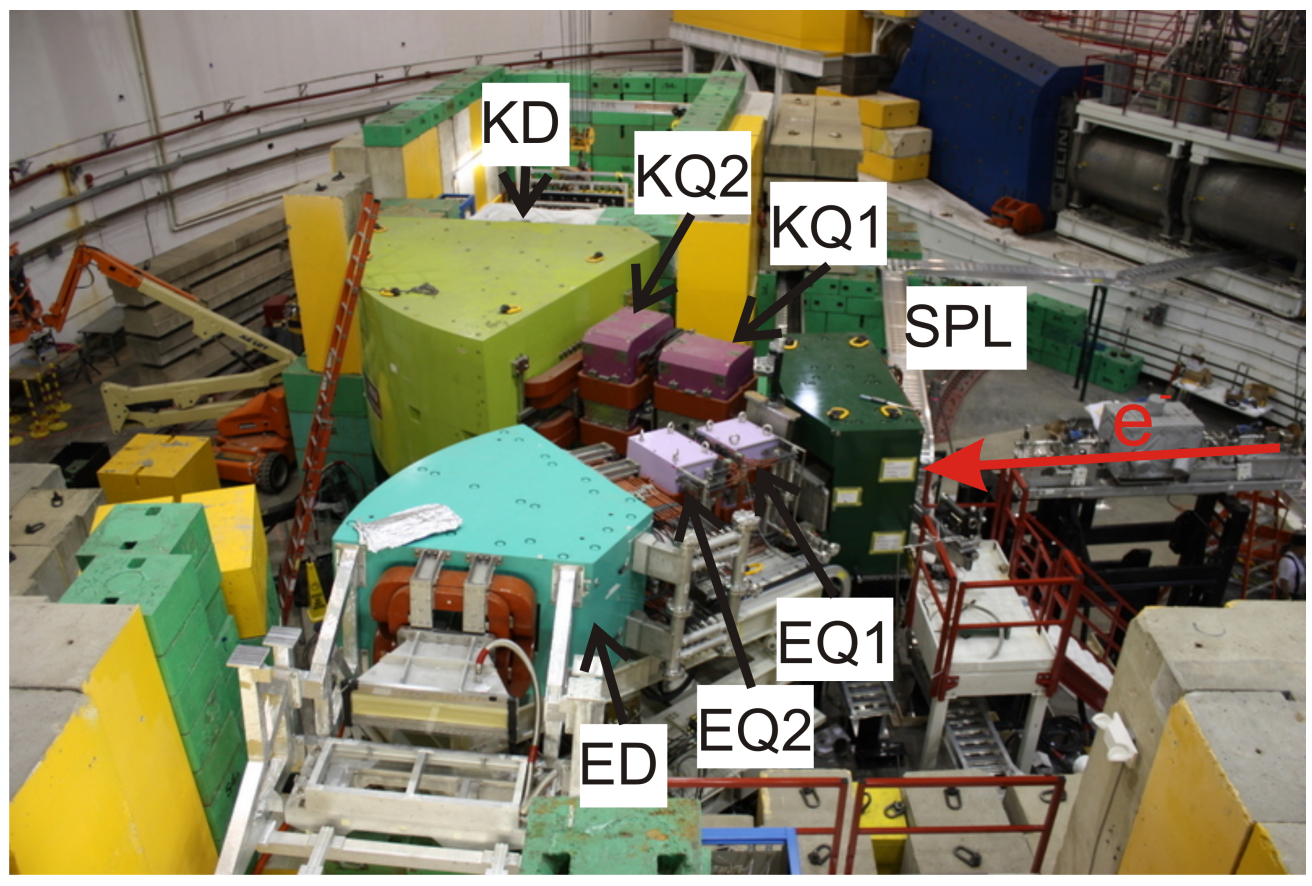

Figure 3.7: Picture of entire setup. 


\subsubsection{Kinematics}

Detail of the $\left(\mathrm{e}, \mathrm{e}^{\prime} \mathrm{K}^{+}\right)$reaction is as explained in chapter 2. Here, important points for the determination of experimental conditions can be summarized as follows.

1. The energy of virtual photon. Experimental data of $\left(\gamma^{*}, \mathrm{~K}^{+}\right)$reaction is shown in figure 2.4 and the total cross section of $\left(\gamma^{*}, \mathrm{~K}^{+}\right)$reaction is expected to be maximum at virtual photon energy of $1.5 \mathrm{GeV}$. Therefore, we set the virtual photon energy as $1.5 \mathrm{GeV}$.

2. The momentum range of kaon and electron. Since the virtual photon energy is determined as $1.5 \mathrm{GeV}$, the kaon momentum assumed by $\mathrm{p}\left(\mathrm{e}, \mathrm{e}^{\prime} \mathrm{K}^{+}\right) \Lambda$ reaction is automatically derived as about $1.2 \mathrm{GeV} / c$. This value is important for the design of Cherencov counters to identify kaon from other particles (see sections 3.6 and 5.2). For electron side, we prefer lower momentum from the viewpoint of better resolution, because the absolute momentum resolution $\Delta p$ become better as long as the momentum resolution of the spectrometer is fixed (usually $\Delta p / p$ is worse than $\left.10^{-4}\right)$.

3. The angular acceptance of kaon and scattered electron. As shown in figures 2.2 and 2.3, the yields of both scattered electron associated with virtual photon and kaon are higher in the forward angles. Therefore, the spectrometers were designed so as to accept kaons and scattered electrons at forward angles. However, because of the positron and electron background events, we must optimize the acceptance of each side so as to avoid the "very" forward angle.

Especially for the scattered electron side, we tilted HES vertically in order to avoid the very fowarded bremsstrahlung background (so called "tilt method", as explained in section 2.3).

Table3.2.1 shows the entire kinematical conditions and specifications of HKS and HES.

\subsubsection{Particles to be detected}

\section{Particles detected in HKS}

Positive charged particles generated at target are transported to the HKS. The main particles are positron, pion, kaon and proton, and kaon must be identified from other particles.

Figure 3.8 shows the correlations between $\beta$ and momentum for pion, kaon and proton at HKS. Kaon can be identified using the difference of time of flight (TOF) and the number of photoelectrons of Cherencov counters. The detail of particle identification is explained at section 3.6 about trigger level suppression and 5.2.2 about off-line analysis suppression.

\subsubsection{Tilt Method}

In order to suppress the huge background, HES was vertically tilted during the experiment by 6.5 degrees, as shown in figure 3.9 . 
Table 3.2.1: E05-115 experimental condition and spectrometer specification.

\begin{tabular}{ll}
\hline \hline Beam condition & \\
Beam energy & $2.344 \mathrm{GeV}$ \\
Beam momentum stability & $<1 \times 10^{-4}$
\end{tabular}

\section{HKS}

Configuration

Central momentum

Momentum acceptance

Momentum resolution $(\Delta p / p)$

Solid Angle
QQD (KQ1+KQ2+KD, horizontal bend)
$1.2 \mathrm{GeV} / \mathrm{c}$
$\pm 12.5 \%$
$2 \times 10^{-4}$
10 msr with a SPL
$30 \mathrm{msr}$ without a SPL
$-30 \mathrm{mrad}$ to $-230 \mathrm{mrad}$ (horizontal)
$-70 \mathrm{mrad}$ to $70 \mathrm{mrad}$ (vetical)
$10 \mathrm{~m}$

Angular acceptance

Flight path length

\section{HES}

Configuration

Central momentum

Momentum acceptance

Momentum resolution $(\Delta p / p)$

Solid Angle

QQD (EQ1+EQ2+ED, horizontal bend)

$0.844 \mathrm{GeV} / \mathrm{c}$

$\pm 17.5 \%$

$3 \times 10^{-4}$

Angular acceptance

10 msr with a SPL

$-200 \mathrm{mrad}$ to $200 \mathrm{mrad}$ (horizontal)

$30 \mathrm{mrad}-90 \mathrm{mrad}$ (vetical) 


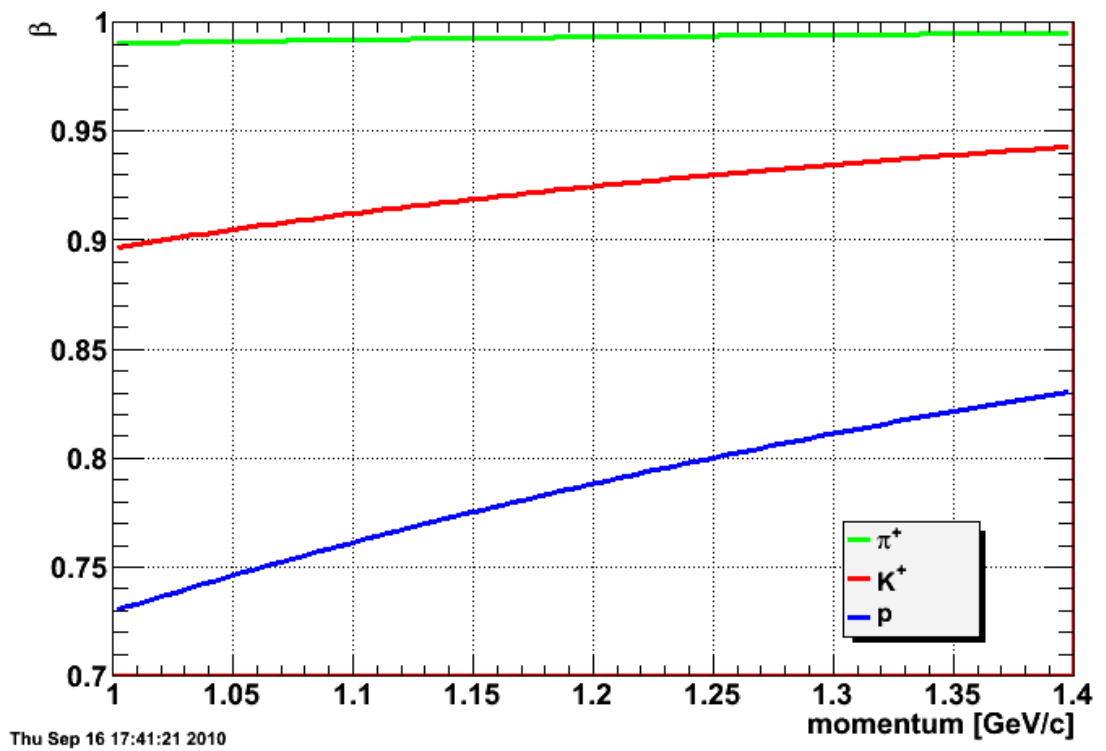

Figure 3.8: $\beta$ vs momentum correlation for pion, kaon and proton in the momentum range of HKS $(1.05[\mathrm{GeV} / c]$ to $1.35[\mathrm{GeV} / c])$. The $\beta$ of pion is almost 1 in this region and they can be suppressed by AC. Proton is suppressed by WC using the $\beta$ difference with kaon.

Although the principle of the tilt method is same as that of the last experiment (E01-011, see 2.3), the tilt angle becomes small. The higher beam energy of E05-115 is higher than that of E01-011, and the electron background is emitted to forwarder angles. Therefore, it becomes possible to extend the electron acceptance with the counting rate level of E01-011. The detail of the tilt angle optimization is explained in Chapter 4.

\subsubsection{Expected singles rate and background suppression}

Table 3.2.4 shows the estimated singles rates for the E05-115 experiment. These values are based on the singles rates of E01-011 experiment, the result of the EPC code calculation [54] and Geant4 simulations ${ }^{1}$ as follows. The EPC is a calculation code of the $(\mathrm{e}, \pi)$ and $(\mathrm{e}, \mathrm{N})$ reactions cross sections with an accuracy of factor 2 for the projectile energy range of $0.5-5.0 \mathrm{GeV}$ and the recoil kinematic energy of $\geq 50 \mathrm{MeV}$. Actually the calculation based on the EPC code provided consistent results with the E01-011 results in its accuracy.

1. The $\pi^{+}$, kaon proton rates in HKS were estimated from E01-011 data and normalized with the new solid angle of the HKS. For the ${ }^{52} \mathrm{Cr}$ target estimation, the ${ }^{51} \mathrm{~V}$ data of the E01-011 were used.

2. The positron rate in HKS was evaluated with the GEANT simulation code using $1.7 \times 10^{4}$ positron events (corresponds to $1.9 \times 10^{9}$ beam electrons hitting $100 \mathrm{mg} / \mathrm{cm}^{2}$

\footnotetext{
${ }^{1} \mathrm{~A}$ toolkit for the monte carlo simulation package which describe the passage of particles through the matter [55].
} 


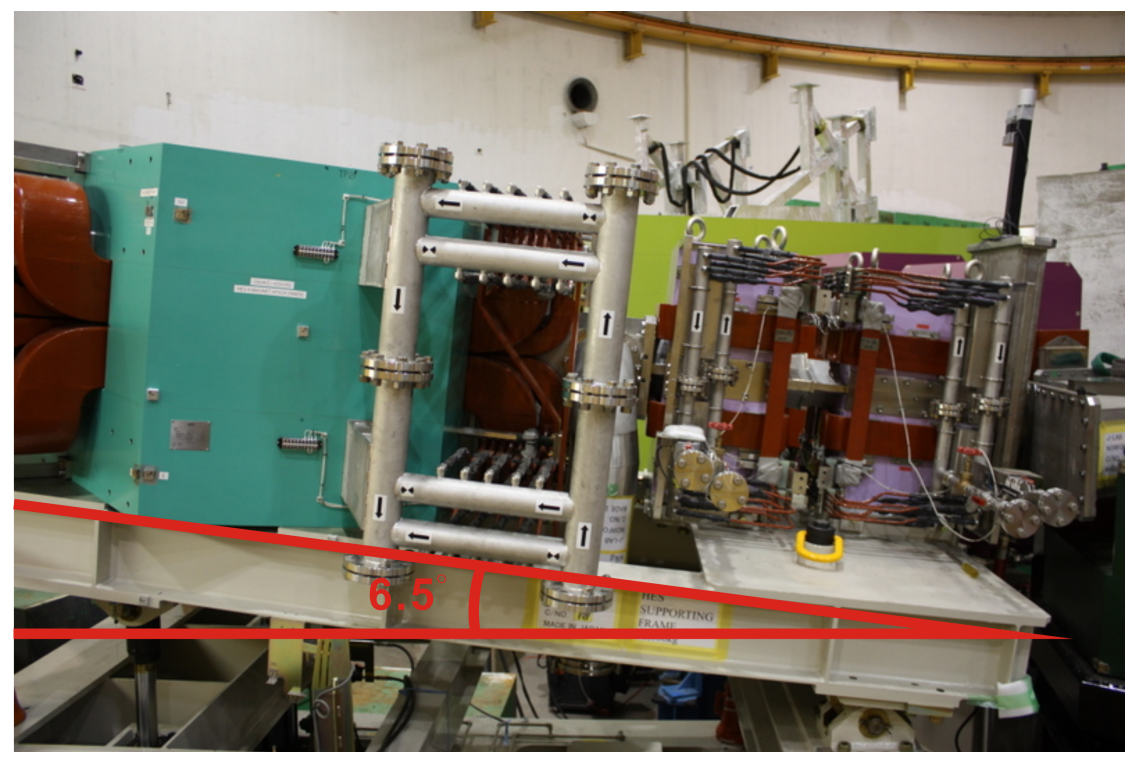

Figure 3.9: Picture of tilted HES.

carbon target, i.e. $30 \mu \mathrm{A}$ beam for $10 \mu \mathrm{s})$ generated by EGS code ${ }^{2}$ [56]. With this condition, it was confirmed that no positrons passed through the dipole.

3. Electron rate in HES was evaluated with Tsai's formula (equation 2.18) and GEANT4. Those estimations gave slightly smaller rate than the actual experimental result of E01-011. This difference was probably caused by scattered-in electrons from M $\phi$ ller scattering which will be avoided in E05-115.

4. $\pi^{-}$rate in HES was calculated based on the EPC code and it was less than $1 \%$ of the electron. Therefore the $\pi^{-}$rate gave no effect on the rate estimation.

Table 3.2.2: Estimated singles rates for each target. These values are based on the singles rate of E01-011 experiment.

\begin{tabular}{c|c|c|c|c|c|c}
\hline \hline Target & Thickness & $\begin{array}{c}\text { Beam } \\
\text { Intensity } \\
{[\mu \mathrm{A}]}\end{array}$ & $\begin{array}{c}\pi \\
{[\mathrm{kHz}]}\end{array}$ & $\begin{array}{c}\mathrm{K}^{+} \\
{[\mathrm{kHz}]}\end{array}$ & $\begin{array}{c}\mathrm{p} \\
{[\mathrm{kHz}]}\end{array}$ & $\begin{array}{c}e^{-} \\
{[\mathrm{kHz}]}\end{array}$ \\
\hline${ }^{7} \mathrm{Li}$ & 200 & 40 & 30 & 0.4 & 110 & 1490 \\
${ }^{10} \mathrm{~B}$ & 50 & 40 & 30 & 0.1 & 10 & 320 \\
${ }^{12} \mathrm{C}$ & 100 & 30 & 20 & 0.1 & 30 & 1240 \\
${ }^{52} \mathrm{Cr}$ & 100 & 10 & 3 & 0.02 & 4 & 590 \\
\hline \hline
\end{tabular}

From these results, it can be said that main background particles are pion and proton. The trigger rate have to be less than $2 \mathrm{kHz}$ which is required from our DAQ system

\footnotetext{
${ }^{2} \mathrm{~A}$ Monte Calro simulation code for the electromagnetic interaction of electron, photon and positron through the material.
} 
(CODA, see section 3.10). Therefore the singles rates of particles should be suppressed by the order of two in order to keep the trigger rate of pion and proton to the order of 100 Hz. This suppression is done by the Aerogel and Water Cherencov counters (see section 3.6) and their suppression power is required to be more than $99 \%$.

For the HES, almost all electrons are associated with bremsstrahlung process and these backgrounds cannot be separated from the scattered electrons associated with the $\Lambda$ production.

\subsubsection{Apparatus requirement from analysis}

\section{Proton target}

It is one of the important merit of the $\left(\mathrm{e}, \mathrm{e}^{\prime} \mathrm{K}^{+}\right)$reaction spectroscopy that the masses of $\Lambda$ and $\Sigma^{0}$ can be measured via the elementary process, $\mathrm{e}^{-}+\mathrm{p} \rightarrow \mathrm{e}^{-}+\mathrm{K}+\Lambda / \Sigma^{0}$. Since the masses of these particles are well known $\left(m_{\Lambda}=1115.683 \pm 0.006[\mathrm{MeV}]\right.$ and $\left.m_{\Sigma^{0}}=1192.642 \pm 0.024[\mathrm{MeV}]\right)$, we use these masses for the energy scale calibration. The energy scale highly depends on the momentum magnitude, then this calibration corresponds to the momentum calibration of kaon and electron.

We prepare $\mathrm{CH}_{2}$ and $\mathrm{H}_{2} \mathrm{O}$ target as proton targets. The technical detail of the target will be described in section 3.3 and the calibration strategy will be explained in chapter 5 .

\section{Energy scan}

Figure 3.10 gives the calculated momentum correlation between kaon and electron in the experiment for the $\Lambda, \Sigma^{0}$, the ground state (gs) of ${ }_{\Lambda}^{12} \mathrm{~B}$ and ${ }_{\Lambda}^{52} \mathrm{~V}$. The region of hypernucleus is existing in the "outside" of the $\Lambda$ and $\Sigma^{0}$ region when we use the beam energy. In other word, the hypernuclear peak corresponds to the "extrapolation" of the calibration using the $\Lambda$ and $\Sigma^{0}$ peak in the missing mass scale.

In order to solve this problem, we took the data with different beam energy as shown in figure 3.10. In the present experiment, we took data with the beam energy of 2.364 $\mathrm{GeV}$ (the nominal beam energy is $2.344 \mathrm{GeV}$ ). The area of ${ }_{\Lambda}^{52} \mathrm{~V}$ gs can be covered by the ${ }_{\Lambda}^{12} \mathrm{~B}$ gs of this beam energy. Since the biding energy of ${ }_{\Lambda}^{52} \mathrm{~V}$ gs is the biggest in the hypernucleus to be measured in the experiment, the $+20 \mathrm{MeV}$ shift is enough to cover the hypernuclear region of the experiment. In order to take the $+20 \mathrm{MeV}$ run, additional optimization of beamline magnets were necessary with keeping the current setting of SPL magnet as ordinary one.

\section{Sieve Slit}

While the proton target tune calibrate the momentum magnitude, the Sieve Slit (SS) is a tool for the emission angle calibration.

Figure 3.11 shows the concept of SS tune in the case of our experiment. SS is basically a metal plate with some holes. Particles pass through the holes make a pattern in the reference plane (FP, a virtual plane at the exit of the magnets. For detail, see section 3.6 and 3.8). This pattern can be studied by the simulation and we can know which line corresponds to which hole. Furthermore, we can know the emission angle by the 


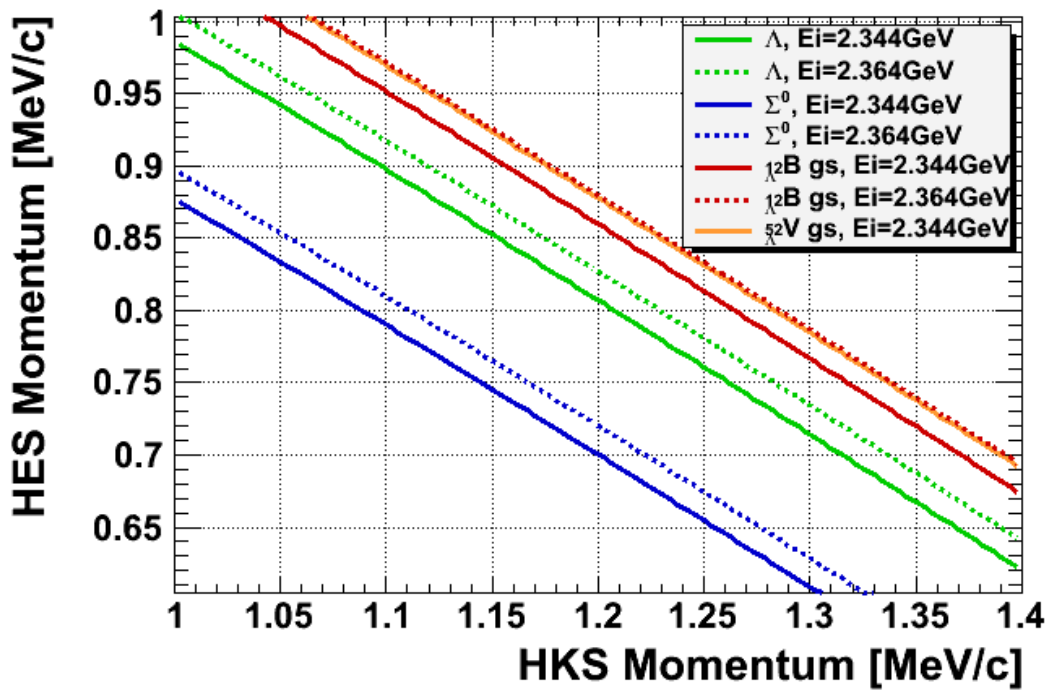

Figure 3.10: The effect of beam energy shift on the $P_{K}$ vs $P_{e^{\prime}}$ correlation.

hole position. Therefore we can specify the emission angle by the FP pattern with some accuracy decided by the hole size.

In the experiment, $\mathrm{SS}$ was placed in the each arms and we took data with SS in some times. The specification of the each SS will be described in section 3.5 and 3.7.

\subsection{Experimental target and target system}

As already mentioned in the last section, the experimental targets were placed at the entrance of the SPL. The radiation level of the experimental hall is quite high during our experiment period because of the high intensity electron beam. In addition, we use several targets which need to be changed during experiment. The target exchange have to be under the high radiation environment and it takes time. Therefore we designed the target so as to be controlled remotely from counting house. The movement of the target system can be checked by the target monitoring system. We also designed the water cell target integrated with the water cooling system. In this section, the motivations for each target and the whole target system will be explained.

\subsubsection{Experimental target}

Table 3.3.1 is a summary of the experimental targets used in E05-115 experiment. $\mathrm{CH}_{2}$ target and $\mathrm{H}_{2} \mathrm{O}$ target are for $\Lambda$ and $\Sigma^{0}$ creation via $\mathrm{p}\left(\mathrm{e}, \mathrm{e}^{\prime} \mathrm{K}^{+}\right) \Lambda$ reaction. The other targets are aiming to produce hypernuclei.

The masses of $\Lambda$ and $\Sigma^{0}$ are well known. These information can be used for the missing mass calibration (see section 5.5.1). The masses separate by about $77 \mathrm{MeV}$ each other and can be measured in the identical experimental setup with that of hypernuclei. This is 


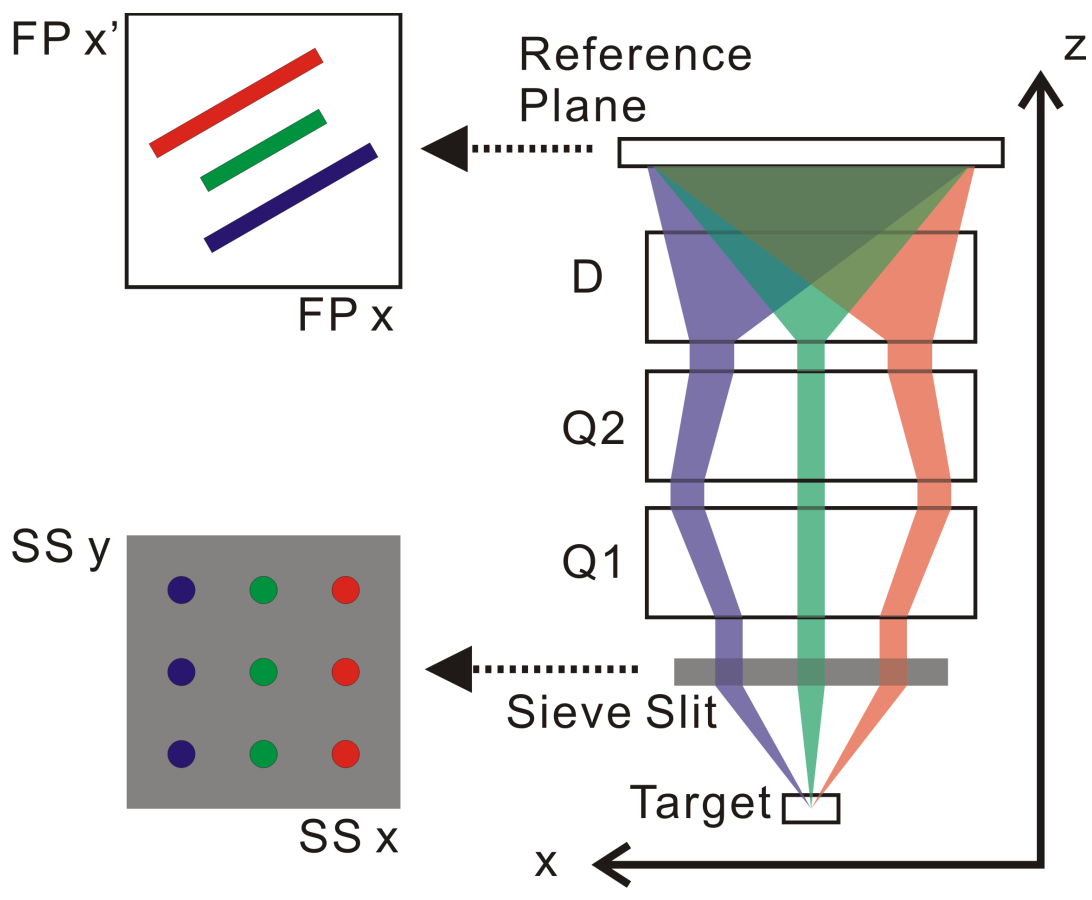

Figure 3.11: Concept of SS tune.

one of the significant merits of the experiment because it becomes possible to determine the absolute mass scale.

There are three differences between $\mathrm{H}_{2} \mathrm{O}$ target and $\mathrm{CH}_{2}$ target:

1. Beam raster is necessary for $\mathrm{CH}_{2}$ target, because the melting point of $\mathrm{CH}_{2}$ is low (about $100{ }^{\circ} \mathrm{C}$ ). The beam raster effect on the mass scale needs to be correct in the offline analysis. On the other hand, the beam raster is not necessary for the $\mathrm{H}_{2} \mathrm{O}$ target, because of the water flow. The $\mathrm{H}_{2} \mathrm{O}$ target is free from ambiguity of raster correction.

2. The $\mathrm{CH}_{2}$ target is dehydrogenated due to the heat deposit of the beam. Meanwhile, we do not have to worry about the dehydronation of $\mathrm{H}_{2} \mathrm{O}$ target. The ratio of dehydrogenation can be estimated by the time dependence of $\Lambda$ yield, but this procedure can be skipped by using the $\mathrm{H}_{2} \mathrm{O}$ target. Although the dehydrogenation has an influence on the calculation of the cross section, the missing mass calculation is not affected.

3. The $\mathrm{H}_{2} \mathrm{O}$ target needs the windows which have to be bore to the water pressure and needs some thickness for the safety reason. The windows will generate background events and make the energy and angular resolution worse because of the multiple scattering, though actually the effect was not serious during experiment. On the other hand, $\mathrm{CH}_{2}$ target does not need the windows, then $\mathrm{CH}_{2}$ target has an advantage in this point. 
The $\mathrm{H}_{2} \mathrm{O}$ and $\mathrm{CH}_{2}$ target have their benefits each other, then the data of these targets can be used complementary. However, the water target system needs to be designed carefully for the operation under the vacuum connected to the beamline. The design detail will be explained in this section.

In the other targets, ${ }^{7} \mathrm{Li},{ }^{10} \mathrm{~B}$ and ${ }^{52} \mathrm{Cr}$ targets were specially prepared. The ${ }^{7} \mathrm{Li}$ target is enriched with the purity of $99 \%$ and made by Cambridge Isotope Laboratories. The ${ }^{10} \mathrm{~B}$ target is also enriched target with the purity pf $99 \%$. This target was made before the E01-011 experiment in the Institute for Materials Research of Tohoku University by the two-tiered sintering method [57].

The ${ }^{52} \mathrm{Cr}$ target was made with the High energy Vibrational Powder Plating (HIVIPP) method in KEK by Dr. Sugai[58]. The principle of the method is the high-energy vibrational motion of powder in the high electric field between parallel electrodes at room temperature and in vacuum.

Table 3.3.1: The list of target and its reaction used in E05115 experiment.

\begin{tabular}{lccccc}
\hline \hline Target & Reaction & $\begin{array}{c}\text { Thickness } \\
{\left[\mathrm{mg} / \mathrm{cm}^{2}\right]}\end{array}$ & $\begin{array}{c}\text { Density } \\
{\left[\mathrm{g} / \mathrm{cm}^{3}\right]}\end{array}$ & Purity & $\begin{array}{c}\text { Radiation Length } \\
{\left[\mathrm{mg} / \mathrm{cm}^{2}\right]}\end{array}$ \\
\hline $\mathrm{CH}_{2}$ & $\mathrm{p}\left(\mathrm{e}, \mathrm{e}^{\prime} \mathrm{K}^{+}\right) \Lambda, \Sigma^{0}$ & 450.8 & 0.92 & - & 44.77 \\
& ${ }^{12} \mathrm{C}\left(\mathrm{e}, \mathrm{e}^{\prime} \mathrm{K}^{+}\right){ }_{\Lambda}^{12} \mathrm{~B}$ & & & & 36.08 \\
$\mathrm{H}_{2} \mathrm{O}$ & $\mathrm{p}\left(\mathrm{e}, \mathrm{e}^{\prime} \mathrm{K}^{+}\right) \Lambda, \Sigma^{0}$ & 500 & 1.00 & - & 82.78 \\
& ${ }^{16} \mathrm{O}\left(\mathrm{e}, \mathrm{e}^{\prime} \mathrm{K}^{+}\right){ }_{\Lambda}^{16} \mathrm{~N}$ & & & & 65.19 \\
${ }^{7} \mathrm{Li}$ & ${ }^{7} \mathrm{Li}\left(\mathrm{e}, \mathrm{e}^{\prime} \mathrm{K}^{+}\right){ }_{\Lambda}^{7} \mathrm{He}$ & 184.0 & 0.54 & 99.9 & 42.70 \\
${ }^{9} \mathrm{Be}$ & ${ }^{9} \mathrm{Be}\left(\mathrm{e}, \mathrm{e}^{\prime} \mathrm{K}^{+}\right){ }_{\Lambda}^{9} \mathrm{Li}$ & 188.1 & 1.848 & 100. & \\
${ }^{10} \mathrm{~B}$ & ${ }^{10} \mathrm{~B}\left(\mathrm{e}, \mathrm{e}^{\prime} \mathrm{K}^{+}\right)_{\Lambda}^{10} \mathrm{Be}$ & 56.1 & 2.16 & 99.9 & 15.3 \\
${ }^{n a t} \mathrm{C}$ & ${ }^{12} \mathrm{C}\left(\mathrm{e}, \mathrm{e}^{\prime} \mathrm{K}^{+}\right){ }_{\Lambda}^{12} \mathrm{~B}$ & 112.5 & 2.25 & ${ }^{12} \mathrm{C}: 98.89$ & \\
& & & & ${ }^{13} \mathrm{C}: 1.11$ & \\
${ }^{52} \mathrm{Cr}$ & ${ }^{52} \mathrm{Cr}\left(\mathrm{e}, \mathrm{e}^{\prime} \mathrm{K}^{+}\right){ }_{\Lambda}^{52} \mathrm{~V}$ & 134.0 & 7.15 & 99.9 & \\
& & 154.0 & & 99.9 & \\
\hline \hline
\end{tabular}

\subsubsection{Target system overview}

Figure3.12 shows whole target system used in the E05-115 experiment. The system can be divided into following 3 parts,

1. Target ladder aiming to support solid targets on the beamline.

2. Driving mechanism aiming to move target ladder and adjust target position.

3. Base of the system supporting whole system to the SPL magnet.

This system is attached to the SPL magnet as shown in figure 3.13. The target ladder is placed on the beamline tilting by 17 degree to the $\mathrm{x}$-axis. The reason of the tilting is the target viewability and mechanical reason. In addition, the effective thickness of the target become thicker by the tilting. There is a vacuum port on the right side of the SPL vacuum chamber in order to keep monitoring the target condition by CCD camera. 


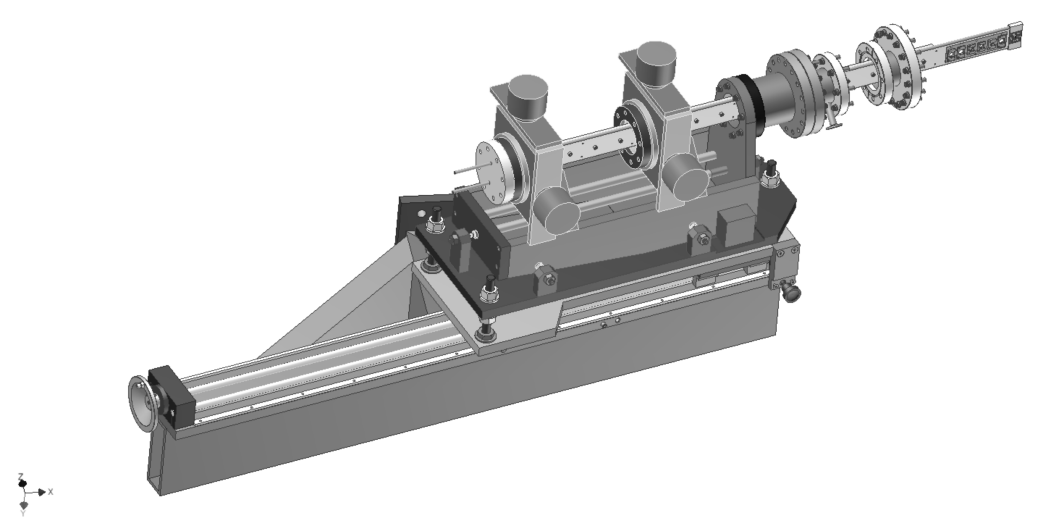

Figure 3.12: Schematic of whole target system.

The beamline, SPL magnet, HKS and HES are connected with the same vacuum system. In order not to break the whole vacuum system, we made the target ladder to be able to be remotely controlled when changing the target during the experiment. However, the number of solid targets which can be mounted on the target plate is limited. Therefore, the plate needs to be exchanged without breaking the vacuum by using the gate valve attached as shown in figure 3.13. Furthermore, the water line should be kept flowing during experiment, because of the safety reason and the system is designed so as for the plate exchange not to affect the line .

The detail of each parts will be described in the following part.

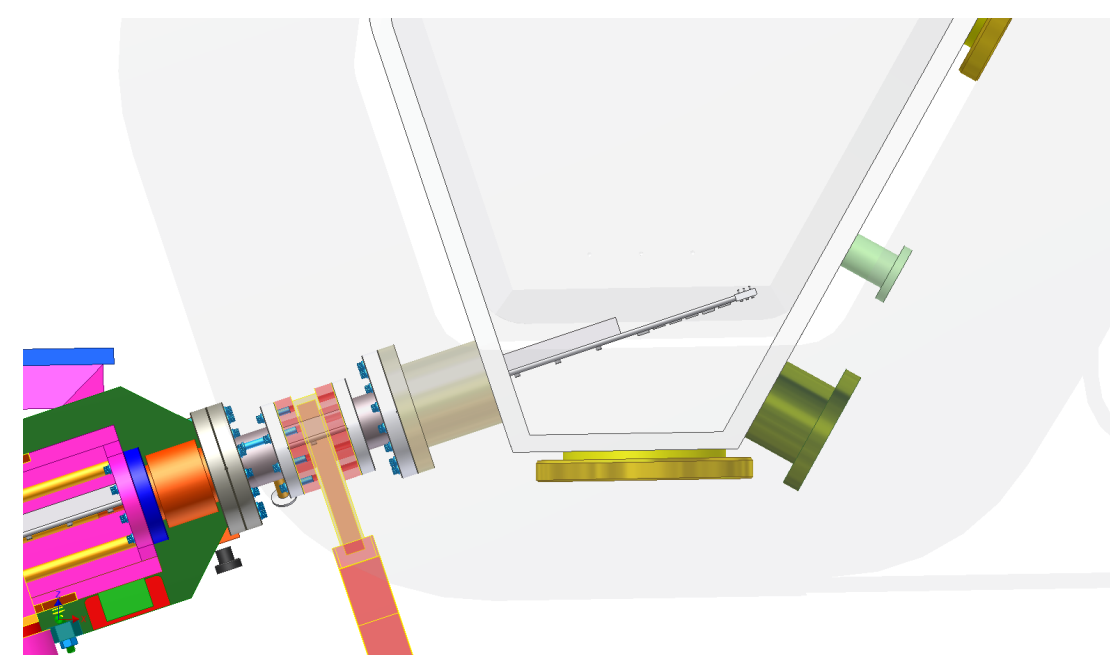

Figure 3.13: Target system in the SPL magnet. 


\subsubsection{Target ladder}

The target ladder is designed as shown in figure 3.14. The water-cell target is attached to the ladder through the water-flowing pipe ( explained in the following part). The other parts of the ladder consists of the water-flowing pipe, the body of ladder, target plate.

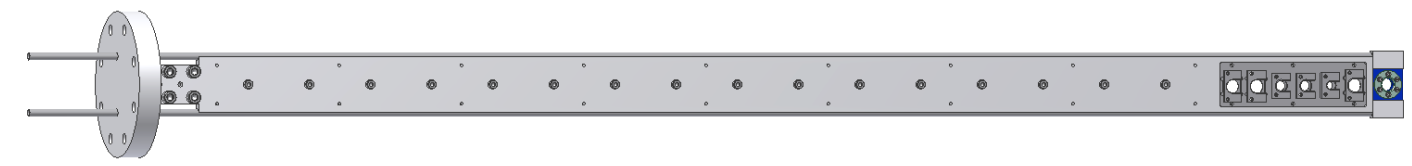

Figure 3.14: Schematic of target ladder.

Water-flowing pipe The water-flowing pipe has two roles, target cooling and water circulation. The target temperature become very high during the beam time as a result of high intensity beam from CEBAF and its high energy deposit in the target. According to the calculation by the finite element method, temperature of some target get near to the melting point (table 3.3.3). Therefore, the water cooling and heat conductance between the target and ladder is important, and the ladder was designed taking care with these points.

The flowing pipe is made of SUS in order to avoid corroding by the water and large backgroud via the bremsstrahlung process whose cross section is roughly proportional to $Z^{2}$. Aiming to the better heat conduction, the pipe is attached to the ladder whose edge shape is fit to the pipe with wires. By flowing the room-temperature water in the cooling pipe, the target temperature was kept under the melting point. As a result, expected intensity beam was available during experiment.

The body of ladder The body consists of two part. One is the board part made of aluminum aiming to conduct heat generated by the energy deposit of electron beam in the target. Another reason to choose aluminium is reduction of electron background compared 


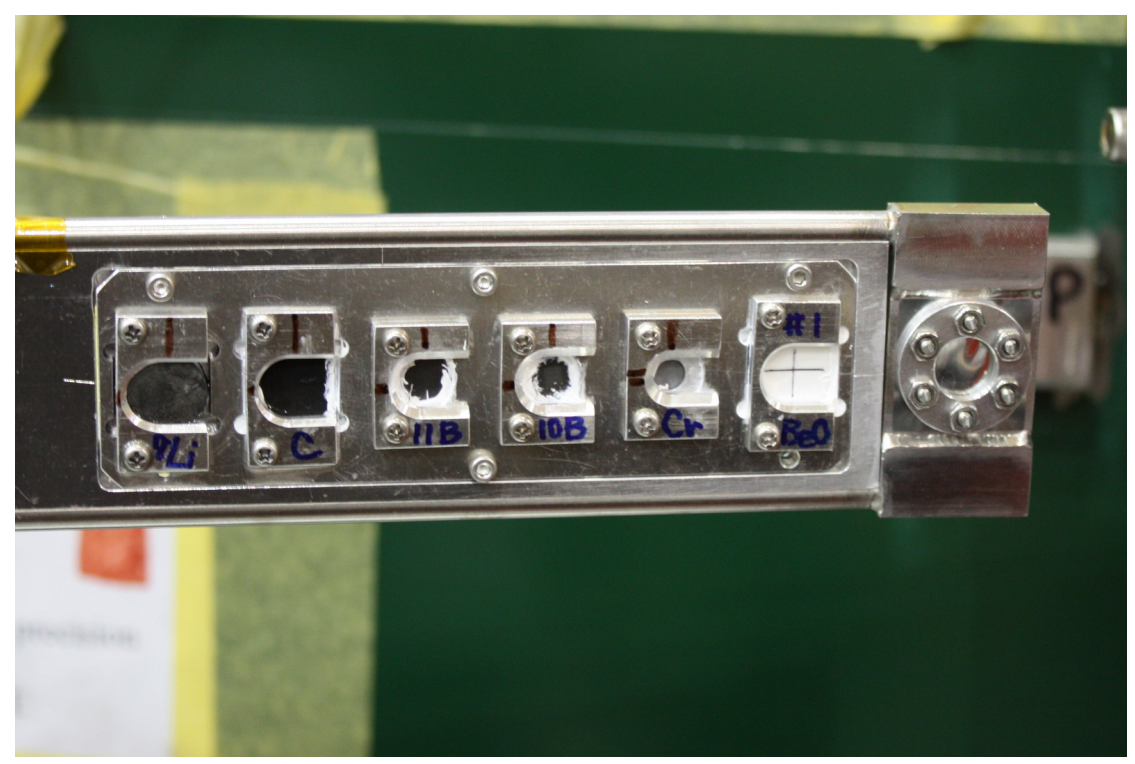

Figure 3.15: Picture of target plate attached in target ladder.

Table 3.3.2: The melting point and expected max. temp. of the target.[59]

\begin{tabular}{ccc}
\hline \hline Target & $\begin{array}{c}\text { Melting point } \\
{[\mathrm{K}]}\end{array}$ & $\begin{array}{c}\text { Expected max. temp. (beam current) } \\
{[\mathrm{K}]([\mu \mathrm{A}])}\end{array}$ \\
\hline $\mathrm{Li}$ & 460 & $386(30)$ \\
$\mathrm{B}$ & 2200 & $970(50)$ \\
$\mathrm{C}$ & 3650 & $521(50)$ \\
$\mathrm{Cr}$ & 1890 & $988(50)$ \\
\hline \hline
\end{tabular}


to other metallic material. The electron background for HES side is also generated by the hit of beam halo on the target ladder. The background events are generated through bremsstrahlung whose cross section is roughly proportional to $Z^{2}$. Therefore it is better to choose small $Z$ material in order to decrease the background events in HES side. In addition, magnetic material cannot be used in the high magnetic field of SPL. As a result of such consideration, we decided to use aluminum as a material of the ladder.

Another part is SUS flange to fix the ladder to the driving arm. It should be notified that the board part can be pulled out without breaking the vacuum. As already mentioned, we need to pull out the target ladder in order to exchange the target plate during the experiment. Since the vacuum of SPL, HKS and HES is connected to the accelerator, it is essential to keep vacuum during the experiment, including the target plate exchanging.

The target plate The target plate is made of aluminum attaching 6 solid target and mounted on the target ladder by screw. We used two type of target ladder during the experiment(table3.3.3). The second one has large pocket for the $\mathrm{CH}_{2}$ plate as shown in picture3.15.

These target plates were exchanged two times during the experiment. This is an effective way to exchange the target group smoothly. In the last experiment, we exchanged target ladder itself to change the target group. However, it is hard to exchange the target ladder in the present experiment, because we have the water circulation system surrounding the target ladder and we have to turn off the circulation system temporarily. The suspension of the circulation might cause some troubles, then we have to do pressure test of the water-cell target in such a case according to the safety regulation of JLab. In order to avoid such a case, we decided to use target plate which can be easily exchanged without breaking the vacuum and the water-flowing line.

Table 3.3.3: The list of target plate in E05115 experiment.

\begin{tabular}{cccc}
\hline \hline Hole number & $\begin{array}{c}\text { Target set 1 } \\
\text { (plate \#1) }\end{array}$ & $\begin{array}{c}\text { Target set 2 } \\
\text { (plate \#2) }\end{array}$ & $\begin{array}{c}\text { Target set 3 } \\
\text { (plate \#2) }\end{array}$ \\
\hline 1 & $\mathrm{BeO}$ & $\mathrm{BeO}$ & $\mathrm{BeO}$ \\
2 & ${ }^{52} \mathrm{Cr}$ & ${ }^{52} \mathrm{Cr}$ & ${ }^{52} \mathrm{Cr}$ \\
3 & ${ }^{10} \mathrm{~B}$ & & \\
4 & ${ }^{11} \mathrm{~B}$ & $\mathrm{CH}_{2}$ & $\mathrm{CH}_{2}$ \\
5 & ${ }^{12} \mathrm{C}$ & & \\
6 & ${ }^{7} \mathrm{Li}$ & ${ }^{9} \mathrm{Be}$ & ${ }^{12} \mathrm{C}$ \\
\hline \hline
\end{tabular}

\subsubsection{Water-cell target}

One of the important point of our experiment is that we can calibrate the hypernuclear mass scale using the known masses of $\Lambda$ and $\Sigma^{0}$. Since the $\Lambda$ and $\Sigma^{0}$ are created by the elementary process: $\mathrm{e}^{-}+\mathrm{p} \rightarrow \mathrm{e}^{-}+\mathrm{K}+\Lambda / \Sigma^{0}$, we need to prepare the proton target. 
We used the $\mathrm{CH}_{2}$ target in the past experiments and the present experiment, but the $\mathrm{CH}_{2}$ target need beam raster. Though the raster effect on the mass spectrum can be corrected well by the raster correction, it is better to remove the uncertainty of the raster effect. This is one of the reasons to use the water-cell target, because the water-cell target can keep the temperature by the flowing water. It will be also possible to perform the spectroscopic study of the ${ }_{\Lambda}^{16} \mathrm{~N}$ by using the ${ }^{16} \mathrm{O}$ target in the water.

Figure 3.16 shows the picture and CAD drawing of the water-cell target. This can be divided into main three parts:

- Water flowing pipe,

- Water-cell which was used as a water target,

- Window of the cell and the flange.

The water flowing pipe has been already mentioned in the last section.

The structure of the water-cell part is shown in the right part of figure 3.16. This part is made by SUS, as well as the flowing pipe, in order to avoid corroding by the water and radiation. The circling water is lead into the cell from the pipe divided into two lines in order to avoid pressure loss and keep the water flow. In addition, the flow route is asymmetry to prevent the accumulation of the air bubble caused by the turbulent flow. The window part of the cell is a hole of $\phi=10 \mathrm{~mm}$. We made a mock-up and confirmed that water is actually flowing.

The window foil is made by an alloyed metal "Havar" ${ }^{3}$. The Havar is stronger for tension than usual SUS and widow thickness could be thin $(25 \mu \mathrm{m})$. This thin window is essential for our experiment because the window is the source of background event and its rate is proportional to the window thickness. The surround of foil is sealed by putting an indium wire $(\phi=0.5 \mathrm{~mm})$ under the foil. These are fixed by screws with an aluminum flange put on the Havar foil. The pressure test of this system was performed in JLab in order to confirm that the window foil can bear the pressure difference of the flowing water and the vacuum surround the target. The system passed the test and successfully used during the experiment.

Further detail about the water-cell target is described in [59].

\subsubsection{BeO target and Target viewer}

It is important to monitor the condition of target during experiment in order to check beam position at target and physical status of the target. Since the electron beam from CEBAF is very high intensity, if beam position is wrong and hit on the materials around the target, we possibly to have many background or there might have safety problem in the worst case.

Figure 3.17 shows our target viewer system in this experiment. Because we use high intensity electron beam from CEBAF, there are a lot of radiation around the target. Therefore, target monitoring camera have to be placed so as to reduce the radiation damage, by increasing the distance from target or shielding the radiation. For this reason, we placed a CCD camera on the floor and monitored the target using a optical system.

\footnotetext{
${ }^{3}$ Havar(R High-Strength Non-Magnetic Alloy : GoodFellow : 382-725-07)
} 

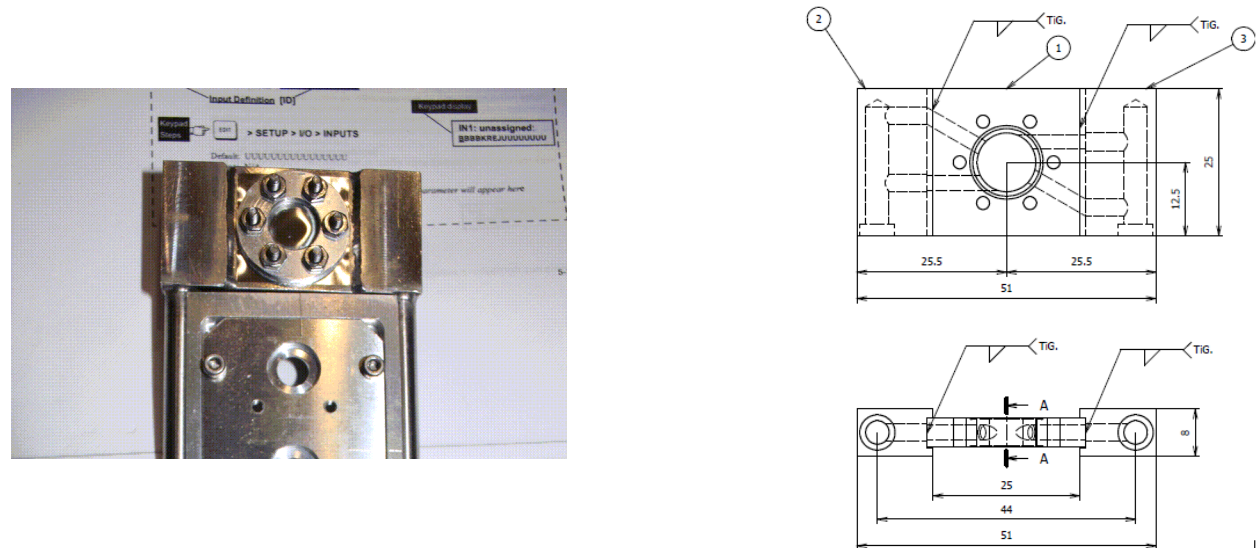

Figure 3.16: Schematic view of the water-cell target. The left figure is a picture of the water-cell target and the right one is a CAD drawing.

The target lighted by LED is monitored from the camera using mirrors and the vacuum is cut off by a quartz glass.

In order to check the beam position at target, not only camera but also $\mathrm{BeO}$ target is important. BeO target is used as a traditional beam position visualizer, because it shines with the electron beam. The cross hairs are marked on the center of the BeO target and the beam hit point is burned out. The beam position on the target can be monitored by this traditional way of electron beam experiment.

\subsection{The SPL magnet}

The SPL magnet(figure3.19 and picture3.20) is a dipole magnet which is used to transport positive charged particles to HKS and negative charged particles to HES. This magnet also needs to separate post-beam (electrons which do not react in the target) and scattered electron. The specification of SPL magnet is summarized in table 3.4.

The magnet was constructed in 2008 at TOKIN (Sendai, JAPAN) with HES magnets and used in E05-115 experiment for the first time. The SPL magnet is dedicated to HES+HKS configuration and has complicated shape, because of its roles to transport the particles to four exits by a dipole magnetic field. The four exits are HKS exit, photon exit, post-beam exit and HES exit. In order to realize that, the pole shape of the SPL magnet was optimized. Detail of SPL design and the optimization will be explained in chapter4. 


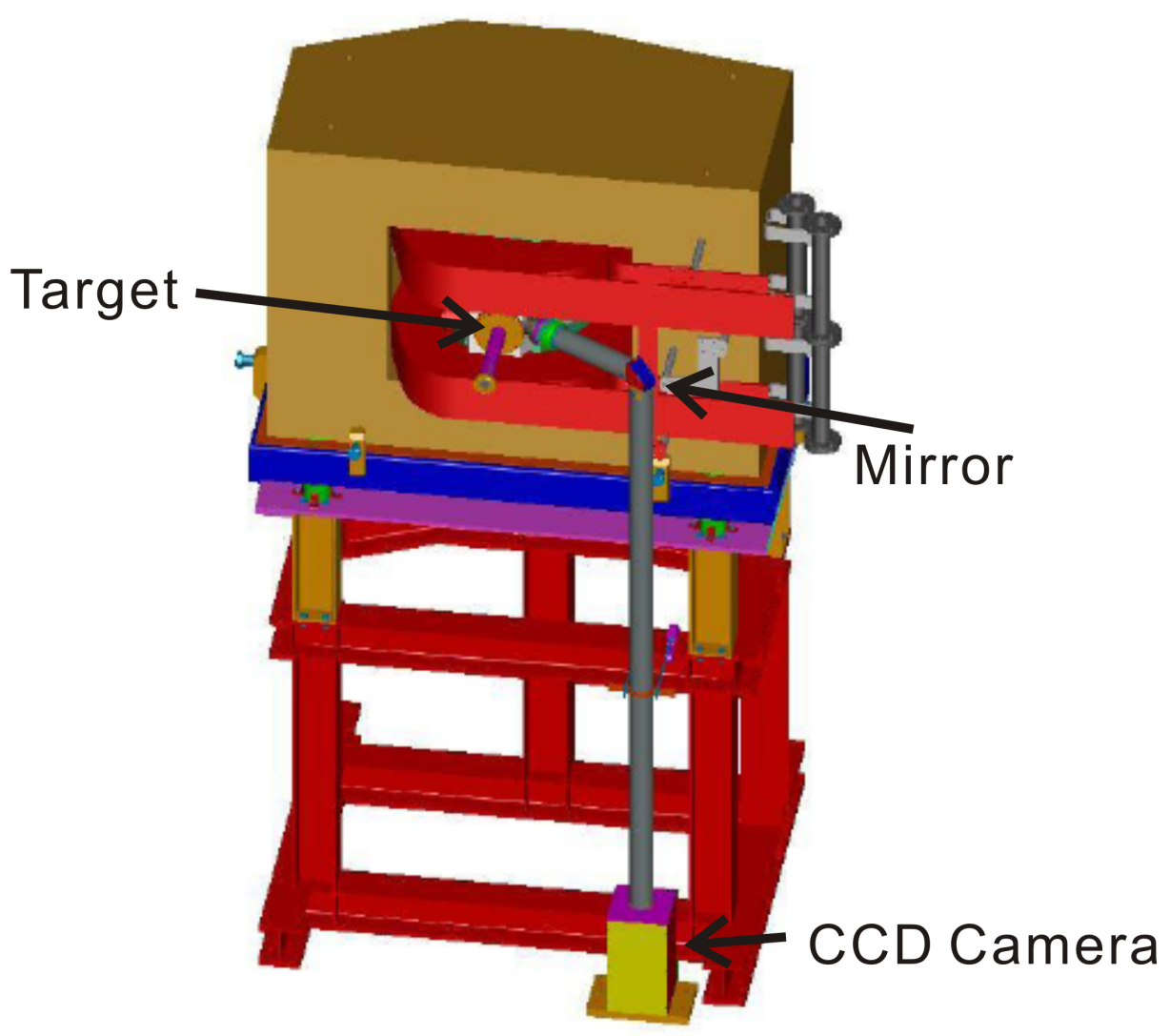

Figure 3.17: Schematic view of Target Viewer.

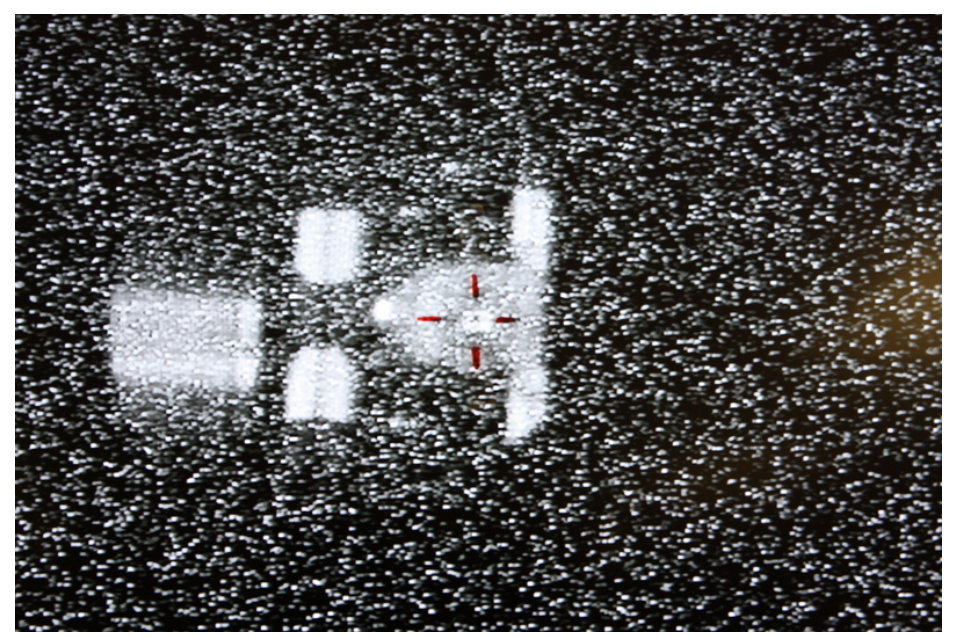

Figure 3.18: BeO target monitoring during experiment. 
Table 3.4.1: SPL magnet parameters.

\begin{tabular}{lc}
\hline \hline Pole Gap Height [mm] & 190 \\
Max. Ampare Turns [A turns] & 214200 \\
Number of Turns & 210 \\
Conductor Size [mm] & $17 \times 17(\phi 11$ hole $)$ \\
Max. Field [T] & 1.74 \\
Max. Current [A] & 1020 \\
Total Magnet Weight [ton] & 31.7 \\
\hline \hline
\end{tabular}

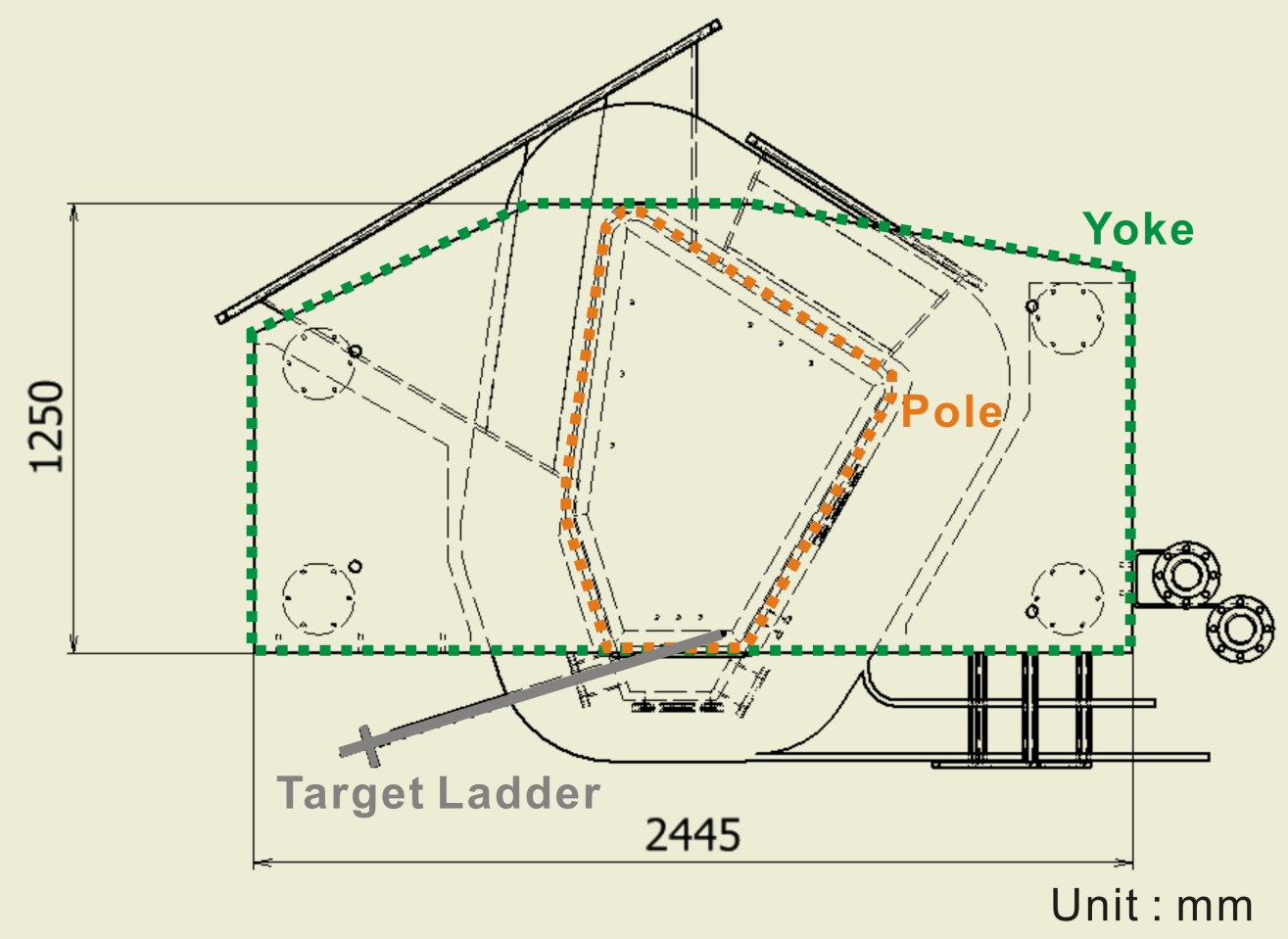

Figure 3.19: Schematic view of SPL magnet. 


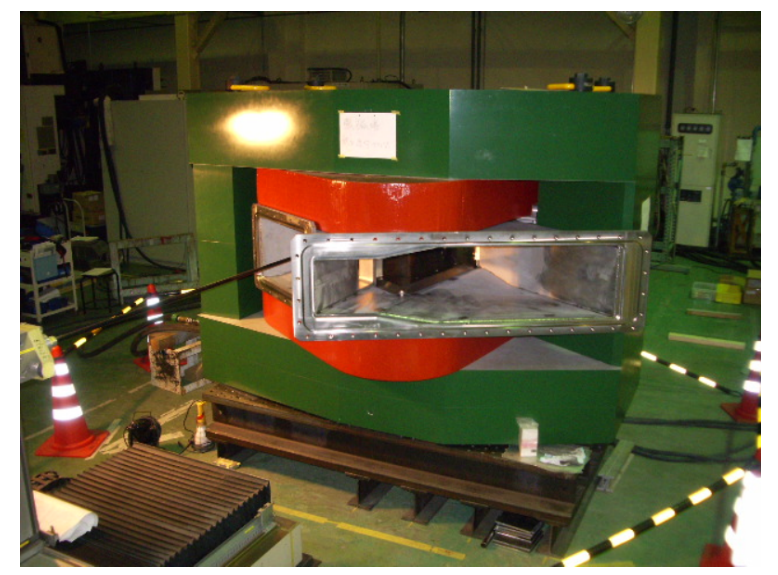

Figure 3.20: Picture of SPL magnet.

\subsection{HKS magnets}

The High resolution Kaon Spectrometer (HKS) was constructed and used in the E01-011 experiment (2005) aiming to high momentum resolution and large kaon yield at the same time. In the E01-011 experiment, the HKS worked as we expected, and its performance is guaranteed. The HKS magnets were manufactured at Mitsubishi Electric Co., Kobe, Japan. HKS consists of two quadrupole magnets (KQ1 and KQ2), one dipole magnet (KD) and a detector package. They are normal conduction magnets. The detail of these components and expected performances are explained in this section.

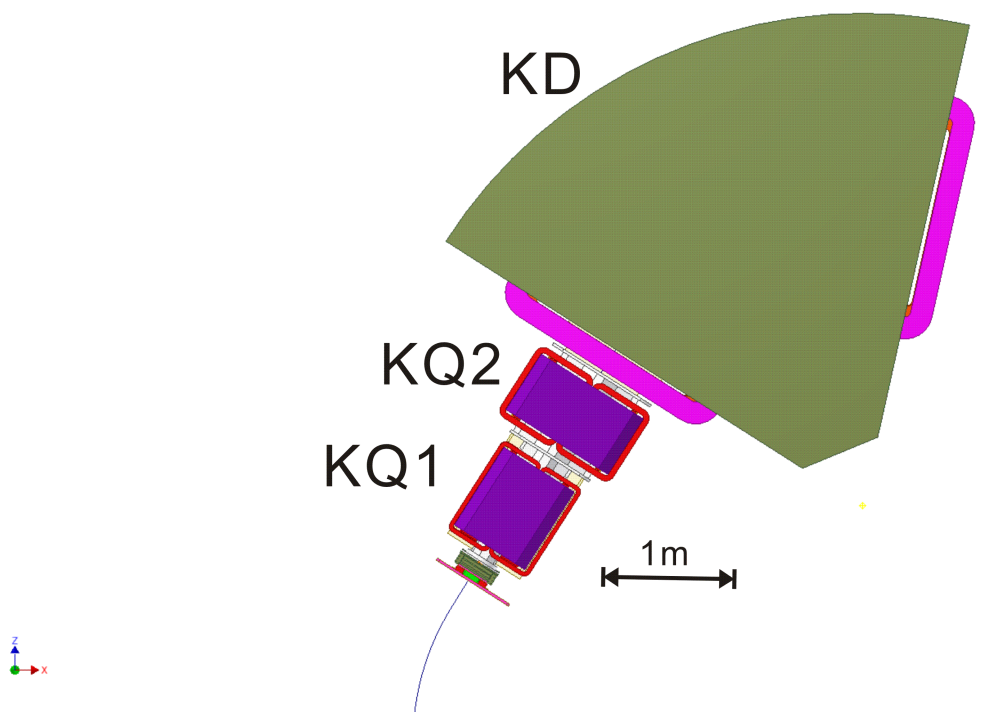

Figure 3.21: Schematic view of HKS magnets. 


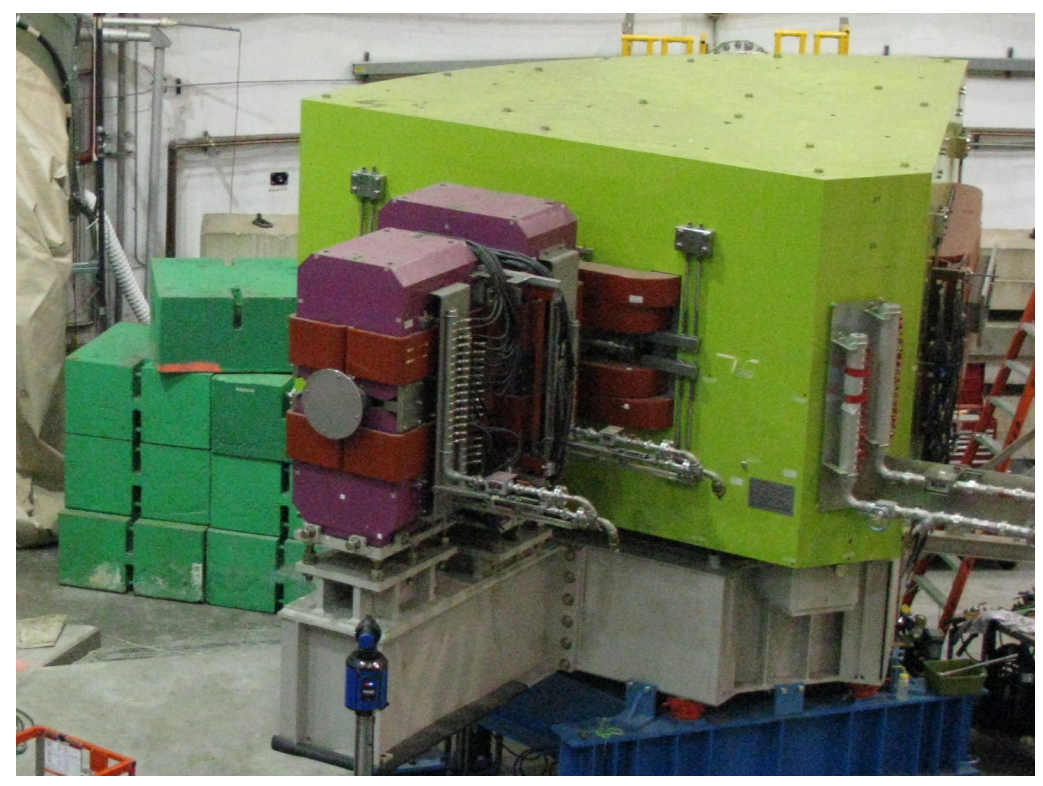

Figure 3.22: Picture of HKS magnets.

\subsubsection{Magents specification and alignment}

KQ1 and KQ2 are vertical and horizontal focusing quadrupole magnets, respectively. KD is a dipole magnet with 70 degree bending angle. It is possible to adjust the focus point and focusing angle separately for vertical and horizontal directions by these two magnets, then the QQD configuration can increase the flexibility of the particle optics. The detail specification of these magnets are summarized in table3.5.1 and table3.5.1.

Figure 3.23 shows the HKS alignment to the new SPL magnet. Basically the HKS is placed on the $1.2 \mathrm{GeV} / c$ kaon track, which is bent in the SPL by 32.53 degree. This angle was determined by the magnetic field of the SPL magnet.

\subsubsection{Magnetic field}

Table 3.5.2 is a list of the typical magnetic field of HKS magnets. As shown in the table, the magnetic rigidity of whole HKS is almost determined by the two dipole magnets ; SPL and KD. It should be noted the HKS sieve slit (see next subsection) is placed just after the SPL dipole magnetic field which has about $30 \%$ of total HKS magnetic rigidity.

\subsubsection{Sieve slit and collimator}

Figure 3.25 is a picture of the HKS Sieve Slit (SS) and figure 3.26 is a CAD drawing of SS and collimator. The SS is a HEAVIMET (alloy of tungsten, nickel and copper) plate which has many holes and collimator is also made from same material. The density of the HEAVIMET is $16.9 \mathrm{~g} / \mathrm{cm}^{3}$ and radiation length is $7.11 \mathrm{~g} / \mathrm{cm}^{2}$. The role of SS is to specify the position of particles at the SS position and the information can be used for the optics calibration of the spectrometer. HKS SS is placed just before the KQ1 magnet in 
Table 3.5.1: KQ1 and KQ2 parameters

\begin{tabular}{l|cc}
\hline \hline & KQ1 & KQ2 \\
\hline Bore Radius [mm] & 120 & 145 \\
Pole Length [mm] & 840 & 600 \\
Max. Ampare Turns [A turns] & 224000 & 144000 \\
Number of Turns & 256 & 320 \\
Conductor Size & $8 \times 8(\phi 6$ hole $)$ & $13.5 \times 11.5(\phi 6.3$ hole $)$ \\
Coil Winding & Double Pancake Winding & Solenoid Winding \\
Max. Field Gradient [T/m] & 6.6 & 4.2 \\
Max. Current [A] & 875 & 450 \\
Resistance [m $\Omega]$ & $181\left(\right.$ at $\left.55^{\circ} \mathrm{C}\right)$ & $119\left(\right.$ at $\left.45^{\circ} \mathrm{C}\right)$ \\
Cooling Water Flow Rate $[\mathrm{l} / \mathrm{m}]$ & 49.6 & 17.3 \\
Pressure Drop [MPa] & 0.36 & 0.38 \\
Number of Coolant Circuits & 16 & 8 \\
Total Magnet Weight [ton] & 8.2 & 10.5 \\
\hline \hline
\end{tabular}

Table 3.5.2: KD parameters

\begin{tabular}{|c|c|c|}
\hline Pole Gap Hight $[\mathrm{mm}]$ & \multicolumn{2}{|c|}{200} \\
\hline Pole Length $[\mathrm{mm}]$ & \multicolumn{2}{|c|}{1560} \\
\hline Max. Ampare Turns [A turns] & \multicolumn{2}{|c|}{291840} \\
\hline Number of Turns & \multicolumn{2}{|c|}{256} \\
\hline Conductor Size [mm] & \multicolumn{2}{|c|}{$22 \times 22(\phi 12$ hole $)$} \\
\hline Max. Field $[\mathrm{T}]$ & \multicolumn{2}{|c|}{1.53} \\
\hline Max. Current $[\mathrm{A}]$ & \multicolumn{2}{|c|}{1140} \\
\hline Resistance $[\mathrm{m} \Omega]$ & \multicolumn{2}{|c|}{$145\left(\right.$ at $\left.47.5^{\circ} \mathrm{C}\right)$} \\
\hline & Gap Side & Yoke side \\
\hline Cooling Water Flow Rate $[\mathrm{l} / \mathrm{m}]$ & 66.3 & 68.8 \\
\hline Pressure Drop $[\mathrm{MPa}]$ & 0.32 & 0.35 \\
\hline Number of Coolant Circuits & 8 & 8 \\
\hline Total Magnet Weight [ton] & \multicolumn{2}{|c|}{210} \\
\hline
\end{tabular}

Table 3.5.3: HKS magnetic field summary

\begin{tabular}{c|ccc}
\hline \hline $\begin{array}{c}\text { Magnet } \\
(\text { Dipole })\end{array}$ & $\begin{array}{c}\text { Path length }(l) \\
{[\mathrm{m}]}\end{array}$ & $\begin{array}{c}\text { Typical magnetic field }(B) \\
{[\mathrm{T}]}\end{array}$ & $\begin{array}{c}\int B \cdot d l \\
{[\mathrm{~T} \cdot \mathrm{m}]}\end{array}$ \\
\hline SPL & 1.60 & 1.7 & 1.85 \\
KD & 4.50 & 1.4 & 4.95 \\
\hline \hline Magnet & Path length $(l)$ & Magnetic field gradient & $\int B \cdot d l$ \\
(Quadrupole) & {$[\mathrm{m}]$} & {$[\mathrm{T} / \mathrm{m}]$} & {$[\mathrm{T} \cdot \mathrm{m}]$} \\
\hline KQ1 & 1.14 & 4.5 & $4.22 \times 10^{-2}$ \\
KQ2 & 0.90 & 2.8 & $1.96 \times 10^{-2}$ \\
\hline \hline
\end{tabular}




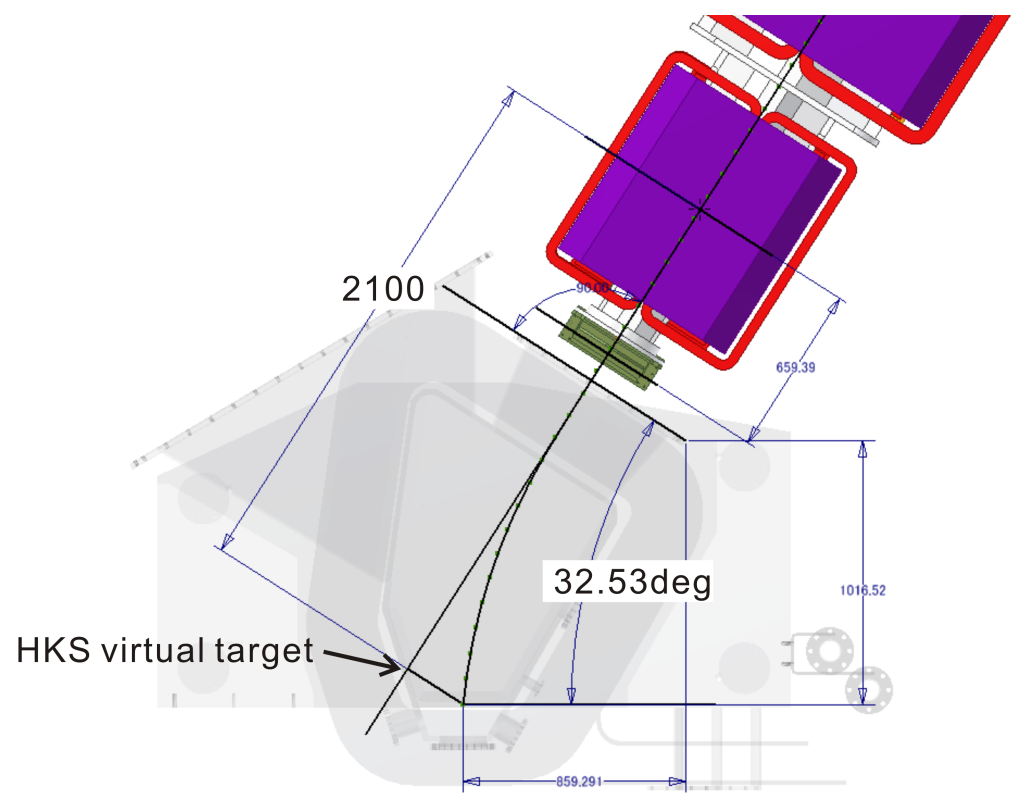

Figure 3.23: Alignment of the HKS to the SPL magnet. Unit is mm.

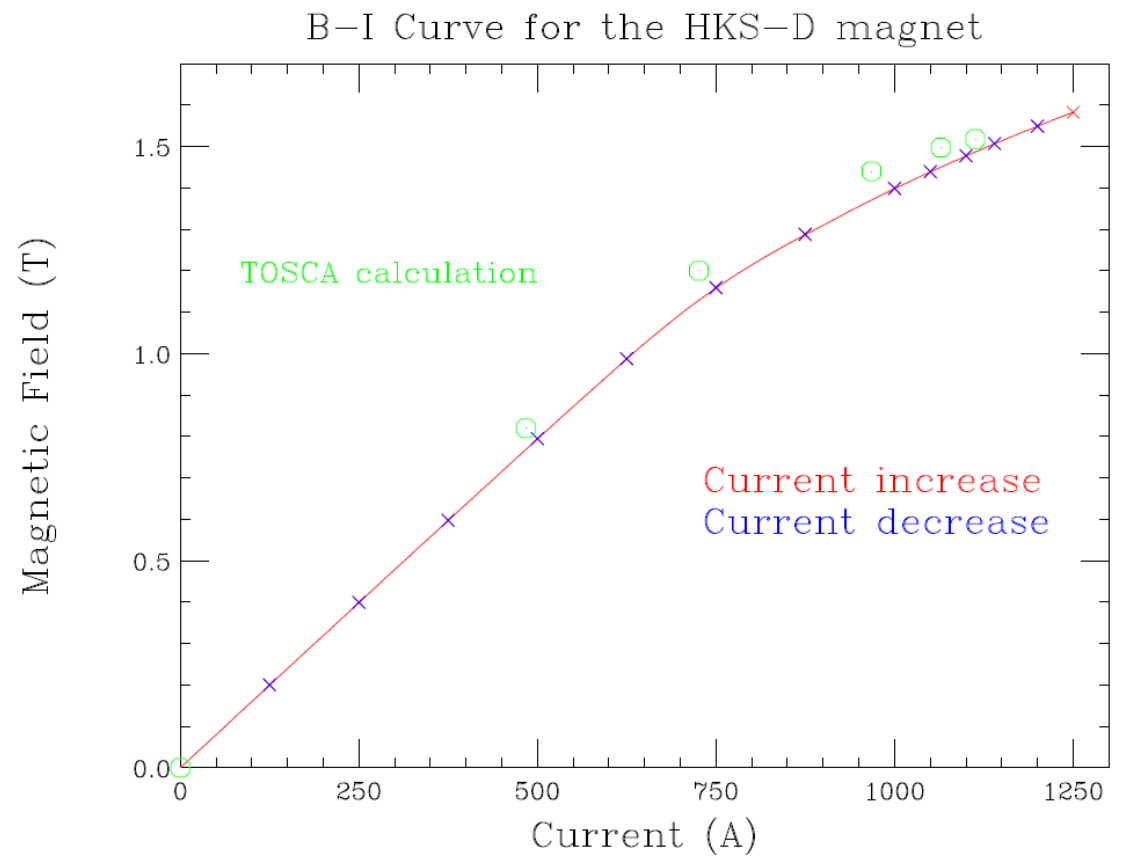

Figure 3.24: Excitation curve of KD magnet. 
several runs during experiment and the particle position can be known with an accuracy of a few $\mathrm{mm}$. The parameter is summarized in table 3.5.3. The hole diameter is 0.632 $\mathrm{cm}(=0.249 \mathrm{inch})$, the interval between holes are $1.524 \mathrm{~cm}$ for horizontal direction and $2.032 \mathrm{~cm}$ for vertical direction and the thickness is 2 inch. The thickness was determined to reduce the punch-through events as much as possible. Furthermore, in order to make asymmetry and avoid misidentification of hole pattern, one hole is filled in.

HKS collimator is placed at the same position as SS in usual physics runs during experiment in order to reject the particles which pass through the immediate area of magnet face. As seen in figure 3.26, the SS and the collimator is on the same plate and the position can be remotely controlled by stepping motor with an accuracy of $0.1 \mathrm{~mm}$. The collimator was placed during the physics production runs.

In the SS run, each event has to be identified which hole the particle run through. The identification is performed by the pattern of reference plane as shown in figure 3.27 which is a result of an actual SS run. Each line of the left (right) figure corresponding to the column (row) of SS and we can surely identify the hole position.

Some events existing between the lines are the punch-through of the particles at SS. Though it is possible to reduce the number of the punch-though event by using thicker SS, we determined present thickness because that is an allowable thickness considering spectrometer configuration. In order to decrease the number of punch-through event, the holes are not parallel to the optics line, but have angles referring the particle track at the SS position. In addition, the SS hole has some angles corresponding to the particle tracks at the SS position, and the hole configuration contributes to decrease the punch-through events.

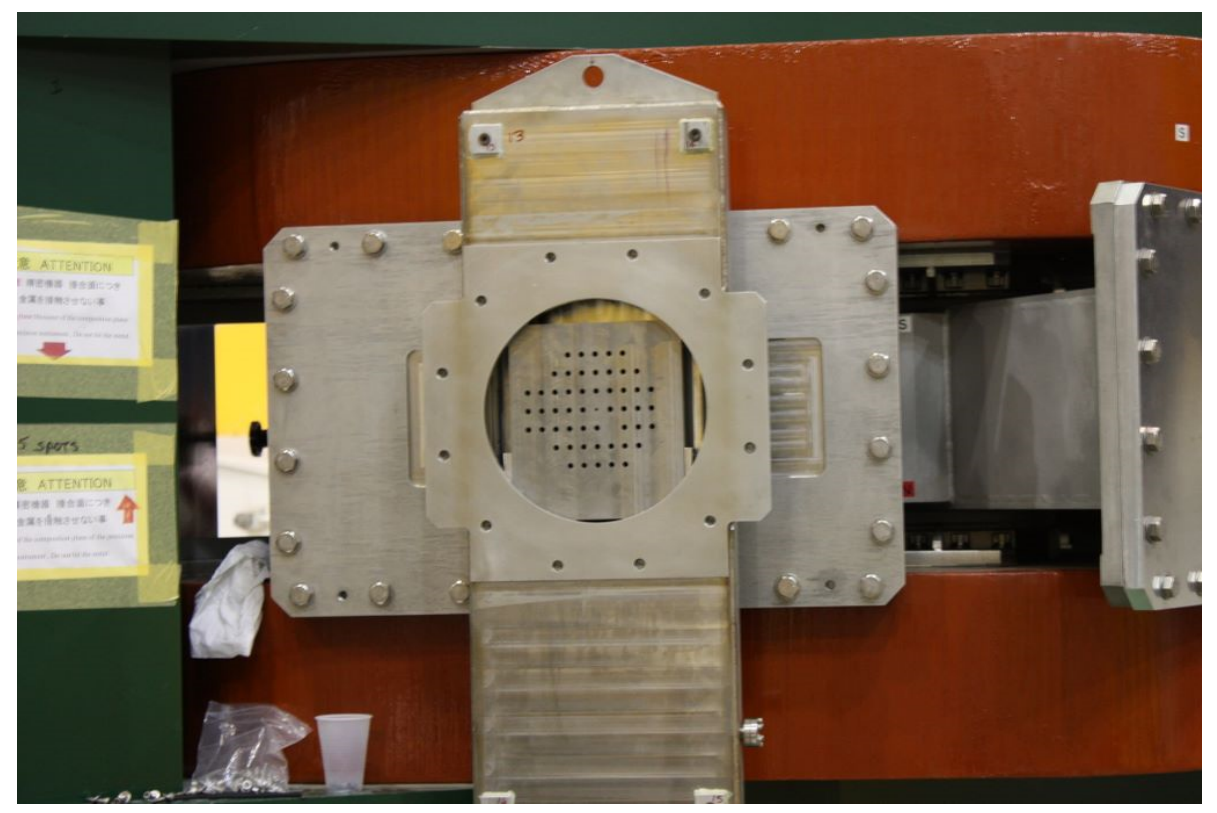

Figure 3.25: Picture of HKS Sieve Slit mounted on the exit of kaon side. 

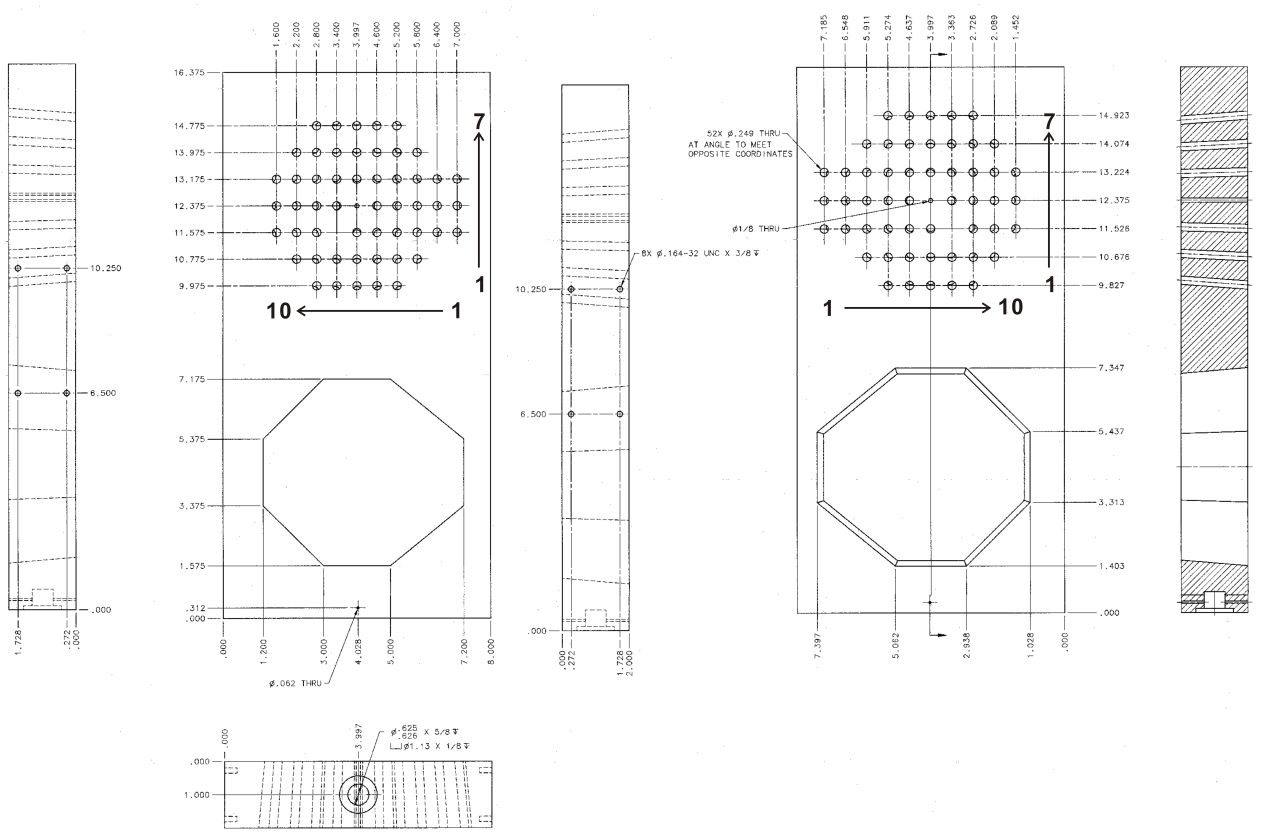

Figure 3.26: CAD drawing of HKS Sieve Slit and collimator. Unit is inch.

Table 3.5.4: HKS SS parameters

\begin{tabular}{lc}
\hline \hline The number of holes & 53 \\
Hole diameter & $0.632 \mathrm{~cm}$ \\
Thickness & 2 inch \\
Interval between holes & $1.524 \mathrm{~cm}$ (horizontal) \\
& $2.032 \mathrm{~cm}$ (vertical) \\
\hline \hline
\end{tabular}
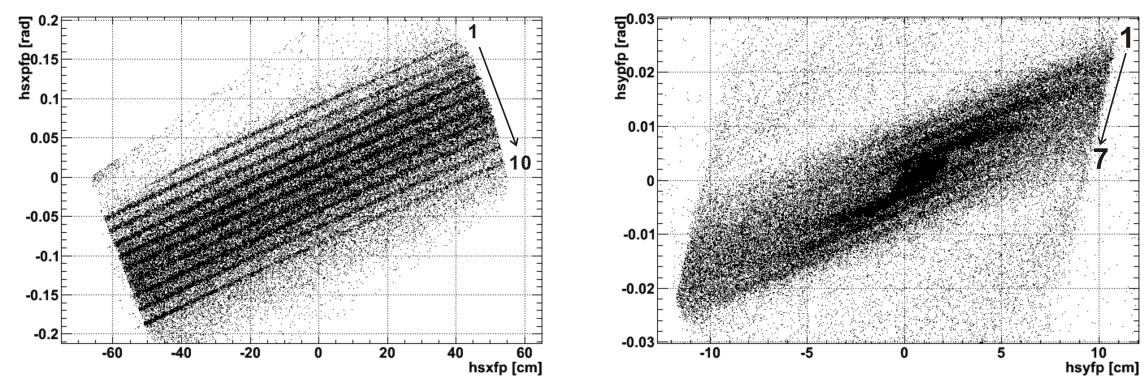

Figure 3.27: HKS Sieve Slit pattern at reference plane. Left figure is scatter plot of $x$ vs $x^{\prime}$ and right figure is that of $y$ vs $y^{\prime}$. The line numbers correspond to the numbers in figure 3.26 . 


\subsubsection{HKS magnets support}

The KQ1, KQ2 and KD magnets are installed on a common movable base. Figure 3.28 is a CAD drawing of the HKS magnets and the common base. The height of the HKS magnet system from the common base (1250 mm height) is $2500 \mathrm{~mm}$ and it was placed on a table with Hillman rollers which allow the HKS to relocate easily with the iron plates laid on concrete floor. The height of the central plane of the HKS magnets from the JLab base is $2530 \mathrm{~mm}$. The spectrometer position could be adjusted by pushing bolts and small-friction sheets (oilless) in the horizontal plane. The height could be adjustable by hydraulic jacks (stroke $30 \mathrm{~mm}$ ) with swivels placed between the common platform and the roller table. The KQ1 and KQ2 magnets are placed on a girder stuck from the common base which support the KD magnet. The center of mass is not expected to shift much by this connection because the KD magnet is much heavier than KQ1 and KQ2.

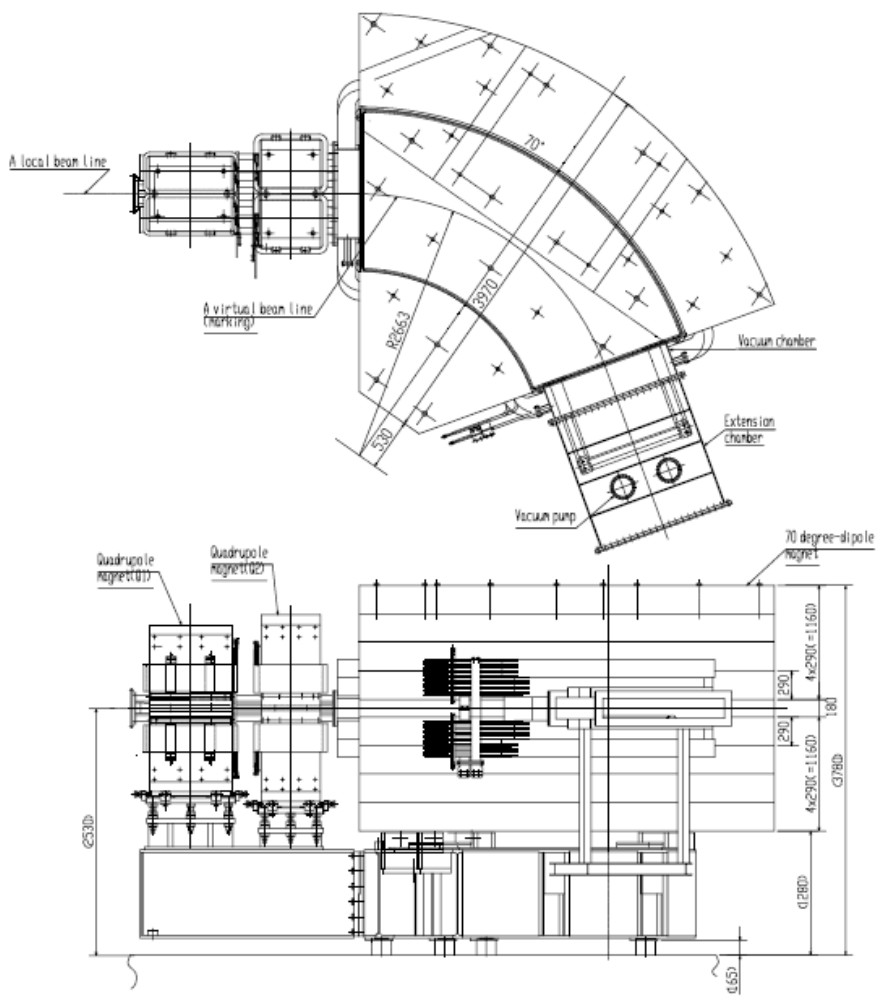

Figure 3.28: HKS magnets and common movable base. Beam height can be adjusted with four hydraulic jacks with a stroke of $\pm 15 \mathrm{~mm}$. 


\subsection{HKS detectors}

Figure 3.29 shows the HKS detector setup and figure 3.30 is its picture. The purpose of these detectors are divided into the particle tracking and the kaon identification. The tracking is done by two drift chambers $(\mathrm{KDC} 1,2)$ and three layers of TOF counters (KTOF-1X, $1 \mathrm{Y}$ and $2 \mathrm{X}$ ). The reference plane (FP, defining $\mathrm{z}=0$ ) of the tracking is defined as shown in the figure 3.29. Two type of Cherencov counters (AC1, 2, 3 and WC1, 2) were used to identify kaons from pion and proton background. In this section, the detail of these counters are explained.

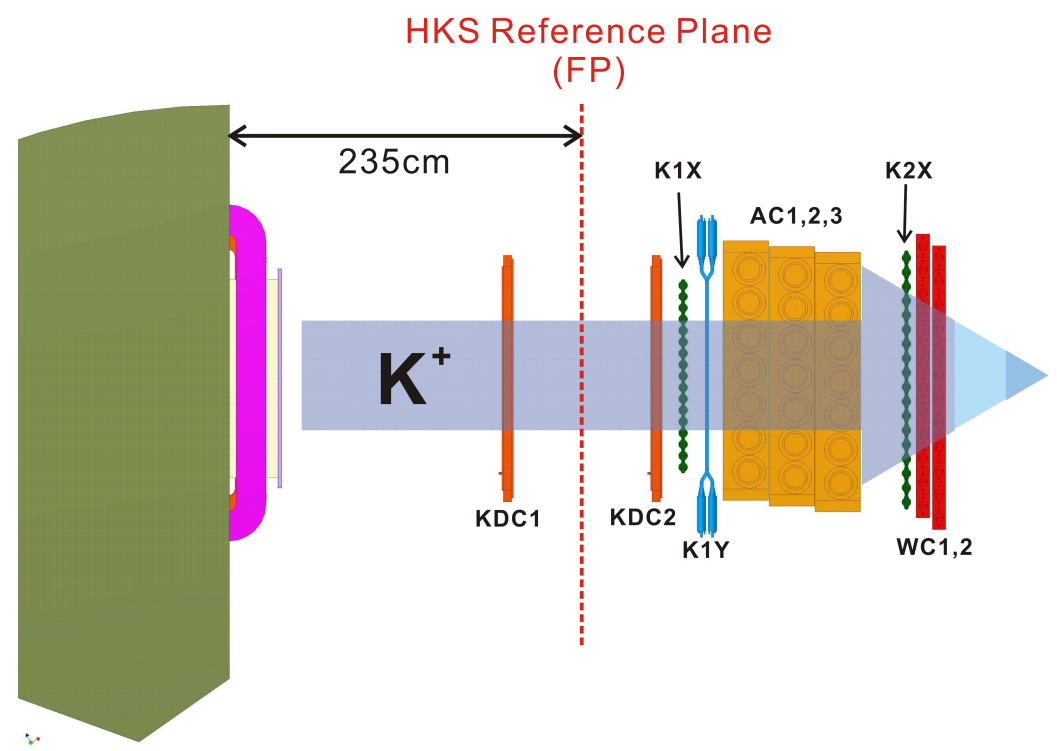

Figure 3.29: HKS detector setup.

\subsubsection{Drift chambers (KDC1, KDC2)}

The KDC1 and KDC2 are drift chambers for particle tracking, mounted directly on the KD magnet, $1 \mathrm{~m}$ apart from their center to center. The FP is defined as the center of KDC1 and KDC2. The position of the particles come from the target through HKS is measured at each KDC plane and the information is extracted to the position and angle at FP by the tracking analysis.

Their schematic of each KDC is as shown in figure 3.31 and its parameters are summarized in table 3.6.1. The KDC1 and KDC2 are identical configuration, consisted of 6 layers; u, u', x, x', v, v' and the cathode plane are placed alternatively with the each wire plane. Wire orientations of $\left(\mathrm{x}, \mathrm{x}^{\prime}\right)$ planes are perpendicular to the scattering plane, and tilted by 30 degree $\left(\mathrm{u}, \mathrm{u}^{\prime}\right)$ and -30 degree $\left(\mathrm{v}, \mathrm{v}^{\prime}\right)$ with respect to the $\mathrm{x}$ wires,respectively. Each pair of wire plane is an offset of half cell size $(0.5 \mathrm{~cm})$ from each other in order to 


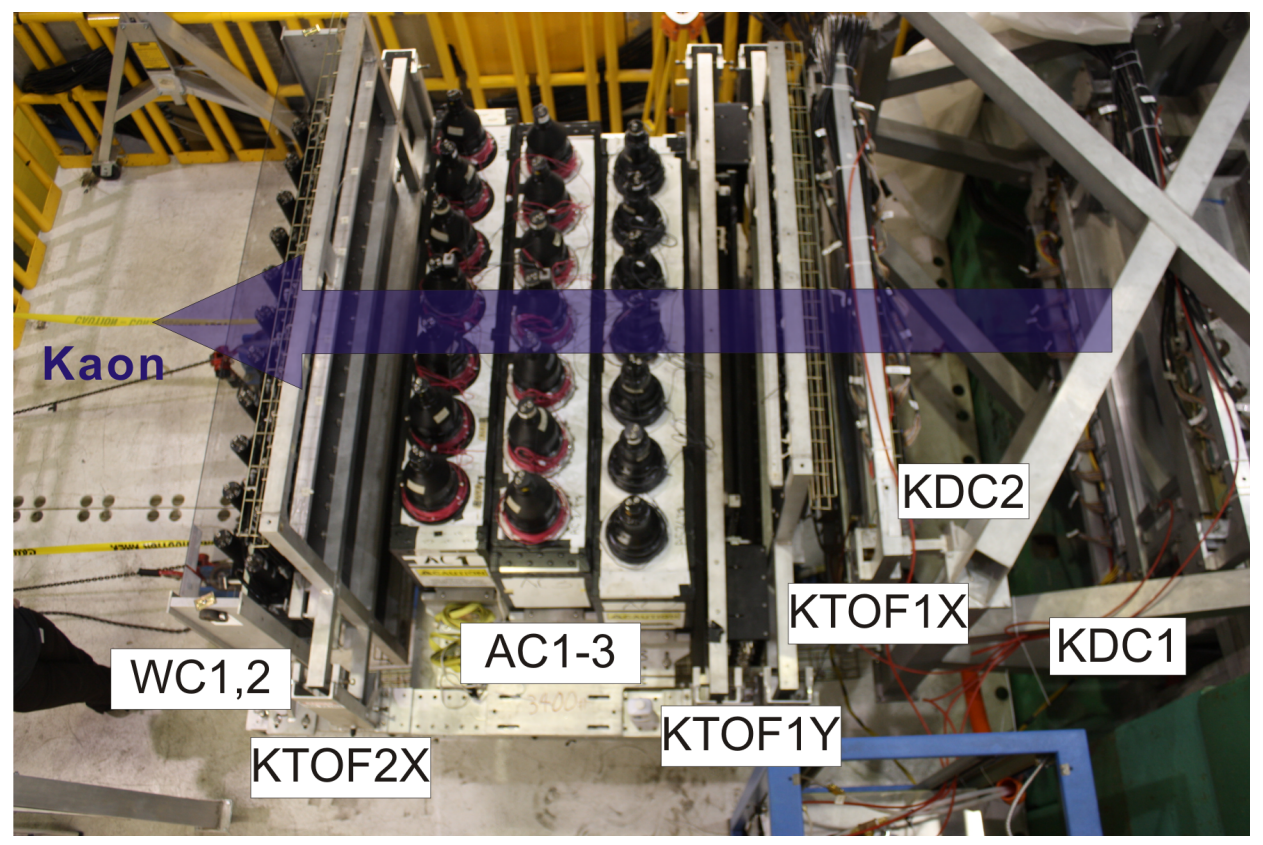

Figure 3.30: Picture of HKS detector setup.

increase the capability to resolve left-right ambiguity. Each wire plane has an effective area of $122 \times 30 \mathrm{~cm}^{2}$.

The coordinate system and detail configuration of each KDC are shown in figure 3.32. They are filled with $\mathrm{Ar}-\mathrm{C}_{2} \mathrm{H}_{6} 50 / 50$ gas. The sense wires are gold plated tungsten with the diameter of $30 \mu \mathrm{m}$ and the potential wires are beryllium copper with the diameter of $90 \mu \mathrm{m}$. The cathode planes are made of double copper coated mylar. The sense wires and the potential wires are placed alternatively as shown in 3.32. The sense wires are connected to the ground and the potential wires and cathode plane are connected to negative high voltages of about $-1970 \mathrm{~V}$.

The signals from sense wires are read and transfered to discriminated ECL signals in the Nanometric N277L (clone of LeCroy part No. 2735PC, [60]) amplifier-discriminator cards which are attached to the KDC frame. Then the signals are sent to FASTBUS multi-hit TDC (LeCroy 1877) located in the Hall-C.

\subsubsection{TOF counters}

The HKS TOF counters consist of three planes of plastic scintillation counters (KTOF$1 \mathrm{X}, 1 \mathrm{Y}$ and $2 \mathrm{X}$ ). They are used for the trigger timing determination and the velocity measurement. The velocity $(\beta)$ of charged particles were measured by the time difference between 1X (1Y) and 2X, or the time difference with HES hodoscope. The velocity information can be used for the particle identification (see section 5.2).

The detail specification is summarized in table3.6.2 and these schematic views are given as figure3.33, 3.34 and 3.35. The KTOF-1X consists of horizontal 17 segments of scintillators, determining the start timing of HKS detectors. The KTOF-1Y consists of 


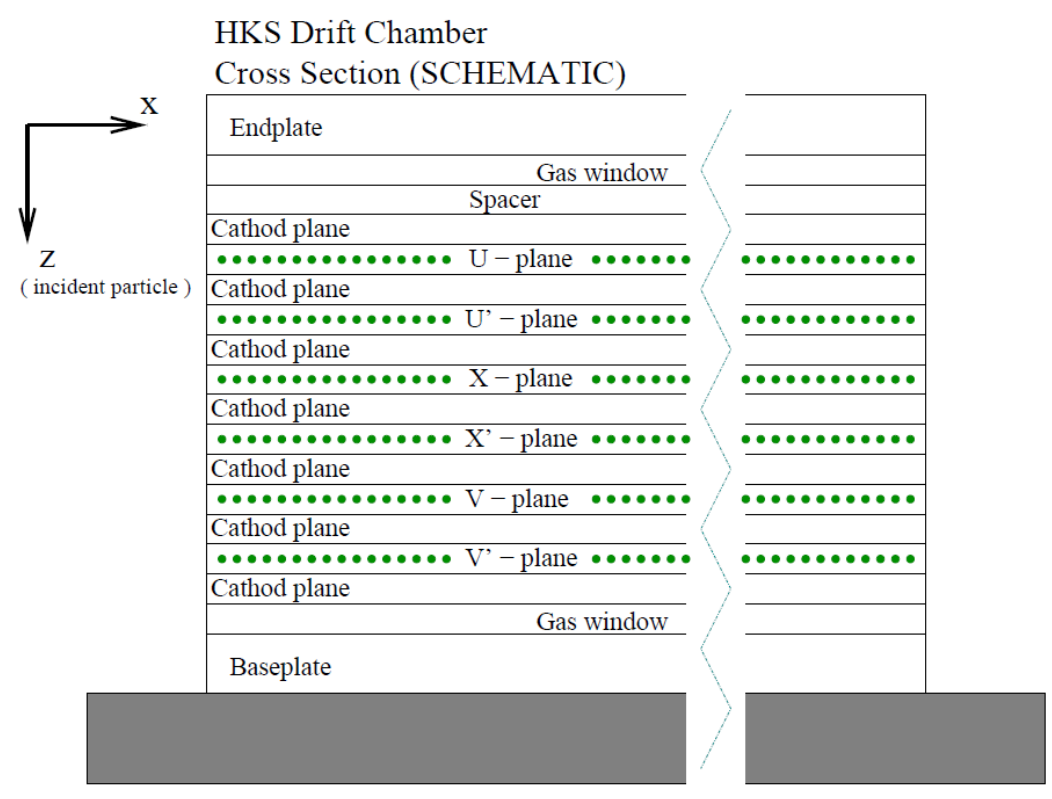

Figure 3.31: Schematic view of KDC.

Table 3.6.1: KDC parameters

\begin{tabular}{cc}
\hline \hline Basic Cnfiguration & \\
Effective area & $122^{W} \mathrm{~cm} \times 30^{H} \mathrm{~cm} \times 5^{T} \mathrm{~cm}$ \\
Number of Layers & $6\left(U U^{\prime}, X X^{\prime}, V V^{\prime}\right)$ \\
Distance between Layer & $0.635 \mathrm{~cm}$ \\
Cell Size & $1.0 \mathrm{~cm}$ \\
Angle of $U U^{\prime}, V V^{\prime}$ for $X X^{\prime}$ & $+60 \mathrm{deg},-60 \mathrm{deg}$ \\
\hline Material & Aluminized Myler $(12.5 \mu \mathrm{m})$ \\
Window & W-Au coated $(30 \mu \mathrm{m} \phi)$ \\
Sense Wire & $\mathrm{Cu}-\mathrm{Be}$ coated $(90 \mu \mathrm{m} \phi)$ \\
Potential Wire & \\
Readout & $\mathrm{Nanometric} \mathrm{N} 277 \mathrm{~L}$ \\
\hline Amplifier Discriminator & $-1970 \mathrm{~V}$ \\
Operation Parameters & $3 \mathrm{~V}$ \\
Operation HV & $\mathrm{Ar}+\mathrm{C}_{2} \mathrm{H}_{6} 50 / 50$ \\
Threshold & $16 \mathrm{psi}(\sim 1.1 \mathrm{~atm})$ \\
Gas Content & \\
Gas Pressure &
\end{tabular}




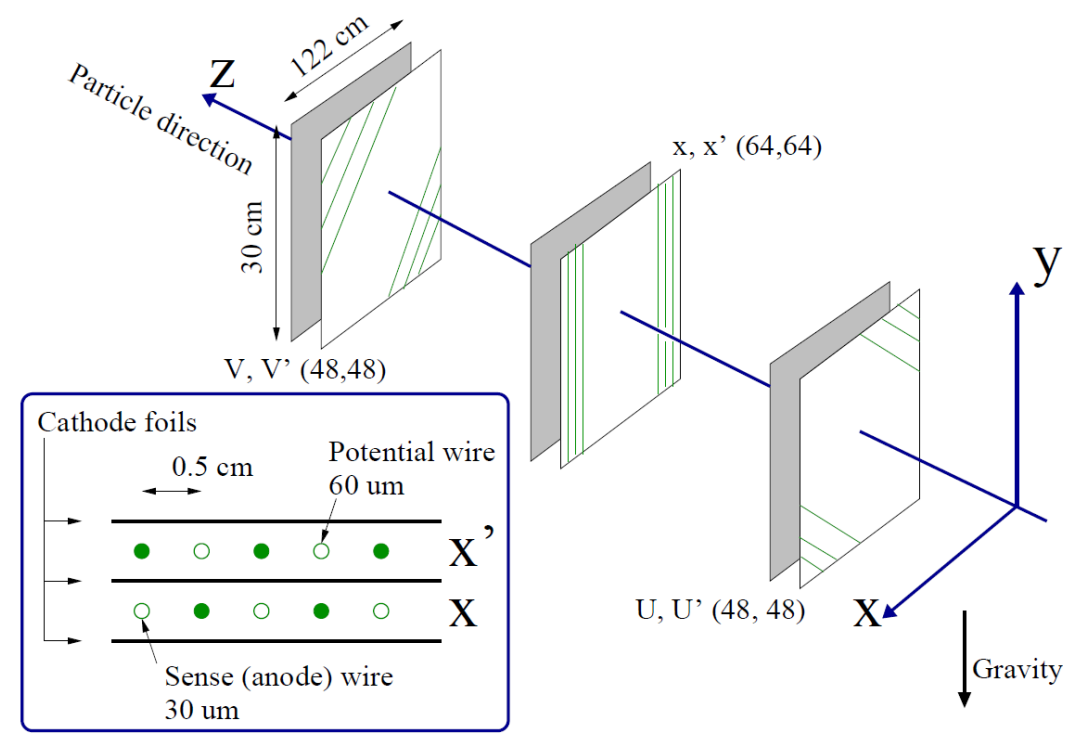

Figure 3.32: The definition of HKS coordinate system with KDC configuration.

vertical 9 segments of scintillators. As shown in figure3.34, each segment has $5 \mathrm{~mm}$ overlap with the neighbor segment in order to avoid missing acceptance. KTOF-2X consist of horizontal 18 segments of scintillators, similar to KTOF-1X.

Each scintillation counter is made of Bicron BC408 scintillators and Hamamatsu H1949-50 phototubes on both ends. The configuration is aiming to obtain good timing resolution, by the good rise time (1.3 ns) and transit time (28 ns) of the H1949-50 [61], and the good rise time $(0.9 \mathrm{~ns})$ and long attenuation length $(210 \mathrm{~cm})$ of BC408. The timing resolutions of each segment were measured by cosmic ray test before experiment. The results are $\sigma=50$ [ps] for KTOF-1X and KTOF2X counters and $\sigma=70$ [ps] for KTOF-1Y. This value corresponds to better than $4 \sigma$ separation between $1.3 \mathrm{GeV} / c$ kaons and pions between $1 \mathrm{X}$ and $2 \mathrm{X}$. 


\section{HKS hodoscope HTF1X}

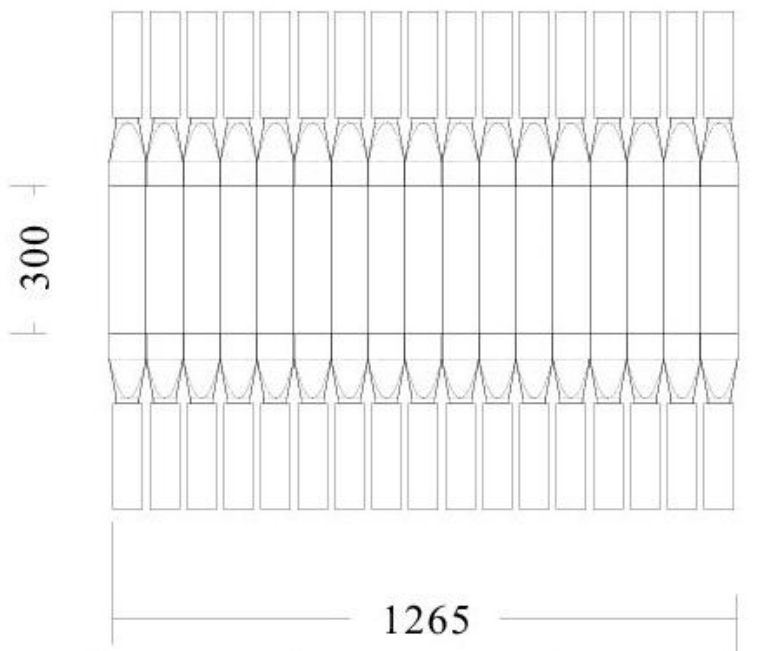

Scintillator : Bicron BC-408, $20 \mathrm{~mm}$ thick

Phototube: Hamamatsu H1949-50

Figure 3.33: Schematic view of KTOF-1X counters.

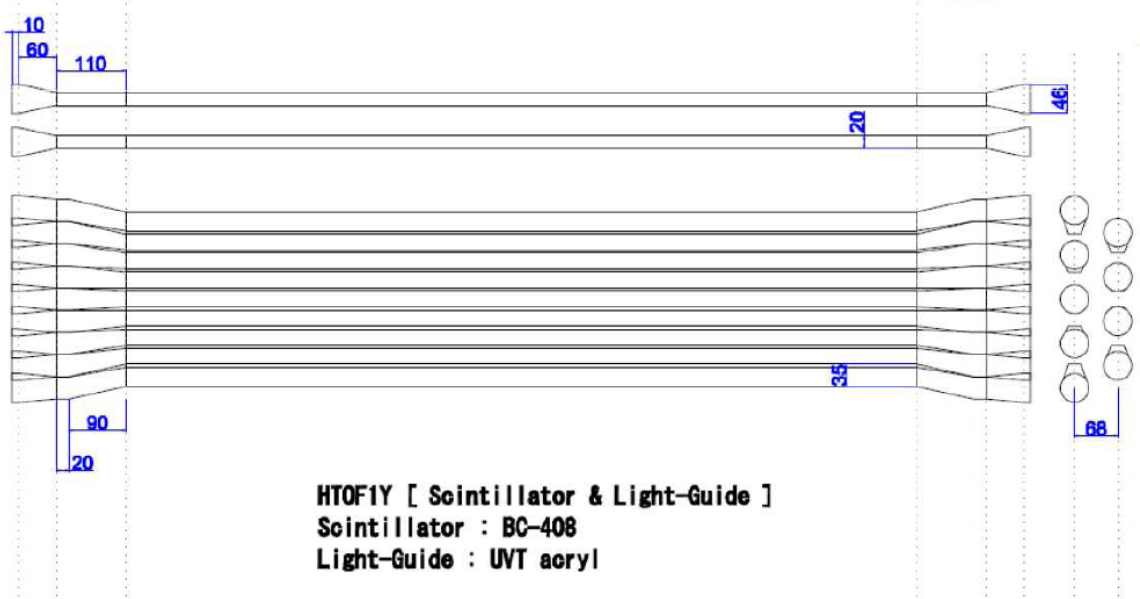

Figure 3.34: Schematic view of KTOF-1Y counters. 


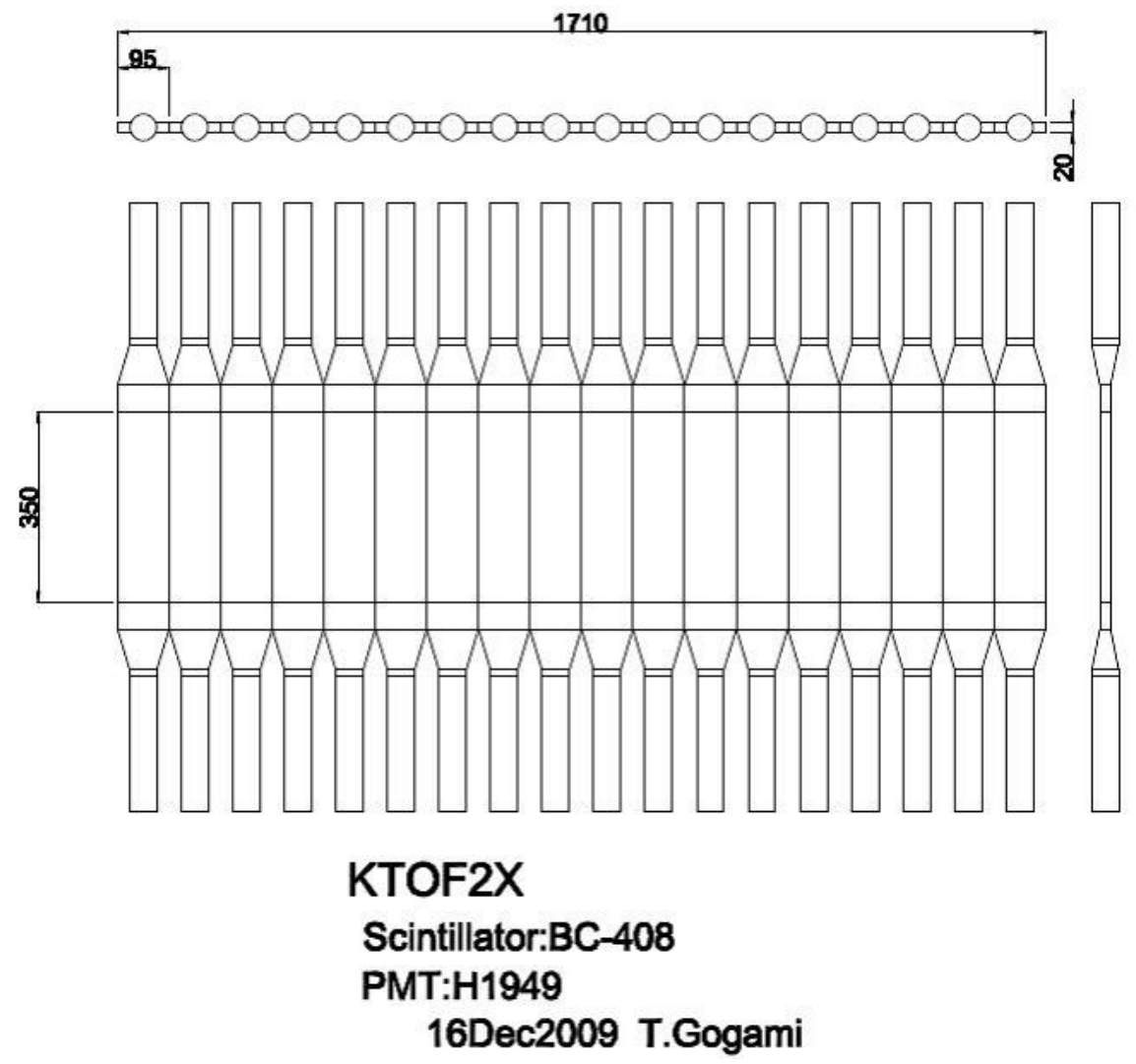

Figure 3.35: Schematic view of KTOF-2X counters.

Table 3.6.2: KTOF parameters

\begin{tabular}{cc}
\hline \hline KTOF-1X & \\
Effective area & $125^{W} \mathrm{~cm} \times 30^{H} \mathrm{~cm} \times 2^{T} \mathrm{~cm}$ \\
Segments & $7.5^{W} \mathrm{~cm} \times 17 \mathrm{segments}$ \\
PMT & $\mathrm{H} 1949-50(2$ inch $)$ \\
Scintillator & BC408 \\
\hline KTOF-1Y & \\
Effective area & $125^{W} \mathrm{~cm} \times 30^{H} \mathrm{~cm} \times 2^{T} \mathrm{~cm}$ \\
Segments & $3.5^{H} \mathrm{~cm} \times 9 \mathrm{segments}$ \\
PMT & $\mathrm{H} 1949-50(2$ inch $)$ \\
Scintillator & BC408 \\
\hline KTOF-2X & \\
Effective area & $170^{W} \mathrm{~cm} \times 35^{H} \mathrm{~cm} \times 2^{T} \mathrm{~cm}$ \\
Segments & $9.5^{W} \mathrm{~cm} \times 18 \mathrm{segments}$ \\
PMT & $\mathrm{H} 1949-50(2 \mathrm{inch})$ \\
Scintillator & BC408 \\
\hline \hline
\end{tabular}




\subsubsection{Aerogel Cherencov counters}

Aerogel Cherencov counters (AC) consist of three sets of layers, placed between KTOF-1Y and KTOF-2X to suppress pion and positron. Each layer consists of optically separated seven segments in a diffusion box with a Millipore memblane (HAHY00010, hole size $\phi=0.45 \mu \mathrm{m})$. They are staggered by $1 / 3$ segmants as shown in figure3.36.

The index of AC radiator; silica aerogel is 1.055. $\beta$ of pions in HKS momentum acceptance is $0.99-1.00$ and $0.92-0.95$ for kaon. The threshold of $\mathrm{AC}$ cherencov radiation is $\beta>1 / n=0.95$, therefore pion can be suppressed with the $\mathrm{AC}$ used as veto counter in trigger level (for detail, see [62]). The pion suppression also can be done in the off-line analysis level (see section 5.2).

The AC radiator consists of 5 layers and each layer consists of $4 \times 2$ silica aerogel tiles (Panasonic Electric Works; SP-50, $113^{H} \times 113^{W} \times 10^{T} \mathrm{~mm}^{3}$ for 1 tile). The tile is hydrophobic in order to avoid the deterioration of light transmission due to water absorption. The AC tile configuration (the thickness of the radiator, box design and reflective material) was designed based on the results of test experiment at KEK-PS held on December 2002 (KEK PS-T530).

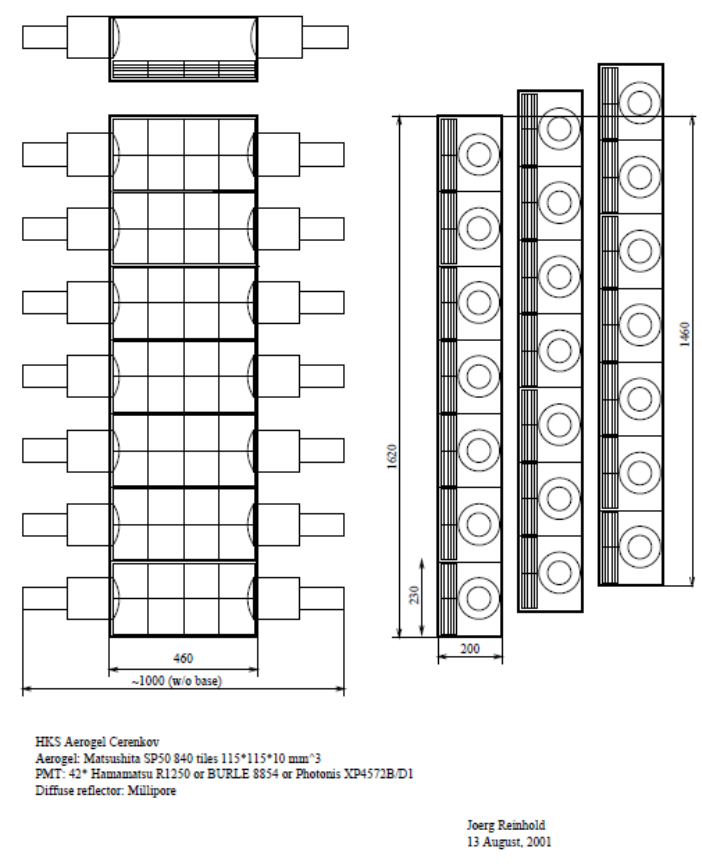

Figure 3.36: Schematic view of AC counters.

\subsubsection{Water Cherencov counters}

The Water Cherencov counters (WC) consist of two sets of layers, placed at just downstream of KTOF-2X to suppress protons. Each layer consists of optically separated twelve segments and each layers are staggered by half segments as shown in figure3.37. 
Table 3.6.3: AC parameters

\begin{tabular}{cc}
\hline \hline Effective area & $169^{W} \mathrm{~cm} \times 46^{H} \mathrm{~cm} \times 31^{T} \mathrm{~cm}$ \\
Segments & $7.5^{W} \mathrm{~cm} \times 7$ segments \\
Layers & 3 \\
PMT & XP4572B $/ \mathrm{D} 1(5$ inch, the third layer $)$ \\
\hline \hline
\end{tabular}

The refraction index of water is 1.33 and $\beta$ of proton in $\mathrm{HKS}$ momentum acceptance is $0.75-0.82$. The threshold of $\mathrm{WC}$ Cherencov radiation is $\beta>1 / n=0.75$, therefore almost all protons can radiate Cherencov light. However, as shown in figure 3.38, the $\beta$ distribution is different between protons and kaons. The protons can be separated from kaons by setting an appropriate threshold in the trigger level (for detail, see [62]). The proton suppression also can be done in the off-line analysis (see section 5.2).

The WC counters were used in the E01-011 experiment with Amino-G salt (7-Amino1. 3naphthalenedisulfonic - acid. monopotassium salt hydrate : $\left.\mathrm{C}_{10} \mathrm{H}_{9} \mathrm{NO}_{6} \mathrm{~S}_{2}\right)$ as a wave length shifter (WLS). However, the solution of Amino-G solt in water hardly suffered from radiation and the number of photo-electron has been decreased during experiment [63]. Therefore we decided to use pure deionized water (specific resistance $18.2[\mathrm{M} \Omega \cdot \mathrm{cm}]$ ) as a Cherencov radiator and developed new box in order to increase the light collection efficiency in the short-wavelength region such as wavelength $\lambda<400 \mathrm{~nm}$ [64]. In the present experiment, we used the new boxes (Box-A) in high momentum side of WC layers where the $\beta$ difference between protons and kaons is small and then high separation power is necessary. For the low momentum side, we used the conventional boxes (Box-B) because the separation power of the Box-B is enough to reject protons.

The specifications of the Box-A and Box-B are summarized in table 3.6.4. In order to increase the light collection efficiency, it is essential to improve the reflectance in UV region, because the Cherencov light is in the short wavelength region. Since the number of Cherencov photon is roughly proportional to $\lambda^{-2}$ ( $\lambda$ is wavelength), the reflectance in UV region is critical for the collection efficiency of Cherencov light. In order to do that, we improved following three points for the Box-A.

1. Teflon is used as a reflection material inside of the Box-A instead of acrylic. As shown in figure 3.39, the reflectance of white acrylic is decreased in the short wavelength region, meanwhile, that of teflon is almost flat in the optical wavelength region.

2. We replaced the material of the WC window which is attached at the end of the box and facing to PMT. Though UVT acrylic was used as the window material for the Box-B, we found that the transmittance of Quartz glass is much better than that of UVT acrylic especially in UV light region, as given in figure 3.40. Therefore we decided to use the Quartz glass for the window of the Box-A.

3. For the PMT of the Box-A, UV-transmission PMT (H7195UV, using UV glass as a window material) was used while that of Box-B is the Bialkali window PMT(H7195). Figure 3.41 is the wavelength dependency of photocathode quantum efficiency, comparing the UV glass (line B in the figure) and Bialkali (line D in the figure). The 
quantum efficiency of UV glass is much higher than that of Bialkali and H7195UV has better sensitivity for Cherencov light.

We tested the Box-B with cosmic ray and the response is shown in figure 3.42. According to the result, the detection efficiency of Box-A is 2.5 times larger than that of Box-B. The results of WC performance during the experiment about the kaon identification will be reported in section 5.2.

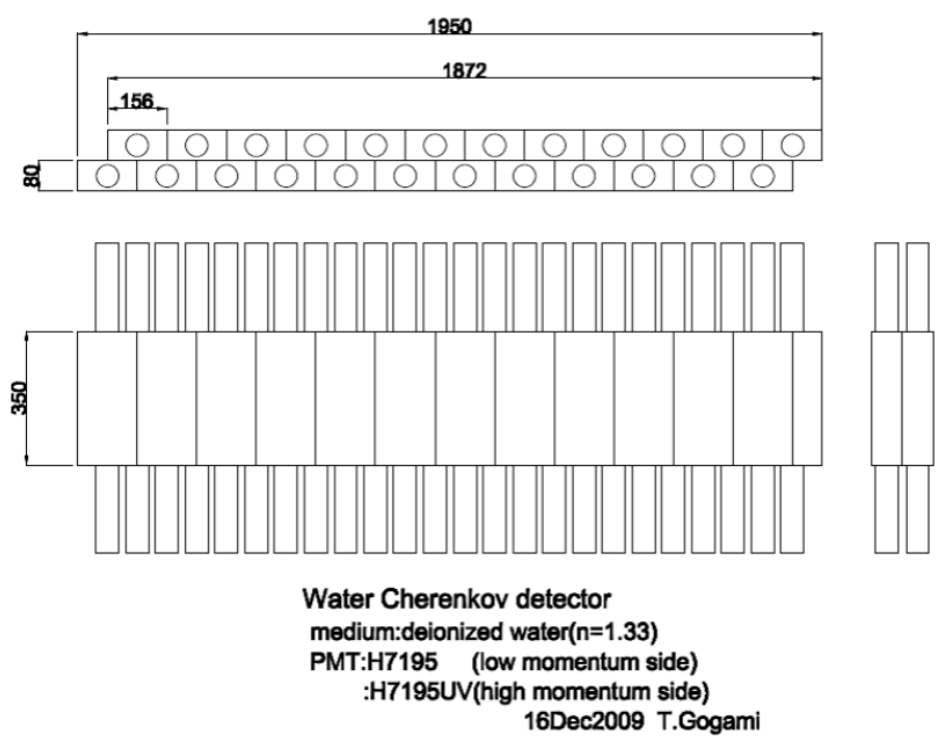

Figure 3.37: Schematic view of WC counters.

\subsubsection{Backing coil for Cherencov counters}

The magnitude of HKS leakage magnetic field in HKS detector package is about 5 gauss. Basically PMT cannot work with its expected performance in the external magnetic field because the external magnetic field will distort the path of the electron inside the PMT. As a result, the HKS leakage will field reduce the gain of PMT. In particular, the small gain is critical problem for Cherencov counters, because the original number of photon (NPE) is small (typically about 100 photons for $\mathrm{WC}$ and 5 photons for $\mathrm{AC}$ ) and the amplified NPE information is used to the pion and proton suppression in the trigger level. If the NPE is too small, we cannot suppress the pion and proton background with the expected level, then we need to recover the PMT gain.

We recovered the PMT gain by putting another magnetic field so as to cancel the HKS leakage field. The magnetic field is generated by coils winded around the PMT (figure 3.43). We call this coil as "backing coil". Figure 3.44 is pictures of backing coil

\footnotetext{
${ }^{1}$ Mitsubishi Rayon, acrylic snow white

${ }^{2}$ Chukoh Flo, Seal tape

${ }^{3}$ Material between the box and the PMT
} 
Table 3.6.4: WC parameters

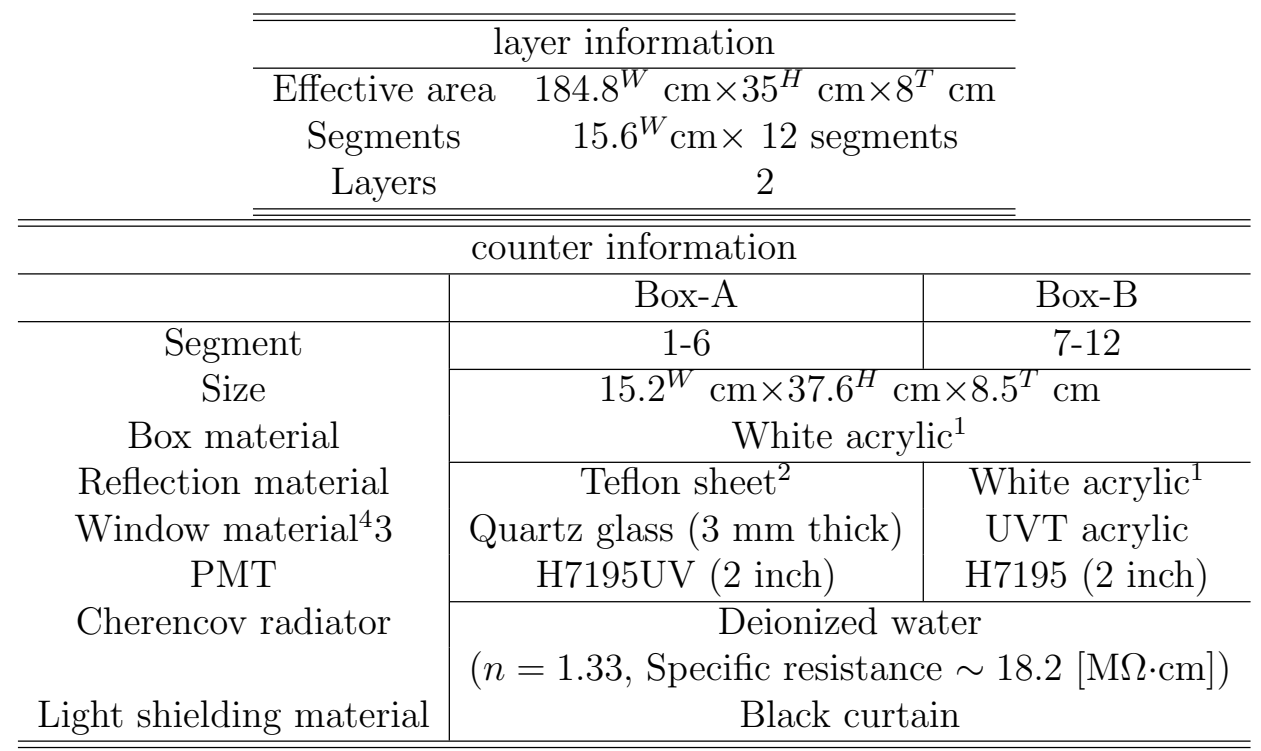

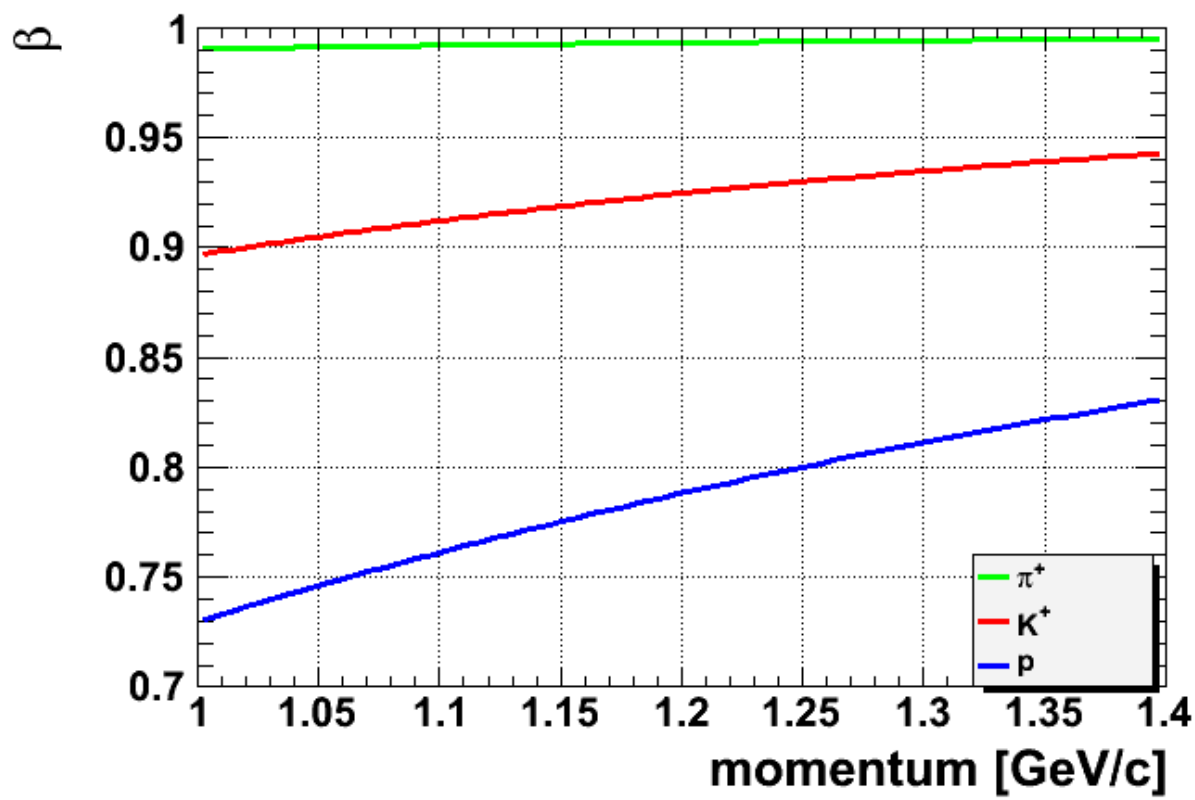

Figure 3.38: Momentum dependence of $\beta$ in HKS acceptance. 


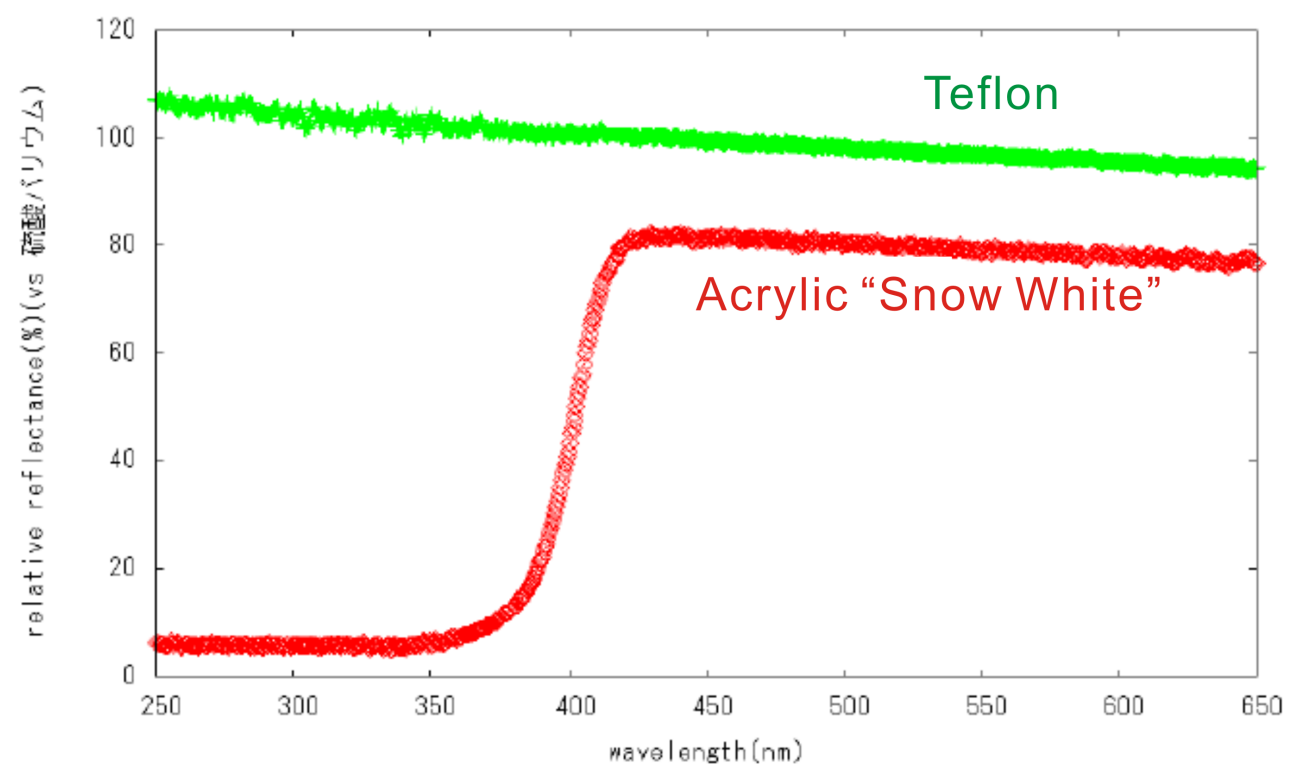

Figure 3.39: Reflectivity comparison between acrylic and teflon.

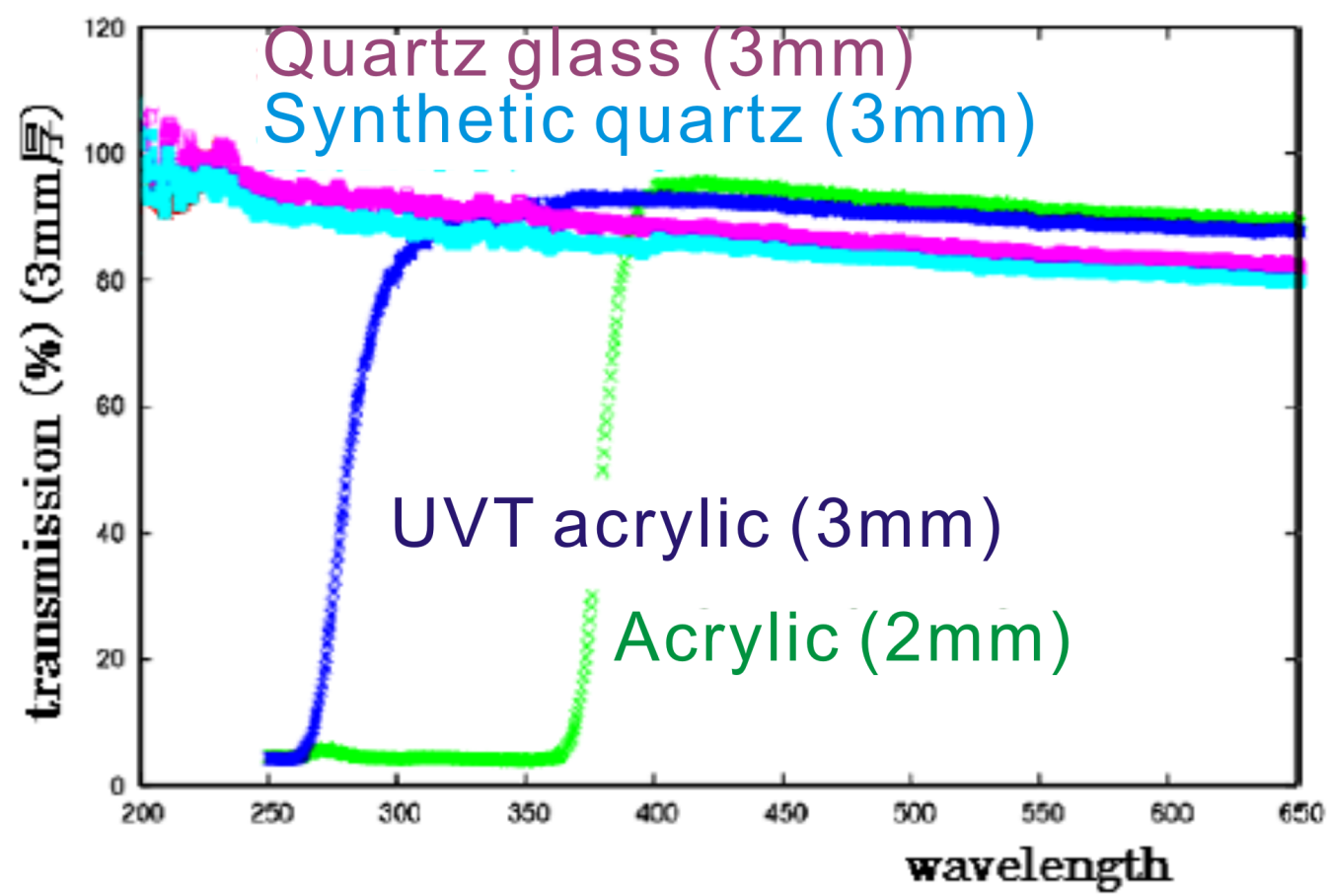

Figure 3.40: Transmittance comparison of WC window materials candidates. 


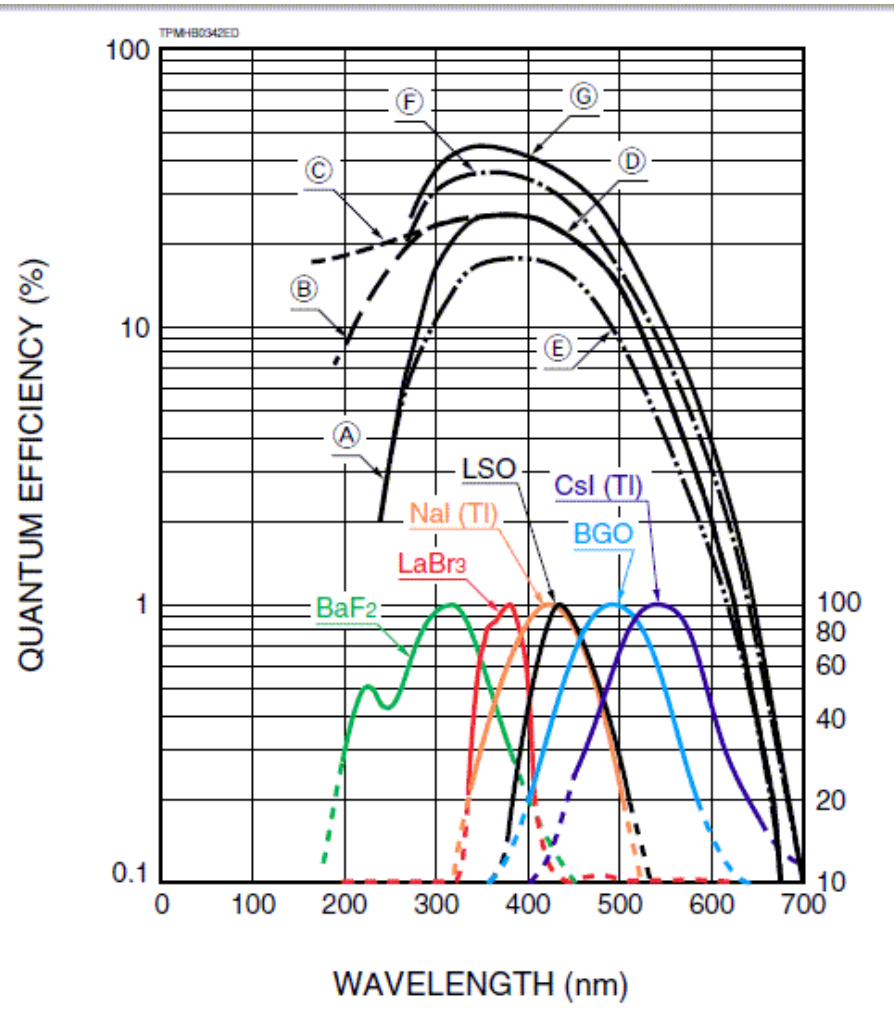

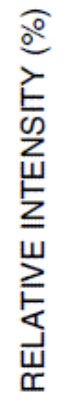

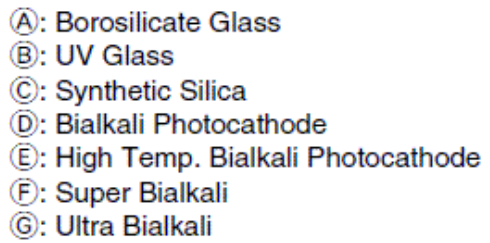

(G): Ultra Bialkali

Figure 3.41: Typical photocathode spectral response [61]. 


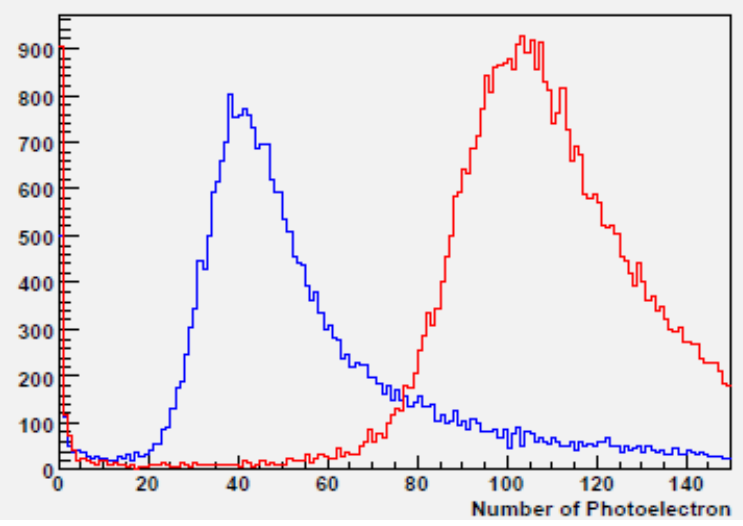

Figure 3.42: Result of WC cosmic ray test. Blue hitogram is old box and red one is new box result.

winded around $\mathrm{AC}$ and WC PMT during the experiment. The condition of the coils are summarized in table 3.6.5. The detail of backing coil test is described in Master's thesis of A.Chiba [62].

Table 3.6.5: List of the backing coil parameters.

\begin{tabular}{c|ccc}
\hline \hline & $\begin{array}{c}\text { Diameter } \\
{[\mathrm{cm}]}\end{array}$ & Turn & $\begin{array}{c}\text { Current } \\
{[\mathrm{A}]}\end{array}$ \\
\hline $\mathrm{AC}$ & 13.97 & 20 & 2 \\
$\mathrm{WC}$ & 6.0 & 40 & 8 \\
\hline \hline
\end{tabular}

\subsubsection{HKS background events and singles rate of HKS detectors}

As already mentioned, the 0-degree direction is suffered from many background of electron and positron because of the pair-creation in the present experiment. HKS is placed to avoid the 0-degree positrons, but a small part of them can come into the KD and hit the vacuum chamber of KD exit (see figure 3.45). Such events generated many positron and electron and increased the singles rates of the detectors and also the rate of HKS single trigger (see section 3.9). We performed a Geant4 simulation after the experiment and the simulation result is almost consistent with the experiment result.

Table 3.6.6 is a summary of the singles rate of HKS detectors during the experiment for several experimental conditions. The singles rate of KDC1 is up to $30 \mathrm{MHz}$. Since the number of wires per a plane is 122 for $\mathrm{x}$ and $\mathrm{x}$ ' layers and 88 for the other layers, the singles rate for each wires seems to be the order of $0.1 \mathrm{MHz}$, which is lower than typical operating limit.

For TOF counters, the singles rate of KTOF1X is up to $35 \mathrm{MHz}$ per layer. Since the number of KTOF1X segments per layer is 17 , the singles rate of a segment is $2-3$ 


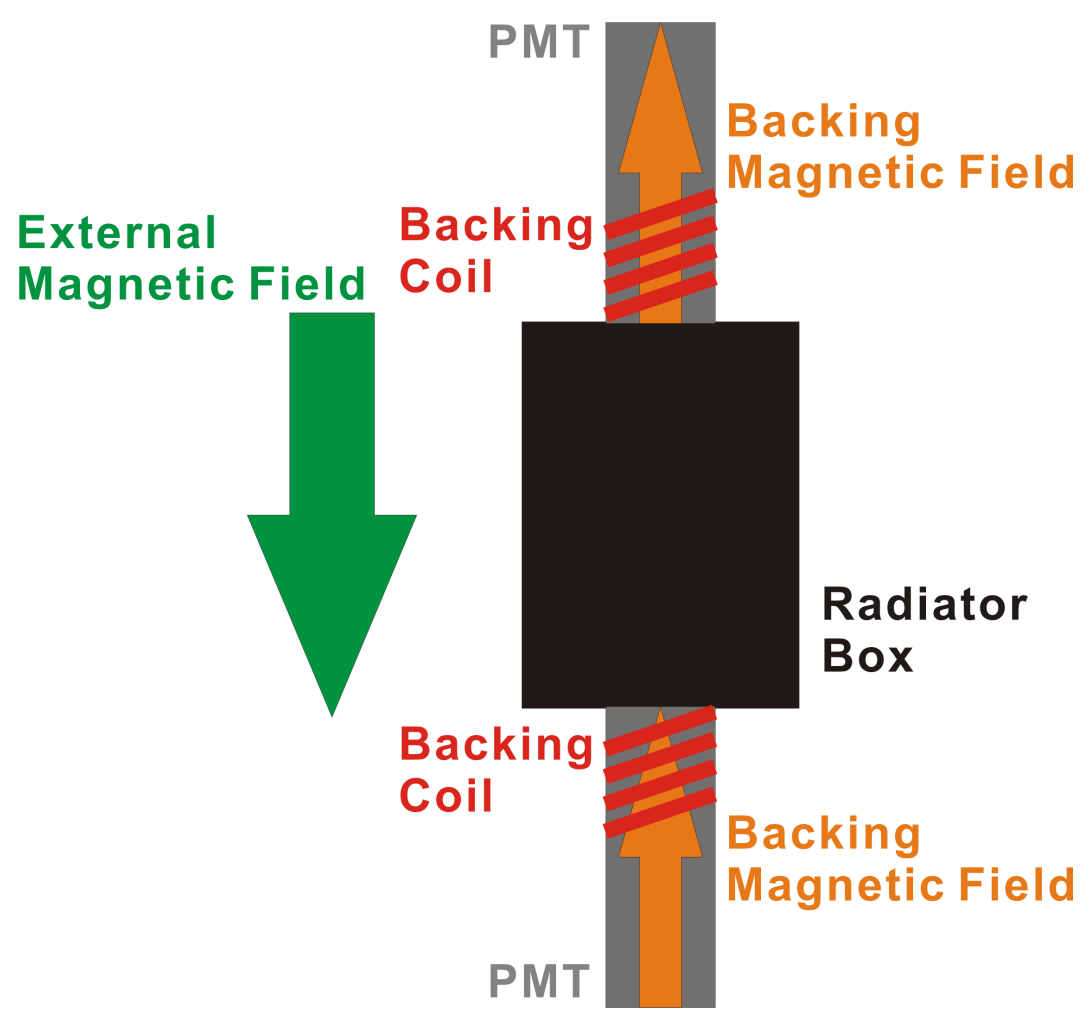

Figure 3.43: Basic concept of backing coil. In order to cancel the external magnetic field, wind a coil around the PMT and generate so as to cancel the external field.
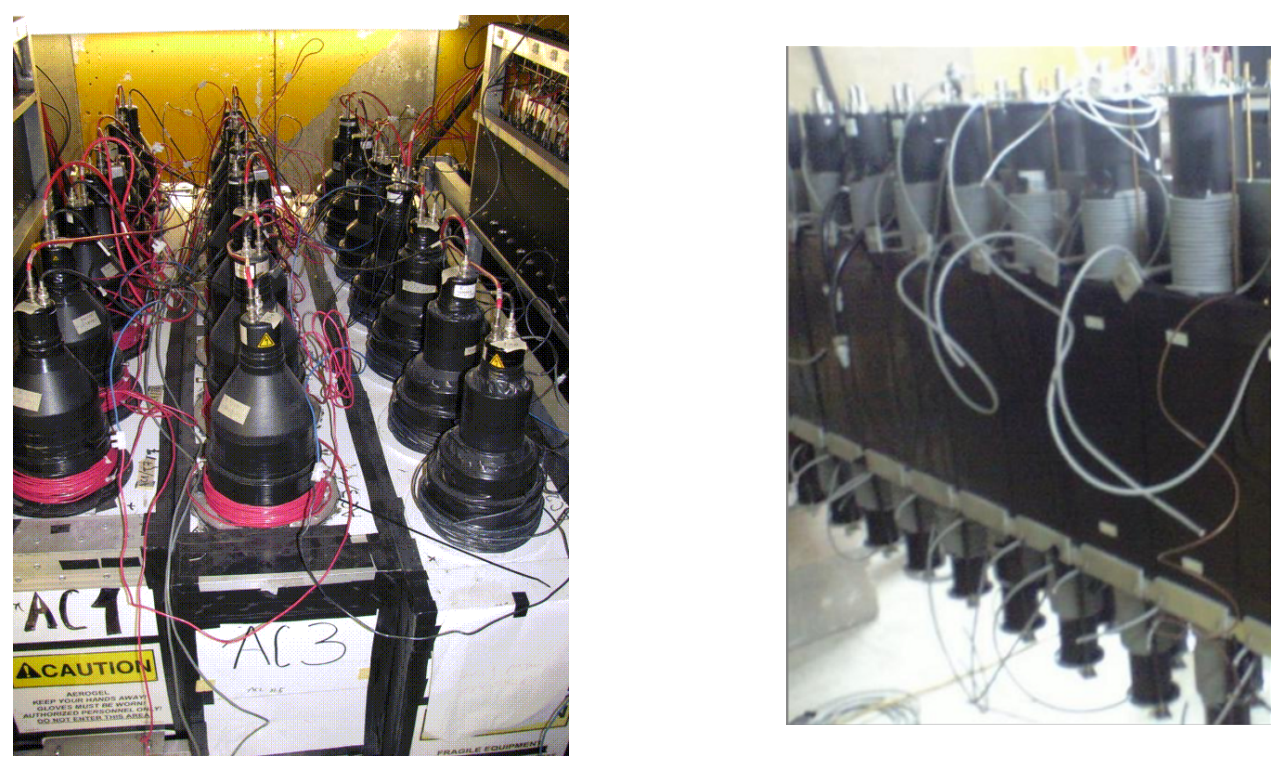

Figure 3.44: Pictures of the backing coil winded around AC (left) and WC (right) PMT 


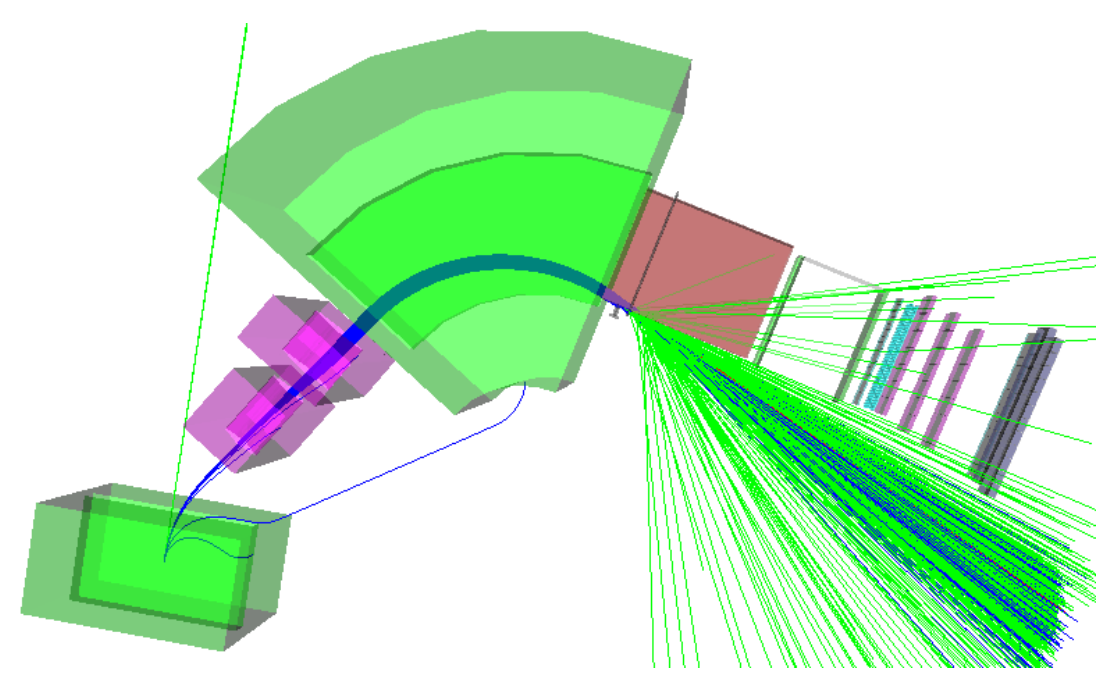

Figure 3.45: Monte Carlo simulation result of HKS background events. Blue tracks are positrons and green lines are photons. Positron generated at target can hit the vacuum extension placed at the exit of KD magnet and photons are generated there. The photon can create new positron and electron, which are one of the main background sources.

MHz. This value is high and the detection efficiencies of the TOF counters are expected to become down. The effect will be estimated in the analysis (see section 5.6).

Table 3.6.6: Summary of HKS detector singles rate.

\begin{tabular}{l|ccccccc}
\hline \hline target & $\begin{array}{c}\text { Thickness } \\
{\left[\mathrm{mg} / \mathrm{cm}^{2}\right]}\end{array}$ & $\begin{array}{c}\text { Beam current } \\
{[\mu \mathrm{A}]}\end{array}$ & KDC1 & KDC2 & $\begin{array}{c}\text { KTOF1X } \\
{[\mathrm{MHz}]}\end{array}$ & KTOF1Y & KTOF2X \\
\hline $\mathrm{CH}_{2}$ & 450.8 & 2.0 & 8.4 & 4.0 & 5.1 & 5.1 & 2.3 \\
$\mathrm{H}_{2} \mathrm{O}$ & 500.0 & 2.8 & 29.3 & 14.8 & 32.2 & 31.9 & 11.3 \\
${ }^{7} \mathrm{Li}$ & 184.0 & 35 & 19.3 & 9.3 & 14.0 & 14.0 & 6.0 \\
${ }^{9} \mathrm{Be}$ & 188.1 & 40 & 23.0 & 11.3 & 18.7 & 19.3 & 7.9 \\
${ }^{10} \mathrm{~B}$ & 56.1 & 40 & 8.2 & 3.9 & 4.9 & 4.9 & 2.3 \\
${ }^{n a t} \mathrm{C}$ & 112.5 & 10 & 5.7 & 2.7 & 3.6 & 3.5 & 1.7 \\
${ }^{n a t} \mathrm{C}$ & 112.5 & 35 & 19.1 & 9.5 & 15.1 & 15.6 & 6.5 \\
${ }^{51} \mathrm{Cr}$ & 134.0 & 8.0 & 29.8 & 15.0 & 34.6 & 33.2 & 11.8 \\
\hline \hline
\end{tabular}




\subsection{HES magnets}

The High resolution Electron Spectrometer (HES) was constructed in 2008 at TOKIN (Sendai, JAPAN), and used in the present experiment for the first time. The feature of HES is high momentum resolution, large angle solid angle and kinematic matching with HKS. The HES consists of two quadrupole magnets (EQ1 and EQ2), one dipole magnet (ED) and a detector package. The reason to choose Q-Q-D configuration is also the flexibility of the spectrometer optics, similarly to the HKS. The detail of these components and expected performance are explained in this section and the detail of magnet design are explained in chapter4.
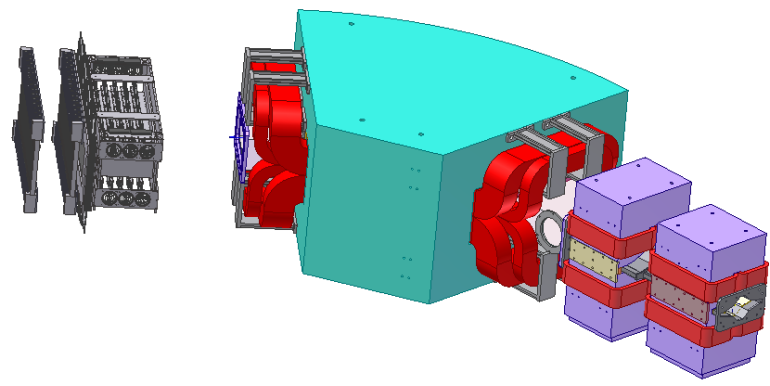

Figure 3.46: Schematic view of HES spectrometer system.

\subsubsection{Magnet specification}

The EQ1 and EQ2 are vertical and horizontal convergence quadrupole magnets, respectively. The ED is a dipole magnet which has 50 degree bending angle for $0.844 \mathrm{GeV} / c$ momentum particles. The detail specification of these magnets are summarized in table3.7.1 and table3.7.1.

\subsubsection{Magnetic field of HES}

The magnetic field information of HES is summarized in section 4.3. 


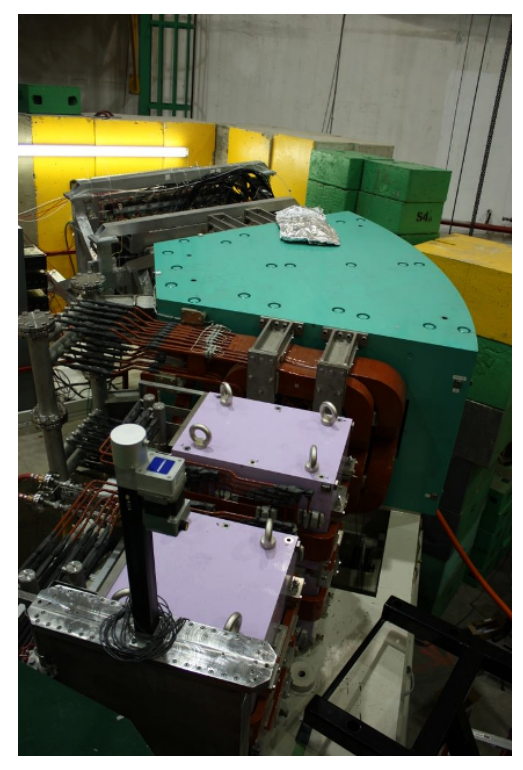

Figure 3.47: Picture of HES magnets.

Table 3.7.1: EQ1 and EQ2 parameters.

\begin{tabular}{lcc}
\hline \hline & EQ1 & EQ2 \\
\hline Bore Radius [mm] & 100 & 125 \\
Pole Length [mm] & 600 & 500 \\
Max. Ampare Turns [A turns] & 144000 & 144000 \\
Number of Turns & 180 & 180 \\
Conductor Size [mm] & $9 \times 9(\phi 6$ hole $)$ & $9 \times 9(\phi 6$ hole $)$ \\
Max. Field Gradient [T/m] & 7.8 & 5.0 \\
Max. Current [A] & 800 & 800 \\
Total Magnet Weight [ton] & 2.8 & 3.1 \\
\hline \hline
\end{tabular}

Table 3.7.2: ED parameters

\begin{tabular}{lc}
\hline \hline Pole Gap Hight [mm] & 194 \\
Pole Length [mm] & 1560 \\
Max. Ampare Turns [A turns] & 289680 \\
Number of Turns & 136 \\
Conductor Size & $17 \times 17(\phi 11$ hole $)$ \\
Max. Field [T] & 1.65 \\
Max. Current [A] & 1065 \\
Total Magnet Weight [ton] & 36.4 \\
\hline \hline
\end{tabular}




\subsubsection{Sieve slit and collimator}

Figure 3.48 and 3.49 are a picture and a CAD drawing of HES Sieve Slit (SS) and collimator. The HES SS is placed just before the EQ1 magnet, and its material and purpose is same as these of the HKS SS. The HES collimator is also in the same plate with the SS.

As seen in figure 3.50, the SS and the collimator is on the same plate and the position can be changed by an electric motor with an accuracy of $0.1 \mathrm{~mm}$.

The collimator position is adjustbable depending on the singles rate of HES detectors, namely, run condition. Actually we took data with three types of collimator positions during the experiment, as shown in the figure 3.51. Basically " $\mathrm{SS}=-300.2 \mathrm{~mm}$ " used for lower rate run and " $\mathrm{SS}=-330.2 \mathrm{~mm}$ " is used for higher rate run. "SS=-347.2 $\mathrm{mm}$ " is not used for the physics run.

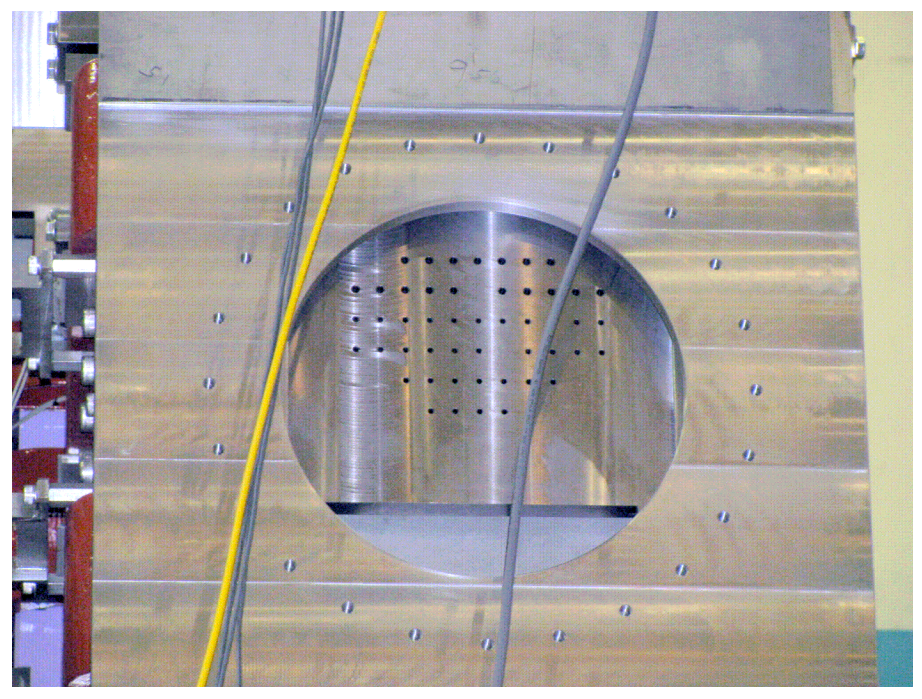

Figure 3.48: Picture of HES Sieve Slit mounted on the entrance of EQ1.

Table 3.7.3 is a summary of HES SS parameter. HES SS has two sizes of holes, 3/16 inch and 1/4 inch, because the particles coming to HES are associated with bremsstrahlung and they distribute forward direction which is corresponding to the bottom side of HES SS. The intervals between holes are $1.905 \mathrm{~cm}$ for horizontal direction and $2.54 \mathrm{~cm}$ for vertical direction and the thickness is 1 inch. 2 holes are filled in order to make asymmetry and avoid misidentification of hale pattern.

Figure 3.52 shows the pattern of reference in actual SS run. Each line of the left (right) figure corresponding to the column (row) of SS and we can surely identify which hole the particle run through. In the HES side, most of the electrons hit to the plate are stopped because of the bremsstrahlung process, then there are almost no events between the lines, differently from HKS side. 


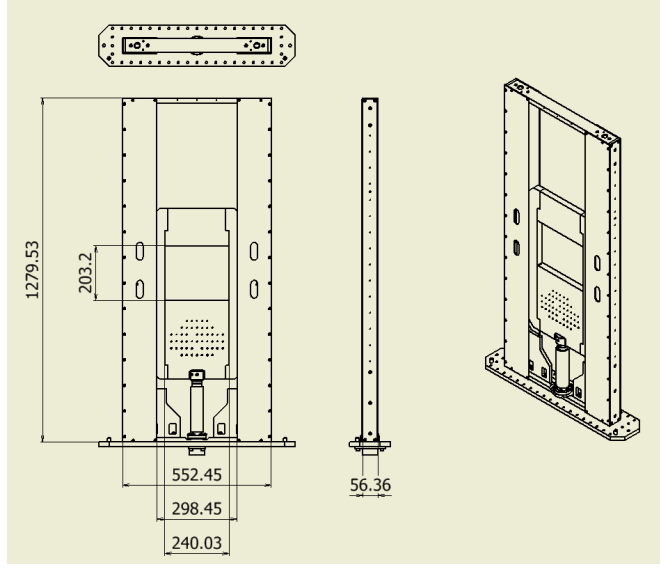

Figure 3.49: CAD drawing of HES Sieve Slit and collimator plate. Unit is mm. Note that this figure is upside-down.

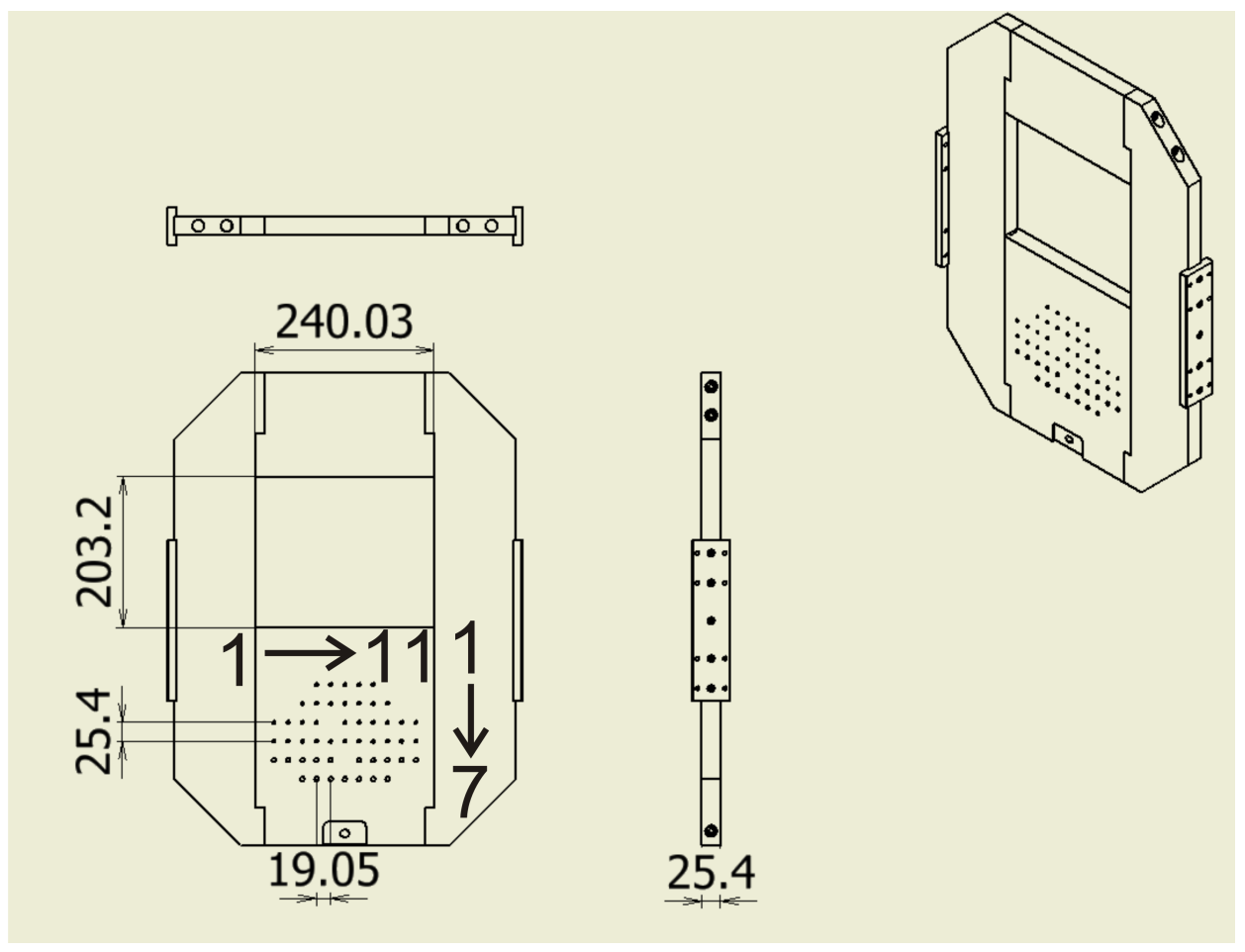

Figure 3.50: CAD drawing of HES Sieve Slit. Unit is $\mathrm{mm}$. Note that this figure is upside-down. 


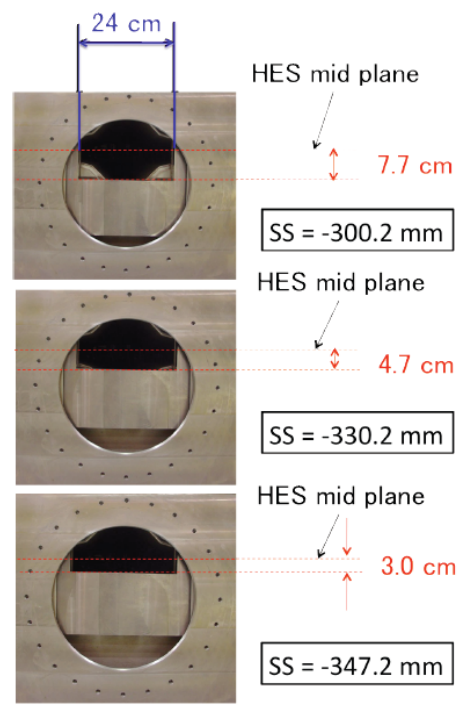

Figure 3.51: HES collimator position during experiment. Collimator position is adjustable depending on the detection rate of HES detectors and we used three types of collimator position during experiment, as shown in the figure. Basically "SS=-300.2 mm" used for lower rate run and " $\mathrm{SS}=-330.2 \mathrm{~mm}$ " is used for higher rate run. " $\mathrm{SS}=-347.2 \mathrm{~mm}$ " is not used for the physics run.

Table 3.7.3: List of HES collimator position for each run conditions.

\begin{tabular}{l|ccc}
\hline \hline Target & $\begin{array}{c}\text { Thickness } \\
{\left[\mathrm{mg} / \mathrm{cm}^{2}\right]}\end{array}$ & $\begin{array}{c}\text { Nominal current } \\
{[\mu \mathrm{A}]}\end{array}$ & $\begin{array}{c}\text { Collimator position } \\
{[\mathrm{mm}]}\end{array}$ \\
\hline $\mathrm{CH}_{2}$ & 450.8 & 2 & -300.2 \\
$\mathrm{H}_{2} \mathrm{O}$ & 500.0 & 2.5 & -330.2 \\
${ }^{7} \mathrm{Li}$ & 184.0 & 35 & -300.2 \\
${ }^{9} \mathrm{Be}$ & 188.1 & 40 & -300.2 \\
${ }^{10} \mathrm{~B}$ & 56.1 & 40 & -300.2 \\
${ }^{n a t} \mathrm{C}$ & 112.5 & 10 & $-300.2 /-330.2$ \\
& & 20 & -300.2 \\
& & 35 & $-300.2 /-330.2$ \\
${ }^{51} \mathrm{Cr}$ & 134.0 & & -330.2 \\
\hline \hline
\end{tabular}


Table 3.7.4: HES SS parameters

\begin{tabular}{lc}
\hline \hline The number of holes & 55 \\
Hole diameter & $0.476 \mathrm{~cm}(3 / 16$ inch) \\
& $0.635 \mathrm{~cm}(1 / 4$ inch) \\
Thickness & 1 inch \\
Interval between holes & $1.905 \mathrm{~cm}$ (horizontal) \\
& $2.54 \mathrm{~cm}$ (vertical) \\
\hline \hline
\end{tabular}
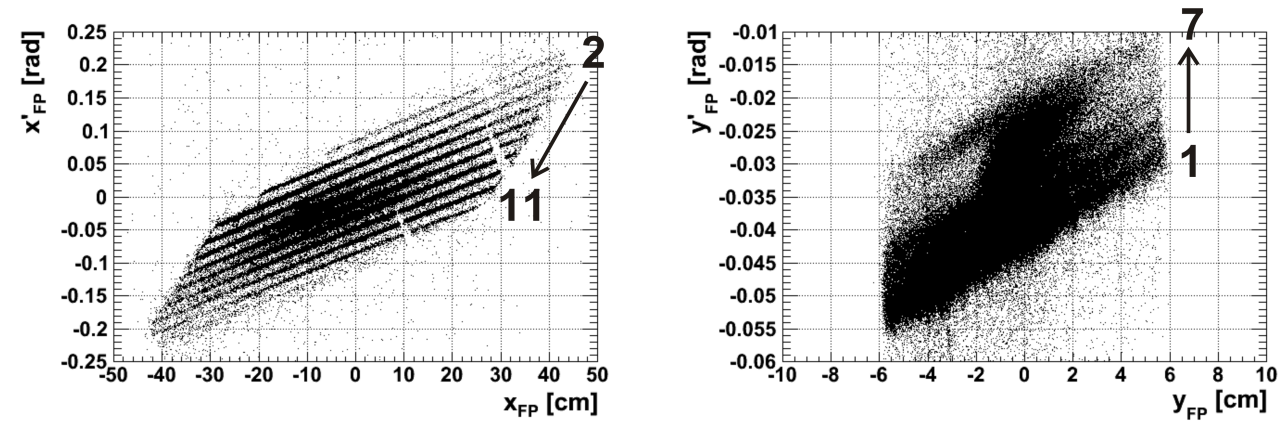

Figure 3.52: HES Sieve Slit pattern at reference plane. Left figure is scatter plot of $x$ vs $x^{\prime}$ and right figure is that of $y$ vs $y^{\prime}$. The line numbers correspond to the numbers in figure 3.50. The line number 1 in the $x$ vs $x^{\prime}$ plot is very hard to see, because the position is out of the acceptance. 


\subsubsection{HES movable base}

The EQ1, EQ2 and ED magnets are also installed on a common base as well as HKS and figure 3.53 show the CAD drawing of the base. The base is equipped with six hydraulic jacks to adjust the HES level and tilting angle. The height of the base can be adjustable by four hydraulic jacks, and another two hydraulic jacks are used to tilt the entire system from 0 to 10 degrees. The whole base system was assembled on the movable table with Hilman rollers.

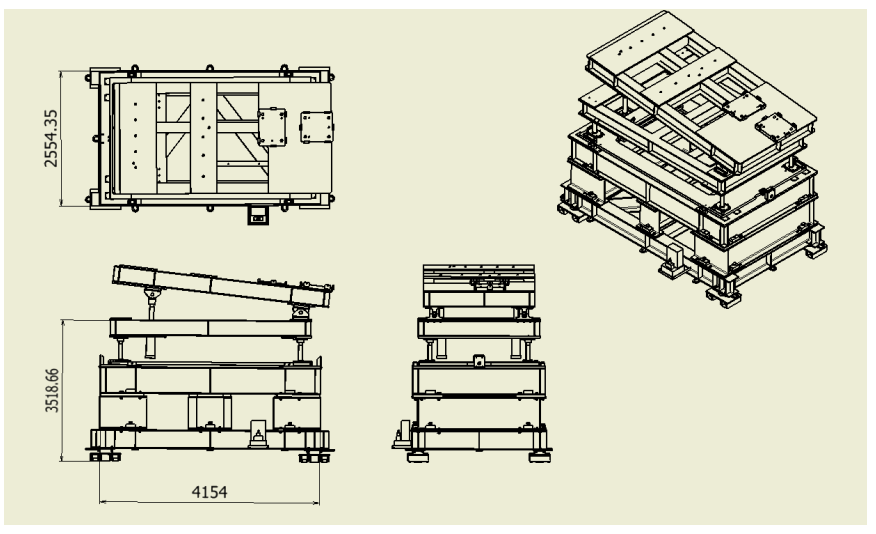

Figure 3.53: CAD drawing of HES movable base. Tilt angle is 6.5 degree. Unit is $\mathrm{mm}$.

\subsection{HES detectors}

Figure 3.54 shows the HES detector setup. These consist of two drift chambers (EDC1, 2) and two layers of TOF counters (ETOF-1, 2), aiming to the particle tracking. The reference plane $(\mathrm{FP})$ of the tracking is defined as shown in figure 3.54. he ETOF counters are also used for the trigger of HES side and making the coincidence time with HKS. In this section, the detail of these counters are explained.

\subsubsection{Drift Chambers}

The HES tracking detectors consist of two types of drift chambers, EDC1 (see figure 3.56 and 3.57) and EDC2, and these are different type each other. They are used for the particle tracking and mounted directly on the ED magnet in order to minimize the uncertainty of the detector position. The reference plane (FP) of HES is defined as the center of EDC1. The position and angle of particles come from target through HES are measured by this EDCs and these valuables are reconstructed to the emission momentum vectors of the particles at the target point by the "F2T function". The particle position and angle at FP are extracted by the measured particle tracks in EDCs. 


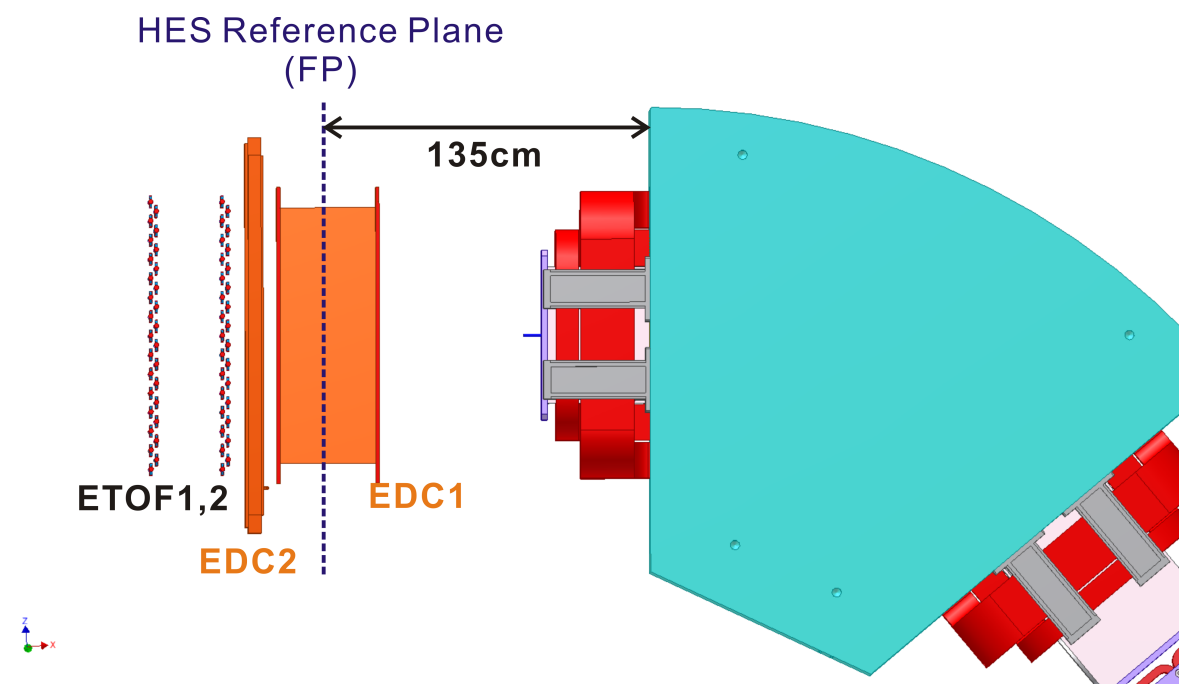

Figure 3.54: HES detector setup.

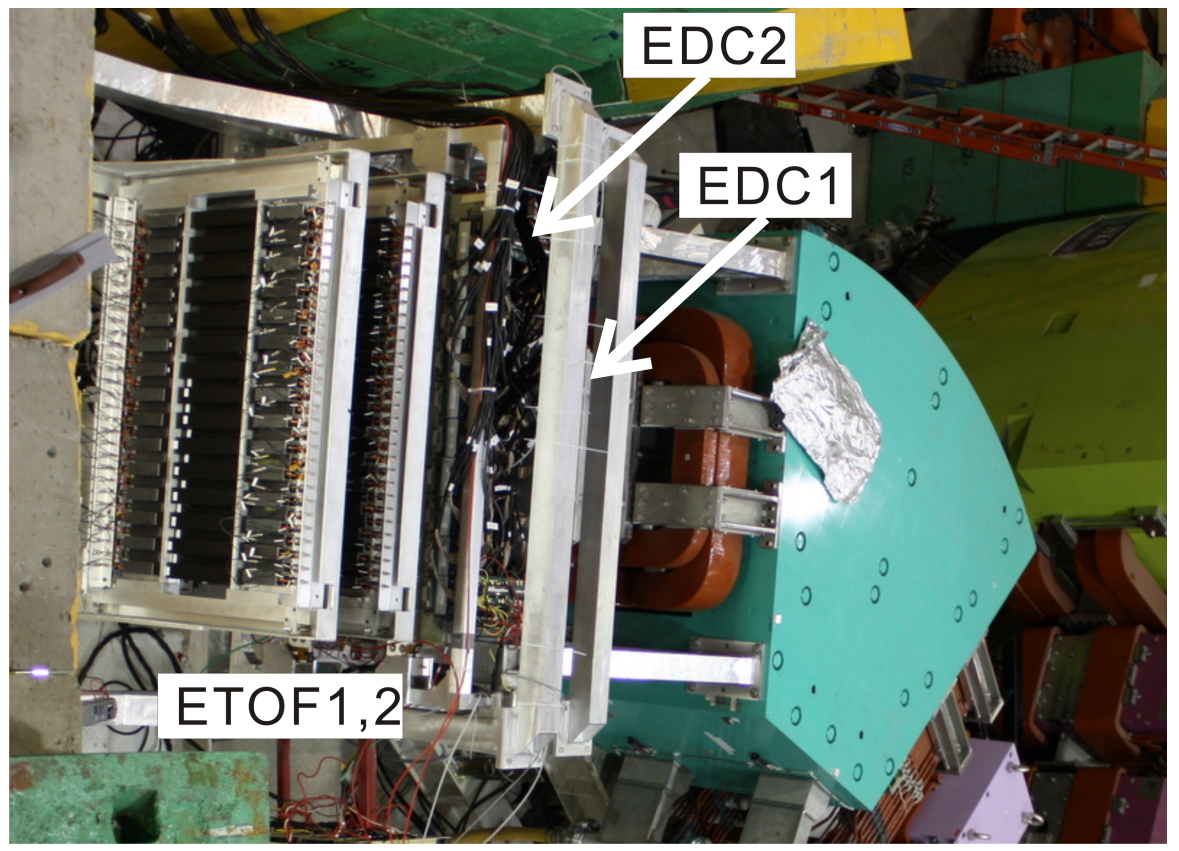

Figure 3.55: Picture of HES detectors. 


\section{EDC1}

The EDC1 is a honeycomb cell type drift chamber, as shown in figure3.58 and its specification is summarized in table3.8.1. It is consisted of 10 layers; $x, x^{\prime}, \mathrm{u}, \mathrm{u}, \mathrm{x}, \mathrm{x}, \mathrm{v}, \mathrm{v}$, $\mathrm{x}, \mathrm{x}^{\prime}$ and the cathod wire plane are placed alternatively with the each wire plane. Wire orientations of $\left(\mathrm{x}, \mathrm{x}^{\prime}\right)$ planes are perpendicular to the scattering plane, and tilted by 30 degree $\left(\mathrm{u}, \mathrm{u}^{\prime}\right)$ and -30 degree $\left(\mathrm{v}, \mathrm{v}^{\prime}\right)$ with respect to the $\mathrm{x}$ wires,respectively. Each pair of wire plane is an offset of half cell size $(0.5 \mathrm{~cm})$ from each other in order to increase the capability to resolve left-right ambiguity. Each wire plane has an effective area of $100 \times$ $12 \mathrm{~cm}^{2}$.

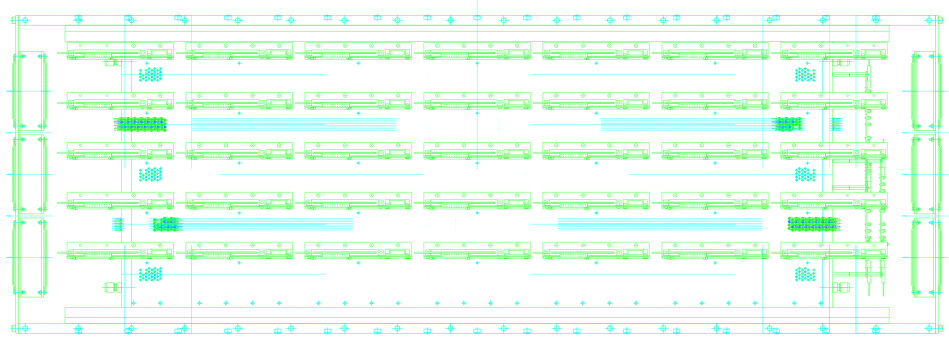

\section{Whole Weight : $160 \mathrm{~kg}$}
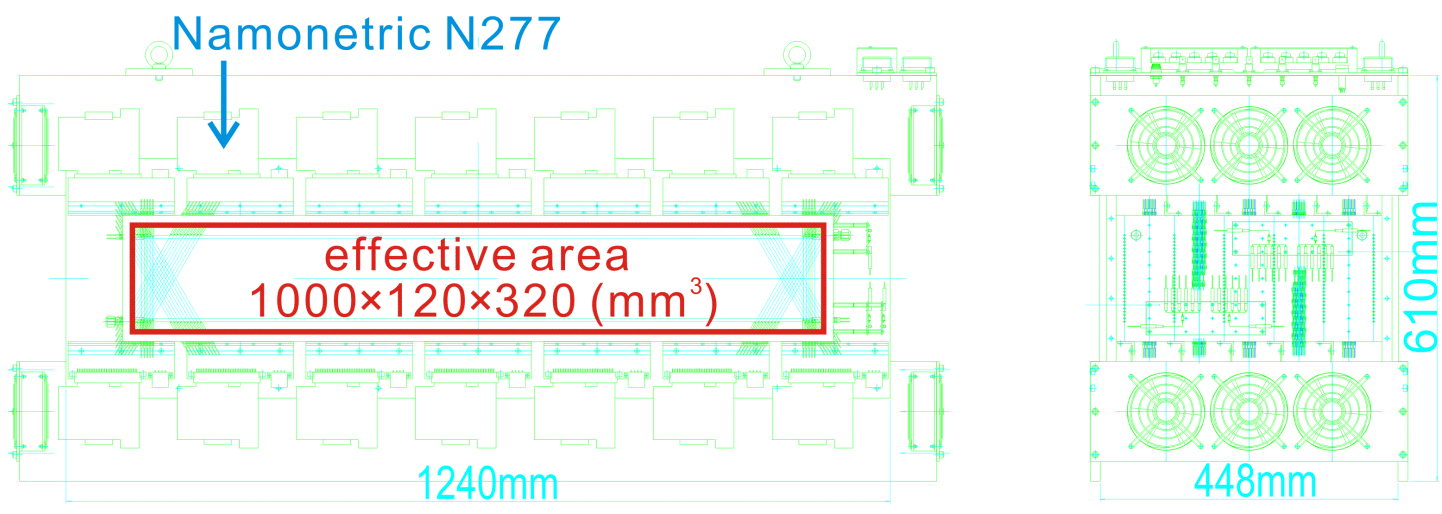

Figure 3.56: Mechanical drawing of EDC1.

The coordinate system and detail configuration of EDC1 is shown in figure 3.58. The EDC1 are filled with $\mathrm{Ar}-\mathrm{C}_{2} \mathrm{H}_{6} 50 / 50$ gas. The sense wires are gold plated tungsten with the diameter of $20 \mu \mathrm{m}$ and the cathode wires are gold plated aluminum with the diameter of $80 \mu \mathrm{m}$. The sense wires and the cathode wires are placed honeycomb-like as shown in 3.58. The sense wires and potential wires are connected to the ground and the cathode wires are connected to negative high voltages of about $-2200 \mathrm{~V}$.

The way of signal readout is same as that of KDCs. The signals from sense sires are read and transfered to discriminated ECL signals in the Nanometric N277L amplifier- 


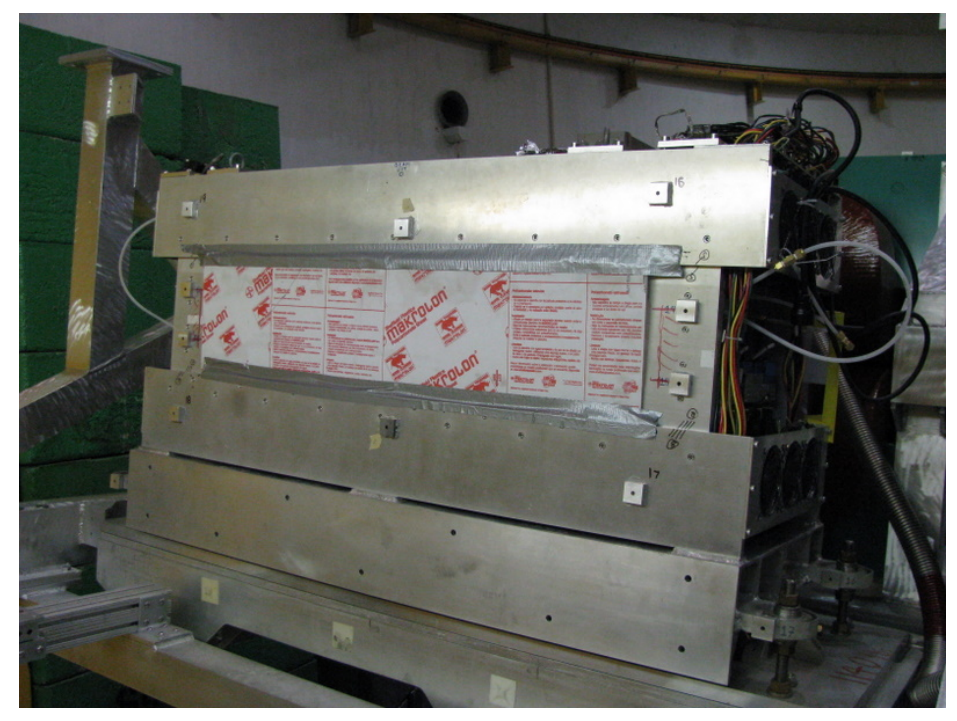

Figure 3.57: Picture of EDC1.

Table 3.8.1: EDC1 parameters

\begin{tabular}{cc}
\hline \hline Effective area & $100^{W} \mathrm{~cm} \times 12^{H} \mathrm{~cm} \times 32^{T} \mathrm{~cm}$ \\
Number of Layers & $10\left(X X^{\prime}, U U^{\prime}, X X^{\prime}, V V^{\prime}, X X^{\prime}\right)$ \\
Number of Cells & $114\left(X, X^{\prime}\right), 107\left(U, V^{\prime}\right), 106\left(U^{\prime}, V\right)$ \\
Distance between Layer & $7.5 \mathrm{~cm}$ \\
Cell Size & $0.5 \mathrm{~cm}$ \\
Angle of $U U^{\prime}, V V^{\prime}$ for $X X^{\prime}$ & $+30 \mathrm{deg},-30 \mathrm{deg}$ \\
\hline Material & Aluminized Myler $(12.5 \mu \mathrm{m})$ \\
Window & W-Au coated $(20 \mu \mathrm{m} \phi)$ \\
Anode Wire & Al-Au coated $(80 \mu \mathrm{m} \phi)$ \\
Cathode Wire & \\
Readout & Nanometric N277L \\
Amplifier Discriminator & $X X^{\prime}=672 \mathrm{ch}, U U^{\prime}=213 \mathrm{ch}, V V^{\prime}=213 \mathrm{ch}$ \\
Number of Channel & $\sigma=86 \mu \mathrm{m}$ \\
Resolution & $\sigma=210 \mu \mathrm{m}$ \\
Position Resolution(horizontal;x) & $\sigma=0.7 \mathrm{mrad}$ \\
Position Resolution(vertical;y) & $\sigma=2.8 \mathrm{mrad}$ \\
Angular Resolution(horizontal; $\left.\mathrm{x}^{\prime}\right)$ & \\
Angular Resolution(vertical; $\left.\mathrm{y}^{\prime}\right)$ & \\
\hline \hline
\end{tabular}


discriminator cards which are attached to the EDC1 frame. Then the signals are sent to FASTBUS multi-hit TDC (LeCroy 1877) located in Hall-C.

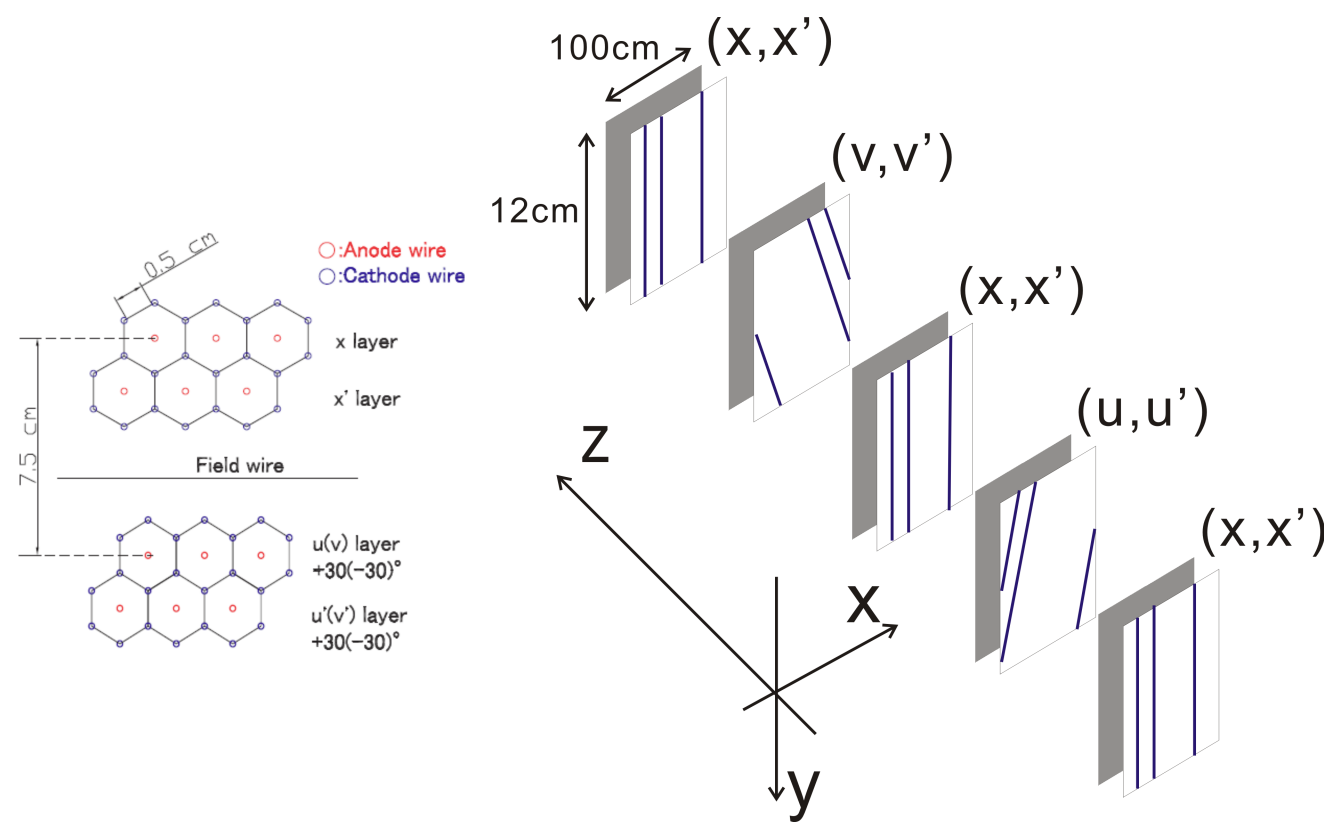

Figure 3.58: Coordinate system and wire configuration of EDC1.

\section{EDC2}

EDC2 is an identical drift chamber with KDCs. Though the tracking of HES side can be done only with EDC1, we installed EDC2 in order to get better resolution. The specification of EDC2 is summarized in table 3.6.1 and configuration is shown in figure 3.32 .

\subsubsection{TOF counters}

The HES TOF counters consist of two sets of plastic scintillation counter layers (ETOF-1 and 2), which determine the trigger timing of HES side. Each scintillation counters are made of Bicron BC408 scintillators and Hamamatsu H6612 phototubes on both ends.

The detail specification is summarized in table3.8.2 and these schematic views are figure3.59. ETOF-1 consist of horizontal 29 segments of scintillators, determining entire start timing of HES detectors. ETOF-2 also consist of horizontal 29 segments of scintillators, similar to ETOF-1. The number of segments were determined by the simulation so that $\mathrm{s}$ the singles rate of each counters was designed to be about $500 \mathrm{kHz}$ at maximum.

The ETOF layers were located at about $170 \mathrm{~cm}$ from ED, but there was about 5 gauss of magnetic field at the PMT position. Therefore, the PMTs attached at the end of each counters were surrounded by iron magnetic shield.

The timing resolutions of each segment were measured by cosmic ray before experiment. The results were about $\sigma=90$ [ps]. 


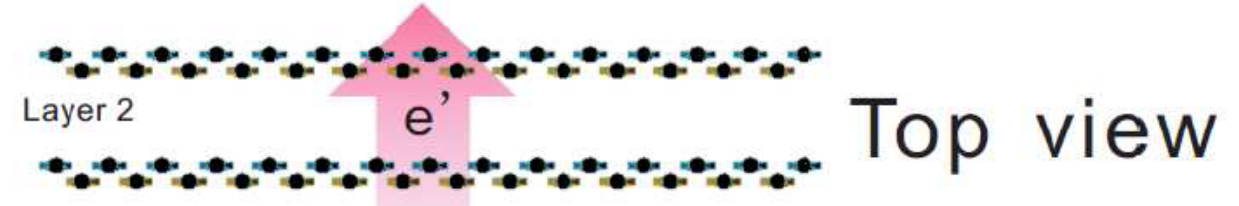

Layer 1

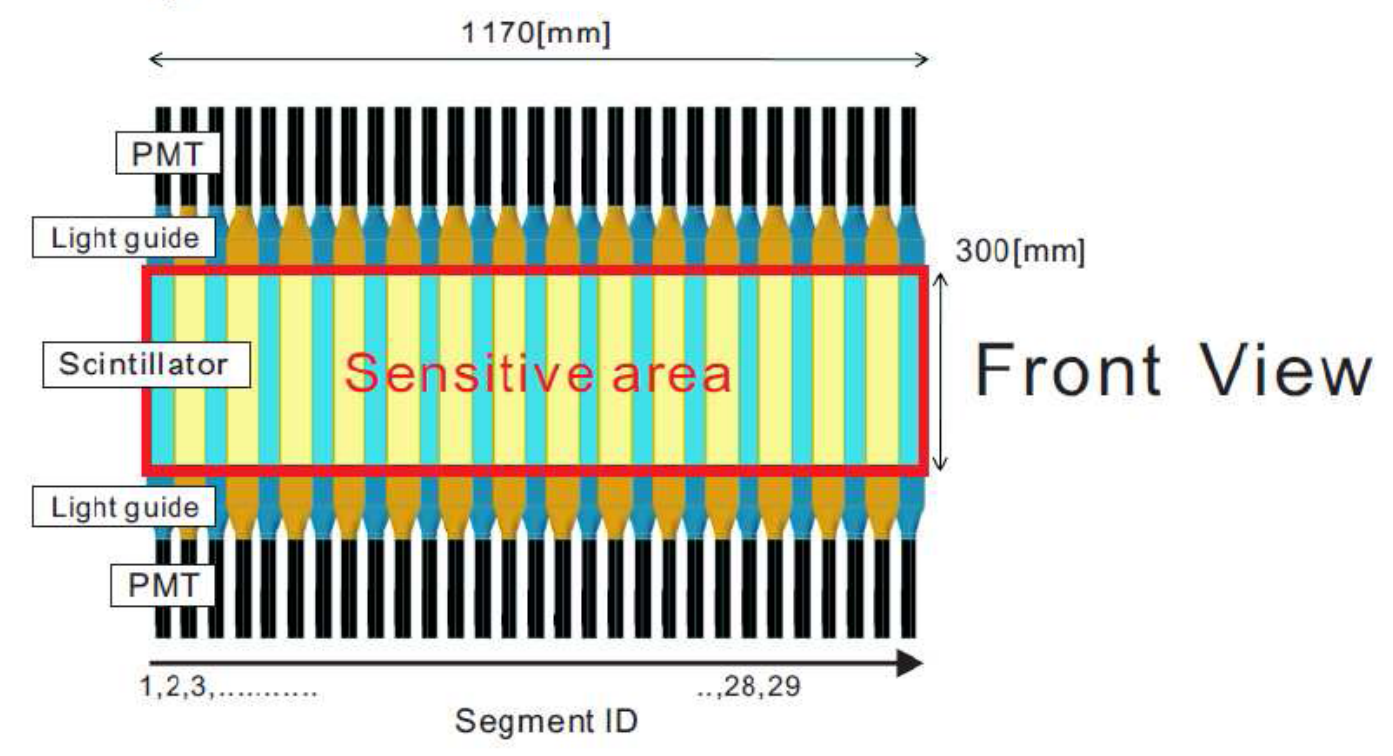

Figure 3.59: Schematic view of EHODO.

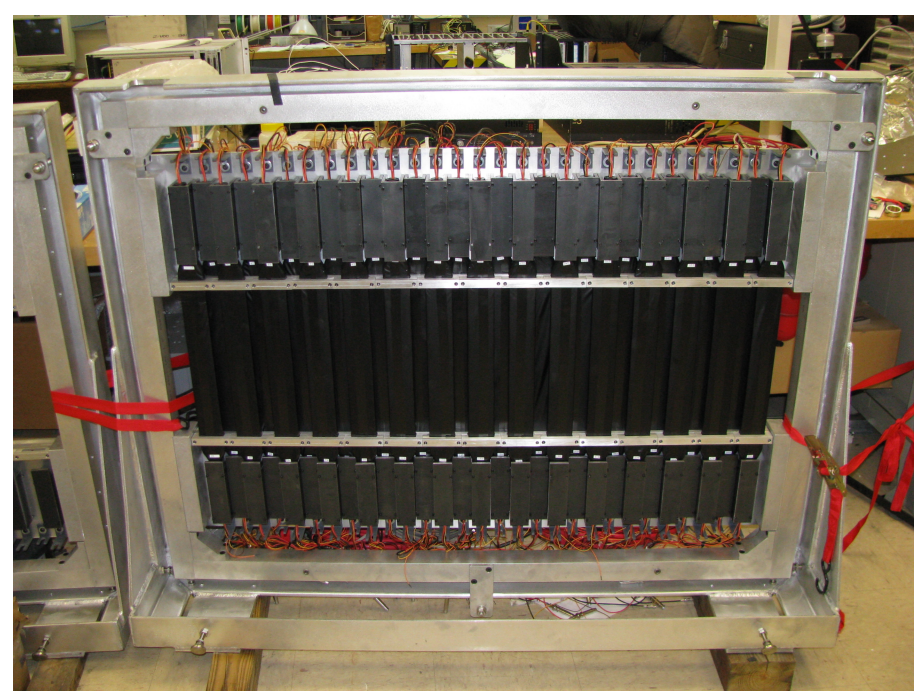

Figure 3.60: Picture of EHODO. 
Table 3.8.2: EHODO parameters

\begin{tabular}{cc}
\hline \hline Effective area & $117^{W} \mathrm{~cm} \times 30^{H} \mathrm{~cm} \times 1^{T} \mathrm{~cm}$ \\
Segments & $5^{W} \mathrm{~cm} \times 29$ segments \\
Layers & 2 \\
PMT & Hamamatsu H6612 (1 inch) \\
Scintillator & BC408 \\
\hline \hline
\end{tabular}

\subsubsection{Singles rate of HES detectors}

Table 3.8.3 is a summary of the singles rate of HES detectors during the experiment for several experimental conditions. The singles rate of EDC1 was up to $15 \mathrm{MHz}$. Since the number of wires per a plane is 114 for $\mathrm{x}$ and x' layers and 106 or 107 for other layers, the singles rate for each wires seems to be the order of a few $100 \mathrm{kHz}$, which did not affect to the detection efficiency.

For TOF counters, the singles rate of ETOF1 is also up to $15 \mathrm{MHz}$. Since the number of ETOF1X segments is 29, the singles rate of a segment is less than $1 \mathrm{MHz}$. Though this value is high, the detection efficiency was not affected.

Table 3.8.3: Summary of HES detector singles rate.

\begin{tabular}{l|cccccc}
\hline \hline Target & $\begin{array}{c}\text { Thickness } \\
{\left[\mathrm{mg} / \mathrm{cm}^{2}\right]}\end{array}$ & $\begin{array}{c}\text { Beam current } \\
{[\mu \mathrm{A}]}\end{array}$ & EDC1 & $\begin{array}{c}\text { EDC2 } \\
{[\mathrm{MHz}]}\end{array}$ & $\begin{array}{c}\text { ETOF1 } \\
{[\mathrm{ETOF} 2}\end{array}$ \\
\hline $\mathrm{CH}_{2}$ & 450.8 & 2.0 & 3.0 & 3.4 & 2.8 & 2.9 \\
$\mathrm{H}_{2} \mathrm{O}$ & 500.0 & 2.8 & 5.3 & 6.0 & 5.5 & 5.6 \\
${ }^{7} \mathrm{Li}$ & 184.0 & 35 & 9.4 & 11.1 & 9.8 & 9.7 \\
${ }^{9} \mathrm{Be}$ & 188.1 & 40 & 12.3 & 14.6 & 12.8 & 12.4 \\
${ }^{10} \mathrm{~B}$ & 56.1 & 40 & 5.0 & 5.9 & 5.1 & 5.1 \\
${ }^{n a t} \mathrm{C}$ & 112.5 & 10 & 1.9 & 2.3 & 2.1 & 2.1 \\
${ }^{n a t} \mathrm{C}$ & 112.5 & 35 & 12.4 & 14.6 & 12.9 & 12.8 \\
${ }^{51} \mathrm{Cr}$ & 134.0 & 8.0 & 6.7 & 7.8 & 7.3 & 7.3 \\
\hline \hline
\end{tabular}




\section{$3.9 \quad$ Trigger}

\subsubsection{Trigger overview}

During the experiment, we used five triggers;

1. HKS CP0 trigger

$$
(C P 0) \equiv(K T O F 1 X) \otimes(K T O F 1 Y) \otimes(K T O F 2 X)
$$

An unbiased trigger aiming to detect chaged particle coming into HKS detectors.

2. HKS single trigger

$$
(H K S) \equiv(K T O F 1 X) \otimes(K T O F 1 Y) \otimes(K T O F 2 X) \otimes(\overline{A C}) \otimes(W C)
$$

where

$$
\begin{aligned}
(A C) & \equiv(A C 1) \otimes(A C 2) \oplus(A C 2) \otimes(A C 3) \oplus(A C 3) \otimes(A C 1) \\
(W C) & \equiv(W C 1) \otimes(W C 2)
\end{aligned}
$$

The kaon trigger is made by the coincidence of the pion rejection by the $\mathrm{AC}$ veto and the proton rejection by the WC. It was divided into the six groups in order to avoid to detect background events (grouping trigger). The detail of grouping trigger will be explained in the following part.

3. HES single trigger

$$
(H E S) \equiv(E T O F 2) \otimes(E T O F 2)
$$

The HES single trigger is the 2-layer coinsidence of ETOF.

4. COIN trigger

$$
(C O I N) \equiv(H K S) \otimes(H E S)
$$

This is the main trigger of the experiment. The coincidence trigger between HKS single trigger and HES single trigger.

5. Pedestal trigger

This is a trigger generated by clock generator in order to take pedestal events. The first thousand triggers of all runs are this trigger.

These triggers are put into trigger supervisor(TS) with suitable prescale factor. The detail of TS is explained at 3.9.6 


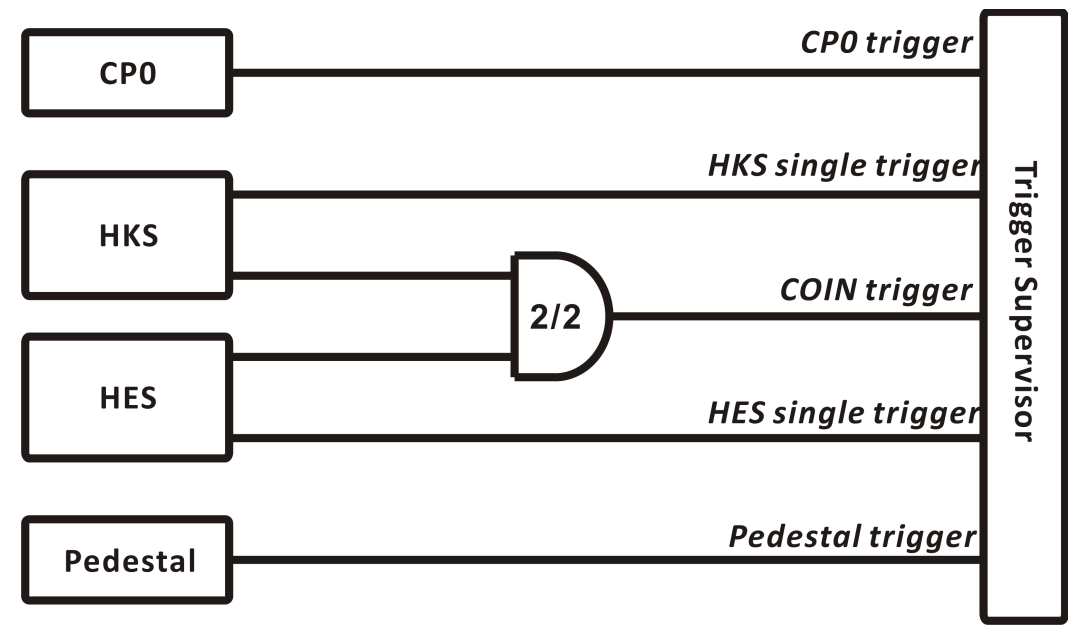

Figure 3.61: E05115 main trigger logic.

\subsubsection{Tohoku Universal Logic module (TUL-8040)}

Nuclear experiments of recent date need lots of electronic equipments due to the use of higher intensity beam. Construction of the electronics using conventional NIM (Nuclear Instruments Modules) logics requires large number of modules and much complicated cabling. We developed and introduced a programmable logic module, so-called Tohoku Universal Logic module (TUL-8040, TUL) in order to solve such kind of problem (Figure3.62). Furthermore, a propagation time in one TUL-8040 module can be suppressed within 50 ns.

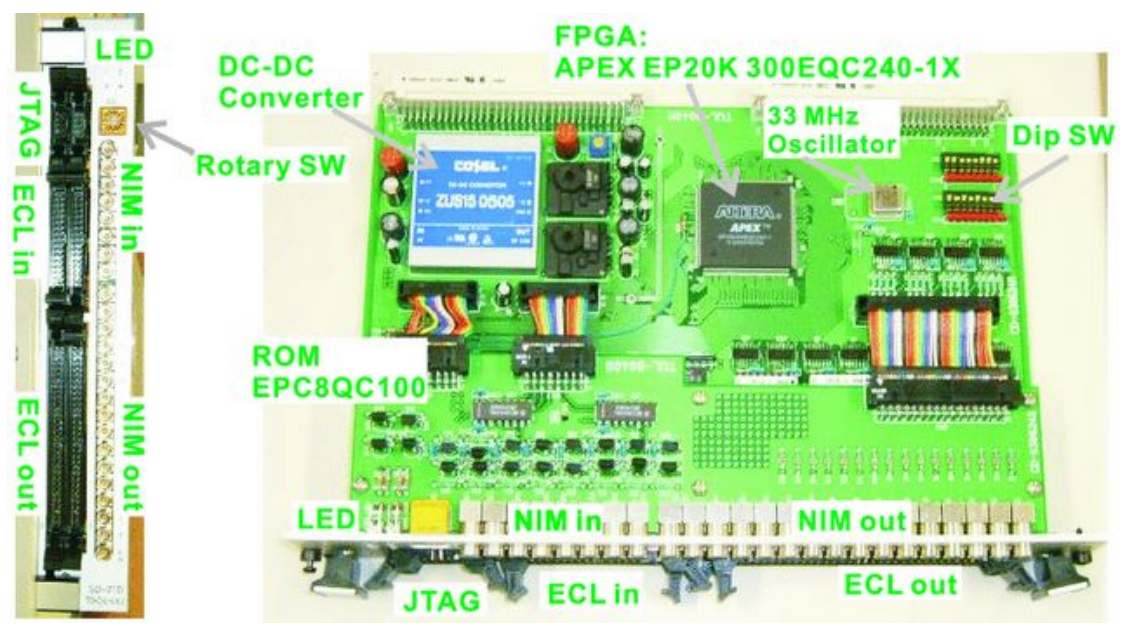

Figure 3.62: Picture of TUL-8040.

The properties of the TUL is summarized in table 3.9.2. The TUL module mounts ALTERA APEX 20K series of FPGA (Field Programable Gate Array). FPGA is an 
extensive programmable logic device, consists of vari-ous numbers of logic gates and flipflop elements on arrays of which we can modify connections. This series of FPGA have already been utilized in several nuclear experiments at practical level [65].

During the experiment, digitized signals from the detectors were converted to the ECL signals. They were transfered to the ECL input of the TUL, and the processed signals were output. The NIM output signals were used as the trigger signals and ECL output signals were input for the scalers.

The HDA programming for FPGA were done with ALTERA Quartus $\mathrm{II}^{5}$. They were downloaded with JTAG from a computer to two types of storage device, SRAM and ROM on the TUL-8040. SRAM is specified for temporarily storage for debug use because SRAM is formatted at power-off. HDL program stored in ROM is loaded to FPGA at power-on, therefore final version or stable version of the HDL program should be stored in ROM.

When the TUL processed the signal from the HKS detectors, there were two type of signals, the TOF signals and the other signals. Since the TOF signals were finally required the time resolution of $100 \mathrm{ps}$, they were not synchronized with the $33 \mathrm{MHz}$ internal clock. However, the other signals (such as the AC and WC signals) were synchronized with the internal clock in order to suppress the time jitter effect between each counter.

Table 3.9.1: Properties of TUL.

\begin{tabular}{cc}
\hline \hline & FPGA \\
\hline products & ALTERA APEX20K300EQC240-1X \\
Maximum Gate & 728,000 \\
LEs(Logic Element) & 11,520 \\
\hline \multicolumn{2}{c}{ I/O } \\
\hline Input & NIM ; 16ch, ECL ; 64ch \\
LED ; 4bit \\
Output & NIM ; 8ch, ECL ; 32ch \\
& RotarySW ; 4bit, DipSW ; 16bit \\
\hline Internal clock & $33 \mathrm{MHz}$ \\
\hline \hline
\end{tabular}

\subsubsection{HKS single trigger}

As explained, the HKS single trigger consists of

$$
(C P) \otimes(\overline{A C}) \otimes(W C)
$$

where

$$
\begin{aligned}
(C P) & \equiv(K T O F 1 X) \otimes(K T O F 1 Y) \otimes(K T O F 2 X) \\
(A C) & \equiv(A C 1) \otimes(A C 2) \oplus(A C 2) \otimes(A C 3) \oplus(A C 3) \otimes(A C 1) \\
(W C) & \equiv(W C 1) \otimes(W C 2)
\end{aligned}
$$

\footnotetext{
${ }^{5}$ A programmable logic device design software from Altera, http://www.altera.co.jp/products/software/quartusii/subscription-edition/qts-se-index.html
} 
However, there are some possibility to accept background particles that are not from HKS. In order to suppress the trigger rate due to such background particles, we introduced a "grouping trigger" as follows. Firstly the correlation of the hit segment on each detector plane was studied by the Monte Carlo simulation with the calculated magnetic field map of the HKS. The figure 3.63 (right) shows a result of the simulation for the TOF1X and TOF2X correlation. According to these studies, we divided each layer of HKS detector into 6 groups as shown in figure 3.63 (right). Finally, during the experiment, make a coincidence in each group and the logical sum of them are adopted as the HKS single trigger.
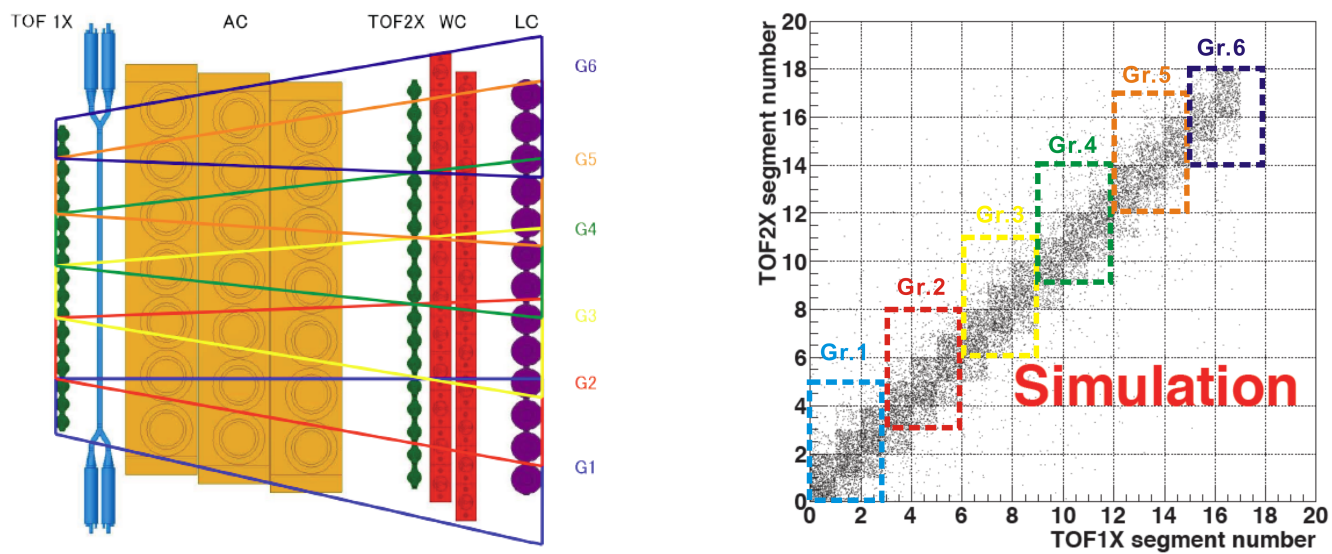

Figure 3.63: Schematics of a HKS grouping (left) and simulated hit segment correlation between KTOF1X and KTOF2X (right).

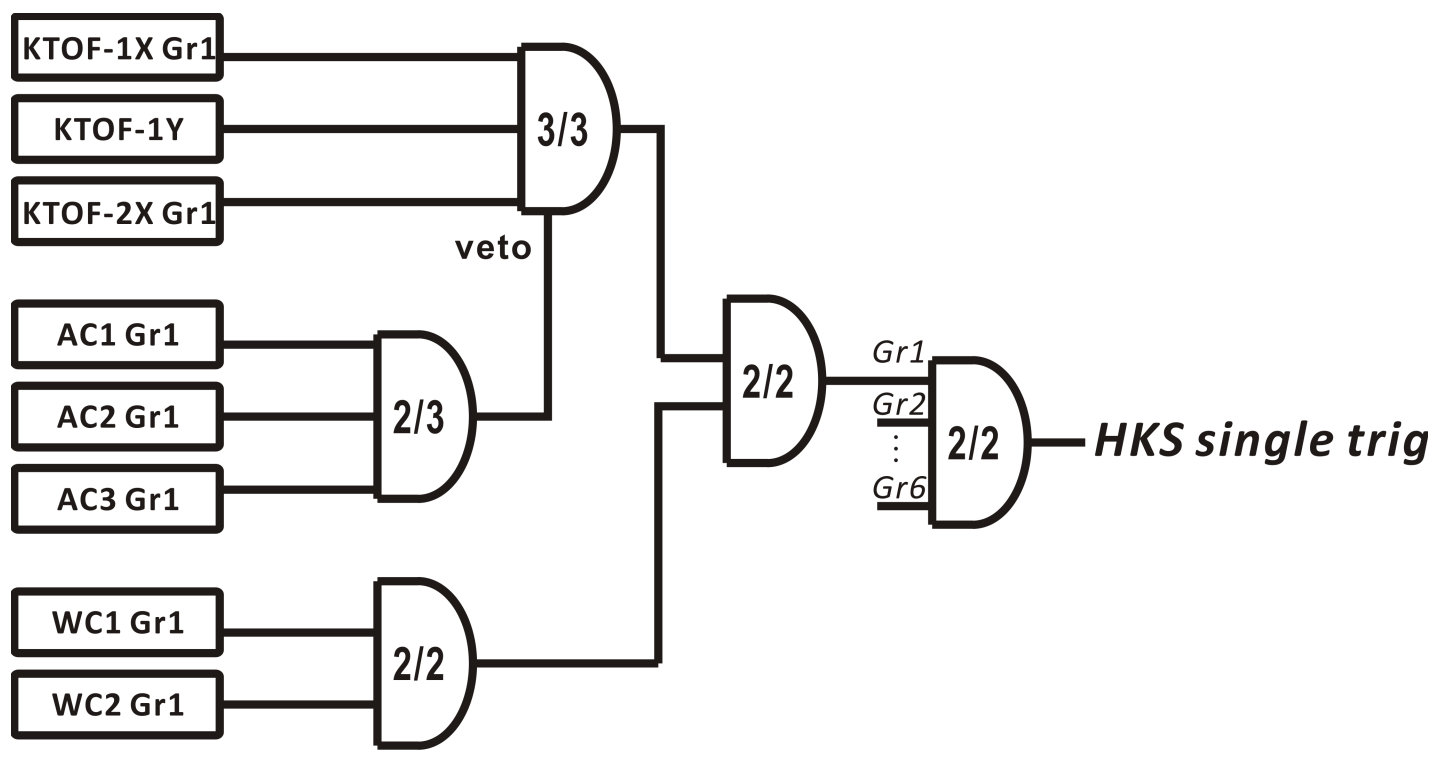

Figure 3.64: HKS trigger logic. 


\subsubsection{HES single trigger}

As already mentioned, the HES single trigger is defined as equation3.5. The grouping trigger for the HES side was also prepared before experiment, but that was not used during experiment, because the grouping was not so effective for the trigger rate suppression.

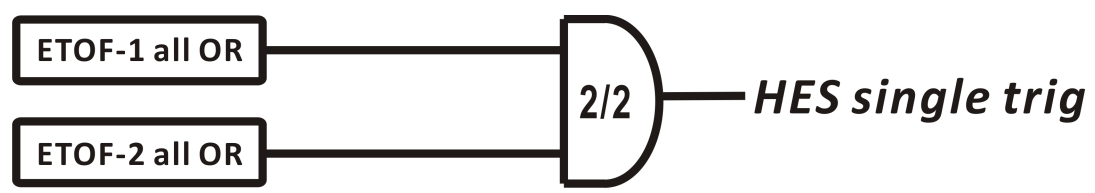

Figure 3.65: HES trigger logic.

\subsubsection{Coincidence trigger}

The coincidence trigger is defined as equation3.6 and its start timing was determined by the HKS trigger. The width of the time window was $40 \mathrm{~ns}$, considering the safety margin and accidental background level study. For the background level study, see section 5.4.

\subsubsection{Typical trigger rate and Trigger Supervisor}

Trigger Supervisor (TS) is a module which can control multiple trigger input signals. The input signals of the TS was controlled during the experiment as shown in figure3.61. Firstly, each signal except for the pedestal trigger signal was made coincidence with the "EN" signal and the "GO" signal, which are sent from the CODA (see section 3.10). The pedestal trigger was made coincidence with EN and GO. The "GO" signal means that the DAQ is ready to take any data. The "EN" signal is sent when the DAQ enable to take physics data. This signal can be programmed by the user. During the experiment, first 1000 events are taken by pedestal trigger and subsequent evets were taken as physics event (and then EN signal was sent to the TS.).

The TS enable to put multiple triggers with each prescale factor (PS) for each signal run by run. The typical trigger rate is shown in table3.9.6. During the experiment, these trigger was mixed by applying each PS so as not to over the trigger rate limit of $2 \mathrm{kHz}$. The PS of the COIN trigger is 1 because the events are what we aim to take. The main parts what we adjust during the experiment was the PS of CP0 and HKS trigger. These trigger is necessary to calculate the kaon kill ratio at the trigger level (the calculation will be described in section 5.6) and the trigger rate is very high because of the positron background. The value was up to 200 for HKS and 12000 for CP0. The PS of HES trigger was very large because the trigger is not used for the analysis. 


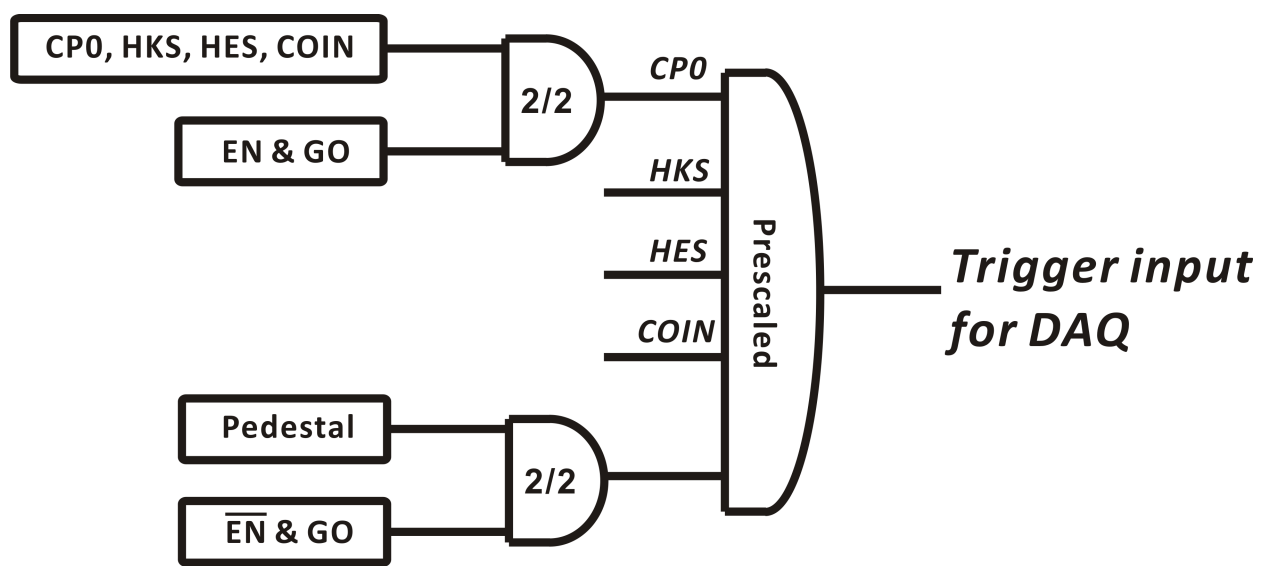

Figure 3.66: Logic around TS.

Table 3.9.2: Typical trigger rate for some conditions

\begin{tabular}{l|cccccc}
\hline \hline target & $\begin{array}{c}\text { thickness } \\
{\left[\mathrm{mg} / \mathrm{cm}^{2}\right]}\end{array}$ & $\begin{array}{c}\text { current } \\
{[\mu \mathrm{A}]}\end{array}$ & $\begin{array}{c}\text { CP0 } \\
{[\mathrm{kHz}]}\end{array}$ & $\begin{array}{c}\text { HKS single } \\
{[\mathrm{kHz}]}\end{array}$ & $\begin{array}{c}\text { HES single } \\
{[\mathrm{MHz}]}\end{array}$ & $\begin{array}{c}\text { COIN } \\
{[\mathrm{kHz}]}\end{array}$ \\
\hline $\mathrm{CH}_{2}$ & 450.8 & 2.0 & 220 & 1.8 & 1.2 & 0.10 \\
$\mathrm{H}_{2} \mathrm{O}$ & 500.0 & 2.8 & 1100 & 20 & 1.5 & 1.5 \\
${ }^{7} \mathrm{Li}$ & 184.0 & 35 & 540 & 7.3 & 2.2 & 0.96 \\
${ }^{9} \mathrm{Be}$ & 188.1 & 40 & 710 & 1.0 & 2.5 & 1.5 \\
${ }^{10} \mathrm{~B}$ & 56.1 & 40 & 190 & 2.0 & 1.6 & 0.17 \\
${ }^{n a t} \mathrm{C}$ & 112.5 & 20 & 160 & 1.3 & 1.5 & 0.97 \\
${ }^{n a t} \mathrm{C}$ & 112.5 & 35 & 630 & 7.9 & 2.3 & 1.3 \\
${ }^{51} \mathrm{Cr}$ & 134.0 & 8.0 & 980 & 11 & 2.5 & 1.8 \\
\hline \hline
\end{tabular}




\subsection{Data acquisition system}

\subsubsection{CODA}

Electronic signals from each counters are digitized and converted to physics data by some ADCs and TDCs and these data is written in HDD by the CEBAF On-line Data Aquisition system (CODA). The data flow of the CODA is as shown in figure3.67.

We used some FASTBUS ADCs (LeCroy 1881M) and VME TDCs (JLab F1TDC) for the PMT signals and FASTBUS TDCs (LeCroy 1877) for the signals of drift chambers (KDCs and EDCs).

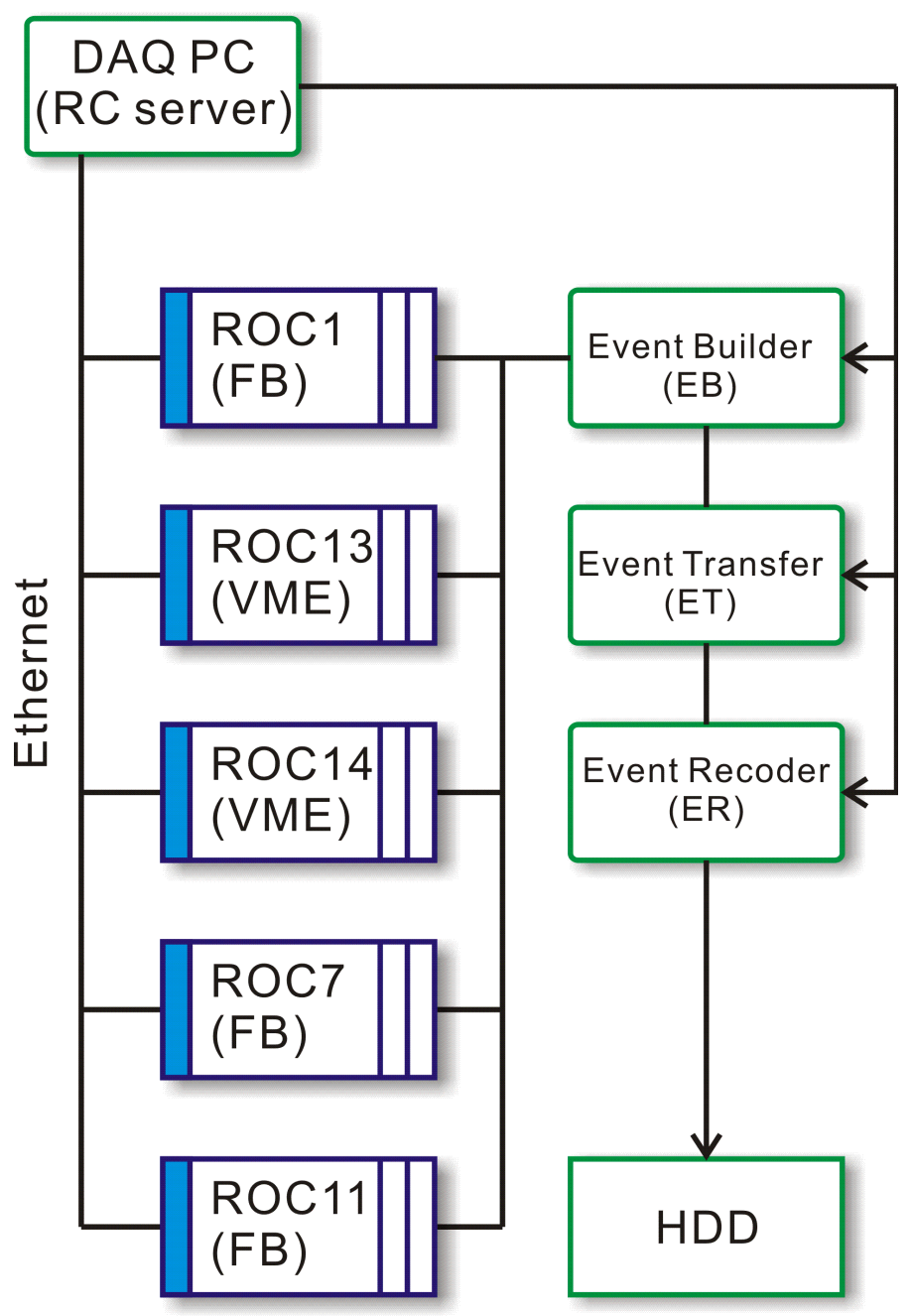

Figure 3.67: CODA flow chart. 


\subsubsection{EPICS}

EPICS (Experimental Physics and Industrial Control System) is a slow readout control system [66] developed by LANL, ANL, CEBAF, LBL and BNL. In the present experiment, EPICS was used to read experimental information as follows.

- Magnet information such as readout of hall probe or NMR. These information can be used to evaluate the reliability of the experimental data. For example, if the magnet field value indicate an unusual value, such data can be excluded.

- Beam information such as BPM, BCM, beam momentum, FFB status. In particular, $\mathrm{BCM}$ information is important for the calculation of the cross section and beam momentum information is essential for the missing mass calculation.

- Sieve Slit (or Collimator) position. This information is also important for the cross section calculation.

The EPICS information was read every 2 second.

\subsection{Data summary}

Experimental data were taken for totally seven targets and ten run conditions as listed in table3.11. The purposes of each targets are described in section 3.3.

Table 3.11.1: Run Summary.

\begin{tabular}{|c|c|c|c|c|c|c|}
\hline \multirow[t]{2}{*}{ Data set } & \multirow[t]{2}{*}{ Target } & \multirow{2}{*}{$\begin{array}{l}\text { Thickness } \\
{\left[\mathrm{mg} / \mathrm{cm}^{2}\right]}\end{array}$} & \multicolumn{2}{|c|}{ Beam current } & \multirow{2}{*}{$\begin{array}{c}\text { Total } \\
\text { charge } \\
{[\mathrm{C}]}\end{array}$} & \multirow{2}{*}{$\begin{array}{c}\text { Run } \\
\text { time } \\
{[\text { hours }}\end{array}$} \\
\hline & & & $\begin{array}{c}\text { Nominal } \\
{[\mu \mathrm{A}]}\end{array}$ & $\begin{array}{c}\text { Average } \\
{[\mu \mathrm{A}]}\end{array}$ & & \\
\hline 1 & $\mathrm{CH}_{2}$ & 450.8 & 2 & 1.990 & 0.281 & 39.24 \\
\hline 2 & $\mathrm{H}_{2} \mathrm{O}$ & 500.0 & 2.5 & 2.681 & 0.204 & 21.13 \\
\hline 3 & ${ }^{7} \mathrm{Li}$ & 184.0 & 35 & 32.01 & 4.839 & 41.98 \\
\hline 4 & ${ }^{9} \mathrm{Be}$ & 188.1 & 40 & 38.28 & 5.332 & 38.69 \\
\hline 5 & ${ }^{10} \mathrm{~B}$ & 56.1 & 40 & 38.71 & 6.253 & 44.88 \\
\hline 6 & ${ }^{n a t} \mathrm{C}$ & 112.5 & 10 & 8.07 & 0.142 & 4.9 \\
\hline 7 & & & 20 & 19.41 & 1.076 & 15.4 \\
\hline 8 & & & 35 & 32.90 & 4.659 & 39.3 \\
\hline 9 & ${ }^{52} \mathrm{Cr}$ & 134.0 & 7.5 & 7.66 & 0.825 & 29.9 \\
\hline 10 & & 154.0 & 7.5 & 7.61 & 5.525 & 201.4 \\
\hline
\end{tabular}




\section{Chapter 4}

\section{Design of the HES}

As already mentioned in chapter3, we newly designed and constructed the SPL and the HES magnets dedicated to the hypernuclear spectroscopy via the $\left(\mathrm{e}, \mathrm{e}^{\prime} \mathrm{K}^{+}\right)$reaction. In this section, the detail of the magnet design is explained, especially its optics design and calculated 3D magnetic field. Firstly in section 4.1, the overview of the present experimental design is shown related to the HES design. Next in section 4.2 and 4.3, detail of the SPL and the HES magnets are explained particularly referring their 3D magnetic field calculation. Section 4.4 represents the principle of "tilt method" and its optimization. Finally in section4.5, the results of yield and resolution estimation of the present experiment are shown.

\subsection{Experimental design of E05-115 experiment}

\subsubsection{Kinematics and optics}

The kinematics of the $\left(\mathrm{e}, \mathrm{e}^{\prime} \mathrm{K}^{+}\right)$hypernuclear production can be simply described as figure 4.1. $P_{e}=\left(E_{e}, \overrightarrow{p_{e}}\right), P_{e^{\prime}}=\left(E_{e^{\prime}}, \overrightarrow{p_{e^{\prime}}}\right), P_{K}=\left(E_{K}, \overrightarrow{p_{K}}\right), P_{N}=\left(E_{N}, \overrightarrow{p_{N}}\right), P_{Y}=\left(E_{Y}, \overrightarrow{p_{Y}}\right)$ in this figure is the four momenta of the electron beam, scattered electron, kaon, proton and hypernuclei. Table4.1.1 is a summary of E05-115 optics and kinematic parameters. These parameters were determined by the properties of elementary process of the $\left(\mathrm{e}, \mathrm{e}^{\prime} \mathrm{K}^{+}\right)$ reaction, as discussed in chapter 2 .

Table 4.1.1: E05-115 optics and kinematic parameters in Lab frame.

\begin{tabular}{ccc}
\hline \hline parameter & $\mathrm{K}^{+}$ & $\mathrm{e}^{\prime}$ \\
\hline Central Momentum $[\mathrm{GeV} / c]$ & 1.20 & 0.844 \\
Momentum Acceptance $[\mathrm{GeV} / c]$ & \pm 0.15 & \pm 0.15 \\
Angular Acceptance $\left(x^{\prime}\right)[\mathrm{mrad}]$ & $-200 \leq x^{\prime} \leq-40$ & $-150 \leq x^{\prime} \leq 150$ \\
Angular Acceptance $\left(y^{\prime}\right)[\mathrm{mrad}]$ & $50 \leq y^{\prime} \leq 50$ & $20 \leq y^{\prime} \leq 100$ \\
$\left\langle x_{f} \mid p\right\rangle[\mathrm{cm} /(\mathrm{MeV} / c)]$ & $\sim 0.25$ & $\sim 0.35$ \\
\hline$Q^{2}$ of virtual photon & $0.01\left[\left(\mathrm{GeV} / c^{2}\right)^{2}\right]$ \\
\hline
\end{tabular}

The opctics parameters can be defined by first order transfer matrix as below; 


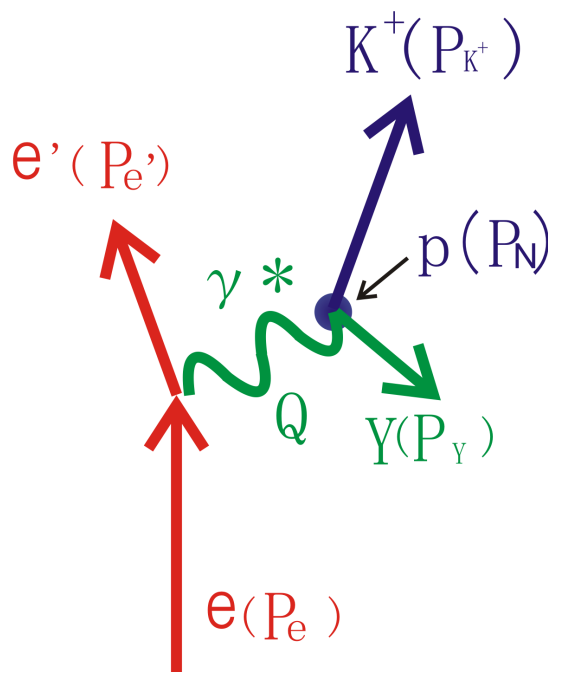

Figure 4.1: Kinematics of the $\left(\mathrm{e}, \mathrm{e}^{\prime} \mathrm{K}^{+}\right)$hypernuclear production reaction.

$$
\left(\begin{array}{c}
x_{f} \\
x_{f}^{\prime} \\
y_{f} \\
y_{f}^{\prime} \\
p
\end{array}\right)=\left(\begin{array}{ccccc}
\cdot & \left\langle x_{f} \mid x_{t}^{\prime}\right\rangle & \cdot & \cdot & \left\langle x_{f} \mid p\right\rangle \\
\cdot & \cdot & \cdot & \cdot & \\
\cdot & \cdot & \cdot & \left\langle y_{f} \mid y_{t}^{\prime}\right\rangle & \\
\cdot & \cdot & \cdot & \cdot & \\
\cdot & \cdot & \cdot & \cdot & 1
\end{array}\right) \cdot\left(\begin{array}{c}
x_{t} \\
x_{t}^{\prime} \\
y_{t} \\
y_{t}^{\prime} \\
p
\end{array}\right)
$$

In the above equation, index "f" means the value at reference plane and "t" means the value at target. The definition of HKS and HES reference planes (FP) are shown in figure 4.2 and 4.3, respectively. The HKS FP is defined as the mid-line between the KDC1 and the KDC2 and the HES FP is the center line of the EDC1. These FP is used as the origin of $z$-axis of each detector packages. $\left\langle x_{f} \mid p\right\rangle$ corresponds to a momentum dispersion of the spectrometer and $\left\langle x_{f} \mid x_{t}^{\prime}\right\rangle$ is an angle dispersion which expresses how $x$-position at the reference plane is depending on the $x^{\prime}$ at target, and the same things is applied to $y$.

As already explained in section 2.4, the SPL is necessary to accept kaons and scattered electrons generated to the very forward angle. Such particles are separated and lead to the spectrometers by the SPL magnetic field. Furthermore, the SPL magnetic field also has to transport post-beam to the Hall-C beam dump. The detail of this characteristic SPL magnet is explained in section 4.2.

\subsubsection{The estimation of momentum resolution by first order}

It is important to estimate the momentum resolution by the first order for the determination of the experimental design. Our hypernuclear spectrum is obtained by the missing mass which can be calculated as

$$
\begin{aligned}
M_{H Y} & =\sqrt{E_{H Y}^{2}-\vec{p}_{H Y}^{2}} \\
& =\sqrt{\left(E_{e}+E_{N}-E_{K}-E_{e^{\prime}}\right)^{2}-\left(\overrightarrow{p_{e}}-\overrightarrow{p_{K}}-\overrightarrow{p_{e^{\prime}}}\right)^{2}} .
\end{aligned}
$$




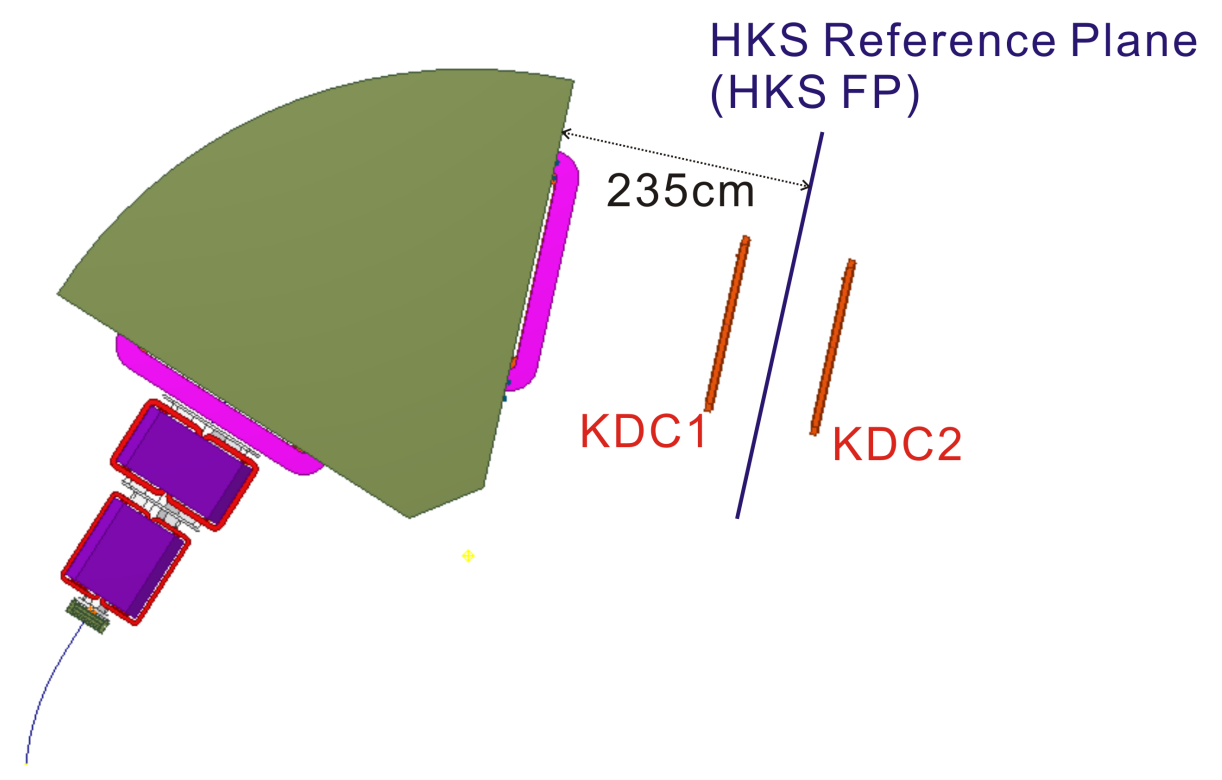

Figure 4.2: Definition of HKS reference plane.

\section{HES Reference Plane (HES FP)}

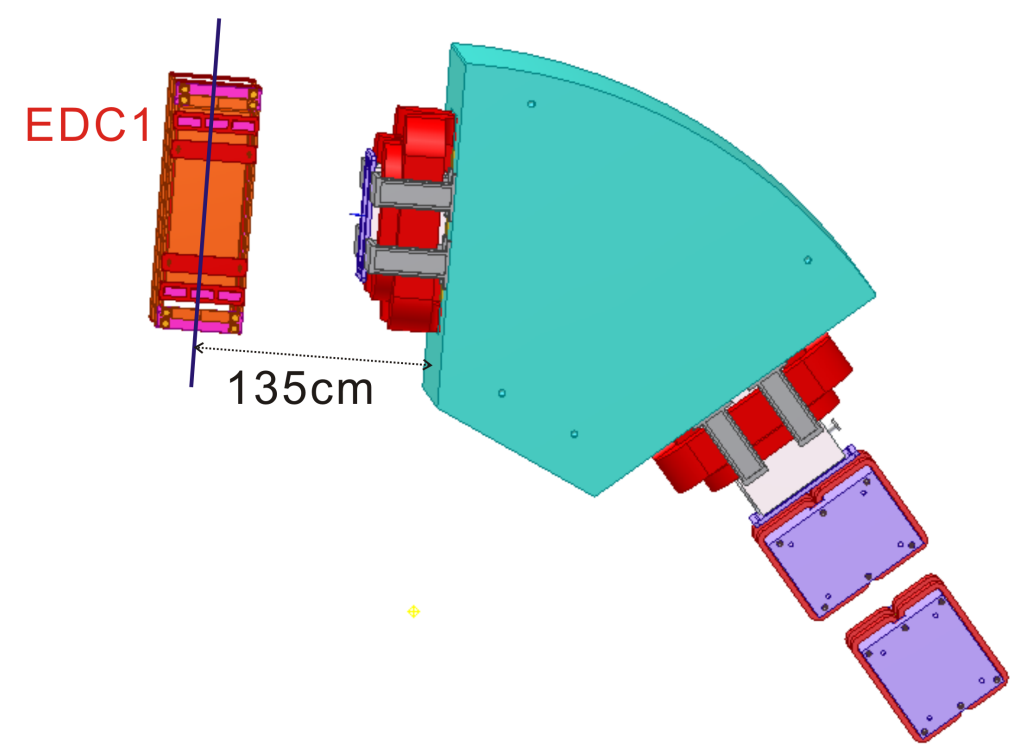

Figure 4.3: Definition of HES reference plane. 
The missing mass resolution is estimated by the low of error propagation. Since the magnification of $\vec{p}_{H Y}$ is much smaller than $E_{H Y}$, the $\vec{p}_{H Y}$ term can be neglected by the first order. Therefore, the error of missing mass can be calculated as

$$
\delta M_{H Y} \sim \sqrt{\delta E_{e}^{2}+\delta E_{K}^{2}+\delta E_{e^{\prime}}^{2}} .
$$

In the above equation, $\delta E_{e}$ is determined by the beamline and the value is required as $d E_{e} / E \sim d p_{e} / p=3 \times 10^{-5}(\mathrm{FWHM})$. Then

$$
\delta E_{e}=70[\mathrm{keV}]
$$

The values $\delta E_{K}$ and $\delta E_{e^{\prime}}$ are defined by the momentum resolution of the HKS and the HES, respectively.

The momentum resolution can be calculated using momentum dispersion by the first order, because $x_{f}$ can be associated with momentum by the following relation;

$$
\begin{aligned}
x_{f} & \sim\left\langle x_{f} \mid p\right\rangle p \\
d p & \sim\left(\left\langle x_{f} \mid p\right\rangle\right)^{-1} d x_{f}
\end{aligned}
$$

$d x_{f}$ is the position resolution at the reference plane which is determined by the drift chambers of each spectrometers, the value is $\sigma=80 \mu \mathrm{m}$ for the HES and $\sigma=60 \mu \mathrm{m}$ for the HKS. Then the momentum resolution of the HKS and the HES are

$$
\begin{aligned}
& d p_{H K S}=23[\mathrm{keV} / c](\mathrm{rms})=54[\mathrm{keV} / c](\mathrm{FWHM}) \\
& d p_{H E S}=24[\mathrm{keV} / c](\mathrm{rms})=56[\mathrm{keV} / c](\mathrm{FWHM}) .
\end{aligned}
$$

The energy resolution $d E$ can be derived as following relation

$$
d E=\frac{p}{E} d p
$$

and the energy resolution of the HKS is

$$
d E_{H K S}=50[\mathrm{keV}],
$$

while $d E_{H E S} \sim d p_{H E S}$ in the HES side. Therefore, the missing mass resolution can be derived as

$$
d M_{H Y} \sim 100[\mathrm{keV}](\mathrm{FWHM}) .
$$

Of course, we need to consider the influence of the multiple scattering or the energy struggling effect in the experimental target or the detector package for the realistic estimation. Further estimation will be performed in section 4.5 .

\subsection{SPL Magnet}

The SPL magnet is a characteristic equipment in the setup of this experiment. This section will explain the role of the SPL magnet and the design of the magnetic field. 


\subsubsection{The role and design of SPL magnet}

The SPL is a dipole magnet to deflect charged particles. The advantages to use the SPL is that the hypernuclear yield become higher because the forwarder angles can be accepted (see section 2.1). However, at the same time, the post-beam and the scattered electron also need to be separated by the same magnetic field.

Figure 4.4 shows the vacuum chamber of SPL magnet, which has four exits, namely, the HKS exit, the HES exit, the beam-dump exit and the photon-dump exit. The SPL is required to bring the particles to these four exit by a dipole magnetic field, and this requirement determine the shape of the vacuum chamber. Figure 4.5 is the particle distribution on the $e^{\prime}$ exit of the SPL obtained by the geant 4 Monte Carlo simulation and this result determine the alignment of three exits on this plane.

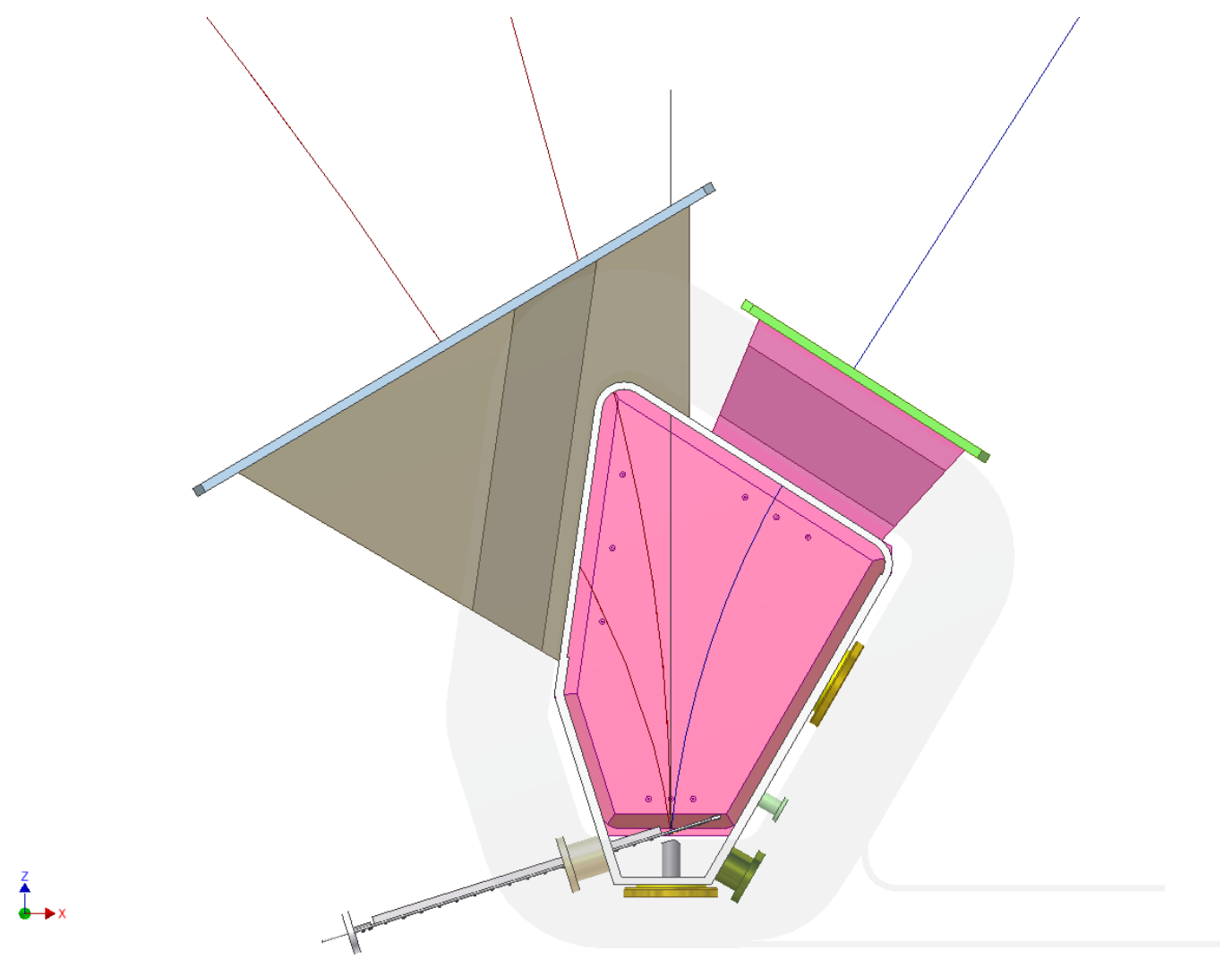

Figure 4.4: Vacuum chamber and pole shape of SPL magnet.

The tracks of each particles are as follows. Firstly, incident beam coming from the CEBAF is lead to the target position through fringe field of SPL. The beamline before target is surrounded by iron shield in order to minimize the effect of SPL fringe field. After the target, positive charged particles go to the HKS side, negative charged particles including post-beam go to the HES side and bremsstrahlung photons go to the photon dump. Especially, negative charged particles going to the HES and beam-dump have to be separated only by its momentum. The construction of the HES and the post-beamline 


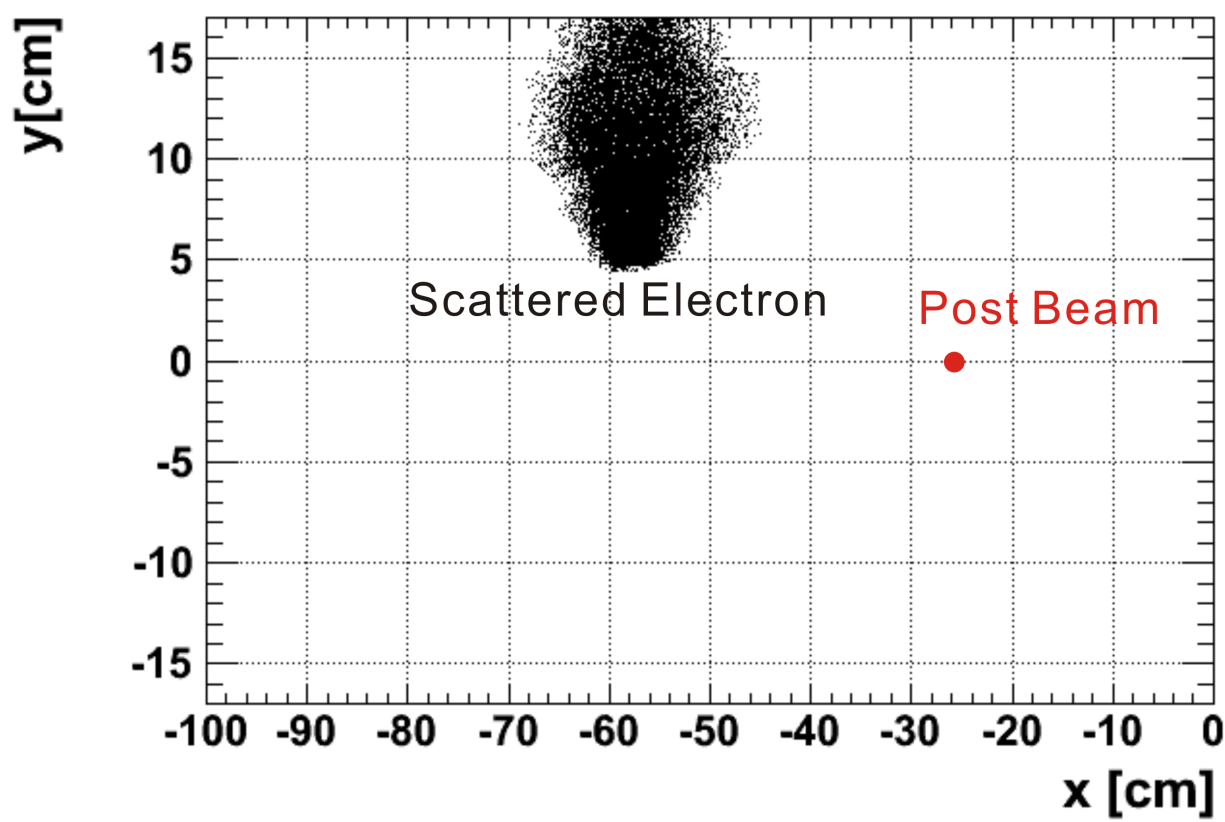

Figure 4.5: Particle coming to the $e^{\prime}$ exit of the SPL.

Table 4.2.1: Momentum and exit angle of SPL magnet.

\begin{tabular}{cccc}
\hline \hline & Particles & Momentum $[\mathrm{GeV} / c]$ & Angle [degree] \\
\hline SPL entrance & $\mathrm{e}^{-}$ & 2.344 & 0 \\
HKS exit & $\mathrm{e}^{+}, \pi^{+}, \mathrm{K}^{+}, \mathrm{p}$ & 1.2 & 32.57 \\
Photon dump exit & $\gamma$ & - & 0 \\
Beam dump exit & $\mathrm{e}^{-}$ & 2.344 & 14.7 \\
HES exit & $\mathrm{e}^{-}$ & 0.844 & 35.45 \\
\hline \hline
\end{tabular}


is based on the simulation using calculated magnetic field, therefore the magnetic field calculation is significantly important. The detail of the calculation will be explained in the part.

\subsubsection{SPL magnetic field calculation}

\section{SPL TOSCA model}

TOSCA is a software for the calculation of $3 \mathrm{D}$ magnetic fields using the finite element method. The procedure of TOSCA calculation is as follows;

1. Input 3D model of the magnet.

2. Set mesh sizes of the finite elements and start meshing.

3. Set boundary conditions and material properties.

4. Start calculation until the iteration of the finite element method is finished.

Figure4.6 shows the 3D model of SPL magnet for the TOSCA calculation. Table4.2.2 is a summary of the maximum mesh sizes of important elements in the model. The number of mesh is limited by the software, then fine mesh is used for important element such as the gap between pole face or pole piece and rough meshes were used for insignificant elements such as the background. Figure 4.7 is the BH curve used in the SPL model. The $\mathrm{BH}$ curve is based on the measured one and adjusted so as to reproduce the measured B-I curve.

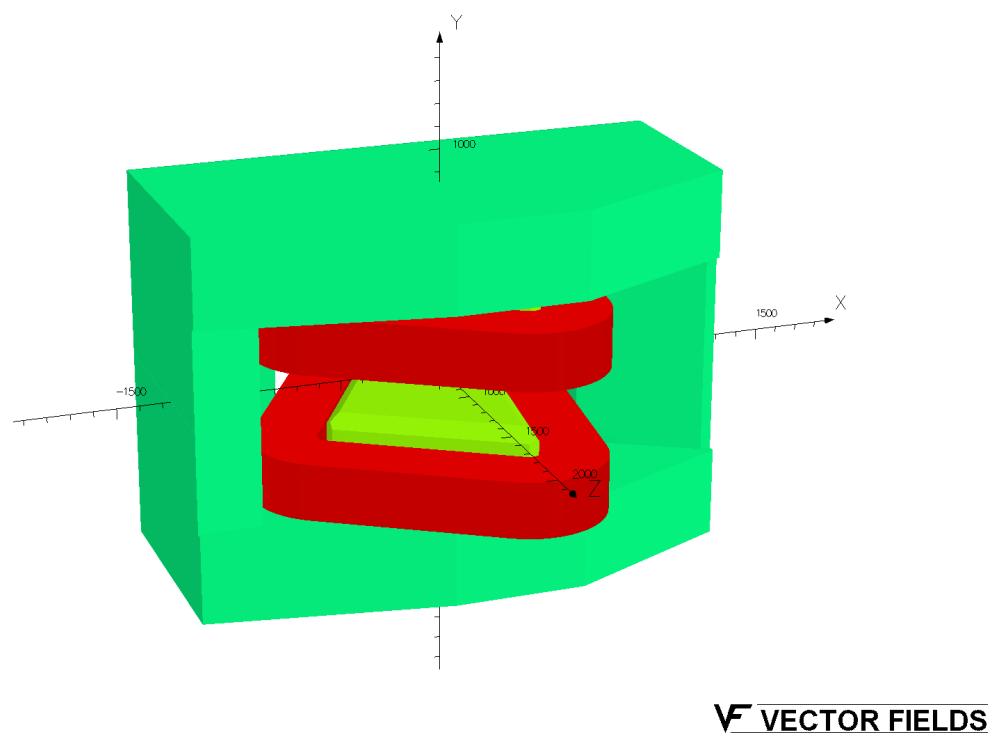

Figure 4.6: SPL 3D model in TOSCA calculation. 
Table 4.2.2: Material and mesh size in TOSCA 3D model.

\begin{tabular}{ccc}
\hline \hline Name & Material & Mesh size $[\mathrm{cm}]$ \\
\hline Yoke & Iron (measured BH) & 10 \\
Pole & Iron (measured BH) & 5 \\
Gap & Air & 2 \\
\hline \hline
\end{tabular}

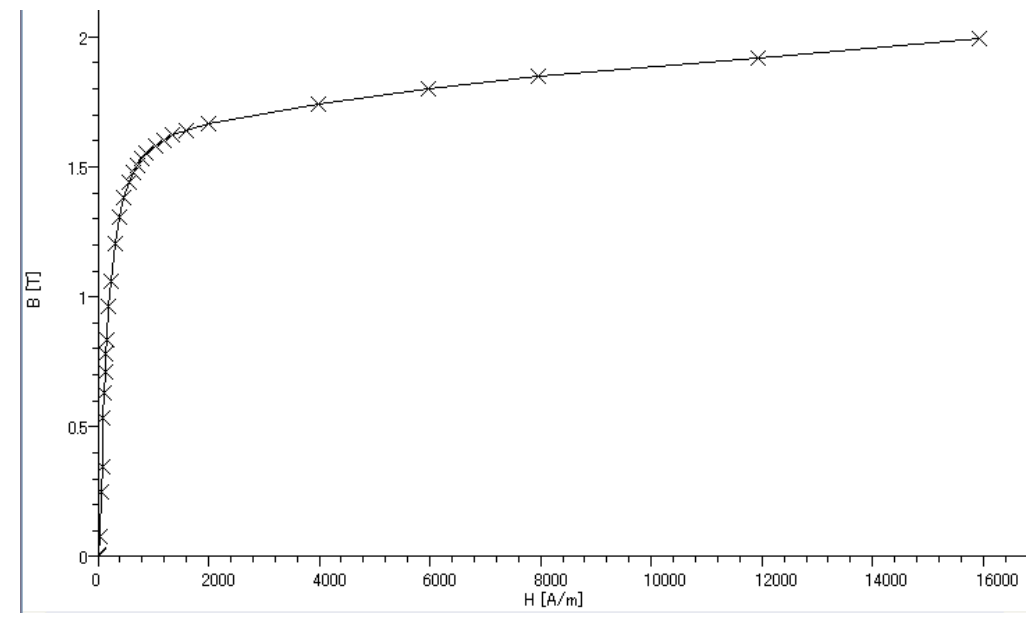

Figure 4.7: BH curve used in SPL model. 


\section{Measurement of SPL magnetic field}

The result of the calculation was compared to the field measured at TOKIN company in 2008. The measured field map cannot be used for the simulation because we did not measure all area of the magnet. However, we can check the result of the TOSCA calculation by the comparison of both map. Figure 4.8 shows the excitation curve by the measurement and the TOSCA calculation. We can see the result of our TOSCA calculation is agree with the measured value by the accuracy of less than $2 \%$.

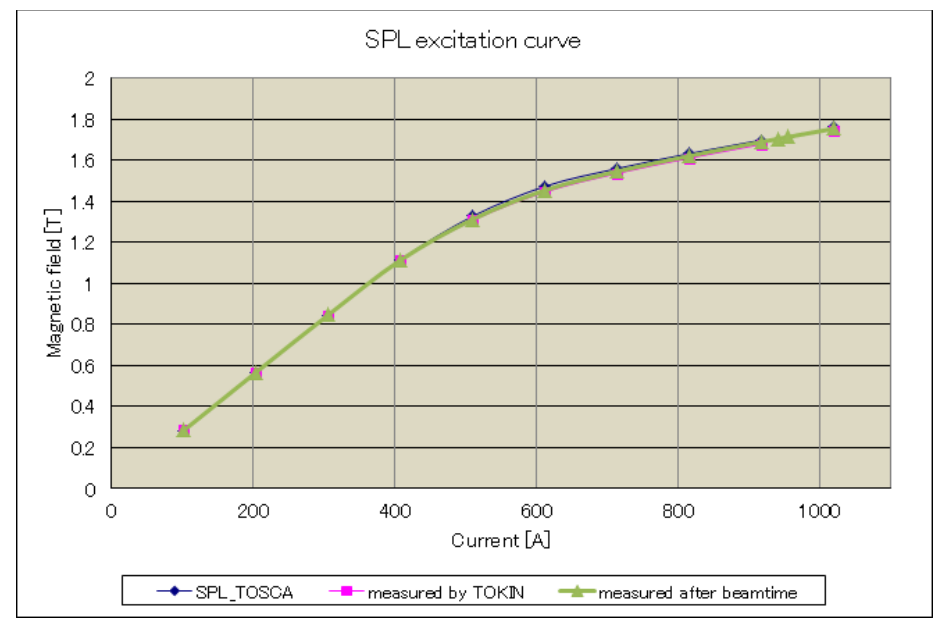

Figure 4.8: Excitation curve of SPL magnet. The measured value and TOSCA calculation result are superimposed. The measured value was taken before and after the beamtime.

\section{Evaluation of the SPL magnetic field}

As already mentioned, the SPL magnet is a part of both the HKS and the HES. However, it has an unique shape because of its role to separate the four particles. In particular, its pole shape has sharp edge around the exit of the post beam, as shown in figure 4.4. Such a sharp edge of an electromagnet is strongly magnetized and the generated magnetic field is significantly affected by the magnetization characteristic of the iron. Figure 4.9 shows the magnetization of the SPL pole piece calculated by TOSCA. The edge part of the pole piece is highly magnetized, more than 3 Tesla. In such a region, it is important to know the accurate saturation curve of the iron, but the B-H curve used in the SPL model (figure 4.7) seems to have inaccuracies compared to the actual one.

It is our basic idea to calibrate such a difference with the actual particle which is transported by the magnetic field. However, we designed the SPL shape so as to decrease the edge effect on the particle transportation, because it is better to obtain the magnetic field as accurate as possible. The inaccuracy of the can be estimated as a few \%, which is not small for our analysis which requires the accuracy of $10^{-3}$ level. It is important to take care about this effect, especially in the momentum reconstruction analysis phase. 

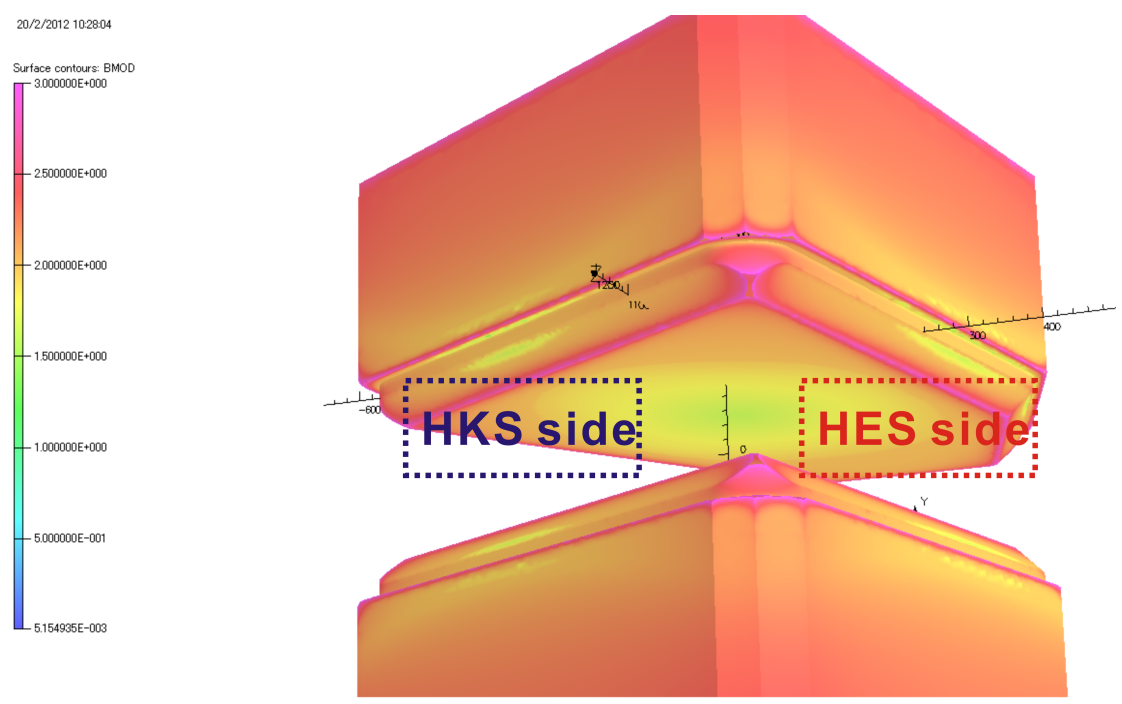

Figure 4.9: Magnetization of the SPL pole piece. The magenta regions are magnetized by more than 3 Tesla.

Table 4.3.1: HES magnetic field summary. The $\int B \cdot d l$ for EQ1 and EQ2 are calculated along a line $10 \mathrm{~cm}$ apart from the center of the magnet.

\begin{tabular}{c|ccc}
\hline \hline $\begin{array}{c}\text { Magnet } \\
\text { (Dipole) }\end{array}$ & $\begin{array}{c}\text { Path length }(l) \\
{[\mathrm{m}]}\end{array}$ & $\begin{array}{c}\text { Typical magnetic field }(B) \\
{[\mathrm{T}]}\end{array}$ & $\begin{array}{c}\int B d l \\
{[\mathrm{~T} \cdot \mathrm{m}]}\end{array}$ \\
\hline SPL & 1.90 & 1.70 & 1.76 \\
ED & 3.20 & 1.14 & 2.50 \\
\hline \hline Magnet & Path length $(l)$ & Magnetic field gradient & $\int B \cdot d l$ \\
(Quadrupole) & {$[\mathrm{m}]$} & {$[\mathrm{T} / \mathrm{m}]$} & {$[\mathrm{T} \cdot \mathrm{cm}]$} \\
\hline EQ1 & 0.90 & 5.6 & $3.88 \times 10^{-2}$ \\
EQ2 & 0.80 & 4.1 & $2.45 \times 10^{-2}$ \\
\hline \hline
\end{tabular}

\subsection{HES Magnets}

HES magnets were also newly constructed for high precision hypernuclear spectroscopic experiments via the $\left(\mathrm{e}, \mathrm{e}^{\prime} \mathrm{K}^{+}\right)$reaction. Table 4.3 is a summary of the magnetic fields of HES magnets. These values are determined by the basic optics requirement, which will be explained in this section. The requirements for the HES are to achieve high momentum resolution $\left(\Delta p / p=2 \times 10^{-4}\right)$, large solid angle as far as possible $(7 \mathrm{mrad}$ with the SPL) and usage with the tilt method for the background reduction. In addition, the kinematic matching with the existing HKS is also important. In this section, the basic optics design of the HES, the kinematic matching with HKS and finally the magnetic field calculation of HES will be described. Tilt method and the optimization of the tilt angle will be also explained in next section. 


\subsubsection{HES optics design}

As already mentioned, the basic design of the HES follows that of HKS. The HES consists of two quadrupole magnets (EQ1 and EQ2) and one dipole magnet (ED). Due to two degrees of freedom of the quadrupole doublet, the horizontal and vertical focusing can be adjusted simultaneously. Figure 4.10 shows the typical tracks of the HES. The EQ1 is vertical forcusing and the EQ2 is horizontal focusing and the optics of the HES is so-called point-to-point focusing, as shown in the figure.

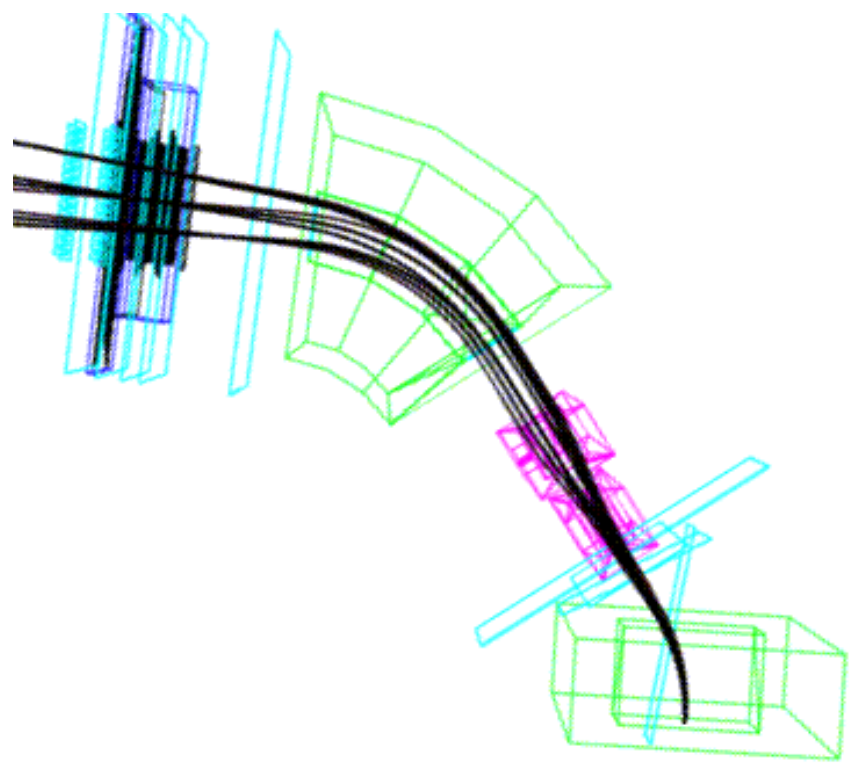

Figure 4.10: Typical tracks of the HES

The basic optics design was performed with the COSY Infinity and the TRANSPORT. These software were used to determine the lower order terms of the transfer matrices of the HES, which corresponds to the typical magnetic field strength as shown in table 4.3. On the other hand, the Geant4 simulation with the calculated magnetic field by the TOSCA was performed to estimate the actual momentum resolution and the solid angle including the higher order term effect of the transfer matrices. The detail of the TOSCA calculation will be described in the following part.

\subsubsection{HES magnetic field}

\section{TOSCA calculation of HES}

The procedure of the 3D magnetic field calculation for HES is almost same as that of the SPL. Figure 4.11 and 4.12 are the 3D model of the ED, the EQ1 and the EQ2. Using these models, the magnetic fields of the magnets were calculated with the mesh condition listed in table 4.3.2. The BH curve used for the calculation is given in figure 4.13. 


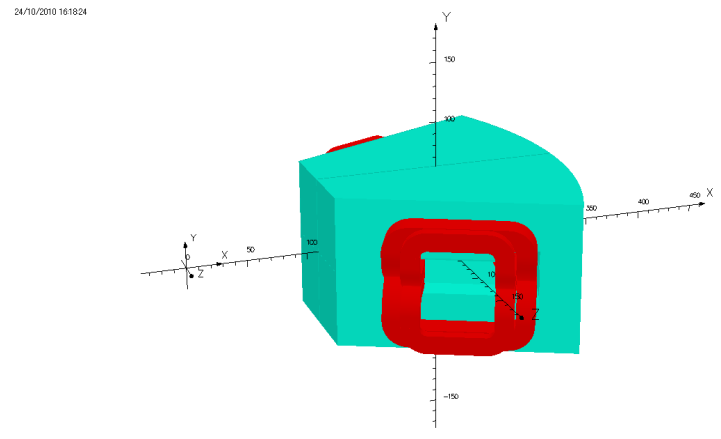

VE VECTOR FIELDS

Figure 4.11: ED 3D model in TOSCA calculation.
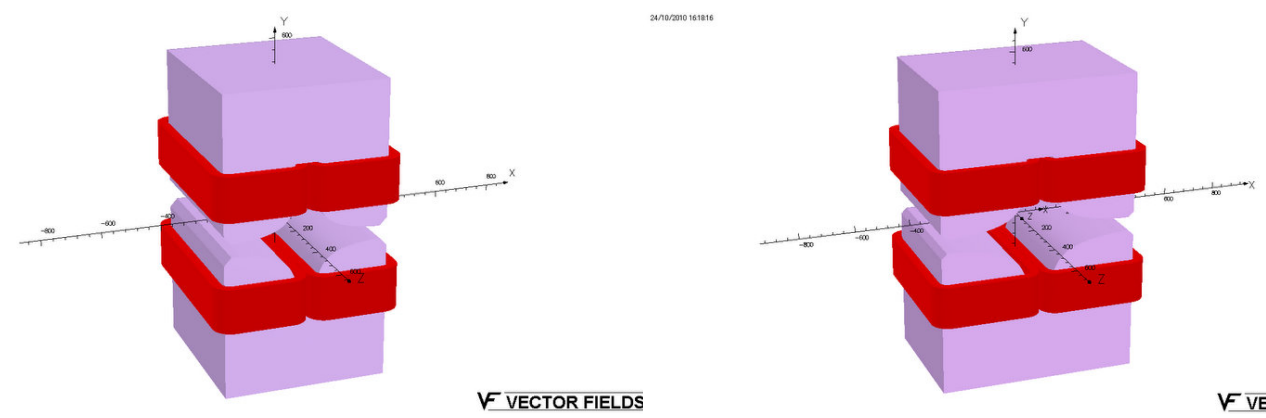

Figure 4.12: EQ1 and EQ2 3D model in TOSCA calculation.

Table 4.3.2: Material and mesh size in HES TOSCA 3D model.

\begin{tabular}{ccc}
\hline Name & Material & Mesh size $[\mathrm{cm}]$ \\
\hline EQ1Yoke & Iron (measured) & 2 \\
EQ1Gap & Air & 1.5 \\
\hline EQ2Yoke & Iron (measured) & 2 \\
EQ2Gap & Air & 3.0 \\
\hline EDYoke & Iron (measured) & 10 \\
EDPole & Iron (measured) & 1.5 \\
EDGap & Air & 3.0 \\
\hline \hline
\end{tabular}




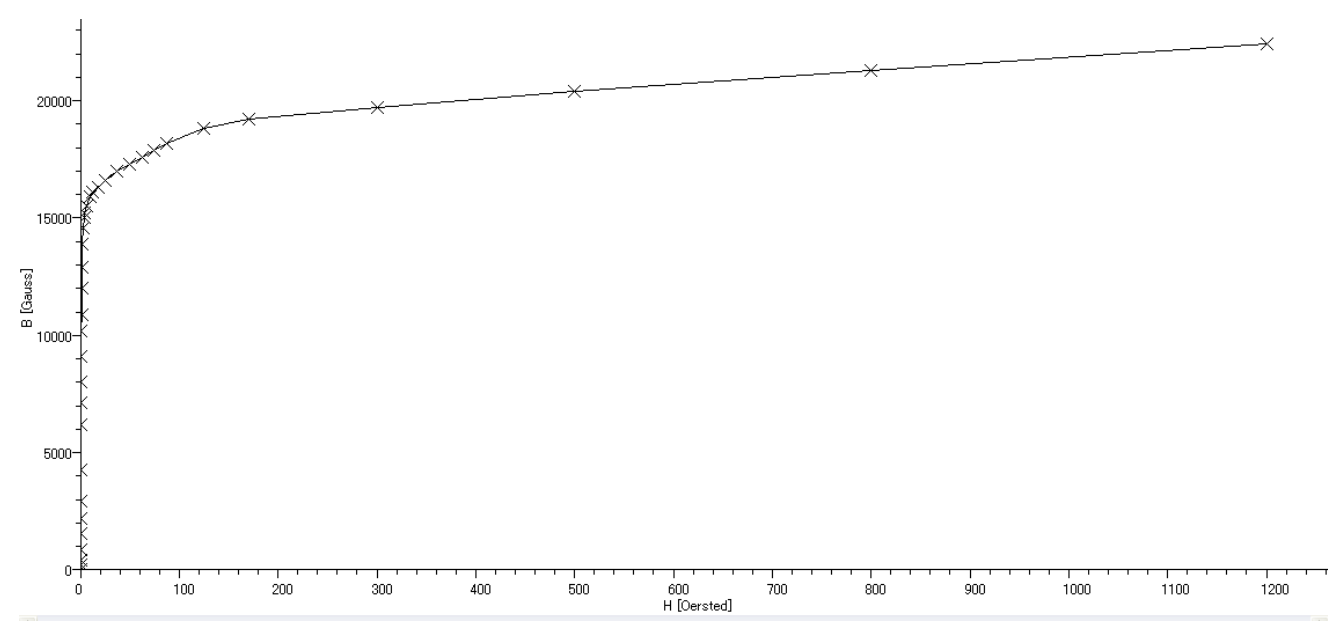

Figure 4.13: BH curve used in HES model.

\section{Measurement of the HES magnetic field}

The magnetic fields of the HES magnets were also measured at TOKIN company, as well as the SPL. Figure 4.14, 4.15 and 4.16 show the comparations of the excitation curves for the EQ1, the EQ2 and the ED magnets, respectively. We can see the result of the TOSCA calculation is agree with the measured value by the accuracy of less than $2 \%$.

\subsubsection{Kinematics matching with HKS}

Hypernuclear spectroscopic experiments via the $\left(\mathrm{e}, \mathrm{e}^{\prime} \mathrm{K}^{+}\right)$reaction is a coincidence experiment between two spectrometers, then the kinematic matching between kaon side and scattered electron side is important for the experiment.

Figure 4.17 shows the correlation between HKS momentum and HES momentum for $\Lambda, \Sigma^{0}$ and ${ }_{\Lambda}^{52} \mathrm{~V}$ ground state in Geant4 simulation. The ground state of ${ }_{\Lambda}^{52} \mathrm{~V}$ seems to be the most deeply bounded in the hypernuclei which we took during the experiment. As shown in this figure, the HES is designed to accept the three peaks and $\Lambda$ hypernucleus taken in the experiment, with the momentum acceptance of existing HKS. In particular, the $\Lambda$ and $\Lambda$ hypernucleus events are made to be located at the center of the acceptance.

\subsection{HES tilt method}

\subsubsection{Fundamental of "tilt method"}

As mentioned in chapter 2, huge background coming into scattered electron side is one of the biggest problem to carry out the present experiment. We can divide the electron in three types by its sources as follwos.

1. Electrons associated with kaon (and $\Lambda$ ) creation.

2. Electrons created by the pair creation through the bremsstrahlung at target. 
EQ1 excitation curve comparison (TOSCA vs measured)

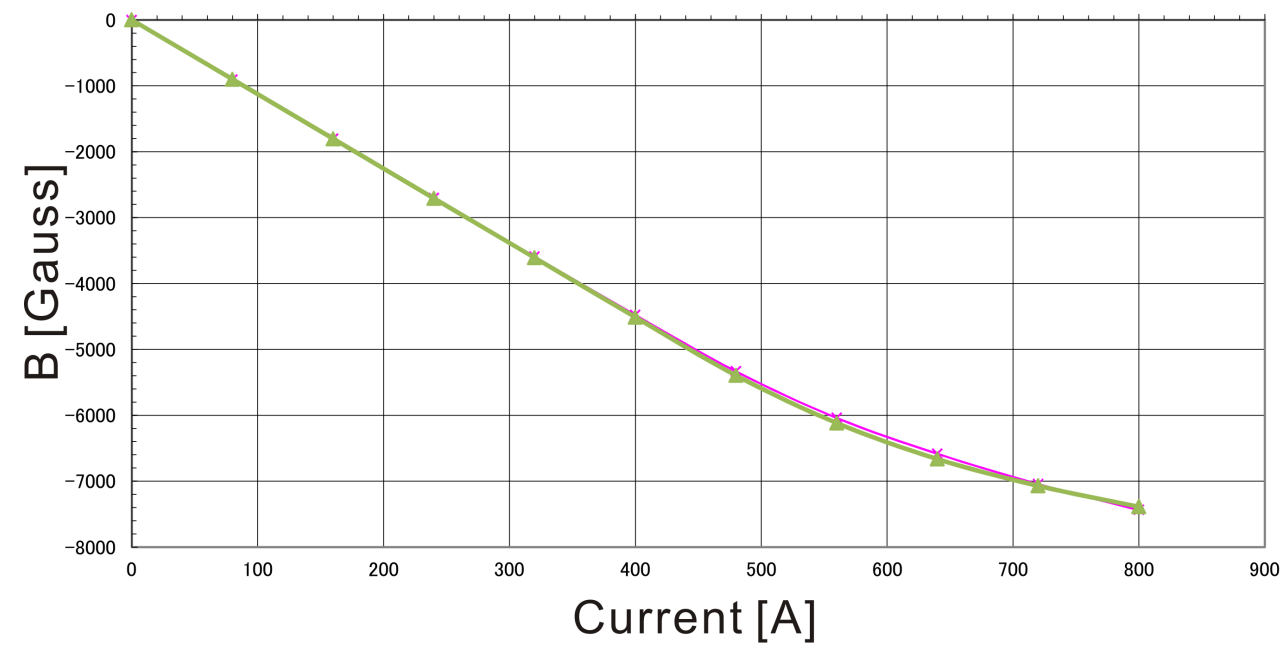

Figure 4.14: Excitation curve of EQ1 magnet. The measured value and TOSCA calculation result are superimposed.

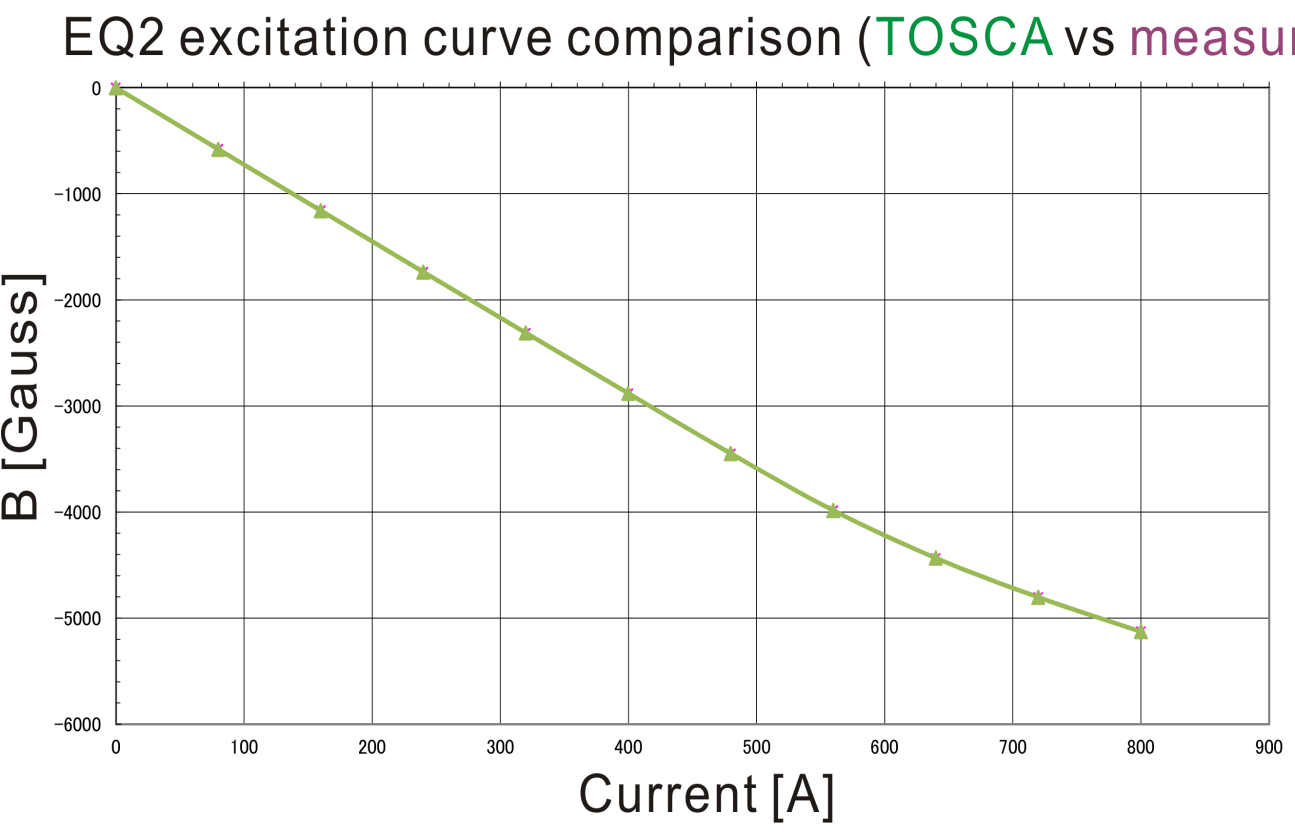

Figure 4.15: Excitation curve of EQ2 magnet. The measured value and TOSCA calculation result are superimposed. 
KD excitation curve comparison (TOSCA vs measured)

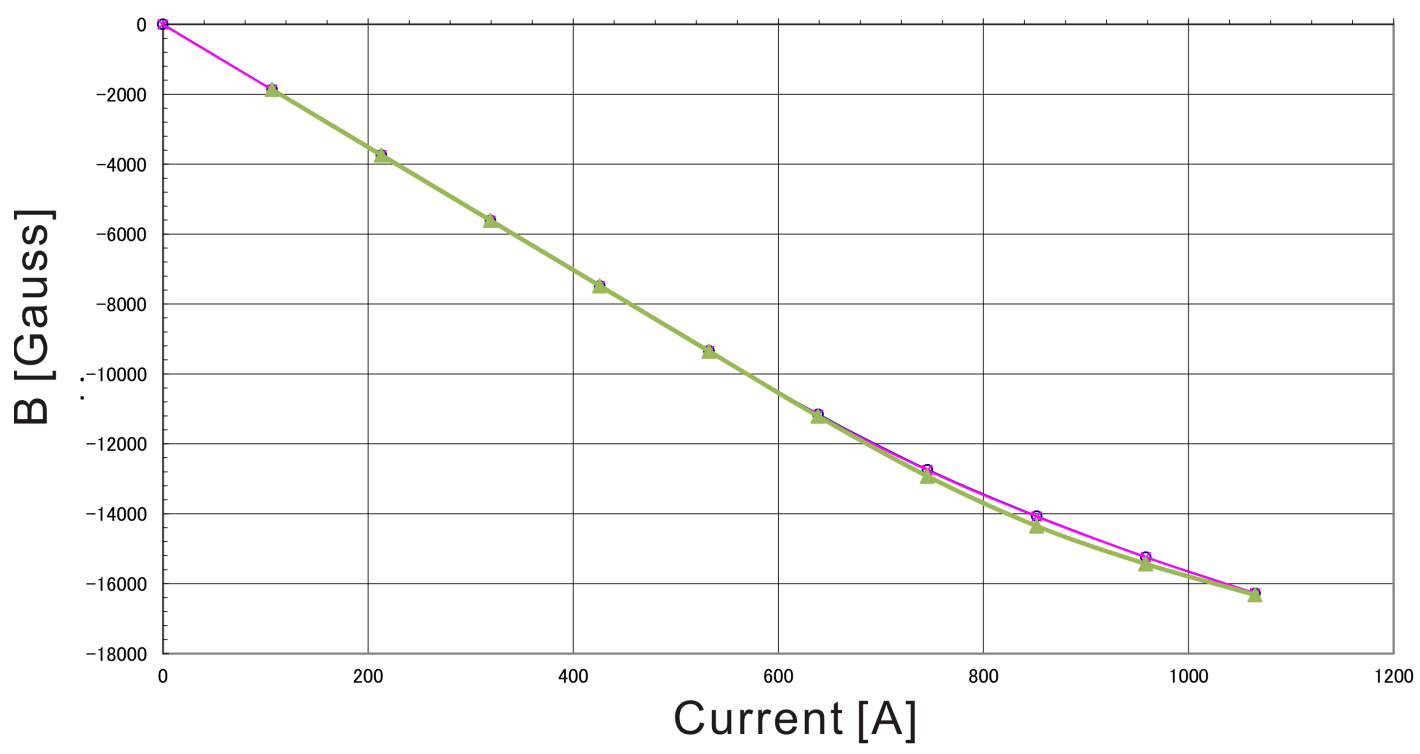

Figure 4.16: Excitation curve of ED magnet. The measured value and TOSCA calculation result are superimposed.

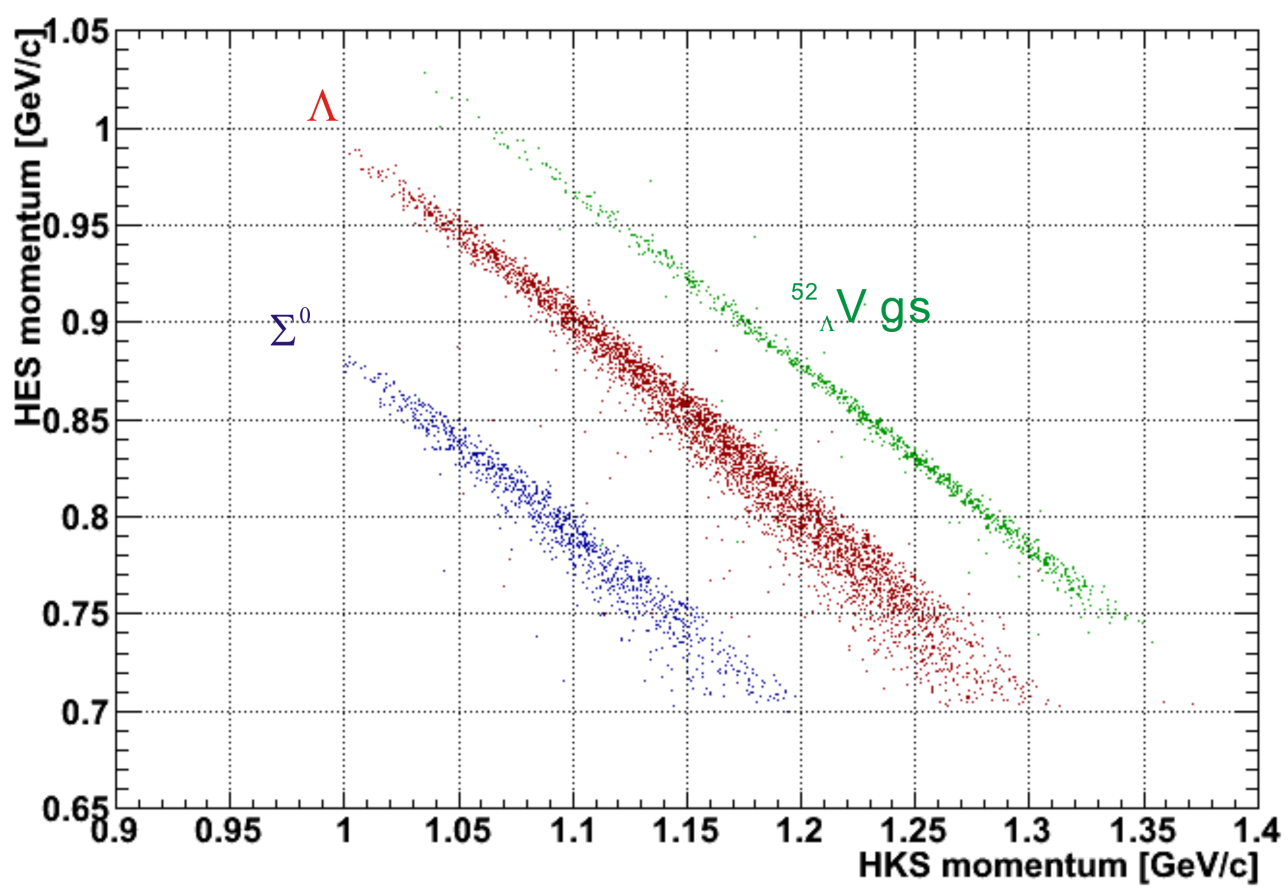

Figure 4.17: Correlation between HKS momentum and HES momentum. 
3. Electrons associated with the $\mathrm{M} \phi$ ller scattering.

In the above types, 1 . is what we aim to detect and will be called " $S$ " electrons in this theis. On the other hand, 2. and 3. are the background electrons and will be called "N" electrons in this thesis. As mentioned in section 2.2 and 3.2, both the $\mathrm{S}$ and the $\mathrm{N}$ electrons concentrate on very forward (see figure 2.5 and 2.7), but the degree of the concentration is different between the $\mathrm{S}$ and the N. Since the N electrons much more concentrate on the zero-degree (see figure 2.12), the ratio of the number of the $\mathrm{S}$ event to that of the $\mathrm{N}$ event increase as the angle become larger. In order to make the angle, we tilt the HES vertically, as mentioned in section 3.2.

However, the yield of the $\mathrm{S}$ electrons decrease in the larger angle and we need to take the S electrons as much as possible. Therefore, we need to optimize the "tilt angle" referring the yield of the $\mathrm{S}$ electrons and the $\mathrm{S} / \mathrm{N}$ ratio. The concrete method to optimize the tilt angle will be explained in the following part.

\subsubsection{Tilt angle optimization}

There are two degrees of freedom when tilting a spectrometer; the tilt angle and offset of the spectrometer (figure 4.18). These two value have to be optimized simultaneously.

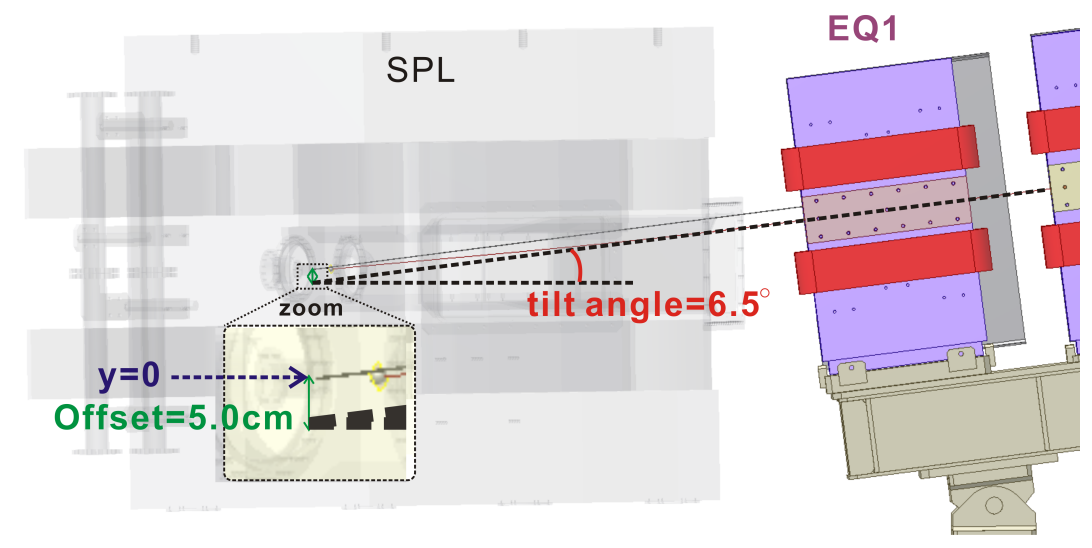

Figure 4.18: Definition of the tilt angle and spectrometer offset

Tilt angle is optimized with the following procedure;

1. Generate scattering electron associated with kaon creation events, bremsstrahlung events and $\mathrm{M} \phi$ ller scattering events source.

2. Put the sources into Geant4 simulation of HES and count the number of events which arrive at detector. Define $S$ as the number of events associated with kaon creation and $N$ as the number of events associated with bremsstrahlung and M $\phi$ ller scattering.

3. Define "Figure of Merit (FoM)" as follows;

$$
F_{O M}=\frac{S}{\sqrt{N}}
$$


4. Repeat procedure 2 and 3 changing tilt angle value in Geant4.

Figure 4.19 show the tilt angle dependence of the FoM. We set the tilt angle 6.5 degree from this result.

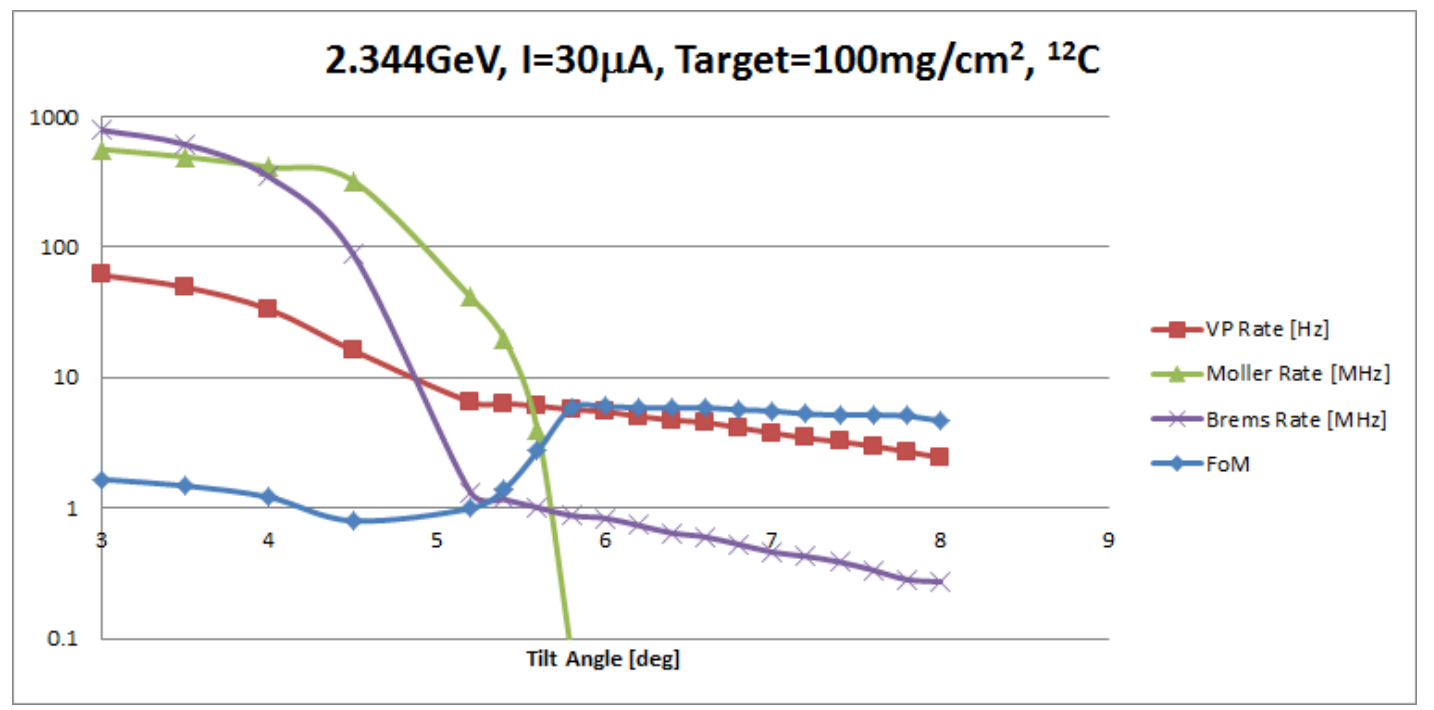

Figure 4.19: Figure of Merit $(F o M)$ changing tilt angle value with $30 \mu \mathrm{A}$ beam and ${ }^{12} \mathrm{C}$ target. The cyan points shows the FoM and the other lines show the FP counting rate associated with the virtual photon, bremsstrahlung and $\mathrm{M} \phi$ ller scattering.

\subsection{Resolution and yield estimation}

The HES was designed as mentioned above, and we estimated the momentum resolution of the HES, the mass resolution of hypernuclear spectrum and the hypernuclear yield. These values were estimated using the Geant4 simulation before the experiment for the detail experiment planning. The results will be described in this section.

\subsubsection{Estimation of HES momentum resolution}

The momentum resolution can be estimated by using the first order transfer matrix of the HES as written in section 4.1. However, this calculation does not include the effect of higher order terms of the matrix and the effect of various materials which cause the energy struggling and multiple scattering. Although it is possible to calculate such effects by had calculation, the Geant 4 simulation can derive much more realistic result.

The materials to be considered for the resolution estimation are the target as listed in table 3.3.1 and FP materials as listed in table 4.5.1. These materials are placed in the Geant4 simulation.

In the simulation, The momentum resolution was estimated in the following way. 


\begin{tabular}{cccc}
\hline \hline & $\mathrm{t}[\mathrm{cm}]$ & $\rho\left[\mathrm{g} / \mathrm{cm}^{3}\right]$ & $\mathrm{X}_{0}\left[\mathrm{~g} / \mathrm{cm}^{2}\right]$ \\
\hline ED Exit Window & & & \\
KeVlar $\left(\mathrm{C}_{14} \mathrm{~N}_{2} \mathrm{O}_{2} \mathrm{H}_{10}\right)$ & 0.02 & 0.74 & 55.2 \\
Mylar $\left(\mathrm{C}_{10} \mathrm{H}_{4} \mathrm{H}_{8}\right)$ & 0.01 & 1.39 & 39.95 \\
\hline EDC1 & & & \\
Mylar $\left(\mathrm{C}_{10} \mathrm{H}_{4} \mathrm{H}_{8}\right)$ & $0.0125 \times 2$ & 1.39 & 39.95 \\
Ar(50\%)+ $\mathrm{C}_{2} \mathrm{H}_{6}(50 \%)$ & 32 & $1.57 \times 10^{-3}$ & 25.95 \\
\hline EDC1-EDC2 gap & \multicolumn{3}{c}{} \\
Air & 7.7 & $1.293 \times 10^{-3}$ & 36.66 \\
\hline EDC2 & & 1.39 & 39.95 \\
Mylar $\left(\mathrm{C}_{10} \mathrm{H}_{4} \mathrm{H}_{8}\right)$ & 0.012 & $1.57 \times 10^{-3}$ & 25.95 \\
Ar $(50 \%)+\mathrm{C}_{2} \mathrm{H}_{6}(50 \%)$ & 5.13 &
\end{tabular}

Table 4.5.1: List of HES materials and their properties used in the simulation.

(1) Generate particles in the target point and detect the positions and angles at FP without any materials and position and angle resolution which should be caused by the EDCs (data-A).

(2) Calculate a sixth order F2T functions from data-A.

(3) Do the same simulation with (1) including materials and resolutions (data-B).

(4) Apply the F2T function calculated in (2) to the data-B and obtain reconstructed momentum $\left(p_{R}\right)$.

(5) Compare $p_{R}$ and real momentum value in the simulation $(p)$.

Table 4.5.1 summarize the simulation results with several conditions. The first condition does not include the effect of material and EDC, therefore the values are affected only from the $F 2 T$ functions. The second and third condition include the effect of materials at the FP and the EDC resolution, respectively. The fourth condition includes the both of them, then this value shows the realistic resolutions during the experiment. The estimated realistic momentum resolution is balanced with that of HKS $\left(d p / p=2.0 \times 10^{-4}\right.$ and $d p=240 \mathrm{keV})$.

Figure 4.20: Estimation of HES momentum resolution in each condition. The resolution value is shown in FWHM.

\begin{tabular}{l|c|c|cccc}
\hline \hline Condition & Material & $\begin{array}{c}\text { EDC } \\
\text { resolution }\end{array}$ & $\begin{array}{c}d p \\
{[\mathrm{keV}]}\end{array}$ & $d p / p$ & $\begin{array}{c}d x^{\prime} \\
{[\mathrm{mrad}]}\end{array}$ & $\begin{array}{c}d y^{\prime} \\
{[\mathrm{mrad}]}\end{array}$ \\
\hline$\# 1$ & OFF & OFF & 130 & $1.5 \times 10^{-4}$ & 0.7 & 3.9 \\
$\# 2$ & ON & OFF & 190 & $2.3 \times 10^{-4}$ & 1.5 & 4.4 \\
$\# 3$ & OFF & ON & 220 & $2.7 \times 10^{-4}$ & 2.3 & 4.0 \\
$\# 4$ & ON & ON & 250 & $3.2 \times 10^{-4}$ & 2.6 & 4.5 \\
\hline \hline
\end{tabular}




\subsubsection{Estimation of mass spectrum resolution}

In order to estimate the mass spectrum resolution, one need to consider the following points.

- Momentum and angular resolution of HES and HKS.

- Momentum resolution of electron beam.

- Energy struggling and multiple scattering effect in the target.

The momentum and angular resolutions of the spectrometer were already discussed above. The momentum resolution of the CEBAF's beam $(d p / p)$ is less than $10^{-4}$ in FWHM (see section 3.1). Therefore, our beam momentum $(p=2344 \mathrm{MeV})$ resolution is less than $240 \mathrm{keV}$, which is balanced with that of both spectrometer.

The energy struggling in target is simulated using Geant4. and the detail results are summarized in table 5.5.2 (section 5.5.1). The energy struggling is less than $200 \mathrm{keV}$ (FWHM) except for $\mathrm{CH}_{2}$ and $\mathrm{H}_{2} \mathrm{O}$ targets, which is much less than the energy resolution of the spectrometers and electron beam.

The missing mass resolution was estimated including these items in the Geant4 simulation. Figure 4.21 shows the result of the estimation. The left figure is the result with the $\mathrm{CH}_{2}$ target and proton kinematics simulation and the missing mass resolution is about $2.3\left[\mathrm{MeV} / \mathrm{c}^{2}\right]$. The right figure is the result with the ${ }^{12} \mathrm{C}$ target simulation and the result is $590\left[\mathrm{keV} / \mathrm{c}^{2}\right]$. This difference of mass resolution between these figures come from the difference of the recoil effect (the effect of the proton kinematics is larger than that of ${ }^{12} \mathrm{C}$ kinematics). The tails shown in these figures is not the radiative tail, but coming from the $F 2 T$ function.
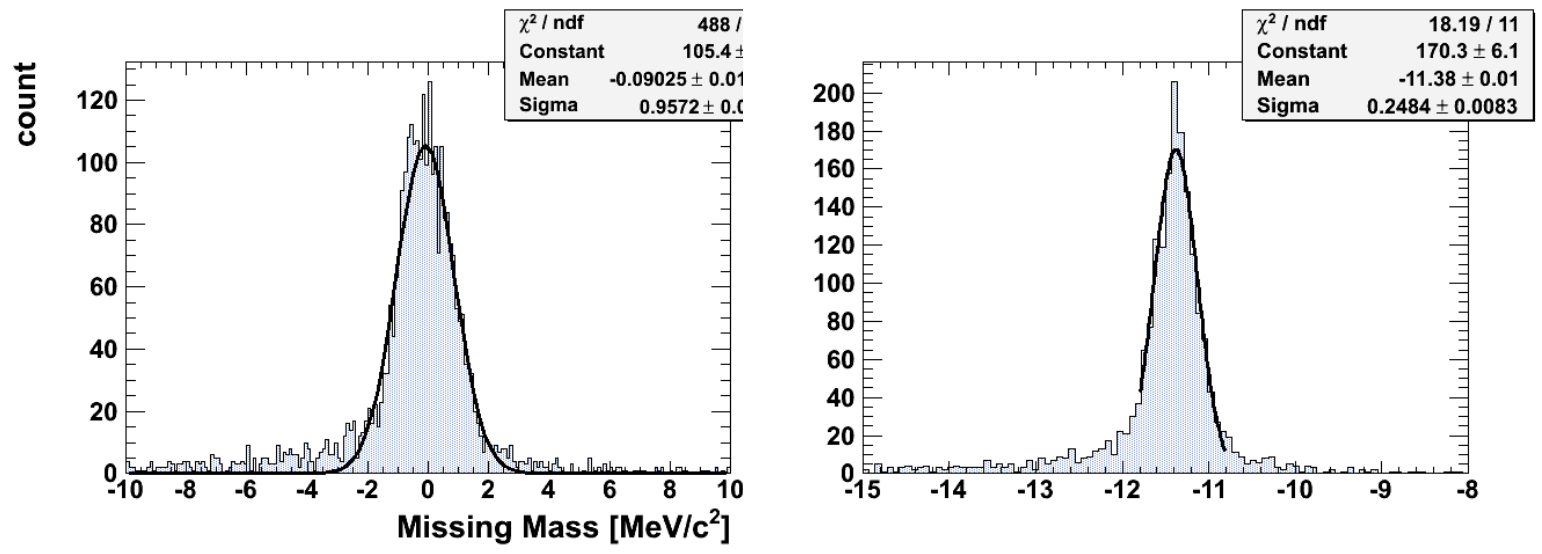

Figure 4.21: Estimated Missing mass resolution in the Geant4 simulation. The left figure and right figure shows the missing mass from $\mathrm{CH}_{2}$ target and ${ }^{12} \mathrm{C}$ target simulation, respectively. 


\subsubsection{Hypernuclear yield estimation}

Basically, the actual hypernuclear yield $\left(N_{H Y}\right)$ can be calculated from the differential cross section of hypernuclei via $\left(\gamma^{*}, \mathrm{~K}^{+}\right)$reaction as follows:

$$
N_{H Y}=N_{T} \cdot N_{v p} \cdot \frac{d \sigma_{\left(\gamma^{*}, \mathrm{~K}^{+}\right)}}{d \Omega_{K}} \cdot \Delta \Omega_{K} \cdot f^{\text {decay }} \cdot \epsilon
$$

The values in the above equation can be obtained as follwos:

- $N_{T}$ is the number of target nuclei which calculated from the target thickness and material as $N_{T}=\frac{T N_{A}}{A}$, where $T$ is the target thickness, $N_{A}=6.02 \times 10^{23}[1 / \mathrm{mol}]$ is the Avogadro number and $A$ is the atomic number of the nuclei.

- $d \sigma_{\left(\gamma^{*}, \mathrm{~K}^{+}\right)} / d \Omega_{K}$ is the the differential cross section of hypernuclei via $\left(\gamma^{*}, \mathrm{~K}^{+}\right)$reaction. This value can be obtained from the previous experiment values or various theoretical calculation.

- $\Delta \Omega_{K}$ is the solid angle of HKS. Here we assume this value as $8.5 \mathrm{mrad}$ not depending on the acceptance. The detail of the solid angle will be described in 5.6.

- $f^{\text {decay }}$ is the kaon decay ratio in HKS. Although this value also depends on the kaon momentum as well as the solid angle, here we assume this value as $30 \%$ for all HKS acceptance. The detail of this values will be also summarized in section 5.6.

- $\epsilon$ is the miscellaneous efficiencies (detection efficiency, computer dead time and so on). we assume this value as 1 .

The remained value, the number of virtual photon $\left(N_{v p}\right)$, needs to be obtained from the Geant4 simulation.

The $N_{v p}$ can be calculated from equation 2.17. The integrated $\Gamma\left(\theta_{e^{\prime}}, E_{e^{\prime}}\right)$ in equation 2.17 through the momentum and angular acceptance of scattered electron, $\int \Gamma\left(\theta_{e^{\prime}}, E_{e^{\prime}}\right) d E_{e^{\prime}} d \Omega_{e^{\prime}}$, shows the number of virtual photons per the beam electrons. The integration is performed using the Geant4 simulation according to following procedure:

1. The seed file which depends on the angle and momentum distribution of the $\Gamma$ is made for the Geant4 simulation. The integration is also performed for the seed file.

2. The Geant4 simulation is performed using the seed file. The number of generated events $\left(N_{\text {gen }}\right)$ and the number of accepted events $\left(N_{\text {acc }}\right)$ are obtained.

3. $\int_{H E S} \Gamma\left(\theta_{e^{\prime}}, E_{e^{\prime}}\right) d E_{e^{\prime}} d \Omega_{e^{\prime}}$ is obtained according to the following equation,

$$
\int_{H E S} \Gamma\left(\theta_{e^{\prime}}, E_{e^{\prime}}\right) d E_{e^{\prime}} d \Omega_{e^{\prime}}=\frac{N_{a c c}}{N_{g e n}} \int_{\text {seed }} \Gamma\left(\theta_{e^{\prime}}, E_{e^{\prime}}\right) d E_{e^{\prime}} d \Omega_{e^{\prime}} .
$$

The value is estimated as $\int_{H E S} \Gamma\left(\theta_{e^{\prime}}, E_{e^{\prime}}\right) d E_{e^{\prime}} d \Omega_{e^{\prime}}=5.2 \times 10^{-5}$ with a typical collimator position.

Using this value, the hypernuclear yield can be calculated with an assumed cross section. The estimation result is summarized in table 4.5.3. In this table, the target 
Table 4.5.2: The list of the estimated yields.

\begin{tabular}{l||c|c|ccc}
\hline \hline Target & $\begin{array}{c}\text { Thickness } \\
{\left[\mathrm{mg} / \mathrm{cm}^{2}\right]}\end{array}$ & $N_{T}$ & $\begin{array}{c}\text { Beam current } \\
{[\mu \mathrm{A}]}\end{array}$ & $\begin{array}{c}\text { Cross section } \\
{[\mathrm{nb} / \mathrm{sr}]}\end{array}$ & $\begin{array}{c}\text { Yield } \\
{[/ \mathrm{h}]}\end{array}$ \\
\hline $\mathrm{CH}_{2}$ & 450.8 & $2.71 \times 10^{23}$ & 2.0 & $200(\Lambda)$ & 320 \\
& & & & $50\left(\Sigma^{0}\right)$ & 80 \\
$\mathrm{H}_{2} \mathrm{O}$ & 500 & $3.01 \times 10^{23}$ & 3.0 & $200(\Lambda)$ & 540 \\
& & & & $50\left(\Sigma^{0}\right)$ & 140 \\
${ }^{7} \mathrm{Li}$ & 184.0 & $1.58 \times 10^{22}$ & 30 & 20 & 40 \\
${ }^{9} \mathrm{Be}$ & 188.1 & $1.26 \times 10^{22}$ & 40 & 20 & 30 \\
${ }^{10} \mathrm{~B}$ & 56.1 & $3.38 \times 10^{21}$ & 40 & 20 & 8 \\
${ }^{12} \mathrm{C}$ & 112.5 & $5.64 \times 10^{21}$ & 30 & 100 & 50 \\
${ }^{52} \mathrm{Cr}$ & 134.0 & $1.55 \times 10^{21}$ & 8 & 70 & 3 \\
${ }^{52} \mathrm{Cr}$ & 154.0 & $1.78 \times 10^{21}$ & 8 & 70 & 3 \\
\hline \hline
\end{tabular}

thickness is actual value in the experiment. The beam current is a typical value for each target and detector efficiencies were assumed as 1 . The cross section values are theoretical values for the ground states of hypernuclei and typical experiment values for $\Lambda$ and $\Sigma^{0}$. These values are five times larger than these of the previous JLab E01-011 experiment. 


\section{Chapter 5}

\section{Data analysis}

In this chapter, data analysis procedure of the present experiment is described. Firstly, in section 5.1, the overview of the analysis is explained. Then, the analysis of each arm is described in section 5.2 and 5.3. After that, coincidence time analysis and the details of missing mass calculation and its calibration procedure represented in section 5.4 and 5.5.1, respectively. Finally, the cross section analysis is explained in section 5.6.

\subsection{Analysis overview}

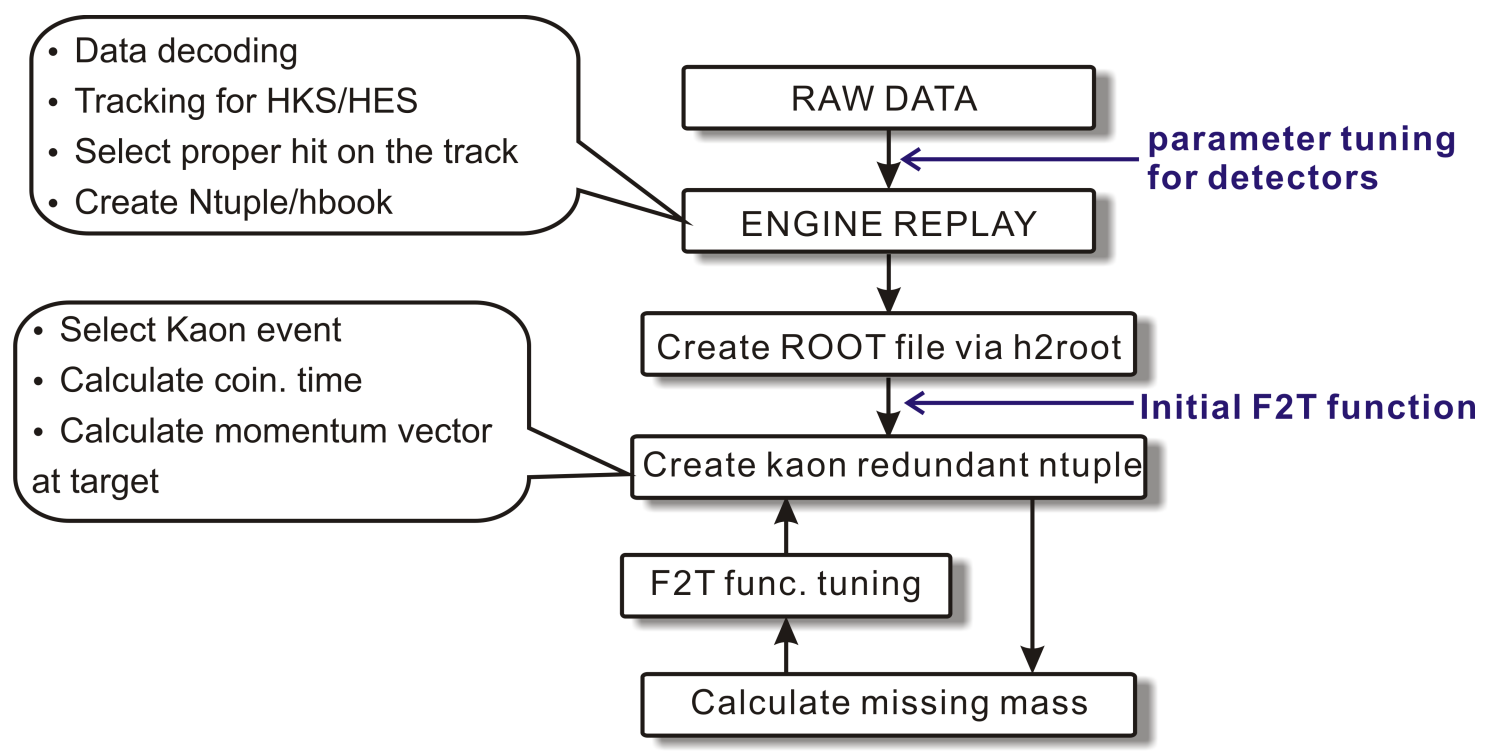

Figure 5.1: E05115 analysis flow chart.

Figure 5.1 is the analysis flowchart of the present experiment. In the first step, raw data of each ADC and TDC are decoded and analyzed by ENGINE, that is an analysis code used in JLab Hall-C traditionally. The main roles of the ENGINE can be summarized as follows. 
- Decode raw data of TDC and ADC.

- Perform tracking analysis using drift chamber and hodoscope information.

- Associate each counter information with tracks.

- Convert TDC and ADC data of each counter into physics information, such as the number of photoelectrons of a Cherencov counter and hit time at a TOF counter.

The detail of tracking and counter data analysis of each arm is explained in section 5.2 and 5.3. These results are output as Ntuple and histogram files.

Ntuple is a set of data array each element of which is associated with each other. and created by fortran program, that is ENGINE in this case. However, the following analysis is carried out using $\mathrm{ROOT}^{1}$ which is described in $\mathrm{C}$ language because of its rich functions. Then, the Ntuple have to be converted to the ROOT file using "h2root" function which is a part of ROOT package.

After that, tight kaon cut is applied for data reduction and files are merged by run condition. At the same time, momentum vector at target point are calculated by F2T function initially given by Geant4 Monte Carlo simulation with three dimension magnetic field map calculated by TOSCA. Finally missing mass is calculated using the momentum vectors at target point.

The F2T function have to be tuned using the missing mass spectrum because the initial F2T function usually does not have sufficient accuracy. Though the accuracy of TOSCA field map is a few $\%$, the accuracy of final missing is required to be the order of $10^{-4}$. The tuning is performed using the $\Lambda$ and $\Sigma^{0}$ mass spectra obtained by the $\mathrm{CH}_{2}$ and $\mathrm{H}_{2} \mathrm{O}$ target data. The mass accuracy and final missing mass resolution is determined by this tuning process, therefore the tuning is the most important part in whole analysis procedure. After the final spectra are obtained, cross section of each spectrum is calculated as the final step of the analysis. The details of the tuning procedure and cross section analysis are explained in section 5.5.1 and 5.6, respectively.

\subsection{Analysis on the HKS side}

The analysis of HKS side consists of mainly two parts; particle tracking and kaon particle identification (PID). In order to perform the kaon PID analysis, the information of each counter must be linked with particle tracks. In this section, firstly the tracking analysis processed in ENGINE and its results are shown. After that, the procedure of kaon identification is described.

\subsubsection{Particle tracking}

The HKS particle tracking is aiming to measure the position $\left(x_{f}\right.$ and $\left.y_{f}\right)$ and angle $\left(x_{f}^{\prime}\right.$ and $\left.y_{f}^{\prime}\right)$ at the HKS FP using the hit information of the KDC1, 2 and the KTOF. The tracking procedure is as follows.

1. Apply proper TDC cut for each wires.

\footnotetext{
${ }^{1}$ An analysis framework supported by CERN[67].
} 
2. Make a series of hits recognizing the hit pattern in each KDC. The obtained position in each KDC is called "space point".

3. Resolve left-right ambiguities and calculating the position in each wire plane using the drift distance obtained by the drift time converted from TDC analysis.

4. Reject inconsistent combination of space points using hodoscope hit information.

5. Fit the positions by straight line in each wire plane and obtain $x_{f}, y_{f}, x_{f}^{\prime}$ and $y_{f}^{\prime}$.

Figure 5.2 shows so-called $x-t$ curve which is the relation between the drift time and drift length obtained by the result of tracking, not by drift time itself. The function to calculate drift distance is a third polynomial and can be obtained by the iteration of fitting of the $x-t$ curve and calculation of $x-t$ curve.

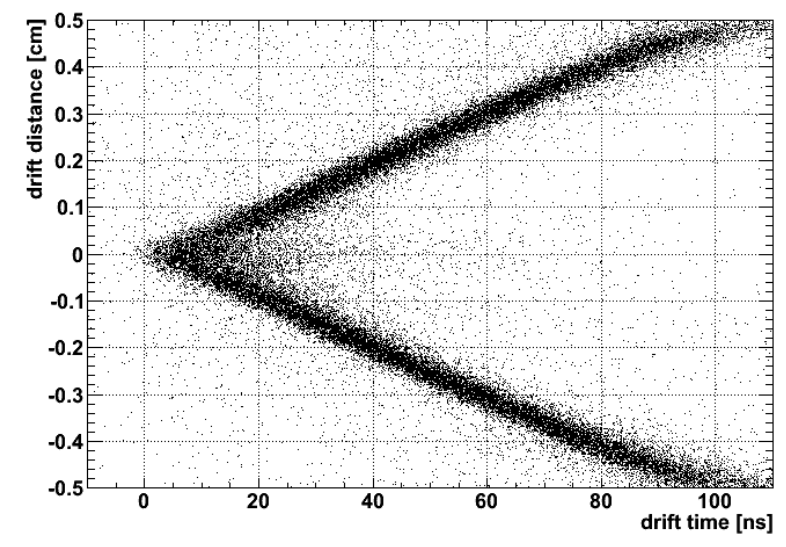

Figure 5.2: The relation drift time between drift length.

Figure 5.3 and 5.4 show the typical results of KDC fitting resolution in a wire plane and tracking $\chi^{2}$, respectively. The average plane resolution during the experiment is about $200 \mu \mathrm{m}$ and FP resolution can be summarized as table 5.2.1.

For the tracking, 5 planes hits are required for each KDC. For the typical $\mathrm{CH}_{2}$ target data set, The typical plane efficiency during experiment is $98 \%$, and the tracking efficiency can be calculated as $99.9 \%$.

Table 5.2.1: Calculated HKS FP resolution from the experimental data $\left(\mathrm{CH}_{2}\right.$ run).

\begin{tabular}{c|c|c|c}
\hline \hline$\delta x$ & $\delta x^{\prime}$ & $\delta y$ & $\delta y^{\prime}$ \\
\hline $100[\mu \mathrm{m}]$ & $0.2[\mathrm{mrad}]$ & $160[\mu \mathrm{m}]$ & $0.3[\mathrm{mrad}]$ \\
\hline \hline
\end{tabular}




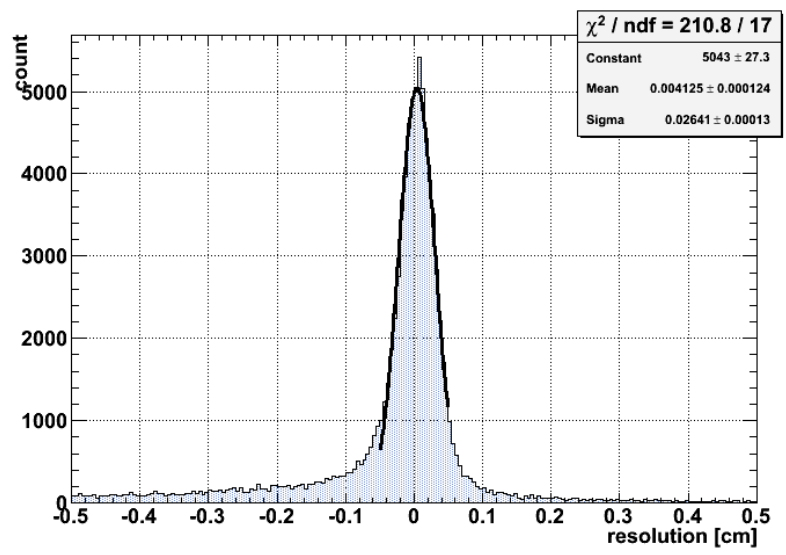

Figure 5.3: Typical plane resolution of KDC.

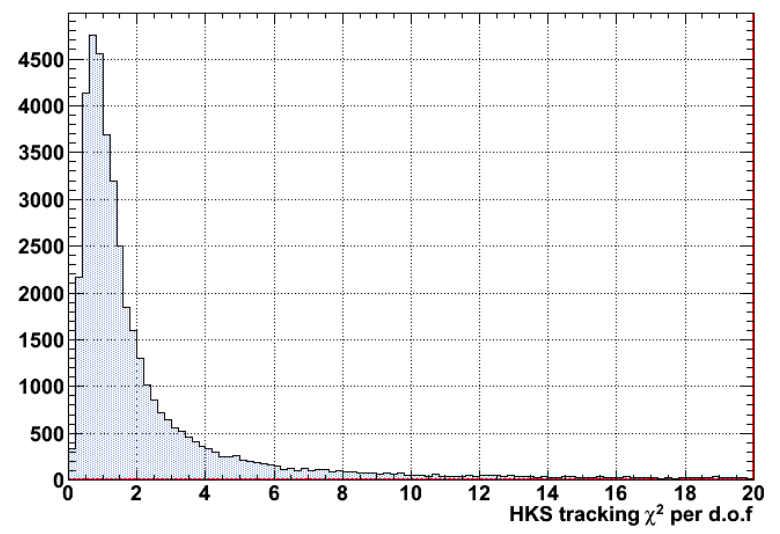

Figure 5.4: HKS tracking $\chi^{2}$. 


\subsubsection{Kaon particle identification}

\section{$\beta$ and $m^{2}$ distribution}

In the HKS side, kaons have to be identified from many background particles; proton, pion and positron. After the tracking, each TOF and Cherencov counter information on a track is linked with each other. Using these informations, the TOF of the track can be calculated.

The procedure of TOF calculation is as follows.

1. Select proper hit on the track.

2. Correct TDC value of the scintillators using its pulse height (ADC) information (slewing correction), the propagation time from hit position to PMT window and any offset caused by the time difference due to electronics and cables.

3. Calculate mean time of TOP and BOTTOM counters.

4. Calculate the time difference $(\Delta t)$ between stop counter and start counter.

The particle velocity $\beta=v / c$ can be calculated by as following:

$$
\beta=\frac{\Delta t}{l \cdot c},
$$

where $l$ is flight path length between the counters which is obtained by the tacking information. Figure 5.5 shows a typical particle $\beta$ distribution calculated by the TOF. In this spectrum, background particles (positrons, pions and protons) are already subtracted at trigger level, using AC signals for pion and positron subtraction and WC signals for proton subtraction.

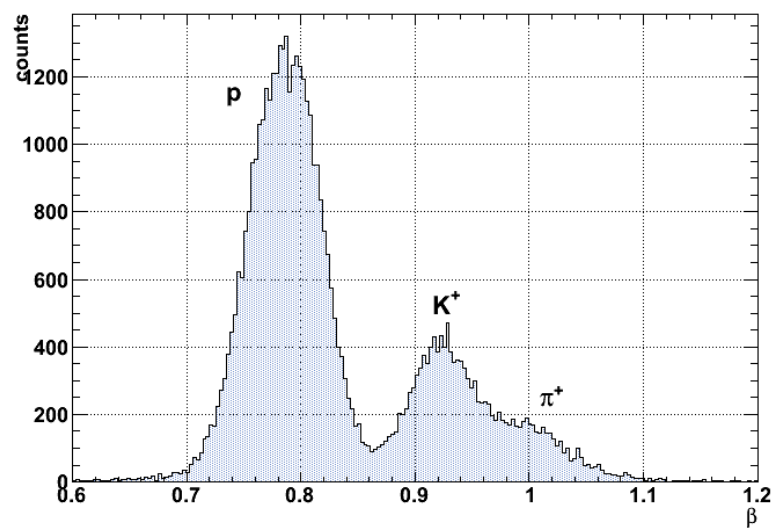

Figure 5.5: Typical $\beta$ distribution.

Since the $\beta$ is depends on the particle momentum which distribute from $1.05 \mathrm{GeV} / c$ to $1.35 \mathrm{GeV} / c, \beta$ is not good index to identify the particles. The particle mass square 
distribution is useful index for the particle identifications because the momentum dependence can be cancelled. The mass square is calculated from $\beta$ and particle momentum $p$ as following equation:

$$
m^{2}=p^{2}\left(\beta^{-2}-1\right)
$$

Figure 5.6 shows the typical mass square distribution. The resolution of $m^{2}$ distribution of proton is $\sigma=0.11\left[\left(\mathrm{GeV} / c^{2}\right)^{2}\right]$ which is due to the $\beta$ resolution.

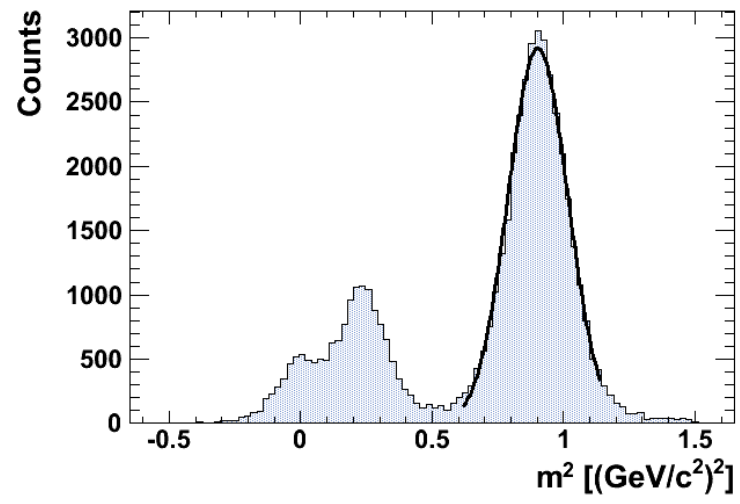

Figure 5.6: Typical $m^{2}$ distribution.

As shown in above, it is possible to separate kaons from protons, pions and positrons using $m^{2}$ spectrum. However, the separation power become more effective by using the number of photoelectrons (NPE) information of AC and WC. In the following sections, the efficiencies of each particle cut using NPE information and $m^{2}$ distribution are discussed.

\section{Pion rejection}

Pions are rejected using $\mathrm{AC}$ NPE and $m^{2}$ distribution. Positrons are also rejected with same cut condition, because the $\beta$ of pions and positrons have almost same. Figure 5.7 shows a typical NPE distribution of a layer of AC and scatter plot with $m^{2}$ distribution. One can see that the NPE of protons and kaons distribute almost around zero and NPE of pions have finite value.

Pions were rejected in online level with safety threshold not to kill kaon and tight cuts are applied in the offline level.. For the offline cut, sum of three layers of AC NPE is used for pion rejection, described as following:

$$
(A C 1 N P E)+(A C 2 N P E)+(A C 3 N P E) \leq X_{A C},
$$

where $X_{A C}$ is a cut value of the AC NPE. Figure 5.9 shows AC NPE cut efficiencies for various $X_{A C}$ values. The number of kaons and pions are obtained by the three gaussian fitting of $m^{2}$ distribution for each $X_{A C}$ value, as shown in figure 5.8. The pion background is fitted with a Pseudo-Voigt function as written in following way:

$$
f(x)=c_{1}\left[c_{2} \frac{2}{\pi} \frac{c_{3}}{4\left(x-c_{4}\right)^{2}+c_{3}^{2}}+\left(1-c_{2}\right) \frac{\sqrt{4 \log 2}}{\sqrt{\pi} c_{3}} \exp \left(-\frac{4 \log 2}{c_{3}^{2}}\left(x-c_{4}\right)^{2}\right)\right]
$$



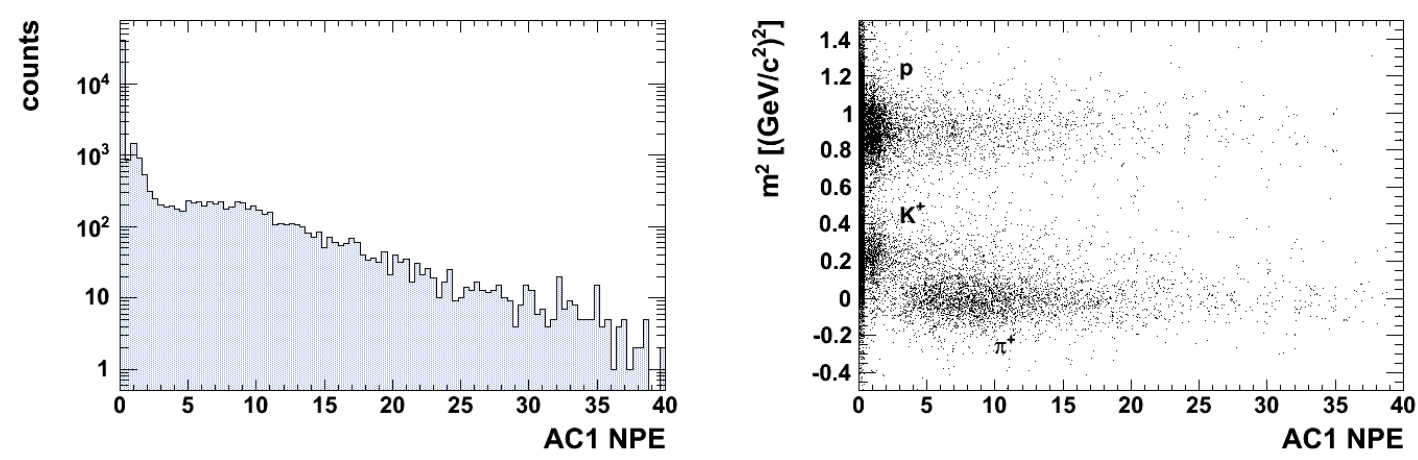

Figure 5.7: A typical NPE distribution of an layer of AC and scatter plot with $m^{2}$ distribution.

The AC NPE cut efficiency is normalized by the number of kaons (pions) with no AC NPE cut. Using these information, $X_{A C}$ value is optimized $X_{A C}=20$ which can reject pions and positrons typically $20-30 \%$ in this level. The kaon cut efficiencies for each run condition is summarized as table 5.2.2.

The remained pions will be rejected by $\mathrm{m}^{2}$ distribution and coin-time information and pions can be almost rejected at missing mass level. The efficiencies of these cuts are explained in following sections.

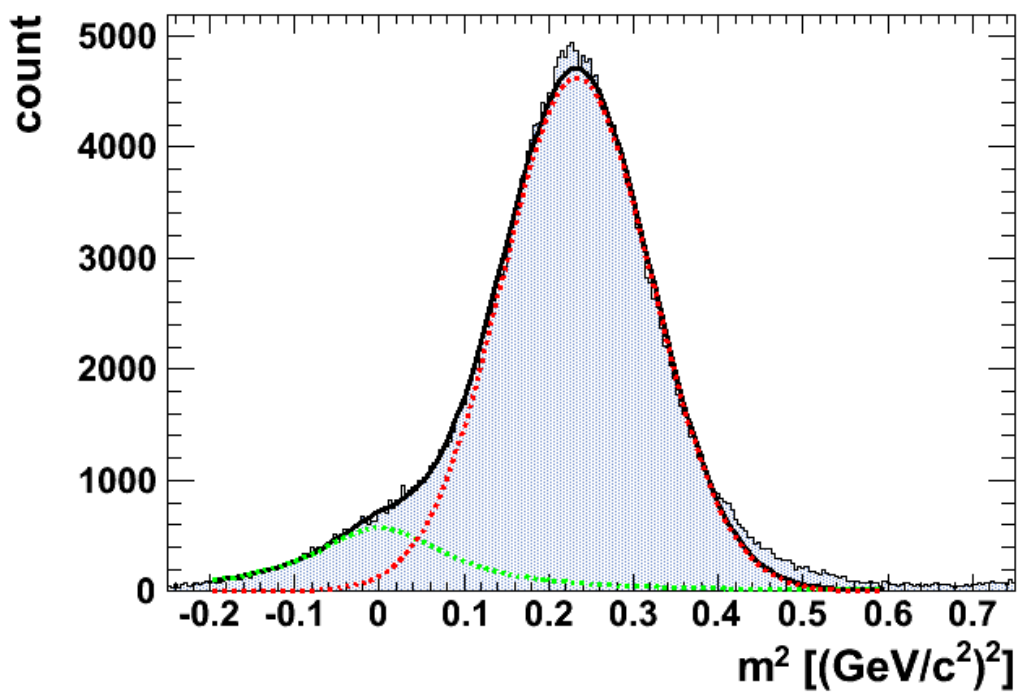

Figure 5.8: Typical fitting result of kaon with remained pion background. 


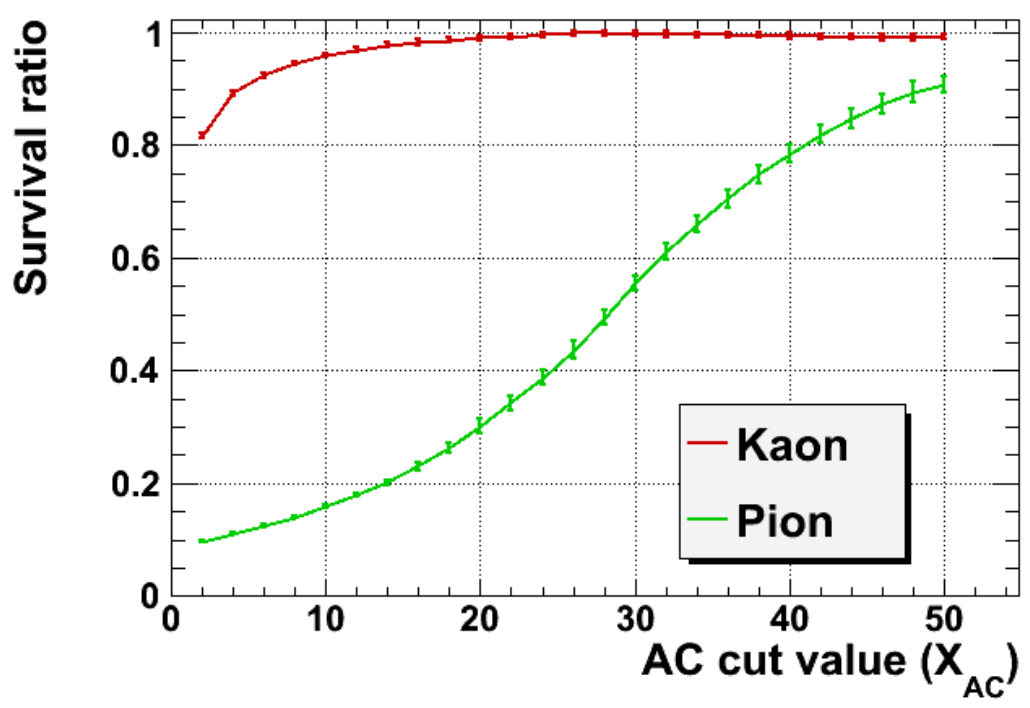

Figure 5.9: Typical AC NPE offline cut efficiency.

Table 5.2.2: Kaon cut efficiencies by AC NPE cut.

\begin{tabular}{ccc}
\hline \hline Data set & $\begin{array}{c}\text { Kaon survival ratio } \\
{[\%]}\end{array}$ & $\begin{array}{c}\text { Pion survival ratio } \\
{[\%]}\end{array}$ \\
\hline 1 & $96.4 \pm 0.5$ & $25.1 \pm 0.4$ \\
2 & $69.2 \pm 1.2$ & $19.2 \pm 0.5$ \\
3 & $88.6 \pm 0.8$ & $18.7 \pm 0.4$ \\
4 & $82.9 \pm 0.8$ & $10.0 \pm 0.3$ \\
5 & $96.9 \pm 0.4$ & $23.4 \pm 0.5$ \\
6,7 & $98.9 \pm 0.5$ & $30.0 \pm 1.3$ \\
8 & $90.2 \pm 1.4$ & $16.2 \pm 0.8$ \\
9,10 & $63.3 \pm 0.5$ & $8.0 \pm 0.1$ \\
\hline \hline
\end{tabular}




\section{Proton rejection}

Protons are rejected using WC NPE and $m^{2}$ distribution. Figure 5.10 shows a typical NPE distribution of a layer of WC and scatter plot with $m^{2}$ distribution. Three particle clusters can be seen in this figure, but each particle cluster has two component because we have two types of WC counter in the same layer and the photon collection efficiency is different each other. This is difficult to treat, then the NPE of each counters are normalized so as the kaon peak to be 1 in the following analysis. Figure 5.11 is the distribution of normalized NPE and the scatter plot with $m^{2}$ distribution. In this figure, pions (positrons), kaons and protons are clearly separated each other.
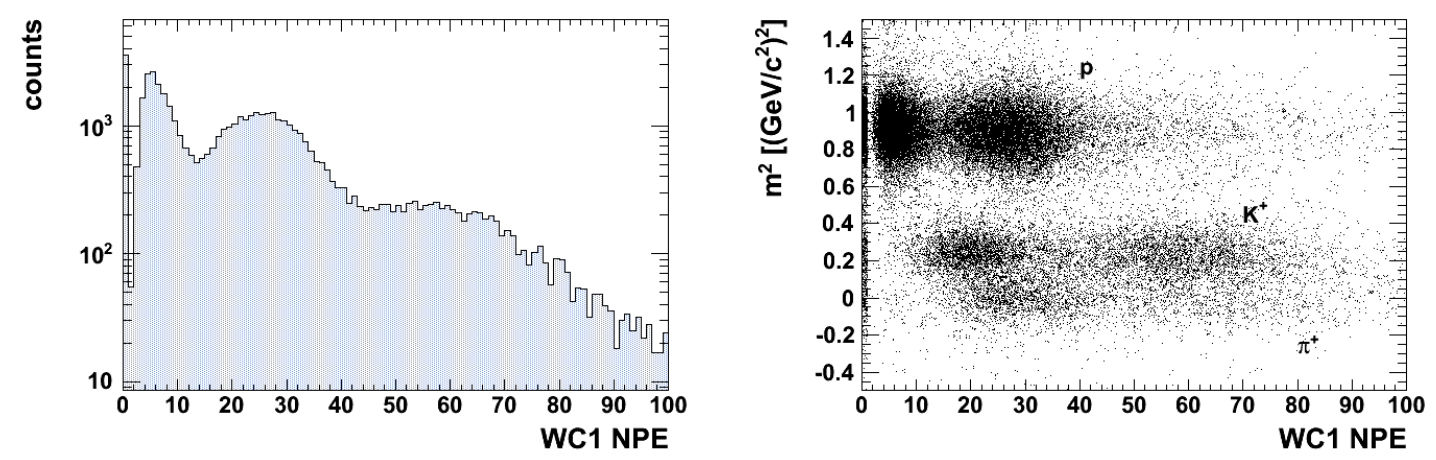

Figure 5.10: A typical NPE distribution of an layer of $\mathrm{WC}$ and scatter plot with $\mathrm{m}^{2}$ distribution. Since we have two types of WC counters in the same layer, each particle cluster has two component corresponding to the each counter.
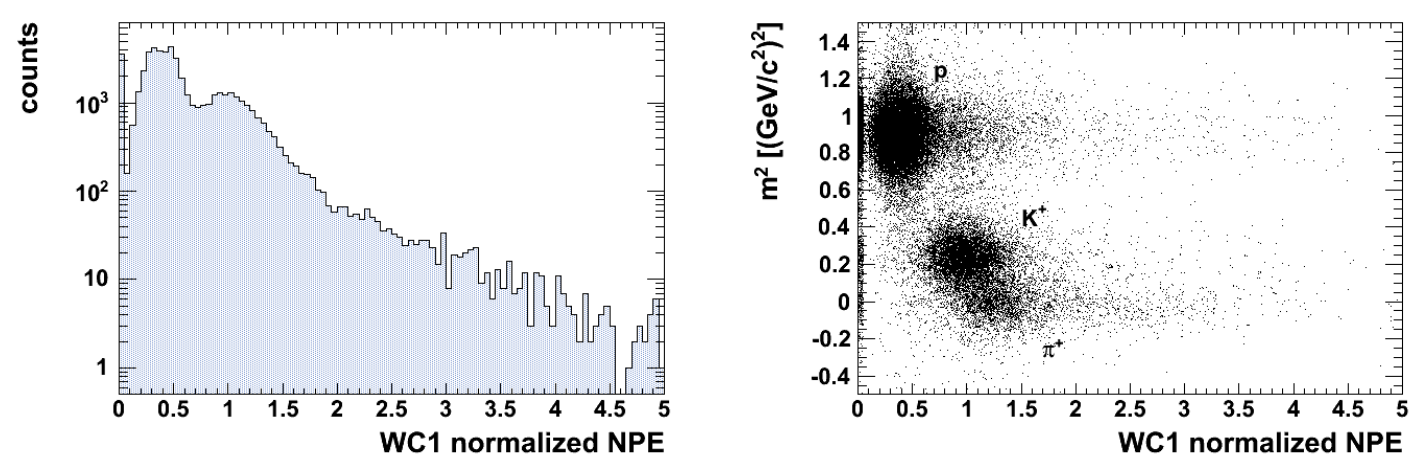

Figure 5.11: A normalized NPE distribution of an layer of the WC and a scatter plot with $m^{2}$ distribution. The NPE is normalized so as the kaon NPE peak of each counter to be 1 .

The procedure of proton cut follows it for the case of pions. For the offline proton rejection, sum of the two layers of WC NPE is used :

$$
(W C 1 N P E)+(W C 2 N P E) \leq X_{W C},
$$


where $X_{W C}$ is cut value of the WC NPE. Figure 5.12 shows the WC NPE cut efficiencies in some $X_{W C}$ values. The number of kaons and protons are obtained by the three Gaussians fitting of $m^{2}$ distribution histogram for various $X_{W C}$ value, as shown in figure 5.13. The proton background in the figure is fitted with 2 Gaussians. The WC cut efficiency is also normalized with the number of kaons (pions) with no WC NPE cut. The applied $X_{W C}$ value is $X_{W C}=1.0$ which can reject protons about $20 \%$ in this level. The kaon cut efficiencies for each run condition is summarized as table 5.2.3. The remained protons are also rejected at missing mass level using $m^{2}$ distribution and coin-time information. The efficiencies of these cuts are explained in following sections.

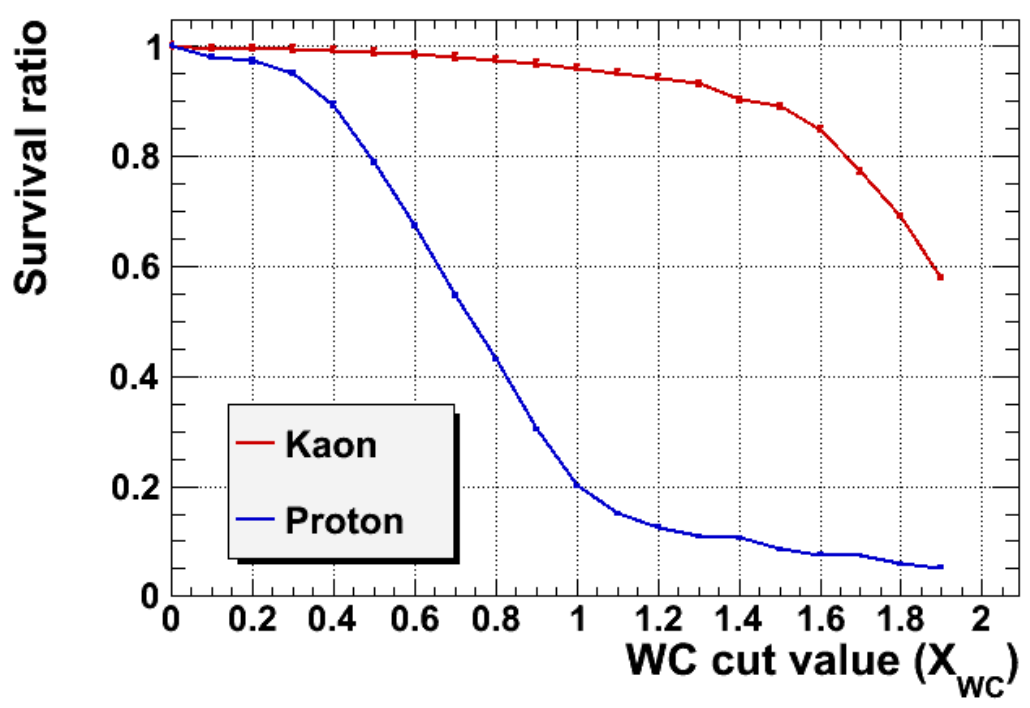

Figure 5.12: Typical WC NPE offline cut efficiency.

Table 5.2.3: Kaon cut efficiencies by WC NPE cut.

\begin{tabular}{ccc}
\hline \hline Data set & $\begin{array}{c}\text { Kaon survival ratio } \\
{[\%]}\end{array}$ & $\begin{array}{c}\text { Pion survival ratio } \\
{[\%]}\end{array}$ \\
\hline$\# 1$ & $94.9 \pm 0.2$ & $18.8 \pm 0.1$ \\
$\# 2$ & $97.6 \pm 0.9$ & $12.7 \pm 0.3$ \\
$\# 3$ & $95.2 \pm 0.3$ & $20.4 \pm 0.4$ \\
$\# 4$ & $95.7 \pm 0.4$ & $21.0 \pm 0.5$ \\
$\# 5$ & $96.7 \pm 0.2$ & $17.4 \pm 0.1$ \\
$\# 6, \# 7$ & $96.0 \pm 0.5$ & $20.1 \pm 0.2$ \\
$\# 8$ & $94.6 \pm 0.6$ & $18.0 \pm 0.5$ \\
$\# 9, \# 10$ & $96.2 \pm 0.6$ & $34.5 \pm 0.1$ \\
\hline \hline
\end{tabular}




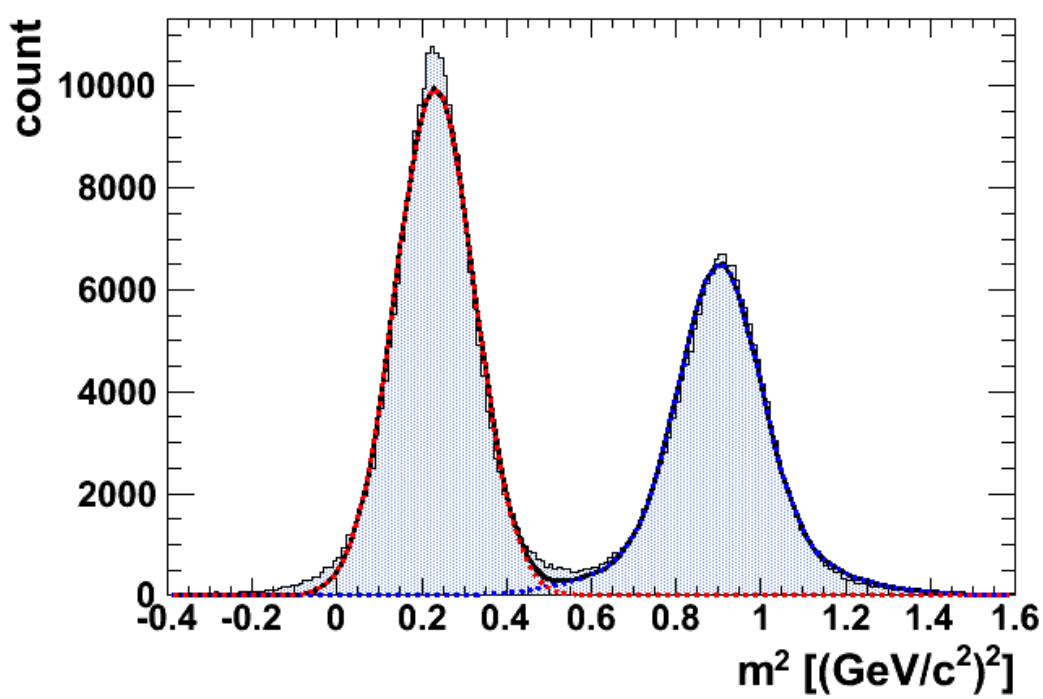

Figure 5.13: Typical fitting result of kaon with remained proton background.

$m^{2}$ cut

Pions (positrons) and protons can be selected the AC and WC NPE information, respectively. However, it is possible to separate these particles much more effectively by using the information of $m^{2}$ distribution. Figure 5.14 shows the pion and proton distribution in the $m^{2}$ spectrum and kaon distribution obtained by subtracting pion and proton from the original $\mathrm{m}^{2}$ distribution. Each particles are selected by using AC and WC cut described above.

Since pions and protons are still existing in the kaon distribution as shown in 5.14, $m^{2}$ cut is applied in order to obtain cleaner kaon events. Figure 5.15 is the result of three Gaussians fit of $m^{2}$ distribution after the subtraction of pions and protons. In this analysis, the $m^{2}$ cut is applied as

$$
\left|m^{2}-m_{K}^{2}\right|<0.3
$$

The cut efficiency of this cut can be calculated by fitting result in some run conditions.

\subsection{Analysis on the HES side}

The main purpose of analysis on the HES side is the particle tracking and obtaining the positions $(x, y)$ and angles $\left(x^{\prime}\right.$ and $\left.y^{\prime}\right)$ of the particles at reference plane $(\mathrm{FP})$. After the tracking, tracking time at the FP is calculated from the TDC information of the ETOF counters. This time information is used for the calculation of coin-time. These analysis is also performed in the ENGINE, as well as the HKS analysis.

The tracking procedure is as follows. This is slightly different from that of the HKS side. 

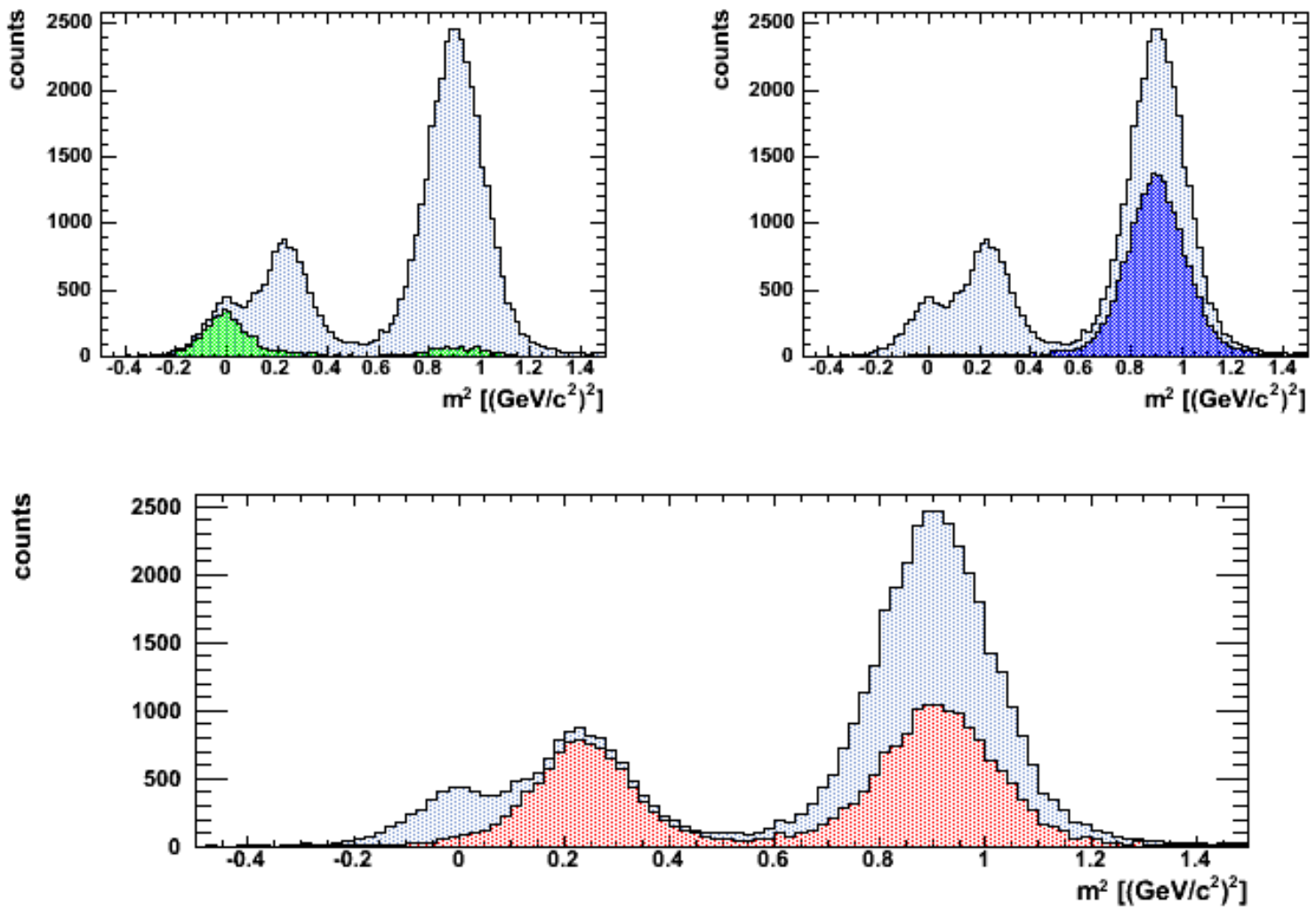

Figure 5.14: Kaon PID on the $m^{2}$ distribution. Upper left and right figures are a pion distribution and a proton distribution drawn over the original $\mathrm{m}^{2}$ distribution, respectively. Lower figure is a result of the subtraction of pion and proton distribution from original $m^{2}$ distribution drawn over the original one.

Table 5.2.4: Kaon cut efficiencies by $m^{2}$ cut.

\begin{tabular}{ccc}
\hline \hline Data set & $\begin{array}{c}\sigma \text { of kaon peak } \\
\left(\times 10^{-2}\right)\end{array}$ & $\begin{array}{c}\text { Kaon cut eff. } \\
{[\%]}\end{array}$ \\
\hline$\# 1$ & $8.8 \pm 0.03$ & $99.9 \pm 0.01$ \\
$\# 2$ & $15.1 \pm 0.13$ & $95.3 \pm 0.05$ \\
$\# 3$ & $11.1 \pm 0.06$ & $99.3 \pm 0.02$ \\
$\# 4$ & $11.6 \pm 0.06$ & $99.0 \pm 0.02$ \\
$\# 5$ & $9.2 \pm 0.02$ & $99.9 \pm 0.01$ \\
$\# 6, \# 7$ & $8.6 \pm 0.03$ & $99.9 \pm 0.01$ \\
$\# 8$ & $10.6 \pm 0.10$ & $99.5 \pm 0.04$ \\
$\# 9$ & $15.2 \pm 0.22$ & $95.2 \pm 0.09$ \\
$\# 10$ & $16.5 \pm 0.36$ & $93.1 \pm 0.14$ \\
\hline \hline
\end{tabular}




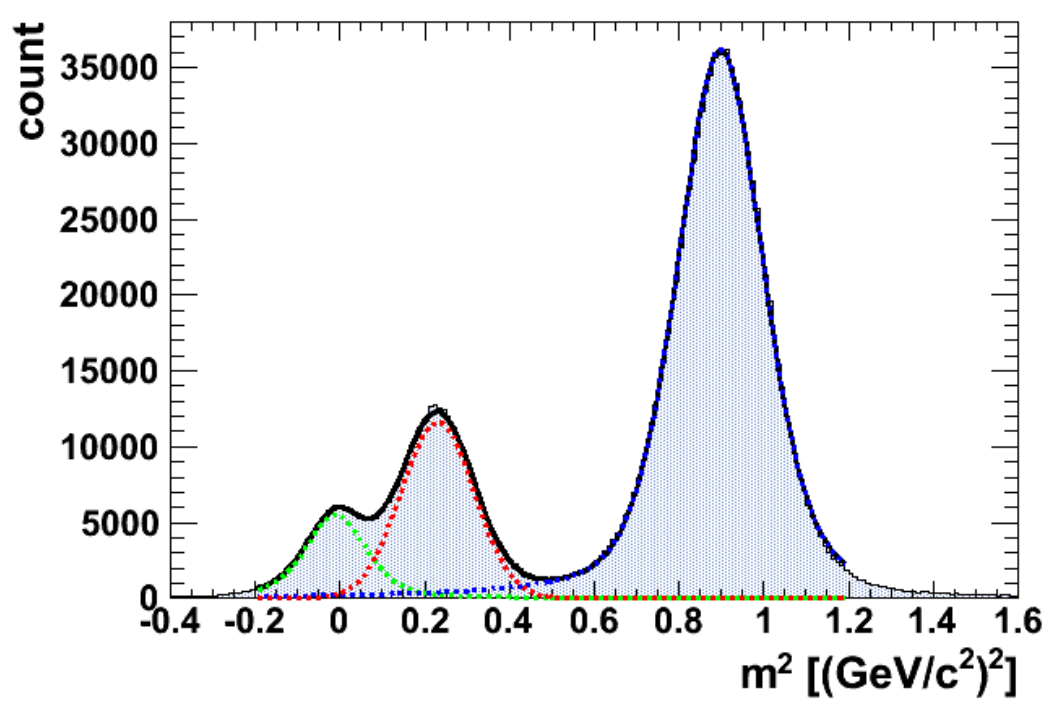

Figure 5.15: Kaon cut using $m^{2}$ distribution.

1. Apply proper TDC cut for each wire of the EDC1 and EDC2.

2. Make a series of hits recognizing the hit pattern in the EDC1.

3. Resolve left-right ambiguities and calculate the reaction point in each cell using the drift distance obtained by the drift time converted from the TDC analysis in the EDC1.

4. Fit the positions in each wire plane of the EDC1 with a line and obtain $x_{f}, y_{f}, x_{f}^{\prime}$ and $y_{f}^{\prime}$ only from the EDC1.

5. Select proper wire hits on the EDC2 layers. The hits far from the track determined by the EDC1 are rejected.

6. Fit the positions in the EDC1 and EDC2 with a line and obtain $x_{f}, y_{f}, x_{f}^{\prime}$ and $y_{f}^{\prime}$ again.

In particular, procedure 3 and 4 is dedicated to the analysis of the EDC1, because the EDC1 is a honeycomb cell type drift chamber and the drift point is not necessarily the center of the plane. In order to obtain the drift point, firstly the left-right ambiguity is solved assuming the drift point is the center of the plane using the hit information of the pair of the adjacent planes staggered by a half cell each other (procedure 3). After that, the tracking is performed by the fitting with a line and the drift point is obtained by the track. The track is obtained after the iteration of drift point calculation and tracking procedure (procedure 4). 


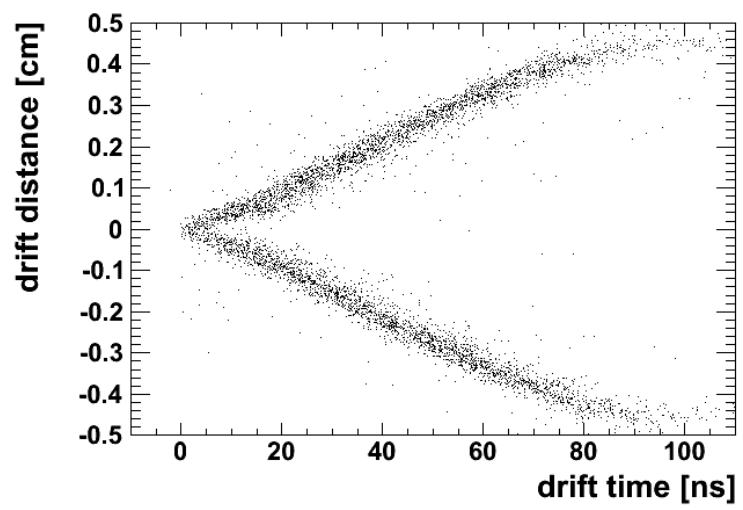

Figure 5.16: The relation drift time between drift length.
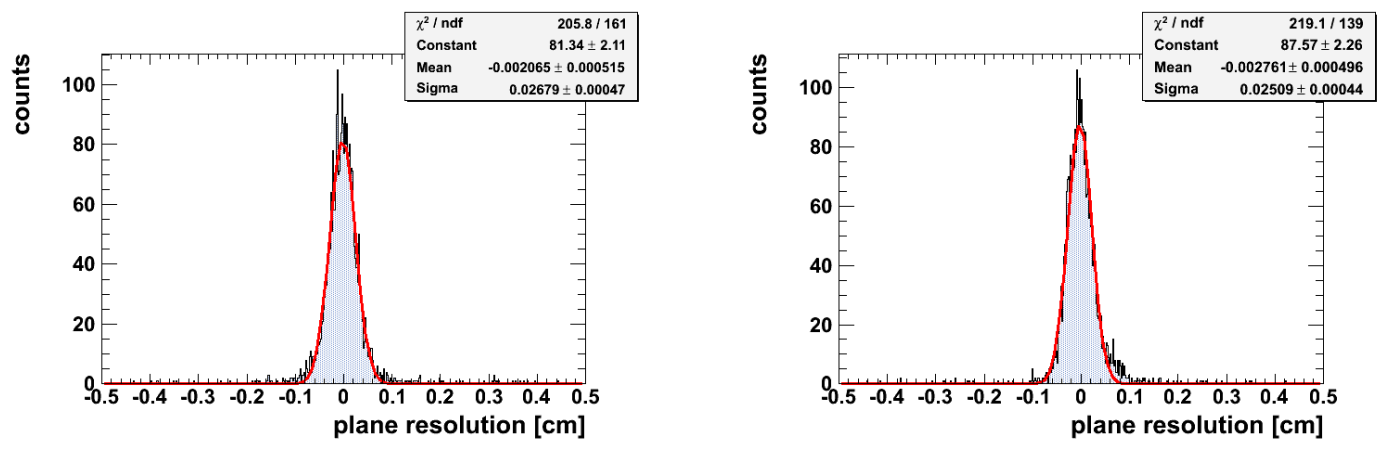

Figure 5.17: Residual of the EDC1 (left) and the EDC2 (right).

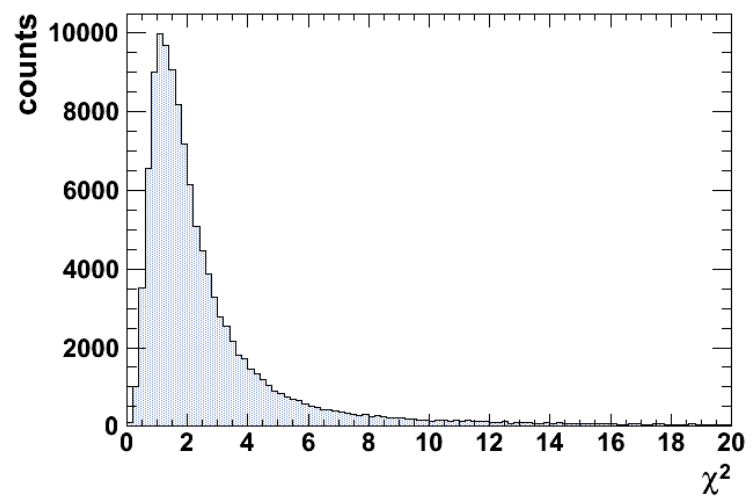

Figure 5.18: HES tracking $\chi^{2}$. 
Table 5.3.1: Calculated HES FP resolution from the experimental data $\left(\mathrm{CH}_{2}\right.$ run).

\begin{tabular}{c|c|c|c}
\hline \hline$\delta x$ & $\delta x^{\prime}$ & $\delta y$ & $\delta y^{\prime}$ \\
\hline $97[\mu \mathrm{m}]$ & $0.8[\mathrm{mrad}]$ & $120[\mu \mathrm{m}]$ & $1.5[\mathrm{mrad}]$ \\
\hline \hline
\end{tabular}

\subsection{Coincidence time analysis of HKS and HES}

\subsubsection{Coincidence time spectrum}

In order to identify true $\left(\mathrm{e}, \mathrm{e}^{\prime} \mathrm{K}^{+}\right)$reaction events, coincidence time (coin-time, $\left.t_{\text {coin }}\right)$ between HKS and HES have to be calculated as following;

$$
t_{\text {coin }}=t_{H K S}(\text { target })-t_{H E S}(\text { target }),
$$

where $t_{H K S}\left(\right.$ target) and $t_{H E S}($ target) are target time reconstructed from reference plane time of HKS and HES, respectively.

Figure 5.19 is a typical coin-time spectrum in narrower range and figure 5.20 is the correlation between $m^{2}$ distribution and coin-time in wider range. Since a bunch of the $\mathrm{CW}$ electron beam is delivered to the Hall $\mathrm{C}$ in every $2 \mathrm{~ns}$, a coin-time spectrum also shows the beam structure. One can see the 6 background peaks and the true coincidence peak on the a background peak in the center of figure 5.19. The background peaks are made from the accidental coincidence between the bremsstrahlung associated events in the HES and kaon events in the HKS. Therefore, we have to select the true coincidence peak in order to obtain the missing mass spectrum. The coin-time selection is as following (unit is ns):

$$
-1 \leq t_{\text {coin }} \leq 1
$$

Since the typical timing resolution in the coin-time spectrum is about $260 \mathrm{ps}$, the cut efficiency is more than $99 \%$.

Table 5.4.1: Kaon cut efficiencies by coin-time cut.

\begin{tabular}{ccc}
\hline \hline Data set & $\sigma$ of coin-time & Kaon cut eff. \\
\hline$\# 1$ & $0.29 \pm 0.002$ & $99.9 \pm 0.08$ \\
$\# 2$ & $0.30 \pm 0.004$ & $99.9 \pm 0.2$ \\
$\# 3$ & $0.28 \pm 0.001$ & $99.9 \pm 0.04$ \\
$\# 4$ & $0.28 \pm 0.003$ & $99.9 \pm 0.1$ \\
$\# 5$ & $0.26 \pm 0.001$ & $99.9 \pm 0.04$ \\
$\# 6, \# 7$ & $0.26 \pm 0.002$ & $99.9 \pm 0.08$ \\
$\# 8$ & $0.27 \pm 0.002$ & $99.9 \pm 0.08$ \\
$\# 9$ & $0.27 \pm 0.006$ & $99.9 \pm 0.2$ \\
$\# 10$ & $0.29 \pm 0.004$ & $99.9 \pm 0.2$ \\
\hline \hline
\end{tabular}




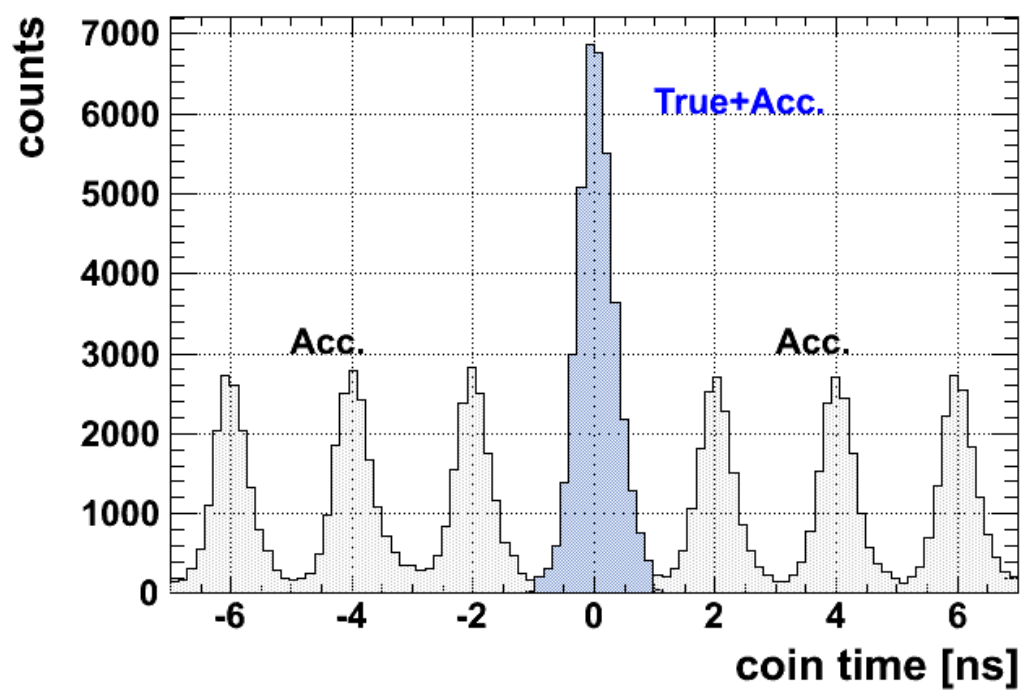

Figure 5.19: Typical coincidence time spectrum.

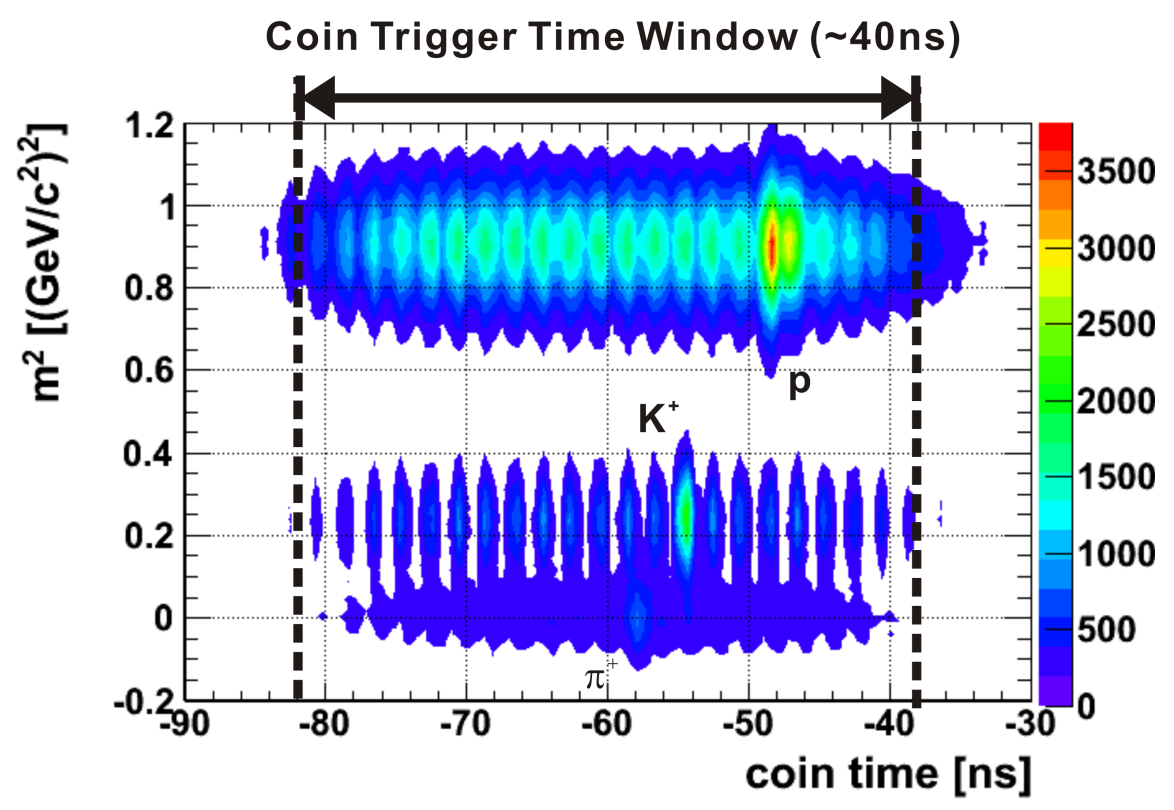

Figure 5.20: The correlation between coincidence time spectrum and $\mathrm{m}^{2}$. 


\subsubsection{Mixed event analysis for background estimation}

Even after selecting the true coincidence peak in 5.19, the accidental background events are remained in the missing mass spectrum. Basically the accidental background level can be estimated by the other bunch peaks and they are subtracted in the final missing mass spectrum. The number of the bunch peaks are limited because the finite trigger window, and the statistics are limited even if we select all peaks in the data.

In order to improve the statistic error of the missing mass due to the background subtraction, we increased the statistics of background event by using the mixed event analysis. Since these background is the random accidental background of the HKS and the HES, it is possible to increase the statistics by paring with the other bunch events, as shown in figure 5.21.

Figure 5.22 shows the result of mixed event analysis in a typical region of the missing mass. One can see the statistical error due to the statistics became drastically small.

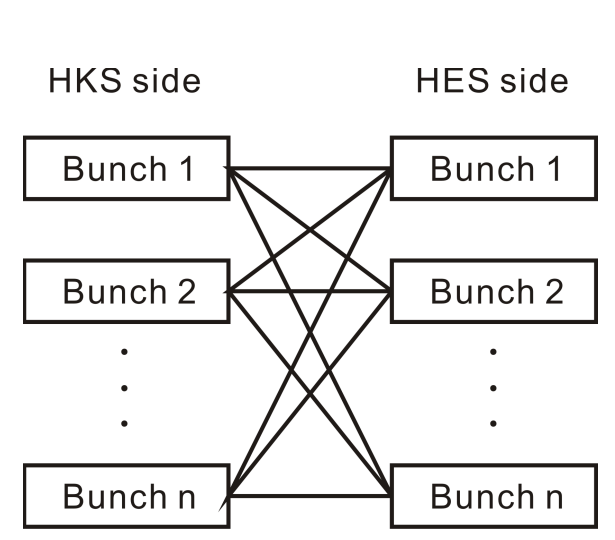

Figure 5.21: Basic idea of the mixed event analysis.

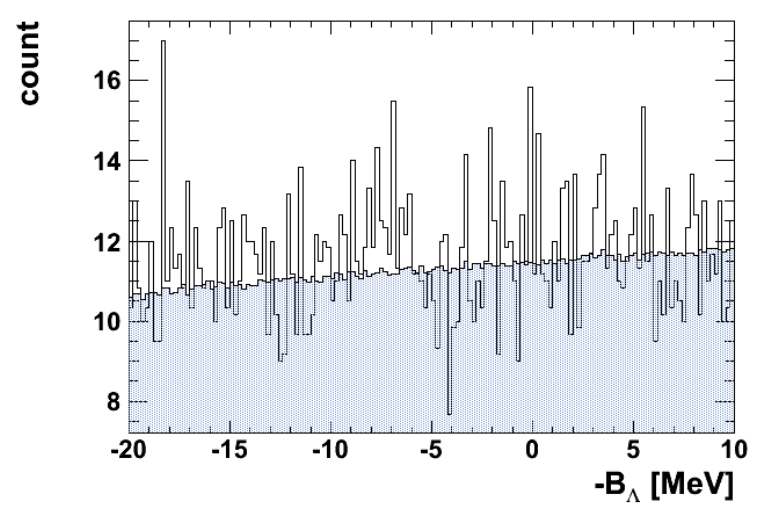

Figure 5.22: Result of the mixed event analysis. The hatched region shows the result of the mixed event analysis and another region is the result from normal background analysis. 


\subsection{Missing mass calibration}

The missing mass calibration is one of the most important process in this study. The procedure will be explained in this section after the calibration overview.

\subsubsection{Momentum reconstruction and calibration overview}

After applying the kaon cut and coin-time cut, missing mass can be obtained as follows:

$$
\begin{aligned}
M_{H Y} & =\sqrt{E_{H Y}^{2}-\vec{p}_{H Y}^{2}} \\
& =\sqrt{\left(E_{e}+M_{T}-E_{K}-E_{e^{\prime}}\right)^{2}-\left(\overrightarrow{p_{e}}-\overrightarrow{p_{K}}-\overrightarrow{p_{e^{\prime}}}\right)^{2}} .
\end{aligned}
$$

The subscripts $e, K$ and $e^{\prime}$ mean the electron beam, respectively, kaon and scattered electron. The three dimensional momentum vectors, $\overrightarrow{p_{e}}, \overrightarrow{p_{K}}$ and $\overrightarrow{p_{e^{\prime}}}$ can be described using the magnitudes and emission angles of the vectors:

$$
\begin{aligned}
\overrightarrow{p_{e}} & =\left(0,0, p_{e}\right), \\
\overrightarrow{p_{K}} & =\left(x_{K}^{\prime} \cdot p_{K z}, y_{K}^{\prime} \cdot p_{K z}, p_{K z}\right), \\
\overrightarrow{p_{e^{\prime}}} & =\left(x_{e^{\prime}}^{\prime} \cdot p_{e^{\prime} z}, y_{K}^{\prime} \cdot p_{e^{\prime}}, p_{e^{\prime}}\right),
\end{aligned}
$$

where $p_{K z}=\left|\overrightarrow{p_{K}}\right| / \sqrt{1+\left(x_{K}^{\prime}\right)^{2}+\left(y_{K}^{\prime}\right)^{2}}$ and $p_{e^{\prime} z}=\left|\overrightarrow{p_{e^{\prime}}}\right| / \sqrt{1+\left(x_{e^{\prime}}^{\prime}\right)^{2}+\left(y_{e^{\prime}}^{\prime}\right)^{2}}$. $p_{e}$ can be obtained by the accelerator information.

The six variables $p_{K}, x_{K}^{\prime}, y_{K}^{\prime}, p_{e^{\prime}}, x_{e^{\prime}}^{\prime}$ and $y_{e^{\prime}}^{\prime}$ which represent the particle momenta and emission angles have to be measured by the spectrometer system, but they cannot be measured directly. The relation between the momentum vector at target and the FP quantities can be written by using the first order transfer matrices $\mathcal{M}_{T 2 F}$, as below (see also chapter 4$)$ :

$$
\left(\begin{array}{c}
x_{f} \\
x_{f}^{\prime} \\
y_{f} \\
y_{f}^{\prime} \\
p
\end{array}\right)=\mathcal{M}_{T 2 F}\left(\begin{array}{c}
x_{t} \\
x_{t}^{\prime} \\
y_{t} \\
y_{t}^{\prime} \\
p
\end{array}\right) .
$$

The matrix $\mathcal{M}_{T 2 F}$ is nothing other than the so called transfer matrix. In the above equation, measured values are the FP quantities, then the target quantities have to be calculated from them using the relation. Therefore, the "inverse" matrix of the transfer matrix is necessary in order to obtain the target quantities, as following:

$$
\left(\begin{array}{c}
x_{t} \\
x_{t}^{\prime} \\
y_{t} \\
y_{t}^{\prime} \\
p
\end{array}\right)=\left(\mathcal{M}_{T 2 F}\right)^{-1}\left(\begin{array}{c}
x_{f} \\
x_{f}^{\prime} \\
y_{f} \\
y_{f}^{\prime} \\
p
\end{array}\right) .
$$

This is the basic idea how to reconstruct the momentum vector from the FP quantities. However, the actual optics of the HKS and HES cannot be expressed in such a simple way, and we need higher order transfer matrices in order to represent the magnetic field 
map in $10^{-4}$. The equations of this case can be represented as follows, corresponding to the equation 5.13 and 5.14 :

$$
\begin{gathered}
\left(\begin{array}{c}
x_{f} \\
x_{f}^{\prime} \\
y_{f} \\
y_{f}^{\prime}
\end{array}\right)=\mathcal{M}_{F 2 T}^{h i g h}\left(\begin{array}{c}
x_{t} \\
x_{t}^{\prime} \\
y_{t} \\
y_{t}^{\prime} \\
p \\
\left(x_{t}\right)^{2} \\
x_{t} \cdot x_{t}^{\prime} \\
\cdot \\
\cdot
\end{array}\right) . \\
\left(\begin{array}{c}
x_{t} \\
x_{t}^{\prime} \\
y_{t} \\
y_{t}^{\prime} \\
p
\end{array}\right)=\mathcal{M}_{T 2 F}^{h i g h}\left(\begin{array}{c}
x_{f} \\
x_{f}^{\prime} \\
y_{f} \\
y_{f}^{\prime} \\
\left(x_{f}\right)^{2} \\
x_{f} \cdot x_{f}^{\prime} \\
\cdot \\
\cdot
\end{array}\right) .
\end{gathered}
$$

In the above equations, matrices $\mathcal{M}_{F 2 T}^{\text {high }}$ and $\mathcal{M}_{T 2 F}^{\text {high }}$ is no longer the inverse matrix each other, because they are not regular matrices ${ }^{2}$.

In order to express the optics of the HKS and HES in $10^{-4}$ accuracy, the third order $\mathcal{M}_{F 2 T}^{\text {high }}$ is enough. However, in general, $\mathcal{M}_{T 2 F}^{\text {high }}$ is not necessary to be the same order, and it contains much higher order terms in this case.

The equation 5.16 can be written as follows, by using $n$th polynomials of $x_{f}, x_{f}^{\prime}, y_{f}$ and $y_{f}^{\prime}$. This expression was applied both for the HKS and HES side. This function is nothing other than the $F 2 T$ function and we adopted sixth order function for the precise description of the spectrometer optics.

$$
\begin{aligned}
p & =F 2 T_{p}\left(\vec{x}_{f}\right) \\
& =\sum_{\mu+\nu+\eta+\xi=0}^{n} C_{p}(\mu, \nu, \eta, \xi)\left(x_{f}\right)^{\mu}\left(x_{f}^{\prime}\right)^{\nu}\left(y_{f}\right)^{\eta}\left(y_{f}^{\prime}\right)^{\xi}, \\
x_{t}^{\prime} & =F 2 T_{x}\left(\vec{x}_{f}\right) \\
& =\sum_{\mu+\nu+\eta+\xi=0}^{n} C_{x}(\mu, \nu, \eta, \xi)\left(x_{f}\right)^{\mu}\left(x_{f}^{\prime}\right)^{\nu}\left(y_{f}\right)^{\eta}\left(y_{f}^{\prime}\right)^{\xi}, \\
y_{t}^{\prime} & =F 2 T_{y}\left(\vec{x}_{f}\right) \\
& =\sum_{\mu+\nu+\eta+\xi=0}^{n} C_{y}(\mu, \nu, \eta, \xi)\left(x_{f}\right)^{\mu}\left(x_{f}^{\prime}\right)^{\nu}\left(y_{f}\right)^{\eta}\left(y_{f}^{\prime}\right)^{\xi} .
\end{aligned}
$$

\footnotetext{
${ }^{2}$ This is also the reason to remove the momentum $p$ from the FP quantities. Conversely, the equation 5.13 and 5.14 contain $p$ in the FP quantities so as to be able to inverse each matrices.
} 
Table 5.5.1: The number of terms of the F2T function.

\begin{tabular}{c|cccccccc}
\hline \hline$n$ & 1 & 2 & 3 & 4 & 5 & 6 & 7 & 8 \\
\hline \# of terms & 5 & 15 & 35 & 70 & 126 & 210 & 330 & 495 \\
\hline \hline
\end{tabular}

The number of terms of a $n$th order $F 2 T$ function can be summarized as table 5.5.1. There are total six F2T functions (momentum, $x^{\prime}$ and $y^{\prime}$ for both side), the total number of terms is six times the number of this table (for example, there are 1260 terms for sixth order $F 2 T$ function). Since the coefficient of the functions will be tuned directly in the analysis procedure (see the following parts), the number of terms are concerned with the tuning time. In addition, it is required to be able to describe the spectrometer optics with the accuracy of $10^{-4}$. From these consideration, we determined $n$ as six.

The functions are initially obtained from the Geant4 simulation which includes the magnetic field map of the HKS and the HES and the detector geometries. Since the tuning result is depending on the initial $F 2 T$ function, they should be calculated as realistic as possible. However, the accuracy of field map obtained by finite element method is a few $\%$, the $F 2 T$ function tuning is essential for the missing mass with an accuracy of $10^{-4}$ order.

In this analysis, the $F 2 T$ functions were tuned by the following procedure.

1. Firstly, we tuned the geometry in the Geant 4 simulation. The geometry of magnets and detectors are measured with an accuracy of $100 \mu \mathrm{m}$ order by JLab survey group, and the information was reflected in the simulation.

2. Secondly, the magnetic parameters were tuned in the Geant4 simulation, looking at the SS pattern and $\Lambda / \Sigma^{0}$ peaks. Here magnetic parameters mean the magnitude of the field and the shape of the fringe field.

3. Direct tuning of $F 2 T$ function terms.

In order to do these tunings, the corrections of target energy loss and beam raster are need to be done. After the description of the corrections, the detail of these tuning procedure will be explained. After that, the systematic error estimation of the tuned missing mass spectrum will be done, using the simulated data from Geant4 simulation.

\subsubsection{Target energy loss correction}

The energy loss correction in a experimental target is essential for the calculation of missing mass spectrum. The energy loss was simulated using the Geant4 as shown in figure 5.23 and the results are summarized in table 5.5.2. In the table, the mean value of energy loss and the energy struggling are described. When we calculate missing mass spectrum, the mean values are added to the scattered electron energy and kaon energy and subtracted from beam energy:

$$
E_{\text {beam }}=E_{\text {beam }}^{\text {measured }}-\Delta E_{\text {beam }},
$$




$$
\begin{aligned}
E_{e^{\prime}} & =E_{e^{\prime}}^{\text {measured }}-\Delta E_{e^{\prime}}, \\
E_{K^{+}} & =E_{K^{+}}^{\text {measured }}-\Delta E_{K^{+}} .
\end{aligned}
$$

$E_{\text {beam }}, E_{e^{\prime}}$ and $E_{K^{+}}$is used for the missing mass calculation.

Since $\mathrm{CH}_{2}$ and $\mathrm{H}_{2} \mathrm{O}$ targets are thick compared to the other targets, the energy loss and energy struggling are larger than these of other targets. For the hypernuclear production target, the energy loss value is around a $100 \mathrm{keV}$ and the energy struggling is smaller than $100 \mathrm{keV}$. These values does not affect to the resolution of missing mass because they are much smaller than the resolution of the spectrometers.

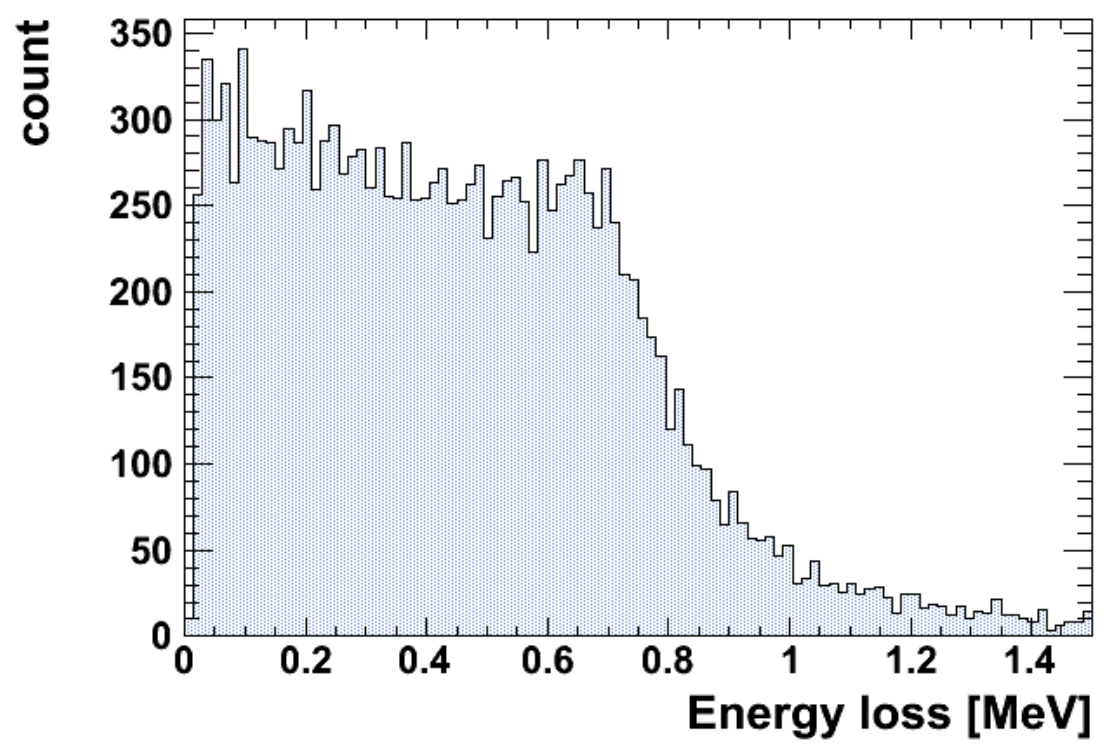

Figure 5.23: Simulated energy loss spectrum in $\mathrm{CH}_{2}$ target.

\subsubsection{Raster correction}

As mentioned above, the F2T functions are calculated assuming the point-target optics. However, the beam raster was performed in $\mathrm{CH}_{2}$ and ${ }^{7} \mathrm{Li}$ target (see section 3.1).

The effect was estimated with the experimental data of $\mathrm{CH}_{2}$ target. Figure 5.24 is the correlation between the missing mass around $\Lambda$ peak and $x$ position at target. There is linear correlation between the $\Lambda$ peak and the $x$.

After fitting this correlation with the first polynomial and applied the function to the real data. Figure 5.25 shows the result for the $\Lambda$ and $\Sigma^{0}$ peaks. The widths of $\Lambda$ and $\Sigma^{0}$ peak were improved from $3.0 \mathrm{MeV}$ (FWHM) to $2.5 \mathrm{MeV}$ (FWHM) and $3.7 \mathrm{MeV}$ (FWHM) to $2.9 \mathrm{MeV}$ (FWHM). 
Table 5.5.2: The result of energy loss calculation in several targets. The energy loss $(\Delta E)$ and energy struggling ( $\delta \Delta E$ ) values are estimated using Geant4 simulation.

\begin{tabular}{|c|c|c|c|c|}
\hline Target & $\begin{array}{l}\text { Thickness } \\
{\left[\mathrm{mg} / \mathrm{cm}^{2}\right]}\end{array}$ & $\begin{array}{l}\text { Density } \\
{\left[\mathrm{g} / \mathrm{cm}^{3}\right]}\end{array}$ & $\begin{array}{l}\Delta E_{K^{+}} \\
{[\mathrm{keV}]}\end{array}$ & $\begin{array}{c}\delta \Delta E_{K^{+}}(\mathrm{RMS}) \\
{[\mathrm{keV}]}\end{array}$ \\
\hline $\mathrm{CH}_{2}$ & 450.8 & 0.92 & 502 & 305 \\
\hline $\mathrm{H}_{2} \mathrm{O}$ & 500 & 1.00 & 487 & 295 \\
\hline${ }^{7} \mathrm{Li}$ & 184.0 & 0.54 & 151 & 95 \\
\hline${ }^{9} \mathrm{Be}$ & 188.1 & 1.848 & 153 & 95 \\
\hline${ }^{10} \mathrm{~B}$ & 56.1 & 2.16 & 52 & 43 \\
\hline${ }^{n a t} \mathrm{C}$ & 112.5 & 2.25 & 103 & 70 \\
\hline \multirow[t]{2}{*}{${ }^{52} \mathrm{Cr}$} & 134.0 & 7.15 & 99 & 67 \\
\hline & \multicolumn{2}{|l|}{154.0} & 113 & 73 \\
\hline$\overline{\text { Target }}$ & \multicolumn{2}{|c|}{$\begin{array}{c}\delta \Delta E_{e^{\prime}}(\mathrm{RMS}) \\
{[\mathrm{keV}]}\end{array}$} & $\begin{array}{c}\Delta E_{\text {Beam }} \\
{[\mathrm{keV}]}\end{array}$ & $\begin{array}{c}\delta \Delta E_{\text {Beam }}(\mathrm{RMS}) \\
{[\mathrm{keV}]}\end{array}$ \\
\hline $\mathrm{CH}_{2}$ & \multicolumn{2}{|c|}{282} & 436 & 2.81 \\
\hline $\mathrm{H}_{2} \mathrm{O}$ & \multicolumn{2}{|c|}{285} & 439 & 283 \\
\hline${ }^{7} \mathrm{Li}$ & \multicolumn{2}{|l|}{133} & 133 & 86 \\
\hline${ }^{9} \mathrm{Be}$ & \multicolumn{2}{|l|}{133} & 133 & 86 \\
\hline${ }^{10} \mathrm{~B}$ & \multicolumn{2}{|l|}{45} & 45 & 40 \\
\hline${ }^{n a t} \mathrm{C}$ & \multicolumn{2}{|l|}{90} & 89 & 63 \\
\hline \multirow[t]{2}{*}{${ }^{52} \mathrm{Cr}$} & 94 & 68 & 93 & 67 \\
\hline & \multicolumn{2}{|l|}{107} & 107 & 72 \\
\hline
\end{tabular}




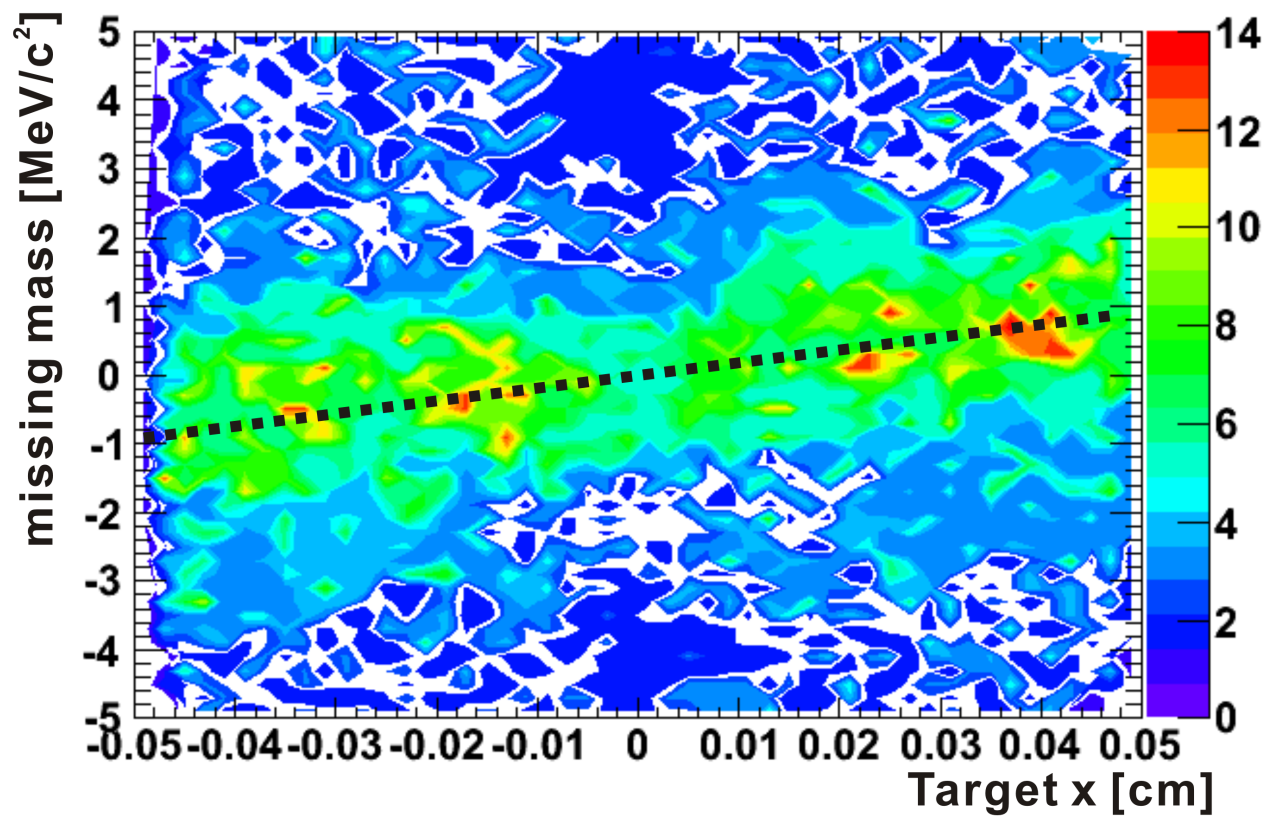

Figure 5.24: Correlation between missing mass $(\Lambda)$ and $x$ at target.
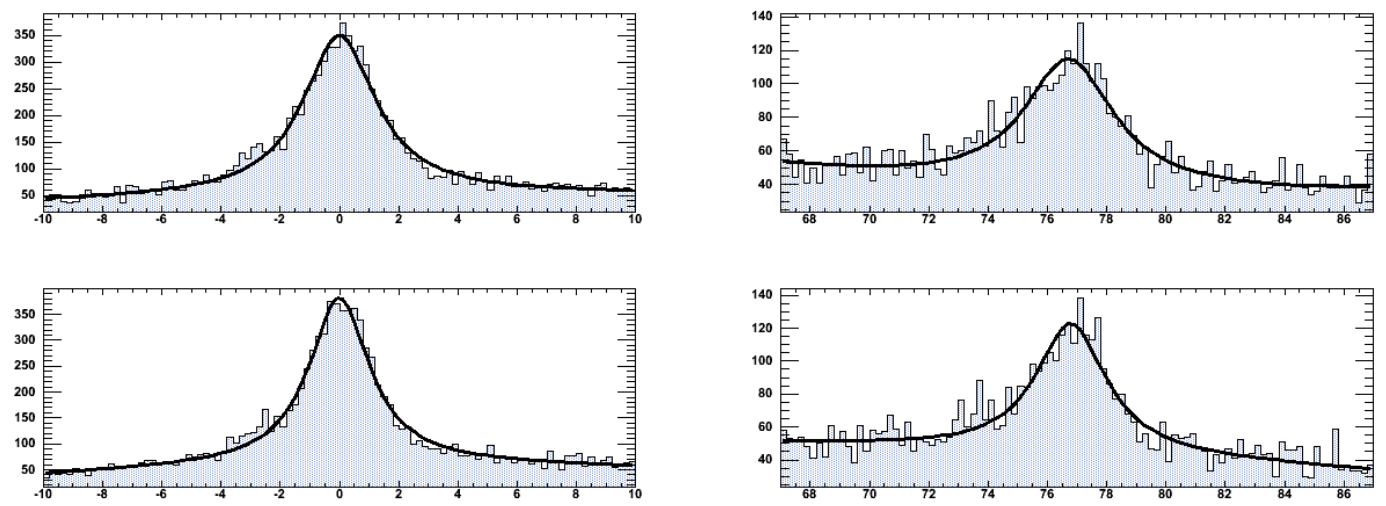

Figure 5.25: Raster correction effect on $\Lambda$ (left) and $\Sigma^{0}$ (right) peak. The top/bottom of each figure is the spectrum before/after correction. 


\subsubsection{Magnetic field improvement with the Sieve Slit data}

As mentioned in the first part of this section, the improvement of magnetic field in the Geant4 simulation has significant roles on the whole calibration procedure. The direct tuning of the $F 2 T$ function possesses an element of risk to distort the linearity of the mass scale. In order to avoid the risk, it is essential to make the "initial" $F 2 T$ function as realistic as possible before starting the direct tuning, because the linearity from $F 2 T$ function made by the real magnetic field is much more reliable than the directly tuned F2T function. However, The magnetic field map obtained by TOSCA not necessary reproduce the actual field map with a required accuracy. In addition, it is difficult to modify the magnet model in TOSCA so as to reproduce the actual field map, because the modification needs accurate measurement of the magnetic field.

Therefore, we used the information of the particle itself which path through the actual magnetic field. We have mainly two references, one is the peak of $\Lambda$ and $\Sigma^{0}$ and another is the Sieve Slit (SS) placed in the HKS and HES (see section 3.5 and 3.7). We modified directly the shape and magnitude of magnetic field map carefully looking at the peaks and the SS hole pattern.

The particle position at SS can be reconstructed from the momentum vector at target. The reconstruction is done by a "T2S" function, just in a similar way with that of $F 2 T$ function as follows:

$$
\begin{aligned}
x_{S} & =T 2 S_{x}\left(\vec{x}_{t}\right) \\
& =\sum_{\mu+\nu+\eta=0}^{n} C_{x}^{T 2 S}(\mu, \nu, \eta)\left(x_{t}^{\prime}\right)^{\mu}\left(y_{t}^{\prime}\right)^{\nu}\left(p_{t}\right)^{\eta}, \\
y_{S} & =T 2 S_{y}\left(\vec{x}_{t}\right) \\
& =\sum_{\mu+\nu+\eta=0}^{n} C_{y}^{T 2 S}(\mu, \nu, \eta)\left(x_{t}^{\prime}\right)^{\mu}\left(y_{t}^{\prime}\right)^{\nu}\left(p_{t}\right)^{\eta} .
\end{aligned}
$$

Here the order of the function was $n=3$. The $T 2 S$ function was also obtained by the Geant4 simulation, mainly depending on the magnetic field of the SPL. The function was used without tuning.

For the magnetic field calibration, we modified the field map shape of the KQ1 and EQ1 magnets and the magnitudes of the other magnets. In actual case, the shape of the all magnetic fields are different from the calculated one, then it is ideal to modify all magnetic fields. However, in this case, the modification of only Q1 magnet is enough to reconstruct the SS hole pattern and $\Lambda / \Sigma^{0}$ peaks, because the purpose of the modification is to obtain the initial $F 2 T$ function for the direct tuning and the necessary order of the accuracy is about $10^{-3}$ level. In fact, the modified "Q1" magnetic field absorb the necessary modification of the each spectrometer. Moreover, the effect of the Q1 magnetic field shape on the SS pattern is easy to see because the both SS is placed just before the Q1 magnet.

The Q1 magnetic field was modified by applying changing the $B_{x}$ and $B_{y}$ component ( $y$ is the vertical axis, $z$ is the beam direction and then $x$ is determined by the righthanded coordinate system, see figure 5.26). The $B_{x}$ was scaled by a function of $x$, such as

$$
B_{x}^{\text {mod. }}=B_{x} \cdot f(x)
$$


where $f(x)$ is a second polynomial function. As a result of this modification, the $B_{x}$ was modified maximumly about $10 \%$ at the edge of the field. In addition, since there were asymmetry which may coming from the fringe field of the SPL magnet in the HKS side, the $f(x)$ for the KQ1 has also asymmetry for $x$ direction. The magnitudes of the other magnets are modified maximumly $3 \%$ from the calculated magnetic field.

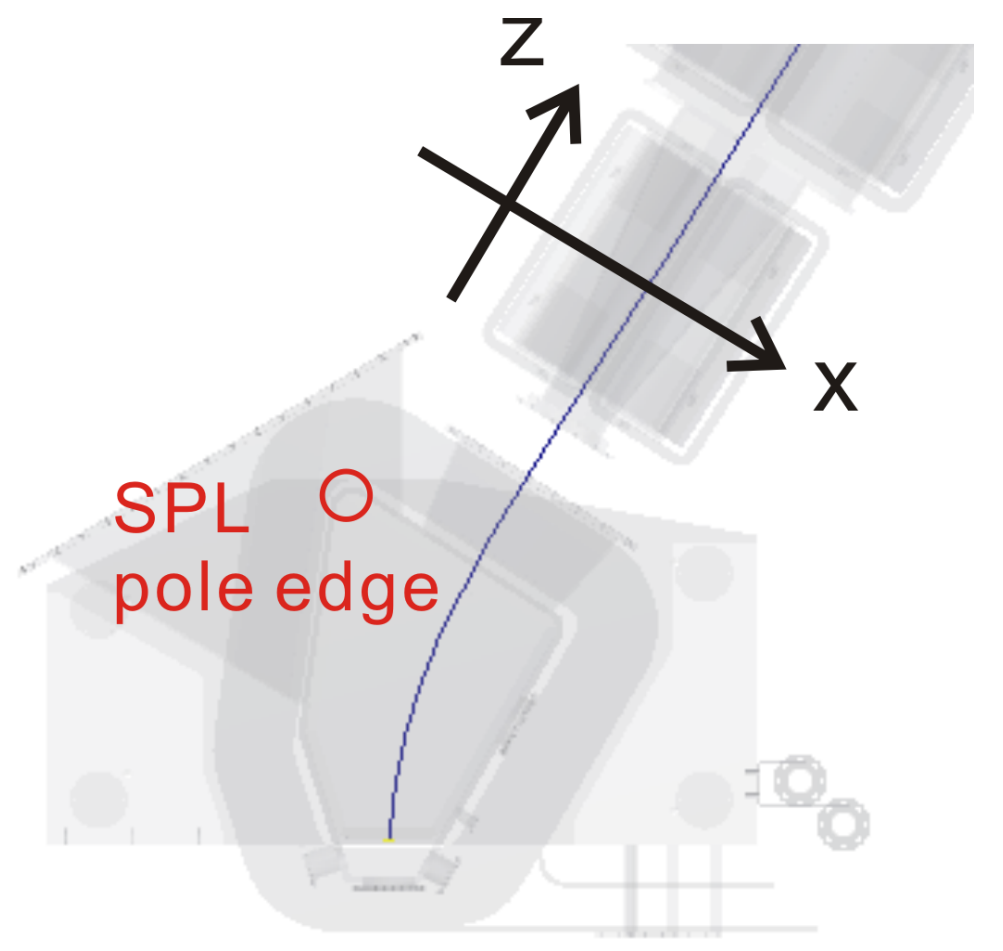

Figure 5.26: Position of the SPL and KQ1 magnet.

Figure 5.27 and 5.28 show the reconstructed SS pattern with the modified magnetic field map in HKS and HES, respectively. The red circles show the actually position of the hole. One can see the SS position was reconstructed with the a resolution which is better than the SS hole size. The occupied holes are also clearly seen, then the left-right ambiguity is solved and the offset of the pattern is well adjusted.

From this result, it is possible to estimate the angular resolution of the particle at target position. Firstly, the position resolution at SS $\left(\delta\left(x_{S S}\right)\right.$ and $\left.\delta\left(y_{S S}\right)\right)$ was calculated as follows:

$$
\begin{aligned}
& \delta\left(x_{S S}\right)=x_{S S}-x_{S S c e n t} \\
& \delta\left(y_{S S}\right)=y_{S S}-y_{S S c e n t}
\end{aligned}
$$

where $x_{\text {SScent }}\left(y_{S \text { Scent }}\right)$ is a center coordinate of corresponding SS holes for the $x(y)$ direction. The obtained results are summarized in table 5.5.3. Next this position resolution can be transformed by the inverse function of the $T 2 S$ function. Here the $T 2 S$ function is assumed as the first polynomial, which was derived as following equations, 

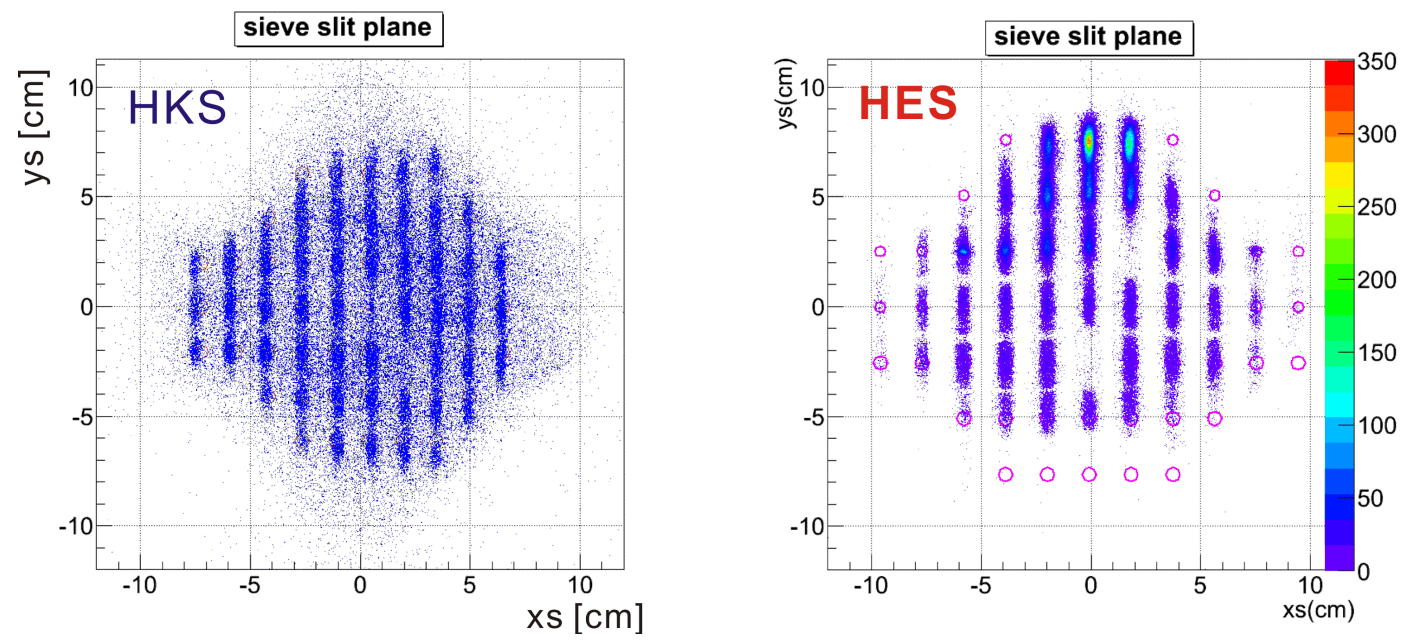

Figure 5.27: The modified HKS SS pattern. Figure 5.28: The modified HES SS pattern.

$$
\begin{aligned}
& x_{K S S}=145 \cdot x_{K^{+}}^{\prime}+37 \cdot p_{K^{+}}, \\
& y_{K S S}=152 \cdot y_{K^{+}}^{\prime} \\
& x_{E S S}=113 \cdot x_{e^{\prime}}^{\prime}+83 \cdot p_{e^{\prime}} \\
& y_{E S S}=123 \cdot y_{e^{\prime}}^{\prime}
\end{aligned}
$$

From these equation, and the SS position equation, the angular resolutions were derived as table 5.5.3. The resolution of the HKS $y^{\prime}$ is not good, but its effect on the missing mass resolution is smaller than that of $x^{\prime}$, especially in hypernuclear case (see section 6.1). The target of SS run was ${ }^{n a t} \mathrm{C}$ with a thickness of about $100 \mathrm{mg} / \mathrm{cm}^{2}$, and the multiple scattering effect in the target is considered to be about $0.8 \mathrm{mrad}$. These values include this effect, but the effect is negligibly small, except for the HKS $x^{\prime}$.

Table 5.5.3: Obtained angular resolution from the SS data. All resolution values are written in rms.

\begin{tabular}{c|c|c}
\hline \hline Item & SS pos. resolution $[\mathrm{cm}]$ & Ang. resolution $[\mathrm{mrad}]$ \\
\hline HKS $x^{\prime}$ & 0.16 & 1 \\
HKS $y^{\prime}$ & 1.52 & 10 \\
HES $x^{\prime}$ & 0.34 & 3 \\
HES $y^{\prime}$ & 0.35 & 3 \\
\hline \hline
\end{tabular}

\subsubsection{Direct calibration of the reconstruction function}

After the magnetic field calibration, the terms of the $F 2 T$ functions are directly calibrated with a non-linear least square method, using the known masses of $\Lambda$ and $\Sigma^{0}$ obtained by 
$\mathrm{CH}_{2}$ target run and the mass of ${ }_{\Lambda}^{12} \mathrm{~B}$ ground state (gs) obtained by ${ }^{12} \mathrm{C}$ target run. In this step, only the momentum part of the $F 2 T$ functions were tuned and the angular parts of the F2T functions were used without tuned. This is because the weight for the momentum become heavier than that of the angular part, because the effect of the angular part on the MM is smaller than that of the momentum part especially in the hypernuclear MM.

The calibration is performed by minimizing $\chi^{2}$ defined by equation $5.33,5.34$ and 5.35 for $\Lambda, \Sigma^{0}$ and gs peak, respectively.

$$
\begin{aligned}
\chi_{\Lambda}^{2} & =\frac{1}{2} \frac{w_{\Lambda}}{(D o F)_{\Lambda}} \sum_{i}^{N_{\Lambda}}\left(\frac{M_{i}^{\text {calc }}-M_{\Lambda}^{P D G}}{\sigma_{i}}\right)^{2} \\
\chi_{\Sigma}^{2} & =\frac{1}{2} \frac{w_{\Sigma}}{(D o F)_{\Sigma}} \sum_{i}^{N_{\Sigma}}\left(\frac{M_{i}^{\text {calc }}-M_{\Sigma}^{P D G}}{\sigma_{i}}\right)^{2} \\
\chi_{g s}^{2} & =\frac{1}{2} \frac{w_{g s}}{(D o F)_{g s}} \sum_{i}^{N_{g s}}\left(\frac{M_{i}^{\text {calc }}-M_{g s}}{\sigma_{i}}\right)^{2}
\end{aligned}
$$

In the equation, $(D o F)$ is the degree of freedom and $N$ is the number of events used for this $\chi^{2}$ calculation. $w$ is the weight of each $\chi^{2}$ which is necessary for the summing up $\chi^{2}$. In the present study, $w_{\Lambda}=w_{\Sigma}=w_{g a}=1 . M_{i}^{\text {calc }}$ is a missing mass value calculated from measured value using $F 2 T$ function. $M_{P D G}$ is the known mass referred from particle data group [68], for $\chi_{\Lambda}^{2}$ and $\chi_{\Sigma}^{2} . M_{g s}$ is the mass of the gs, which is not well-known but measured several times in the past experiments. The treatment of $M_{g s}$ will be explained in the following part.

For the $\chi_{\Lambda}^{2}$, the equation 5.33 was calculated for the several region of momentum and angular acceptance, as shown in figure 5.29. Therefore finally there were total $18 \chi^{2}$. They were summed up as

$$
\chi_{\Lambda}^{\prime 2}=\frac{1}{18} \sum_{i=0}^{18}\left(\chi_{\Lambda}^{2}\right)_{i}
$$

The reason to divide the $\chi^{2}$ is to avoid the distortion of $F 2 T$ functions in the edge of the acceptance. In the present case, the events are converged on the center of the acceptance (see figure 5.29). Then, if there is only single $\chi^{2}$, the "weight" of the events in the edge region will become small and the $\chi^{2}$ of the edge region is not necessary correct. It is better to apply this method to other peaks ( $\Sigma^{0}$ and gs), but their statistics were not enough to apply this method.

The optimization procedure is as shown in figure 5.30. The explanations or each part of the procedure are as follows:

1. Firstly, the $\Lambda, \Sigma^{0}$ and gs peaks are fitted with a Gaussian and $\pm 2 \sigma$ region of each peak was selected.

2. The minimization of $\chi^{2}=\chi_{\Lambda}^{2}+\chi_{\Sigma}^{2}+\chi_{g s}^{2}$ was iterated for ten times for selected events and ten $F 2 T$ functions are obtained during this iteration.

3. The width of the gs was checked by a single Gaussian fitting for the ten spectra made by the ten $F 2 T$ functions. After that, the $F 2 T$ function which made the gs peak narrowest was selected. 

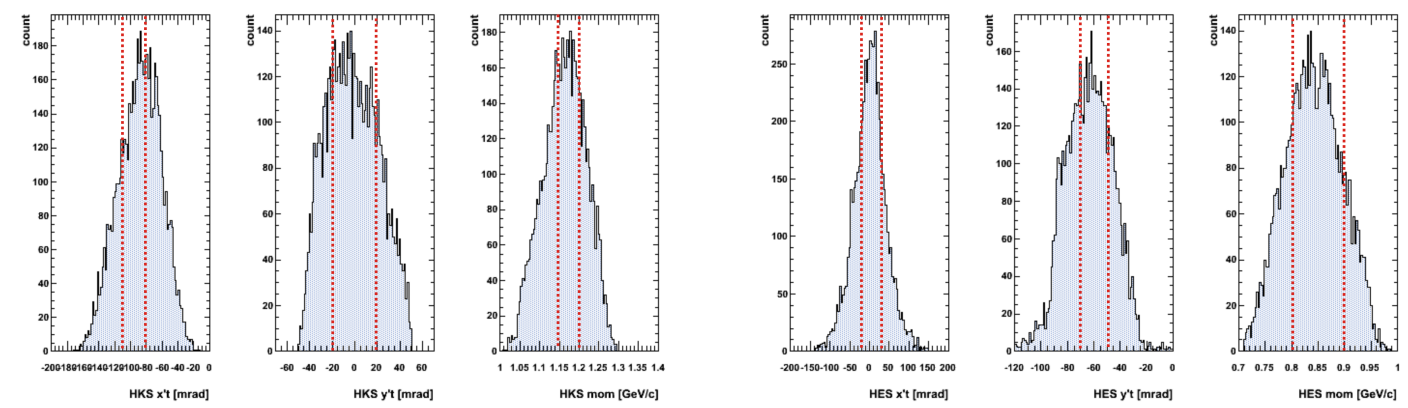

Figure 5.29: The acceptance division of HKS (left) and HES (right) for the $\chi^{2}$ calculation.

4. The procedure 1-3 is iterated for the selected F2T functions. This procedure is iterated until the finest gs peak was obtained.

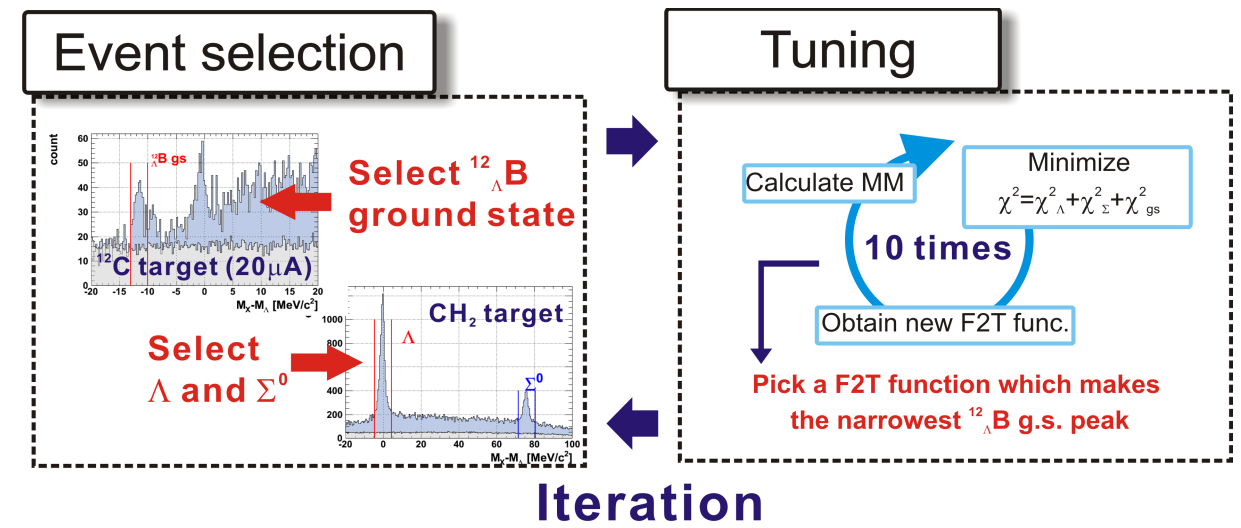

Figure 5.30: Procedure of momentum tuning.

\subsubsection{Systematic errors of mass scale}

\section{Calibration with simulation data (CSD)}

The calibration procedure discussed above was applied to the real $\mathrm{CH}_{2}$ and ${ }^{12} \mathrm{C}$ target data. However, it is very difficult to estimate how effective the calibration procedure works; how the systematic error of the mass scale and cross sections affected from the calibration procedure. In order to solve the problem, the calibration procedure was applied to the simulation data created by Geant4. The followings are the conditions of the calibration with simulation data (call this as "CSD") and its flowchart is shown in figure 5.31 .

1. For $\Lambda$ and $\Sigma^{0}$, the published PDG mass values were used (see Appendix A). The mass of hypernucleus, the mass of ${ }_{\Lambda}^{12} \mathrm{~B}$ in this case, was defined arbitrary and the value can be compared with that obtained from the calibration result. 
2. Momentum vectors corresponds to the particle peaks were calculated from the momentum and energy conservation laws so as to create the peaks.

3. The background events were generated from the random coincidence between the following two event groups. One corresponds to the HKS events which was generated uniformly at target and detected at HKS FP. Another one is the HES events generated in a similar way. However, particles were generated so as to be associated with virtual photon.

4. FP quantities were obtained by the HKS and HES Geant4 simulation system with the momentum vectors at target. The Geant4 simulation includes magnetic field, target and detector materials and expected position and angle resolutions at FP. This momentum vectors and FP quantities were treated as one data set.

5. There were two types of data set. One is a "nominal" condition data set (data N) whose FP quantities were used for the calibration. Another is a "deformed" data set (data D) which was created with arbitrary distorted experimental conditions such as magnetic fields and detector positions. The data D was used only for calculate the deformed initial F2T function used for the calibration. The degree of the deformation was decided so as to reflect the uncertainty of our "real" experimental condition. For example, the uncertainty of the magnetic field is about the order of $10^{-2}$ level, therefore the magnetic field was deformed by a few $\%$ in the data D.

Figure 5.5.6 represents the CSD results with the simulated ${ }_{\Lambda}^{12} \mathrm{~B}$ spectrum. As shown in the figure, some of the events moved significantly and leaked out from the "real" peak position and this leakage affect on the cross section of the events. Table 5.5.4 is a summarized ratio of the leakage which is determined as the ratio of the number of counted event and the number of generated event. From these results, the leakage effect was determined as $96 \pm 1 \%$ for $\Lambda$ and $\Sigma^{0}$, and $91 \pm 2 \%$ for the other hypernuclear states.

It is also important to estimate how accurately the missing mass scale was calibrated. This accuracy can be estimated by

$$
\delta M M=M M_{\text {tuned }}-M M_{\text {gen }},
$$

where $M M_{\text {tuned }}$ is the tuned missing mass and $M M_{\text {gen }}$ is the "ideal" missing mass position which was determined when the event was generated. Figure 5.33 shows the $\delta M M$ distribution for the $-B_{\Lambda}$ calculated by the ${ }^{12} \mathrm{C}$ target kinematics. The bound region of the hypernuclei is roughly from $-20 \mathrm{MeV}$ to $0 \mathrm{MeV}$, and the $\delta M M$ distribution in this region shows the accuracy of the missing mass scale. From this result, the accuracy was determined as $0.2 \mathrm{MeV}$ and this will be applied as the systematic error of the mass scale. 


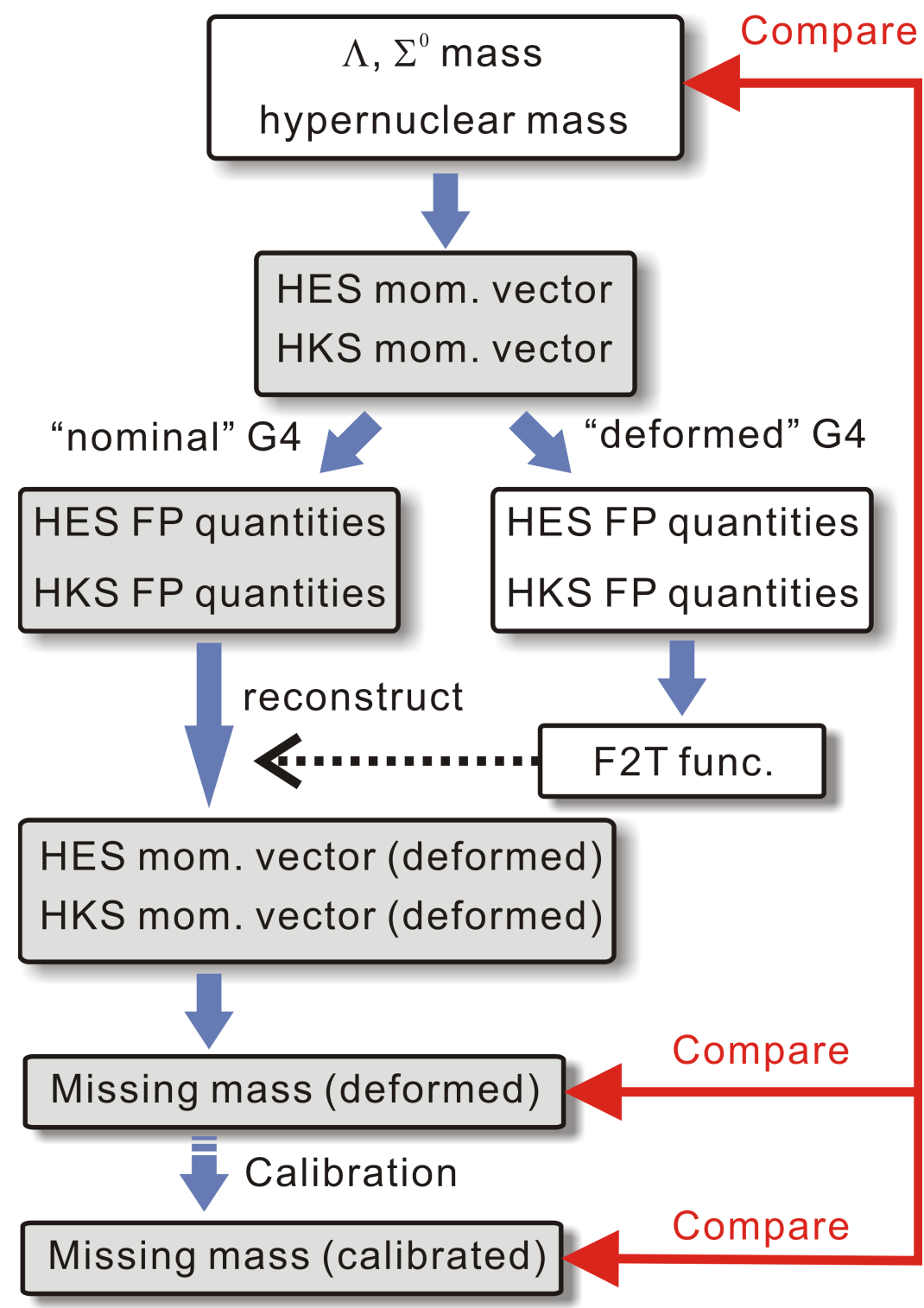

Figure 5.31: Flowchart of simulation data analysis.

Table 5.5.4: The leakage effect for each peak.

\begin{tabular}{c|c|c|c|c}
\hline \hline & $\Lambda$ & $\Sigma$ & ${ }_{\Lambda}^{12} \mathrm{~B}$ peak 1 & ${ }_{\Lambda}^{12} \mathrm{~B}$ peak 2 \\
\hline Generated & 4000 & 1000 & 350 & 350 \\
Counted & 3844 & 964 & 311 & 324 \\
\hline Ratio & 0.96 & 0.96 & 0.89 & 0.93 \\
\hline \hline
\end{tabular}



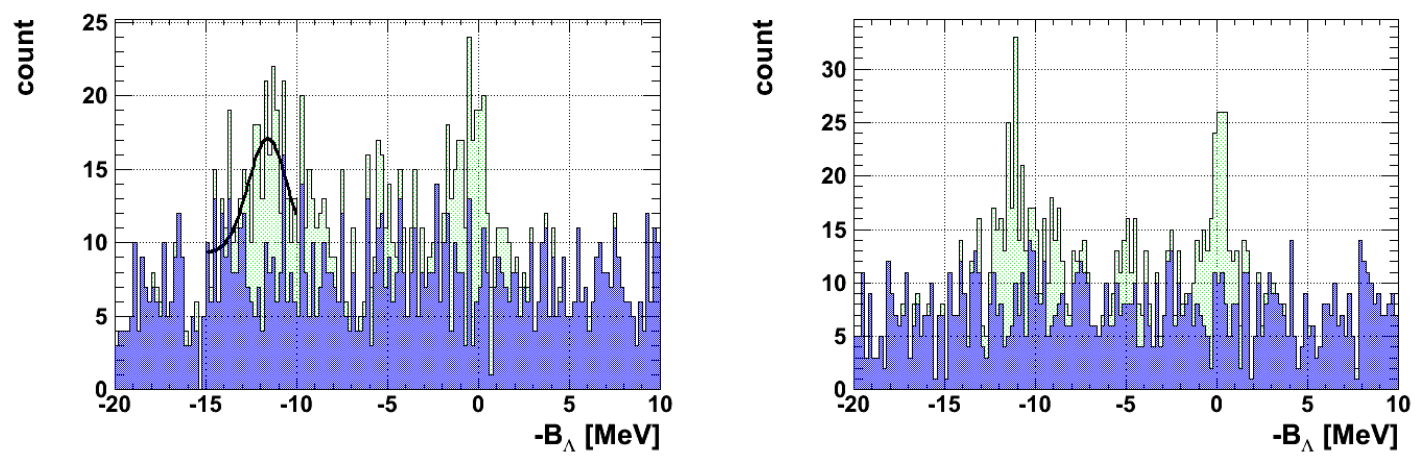

Figure 5.32: The result of the calibration with simulated ${ }_{\Lambda}^{12} \mathrm{~B}$. The left one is a missing mass with distorted $F 2 T$ function. The right one is a missing mass after tuning.

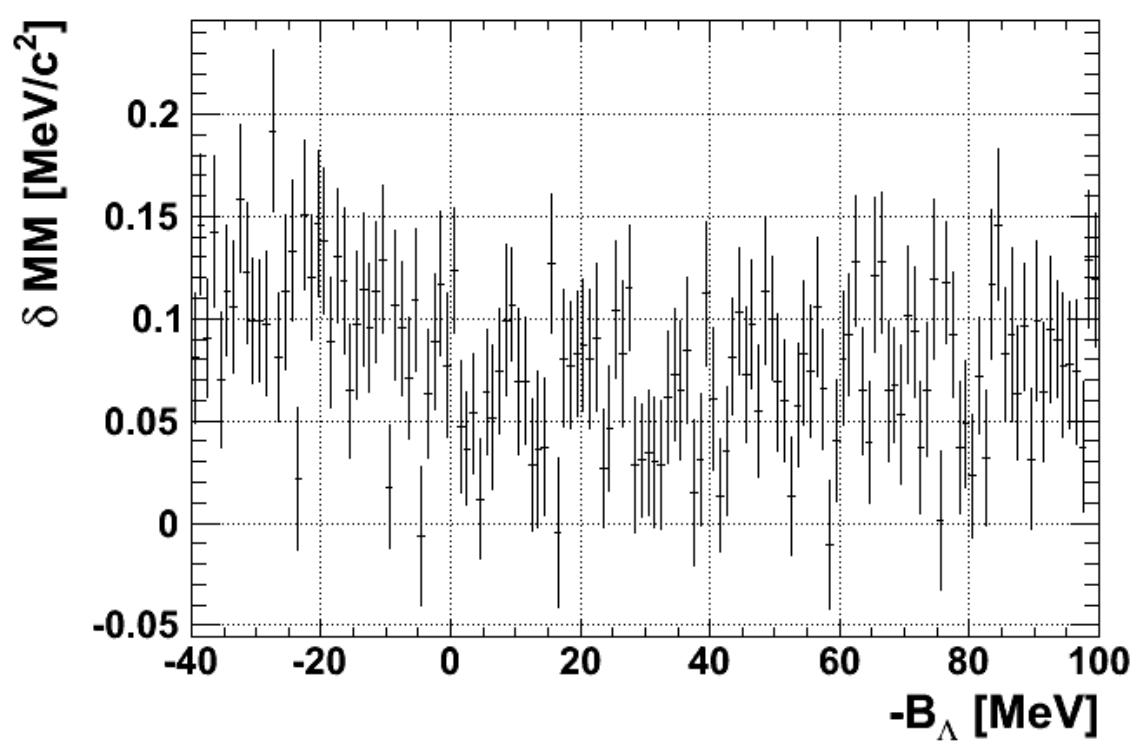

Figure 5.33: Correlation between $\delta \mathrm{MM}$ vs missing mass. 


\subsection{Cross section}

As already discussed in section 2.1, two types of differential cross section can be deduced from the experimental yields, one is the cross section for the $\left(\gamma^{*}, \mathrm{~K}^{+}\right)$reaction and another is that for the $\left(\mathrm{e}, \mathrm{e}^{\prime} \mathrm{K}^{+}\right)$reaction.

For the $\left(\gamma^{*}, \mathrm{~K}^{+}\right)$reaction, the averaged differential cross section over an arbitrary acceptance of kaon and scattered electron can be deduced from experimental yields as follows:

$$
\begin{aligned}
\left.\overline{\left(\frac{d \sigma}{d \Omega_{K}}\right)}\right|_{1^{\circ}-13^{\circ}} & =\frac{\int_{H K S} d \Omega_{K} \frac{d \sigma}{d \Omega_{K}}}{\int_{H K S} d \Omega_{K}} \\
& =\frac{1}{N_{T}} \frac{1}{N_{\gamma^{*}}} \frac{1}{\epsilon^{\text {common }}} \sum_{i}^{N_{H Y}} \frac{1}{\epsilon_{i}^{H E S} \epsilon_{i}^{H K S} \Delta \Omega_{i}^{H K S}} .
\end{aligned}
$$

For the $\left(\mathrm{e}, \mathrm{e}^{\prime} \mathrm{K}^{+}\right)$reaction, the triple differential cross section is also obtained from experimental yields as follows substituting $N_{\gamma}$ by $N_{e}$, the number of incoming electrons:

$$
\begin{aligned}
\overline{\left(\frac{d^{3} \sigma}{d E_{e^{\prime}} d \Omega_{e^{\prime}} d \Omega_{K}}\right)} & =\frac{\int_{H E S} d E \int_{H E S} d \Omega_{e^{\prime}} \int_{H K S} d \Omega_{K} \frac{d^{3} \sigma}{d E_{e^{\prime}} d \Omega_{e^{\prime}} d \Omega_{K}}}{\int_{H E S} d E \int_{H E S} \Delta \Omega_{e^{\prime}} \int_{H K S} d \Omega_{K}} \\
& =\frac{1}{N_{T}} \frac{1}{N_{e} \Delta E_{e^{\prime}}} \frac{1}{\epsilon^{\text {common }}} \sum_{i}^{N_{H Y}} \frac{1}{\epsilon_{i}^{H K S} \Delta \Omega_{i}^{H K S} \epsilon_{i}^{H E S} \Delta \Omega_{i}^{H E S}}
\end{aligned}
$$

This cross section is also averaged over an arbitrary acceptance. The explanations of the values in the above equation are as follows.

- $N_{T}$ is the number of target nuclei which calculated from the target thickness and material as following:

$$
N_{T}=\frac{T N_{A}}{A},
$$

where $T$ is the target thickness, $N_{A}=6.02 \times 10^{23}[1 / \mathrm{mol}]$ is the Avogadro number and $A$ is the atomic number of the nuclei.

- $N_{H Y}$ is the yield of hypernucleus obtained by the experimental result.

- $N_{e}$ is the number of incoming electron measured as the total beam charge in the data set.

- $N_{\gamma^{*}}$ is the number of virtual photons of which the associated scattered electrons is detected in HES in the run condition. This value is calculated by Geant4 simulation with the scattered photon distribution associated with virtual photon. The detail will be explained in this section.

- $\Delta \Omega_{K}$ and $\Delta \Omega_{e^{\prime}}$ are the solid angle of the HKS and the HES, respectively. They depend on the momentum and emission angle of the particle. Detail will be described in this section. 
- $\Delta E_{e^{\prime}}$ is the momentum range of scattered electron.

- $\epsilon_{i}^{H E S}$ is the experimental efficiency of the HES side. Since this value depends on the segment number of the ETOF, these values are applied event by event.

- $\epsilon_{i}^{H K S}$ is the experimental efficiency of the HKS side. Since this value depends on the momentum vector of kaon and the segment numbers of the HKS counter, these values are also applied event by event.

- $\epsilon^{\text {common }}$ is the experimental efficiency which is not included in $\epsilon_{i}^{H K S}$ and $\epsilon_{i}^{H E S}$. This value does not applied event by event, but data set by data set.

The efficiencies, $\epsilon_{i}^{H E S}, \epsilon_{i}^{H K S}$ and $\epsilon^{\text {common }}$ are defined as

$$
\begin{aligned}
\epsilon^{H E S} & =\epsilon^{\text {etrig }} \cdot \epsilon^{\text {etrack }}, \\
\epsilon^{H K S} & =\epsilon^{k t r i g} \cdot \epsilon^{\text {ktrack }} \cdot \epsilon^{A C} \cdot \epsilon^{W C} \cdot \epsilon^{m^{2}} \cdot f^{a b s} \cdot f^{\text {decay }}, \\
\epsilon^{\text {common }} & =\epsilon^{\text {ctime }} \cdot f^{\text {comp }} .
\end{aligned}
$$

The meaning of each value and typical values are summarized in table 5.6.1.

Table 5.6.1: List of the experimental efficiencies and factors. Typical values in the table are under the condition of ${ }^{12} \mathrm{C}$ target and $20 \mu \mathrm{A}$ beam current.

\begin{tabular}{l|c|c}
\hline \hline Name & Meanings & Typical value and error \\
\hline$\epsilon^{\text {etrig }}$ & HES trigger efficiency & $98.2 \pm 1.2$ \\
$\epsilon^{\text {etrack }}$ & HES tracking efficiency & $91.3 \pm 1.8$ \\
$\epsilon^{\text {ktrig }}$ & HKS trigger efficiency & $91.4 \pm 3.1$ \\
$\epsilon^{\text {ktrack }}$ & HKS tracking efficiency & $98.8 \pm 0.2$ \\
$\epsilon^{A C}$ & AC cut efficiency & $98.9 \pm 0.5$ \\
$\epsilon^{W C}$ & WC cut efficiency & $96.0 \pm 0.5$ \\
$\epsilon^{m^{2}}$ & $m^{2}$ cut efficiency & $99.9 \pm 0.01$ \\
$\epsilon^{\text {ctime }}$ & coin-time cut efficiency & $99.9 \pm 0.08$ \\
$f^{\text {abs }}$ & Kaon absorbtion factor & $95.2 \pm 0.1$ \\
$f^{\text {decay }}$ & Kaon live time factor & $32.8 \pm 0.05$ \\
$\epsilon^{\text {comp }}$ & Computer live time factor & $99.4 \pm 0.3$ \\
\hline$\epsilon^{\text {total }}$ & & $23.8 \pm 1.0$ \\
\hline \hline
\end{tabular}

The detail explanation of these efficiency values are as follows.

- $\epsilon^{\text {etrig }}$ is the efficiency of the HES single trigger. Basically this is determined by the geometrical coverage of the ETOF counters and detection efficiency of each the ETOF counter.

- $\epsilon^{\text {etrack }}$ is the efficiency of the EDC1 tracking. This is calculated by the plane efficiency (efficiency of $i$ th layer $=\epsilon_{i}^{E D C 1}$ ) of EDC1 and the number of required layers 
for tracking. Since the number of required layers is 9 out of 10 in this analysis, $\epsilon^{\text {etrack }}$ can be described as following:

$$
\epsilon^{\text {etrack }}=\prod_{i=1}^{10} \epsilon_{i}^{E D C}+\sum_{i=1}^{10}\left(1-\epsilon_{i}^{E D C}\right) \prod_{j=1, j \neq i}^{10} \epsilon_{j}^{E D C}
$$

- $\epsilon^{k t r i g}$ is the efficiency of the HKS single trigger. Since HKS single trigger consists of three layers of the $\mathrm{KTOF}, 3$ layers of $\mathrm{AC}$ and 2 layers of $\mathrm{WC}$, the geometrical coverage of each layer, the detection efficiency of the KTOF and WC counters and the overkill ratio of AC counters are considered to calculate $\epsilon^{k t r i g}$.

- $\epsilon^{k t r a c k}$ is the efficiency of tracking by KDCs. This is calculated by the plane efficiency of KDCs and the number of required layers for tracking, as well as EDC1. The efficiency of $i$ th layer is defined as $\epsilon_{i}^{E D C 1}$ and the tracking efficiency requiring 11 layers out of 12 can be described as following:

$$
\epsilon^{k t r a c k}=\prod_{i=1}^{12} \epsilon_{i}^{K D C}+\sum_{i=1}^{12}\left(1-\epsilon_{i}^{K D C}\right) \prod_{j=1, j \neq i}^{12} \epsilon_{j}^{K D C}
$$

- $\epsilon^{A C}$ is the cut efficiency of AC cut. Detail of this cut efficiency is described in section 5.2 .

- $\epsilon^{W C}$ is the cut efficiency of the WC cut. Detail of this cut efficiency is described in section 5.2, as well as AC cut efficiency.

- $\epsilon^{m^{2}}$ is the cut efficiency of $m^{2}$ cut. Detail of this cut efficiency is also described in section 5.2.

- $\epsilon^{\text {ctime }}$ is the cut efficiency of coin-time cut. Detail of this cut efficiency is also described in section 5.2.

- $f^{a b s}$ is the kaon absorption factor in the material of HKS and target. Some of the kaon is absorbed in the materials such as detectors and target by the inelastic scattering in the material. This value can be calculated by the Geant4 simulation. The detail will be explained in this section.

- $f^{\text {decay }}$ is the kaon decay factor in the HKS. This value can be also calculated by the Geant4 simulation and the detail will be explained in this section.

- $f^{c o m p}$ is the live time factor of the DAQ, that is the ratio of a time interval when DAQ processes a previous event. This value will be calculated and summarized in this section.

The way to estimate these values will be explained in this section. 


\subsubsection{Acceptance}

The acceptances of the HKS and the HES are obtained from the Geant4 simulation of each arm. Figure 5.34 shows the momentum and angular dependencies of solid angle for the HKS and figure 5.35 is the same correlations for the HES. The typical solid angle of HKS is about $8.5 \mathrm{msr}$ and that of the HES is about $8.0 \mathrm{msr}$.

The solid angle values used for the calculation of cross section refer the momentum $\left(p_{K}\right.$ and $\left.p_{e^{\prime}}\right)$ and angle $\left(\theta_{K}\right.$ and $\left.\theta_{e^{\prime}}\right)$. In the present analysis, the solid angle values $\left(\Delta \Omega_{K}\right.$ and $\Delta \Omega_{e^{\prime}}$ ) are obtained by every $5 \mathrm{MeV} / c$ for the momenta and every $2 \mathrm{mrad}$ for the angles and the value is used for the calculation of cross section. The z-axis of the scatter plots in figure 5.34 and 5.35 corresponds to the $\Delta \Omega_{K}$ and $\Delta \Omega_{e^{\prime}}$.

Figure 5.36 and 5.37 show the angular acceptances of the HKS and figure 5.38 and 5.39 are same figures for the HES side. These figures are obtained from real data with the selection of $\left(\mathrm{e}, \mathrm{e}^{\prime} \mathrm{K}^{+}\right)$events.
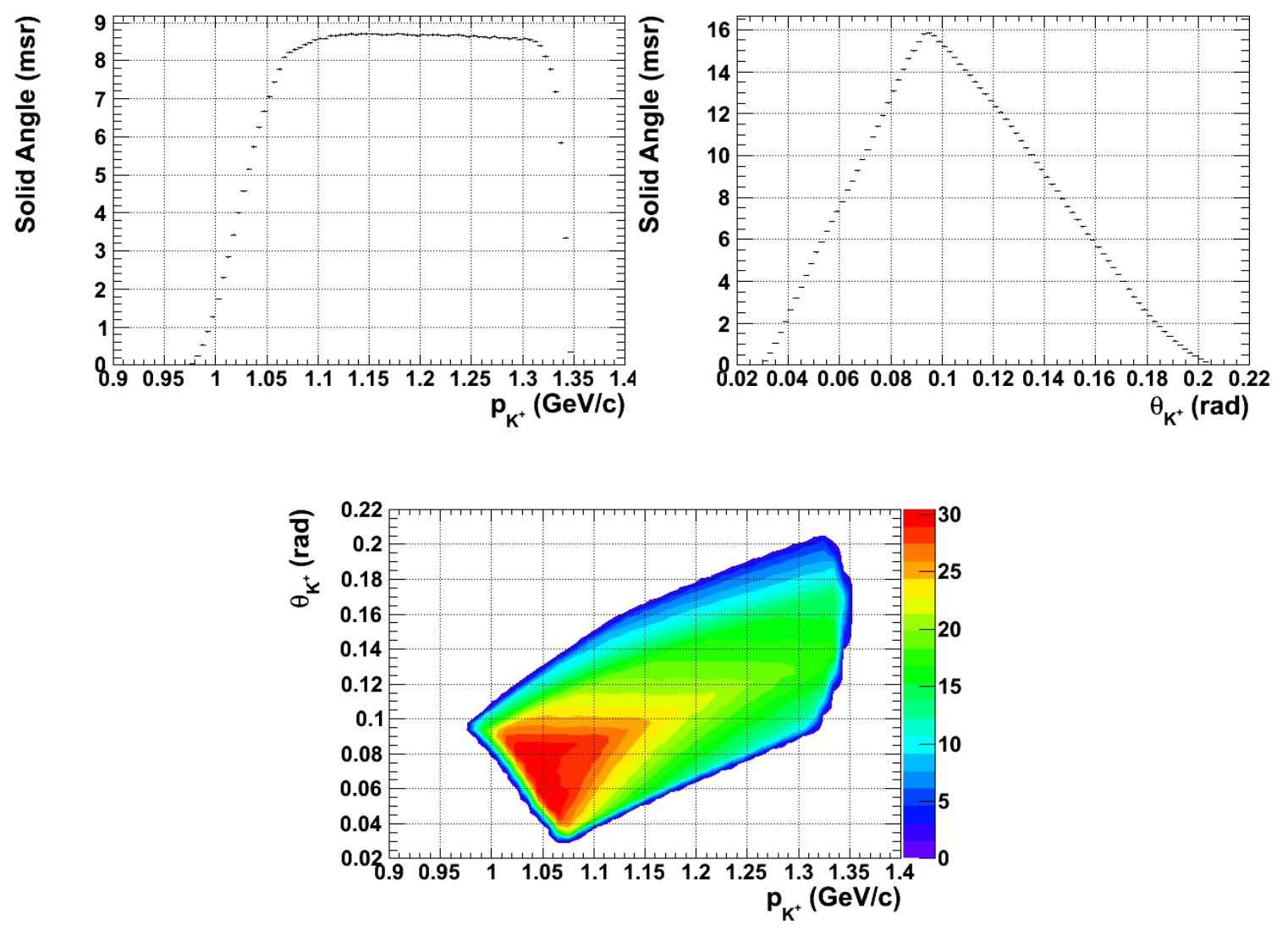

Figure 5.34: HKS solid angle. The top-left figure shows the correlation with $p_{K}$ and the top-right figure shows the correlation with $\theta_{K}$. The bottom figure shows the correlation between these three values. The $z$-axis is the solid angle.

For the cross section calculation, momentum cuts were applied both in the HKS and the HES, as shown in figure $5.40\left(1.1<p_{K}<1.3[\mathrm{GeV} / c]\right.$ and $\left.0.8<p_{e^{\prime}}<0.9[\mathrm{GeV} / c]\right)$. 

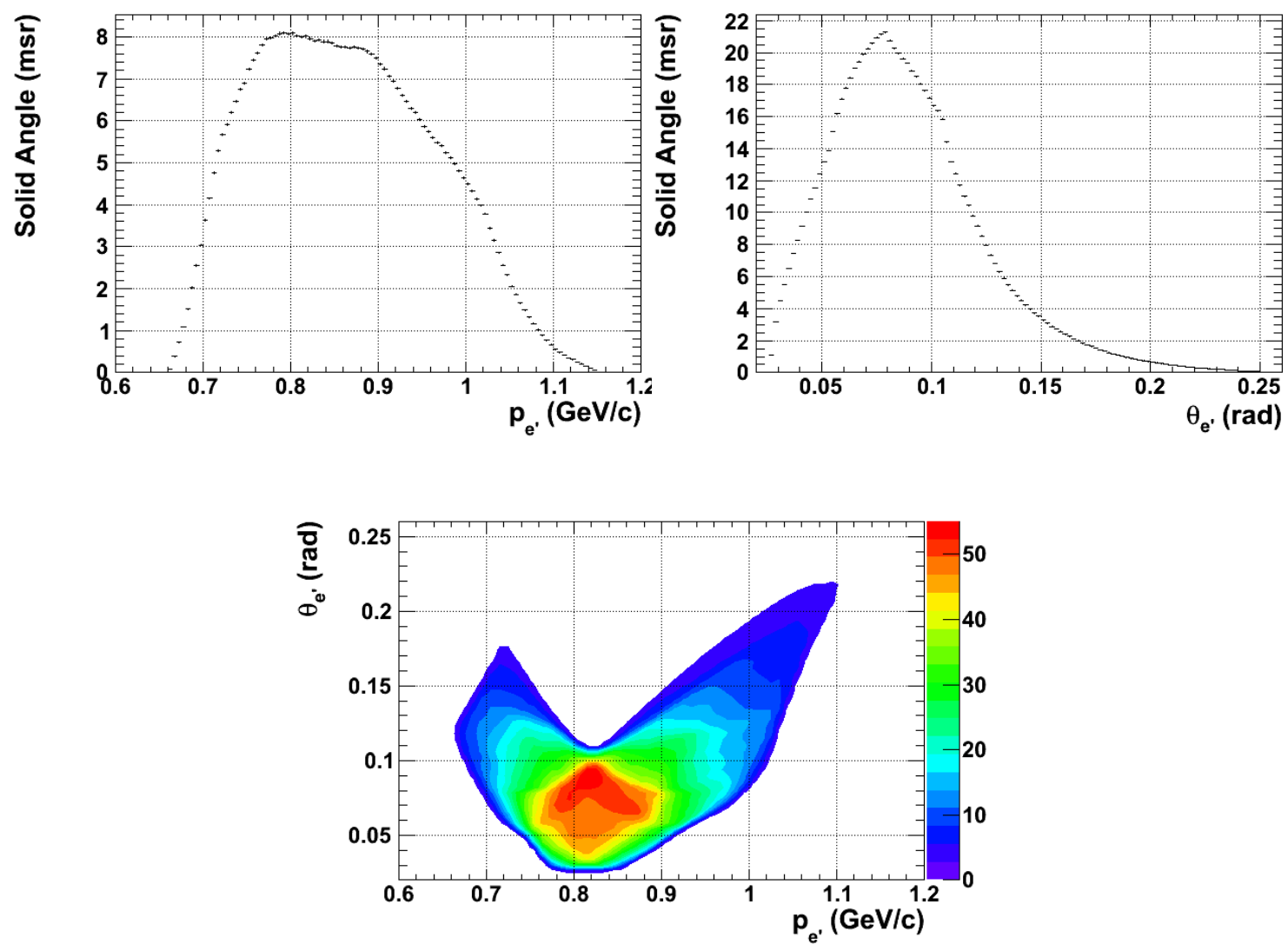

Figure 5.35: HES solid angle. The top-left figure shows the correlation with $p_{e^{\prime}}$ and the top-right figure shows the correlation with $\theta_{e^{\prime}}$. The bottom figure shows the correlation between these three values. The $z$-axis is the solid angle.
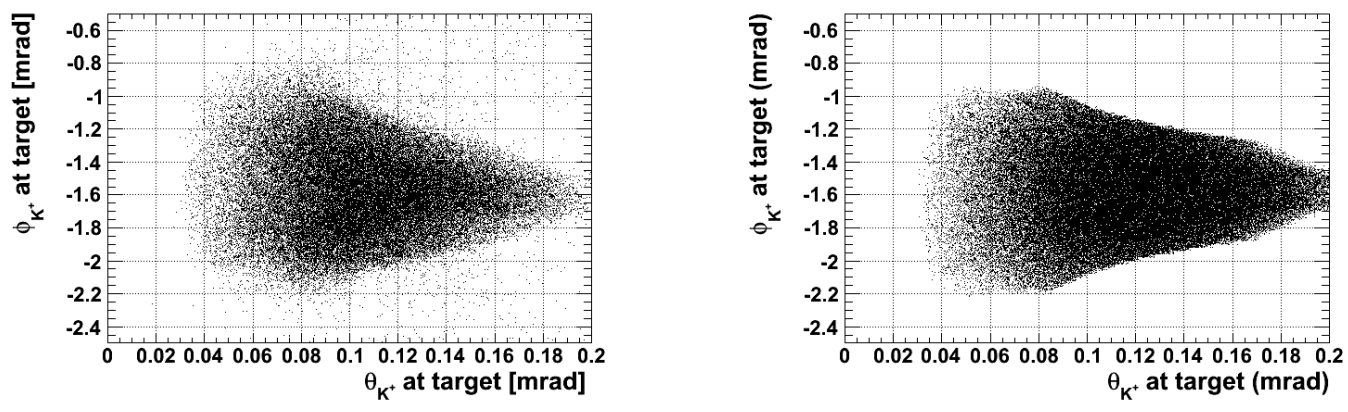

Figure 5.36: Correlation between $\theta_{K^{+}}$and $\phi_{K^{+}}$. 

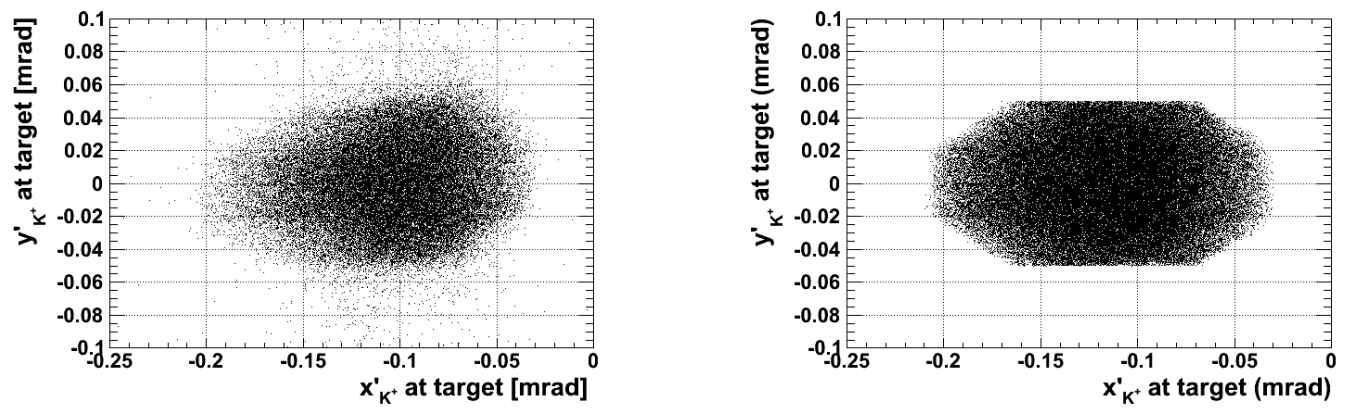

Figure 5.37: Correlation between $x_{K^{+}}^{\prime}$ and $y_{K^{+}}^{\prime}$.
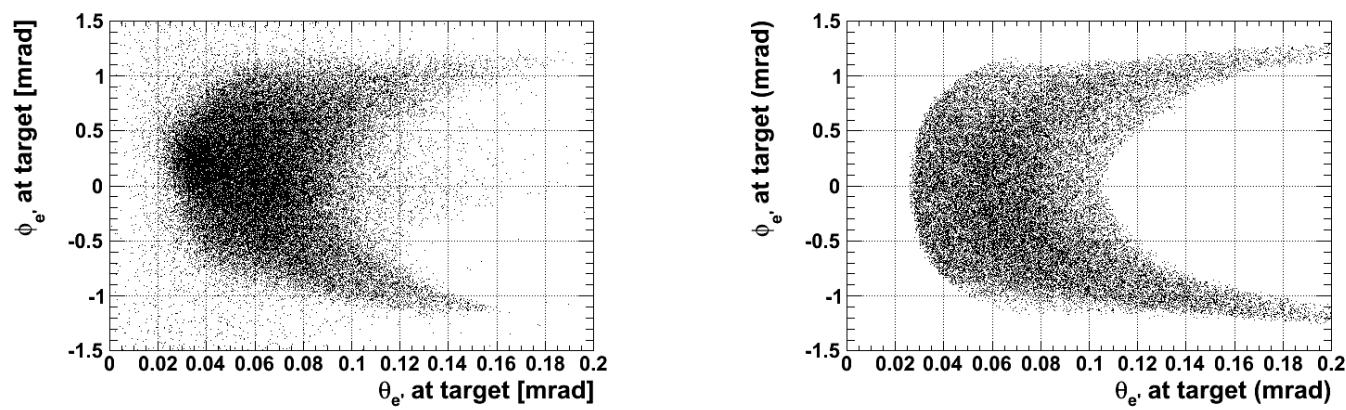

Figure 5.38: Correlation between $\theta_{e^{\prime}}$ and $\phi_{e^{\prime}}$.
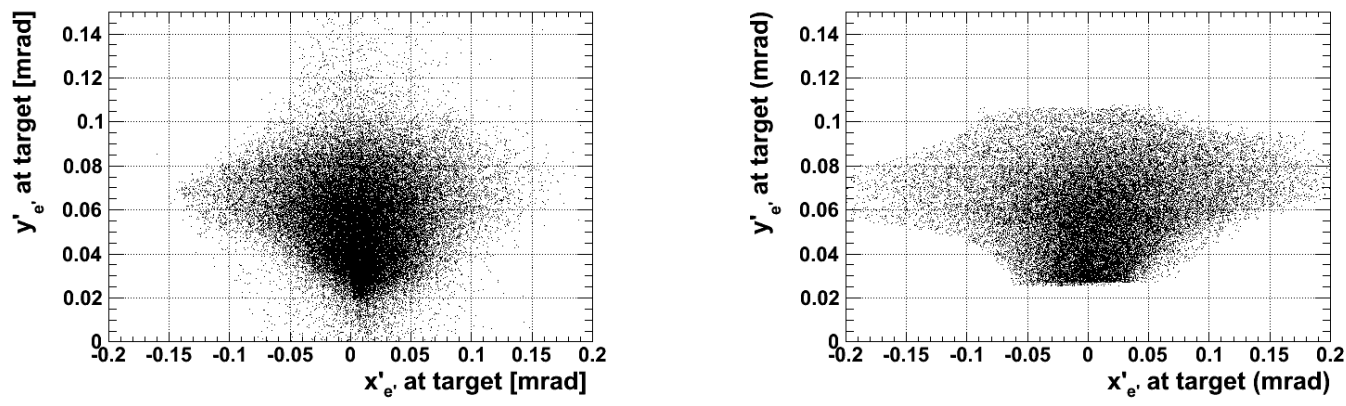

Figure 5.39: Correlation between $x_{e^{\prime}}^{\prime}$ and $y_{e^{\prime}}^{\prime}$. 
The reason is that this central region has less uncertainty about the magnetic fields and the momentum is reconstructed correctly. In order to expand the cut region, it will be necessary to do further study about the $F 2 T$ function, such as the usage of higher order $F 2 T$ functions.

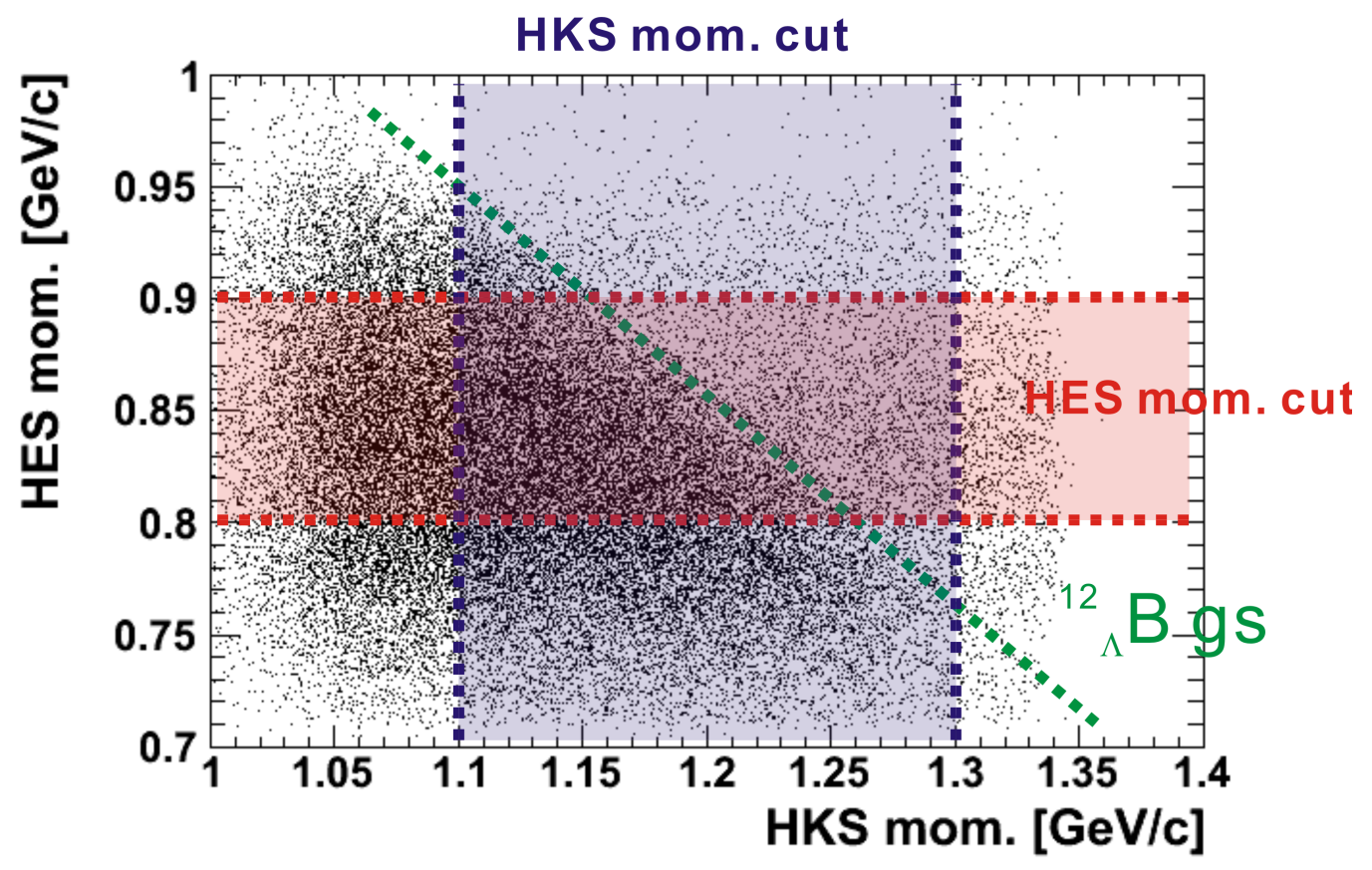

Figure 5.40: The selected region for the cross section calculation.

\subsubsection{The number of incoming electron and virtual photon}

The number of incoming electron $\left(N_{e}\right)$ and virtual photon $\left(N_{\gamma^{*}}\right)$ can be calculated as following equations:

$$
\begin{aligned}
N_{e} & =\frac{Q_{t o t}}{Q_{e}} \\
N_{\gamma^{*}} & =\frac{Q_{t o t}}{Q_{e}} \int d \Omega \int d \omega \Gamma(\theta, \omega) .
\end{aligned}
$$

In the above equation, $Q_{t o t}$ is the total charge of the run, $Q_{e}$ is the elementally charge and $\Gamma(\theta, \omega)$ is the virtual photon flux (see section 2.1). The virtual photon flux is integrated over a certain solid angle and momentum region of scattered electron. Figure 5.41 shows the momentum distribution of $\Gamma(\theta, \omega)$ integrated over the all HES acceptance. The integrated virtual photon flux for whole the HES acceptance is $5.2 \times 10^{-5}$ [1/electron].

$Q_{t o t}$ is measured by beam current monitor (see section 3.1) which was calibrated during the beam time using the Unser. The systematic error from this measurement is about 0.2 


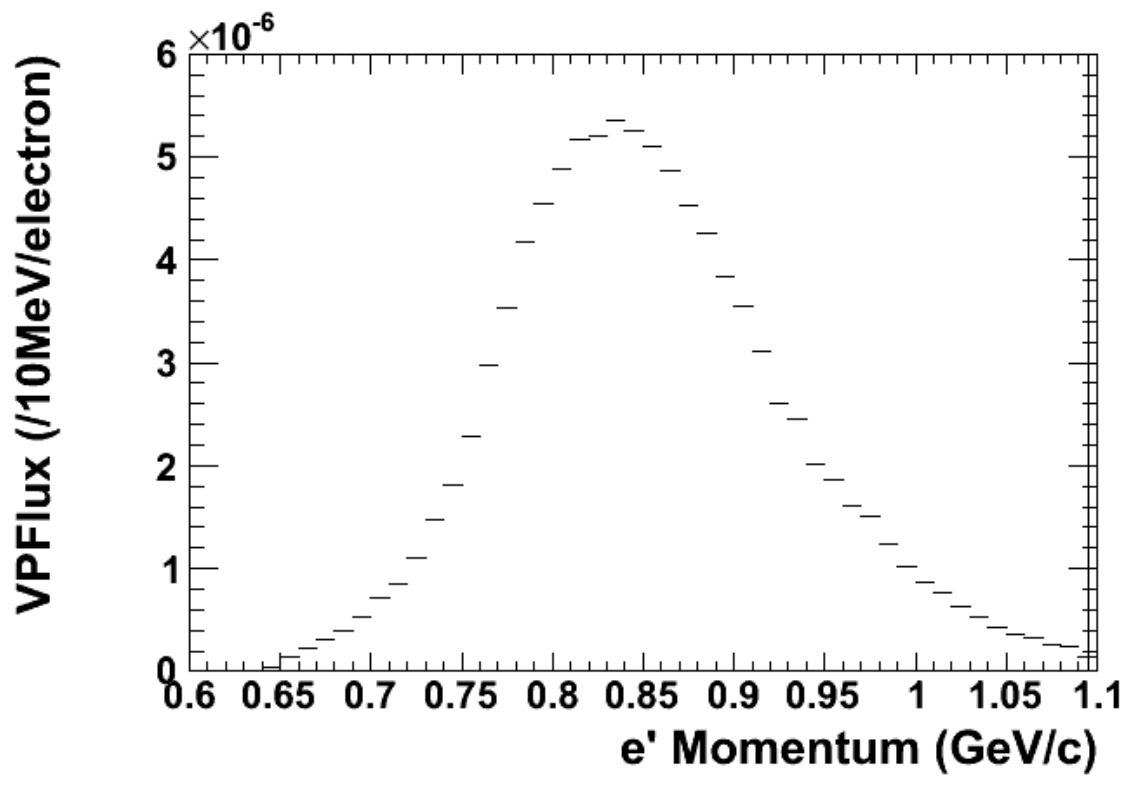

Figure 5.41: The virtual photon flux, $\Gamma(\omega)$ integrated with $e^{\prime}$ scattering angle $\theta_{e}$.

$\mu \mathrm{A}$ for all run conditions. This means the systematic error of $Q_{t o t}$ is less that $1 \%$ because the lowest beam current during the experiment is that if data set $\# 1\left(\mathrm{CH}_{2}\right.$ target, $\left.2 \mu \mathrm{A}\right)$.

On the other hand, $N_{\gamma}$ depends on the integral region of $\Gamma(\theta, \omega)$. The integral is done by the Geant 4 simulation, already mentioned in section 4.5 , but the momentum cut shown in figure 5.40 is applied this time. Furthermore, we need to calculate two types of the integral corresponding to the collimator positions applied during the experiment. The values are summarized in table 5.6.2.

For the consideration of the systematic error of $\Gamma(\theta, \omega)$, the effect of the difference between the simulation and the real experiment was considered. One is the accuracy of alignment of experimental apparatus. The positions of the apparatuses were surveyed before and after the experiment, and the position is determined with an accuracy of about $100 \mu \mathrm{m}$. Another is the accuracy of the magnetic fields, which has uncertainty of a few $\%$.

Therefore, the uncertainty of the magnetic fields is much larger than the position accuracy. In order to estimate the systematic error of $\Gamma(\theta, \omega)$, we compared the difference of $\Gamma(\theta, \omega)$ value between two type of simulations: using the TOSCA field map and the improved field map (see the last section 5.5.1). The difference of these two simulations was about $10 \%$. We adopted this value as a systematic error of $\Gamma(\theta, \omega)$.

The reason of the big difference is coming from the distribution of the electrons associated with the virtual photon. As shown in 2.2, the distribution is concentrated on the very forward angles. Therefore, its integration, $\Gamma(\theta, \omega)$ is highly depending on the "ratio" which the electrons hit collimator position or not. Since this ratio significantly change by the fringe field of the EQ1 and the SPL which has a lot of uncertainty, the difference became big. 
Table 5.6.2: $N_{\gamma^{*}}$ values used for the calculation of cross section. See figure 3.51 for the explanation of the collimator position.

\begin{tabular}{c|c}
\hline \hline HES collimator position $[\mathrm{cm}]$ & $N_{\gamma^{*}}$ \\
\hline-7.7 & $3.54 \times 10^{-5}$ \\
-4.7 & $2.71 \times 10^{-5}$ \\
\hline \hline
\end{tabular}

\subsubsection{Efficiencies and correction factors}

\section{HES trigger efficiency}

HES single trigger is constructed as $(E T O F 1) \otimes(E T O F 2)$, as described in section 3.9. Basically, the HES trigger efficiency can be determined by the detection efficiency of ETOF counters and it is assumed as 1.

However, inefficient counters were found in the ETOF1. Figure 5.42 is the hit pattern of the ETOF1 in ${ }^{12} \mathrm{C}$ target run. One can see there are two inefficient counters, \#13 and \#17. The reason of inefficiency was estimated to be the trouble of the discriminator making the logic signal of the ETOF1.

The inefficiency was estimated as follows. For the counter \#i, the ideal number of hit was estimated as the average between $n_{i-1}$ and $n_{i+1}$, where $n_{i}$ is the number of hit of the ith counter. Therefore, the efficiency of the counter \#i $\left(\epsilon_{i}\right)$ is

$$
\epsilon_{i}=n_{i} \cdot 2 /\left(n_{i-1}+n_{i+1}\right) .
$$

Using this $\epsilon_{i}, n_{13}$ and $n_{17}$ was modified to $n_{13} / \epsilon_{13}$ and $n_{17} / \epsilon_{17}$, respectively. The same method was applied to the double hit counters (shown in half integer in the figure 5.42).

The estimation result is summarized in table 5.6.3. The error shows systematic error, which was estimated by the uncertainty of the $n_{17}$. As shown in figure 5.42 , the counter \#17 is near to the mode center, it is difficult to estimate the efficiency with the above method.

Table 5.6.3: HES trigger efficiency for several run conditions.

\begin{tabular}{c|c|c|c}
\hline \hline Data set & Target & Beam current $[\mu \mathrm{A}]$ & Efficiency [\%] \\
\hline$\# 1$ & $\mathrm{CH}_{2}$ & 2 & $97.7 \pm 1.7$ \\
$\# 2$ & $\mathrm{H}_{2} \mathrm{O}$ & 2.5 & $87.3 \pm 1.4$ \\
$\# 3$ & ${ }^{7} \mathrm{Li}$ & 35 & $95.9 \pm 3.7$ \\
$\# 4$ & ${ }^{9} \mathrm{Be}$ & 40 & $95.3 \pm 4.4$ \\
$\# 5$ & ${ }^{10} \mathrm{~B}$ & 40 & $97.8 \pm 1.6$ \\
$\# 6, \# 7$ & ${ }^{n a t} \mathrm{C}$ & 20 & $98.2 \pm 1.2$ \\
$\# 8$ & ${ }^{n a t} \mathrm{C}$ & 35 & $95.6 \pm 3.1$ \\
$\# 9$ & ${ }^{52} \mathrm{Cr}$ & 7.5 & $97.3 \pm 1.9$ \\
$\# 10$ & ${ }^{52} \mathrm{Cr}$ & 7.5 & $94.7 \pm 5.0$ \\
\hline \hline
\end{tabular}




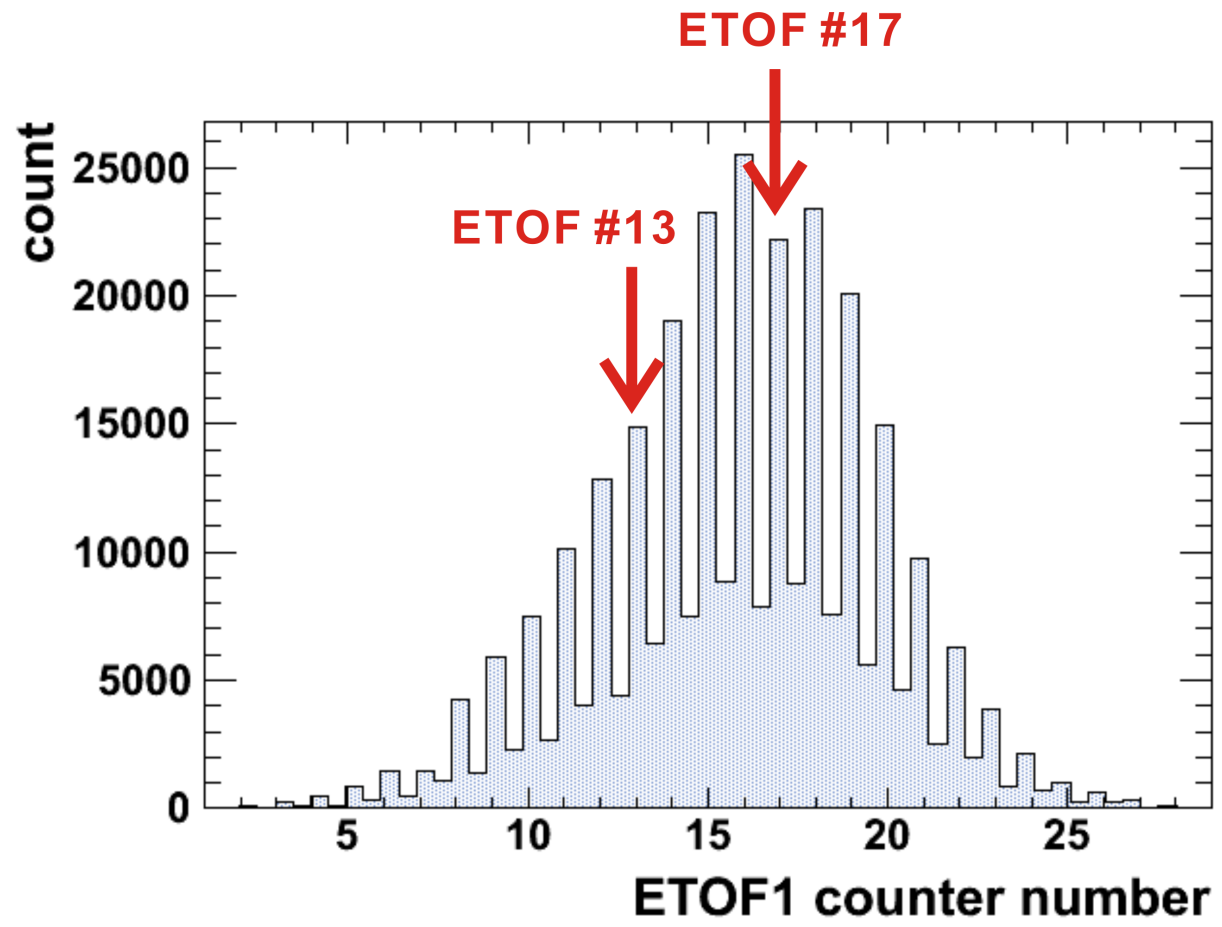

Figure 5.42: ETOF1 hit pattern.

\section{HES tracking efficiency}

The tracking efficiency was calculated by the hit efficiency of each EDC plane. The tracking required 8 hit planes out of 10 . The calculated tracking efficiencies for the various run conditions are summarized in table 5.6.3.

\section{HKS trigger efficiency}

The HKS single trigger is consists as $(C P) \otimes(\overline{A C}) \otimes(W C)$ (see section 3.9). This trigger aim to take kaons with rejecting pions and protons, and the threshold level of the $\mathrm{AC}$ and the WC is set with some margin. However, still there are some possibility to kill kaons due to the skimming at detectors, accidental kill in the $\mathrm{AC}$, and so on. The kill ratio is considered to be the main contribution to the HKS trigger efficiency.

Though the $\mathrm{WC}$ and $\mathrm{AC}$ were included in the trigger, we took the prescaled unbiased trigger $(C P 0$, see section 3.9) and the kaon kill ratio can be estimated from this $C P 0$ data [62]. The efficiencies for several conditions are summarized in table 5.6.3.

\section{HKS tracking efficiency}

The tracking efficiency was calculated by the hit efficiency of each KDC plane. Fof the tracking, 5 hit planes were required for KDC1 and KDC2. The calculated tracking efficiencies for the various run conditions are summarized in table 5.6.3. 
Table 5.6.4: HES tracking efficiency for several run conditions.

\begin{tabular}{c|c|c|c}
\hline \hline Data set & Target & Beam current $[\mu \mathrm{A}]$ & Efficiency [\%] \\
\hline$\# 1$ & $\mathrm{CH}_{2}$ & 2 & $92.9 \pm 1.7$ \\
$\# 2$ & $\mathrm{H}_{2} \mathrm{O}$ & 2.5 & $90.3 \pm 2.1$ \\
$\# 3$ & ${ }^{7} \mathrm{Li}$ & 35 & $82.8 \pm 3.3$ \\
$\# 4$ & ${ }^{9} \mathrm{Be}$ & 40 & $90.9 \pm 1.8$ \\
$\# 5$ & ${ }^{10} \mathrm{~B}$ & 40 & $94.2 \pm 1.7$ \\
$\# 6, \# 7$ & ${ }^{n a t} \mathrm{C}$ & 20 & $91.3 \pm 1.8$ \\
$\# 8$ & ${ }^{n a t} \mathrm{C}$ & 35 & $77.2 \pm 2.5$ \\
$\# 9, \# 10$ & ${ }^{52} \mathrm{Cr}$ & 7.5 & $87.5 \pm 1.9$ \\
\hline \hline
\end{tabular}

Table 5.6.5: HKS trigger efficiency for several run conditions.

\begin{tabular}{c|c|c|c}
\hline \hline Data set & Target & Beam current $[\mu \mathrm{A}]$ & Efficiency [\%] \\
\hline$\# 1$ & $\mathrm{CH}_{2}$ & 2 & $92.3 \pm 1.8$ \\
$\# 3$ & ${ }^{7} \mathrm{Li}$ & 35 & $90.9 \pm 13.7$ \\
$\# 4$ & ${ }^{9} \mathrm{Be}$ & 40 & $93.1 \pm 6.4$ \\
$\# 5$ & ${ }^{10} \mathrm{~B}$ & 40 & $94.1 \pm 1.2$ \\
$\# 6, \# 7$ & ${ }^{n a t} \mathrm{C}$ & 20 & $91.4 \pm 3.1$ \\
$\# 8$ & ${ }^{n a t} \mathrm{C}$ & 35 & $85.4 \pm 6.1$ \\
\hline \hline
\end{tabular}

Table 5.6.6: HKS tracking efficiency for several run conditions.

\begin{tabular}{c|c|c|c}
\hline \hline Data set & Target & Beam current $[\mu \mathrm{A}]$ & Efficiency [\%] \\
\hline$\# 1$ & $\mathrm{CH}_{2}$ & 2 & $99.9 \pm 0.8$ \\
$\# 2$ & $\mathrm{H}_{2} \mathrm{O}$ & 2.5 & $96.1 \pm 0.2$ \\
$\# 3$ & ${ }^{7} \mathrm{Li}$ & 35 & $99.6 \pm 2.1$ \\
$\# 4$ & ${ }^{9} \mathrm{Be}$ & 40 & $99.5 \pm 0.5$ \\
$\# 5$ & ${ }^{10} \mathrm{~B}$ & 40 & $99.9 \pm 0.9$ \\
$\# 6, \# 7$ & ${ }^{n a t} \mathrm{C}$ & 20 & $98.8 \pm 0.2$ \\
$\# 8$ & ${ }^{n a t} \mathrm{C}$ & 35 & $99.0 \pm 0.5$ \\
$\# 9$ & ${ }^{2} \mathrm{Cr}$ & 7.5 & $97.5 \pm 0.3$ \\
$\# 10$ & ${ }^{52} \mathrm{Cr}$ & 7.5 & $96.6 \pm 0.3$ \\
\hline \hline
\end{tabular}




\section{AC, WC, $m^{2}$ cut efficiency}

The cut efficiencies for each run condition is summarized in table 5.2.2, 5.2.3 and 5.2.4 about $\mathrm{AC}, \mathrm{WC}$ and $m^{2}$ cut, respectively.

\section{Kaon absorbtion factor}

Kaon absorption factor can be calculated with the Geant 4 simulation including the properties of materials which kaons passed through. The list of the materials included in the Geant4 simulation are summarized in table 5.6.7 with their thickness and densities. The kaon absorption reaction rate, $\left(1-f^{a b s}\right)$ can be described using inelastic scattering cross section of kaon $\left(\sigma_{\text {inel }}\right)$ :

$$
1-f^{a b s}=\sigma_{\text {inel }} \frac{N_{A} \rho t}{A},
$$

where $A$ is the atomic number, $N_{A}$ is the Avogadro's number, $\rho$ is the material density and $t$ is the thickness of the material.

The total cross section and the elastic cross section around $1.05 \mathrm{GeV} / c<p_{K^{+}}<$ $1.35 \mathrm{GeV} / c$ has been measured as $16.95 \mathrm{mb}<\sigma^{\text {total }}<19.85 \mathrm{mb}[69,70,71]$, and 10.06 $<\sigma^{e l}<11.81 \mathrm{mb}[71,72,73]$, respectively. Therefore the inelastic cross section $\left(\sigma^{\text {inel }}\right)$ can be expected to be less than $10 \mathrm{mb}$. The kaon absorption factors depending on target is summarized in table 5.6.8 where $\sigma^{\text {inel }}$ is fixed as $10 \mathrm{mb}$. Actually the absorption in the target is negligibly small and the factors were determined by the other materials. The uncertainty associated with this correction is based only on a measurement accuracy of $<1 \mathrm{~mm}$ for the detector size, which corresponds to $0.01 \%$.

\section{Kaon live time factor}

The kaon is an unstable particle with a mean life time $\tau=(1.2380 \pm 0.0021) \times 10^{-8} \mathrm{sec}$ [68]. Therefore, some of kaons decay before arriving at the HKS detectors. This kaon decay factor $\left(f^{\text {decay }}\right)$ is estimated by the Geant 4 simulation. As a reference, the kaon decay channels and branching ratios are summarized in table 5.6.9. The kaon decay factor is calculated as following:

$$
f^{\text {decay }}=\frac{N_{a c c}}{N_{g e n}},
$$

where $N_{\text {gen }}$ is the number of generated kaons at target point and $N_{a c c}$ is the number of kaons which create the HKS trigger. This value depends on the kaon momentum as shown in figure 5.43. The averaged $f^{\text {decay }}$ over the HKS momentum acceptance is $32.8 \pm 0.05 \%$ (error is statistic only).

\section{Computer live time factor}

The hall-C DAQ processes the events with $500 \mu$ s time intervals, that is corresponding to $2.0 \mathrm{kHz}$ trigger rate. The computer live time factor can be calculated by the ratio between the number of accepted trigger $\left(N_{a c c}\right)$ and the number of requested trigger $\left(N_{\text {req }}\right)$ :

$$
f^{c o m p}=\frac{N_{a c c}}{N_{g e n}} .
$$


Table 5.6.7: Property of the HKS materials. These parameters are used to calculate the kaon absorption factor. For $p$ [Torr], $t\left[{ }^{\circ} \mathrm{C}\right]$, air density $(\rho)$ is written as $\rho=101.325 /\{287.055 \times(273.15+t)\} \times p / 761.842\left[\mathrm{~g} / \mathrm{cm}^{3}\right]$.

\begin{tabular}{|c|c|c|c|}
\hline Material & $\mathrm{t}[\mathrm{cm}]$ & $\rho\left[\mathrm{g} / \mathrm{cm}^{3}\right]$ & Notes \\
\hline \multicolumn{4}{|l|}{ Target } \\
\hline $\mathrm{CH}_{2}$ & 0.4507 & 0.92 & \\
\hline $\mathrm{H}_{2} \mathrm{O}$ & 0.500 & 1.00 & \\
\hline${ }^{7} \mathrm{Li}$ & 0.345 & 0.534 & \\
\hline${ }^{9} \mathrm{Be}$ & 0.102 & 1.848 & \\
\hline${ }^{10} \mathrm{~B}$ & 0.0240 & 2.340 & \\
\hline${ }^{12} \mathrm{C}$ & 0.0500 & 2.25 & \\
\hline${ }^{52} \mathrm{Cr}$ & 0.0187 & 7.15 & \\
\hline${ }^{52} \mathrm{Cr}$ & 0.0215 & 7.15 & \\
\hline \multicolumn{4}{|l|}{ Spectrometer } \\
\hline Kevlar $\left(\mathrm{C}_{14} \mathrm{~N}_{2} \mathrm{O}_{2} \mathrm{H}_{10}\right)$ exit & 0.02 & 0.74 & \\
\hline Mylar $\left(\mathrm{C}_{10} \mathrm{O}_{4} \mathrm{H}_{8}\right)$ exit & 0.0125 & 1.39 & \\
\hline \multicolumn{4}{|l|}{ Detector Hut } \\
\hline$*$ He bag & & & Placed between \\
\hline He gas & 100 & $1.79 \times 10^{-4}$ & HDC1 \& 2 \\
\hline Kapton bag & 0.004 & 1.42 & \\
\hline \multicolumn{4}{|l|}{$*$ HDC1 and 2} \\
\hline DC Mylar cathode & 0.0176 & 1.39 & \\
\hline $\mathrm{Ar} / \mathrm{C}_{2} \mathrm{H}_{6}$ gas & 7.41 & 0.00154 & \\
\hline Sense wires $(\mathrm{W})$ & 0.00008 & 19.3 & \\
\hline Field wires (W) & 0.00034 & 19.3 & \\
\hline$*$ HTOF $1 \mathrm{X}, 1 \mathrm{Y}$ and $2 \mathrm{X}$ & 6 & 1.032 & \\
\hline \multicolumn{4}{|l|}{$* \mathrm{AC \check {C }} 1,2$ and 3} \\
\hline Paper wall for AČ & 1.8 & 1.42 & \\
\hline Silica aerogel & 15 & 0.2 & \\
\hline \multicolumn{4}{|l|}{$*$ WČ 1 and 2} \\
\hline Plastic wall for WČ & 0.9 & 1.19 & Polymethylmethacrylate \\
\hline Water & 15 & 1 & \\
\hline \multicolumn{4}{|l|}{$*$ Miscellaneous } \\
\hline Air & $\sim 115$ & 0.0012 & HDC2 through WČ2 \\
\hline Aluminum foil for light shielding & 0.312 & 2.70 & \\
\hline Plastic sheet for light shielding & 1.04 & 1.30 & Polyvinyl-chloride \\
\hline
\end{tabular}

The $N_{a c c}$ and $N_{r e q}$ is recorded by a scaler for all runs, then $f^{\text {comp }}$ can be calculated in the offline analysis. The $f^{\text {comp }}$ values for several run conditions are summarized in figure 5.6 .3 . 


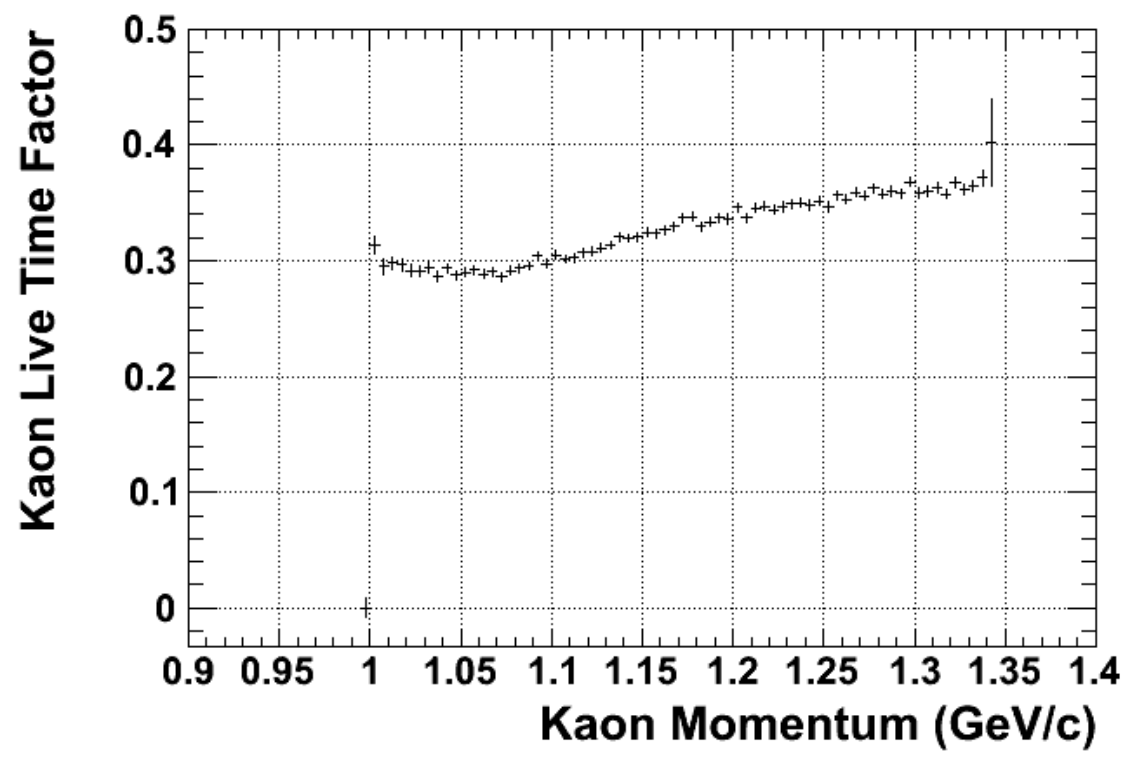

Figure 5.43: The momentum dependence of kaon decay factor in the HKS momentum acceptance calculated by the Geant 4 simulation.

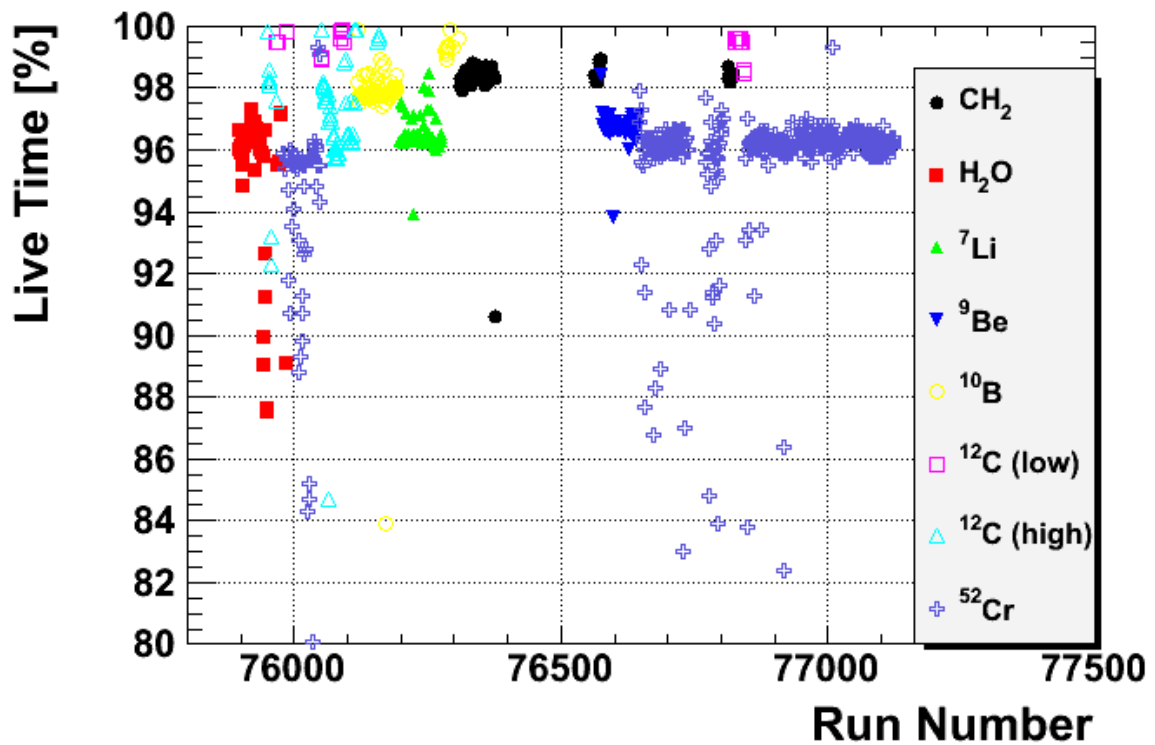

Figure 5.44: Computer live time factor for several run conditions. 
Table 5.6.8: List of the kaon absorption factors.

\begin{tabular}{lcc}
\hline \hline Material & $\mathrm{t}[\mathrm{cm}]$ & Absorption factor [\%] \\
\hline $\mathrm{CH}_{2}$ & 0.489 & 95.2 \\
$\mathrm{H}_{2} \mathrm{O}$ & 0.500 & 95.2 \\
${ }^{7} \mathrm{Li}$ & 0.341 & 95.2 \\
${ }^{9} \mathrm{Be}$ & 0.102 & 95.2 \\
${ }^{10} \mathrm{~B}$ & 0.026 & 95.2 \\
${ }^{12} \mathrm{C}$ & 0.050 & 95.2 \\
${ }^{52} \mathrm{Cr}$ & 0.0187 & 95.2 \\
${ }^{52} \mathrm{Cr}$ & 0.0215 & 95.2 \\
\hline \hline
\end{tabular}

\begin{tabular}{cc}
\hline \hline Decay Modes & Fraction [\%] \\
\hline$\mu^{+} \nu_{\mu}$ & $63.54 \pm 0.14$ \\
$\pi^{+} \pi^{0}$ & $20.68 \pm 0.13$ \\
$\pi^{+} \pi^{+} \pi^{-}$ & $5.59 \pm 0.04$ \\
$\pi^{0} e^{+} \nu_{e}$ & $5.08 \pm 0.05$ \\
$\pi^{0} \mu^{+} \nu_{\mu}$ & $3.35 \pm 0.04$ \\
$\pi^{+} \pi^{0} \pi^{0}$ & $1.76 \pm 0.02$ \\
\hline \hline
\end{tabular}

Table 5.6.9: Summary of $\mathrm{K}^{+}$decay channels and fraction [68].

\subsubsection{Systematic errors for cross section}

The systematic errors derived from experimental conditions are summarized in table 5.6.4. The error of the beam intensity was $0.2 \mu \mathrm{A}$ from the systematic error of the BCM (see section 3.1). This value is about $10 \%$ for $\mathrm{CH}_{2}$ target data and less than $1 \%$ for hypernuclear data. The target thickness was measured by a laser displacement meter with the systematic error of $2 \%$. The systematic errors of the HKS acceptance and the number of virtual photons were determined by the accuracy of the Geant4 simulation compared to the actual experimental condition. In particular, the number of virtual photons is strongly depending on the accuracy of the HES magnetic field. The error was determined as $10 \%$ in the present study.

Table 5.6.10: The systematic errors derived from experimental conditions.

\begin{tabular}{l|c}
\hline \hline Item & Error $[\%]$ \\
\hline Beam Intensity & 1 (hypernuclei) / $10\left(\mathrm{CH}_{2}\right.$ target $)$ \\
Target thickness & 2 \\
Number of virtual photon & 10 \\
HKS acceptance & 1 \\
Efficiencies & 5 \\
\hline \hline
\end{tabular}




\section{Chapter 6}

\section{Experimental results and discussion}

In this chapter, firstly the resolution of the whole system will be evaluated using the obtained spectra using the $\mathrm{CH}_{2}$ target data. This evaluation is necessary as a conclusion of the whole system design including the newly constructed HES and SPL. In the next section, the obtained missing mass spectrum of ${ }_{\Lambda}^{12} \mathrm{~B}$ from the E05-115 experimental data will be shown with the binding energies and cross sections of $\Lambda$ hypernuclei. After that, these values are compared with other experimental data and theoretical calculations. The newly constructed experimental setup will be evaluated through these discussion.

\subsection{Analysis of the $\mathrm{CH}_{2}$ target data}

As already described, we designed and constructed new high resolution electron spectrometer, the HES, which was firstly used in the present experiment. Since its calibration was done with the SS data and the $\mathrm{CH}_{2}$ target spectrum, it is hard to evaluate the performance of the HES as a single spectrometer. However, it is possible to evaluate the resolution of whole system and derive the performance of the HES. The evaluation will be done using the $\mathrm{CH}_{2}$ target data in this section. Moreover, the yield will be also evaluated by the cross section of the elementary process, $\mathrm{p}\left(\mathrm{e}, \mathrm{e}^{\prime} \mathrm{K}^{+}\right) \Lambda / \Sigma^{0}$ reaction.

\subsubsection{Fitting function for mass spectrum}

Basically, the all mass spectrum was fitted with Cauchy functions. In order to evaluate the response function, a missing mass spectrum was make by the Geant4 simulation assuming ${ }^{12} \mathrm{C}$ target and fitted by a Gaussian and a Cauchy function. The comparison result is shown in figure 6.1. From this result, we decided to use a Cauchy function for the fitting of the spectrum.

\subsection{2 $\mathrm{CH}_{2}$ target missing mass spectrum}

Figure 6.2 is the obtained missing mass spectrum of the $\mathrm{CH}_{2}$ target with the vertical axis of counts. The horizontal axis $M_{X}-M_{\Lambda}$ is the difference between the obtained missing mass and known $\Lambda$ mass. The background was originated from the accidental coincidence events and quasi-free kaon production events in the carbon nuclei in $\mathrm{CH}_{2}$ target. 

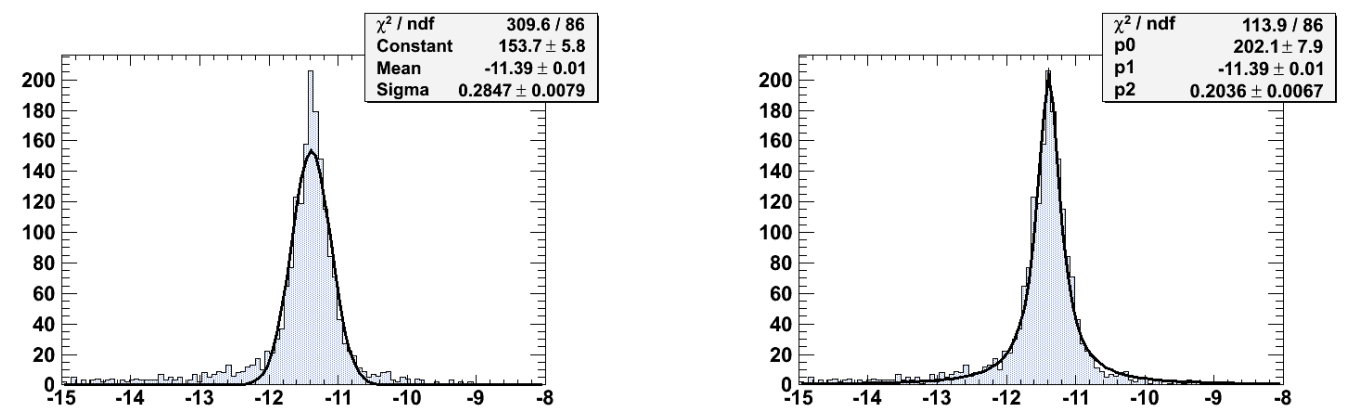

Figure 6.1: Comparison of the fitting function with simulated ${ }_{\Lambda}^{12} \mathrm{~B}$ missing mass. The fitting function of left figure is a Gaussian and the right figure is a Cauchy function.

In this figure, additional acceptance cut of $-0.05<x_{f K}^{\prime}<0.05$ was applied, because the energy resolution was worse in the outer side of the region. There are the possibility to improve the resolution of the outside region, by the further tuning of the $F 2 T$ function or usage of higher order $F 2 T$ functions.

Each peaks were fitted with a Cauchy function and the background shape was assumed as a second polynomial, therefore the fitting function is as following:

$$
f(x)=c_{0}\left[1+\left(\frac{x-c_{1}}{c_{2}}\right)^{2}\right]+c_{3} x^{2}+c_{4} x+c_{5} .
$$

The fitting results are summarized in table 6.1.1, and the width of the $\Lambda$ peak is $1.5 \mathrm{MeV}$ (FWHM). This value will be evaluated in the following part.

Table 6.1.1: Fitting results of $\Lambda / \Sigma^{0}$ peaks. Errors are statistic only.

\begin{tabular}{c|c|c}
\hline \hline Peak & Mean $\left(M_{X}-M_{\Lambda / \Sigma^{0}}\right)[\mathrm{MeV}]$ & Width $(\mathrm{FWHM})[\mathrm{MeV}]$ \\
\hline$\Lambda$ & $0.05 \pm 0.02$ & $1.51 \pm 0.05$ \\
$\Sigma^{0}$ & $0.07 \pm 0.05$ & $1.43 \pm 0.12$ \\
\hline \hline
\end{tabular}

\subsubsection{Evaluation of the resolution}

The obtained missing mass resolution in the $\mathrm{CH}_{2}$ spectrum is originated from several factors, that is, the momentum and angular resolution of each particle. The factors are summarized in table 6.1.2 and they are basically the momenta and angles of the particles. The "typical resolutions" in the table are estimated from the experimental data and simulation. The angular resolutions were obtained by the SS data analysis (see section 5.5.1), and the target energy struggling were estimated by the Geant4 simulation (see table 5.5.2).

The "correlation factors" mean the values how the resolution affect on the MM, calculated as following. When one represents the missing mass equation as

$$
M M=f\left(p_{\text {Beam }}, p_{e^{\prime}}, x_{e^{\prime}}^{\prime}, y_{e^{\prime}}^{\prime}, p_{K^{+}}, x_{K^{+}}^{\prime}, y_{K^{+}}^{\prime}\right),
$$



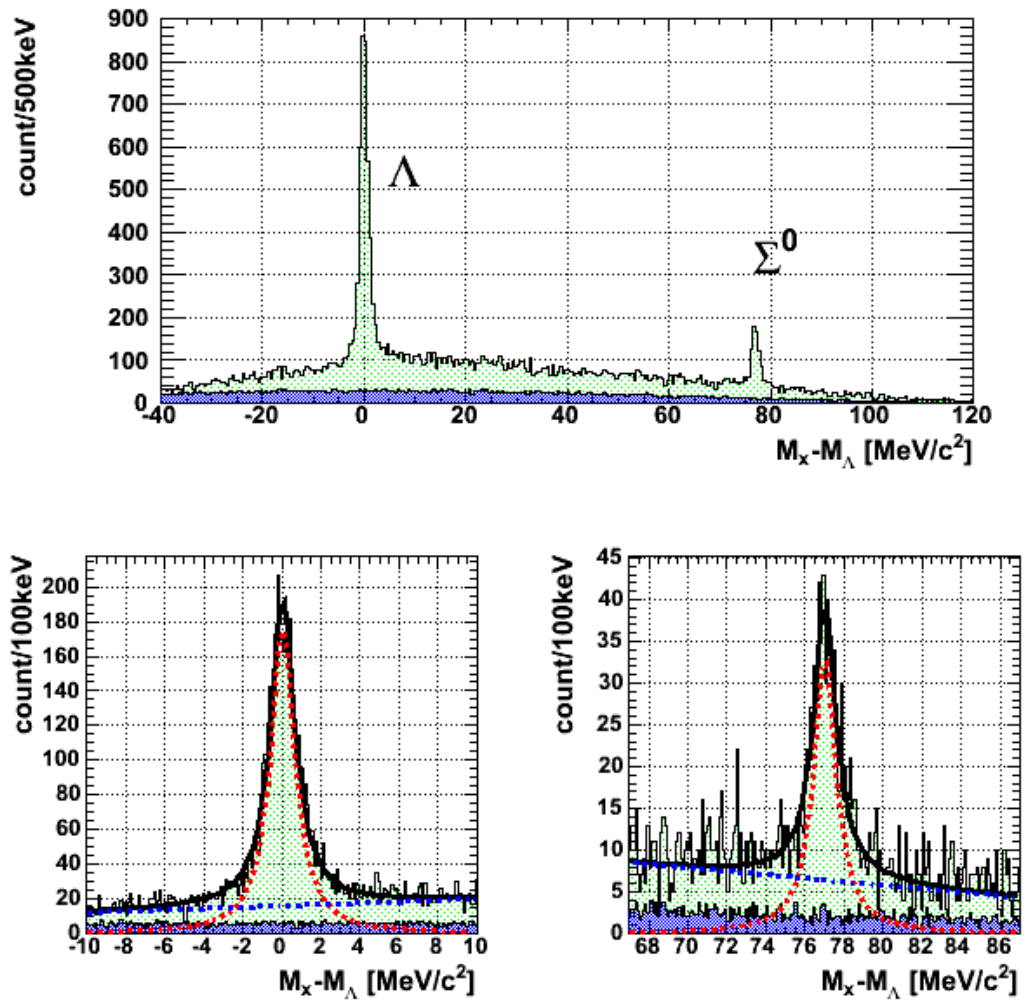

Figure 6.2: The missing mass spectrum of $\mathrm{CH}_{2}$ target data. 
the error of the missing mass $\left(\delta_{M M}\right)$ can be calculated as

$$
\begin{aligned}
\delta_{M M}^{2}= & \left(\frac{\partial f}{\partial p_{\text {Beam }}} \sqrt{\delta\left(p_{\text {Beam }}\right)^{2}+\delta\left(\Delta E_{\text {Beam }}\right)^{2}}\right)^{2}+ \\
& \left(\frac{\partial f}{\partial p_{e^{\prime}}} \sqrt{\delta\left(p_{e^{\prime}}\right)^{2}+\delta\left(\Delta E_{e^{\prime}}\right)^{2}}\right)^{2}+\left(\frac{\partial f}{\partial x_{e^{\prime}}^{\prime}} \delta\left(x_{e^{\prime}}^{\prime}\right)\right)^{2}+\left(\frac{\partial f}{\partial y_{e^{\prime}}^{\prime}} \delta\left(y_{e^{\prime}}^{\prime}\right)\right)^{2}+ \\
& \left(\frac{\partial f}{\partial p_{K^{+}}} \sqrt{\delta\left(p_{K^{+}}\right)^{2}+\delta\left(\Delta E_{K^{+}}\right)^{2}}\right)^{2}+\left(\frac{\partial f}{\partial x_{K^{+}}^{\prime}} \delta\left(x_{K^{+}}^{\prime}\right)\right)^{2}+\left(\frac{\partial f}{\partial y_{K^{+}}^{\prime}} \delta\left(y_{K^{+}}^{\prime}\right)\right)^{2}(6.3)
\end{aligned}
$$

from the error propagation low. Here the energy struggling effect $\delta(\Delta E)$ at the target is divided from the optical effect $\delta(p)$. The "correlation factor" is represented as the coefficient of each error (for example, $\frac{\partial f}{\partial p_{e^{\prime}}}$ for $\delta_{e^{\prime}}(p)$ ). This factors is calculated from the partial differential of the missing mass equation (5.9) and determined by the experimental condition.

Table 6.1.2: Considerable factors for the missing mass resolution.

\begin{tabular}{c|c|c}
\hline \hline Item & Typical resolutions in RMS & Correlation factors \\
\hline Beam momentum $\left(\delta\left(p_{\text {Beam }}\right)\right)$ & $<0.1[\mathrm{MeV} / c]$ & $1.7[\mathrm{MeV} /(\mathrm{MeV} / c)]$ \\
Beam energy loss $\left(\delta\left(\Delta E_{\text {Beam }}\right)\right)$ & $0.28[\mathrm{MeV}]$ & \\
\hline$K^{+}$momentum $\left(\delta\left(p_{K^{+}}\right)\right)$ & - & $1.6[\mathrm{MeV} /(\mathrm{MeV} / c)]$ \\
$K^{+}$energy loss $\left(\delta\left(\Delta E_{K^{+}}\right)\right)$ & $0.30[\mathrm{MeV}]$ & \\
\hline$K^{+}$horizontal angle $\left(\delta\left(x_{K^{+}}^{\prime}\right)\right)$ & $1[\mathrm{mrad}]$ & $0.20[\mathrm{MeV} / \mathrm{mrad}]$ \\
$K^{+}$vertical angle $\left(\delta\left(y_{K^{+}}^{\prime}\right)\right)$ & $10[\mathrm{mrad}]$ & $0.057[\mathrm{MeV} / \mathrm{mrad}]$ \\
$K^{+}$multiple scattering $\left(\delta\left(\theta_{K^{+}}\right)\right)$ & $<1[\mathrm{mrad}]$ & $1.7[\mathrm{MeV} /(\mathrm{MeV} / c)]$ \\
\hline$e^{\prime}$ momentum $\left(\delta\left(p_{e^{\prime}}\right)\right)$ & - & \\
$e^{\prime}$ energy loss $\left(\delta\left(\Delta E_{e^{\prime}}\right)\right)$ & $0.28[\mathrm{MeV}]$ & $0.10[\mathrm{MeV} / \mathrm{mrad}]$ \\
\hline$e^{\prime}$ horizontal angle $\left(\delta\left(x_{e^{\prime}}^{\prime}\right)\right)$ & $3[\mathrm{mrad}]$ & $0.054[\mathrm{MeV} / \mathrm{mrad}]$ \\
$e^{\prime}$ vertical angle $\left(\delta\left(y_{e^{\prime}}^{\prime}\right)\right)$ & $3[\mathrm{mrad}]$ & \\
$e^{\prime}$ multiple scattering $\left(\delta\left(\theta_{K^{+}}\right)\right)$ & $<1[\mathrm{mrad}]$ & \\
\hline \hline
\end{tabular}

Using above information and the missing mass resolution of $\mathrm{CH}_{2}$ spectrum, the relation between $\delta\left(p_{K^{+}}\right)$and $\delta\left(p_{e^{\prime}}\right)$ can be derived from the equation 6.3. Figure 6.3 shows the relation with the z-axis of the missing mass resolution of $\mathrm{CH}_{2}$ spectrum. The red line shows the missing mass resolution of $1.6 \mathrm{MeV}$ (FWHM), and it is possible to read the relation of $\delta\left(p_{K^{+}}\right)$and $\delta\left(p_{K^{+}}\right)$. For example, if the $\delta\left(p_{K^{+}}\right)$and $\delta\left(p_{K^{+}}\right)$are assumed to be almost balanced, we can say that $\delta\left(p_{K^{+}}\right) \sim 4 \times 10^{-4}$ and $\delta\left(p_{e^{\prime}}\right) \sim 6 \times 10^{-4}$. These values are almost two time larger than the designed value. This result indicates that there is the possibility to improve the mass accuracy..

There are two considerable way which is possible to obtain much more accurate mass scale. The first one is the usage of higher order $F 2 T$ function. In general, the edge part of the spectrometer has complicated magnetic field and higher order magnetic filed is necessary compared to the description of the central part. In fact, as already mentioned, the spectrum shown in figure 6.2 was applied a cut of $x^{\prime}$ at HKS FP, because the energy resolution is bad in the edge part of the $x^{\prime}$. 
Next one is the improvement of magnetic field with the SS data. As far as looking at reconstructed SS pattern (see figure 5.27 and 5.28), the central part of the SS is calibrated well, but the misalignment of the edge part can be seen. The angular part of the F2T function was not tuned in the direct tuning, then this ambiguity is still remained in the final mass spectrum in this study. In any sense, further calibration of the "edge" part of the acceptance might be effective to improve the precision of the mass spectrum.

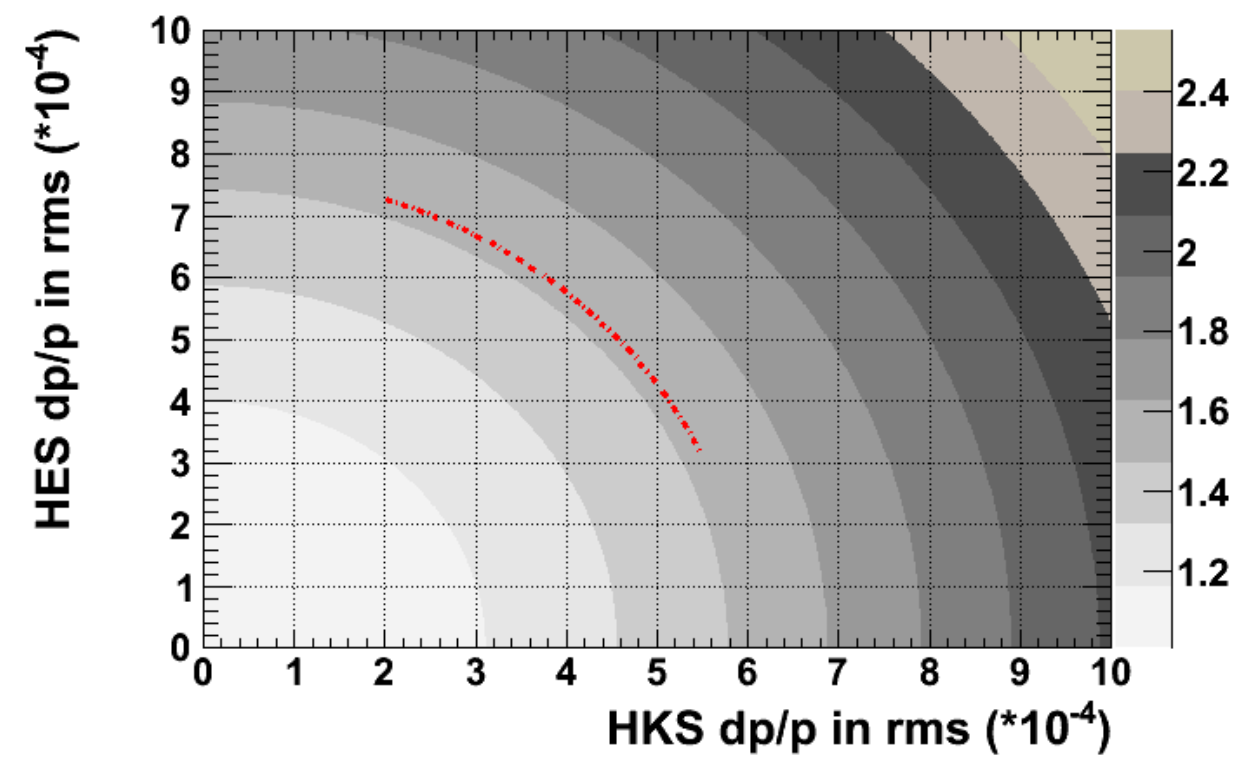

Figure 6.3: The relation between $\delta\left(p_{K^{+}}\right)$and $\delta\left(p_{e^{\prime}}\right)$. The z-axis shows the resolution of $\mathrm{CH}_{2}$ missing mass spectrum (MeV, FWHM). The red line shows the missing mass resolution of $1.6 \mathrm{MeV}$ (FWHM).

\subsubsection{Cross section of the $\mathbf{p}\left(\gamma^{*}, \mathrm{~K}^{+}\right) \Lambda / \Sigma^{0}$ reaction}

Figure 6.4 is the missing mass spectrum for $\Lambda$ and $\Sigma^{0}$ with the vertical scale of differential cross section. The cut of $x_{f K}^{\prime}$ was not applied for this figure, then its cut efficiency has not to be concerned.

For the cross section calculation, the proton decrease effect was concerned. During the experiment, the proton targets were leaked away from the target because of the significant heat deposit of the beam. We estimated this effect by the time dependence of $\Lambda$ yield, and the number of proton was decreased about $83 \pm 15 \%$ in average [59].

The fitting function was same one as equation 6.1 and the Integral of the function was converted to the cross sections. The obtained cross section (averaged in the HKS acceptance) is summarized in table 6.1.3. In the systematic error shown in the table, the error by the background subtraction is considered. The cross section was obtained by the function 6.1 and the background shape was assumed as the second polynomial. However, the background shape can be also obtained by the ${ }^{12} \mathrm{C}$ target data. The shape 
was compared to the fitted function, and the difference was obtained as $5 \%$ of the $\Lambda$ peak and $7 \%$ for $\Sigma^{0}$ peak. These differences were considered as a component of the systematic error.
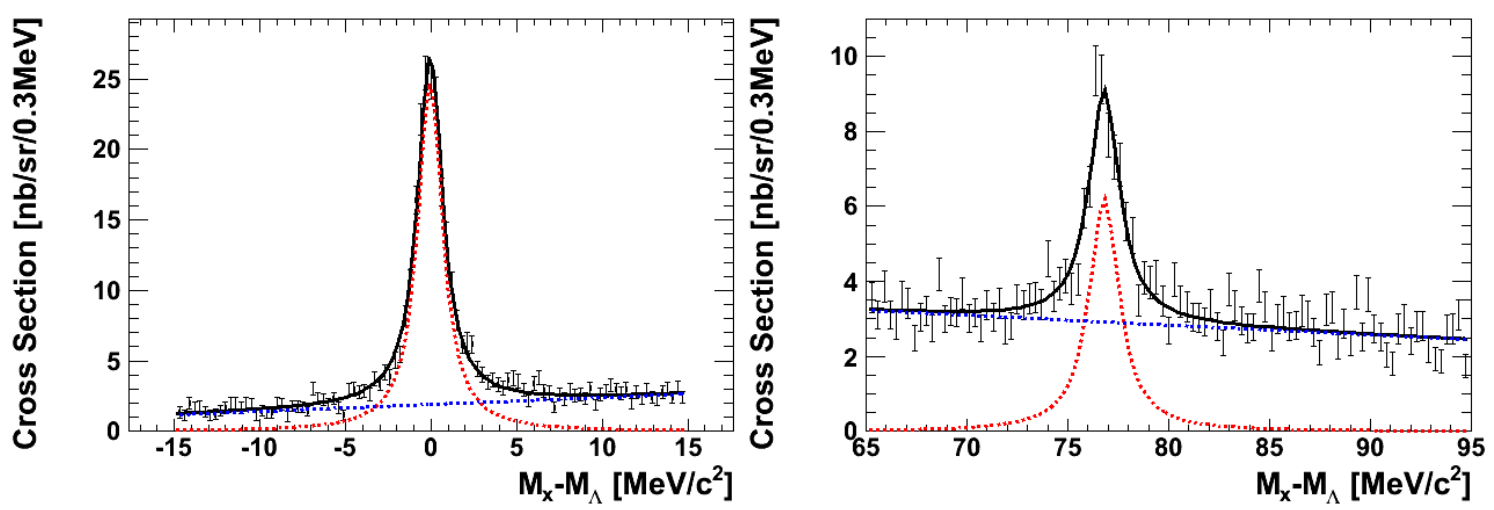

Figure 6.4: The missing mass spectrum for $\Lambda$ (left) and $\Sigma^{0}$ (right) with the vertical scale of differential cross section.

Table 6.1.3: Obtained differential cross sections and total cross sections of the $\mathrm{p}\left(\gamma^{*}, \mathrm{~K}^{+}\right) \Lambda / \Sigma^{0}$ reactions.

\begin{tabular}{c|c|c|c}
\hline \hline Peak & $\begin{array}{c}\left.\overline{\left(d \sigma / d \Omega_{K}^{c m}\right)}\right|_{1^{\circ}-13^{\circ}[\mathrm{nb} / \mathrm{sr}]} \\
\pm(\text { stat. }) \pm(\text { sys. })\end{array}$ & $\begin{array}{c}\sigma_{1^{\circ}-13^{\circ}}[\mathrm{nb}] \\
\pm(\text { stat. }) \pm(\text { sys. })\end{array}$ & $\chi^{2} / \mathrm{ndf}$ \\
\hline$\Lambda$ & $227 \pm 12 \pm 26$ & $1.74 \pm 0.09 \pm 0.19$ & $115.6 / 93$ \\
$\Sigma^{0}$ & $53 \pm 6 \pm 6$ & $0.39 \pm 0.04 \pm 0.05$ & $131.6 / 93$ \\
\hline \hline
\end{tabular}

\section{Comparison with the cross section of the $\mathbf{p}\left(\gamma, K^{+}\right) \Lambda / \Sigma^{0}$ reaction}

Since our $Q^{2}$ of the virtual is almost "real", the obtained data will be compared to the real photon reaction, $\left(\gamma, K^{+}\right)$. For the comparison, the kinematic condition of our experiment is important and shown in figure 6.5. The typical value of each parameter is $\theta_{K}^{c m}=0.3$ $[\mathrm{rad}]\left(\cos \theta_{K}^{c m}=0.95\right),-Q^{2}=0.01\left[(\mathrm{GeV} / c)^{2}\right]$ and $W=1.93[\mathrm{GeV}]\left(E_{\gamma}=1.5[\mathrm{GeV}]\right)$. Here $W$ is the total energy of the $\left(\gamma^{*}, \mathrm{~K}^{+}\right)$reaction.

The cross section is compared with the experimental data of CLAS (JLab)[43] and SAPHIR (ELSA) [74] (table 6.1.4). The obtained cross section is slightly smaller than these results, but almost consistent within the error bar. It can be expected the difference of $Q^{2}$ affect the cross section value, but at least there are no significant difference. From this consistent result with the other experiments, the yield of $\Lambda$ and is confirmed as the same level with the designed value.

From these analysis results of the $\mathrm{CH}_{2}$ target, the experimental system containing the HES worked at the point of both resolution and yield. 

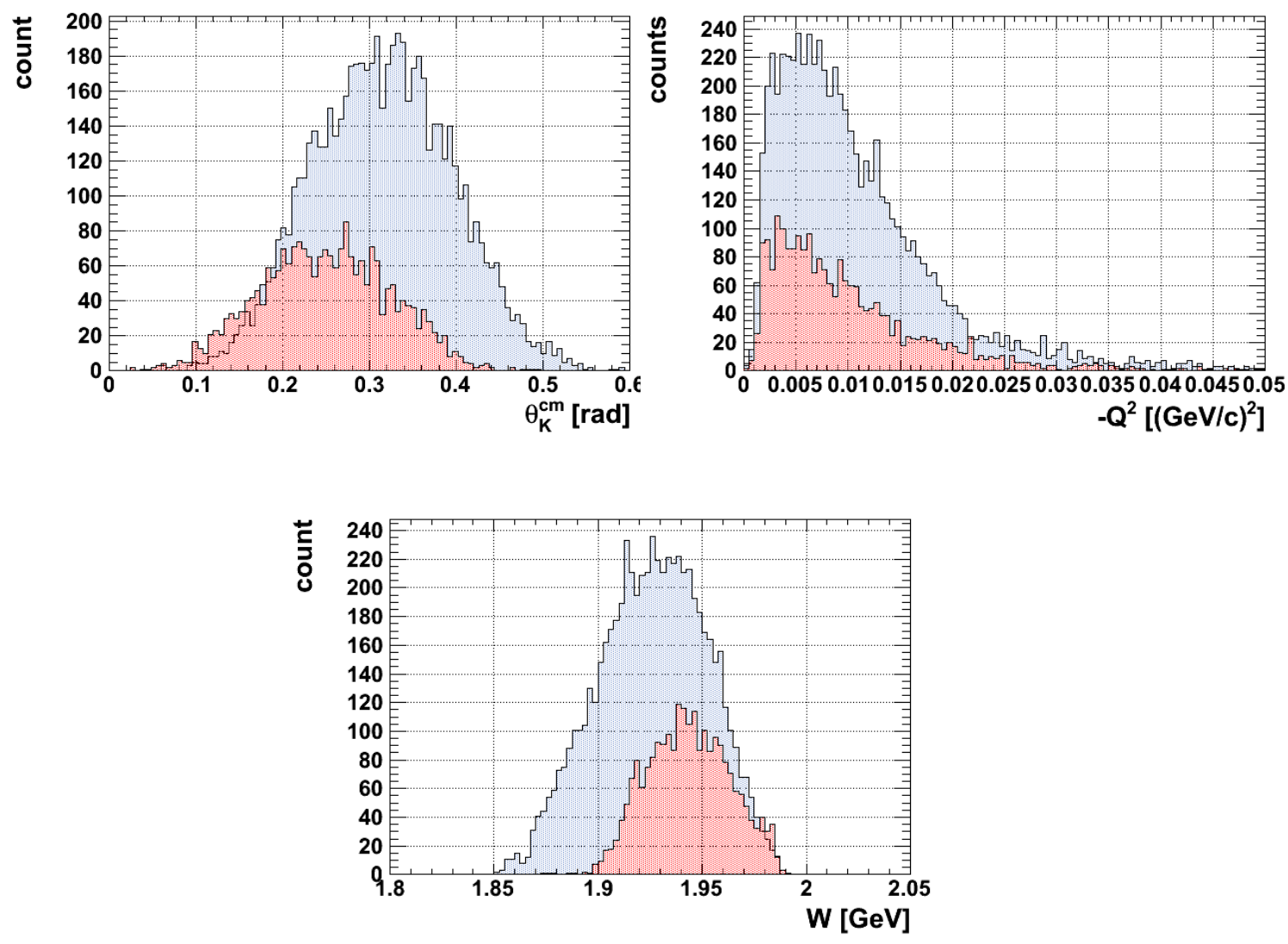

Figure 6.5: Important kinematic parameters for $\Lambda$ and $\Sigma^{0}$. The blue histogram shows the parameter of $\Lambda$ and red one is that of $\Sigma^{0}$.

Table 6.1.4: Obtained differential cross sections and total cross sections of the $\mathrm{p}\left(\gamma^{*}, \mathrm{~K}^{+}\right) \Lambda$ reactions.

\begin{tabular}{c|c|c|c|c}
\hline \hline Experiment & $-Q^{2}\left[(\mathrm{GeV} / c)^{2}\right]$ & $\cos \theta_{K}^{c m}$ & $E_{\gamma}[\mathrm{GeV}]$ & $\overline{\left(d \sigma / d \Omega_{K}^{c m}\right)}[\mathrm{nb} / \mathrm{sr}]$ \\
\hline E05-115 & 0.001 & 0.95 & 1.5 & $227 \pm 12 \pm 26$ \\
SAPHIR & 0 & $0.9-1.0$ & $1.45-1.5$ & $240 \pm 39 \pm 27$ \\
& 0 & $0.9-1.0$ & $1.5-1.55$ & $225 \pm 40 \pm 26$ \\
CLAS & 0 & 0.9 & 1.496 & $318 \pm 21$ \\
\hline \hline
\end{tabular}




\subsection{Experimental results and discussion for ${ }_{\Lambda}^{12} \mathrm{~B}$}

Spectroscopy of ${ }_{\Lambda}^{12} \mathrm{~B}$ will be performed in this section. Obtained production cross section and $\Lambda$ binding energy of hypernuclear states will be summarized and compared with theoretical calculations and other experimental results.

\subsubsection{Mass spectra}

The missing mass spectra are obtained from the experimental data summarized in 3.11. The missing mass was calculated by equation 5.9 for these target run, and the $\Lambda$ binding energy $-B_{\Lambda}$ is defined as the mass difference between the $\Lambda$ hypernuclear mass $\left(M_{H Y}\right)$ and the sum of $\Lambda$ mass $\left(M_{\Lambda}\right)$ and core nuclear mass $\left(M_{\text {core }}\right)$ :

$$
-B_{\Lambda}=M_{H Y}-\left(M_{\Lambda}+M_{\text {core }}\right) .
$$

The $M_{\Lambda}$ and $M_{\text {core }}$ values used in this calculation are summarized in appendix A.

Figure 6.6 is the obtained spectrum of ${ }_{\Lambda}^{12} \mathrm{~B}$ (data set \#6, 7, data set \#8 was not included in this figure). The accidental background spectrum obtained from mixed event analysis is also shown in the same figure as the blue region.

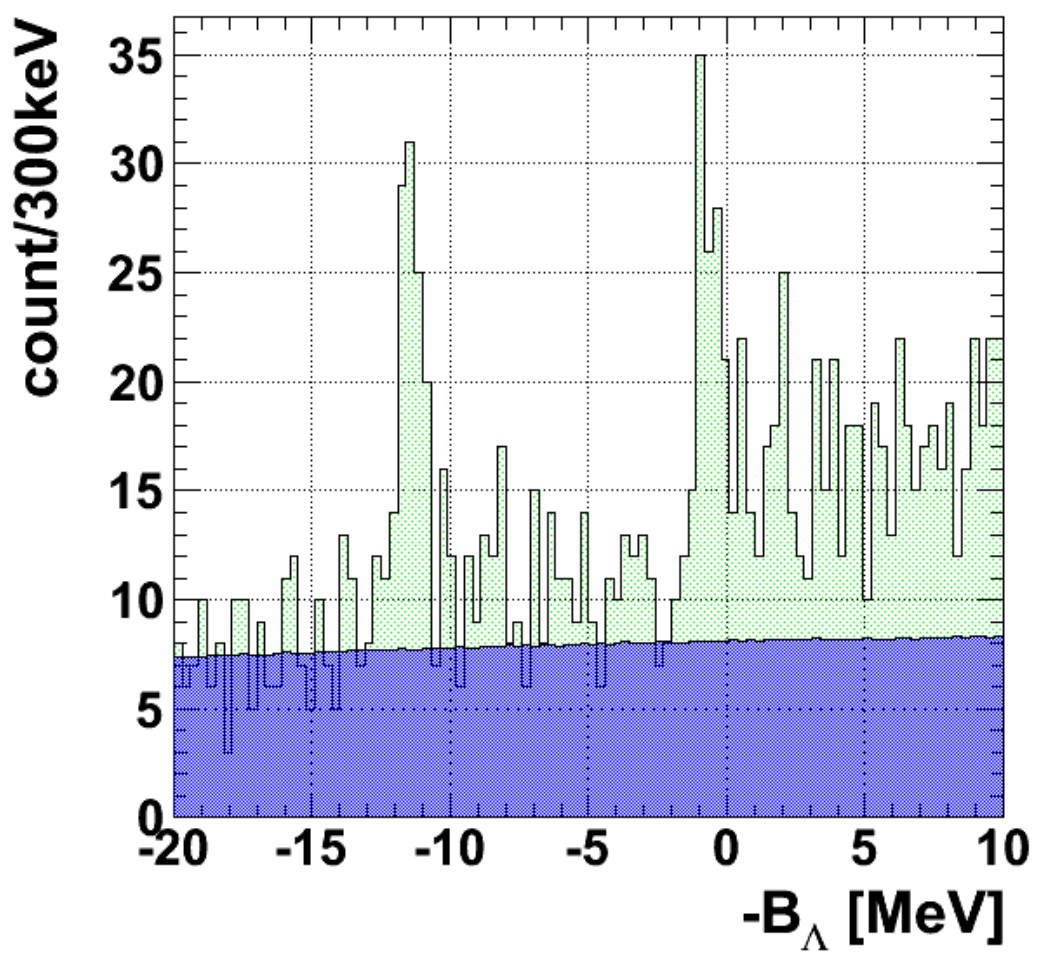

Figure 6.6: The missing mass spectrum for ${ }_{\Lambda}^{12} \mathrm{~B}$ with counts on the vertical scale. 


\subsubsection{Spectroscopy of ${ }_{\Lambda}^{12} \mathrm{~B}$}

${ }_{\Lambda}^{12} \mathrm{~B}$ is a good reference for the confirmation of the experimental system, because the spectra were observed several times in the past $\left(\mathrm{e}, \mathrm{e}^{\prime} \mathrm{K}^{+}\right)$experiments. In particular, it is important to check the consistency of the cross section and binding energy of the ground state (gs) with the past experiments. The gs events were used for the mass scale calibration (see section 5.5.1).

Figure 6.7 is the ${ }_{\Lambda}^{12} \mathrm{~B}$ missing mass spectrum (corresponds to data set \#6 and \#7, only for the lower current runs) with the vertical scale of differential cross section. The error bar represent statistic error and the accidental background estimated by mix event analysis was subtracted.

Two major peaks are observed around $-B_{\Lambda}=-11 \mathrm{MeV}$ and $-1 \mathrm{MeV}$. They are interpreted as ${ }_{\Lambda}^{12} \mathrm{~B}$ hypernuclear states which have configurations of a proton-hole in the $p$ orbit of ${ }^{12} \mathrm{C}$ and a $\Lambda$ hyperon in the $s$ and $p$ orbit couples as $\left[\left(p_{3 / 2}\right)_{p}^{-1} \otimes\left(s_{1 / 2}\right)_{\Lambda}\right]$ and $\left[\left(p_{3 / 2}\right)_{p}^{-1} \otimes\left(p_{3 / 2}, p_{1 / 2}\right)_{\Lambda}\right]$, respectively.

In order to obtain the $\Lambda$ binding energy and cross section of each peak, six Cauchy functions were used. The $\mathrm{QF}$ part $\left(-B_{\Lambda}>0\right.$ region) was fitted with a function proportional to $\sqrt{-B_{\Lambda}}$. In order to do the fitting, the past experimental results were referred to set the limits to the fitting parameter, particularly for the central value of each Cauchy function, except for peak \#4. The referred data is the result of JLab E94-107 experiment (see figure 6.10) and JLab E01-011 experiment (see figure 6.11). About the peak \#4, the peak can be clearly seen, then it was taken part in the fitting. In addition, the widths of the Cauchy functions were fixed for the peak \#2, \#3, \#4 and \#6. With the above condition, the spectrum was fitted with $\chi^{2} / \mathrm{ndf}=55.05 / 75$. The obtained $\Lambda$ binding energies and cross sections are summarized in table 6.2.1 and 6.2.2.

Table 6.2.1: Obtained binding energies, excitation energies and width of ${ }_{\Lambda}^{12} \mathrm{~B}$.

\begin{tabular}{c|c|c|c}
\hline \hline $\begin{array}{c}\text { Peak } \\
\text { No. }\end{array}$ & $\begin{array}{c}-B_{\Lambda}[\mathrm{MeV}] \\
(\text { stat.) } \pm \text { (sys.) }\end{array}$ & $\begin{array}{c}E x[\mathrm{MeV}] \\
\pm \text { (stat.) } \pm(\text { sys. })\end{array}$ & $\begin{array}{c}\text { FWHM } \\
{[\mathrm{MeV}]}\end{array}$ \\
\hline$\# 1$ & $-11.50 \pm 0.10 \pm 0.2$ & 0 & 1.1 \\
$\# 2$ & $-8.37 \pm 0.19 \pm 0.2$ & $3.13 \pm 0.46 \pm 0.2$ & 1.1 (fixed) \\
$\# 3$ & $-6.14 \pm 0.30 \pm 0.2$ & $5.36 \pm 0.56 \pm 0.2$ & 1.1 (fixed) \\
$\# 4$ & $-3.86 \pm 0.26 \pm 0.2$ & $7.63 \pm 0.51 \pm 0.2$ & 1.1 (fixed) \\
$\# 5$ & $-0.82 \pm 0.13 \pm 0.2$ & $10.68 \pm 0.38 \pm 0.2$ & 1.3 \\
$\# 6$ & $1.14 \pm 0.62 \pm 0.2$ & $12.68 \pm 0.80 \pm 0.2$ & 1.1 (fixed) \\
\hline \hline
\end{tabular}

\section{Comparison with other ${ }_{\Lambda}^{12} \mathrm{~B}$ data}

As mentioned in chapter 1 and 2, there are three experimental results of ${ }_{\Lambda}^{12} \mathrm{~B}$ via $\left(\mathrm{e}, \mathrm{e}^{\prime} \mathrm{K}^{+}\right)$ reaction spectroscopy. The first one is the result from the first generation $\left(\mathrm{e}, \mathrm{e}^{\prime} \mathrm{K}^{+}\right) \Lambda$ hypernuclear spectroscopic experiment in JLab Hall-C (E89-009, 2000) [46, 47], and the spectrum is shown in figure 6.9. The second one is the result of JLab Hall-A (E94-107, 2004) [75]. The spectrum is represented in figure 6.10 with the vertical axis of five-fold differential cross sections as $\left(\mathrm{e}, \mathrm{e}^{\prime} \mathrm{K}^{+}\right)$reaction, while the other two spectrum are drawn 


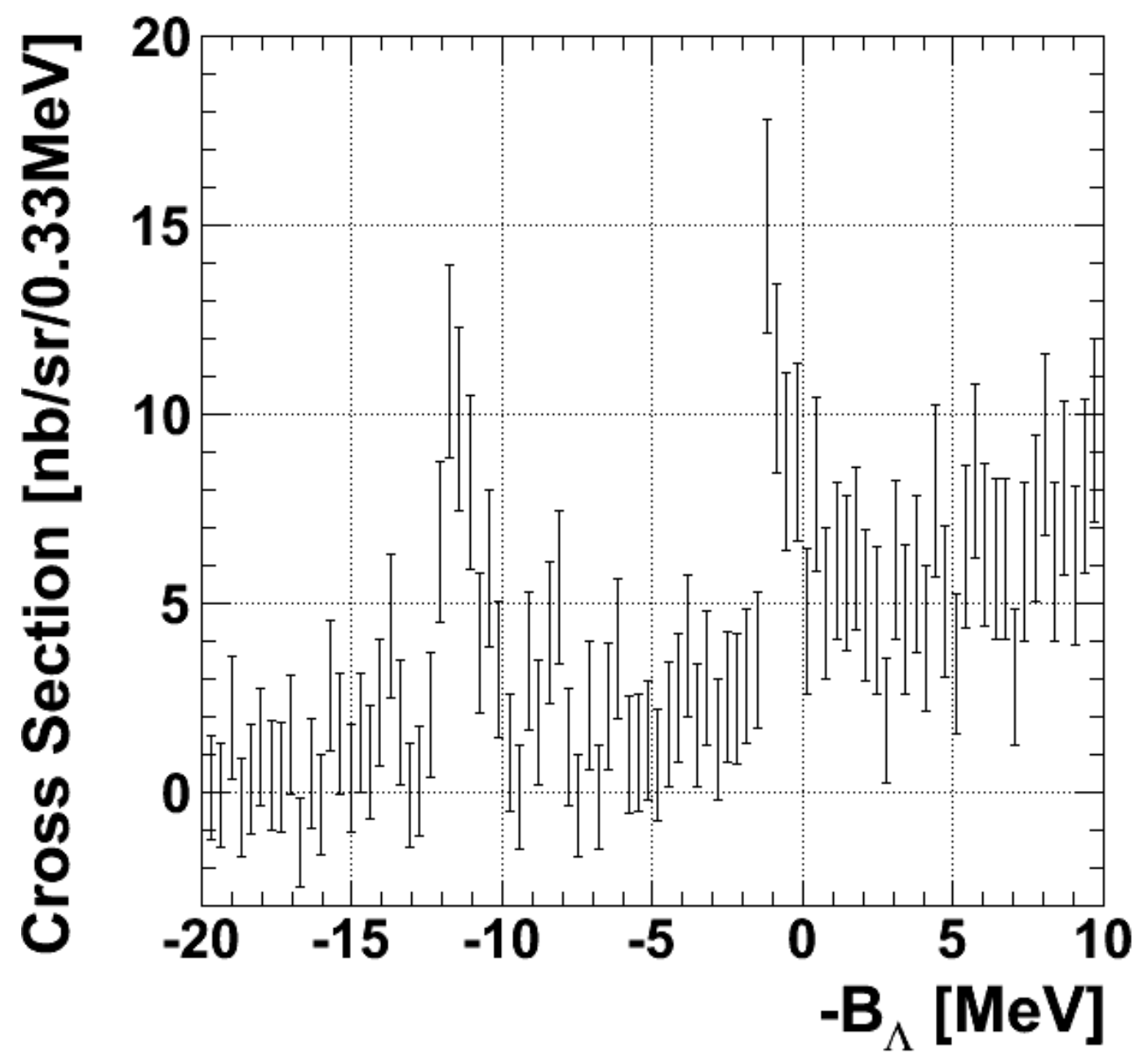

Figure 6.7: The missing mass spectrum for ${ }_{\Lambda}^{12} \mathrm{~B}$ with the vertical scale of differential cross section.

Table 6.2.2: Obtained differential cross sections and total cross sections of ${ }_{\Lambda}^{12} \mathrm{~B}$.

\begin{tabular}{c|c|c}
\hline \hline $\begin{array}{c}\text { Peak } \\
\text { No. }\end{array}$ & $\begin{array}{c}\left.\overline{(d \sigma / d \omega)}\right|_{1^{\circ}-13^{\circ}[\mathrm{nb} / \mathrm{sr}]} \pm(\text { stat. }) \pm(\text { sys. }) \\
\# 1\end{array}$ & $\begin{array}{c}\sigma_{1^{\circ}-13^{\circ}}[\mathrm{nb}] \\
(\text { stat. }) \pm(\text { sys. })\end{array}$ \\
\hline$\# 2$ & $16 \pm 6 \pm 2$ & $0.53 \pm 0.15 \pm 0.06$ \\
$\# 3$ & $8 \pm 6 \pm 1$ & $0.14 \pm 0.05 \pm 0.02$ \\
$\# 4$ & $13 \pm 6 \pm 2$ & $0.07 \pm 0.05 \pm 0.01$ \\
$\# 5$ & $72 \pm 23 \pm 9$ & $0.11 \pm 0.05 \pm 0.01$ \\
$\# 6$ & $15 \pm 9 \pm 2$ & $0.13 \pm 0.19 \pm 0.07 \pm 0.02$ \\
\hline \hline
\end{tabular}




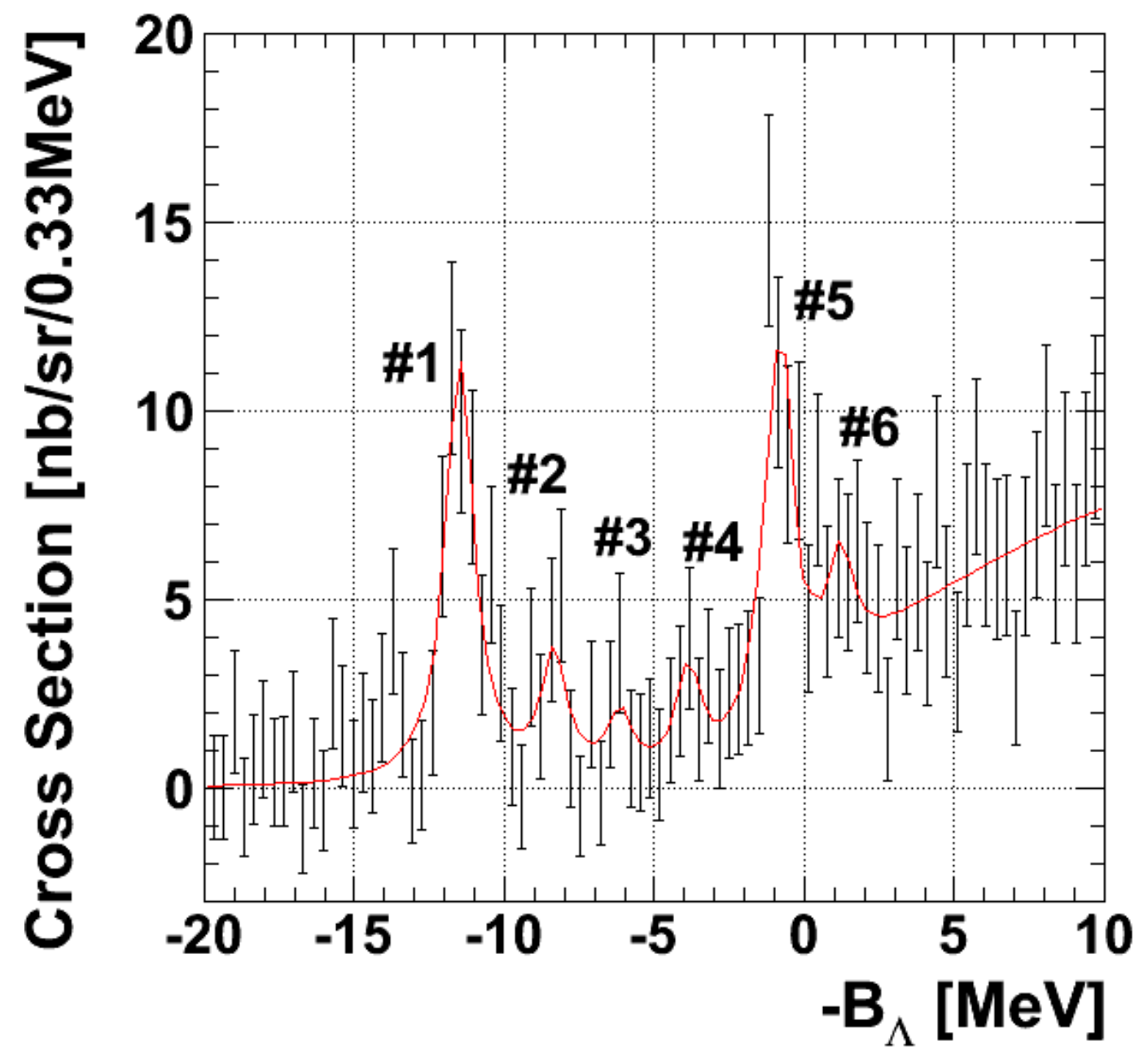

Figure 6.8: The fitted missing mass spectrum of ${ }_{\Lambda}^{12} \mathrm{~B}$. The vertical scale is differential cross section. 
with three-fold differential cross sections as $\left(\gamma^{*}, \mathrm{~K}^{+}\right)$reaction. The third one is the result from the second generation experiment in JLab Hall-C (E01-011, 2005), and the spectrum is shown in figure 6.11 .

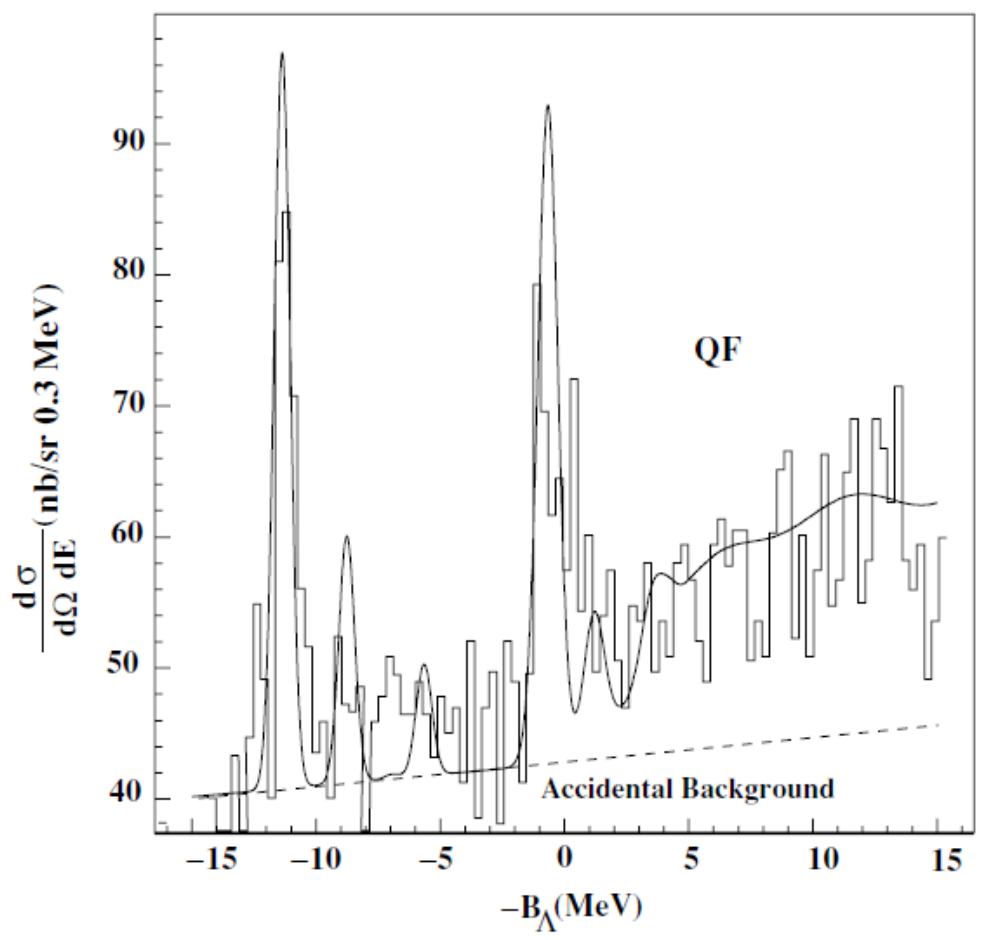

Figure 6.9: The missing mass spectrum of ${ }_{\Lambda}^{12} \mathrm{~B}$ obtained in JLab E89-009 experiment [47].

The kinematic conditions of these three experiments and the present E05-115 experiments are summarized in table 6.2.3. The cross sections are affected by these kinematic conditions, in particular, the energy and $Q^{2}$ of virtual photon and kaon scattered angle $\left(\theta_{K}\right)$.

The measured excitation (binding) energies and cross sections of $s_{\Lambda}$ state and $p_{\Lambda}$ state in these experiments are summarized in table 6.2.4 and 6.2.5, respectively. The $\Lambda$ binding energy measured in emulsion experiments [27] is also added to the table.

These values of the binding energies are almost consistent with the other experiments within the error bar. In particular, the obtained binding energy of $-11.50 \pm 0.10 \pm 0.2$ $\mathrm{MeV}$ for the ground state $\left(s_{\Lambda}\right)$ is in good agreement with the E01-011 value of $-11.40 \pm$ $0.01 \pm 0.04 \mathrm{MeV}$ which were determined with very small systematic error. For the energy spacing between $s_{\Lambda}$ and $p_{\Lambda}$, the obtained value of $10.68 \pm 0.38 \pm 0.2 \mathrm{MeV}$ is slightly smaller than that of E01-011 value $(11.05 \pm 0.01 \pm 0.19 \mathrm{MeV})$ and E94-107 value (10.93 \pm 0.03$)$. However, the value is within the large systematic error.

On the other hand, there are large inconsistency with the E01-011 experiment data about the cross section. This inconsistency is coming from the shape of the HKS acceptance. The "cross section" $\left.\overline{(d \sigma / d \omega)}\right|_{1^{\circ}-13^{\circ}}$ is not a physical quantity. It was integrated in 


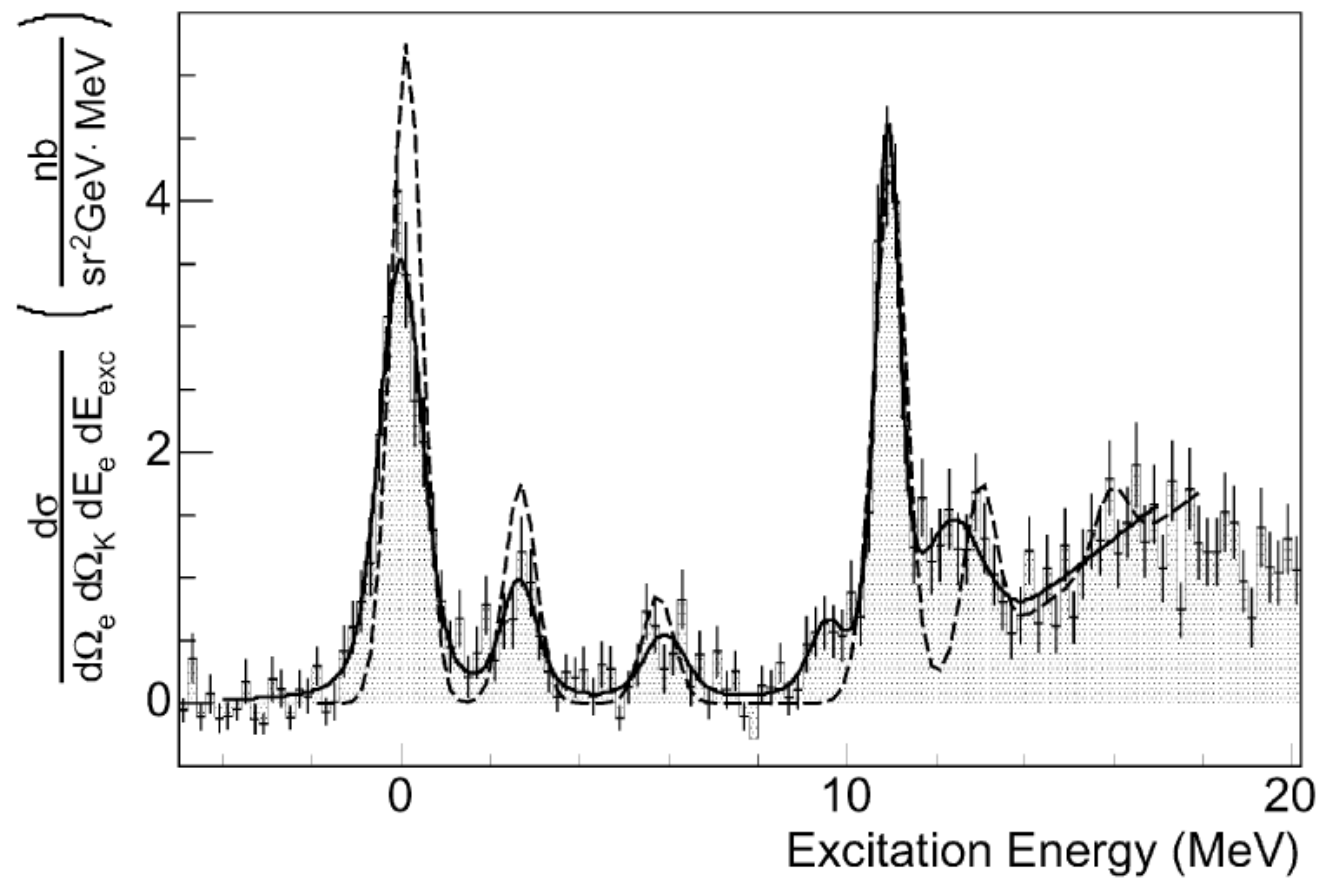

Figure 6.10: The missing mass spectrum of ${ }_{\Lambda}^{12} \mathrm{~B}$ obtained in JLab E94-107 experiment $[75]$.

Table 6.2.3: Comparison of kinematic conditions between hypernuclear experiments at JLab.

\begin{tabular}{c|c|c|c|c|c|c|c}
\hline $\begin{array}{c}\text { Experiment } \\
(\text { Year })\end{array}$ & $\begin{array}{c}E_{e} \\
{[\mathrm{GeV}]}\end{array}$ & $\begin{array}{c}E_{\gamma} \\
{[\mathrm{GeV}]}\end{array}$ & $\begin{array}{c}Q^{2} \\
{\left[(\mathrm{GeV} / c)^{2}\right]}\end{array}$ & $\begin{array}{c}P_{e^{\prime}} \\
{[\mathrm{GeV} / c]}\end{array}$ & $\begin{array}{c}\theta_{e^{\prime}} \\
{[\mathrm{deg}]}\end{array}$ & $\begin{array}{c}P_{K} \\
{[\mathrm{GeV} / c]}\end{array}$ & $\begin{array}{c}\theta_{K} \\
{[\mathrm{deg}]}\end{array}$ \\
\hline $\begin{array}{c}\text { E05-115 } \\
(2009)\end{array}$ & 2.34 & 1.5 & 0.01 & 0.844 & 4.0 & 1.2 & 7.0 \\
\hline $\begin{array}{c}\text { E01-011 } \\
(2005)\end{array}$ & 1.85 & 1.5 & 0.01 & 0.32 & 4.5 & 1.2 & 7.0 \\
\hline $\begin{array}{c}\mathrm{E} 94-107 \\
(2004,2005)\end{array}$ & $\begin{array}{c}4.92 / 3.78 \\
/ 3.66\end{array}$ & 2.2 & 0.07 & $\begin{array}{c}1.8 / 1.57 / \\
/ 1.44\end{array}$ & 6.0 & 1.96 & 6.0 \\
\hline $\begin{array}{c}\mathrm{E} 89-009 \\
(2000)\end{array}$ & $\begin{array}{c}1.86 \\
1.72\end{array}$ & 1.5 & 0.01 & 0.28 & 0.0 & 1.2 & 0.0 \\
\hline \hline
\end{tabular}




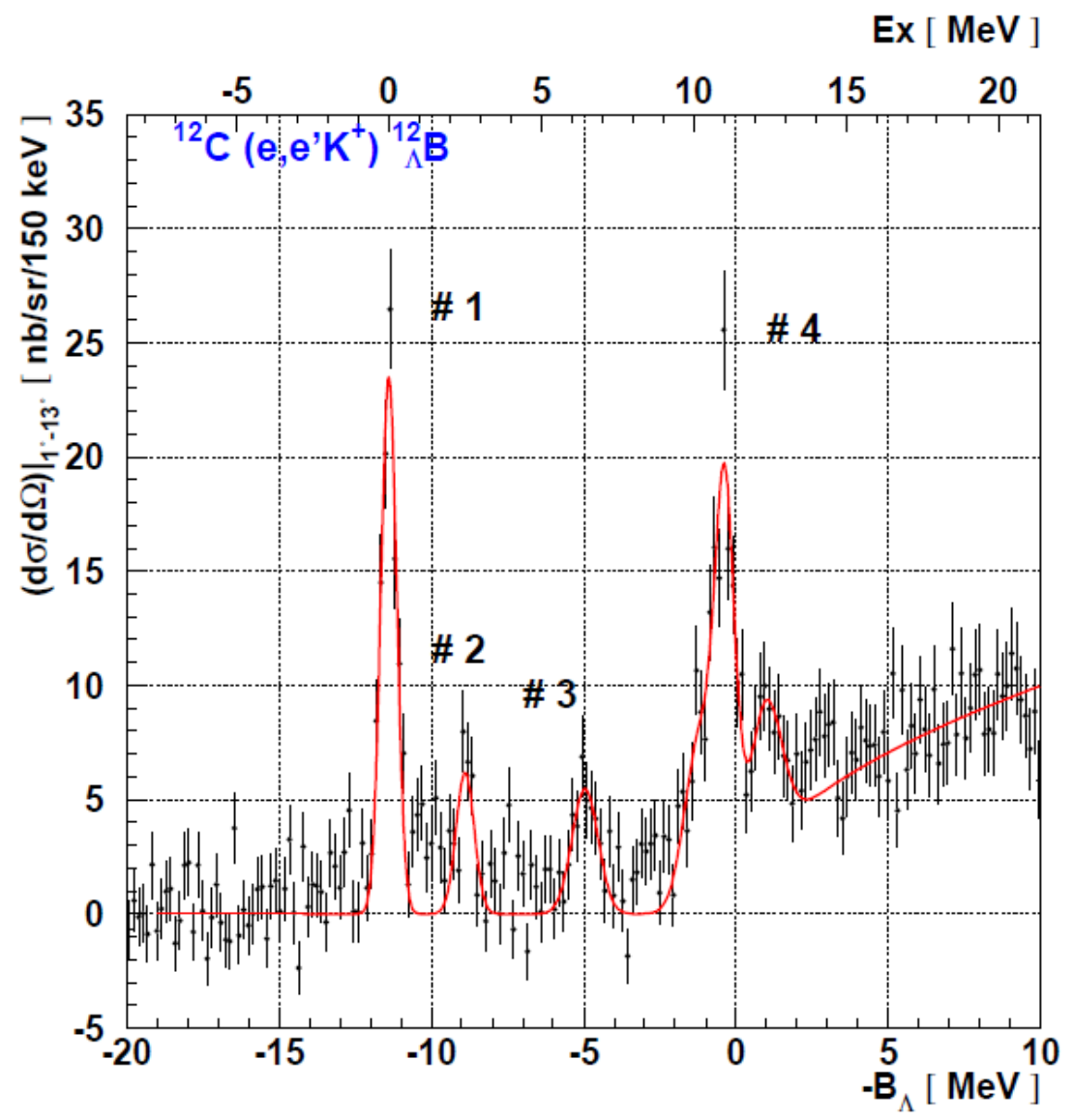

Figure 6.11: The missing mass spectrum of ${ }_{\Lambda}^{12} \mathrm{~B}$ obtained in JLab E01-011 experiment. 
Table 6.2.4: Comparison of excitation energies and width of ${ }_{\Lambda}^{12} \mathrm{~B}-s_{\Lambda}$ state for several experiments. The kinematic conditions of each experiment is summarized in table 6.2.3.

\begin{tabular}{c|c|c|c}
\hline \hline Experiment & $\begin{array}{c}E x\left(-B_{\Lambda}\right)[\mathrm{MeV}] \\
\pm(\text { stat. }) \pm(\text { sys. })\end{array}$ & $\begin{array}{c}\text { FWHM }[\mathrm{MeV}] \\
\pm(\text { stat. })\end{array}$ & $\left.\overline{(d \sigma / d \omega)}\right|_{1^{\circ}-13^{\circ}[\mathrm{nb} / \mathrm{sr}]}$ \\
\hline E05-115 & 0 & $1.1 \pm 0.2$ & $62 \pm 17 \pm 7$ \\
\hline E01-011 & 0 & $0.61 \pm 0.05$ & $101 \pm 4.2_{-31}^{+38}$ \\
\hline E94-107[75] & 0 & $1.15 \pm 0.18$ & $\mathrm{~N} / \mathrm{A}$ \\
\hline $\begin{array}{c}\text { E89-009 } \\
{[47]}\end{array}$ & 0 & 0.75 & $140 \pm 17 \pm 18$ \\
\hline $\begin{array}{c}\text { Emulsion } \\
{[27]}\end{array}$ & $(-11.40 \pm 0.01 \pm 0.04)$ & & $\mathrm{N} / \mathrm{A}$ \\
\hline \hline
\end{tabular}

Table 6.2.5: Comparison of excitation energies and width of ${ }_{\Lambda}^{12} \mathrm{~B}-p_{\Lambda}$ for several experiments. The kinematic conditions of each experiment is summarized in table 6.2.3.

\begin{tabular}{c|c|c|c}
\hline \hline Experiment & $\begin{array}{c}E x\left(-B_{\Lambda}\right)[\mathrm{MeV}] \\
\pm(\text { stat. }) \pm(\text { sys. })\end{array}$ & $\begin{array}{c}\text { FWHM }[\mathrm{MeV}] \\
\pm(\text { stat. })\end{array}$ & $\left.\overline{(d \sigma / d \omega)}\right|_{1^{\circ}-13^{\circ}[\mathrm{nb} / \mathrm{sr}]}$ \\
\hline E05-115 & $\begin{array}{c}10.68 \pm 0.38 \pm 0.2 \\
(-0.82 \pm 0.13 \pm 0.2)\end{array}$ & $1.3 \pm 0.3$ & $72 \pm 23 \pm 9$ \\
\hline E01-011 & $\begin{array}{c}11.05 \pm 0.01 \pm 0.19 \\
(-0.41 \pm 0.01 \pm 0.13)\end{array}$ & $0.87 \pm 0.13$ & $94 \pm 4.0 \pm 35$ \\
\hline E94-107[75] & $10.93 \pm 0.03$ & $0.67 \pm 0.15$ & $\mathrm{~N} / \mathrm{A}$ \\
\hline E89-009 & & $\mathrm{N} / \mathrm{A}$ & $\mathrm{N} / \mathrm{A}$ \\
{$[46],[47]$} & $(-0.49 \pm 0.16)$ & & \\
\hline \hline
\end{tabular}

the HKS acceptance and the value can be changed with its shape. In order to compare with the last experiment data, it is better to divide the angular acceptance as far as the statistics allow.

\section{Comparison with mirror symmetric ${ }_{\Lambda}^{12} \mathrm{C}$ data}

It is also possible to compare the obtained ${ }_{\Lambda}^{12} \mathrm{~B}$ spectrum with its mirror hypernuclear data; ${ }_{\Lambda}^{12} \mathrm{C}$. The spectrum of ${ }_{\Lambda}^{12} \mathrm{C}$ was measured via $\left(\pi^{+}, \mathrm{K}^{+}\right)$reaction spectroscopy in $\mathrm{KEK}$ E336 experiment $[21,23]$ The spectrum is shown in figure 6.12 with its excitation energy and cross section and the result of the fitting to observed peaks is summarized in table 6.2.6. The binding energy of ${ }_{\Lambda}^{12} \mathrm{C}$ ground state was determined by the result of emulsion experiment to be $B_{\Lambda}=10.76 \mathrm{MeV}$ [27].

The first major peak assigned as \#1 in figure 6.12 is interpreted as the ground state of ${ }_{\Lambda}^{12} \mathrm{C}$ which have the configuration of a neutron-hole and a $\Lambda$ particle in the $s$ orbit $\left(s_{\lambda}\right.$ state). Similarly, the second major peak assigned as \#4 is interpreted as $p_{\Lambda}$ state. The energy spacing between these two states is $11.00 \pm 0.03 \mathrm{MeV}$, which can be compared 
with that of ${ }_{\Lambda}^{12} \mathrm{~B}$. This value is almost consistent with the value of E01-011 and E94-107, and the obtained value of $10.68 \pm 0.38 \pm 0.2$ is smaller than this value.

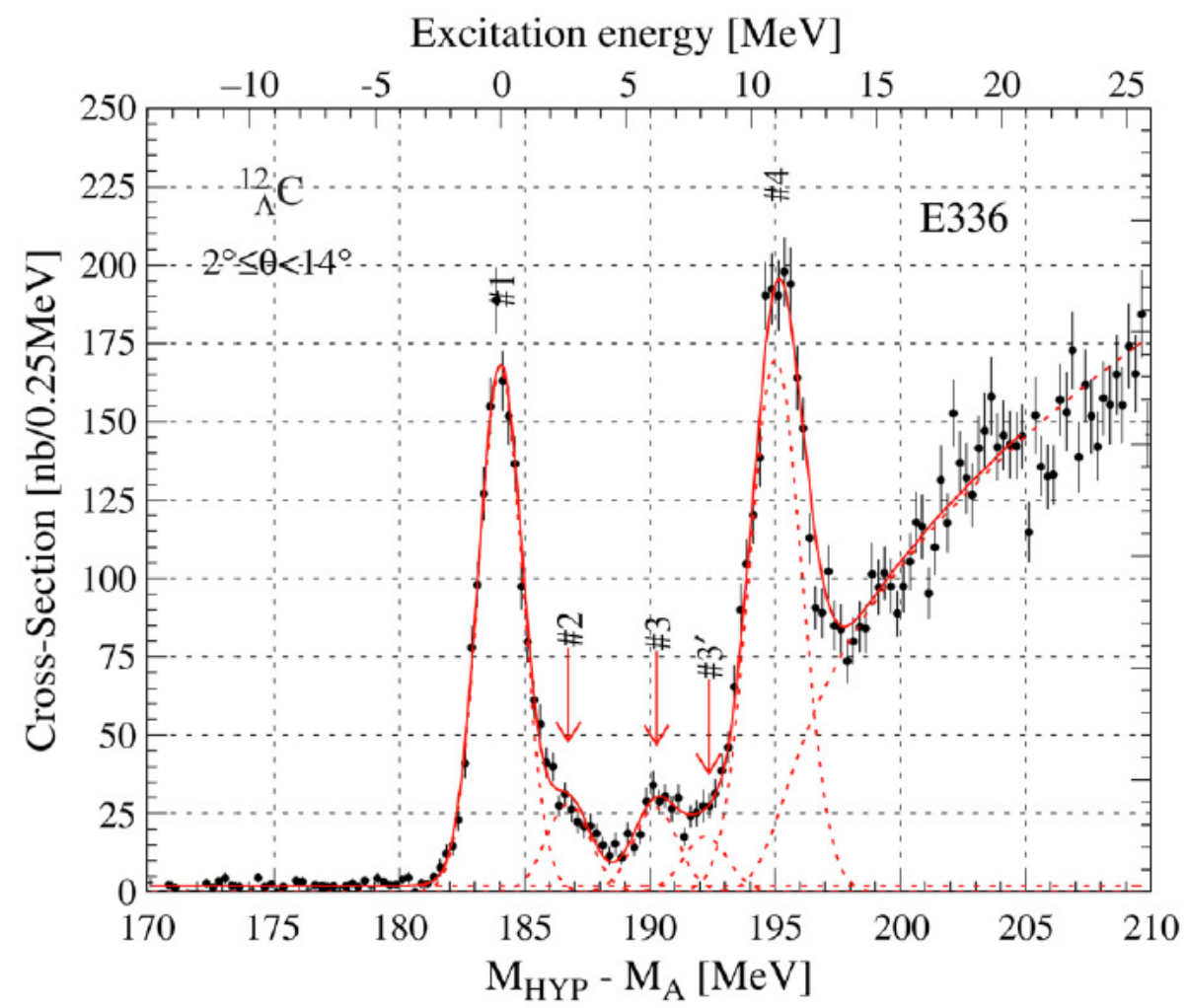

Figure 6.12: The missing mass spectrum of ${ }_{\Lambda}^{12} \mathrm{C}$ obtained by $\left(\pi^{+}, \mathrm{K}^{+}\right)$reaction, [21, 23].

These results are summarized in figure 6.13 with theoretical calculations. The shellmodel calculation results for ${ }_{\Lambda}^{12} \mathrm{C}$ by Millener [76] used the experimental results from $\gamma$-ray spectroscopy [23] to constrain the parameters for $\Lambda \mathrm{N}$ interaction. The predictions for ${ }_{\Lambda}^{12} \mathrm{~B}$ by electro- and photon-production was performed by Motoba etal. [77] in the DWIA framework with shell-model.

The obtained cross sections can be compared with the work of Sotona et al. [78] They compared some elemental production models : Scalay-Lyon A (SLA) [79] and KaonMAID [80]. The result of the comparison is summarized in table 6.2.7, for the two major peaks $\left(s_{\Lambda}\right.$ and $\left.p_{\Lambda}\right)$. The Kaon-MAID model includes four nucleon and two kaon resonances as intermediate state in resonance terms of s-channel and t-channel. The SLA model excludes the nucleon resonances with spin $1 / 2$ and $5 / 2$ and includes one nucleon resonance in s-channel, two kaon resonances in t-channel and four hyperon resonances in $\mathrm{u}$-channel. Cross sections for the virtual photon energies of $1.3<E<1.6 \mathrm{GeV}$ and the kaon scattering angle of $1^{\circ}<\theta_{K^{+}}^{L a b}<13^{\circ}$, which correspond to the present experimental conditions with a beam energy of $\mathrm{E}=2.3 \mathrm{GeV}$, are averaged for each theoretical The result shows that the present result preferable the SLA model for the $s_{\Lambda}$, and is consistent with the both calculations for the $p_{\Lambda}$. 
Table 6.2.6: Obtained differential cross sections and total cross sections of ${ }_{\Lambda}^{12} \mathrm{C}$.

\begin{tabular}{c|c|c|c}
\hline \hline $\begin{array}{c}\text { Peak } \\
\text { No. }\end{array}$ & $\begin{array}{c}E x \\
{[\mathrm{MeV}]}\end{array}$ & $\begin{array}{c}\text { FWHM } \\
{[\mathrm{MeV}]}\end{array}$ & $\begin{array}{c}\text { Cross section } \\
\sigma_{2^{\circ}-14^{\circ}}[\mu \mathrm{b} / \mathrm{sr}]\end{array}$ \\
\hline$\# 1$ & $0\left(B_{\Lambda}=10.76\right)$ & $1.97 \pm 0.03$ & $1.44 \pm 0.03$ \\
$\# 2$ & $2.63 \pm 0.06$ & $1.97 \pm 0.03$ & $0.24 \pm 0.02$ \\
$\# 3$ & $6.09 \pm 0.08$ & $1.97 \pm 0.03$ & $0.23 \pm 0.02$ \\
$\# 3$ & $8.12 \pm 0.17$ & $1.97 \pm 0.03$ & $0.18 \pm 0.02$ \\
$\# 4$ & $11.00 \pm 0.03$ & $2.39 \pm 0.06$ & $1.81 \pm 0.05$ \\
\hline \hline
\end{tabular}

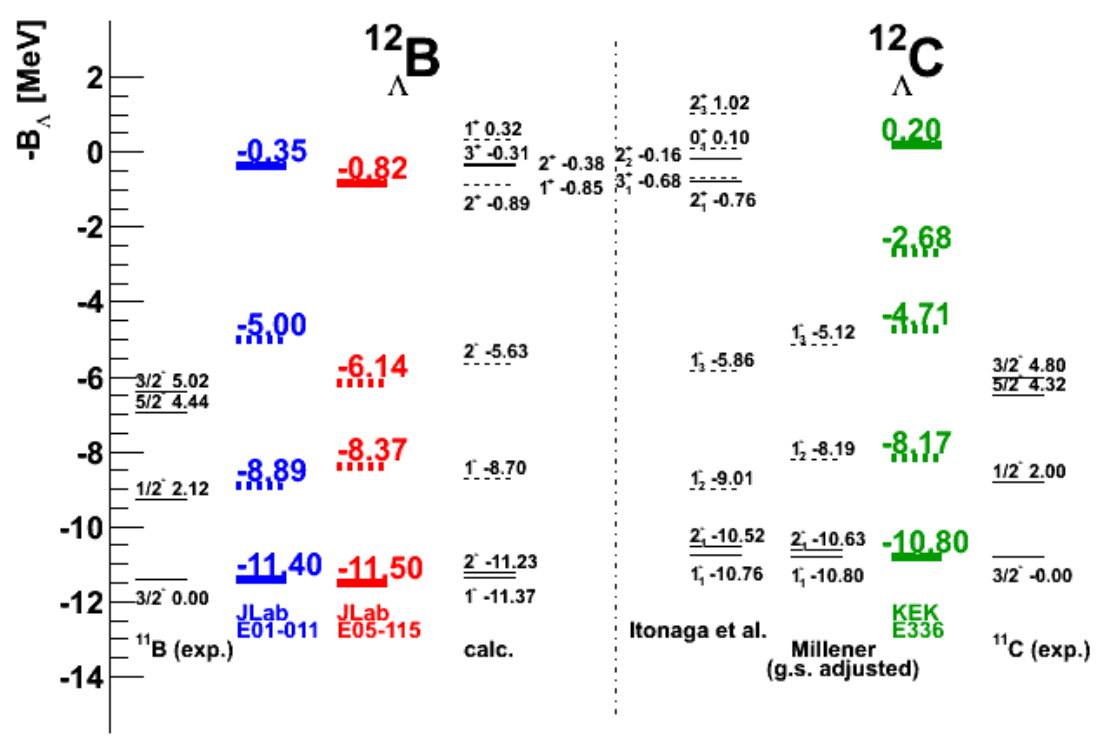

Figure 6.13: Comparison of the level schemes for ${ }_{\Lambda}^{12} \mathrm{~B}$ and ${ }_{\Lambda}^{12} \mathrm{C}$ and their core nuclei. The calculated binding energies of the ground states are adjusted to that of the emulsion data. 
It is interesting that the previous E01-011 data conclude the similar result [33]. As mentioned above, since the HKS angular acceptance is different from that of E01-011, the integration of theoretical result is different in both experimental condition.

Table 6.2.7: Comparison of the differential cross section with the present results and theoretical calculation. Note that the "cross section" is normalized with the HKS angular acceptance, $1^{\circ}-13^{\circ}$.

\begin{tabular}{|c|c|c|c|c|c|}
\hline Peak & $\begin{array}{c}\text { Exp. } \\
{[\mathrm{nb} / \mathrm{sr}]}\end{array}$ & $J^{\pi}$ & $\begin{array}{c}\mathrm{Ex} \\
{[\mathrm{MeV}]}\end{array}$ & $\begin{array}{c}\mathrm{SLA} \\
{[\mathrm{nb} / \mathrm{sr}]}\end{array}$ & $\begin{array}{c}\text { KMAID } \\
{[\mathrm{nb} / \mathrm{sr}]}\end{array}$ \\
\hline \multicolumn{6}{|c|}{ E05-115 } \\
\hline \multirow[t]{2}{*}{$s_{\Lambda}$} & $62 \pm 17 \pm 7$ & $1^{-}$ & 0 & 12.8 & 12.2 \\
\hline & & $2^{-}$ & 0.14 & 43.4 & 27.8 \\
\hline \multirow[t]{2}{*}{$p_{\Lambda}$} & $72 \pm 23 \pm 9$ & $2^{+}$ & 10.99 & 30.4 & 22.5 \\
\hline & & $3^{+}$ & 11.06 & 48.7 & 42.0 \\
\hline \multicolumn{6}{|c|}{ E01-011[33] } \\
\hline \multirow[t]{2}{*}{$s_{\Lambda}$} & $101 \pm 4.2_{-31}^{+38}$ & $1^{-}$ & 0 & 19.7 & 20.7 \\
\hline & & $2^{-}$ & 0.14 & 65.7 & 43.0 \\
\hline \multirow[t]{2}{*}{$p_{\Lambda}$} & $94 \pm 4.0 \pm 35$ & $2^{+}$ & 10.99 & 48.3 & 38.0 \\
\hline & & $3^{+}$ & 11.06 & 75.3 & 68.5 \\
\hline
\end{tabular}

From these comparison, it can be said that the obtained spectrum is consistent with the other experimental results and theoretical calculations within the error bar. However, still systematic error coming from the analysis procedure is not small in the present status. In particular, the evaluation of the core excited states of ${ }_{\Lambda}^{12} \mathrm{~B}$ involve large error coupled with the low statistics. This will be improved by the analysis of high rate data, including data set \#8. In this analysis, it was demonstrated that the calibration procedure of the mass scale is working, although there is still some possibility to be improved by the further calibration study. 


\section{Chapter 7}

\section{Conclusion and outlook}

Conclusion The investigation of hypernuclei is an important tool for the study of the strong interaction in the low energy region. In addition, the structure of hypernuclei itself has unique character because of the bound hyperon and its interactions with nucleons. The study of $\Lambda$ hypernuclei was historically performed via the $\left(\mathrm{K}^{-}, \pi^{-}\right)$and $\left(\pi^{+}, \mathrm{K}^{+}\right)$reaction, and the importance of precise hypernuclear spectroscopy increase its importance. The $\left(\mathrm{e}, \mathrm{e}^{\prime} \mathrm{K}^{+}\right)$reaction spectroscopy was started at 2000 using the CEBAF electron accelerator in JLab. This new generation reaction spectroscopy of hypernuclei can investigate various hypernuclear structures with an energy resolution of sub-MeV.

A goal of the present study is to establish the experimental way to investigate hypernuclei via the $\left(\mathrm{e}, \mathrm{e}^{\prime} \mathrm{K}^{+}\right)$reaction precisely, in the wide mass region. This is a challenging experiment because of the huge background events caused by the bremsstrahlung and $\mathrm{M} \phi$ ller scattering process, whose counting rate is roughly proportional to the $Z^{2}$ of the experimental target. In the last experiments performed at JLab Hall-C (E01-011), we applied the "tilt method" in order to optimize the counting rate of the background and hypernuclear yield. In addition, we designed and constructed a new kaon spectrometer (HKS) dedicated to the hypernuclear spectroscopy. The experimental data of ${ }^{7} \mathrm{Li},{ }^{12} \mathrm{C}$ and ${ }^{28} \mathrm{Si}$ target was successfully taken thanks to these techniques. In the present study, we improved the experimental setup in order to be able to expand the target mass region, using these accumulated knowledges. As a result, we used higher energy $(2.344 \mathrm{GeV})$ beam compared to that of the last experiment and newly constructed the HES and SPL aiming to effective data taking with medium heavy target $\left({ }^{52} \mathrm{C}\right.$, in the present case) with the high energy resolution of several handled keV.

The SPL magnet is a part of the beam line, the HKS and the HES, simultaneously. Therefore the magnet shape and magnetic field was designed carefully so as to achieve these roles. The SPL was working fine during the experiment after the parameter tuning of the beam line. The HES was designed as a Q-Q-D type spectrometer, as well as the HKS, aimed to have the degree of freedom for the vertical and horizontal focusing. The tilt method was also applied to the HES, and the tilt angle was decided to be 6.5 degree after the optimization using the Geant4 simulation with calculated 3D magnetic field. By the construction of the HES and the SPL, the hypernuclear yield was expected to be about five times larger than that of the last experiment (E01-011). The momentum resolution of the HES is simulated to be about $\delta p / p=3 \times 10^{-4}$, and the missing mass resolution is estimated to be $600 \mathrm{keV}$ (FWHM). 
The experiment was done in 2009 with the setup of the HKS, the HES and the SPL in JLab Hall-C, aiming to do the spectroscopy of ${ }_{\Lambda}^{7} \mathrm{He},{ }_{\Lambda}^{9} \mathrm{Li},{ }_{\Lambda}^{10} \mathrm{~B},{ }_{\Lambda}^{12} \mathrm{~B}$ and ${ }_{\Lambda}^{52} \mathrm{~V}$. The elementary reaction data of $\mathrm{p}\left(\mathrm{e}, \mathrm{e}^{\prime} \mathrm{K}^{+}\right) \Lambda / \Sigma^{0}$ was also taken using the $\mathrm{CH}_{2}$ and $\mathrm{H}_{2} \mathrm{O}$ target. For the $\mathrm{H}_{2} \mathrm{O}$ target, water-cell target was newly designed. The data was successfully taken with this setup and high intensity beam up to $40 \mu \mathrm{A}$.

The data analysis was performed with the following steps:

- Particle tracking,

- Kaon identification,

- Missing mass scale calibration,

- Cross section calculation.

There are traditional analysis method for the particle tracking and kaon identification, and it worked well for the low intensity data.

For the missing mass scale calibration, we used the two information, one is the Sieve Slit (SS) data and another is the known mass peaks. The SS data was used to tune the shape and magnitude of the calculated magnetic field. The used peaks for this procedure were the $\Lambda$ and $\Sigma^{0}$ peaks via the $\mathrm{p}\left(\mathrm{e}, \mathrm{e}^{\prime} \mathrm{K}^{+}\right) \Lambda / \Sigma^{0}$ reaction and the hypernuclear peak of ${ }_{\Lambda}^{12} \mathrm{~B}$ ground state. These peak information was used for the direct tuning of the reconstruction function. As a result of this calibration procedure and the analysis of the $\mathrm{CH}_{2}$ missing mass spectrum, we estimated the momentum resolution of $\delta p / p \sim 4 \times 10^{-4}$ for the HKS and $\delta p / p \sim 6 \times 10^{-4}$ for the HES (both of them in rms) in the current situation. These values are the double of the designed value, therefore there is a room to improve the momentum resolution by the further study of the spectrometer optics. However, the calibration procedure itself could obtain the $\Lambda / \Sigma^{0}$ and hypernuclear peaks with the same order of the designed energy resolution from the original reconstruction function made by the calculated magnetic field.

The cross section was calculated with the several estimated factors. The averaged $\mathrm{p}\left(\gamma^{*}, \mathrm{~K}^{+}\right) \Lambda$ cross section in the HKS acceptance, $\left(0.90<\cos \left(\theta_{K^{+}}^{C M}\right)<1.0\right)$ was calculated as $227 \pm 12 \pm 26$ [nb/sr], which is consistent within the error bar with the other experiment results of $\mathrm{p}\left(\gamma, K^{+}\right) \Lambda$. The obtained yield of the peak was almost same as the designed value with the considered detector efficiencies.

The hypernuclear spectrum of ${ }_{\Lambda}^{12} \mathrm{~B}$ was also obtained. However, there are the possibility of improvement for the calibration of the mass scale for the precise measurement of the ${ }_{\Lambda}^{12} \mathrm{~B}$ and other hypernuclei.

These analysis result represents that the experimental setup including the newly constructed HES and SPL worked and the calibration procedure of this unique experimental setup is basically established. One of the experimental advantages of the $\left(\mathrm{e}, \mathrm{e}^{\prime} \mathrm{K}^{+}\right)$reaction spectroscopy is that the absolute mass scale can be calibrated by the known mass of $\Lambda / \Sigma^{0}$. This works actually showed that it is possible to determined the absolute mass scale with a few handled keV level.

Outlook of the analysis The accurate absolute mass scale is significantly important for the comparison with the theoretical calculations. In order to obtain much more accurate 
mass spectrum with the designed energy resolution, the following calibration should be done.

1. Further calibration of the magnetic field, which corresponds to the "forward" transfer matrix tuning. The accuracy SS pattern (figure 5.27 and 5.28) is still not enough, especially at the edge of the acceptance. In order to improve the SS pattern, much more complicated correction function for the magnetic field is likely to be effective.

2. Calibration of the F2T function, which corresponds to the "backward" matrix tuning. In particular, it is important to add some weights to the events in the acceptance edge. In addition, higher order $F 2 T$ function or the usage of multiple functions might be effective for this purpose.

After such calibrations, not only the mass scale, but also the accuracy of the cross section will be improved.

In addition, it become possible to approach following topics about hypernuclei.

- Study of the CSB from the $B_{\Lambda}$ measurement of ${ }_{\Lambda}^{7} \mathrm{He}$ and ${ }_{\Lambda}^{10} \mathrm{Be}$. The comparison between the ${ }_{\Lambda}^{12} \mathrm{~B}$ structure with that of ${ }_{\Lambda}^{12} \mathrm{C}$ also can provide information about the CSB.

- Study of the ${ }_{\Lambda}^{52} \mathrm{~V}$ structure for the study of $\Lambda$ single particle energies and other fine structures.

- Comparison of the excitation energies of core excited states with that of core nuclei. From this information, it is possible to investigate the "deformation" of nuclei by inputting $\Lambda$ hyperon as a impurity.

- Cross section study. In particular, our experiment can provide the kaon and scattered electron angular (that is, $Q^{2}$ of the virtual photon) dependence of the cross section at the very forward angles. It is interesting to compare such experimental data with the theoretical calculation and determine which model is preferable from the obtained result. Since there are no experimental data at the very forward angles, this will be a valuable data for the study of electromagnetic strangeness production.

The present experimental method provides us with an opportunity to measure the hypernuclear data by the reaction spectroscopy with an accurate mass scale. It is notable that it becomes possible to approach to the past emulsion hypernuclear data by the reaction spectroscopy with the absolute mass scale. These works opened the door to a new experimental technique for the precise hypernuclear spectroscopy via the $\left(\mathrm{e}, \mathrm{e}^{\prime} \mathrm{K}^{+}\right)$ reaction in the wide mass region. 


\section{Acknowledgement}

I would like to express the best appreciation to my supervisor, Prof. O. Hashimoto. It is the greatest sorrow that I could never submit this thesis to him, because he passed away when I was writing it. My study started when he gave me the opportunity to take part in the E01-011 experiment and to do the experimental design of the E05-115 experiment. When I went to US for the E01-011 experiment at the first time, he said to me, "Always think about all experimental apparatuses and understand the reason why that is necessary". This is very simple, but the most important statement for the experimentalist. After the E05-115 experiment, I discussed with him about the experimental data analysis many times, and he was always thinking how we can provide the reliable data. This is also the most significant attitude for the experimental physicists. I would like to respect what he told me and make them as a foundation of my researcher life.

I also thank sincerely to Prof. S. N. Nakamura. We stayed about one and half year in US for the experimental preparation, but the station and experiment itself was the first experience for me. In such a situation, he always gave me appropriate advises, including information a lot of delicious American foods. During experiment, he worked hardly to lead the experimental group. and also help me in general about this thesis until its completion. I would like to follow his researcher style in future.

It was happy for me to have two more supervisors about this experiment in US, prof. L. Tang and Prof. J. Reinhold, as Profs. Hashimoto and Nakamura. They are the spokesperson of the experiment and gave me many knowledges based on their experience. I also appreciate Dr. S. A. Wood, the JLab Hall-C leader. His leadership and valuable advices played significant roles for our experiment. In particular, he taught me the knowledge of the Hall-C DAQ system, which was important not only as our experimental apparatus, but also as my personal experience. For the DAQ system, I would like to give my thanks to Dr. M. Jones who worked together with me.

Prof. S. Kato help me in the spectrometer design. His knowledge about the spectrometer optics and the characteristic of the magnets were essential for the magnet design. For the detail mechanic design of the HES and SPL, it was essentially important to discuss with Mr. K. Ogata, Mr. T. Yokoi and members of NEC TOKIN Corporation.

I would like to thank the support of Dr. T. Maruta during the experiment. His effort was the most significant for the construction of the HES and SPL. In addition, he supported our life in US, not only the experimental side. In particular, his Audi was another essential apparatus for this work.

The contribution of Mr. T. Gogami to this study was also essential. He participated in our group in the preparation phase and worked hard during experiment. He was the

only person for me whomI can discuss physics and the experimental issues on even ground 
as a doctor candidate student. His friendly character was helpful for me in many times, and that will be also valuable for his researcher life. He will take over this work and I believe he will be able to complete the goal of this experiment.

Ms. A. Shichijo, Mr. N. Taniya, Mr. A. Chiba, Mr. D. Doi and Mr. S. Nagao also worked hard during the experiment. They wrote great master's theses and the large portion of this work is based on their works. Mr. M. Kawai, Mr. K. Yokota and Mr. T. Yamamoto also did good jobs about the detector design. Dr. L. Yuan, Ms. C. Chunhua and Y. Zhuhong worked with me for the experiment. Their knowledge about the experiment and data analysis was necessary for this work.

From the E01-011 experiment, Dr. Y. Okayasu and Dr. A. Matsumura told me many things. Their experiences and advices both for the experiment and life in US were very helpful for this study.

Prof. H. Tamura is another person who guided me to this hypernuclear physics world. His knowledge about hypernuclei and hypernuclear experiment was greatly valuable for this study. Dr. Y. Fujii has a lot of various experiences for the $\left(\mathrm{e}, \mathrm{e}^{\prime} \mathrm{K}^{+}\right)$reaction experiment, those were significant for me. Dr. M. Kaneta, Dr. T. Koike, Dr. M. Ukai, Dr. K. Tsukada and Dr. K. Miwa discussed hypernuclear physics, experimental apparatus and analysis tools. Ms. N. Kawamura, Ms. M. Shimaya and Ms. A. Yamanouchi greatly supported me from the point of the office duty.

The discussion with Prof. T. Motoba and Dr. E. Hiyama was important for me to understand the latest theoretical result of the hypernuclear physics. I would like to express my thanks to Prof. K. Maeda, Prof. K. Hagino and Prof. T. Sanuki for the reviewing of this thesis and giving me a lot of helpful ideas to improve this thesis.

Mr. N. Chiga was one of the most important persons for the construction of HES. He came to JLab as a technician for the experimental preparation and worked hard for the construction of the spectrometers, target systems and so on. More importantly, his cheerful nature and the passion for the sweet stuffs was necessary for our experimental group. I express my gratitude for Mr. W. Kellner, Mr. A. Kenyon and all Hall-C technicians. They worked together with us for the experimental preparation. Mr. W. Vulcan and J. Beaufait kindly arranged our electronic staffs. In the detector design phase, Mr. P. Brindza, Mr. M.Fowler and their group made great design satisfying our request. I also thank to Ms. E. Jones who supported my station in JLab from the point of the office duty, including the visa procedure. We would be not able to perform the experiment without their excellent contributions.

For the experiment, we newly designed the beamline of the Hall-C for this experiment and that was a big job. Dr. M. Spata made great effort for the controlling of the accelerator issues from the beginning of the experiment preparation. For the detail of beamline and spectrometer design, collaboration of Mr. B. Dillon-Towns and his group was very powerful. I also appreciate Dr. J. Benesch for his detail magnetic field calculation of the whole beamline.

This study could not be done without a lot of supports. I would like to thank all collaboration members including people who cannot be written here.

Finally, I would like to give my special appreciation to my proudest Family. 
Appendix 


\section{Appendix A}

\section{Mass references used in spectra calculation}

TableA.0.1 is the mass references used for the calculations. For the lepton and hadron masses, the values of the particle data group [68] were used. For the nuclear masses, the values in [81] were used.

Table A.0.1: Particle mass references. The left shows the lepton and hadron mass and the right shows the nuclear mass.

\begin{tabular}{c|c}
\hline \hline Item & Mass $\left[\mathrm{MeV} / c^{2}\right]$ \\
\hline electron & 0.511 \\
$\mathrm{~K}^{+}$ & 493.667 \\
proton & 938.272 \\
neutron & 939.565 \\
$\Lambda$ & 1115.683 \\
$\Sigma$ & 1192.642 \\
\hline \hline
\end{tabular}

\begin{tabular}{c|c}
\hline \hline Item & Mass $\left[\mathrm{MeV} / c^{2}\right]$ \\
\hline${ }^{5} \mathrm{He}$ nucl. & 4667.831 \\
${ }^{6} \mathrm{Li}$ nucl. & 5601.518 \\
\hline${ }^{6} \mathrm{He}$ nucl. & 5605.537 \\
${ }^{7} \mathrm{Li}$ nucl. & 6533.834 \\
\hline${ }^{9}$ Be nucl. & 8392.750 \\
${ }^{10} \mathrm{~B}$ nucl. & 9324.437 \\
\hline${ }^{11} \mathrm{~B}$ nucl. & 10252.548 \\
${ }^{12} \mathrm{C}$ nucl. & 11174.864 \\
\hline${ }^{27} \mathrm{Al}$ nucl. & 25126.506 \\
${ }^{28} \mathrm{Si}$ nucl. & 26053.194 \\
\hline${ }^{51} \mathrm{~V}$ nucl. & 47442.268 \\
${ }^{52} \mathrm{Cr}$ nucl. & 48370.038 \\
\hline${ }^{88} \mathrm{Sr}$ nucl. & 81864.223 \\
${ }^{89} \mathrm{Y}$ nucl. & 82795.431 \\
\hline \hline
\end{tabular}




\section{Appendix B}

\section{Experimental results and discussion for ${ }_{\Lambda}^{7} \mathrm{He}$ and ${ }_{\Lambda}^{10} \mathrm{Be}$}

In this chapter, the obtained mass spectra of ${ }_{\Lambda}^{7} \mathrm{He}$ and ${ }_{\Lambda}^{10} \mathrm{Be}$ will be shown. These hypernuclear data are important for the discussion of the CSB effect in the $\Lambda$-N interaction, but it is difficult to discuss the topic using the present data, because of the large systematic error of the mass scale. Here the obtained $\Lambda$ binding energies and cross sections will be just summarized and compared to the other experimental data.

\section{B.1 Mass spectra}

Figure B.1 and B.2 shows the spectrum of ${ }_{\Lambda}^{7} \mathrm{He}$ and ${ }_{\Lambda}^{10} \mathrm{Be}$, respectively. The accidental background spectrum obtained from mixed event analysis is also shown in the same figure as the blue region.

Additional analysis efficiencies for the spectra There are some event excess in the region of $-10<-B_{\Lambda}<6[\mathrm{MeV}]$ in figure B.1. They can be interpreted in two way, to consider them as the "leakage" from the peak around $-B_{\Lambda}=-5.5$ and $-B_{\Lambda}=-4.0$ $\mathrm{MeV}$, or the simply remained background. The number of these excess events are about $25 \%$ of these two peaks. In the present analysis, this ratio of $25 \%$ was counted in the systematic error for the cross section of these two peaks.

In a similar way, the event excess seen in around $-B_{\Lambda}=-12[\mathrm{MeV}]$ in figure B.2 is about $20 \%$ of the two peaks seen around $-B_{\Lambda}=-8.5$ and $-B_{\Lambda}=-6.5 \mathrm{MeV}$. This was also counted as systematic error of the cross section. 




Figure B.1: The missing mass spectrum for ${ }_{\Lambda}^{7} \mathrm{He}$ with counts on the vertical scale. 


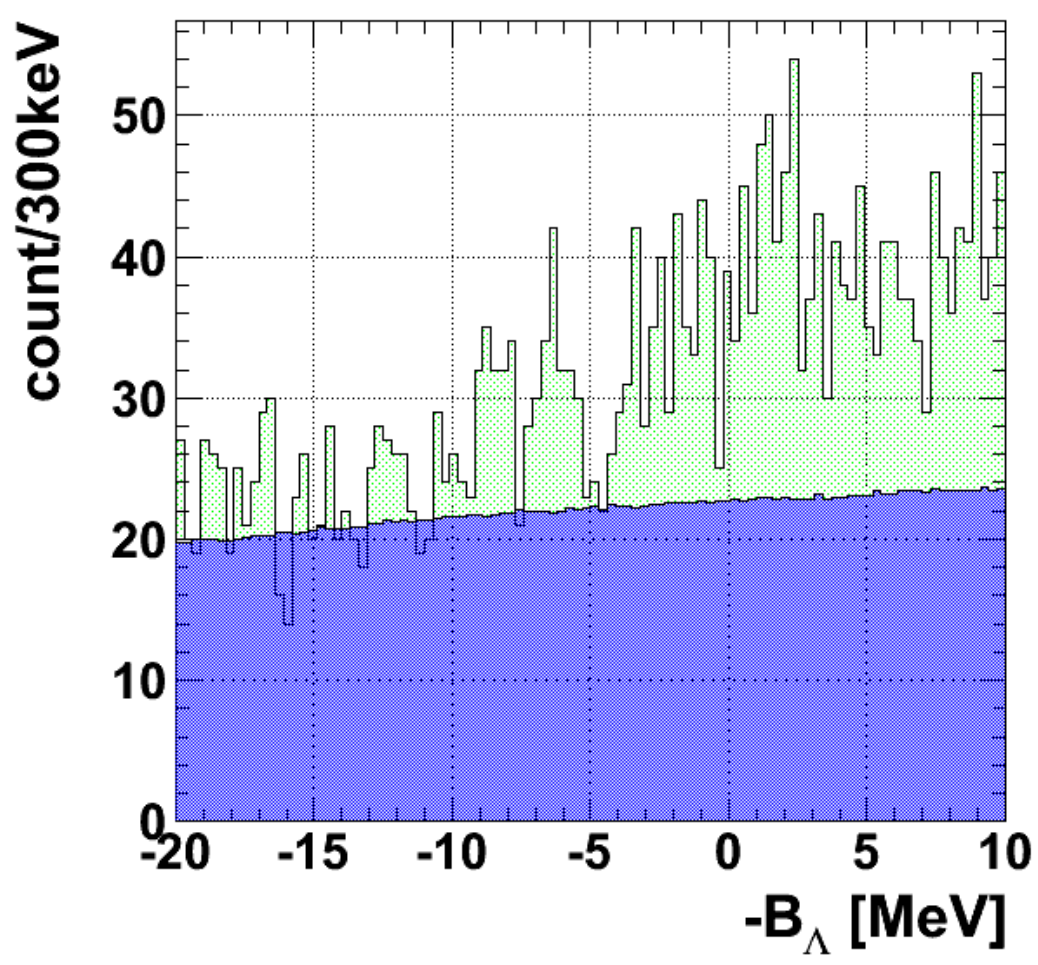

Figure B.2: The missing mass spectrum for ${ }_{\Lambda}^{10}$ Be with counts on the vertical scale. 


\section{B.2 Spectroscopy of ${ }_{\Lambda}^{7} \mathrm{He}$}

As described in section 1.4, it is important to measure the binding energy of the ${ }_{\Lambda}^{7} \mathrm{He}$ ground state for the study of the charge symmetry breaking (CSB) in $\Lambda$-N interaction. For the CSB study, very precise measurement of the mass scale was required to compare with the theoretical calculation. Though the binding energy was measured in the JLab E01-011 experiment, the measurement in the present experiment with the additional statistics and differential experimental setup is very significant.

Figure B.3 is the missing mass spectrum for ${ }_{\Lambda}^{7} \mathrm{He}$ with the vertical scale of differential cross section. The error bar represent statistic error and the accidental background estimated by mix event analysis was subtracted. In the figure, two peaks are observed around $-B_{\Lambda}=-5.5 \mathrm{MeV}$ and $-4.0 \mathrm{MeV}$. The first one can be interpreted as ${ }_{\Lambda}^{7}$ He hypernuclear states whose configuration of ${ }^{6} \mathrm{He}$ core and a $\Lambda$ hyperon in the $s$ orbit couples as $\left[{ }^{6} \mathrm{He}\left(0^{+}\right) \otimes\left(\mathrm{s}_{1 / 2}\right)_{\Lambda}\right]$. Another peak is likely to be the doublet of $\left[{ }^{6} \mathrm{He}\left(2^{+}\right) \otimes\left(\mathrm{s}_{1 / 2}\right)_{\Lambda}\right]$. The graph was fitted with two Cauchy functions in order to obtain the $\Lambda$ binding energy and cross section of each peak, with $\chi^{2} / \mathrm{ndf}=22.1 / 17$. The obtained $\Lambda$ binding energies and cross sections are summarized in table 6.2.1 and 6.2.2.

As a result, the systematic error of the cross section become very large in the present study, because of the $\chi^{2}$ cut of the HES side and $x_{f K}$ cut. These reduced events would be restored by the modification of the HES tracking and better $F 2 T$ functions. The systematic error will be small at that time, we need further study to obtain precise result.

Table B.2.1: Obtained binding energies, excitation energies and width of ${ }_{\Lambda}^{7} \mathrm{He}$.

\begin{tabular}{c|c|c|c}
\hline \hline Peak & $-B_{\Lambda}[\mathrm{MeV}]$ & $E x[\mathrm{MeV}]$ & FWHM \\
No. & $\pm($ stat. $) \pm($ sys. $)$ & \pm (stat.) $\pm($ sys. $)$ & {$[\mathrm{MeV}]$} \\
\hline$\# 1$ & $-5.90 \pm 0.1 \pm 0.2$ & 0 & 1.1 (fixed) \\
$\# 2$ & $-3.87 \pm 0.2 \pm 0.2$ & $2.03 \pm 0.2 \pm 0.3$ & 1.1 (fixed) \\
\hline \hline
\end{tabular}

Table B.2.2: Obtained differential cross sections and total cross sections of ${ }_{\Lambda}^{7} \mathrm{He}$.

\begin{tabular}{c|c|c}
\hline \hline $\begin{array}{c}\text { Peak } \\
\text { No. }\end{array}$ & $\begin{array}{c}\left.(d \sigma / d \Omega)\right|_{1^{\circ}-13^{\circ}}[\mathrm{nb} / \mathrm{sr}] \\
\pm(\text { stat. }) \pm(\text { sys. })\end{array}$ & $\begin{array}{c}\sigma_{1^{\circ}-13^{\circ}}[\mathrm{nb}] \\
\pm(\text { stat. }) \pm(\text { sys. })\end{array}$ \\
\hline$\# 1$ & $12 \pm 2 \pm 4$ & $(6.4 \pm 1.2 \pm 1.9) \times 10^{-2}$ \\
$\# 2$ & $11 \pm 2 \pm 3$ & $(5.6 \pm 1.2 \pm 1.7) \times 10^{-2}$ \\
\hline \hline
\end{tabular}

\section{Comparison with other ${ }_{\Lambda}^{7} \mathrm{He}$ data}

Two types of the experimental data is existing for the study of ${ }_{\Lambda}^{7} \mathrm{He}$. One is the emulsion data by Juric et al. in 1970s[27]. Figure B.5 shows the all ${ }_{\Lambda}^{7} \mathrm{He}$ emulsion data. However, it was impossible to determine the binding energy of ${ }_{\Lambda}^{7} \mathrm{He}$ from the data due to insufficient statistics. 


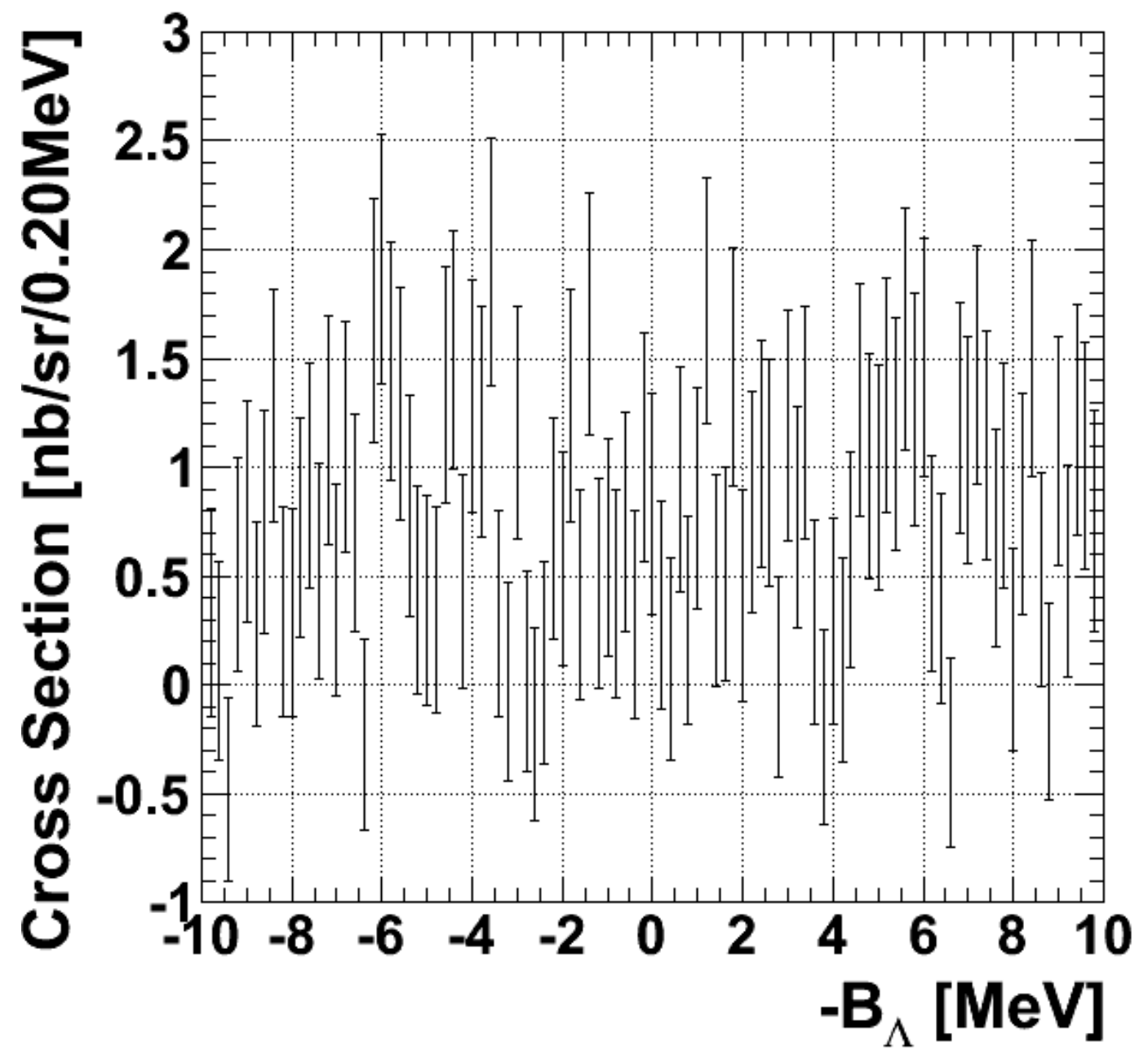

Figure B.3: The missing mass spectrum for ${ }_{\Lambda}^{7} \mathrm{He}$ with the vertical scale of differential cross section. 


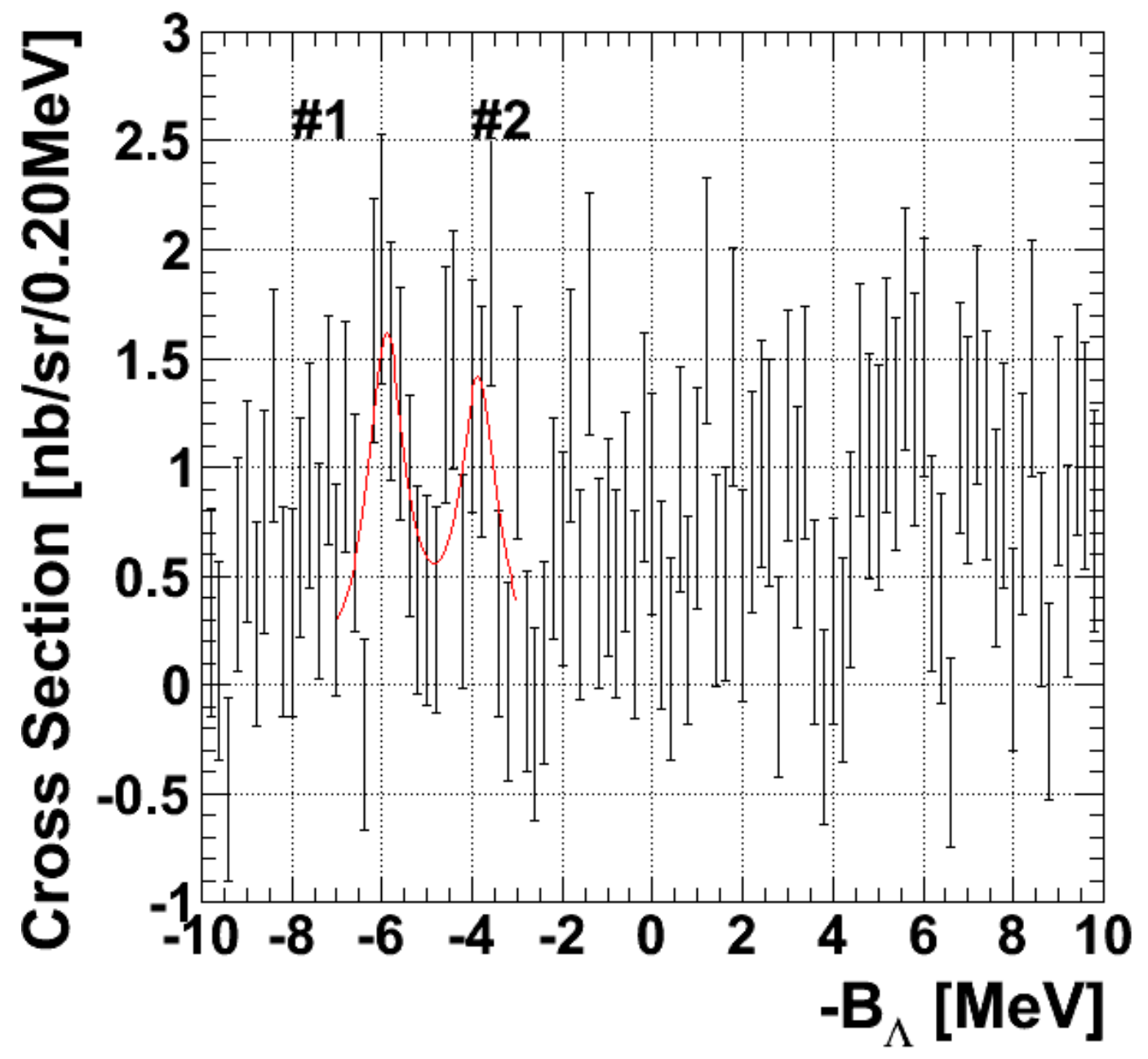

Figure B.4: The fitted missing mass spectrum for ${ }_{\Lambda}^{7} \mathrm{He}$. The vertical scale is differential cross section. 

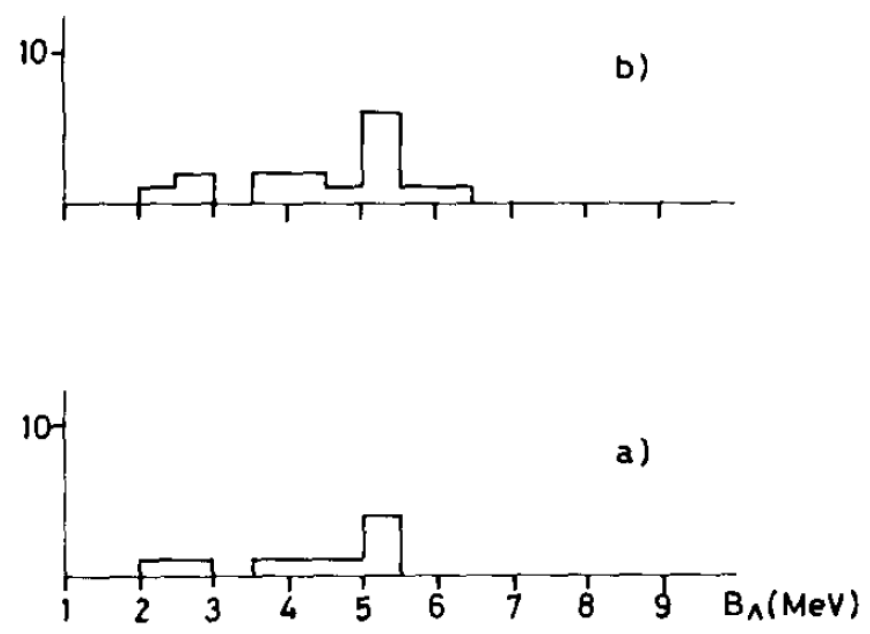

Figure B.5: Emulsion data of ${ }_{\Lambda}^{7} \mathrm{He}$ [27]. The top figure shows all the world data. Bottom figure is the experimental result in Julic's paper.

Another data was provide by the previous $\left(\mathrm{e}, \mathrm{e}^{\prime} \mathrm{K}^{+}\right)$reaction spectroscopic experiment. In the first generation experiment (E89-009), the ${ }_{\Lambda}^{7} \mathrm{He}$ data was taken, but the ground state was not measured because of the low statistics. In the E01-011 experiment, we measured clear ground state peak, as shown in figure B.6. However, only the first peak corresponds to the \#1 in figure B.4 is fitted and no comment about the second peak. As far as looking at figure B.4, there seems to be some excesses around $-B_{\Lambda}=-4 \mathrm{MeV}$ in the E01-011 data, then it is better to analyze this data again. Moreover, the cross section of the peak \#2 is almost same level as that of peak \#1. As far as looking at figure B.4, the yield of the "second peak" does not seems to be same level as that of the first peak in the E01-011 data. This is open question the difference is due to some inaccuracy in the present data, or the difference of the experimental condition. In order to answer this question, further study about the mass spectrum accuracy is essential.

The comparison with the E01-011 result is summarized in table B.2.3. The $\Lambda$ binding energy is consistent in both of them. The cross section obtained by the present data is smaller than that of the E01-011 result. This is due to the difference of HKS angular acceptance, as well as the case of ${ }_{\Lambda}^{12} \mathrm{~B}$. However, the present result has large error both in the binding energy and cross section, and we need further analysis about the present data. 


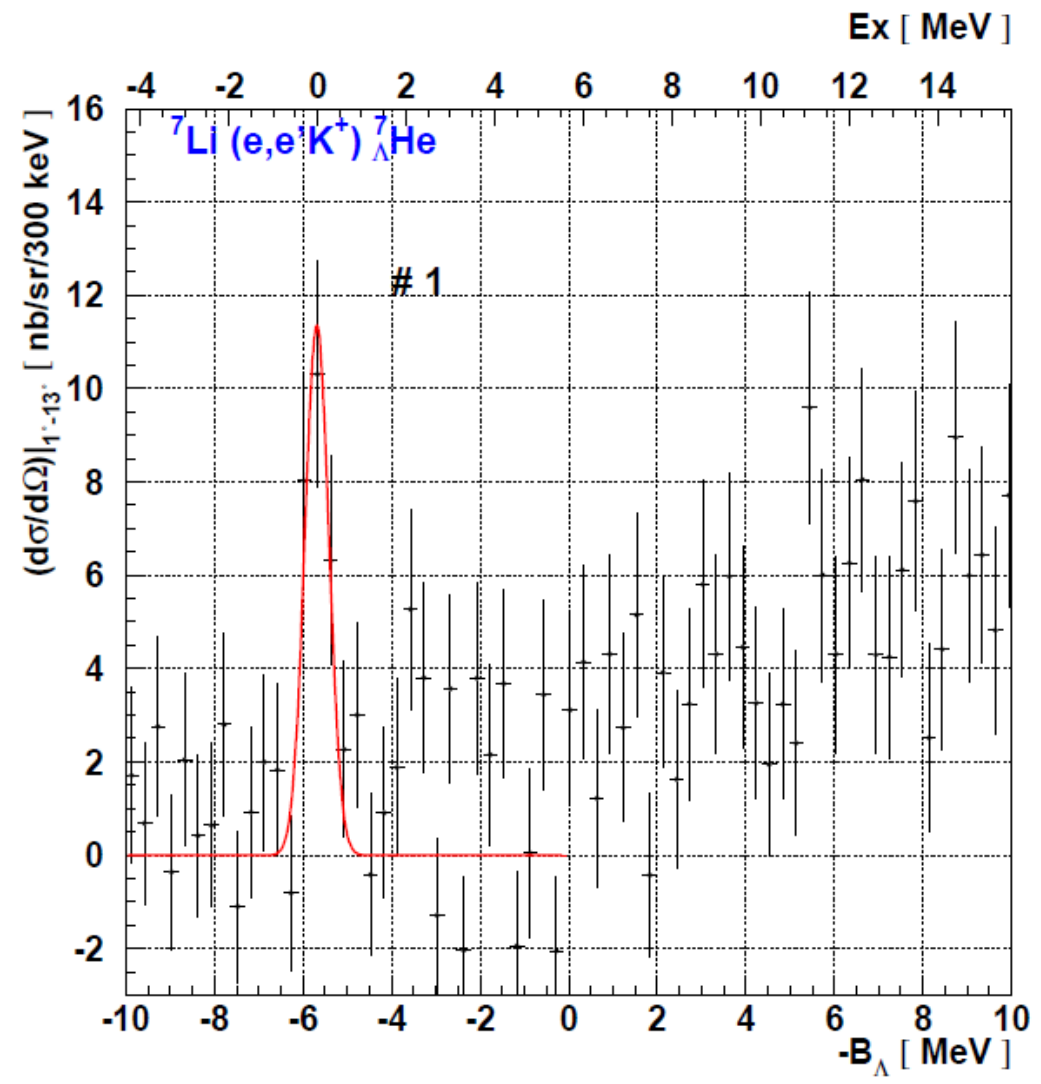

Figure B.6: The missing mass spectrum for ${ }_{\Lambda}^{7} \mathrm{He}$ obtained in JLab E01-011 experiment.

Table B.2.3: Comparison of excitation energies and width of ${ }_{\Lambda}^{7} \mathrm{He}$ for several experiments. The kinematic conditions of each experiment is summarized in table 6.2.3.

\begin{tabular}{c|c|c|c|c}
\hline \hline \multirow{2}{*}{ Experiment } & \multicolumn{2}{|c|}{$1 / 2^{+}$} & \multicolumn{2}{c}{$3 / 2^{+}, 5 / 2^{+}$doublet } \\
\cline { 2 - 5 } & $\begin{array}{c}-B_{\Lambda}[\mathrm{MeV}] \\
\pm(\text { stat.) } \pm \text { (sys.) }\end{array}$ & FWHM $[\mathrm{MeV}]$ & $\begin{array}{c}E x\left(-B_{\Lambda}\right)[\mathrm{MeV}] \\
\pm(\text { stat.) } \pm(\text { sys.) }\end{array}$ & FWHM [MeV] \\
\hline E05-115 & $-5.90 \pm 0.1 \pm 0.2$ & 1.00 (fixed) & $-3.87 \pm 0.2 \pm 0.2$ & 1.1 (fixed) \\
\hline E01-011 & $-5.68 \pm 0.03 \pm 0.22$ & 0.63 & $\mathrm{~N} / \mathrm{A}$ & $\mathrm{N} / \mathrm{A}$ \\
\hline \hline
\end{tabular}




\section{B.3 Spectroscopy of ${ }_{\Lambda}^{10} \mathrm{Be}$}

The spectroscopy of ${ }_{\Lambda}^{10} \mathrm{Be}$ is also important for the CSB study, as well as the ${ }_{\Lambda}^{7} \mathrm{He}$ spectrum. This is the first observation of ${ }_{\Lambda}^{10} \mathrm{Be}$ by the reaction spectroscopy. There is only one experimental data of one event via the emulsion experiment which shows the binding energy as $9.30 \pm 0.26[27]$.

Figure B.7 is the missing mass spectrum for ${ }_{\Lambda}^{10} \mathrm{Be}$ with the vertical scale of differential cross section. The error bar represent statistic error and the accidental background estimated by mix event analysis was subtracted. In the figure, two peaks are observed around $-B_{\Lambda}=-8.5 \mathrm{MeV}$ and $-6.5 \mathrm{MeV}$. These peaks were fitted with two Cauchy functions in order to obtain the $\Lambda$ binding energy and cross section of each peak. The width of the Cauchy functions are fixed as $1.1 \mathrm{MeV}$ (FWHM) from the fitting result of ${ }_{\Lambda}^{12} \mathrm{~B}$ spectrum. The spectrum was fitted with $\chi^{2} / \mathrm{ndf}=4.8 / 13$, and the obtained $\Lambda$ binding energies and cross sections are summarized in table B.3.1 and B.3.2. The interpretation of these peaks will be done in the following part.

Table B.3.1: Obtained binding energies, excitation energies and width of ${ }_{\Lambda}^{10} \mathrm{Be}$.

\begin{tabular}{c|c|c|c}
\hline \hline Peak & $-B_{\Lambda}[\mathrm{MeV}]$ & $E x[\mathrm{MeV}]$ & FWHM \\
No. & $\pm($ stat.) $\pm($ sys. $)$ & $\pm($ stat. $) \pm($ sys. $)$ & {$[\mathrm{MeV}]$} \\
\hline$\# 1$ & $-8.72 \pm 0.2 \pm 0.2$ & 0 & 1.1 (fixed) \\
$\# 2$ & $-6.49 \pm 0.2 \pm 0.2$ & $2.23 \pm 0.3 \pm 0.3$ & 1.1 (fixed) \\
\hline \hline
\end{tabular}

Table B.3.2: Obtained differential cross sections and total cross sections of ${ }_{\Lambda}^{10} \mathrm{Be}$.

\begin{tabular}{c|c|c}
\hline \hline $\begin{array}{c}\text { Peak } \\
\text { No. }\end{array}$ & $\begin{array}{c}\left.(d \sigma / d \omega)\right|_{1^{\circ}-13^{\circ}}[\mathrm{nb} / \mathrm{sr}] \\
\pm(\text { stat. }) \pm(\text { sys. })\end{array}$ & $\begin{array}{c}\sigma_{1^{\circ}-13^{\circ}}[\mathrm{nb}] \\
\pm(\text { stat. }) \pm(\text { sys. })\end{array}$ \\
\hline$\# 1$ & $11 \pm 3 \pm 3$ & $(5.7 \pm 1.6 \pm 1.3) \times 10^{-2}$ \\
$\# 2$ & $15 \pm 3 \pm 4$ & $(7.6 \pm 1.7 \pm 1.8) \times 10^{-2}$ \\
\hline \hline
\end{tabular}

\section{B.3.1 Comparison with mirror symmetric ${ }_{\Lambda}^{10} \mathrm{~B}$ data and theoret- ical calculation}

Though there are no experimental data of ${ }_{\Lambda}^{10} \mathrm{Be}$ via the reaction spectroscopy, the mirror symmetric ${ }_{\Lambda}^{10} \mathrm{~B}$ data via the $\left(\pi^{+}, \mathrm{K}^{+}\right)$reaction is existing $[20,23]$. Figure B.9 is the spectrum and the fitting results are summarized in table B.3.3. These peaks can be interpreted as having configurations of a $\Lambda$ hyperon in the $s$ orbit coupled to the ground and excited states of ${ }^{9} \mathrm{~B}$ core nucleus.

The interpretation of ${ }_{\Lambda}^{10} \mathrm{Be}$ can be done in the similar way. In table B.10, the experimental results and theoretical calculation of ${ }_{\Lambda}^{10} \mathrm{Be}$ and ${ }_{\Lambda}^{10} \mathrm{~B}$ are summarized with the excited states of their core nuclei. The theoretical calculations of ${ }_{\Lambda}^{10} \mathrm{Be}$ have been performed by Hiyama [82] and Millener [83]. From this figure, the fitted peak can be interpreted as ${ }_{\Lambda}^{10} \mathrm{Be}$ 


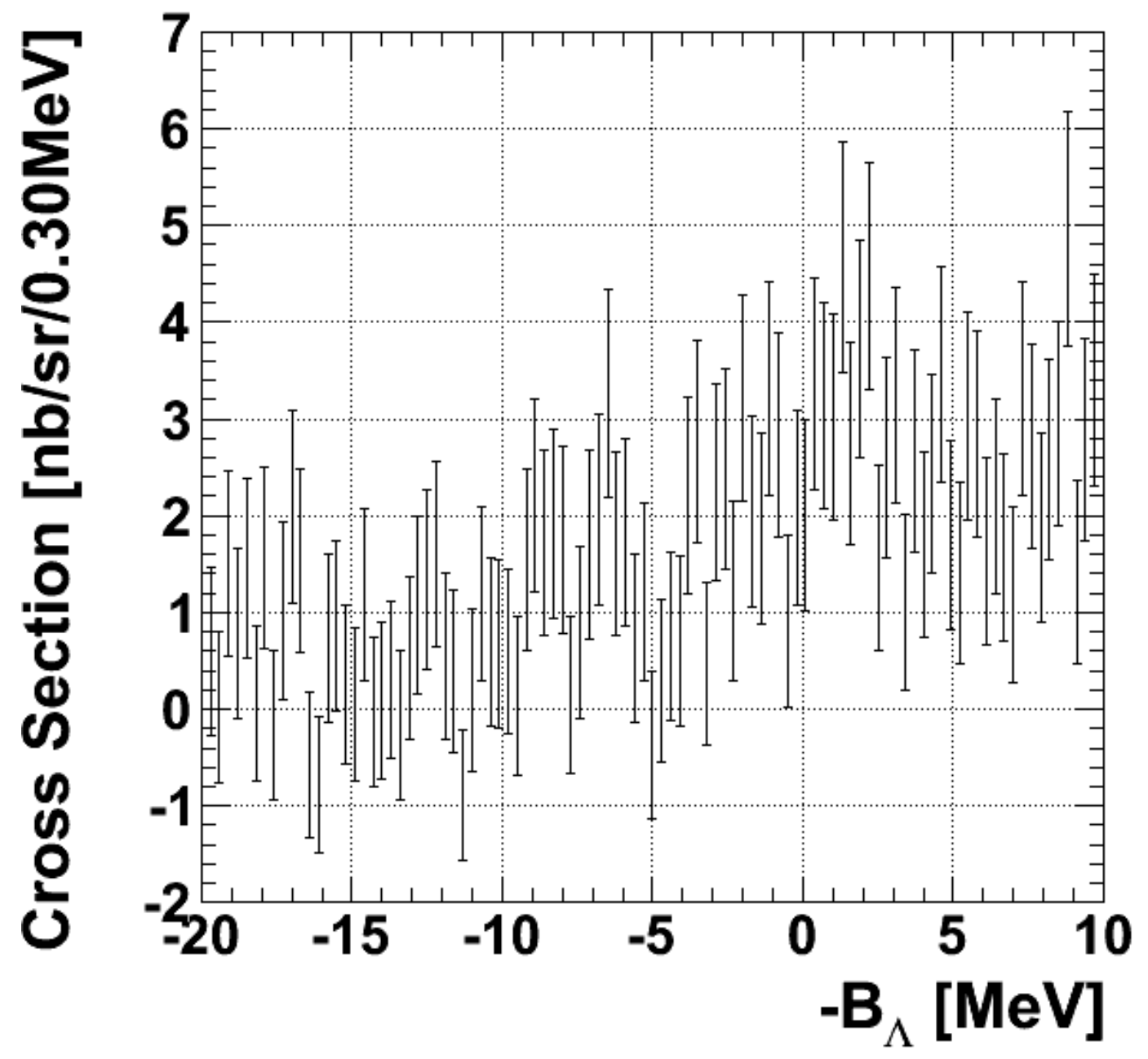

Figure B.7: The missing mass spectrum for ${ }_{\Lambda}^{10} \mathrm{Be}$ with the vertical scale of differential cross section. 


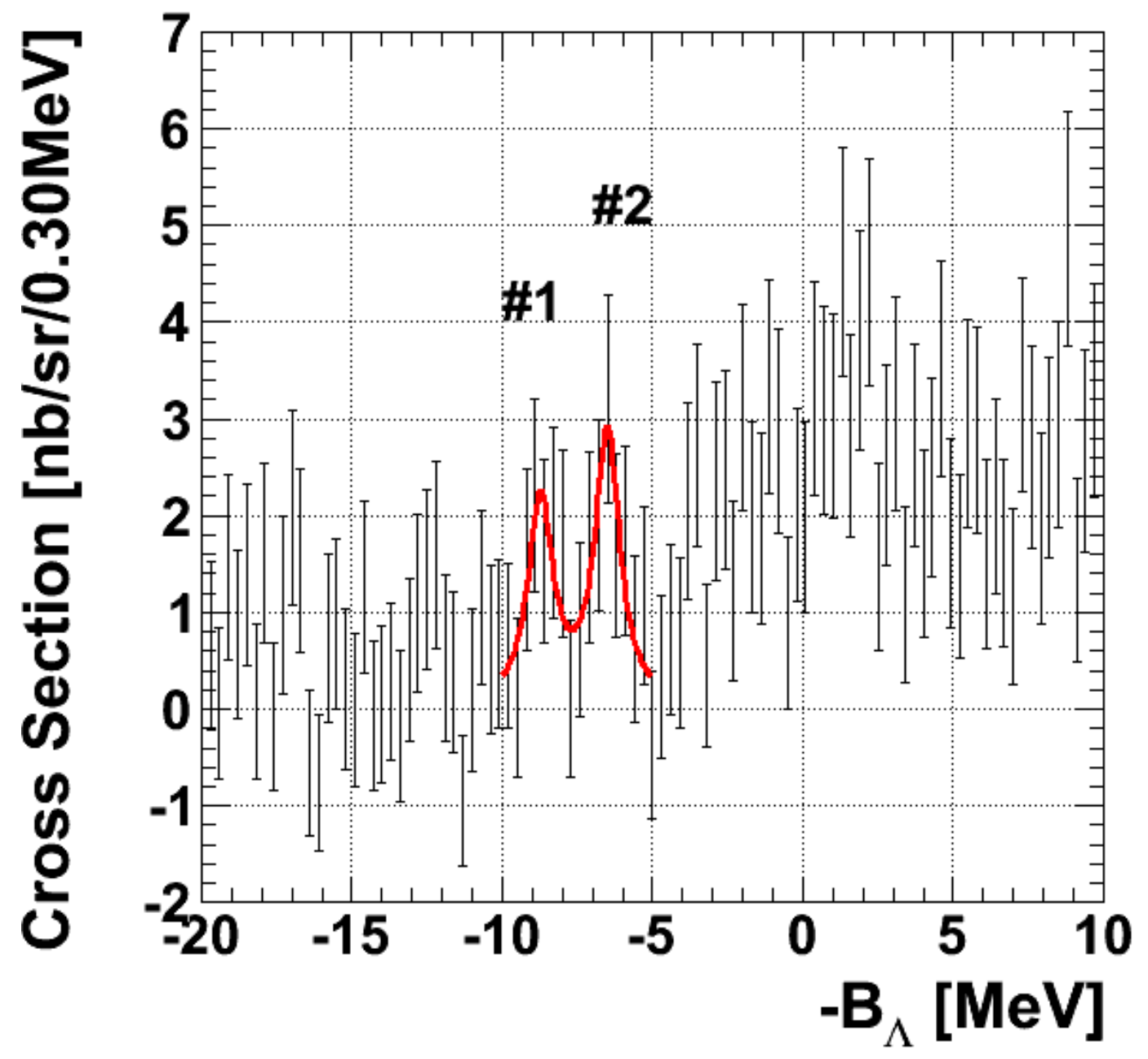

Figure B.8: The fitted missing mass spectrum of ${ }_{\Lambda}^{10} \mathrm{Be}$. The vertical scale is differential cross section. 
states of a $\Lambda$ hyperon in the $s$ orbit coupled to the ground state $\left(3 / 2^{-}\right)$and the first excited states $\left(5 / 2^{-}\right)$of ${ }^{9} \mathrm{Be}$ core nucleus. According to the calculation, there should be other two peaks corresponding to the two $7 / 2^{-}$states of the core nucleus around $-B_{\Lambda}=-2$ $\mathrm{MeV}$ and $-B_{\Lambda}=2 \mathrm{MeV}$. Actually there seems to be some excesses in the corresponding position of figure B.8, but they are not taken part in the fitting. Further calibration will make it possible to include these excesses in the fitting.

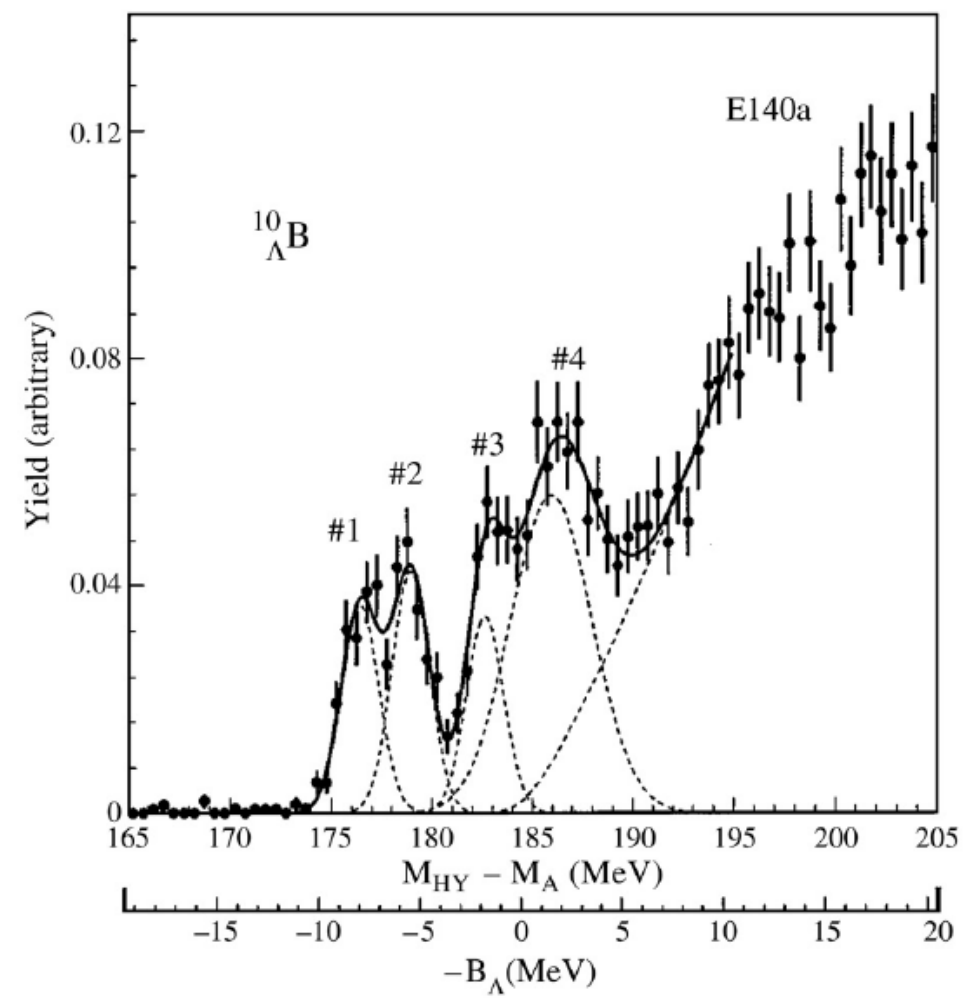

Figure B.9: The missing mass spectrum of ${ }_{\Lambda}^{10} \mathrm{~B}$ obtained by $\left(\pi^{+}, \mathrm{K}^{+}\right)$reaction $[20,23]$.

Table B.3.3: Obtained excitation energies and relative yields of ${ }_{\Lambda}^{10} \mathrm{~B}$.

\begin{tabular}{c|c|c|c}
\hline \hline $\begin{array}{c}\text { Peak } \\
\text { No. }\end{array}$ & $\begin{array}{c}E x \\
{[\mathrm{MeV}]}\end{array}$ & $\begin{array}{c}\text { FWHM } \\
{[\mathrm{MeV}]}\end{array}$ & $\begin{array}{c}\text { Relative yield } \\
\text { (arbitrary unit) }\end{array}$ \\
\hline$\# 1$ & $0\left(B_{\Lambda}=8.1 \pm 0.1\right)$ & 2.2 (fixed) & $0.17 \pm 0.02$ \\
$\# 2$ & $2.5 \pm 0.2$ & 2.2 (fined) & $0.20 \pm 0.02$ \\
$\# 3$ & $6.2 \pm 0.3$ & 2.2 (fixed) & $0.16 \pm 0.05$ \\
$\# 4$ & $9.5 \pm 0.3$ & $4.8 \pm 0.8$ & $0.57 \pm 0.14$ \\
\hline \hline
\end{tabular}




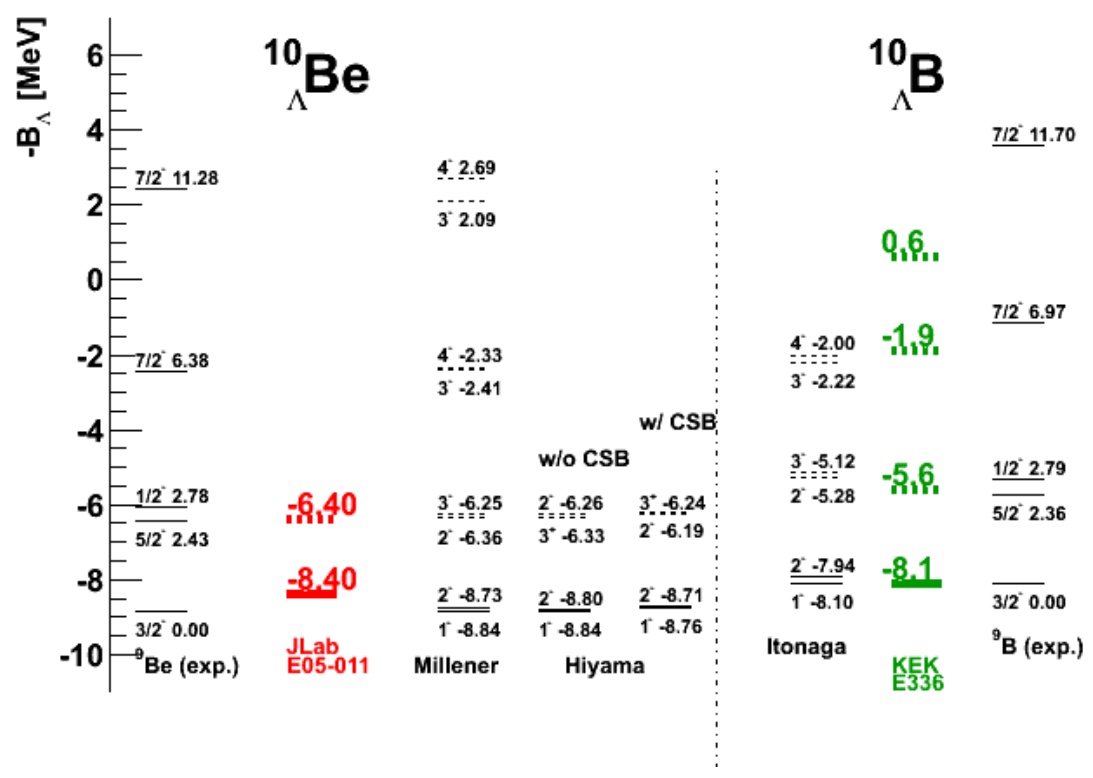

Figure B.10: Level scheme of ${ }_{\Lambda}^{10} \mathrm{Be},{ }_{\Lambda}^{10} \mathrm{~B}$ and their core nuclei. 


\section{Appendix C}

\section{Parameters for the mass scale calibration}

The mass scale calibration explained in section 5.5.1 is an unique procedure in our data analysis. This procedure can affect the missing mass scale by $100 \mathrm{keV}$ order, then it needs very delicate parameter tuning. In particular, the kinematic parameter such as the central momenta and angles is essential parameter which determine the "offset" of the mass scale. In addition, the background of the $\mathrm{CH}_{2}$ spectrum affect the mass scale. In this chapter, such parameters will be summarize for the further calibration study.

\section{C.1 Kinematic parameters}

The basic parameters to be determined by the calibration process is summarized as table C.1.1 with their determined value. See section 5.5.1 for the meaning of the notation. Note that these central values are corresponding to the center of the FP value, not the mean of the distribution. The FP center was determined as 0 for every value, except for the HES $y^{\prime}$ whose center was determined as $-0.03[\mathrm{mrad}]$.

Table C.1.1: The number of events with and without applying the $\chi^{2}$ cut in three regions.

\begin{tabular}{c|c|c|c|c|c|c}
\hline \hline & $\begin{array}{c}p_{K} \\
{[\mathrm{GeV} / c]}\end{array}$ & $\begin{array}{c}x_{K}^{\prime} \\
{[\mathrm{mrad}]}\end{array}$ & $\begin{array}{c}y_{K}^{\prime} \\
{[\mathrm{mrad}]}\end{array}$ & $\begin{array}{c}p_{e^{\prime}} \\
{[\mathrm{GeV} / c]}\end{array}$ & $\begin{array}{c}x_{e^{\prime}}^{\prime} \\
{[\mathrm{mrad}]}\end{array}$ & $\begin{array}{c}y_{e^{\prime}}^{\prime} \\
{[\mathrm{mrad}]}\end{array}$ \\
\hline \hline & 1.18495 & -101.6 & 8.5 & 0.84125 & 4.5 & 68.1 \\
\hline \hline
\end{tabular}

\section{C.2 Offset of the missing mass}

As shown in figure 6.2, the $\mathrm{CH}_{2}$ spectrum has asymmetric shape background events under the $\Lambda / \Sigma^{0}$ peaks. Since the $\Lambda / \Sigma^{0}$ peaks were used for the mass scale calibration with these background events, the shape affects the calibration procedure. Figure C.1 shows the schematic of the $\Lambda$ peak on the background events. One can see the mean of the $\Lambda$ events only is different from the mean of all events containing the background. The calibration 
$\chi^{2}$ for $\Lambda$ events were defined as equation 5.33, the $m_{\Lambda}$ in the equation needs to be modified considering this effect.

In the present study, the $m_{\Lambda}$ is the value of the PDG value, but there is still about $50 \mathrm{keV}$ offset, as seen in table 6.1.1. This $50 \mathrm{keV}$ effect is probably derived from this background effect. It is possible to improve the mass scale by introducing the new $m_{\Lambda}$ value so as to cancel this $50 \mathrm{keV}$ offset.

The improvement of these parameters will improve the mass scale accuracy.

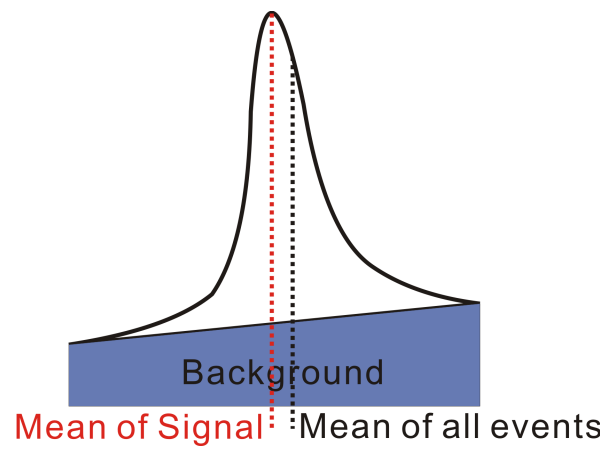

Figure C.1: Background effect on the mass scale calibration. 


\section{Reference}

[1] B. Povh, Progress in Particle and Nuclear Physics 18, 183 (1987).

[2] S. Cohen, D. Kurath, Nuclear Physics 73, 1 (1965).

[3] S. Cohen, D. Kurath, Nuclear Physics A 101, 1 (1967).

[4] P. M. M. Maessen, T. A. Rijken, J. J. de Swart, Phys. Rev. C 40, 2226(Nov 1989).

[5] T. A. Rijken, V. G. J. Stoks, Y. Yamamoto, Phys. Rev. C 59, 21(Jan 1999).

[6] B. Holzenkamp, K. Holinde, J. Speth, Nuclear Physics A 500, 485 (1989).

[7] T. Rijken, P. Maessen, J. de Swart, Nuclear Physics A 547, 245 (1992).

[8] R. H. Dalitz, A. Gal, Annals of Physics 116, 167 (1978).

[9] D. J. Millener et al., Phys. Rev. C 31, 499(Feb 1985).

[10] M. Danysza, J. Pniewskia, Phylisophical Magazine 44, p. 348 (1953).

[11] A. Bamberger et al., Physics Letters B 36, 412 (1971).

[12] M. Faessler et al., Physics Letters B 46, 468 (1973), cited By (since 1996) 8.

[13] R. Bertini et al., Nuclear Physics A 360, 315 (1981).

[14] R. Bertini et al., Nuclear Physics A 368, 365 (1981).

[15] W. Brükner et al., Physics Letters B 79, 157 (1978).

[16] P. H. Pile et al., Phys. Rev. Lett. 66, 2585(May 1991).

[17] C. Milner et al., Phys. Rev. Lett. 54, 1237(Mar 1985).

[18] M. Akel et al., Nuclear Physics A 534, 478 (1991).

[19] T. Hasegawa et al., Phys. Rev. Lett. 74, 224(Jan 1995).

[20] T. Hasegawa et al., Phys. Rev. C 53, 1210(Mar 1996).

[21] S. Ajimura et al., Nuclear Physics A 639, 93c (1998), Proceedings of the International Conference on Hypernuclear and Strange Particle Physics. 
[22] H. Hotchi et al., Phys. Rev. C 64, p. 044302(Sep 2001).

[23] O. Hashimoto, H. Tamura, Progress in Particle and Nuclear Physics 57, 564 (2006).

[24] H. Tamura et al., Nuclear Physics A 479, 161 (1988).

[25] E. Hiyama et al., Physical Review C 74, p. 54312 (2006).

[26] E. Hiyama et al., Phys. Rev. C 80, p. 054321(Nov 2009).

[27] M. Juric et al., Nuclear Physics B 52, 1 (1973).

[28] M. Bedjidian et al., Physics Letters B 62, 467 (1976).

[29] M. Bedjidian et al., Physics Letters B 83, 252 (1979).

[30] E. Hiyama et al., Phys. Rev. C 65, p. 011301(Dec 2001).

[31] P. Bydzovsky et al., ArXiv e-prints (June 2007).

[32] D.H., Davis, Nuclear Physics A 547, 369 (1992).

[33] M. Akihiko, Spectroscopy of ${ }_{\Lambda}^{28} \mathrm{al},{ }_{\Lambda}^{12} \mathrm{~b}$ and ${ }_{\Lambda}^{7}$ he by the $\left(\mathrm{e}, \mathrm{e}^{\prime} \mathrm{K}^{+}\right)$reaction, $\mathrm{PhD}$ thesis, Tohoku University2010.

[34] D. J. Millener, C. B. Dover, A. Gal, Phys. Rev. C 38, 2700(Dec 1988).

[35] Y. Yamamoto, H. Bandō, J. Žofka, Progress of Theoretical Physics 80, 757 (1988).

[36] D. E. Lanskoy, Y. Yamamoto, Phys. Rev. C 55, 2330(May 1997).

[37] K. Tsushima et al., Nuclear Physics A 630, 691 (1998).

[38] C. M. Keil, F. Hofmann, H. Lenske, Phys. Rev. C 61, p. 064309(May 2000).

[39] H. Lenske et al., Hypernuclear density functional theory, in Proceedings of The IX International Conference on Hypernuclear and Strange Particle Physics, 2007.

[40] S. Nozawa, T. S. H. Lee, Nuclear Physics A 513, 511 (1990).

[41] J. Adam et al., Czechoslovak Journal of Physics 42, 1167 (1992), 10.1007/BF01591400.

[42] T. Motoba, M. Sotona, K. Itonaga, Progress of Theoretical Physics Supplement 117, 123 (1994).

[43] R. Bradford et al., Physical Review C 73, p. 035202 (2006).

[44] Y. Tsai, Reviews of Modern Physics 46, 815 (1974).

[45] C. Itzykson, J. Zuber, Quantum Field Theory (Dover, 1980).

[46] T. Miyoshi et al., Physical Review Letters 90, p. 232502 (2003). 
[47] L. Yuan et al., Physical Review C 73, p. 44607 (2006).

[48] P. Achenbach et al., Status of strangeness electro-production at mami, in Strangeness in Nuclear and Hadronic Systems: Sendai08-Proceedingsof the Sendai International Symposium, 2009.

[49] C. Yan et al., Nuclear Instruments and Methods in Physics Research Section A: Accelerators, Spectrometers, Detectors and Associated Equipment 365, 261 (1995).

[50] K.B.Unser, CERN Report SL/91-42 (1991).

[51] P. Chevtsov et al., Synchrotron light interferometer at Jefferson Lab, in Particle Accelerator Conference, 2003. PAC 2003. Proceedings of the, 2004.

[52] R. Dickson, V. Lebedev, Fast digital feedback system for energy and beam position stabilization, in Particle Accelerator Conference, 1999. Proceedings of the 1999, 1999.

[53] R. Wojcik, C. Yan, Nuclear Instruments and Methods in Physics Research Section A: Accelerators, Spectrometers, Detectors and Associated Equipment 484, 690 (2002).

[54] J. J.W.Lightbody, J. Connell, Computers in Physics, 57 (May/June 1988).

[55] Geant4 Webpage. http://geant4.web.cern.ch/geant4/.

[56] H. Hirayama et al., The EGS5 code system (United States. Dept. of Energy, 2005).

[57] D.Uchida, $\lambda$ hypernuclear spectroscopy via high intensity electron beam -the study of high resolution kaon spectrometer based on the requirement of target temparature-, Master's thesis, Tohoku University (2005).

[58] I. Sugai et al., Nuclear Instruments and Methods in Physics Research Section A: Accelerators, Spectrometers, Detectors and Associated Equipment 590, 83 (2008), Toward the Realization of Target and Stripper Foil Technologies for High-Power Proton and Radioactive Ion Accelerators, Proceedings of the 23rd World Conference of the International Nuclear Target Development Society.

[59] A.Shichijo, Development of the target system and analysis of the $\mathrm{p}\left(\mathrm{e}, \mathrm{e}^{\prime} \mathrm{K}^{+}\right) \Lambda, \Sigma$ reactions in jlab e05-115 experiment, Master's thesis, Tohoku University (2009).

[60] Nanometric Systems Webpage. http://www.nanometricsystems.com/.

[61] Hamamatsu, Photomultiplier tubes and assemblies.

[62] A.Chiba, Development and evaluation of cherenkov detectors for hypernuclear spectroscopy experiments via the $\left(\mathrm{e}, \mathrm{e}^{\prime} \mathrm{K}^{+}\right)$reaction, Master's thesis, Tohoku University (2011).

[63] M. Kawai, Development of kaon detectors for the next generation lambda hypernuclear spectroscopic experiment via $\left(\mathrm{e}, \mathrm{e}^{\prime} \mathrm{k}^{+}\right)$reaction, Master's thesis, Tohoku University (2008). 
[64] T. Yamamoto, Development of kaon detectors for the next generation lambda hypernuclear spectroscopic experiment via the $\left(\mathrm{e}, \mathrm{e}^{\prime} \mathrm{K}^{+}\right)$reaction, Master's thesis, Tohoku University (2009).

[65] M. Nomachi, S. Ajimura, Serial data link on advanced tca back plane Real Time Conference 2005. 14th IEEE-NPSS June 2005.

[66] L. R. Dalesio et al., Nuclear Instruments and Methods in Physics Research Section A: Accelerators, Spectrometers, Detectors and Associated Equipment 352, 179 (1994).

[67] ROOT Webpage. http://root.cern.ch/drupal/.

[68] C. Amsler et al., Physics Letters B 667, 1 (2008), Review of Particle Physics.

[69] T. Bowen et al., Phys. Rev. D 7, 22(Jan 1973).

[70] D. V. Bugg et al., Phys. Rev. 168, 1466(Apr 1968).

[71] R. W. Bland et al., Nuclear Physics B 13, 595 (1969).

[72] G. Giacomelli et al., Nuclear Physics B 20, 301 (1970).

[73] K. Abe et al., Phys. Rev. D 11, 1719(Apr 1975).

[74] K.-H. Glander et al., The European Physical Journal A - Hadrons and Nuclei 19, 251 (2004), 10.1140/epja/i2003-10119-x.

[75] J. J. LeRose et al., Nuclear Physics A 804, 116 (2008), Special Issue on Recent Advances in Strangeness Nuclear Physics.

[76] D.J., Millener, Nuclear Physics A 691, 93 (2001), jce:title¿Proc. 7th Int. Conf. on Hypernuclear and Strange Particle Physicsi/ce:titlę.

[77] T. Motoba et al., Progress of Theoretical Physics Supplement 185, 224 (2010).

[78] M. Sotona, Private communications.

[79] T. Mizutani et al., Phys. Rev. C 58, 75(Jul 1998).

[80] T. Mart, C. Bennhold, Phys. Rev. C 61, p. 012201(Nov 1999).

[81] G. Audi, A. Wapstra, C. Thibault, Nuclear Physics A 729, 337 (2003), ¡ce:title $T h e$ 2003 NUBASE and Atomic Mass Evaluationsi/ce:titlę.

[82] E. Hiyama, Private communications.

[83] D.J., Millener, Private communications. 